\title{
Bacterial NanoCellulose
} A Sophisticated Multifunctional Material

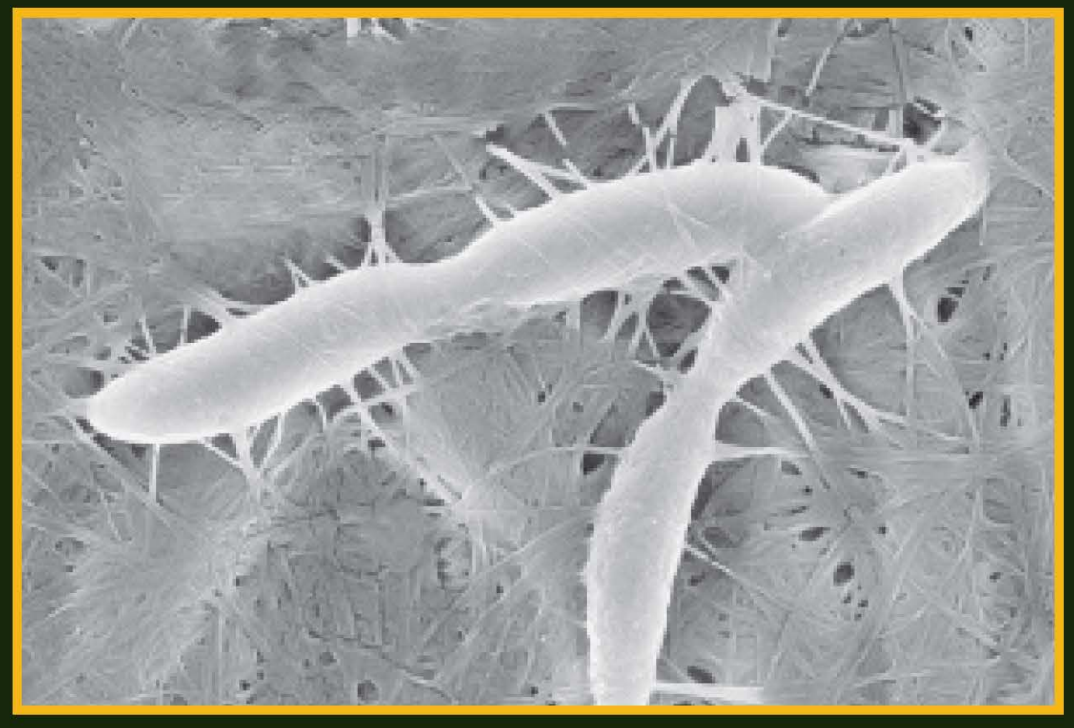

Edited by

\author{
Miguel Gama \\ Paul Gatenholm \\ Dieter Klemm
}




\section{Bacterial NanoCellulose}

A Sophisticated Multifunctional Material 
PERSPECTIVES IN NANOTECHNOLOGY

\section{Series Editor}

Gabor L. Hornyak

Bacterial NanoCellulose: A Sophisticated Multifunctional Material

Miguel Gama, Paul Gatenholm, and Dieter Klemm

Medical Nanotechnology and Nanomedicine

Harry F. Tibbals

Nanotechnology: The Business

Michael T. Burke

Nanotechnology: Ethics and Society

Deb Bennett-Woods

Nanotechnology: Health and Environmental Risks, Second Edition

Jo Anne Shatkin

Nanotechnology: Legal Aspects

Patrick M. Boucher

Nanotechnology: The Future

Thomas J. Frey

Nanotechnology and Global Sustainability

Donald Maclurcan

Nanotechnology and the Public: Risk Perception and Risk Communication

Susanna Hornig Priest

Nanotechnology Intellectual Property Rights: Research, Design, and Commercialization

Prabuddha Ganguli and Siddharth Jabade 


\title{
Bacterial NanoCellulose
} A Sophisticated Multifunctional Material

\author{
Edited by \\ Miguel Gama \\ Paul Gatenholm \\ Dieter Klemm
}

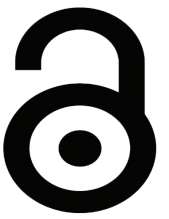

(CRC

CRC Press 
The SEM image on the cover of the bacteria Gluconacetobacter xylinus producing nanocellulose is courtesy of M. Sano, R. Davalos, and P. Gatenholm, Virginia Tech, USA.

\section{CRC Press}

Taylor \& Francis Group

6000 Broken Sound Parkway NW, Suite 300

Boca Raton, FL 33487-2742

() 2013 by Taylor \& Francis Group, LLC

CRC Press is an imprint of Taylor \& Francis Group, an Informa business

No claim to original U.S. Government works

Printed in the United States of America on acid-free paper

Version Date: 20121003

International Standard Book Number: 978-1-4398-6991-8 (Hardback)

This book contains information obtained from authentic and highly regarded sources. Reasonable efforts have been made to publish reliable data and information, but the author and publisher cannot assume responsibility for the validity of all materials or the consequences of their use. The authors and publishers have attempted to trace the copyright holders of all material reproduced in this publication and apologize to copyright holders if permission to publish in this form has not been obtained. If any copyright material has not been acknowledged please write and let us know so we may rectify in any future reprint.

The Open Access version of this book, available at www.taylorfrancis.com, has been made available under a Creative Commons Attribution-Non Commercial-No Derivatives 4.0 license.

Trademark Notice: Product or corporate names may be trademarks or registered trademarks, and are used only for identification and explanation without intent to infringe.

\section{Library of Congress Cataloging-in-Publication Data}

Bacterial nanocellulose : a sophisticated multifunctional material / editors: Miguel Gama, Paul Gatenholm, Dieter Klemm.

p.; cm. -- (Perspectives in nanotechnology)

Includes bibliographical references and index.

ISBN 978-1-4398-6991-8 (hardback : alk. paper)

I. Gama, Miguel. II. Gatenholm, Paul. III. Klemm, D. (Dieter) IV. Series: Perspectives in nanotechnology.

[DNLM: 1. Bacteria--metabolism. 2. Cellulose--chemistry. 3. Drug

Discovery--economics. 4. Nanostructures--chemistry. QU 83]

\section{Visit the Taylor \& Francis Web site at http://www.taylorandfrancis.com}

and the CRC Press Web site at

http://www.crcpress.com 
This book is dedicated to R. Malcolm Brown, Jr.,

for his contributions to our current knowledge on the biosynthesis, production, and applications of bacterial nanocellulose. 



\section{Contents}

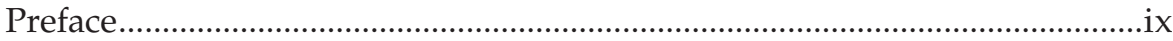

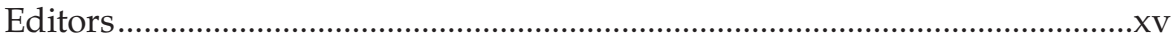

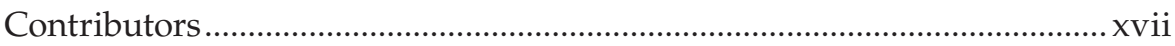

1 Biosynthesis of Bacterial Cellulose .......................................................... 1

Inder M. Saxena and R. Malcolm Brown, Jr.

2 Effect of Cultivation Conditions on the Structure and Morphological Properties of BNC Biomaterials with a Focus on Vascular Grafts

Paul Gatenholm, Kim Höglund, Sara Johannesson, Maja Puchades, Christian Brackmann, Annika Enejder, and Lisbeth Olsson

3 Large-Scale Production of BNC: State and Challenges 43

Dana Kralisch and Nadine Hessler

4 Tough Bacterial Nanocellulose Hydrogels Based on the Double-Network Technique.

Anamul Haque, Takayuki Kurokawa, and Jian Ping Gong

5 Bacterial Cellulose Surface Modifications

João P. Silva, Fábia K. Andrade, and Francisco Miguel Gama

6 Nematic Ordered Cellulose Templates

Tetsuo Kondo

7 Applications and Products-Nata de Coco

Muenduen Phisalaphong and Nadda Chiaoprakobkij

8 Wound Dressings and Cosmetic Materials from

Bacterial Nanocellulose

Stanislaw Bielecki, Halina Kalinowska, Alina Krystynowicz, Katarzyna

Kubiak, Marek Kołodziejczyk, and Manu de Groeve

9 Bacterial Nanocellulose Hydrogels Designed as

Bioartificial Medical Implants.

Dieter Klemm, Hannes Ahrem, Friederike Kramer, Wolfgang Fried, Jens Wippermann, and Raimund W. Kinne 
10 Bacterial Nanocellulose Biomaterials with Controlled Architecture for Tissue Engineering Scaffolds and Customizable Implants

Paul Gatenholm, Joel Berry, Andrea Rojas, Michael B. Sano, Rafael V. Davalos, Kara Johnson, and Laurie O’Rourke

11 Biomimetic Mineralization of Apatite on Bacterial Cellulose. Thi Thi Nge and Junji Sugiyama

12 Bacterial Nanocellulose as a Structured Platform for Conductive Biopolymers. 239

Fernando Dourado, Daliana Muller, Catarina Nunes, Carlos Rambo, and Guilherme Mariz de Oliveira Barra

Index 265 


\section{Preface}

Bacterial nanocellulose (BNC) is an emerging nanomaterial with unique properties produced by several species of bacteria. The most important of these is Acetobacter xylinum, now renamed Gluconacetobacter xylinus, discovered in 1886 by A. J. Brown. These bacteria are highly ubiquitous. They are found wherever the fermentation of sugars and plant carbohydrates takes place; for example, on the surfaces of rotting fruits and in unpasteurized or unsterilized juice, beer, and wine.

Although the molecular formula and morphology of BNC is similar to nanocellulose produced from wood, the former present several advantages. Bacteria produce pure cellulose, free from other plant components such as hemicelluloses and lignin, and form a three-dimensional network that provides unique mechanical properties. Due to their large surface area and hydrophilic nature, cellulose nanofibrils produced by bacteria hold a large amount of water making them a very strong hydrogel. BNC has been used for a variety of commercial applications including textiles, cosmetics, and food products, and has a high potential for medical applications. This book provides state-of-art scientific knowledge about the mechanism of cellulose production by bacteria and points out the challenges in the expansion of $\mathrm{BNC}$ production to a large scale. It also provides the latest information on BNC structure and its modification, as well as comprehensive information concerning current and future applications of BNC.

Since the first identification of genes for cellulose biosynthesis in Gluconacetobacter xylinus more than twenty years ago, homologous sequences have been identified in the genomes of a large number of bacterial species. The genes for cellulose biosynthesis are organized in an operon consisting of three to four genes and this organization is conserved in most bacteria. The first gene in the operon encodes the enzyme cellulose synthase and, so far, this is the only protein with a known function in cellulose biosynthesis. Although genetic analysis demonstrated a role in cellulose biosynthesis for the other genes in the operon, the exact function of the proteins encoded by these genes remains to be determined. Chapter 1 introduces the field of biosynthesis of BNC.

BNC is a unique, nontoxic hydrogel with good mechanical properties. It has been shown to be biocompatible and, therefore, it has found several applications in the medical field as wound and burn dressings and has high potential for development as medical implants and scaffolds for tissue engineering. Another unique property is its ability to be shaped into three-dimensional structures during biosynthesis. For instance, in the case of vascular grafts, the material synthesis and tubular product formation takes 
place simultaneously. The biomechanical performance (e.g., rupture pressure) and compliance, along with biological response (endothelialization, blood compatibility), are dependent on the morphology of the fibrillar network. The network formation is affected by the cellulose assembly, bacteria motion, and proliferation rate. An understanding of the effects of cultivation conditions on BNC network formation is, therefore, of great importance. This is described in Chapter 2.

A shortcoming limiting broad commercialization has been the lack of large-scale production capacity. Chapter 3 reviews a variety of approaches that are available for the generation of BNC in different forms, ranging from batch, to fed batch, to continuous cultivation methods applying typical bioreactors but also several specific devices. The choice of a cultivation technique depends on the envisaged application, since the cellulose supramolecular structure and its physical and mechanical properties are strictly influenced by the production method.

Because of its unique structure, BNC shows mechanical anisotropy with a high tensile modulus along the fiber layer direction, but a low compressive modulus perpendicular to the stratified direction. Consequently, water is easily squeezed out of the gel under slight compression and the swelling property is not fully recovered due to hydrogen-bond formation between cellulose fibrils. In Chapter 4, a BNC-based biocompatible hydrogel with high mechanical strength and reversible swelling following repeated compression cycles is described. It was developed by combining the BNC with natural polymers such as gelatin, as well as synthetic polymers such as polyacrylamide, using a method known as the double-network technique. These gels exhibit high compressive fracture stress comparable to cartilage and high tensile strength, comparable to ligament or tendon. In addition, some biological properties of these gels, such as resistance against wear, biodegradation, and biological interactions within the living body, are discussed with a perspective toward its applicability as a potential biomaterial.

The unique properties of BNC provide the basis for a wide range of applications in human and veterinary medicine, odontology, pharmaceuticals, acoustic and filter membranes, biotechnological devices, and in the food and paper industries. Chapter 5 provides an introduction to the surface modifications of BNC. Depending on the applications, chemical modifications, incorporation of bioactive molecules, and modification of the porosity, crystallinity, and biodegradability may be obtained, further increasing the potential of BNC.

Chapter 6 reviews attempts to reveal the exclusive structure-property relationship in order to extend the use of a nematic ordered cellulose film as a functional template for bacterium culture and biomimetic mineralization. In addition, a review is provided on carbohydrate polymers with a variety of hierarchical nematic ordered states on various scales-so-called nano/micro structures - that would allow development of new functional ordered scaffolds. The regulating factors for the rate of the microbial three-dimensional buildup are also discussed. 
Chapter 7 describes one of the first commercially available products of BNC: nata de coco. This is a white, gelatinous, chewy material used for the production of sweets and desserts. It is produced from coconut water or coconut milk through static fermentation. Nata de coco production originated in the Philippines during the 1990s and spread to nearby countries. In 1991 nata de coco was introduced to Japan through its use in diet drinks and became very popular, especially among young girls. Production of nata de coco has increased in recent years not only for domestic markets, but also export markets, especially Europe, Japan, the United States, and the Middle East. Nata de coco is usually produced in the form of thick sheets of pure cellulose, which are then cut into small uniform cubes, washed, and boiled in water before cooking in sugar syrup for food applications. Nata de coco is used in food products such as low-calorie desserts, salads, and high-fiber foods. It is highly regarded for its high content of dietary fiber and low fat and cholesterol content. Commercial nata de coco is made by large and midsize companies and small or home industries in East Asian countries including the Philippines, Indonesia, China, Vietnam, Malaysia, and Thailand.

In Chapter 8, wound dressings and cosmetic materials fabricated from BNC are reviewed. $\mathrm{BNC}$ has proven to be a versatile biomaterial that can be used to fabricate excellent wound dressings that promote rapid and virtually painless wound healing as well as cosmetics with moisturizing and antiaging properties. As mentioned before, it is a natural, nontoxic, biocompatible, and stable hydrogel with an excellent affinity to connective tissue. BNC has ideal wound care dressing properties: it causes neither toxic nor allergic side effects when remaining in long-term contact with wounds; its high water-holding capacity (up to 100 times its dry mass) moisturizes the wound surface preventing additional tissue loss from dehydration; it stimulates the action of lytic enzymes that remove residual debris in the early stages of wound healing, speeds restoration of the wounded tissue to its normal state, and significantly reduces pain. It is highly porous and hydrated, simultaneously moisturizing the environment and absorbing exudates; it conforms to any wound shape and is very light and transparent, forming a mechanically durable and tight physical barrier to microbial contamination and additional injuries, while providing gaseous exchange; it allows easy and painless removal of wound coverings; it is sterilizable, nondegradable in mammalian systems, flexible, elastic, and available in any size and shape; it can be infused with other therapeutic substances without deterioration of its inherent features; its properties can be tailored to individual needs through deliberate changes in the bacterial culture conditions, genetic modifications, or postculture treatments of the polymer; and it forms homogeneous blends with other biocompatible polymers like alginate, hyaluronic acid, or soluble cellulose derivatives.

In Chapter 9, the suitability of BNC as a biomaterial is presented in detail. This chapter should be of interest to readers from polymer sciences and applications, medicine, pharmaceutics, galenics, and cosmetics. Examples of flat BNC implants for cartilage repair and tubular BNC blood vessel grafts are 
described. The biotechnological production not only allows control of the network structure, but also the shaping and surface design of the BNC implants. In postprocessing steps, partial or total drying of the implants can be performed, in a vacuum, by heating or hot pressing, or by freeze-drying or solvent exchange. In addition, specific perforation of the material with channels up to $300 \mu \mathrm{m}$ in diameter can be reliably carried out. By combining BNC with other polymers or inorganic compounds (such as hydroxyapatite) during biosynthesis or aftertreatment, anocomposites are formed or material surfaces can be coated with BNC. In addition, metals and metal oxides can be easily precipitated on the BNC nanofiber network and active agents of various kinds, such as pharmaceuticals and dyes, can be incorporated reversibly or irreversibly.

Chapter 10 describes BNC biomaterials with controlled architectures for tissue engineering scaffolds and customizable implants. Through careful control of bacteria motion, it is possible to produce well-defined, three-dimensional scaffolds for tissue reconstruction. A particular focus is the use of electric fields to produce customized and highly oriented cellulose networks and also the development of microvascular networks within BNC structures. This is the first attempt to control a bottom-up biofabrication process in three dimensions. The manipulation of electrokinetic forces acting upon a bacterial cell can produce complex cellulose patterns on the nanoscale not achievable in static culture. The ability to control the direction of fiber orientation could be readily expanded to weave structures of multiple fiber layers by changing the orientation of the applied electric field for each layer. Using this method, structures could be constructed with desired mechanical properties for a variety of applications including tissue engineering, microelectromechanical systems (MEMS), textiles, and electronics.

$\mathrm{BNC}$ has been recently shown to have the capacity to induce crystal growth. Chapter 11 describes biomimetic mineralization of apatite on BNC. Strategies for the surface modification of BNC include TEMPO (2,2,6,6-tetramethylpyperidine-1-oxyl)-mediated oxidation and medium modification by the addition of amino sugar $\mathrm{N}$-acetylglucosamine (GlcNAc) during biosynthesis. TEMPO-modified BNC having active carboxyl functional groups showed a faster nucleation rate than native BNC and GlcNAc-incorporated $\mathrm{BNC}$. It was found that the ability to induce mineral nucleation differed according to the BNC template surface structure, which strongly influenced the growth behavior of the apatite crystals.

One of the promising nonmedical applications of BNC is its use as electronic paper. Since the discovery that polyacetylene could be doped to the metallic state more than three decades ago, an ever-growing body of multidisciplinary approaches to material design, synthesis, and system integration has been produced. Chapter 12 reviews some of the potential areas for application of conductive polymer blends. Current results concerning the chemical polymerization of conducting polymers on BNC are presented, including a brief remark on the rationale for the use of conductive $\mathrm{BNC}$ blends. This is followed by a discussion of their properties and potential applications. 
This book presents the current state of knowledge and important developments and perspectives in the field of BNC. The contributions clearly demonstrate the exciting structure and properties of the green nanomaterial BNC. As the most important member of the nanocellulose family, BNC opens the expanding fields of sustainable materials and nanocomposites, as well as medical and life science devices.

It is our intention to broaden knowledge in this subject area and to stimulate the development of practical uses of BNC. Science and technology continue to move toward the use of renewable raw materials and more environmentally friendly and sustainable resources and processes. The development of BNC is an important component of this movement and all signs seem to indicate that the impressive rate of development in the field of BNC will increase further.

Dieter Klemm, Francisco Miguel Gama, and Paul Gatenholm 



\section{Editors}

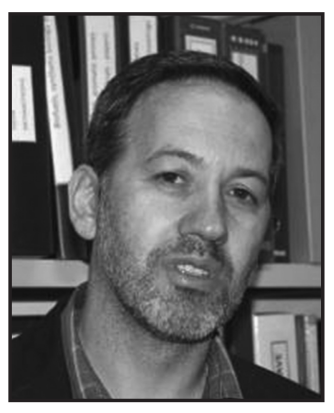

Francisco Miguel Gama received his $\mathrm{PhD}$ in biological engineering from Minho University. He is currently an associate professor in habilitation at Minho University, where he directs the FUNCARB Laboratory, a research group with about 20 researchers. He has authored or edited two books, more than eighty papers, and two patents. His research interests include enzyme technology (enzymatic degradation of biomass) and the development of biomaterials, in particular bacterial cellulose, self-assembled amphiphilic nanogels and injectable hydrogels, developing applications for tissue regeneration and drug delivery.

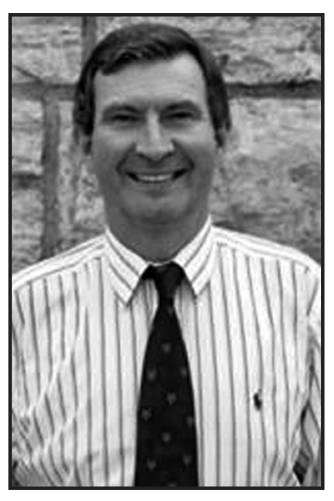

Paul Gatenholm received his $\mathrm{PhD}$ in material science from Chalmers University of Technology. He is currently a professor of biopolymer technology at Chalmers University, director of the Biosynthetic Blood Vessels Laboratory, coordinator of the EAREG program, and director of the Graduate School at Wallenberg Wood Science Center. He is also an adjunct professor at the joint School of Biomedical Engineering and Sciences at Virginia Tech and Wake Forest University and an adjunct professor of biomaterials at the Wake Forest Institute for Regenerative Medicine in Winston-Salem, North Carolina, USA. He has authored or edited two books, more than two hundred papers, and fifteen patents. His research interest is in the biomimetic design of future materials. This requires an understanding of structure-property relationships at all length scales. His research includes biological fabrication through the use of enzymes, cells, and the coordination of biological systems. 


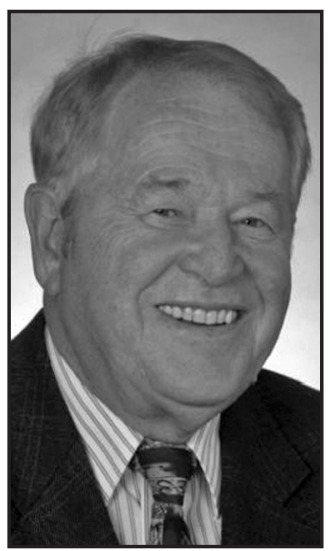

Dieter Klemm received his $\mathrm{PhD}$ for his work on steroids and his habilitation in 1977 based on research on synthetic polymers. After working in the pharmaceutical industry, he has been a professor of organic chemistry at the University of Jena since 1987, engaged in cellulose chemistry and carbohydrate metal complexes. Currently, he is active in the research and development of nanocelluloses and medical implants in the Polymet Jena Association and Jenpolymers Ltd. His honors include the Anselme Payen Award of the American Chemical Society for the development of new cellulose-based materials. 


\section{Contributors}
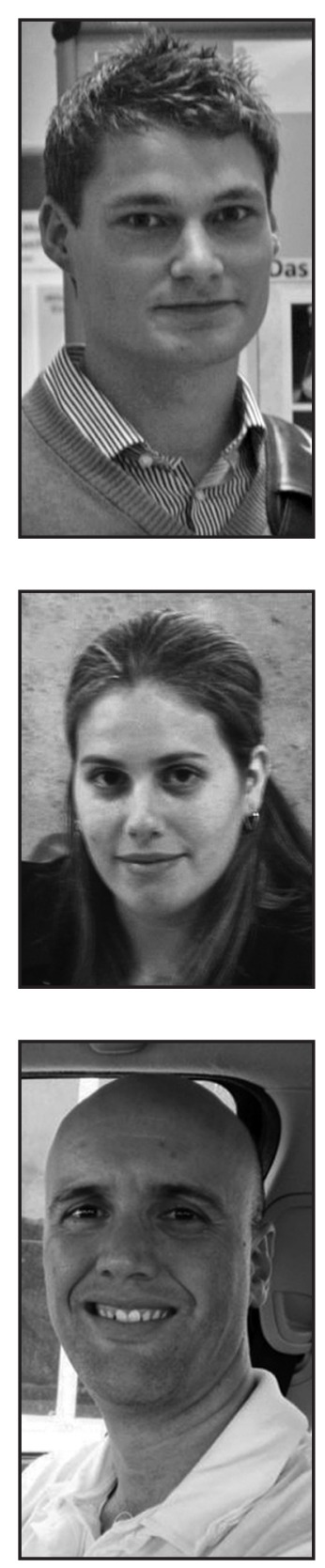

Hannes Ahrem studied biology at Friedrich Schiller University where his main focus was on microbiology. With the topic of structural design of BNC for improved cell migration, he graduated in 2009 in the group of D. Klemm and has worked since that time on the development of BNC-based implants as a PhD student at Jenpolymer Materials Ltd. \& Co. KG.

Fábia Karine Andrade has a degree in biological sciences from the Universidade Federal do Ceará (UFC). She received her master's in biochemistry from UFC in 2006 and a $\mathrm{PhD}$ in biomedical engineering from the University of Minho in 2010.

Guilherme Mariz de Oliveira Barra received a degree in material science and engineering from the Federal University of São Carlos, Brazil (1994). He has a master's degree in material and metallurgical engineering from the Federal University of Santa Catarina (1997) and a PhD in polymer science and technology from the Federal University of Rio de Janeiro, Brazil (2001). Since 2004, he has worked in the Mechanical Engineering Department of the Federal University of Santa Catarina where he has focused on the development of intrinsically conducting polymers, nanocomposites, conducting polymer blends, and vegetal fiber-reinforced polymer materials. 


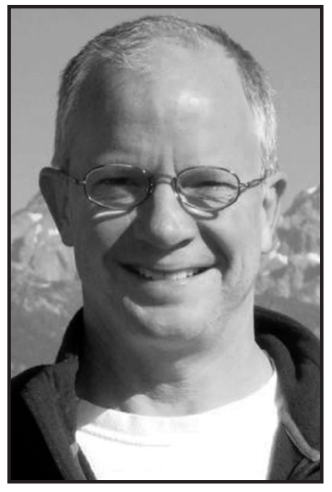

Joel Berry is an associate professor in the Department of Biomedical Engineering at the University of Alabama at Birmingham. His research has focused on the development of experimental and computational flow models for vascular stent research. This work led to the discovery that most vascular stents create adverse blood flow disturbances, potentially leading to their clinical failure. This also led to the invention of a stent design that eliminates these disturbances. This flow modeling experience further led to the development of tissue bioreactors for engineered arteries and heart valves. He is currently developing mechanical testing methodologies for analyzing the tensile behavior of individual electrospun fibers used in tissue engineering applications. Knowledge of the mechanical properties of individual fibers is essential in designing tissue scaffolds that allow effective mechanotransduction of seeded cells. His most recent work includes the development of bioreactors to promote the growth of tissue scaffolds using bacterial-derived cellulose.

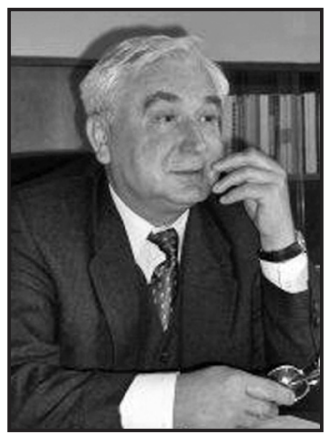

Stanislaw Bielecki has been employed since 1970 as academic teacher in the Institute of Technical Biochemistry at the Technical University of Lodz, where in 1978 he received a PhD and in 1999 became a full professor. He is a member of several Polish and European federations and societies for biotechnology. His scientific interests focus on biocatalysis (also in nonconventional systems), industrial biotechnology, engineering of biocatalysts, enzymatic synthesis, and applications of oligosaccharides and microbial biopolymers including bacterial cellulose. He is an author and coauthor of numerous international patents and more than two hundred papers in these fields. The achievements of research groups he has headed have been awarded prizes and medals both in Poland and abroad.

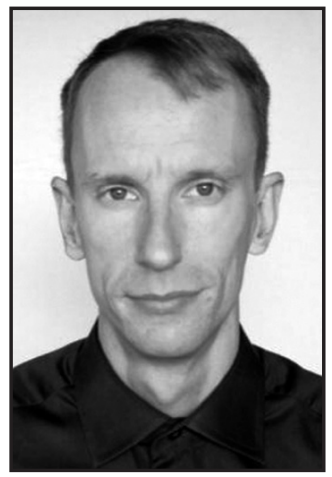

Christian Brackmann received his $\mathrm{PhD}$ from the Division of Combustion Physics, Department of Physics, Lund University in 2004. During 2005-2010 he worked in the group of Molecular Microscopy, Chalmers University of Technology. His research during that time was on laser-based nonlinear optical microscopy and included both method development as well as applications (e.g., in lipidomics, food science, and tissue engineering). 


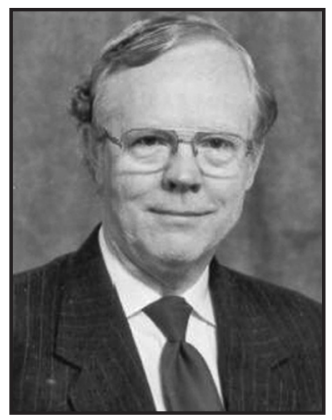

R. Malcolm Brown, Jr., graduated with a major in botany at the University of Texas at Austin in 1961 and obtained his $\mathrm{PhD}$ at the same university in 1964 . Dr. Brown accepted a postdoctoral position at Freiburg University in 1968. He taught at the University of North Carolina at Chapel Hill for 13 years before being invited to return to his alma mater in 1981 . He is currently a professor of biology in the Section of Molecular Genetics and Microbiology at the University of Texas at Austin, and holds the Johnson \& Johnson Centennial Chair in Plant Cell Biology at the same university. He received the Darbaker Prize in phycology in 1978, the Lamb Award (University of Nebraska) in 1980, and the Anselme Payen Award for cellulose from the American Chemical Society in 1986. He has been a fellow of the International Academy of Wood Science since August 1983. Dr. Brown has had grants from different sources including the National Science Foundation, U.S. Department of Agriculture, Department of Energy, NASA, Johnson \& Johnson, Welch Foundation, National Institutes of Health, Office of Naval Research, Texas Advanced Technology Program, and various corporations, totaling \$11,898,426. $\mathrm{He}$ is the author of more than 220 peer-reviewed papers in international journals, and holds more than forty patents, including U.S. and international patents. He is the inventor of electronic paper. He has had more than twenty-five students complete their doctorates with him over the years. He is on the editorial board of the journal Cellulose and coedited the book Cellulose: Molecular and Structural Biology with his long-time colleague, Inder Saxena. His hobbies are music composition and gardening.

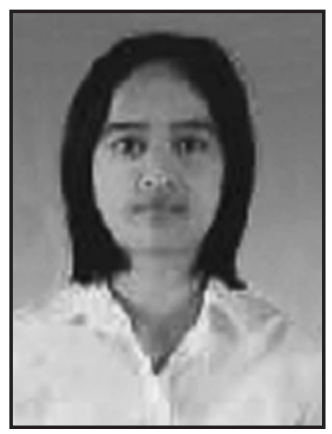

Nadda Chiaoprakobkij earned a master's degree in chemical engineering from Chulalongkorn University in 2008. At present she is a PhD student and a member of the Biochemical Engineering Research Group, Department of Chemical Engineering, Chulalongkorn University. She has been involved in research projects on the development of bacterial cellulose for biomedical applications. Her research works, in collaboration with the Faculty of Dentistry at Chulalongkorn University, on the development of novel composite sponges to cover surgical wounds in oral cavities have been presented at national and international conferences and published in an international journal. 


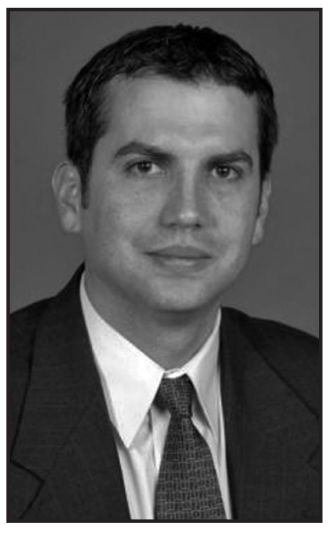

Rafael V. Davalos is an associate professor at the joint School of Biomedical Engineering and Sciences at Virginia Tech and Wake Forest University and a College of Engineering faculty fellow. He also holds adjunct appointments in the Departments of Engineering Science and Mechanics, the Wake Forest Comprehensive Cancer Center, and the Wake Forest Institute of Regenerative Medicine. He received his BS from Cornell University and PhD from the University of California, Berkeley. Prior to his career as a faculty member, he was a principal member of the technical staff at Sandia National Laboratories. He is the recipient of the 2006 HENAAC award for the nation's most promising engineer, the NSF CAREER, and the Coulter Foundation's Early Career Award. He has authored or coauthored more than forty-five peer-reviewed papers, twelve pending or issued patents, and five book chapters. His research interests are in the effects of electric fields on biological systems, specifically with regard to dielectrophoresis, electroporation, and biotransport.

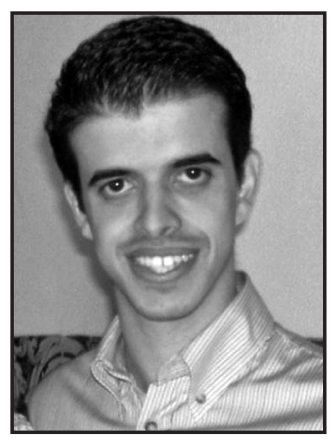

João Pedro Martins Soares de Castro e Silva has a degree in applied biology from the University of Minho. He received a master's in molecular genetics from the University of Minho in 2004 and a PhD in biology from the University of Minho in 2009. Currently, he is a postdoctoral researcher in biochemical and biotechnological engineering in the Department of Biological Engineering at the University of Minho, working on the production and modification of bacterial cellulose tubes to be used as artificial vascular prosthesis.

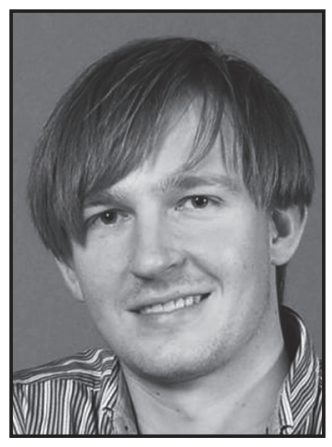

Manu de Groeve has a degree in bioengineering (2005, cell and gene biotechnology) and a PhD in applied biological sciences (2009), both obtained at Ghent University. At present he is an assistant professor at the Technical University of Lodz. His research interests include industrial biotechnology, and, more specifically, biocatalysis, enzyme engineering and the biotechnological production of biomaterials. His research results have been presented at national and international conferences and he is author or coauthor of several scientific publications in peer-reviewed international journals. He also holds an international patent. 


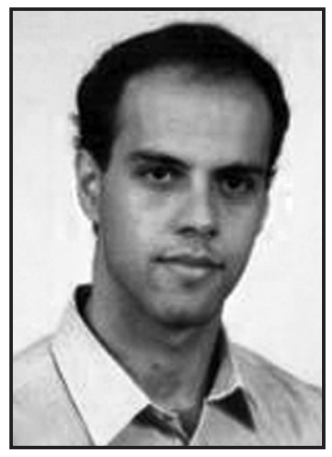

Fernando Dourado is a faculty member in the Department of Biological Engineering at the University of Minho. With a PhD in chemical and biological engineering, he is a member of the FUNCARB (FUNctional CARBohydrates Nanobiotechnology) Group, integrating the Centre of Biological Engineering (CEB) of University of Minho, a part of the Associated Laboratory Institute of Biotechnology and Bioengineering (IBB). He has been working on the development of bacterial cellulose (BC)-based films for biotechnological and biomedical applications. These activities include the production of BC composite films and the surface modification of BC nanofibers. In parallel, he has been working on the development of a large-scale BC production system.

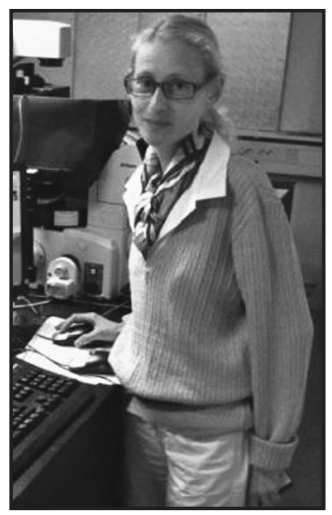

Annika Enejder received her $\mathrm{PhD}$ in physics at the Department of Atomic Physics, Lund University. In subsequent years, she was a postdoctoral fellow at MIT and Ludwig-Maximillian University. She is currently an associate professor in molecular microscopy at Chalmers University of Technology, a visiting associate professor in materials science and engineering at Stanford University, as Marie Curie Fellow, chair of the European Network for Coherent Raman Microscopy (microCoR), and national coordinator for Sweden in EuroBioImaging, promoting access to infrastructure for biological and medical imaging. Her current research interests include nonlinear optical microscopy, nonlinear plasmonics and near-field microscopy, Raman spectroscopy, and their applications within cell and molecular biology, medicine, and tissue engineering.

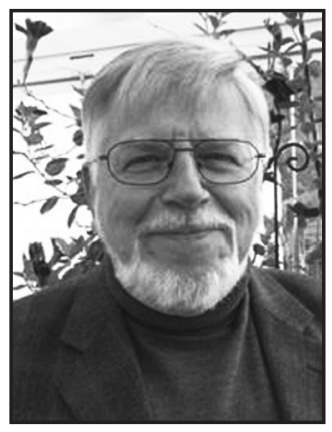

Wolfgang Fried was educated at the Technical University Ilmenau and at Friedrich Schiller University, where he received both his $\mathrm{PhD}$ and Habilitation. Throughout his career, he has worked on research projects as diverse as the development of software for computer-animated manufacturing, high-temperature sensors for space technology, and patient-specific ceramic implants in surgical reconstruction for humans. For sixteen years, he headed the working group "Modelling and Simulation" at the Faculty of Physics and Astronomy at Friedrich Schiller University and is now on the board of directors of the Polymet Jena Association. He has conducted research on bacterial cellulose for more than a decade. 


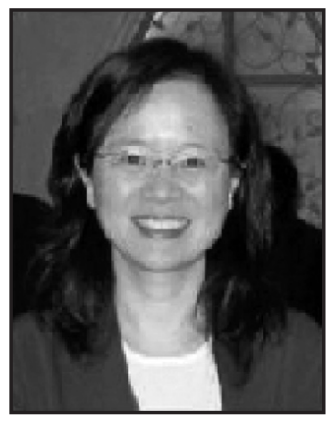

Jian Ping Gong is a professor in the faculty of Advanced Life Science at Hokkaido University. She earned her doctor of engineering from the Tokyo Institute of Technology and joined the faculty of Hokkaido University in 1993. She received the Wiley Polymer Science Award (2001), Society of Polymer Science Japan Award (2006), and the Chemical Society of Japan Award (2011). She serves on the editorial and advisory boards of Soft Matter, Macromolecules, Biointerphases, and Asia Materials. She is currently concentrating on novel hydrogels with high mechanical performances and applications as biomaterials.

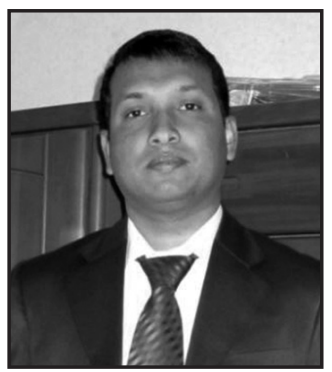

Anamul Haque graduated with a master's in science from the University of Dhaka in 2008 and received his PhD from Hokkaido University in 2011. During his doctoral research, he studied the creation of an anisotropic hydrogel with a well-defined hierarchical structure. Now he is concentrating on the functionalization of this anisotropic hydrogel, such as toughness and multistimuli color sensors.

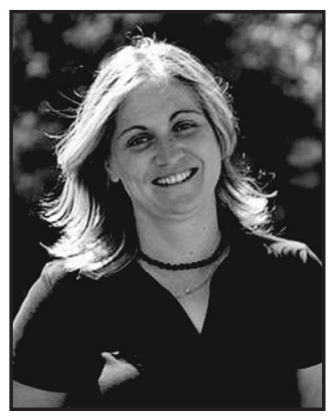

Nadine Hessler studied chemistry a with specialization in bioorganic chemistry at Friedrich Schiller University (FSU) from 1999 to 2004. In 2008, she received her PhD under the supervision of D. Klemm. The title of her PhD thesis was "Bacterial nanocellulose: Structural and form design during biosynthesis." Currently, she works in the group of D. Kralisch at the Institute of Technical Chemistry and Environmental Chemistry at FSU, studying process optimization of BNC in the research and development phase, production of nanocellulose in different forms, as well as its structural design. 


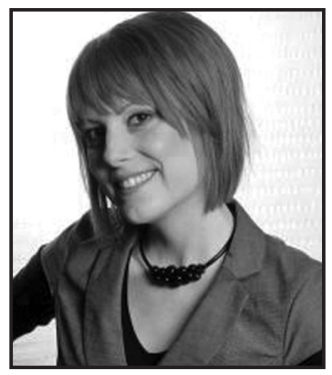

Kim Höglund received her degree in biotechnology from Chalmers University of Technology in 2011. She did her master's thesis in Professor Paul Gatenholm's group at the Biosynthetic Blood Vessels Laboratory during the autumn of 2010. Her master's thesis included the optimization of cellulose production for the production of bacterial cellulose blood vessels. Her interest in biomaterials and their biological effect on cells has led her into the area of tissue engineering. Today she works with cell culturing on starch particles to determine how different surface modifications affect cell growth.

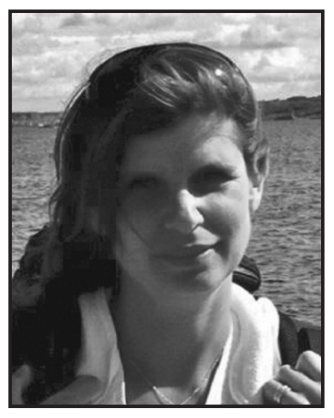

Sara Johannesson received her $\mathrm{PhD}$ in 2005 at the Karolinska Institutet. In collaboration with the pharmaceutical company AstraZeneca, she developed an in vitro organotypic rat hippocampal model for investigations of neurodegenerative processes in Alzheimer's disease. After moving to Gothenburg, Sweden, she worked in Eric Hanses's research group at Gothenburg University on electrophysiological and immunohistochemical studies of the N-methyl-Daspartate (NMDA) receptors in the hippocampal region of the rat brain. Since early 2011, she has worked as a laboratory manager in Paul Gatenholm's group at Chalmers University of Technology. She has mainly worked with the biosynthetic blood vessel project, and most recently she started a research project where the main goal is to develop neuronal networks on BNC.

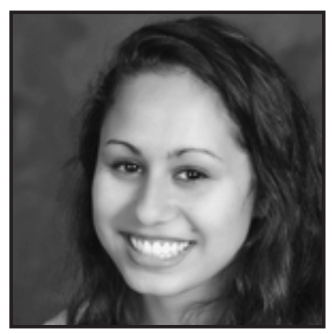

Kara Johnson is a MRI Research Technologists at the Virginia Tech Carilion Research Institute, USA. She works in the Montague Lab studying decision-making using fMRI analysis of brain function. Kara graduated in May 2011 with a degree in electrical engineering from Virginia Tech. She has assisted at BC Genesis in designing the prototype for a biomechanical microweaver in order to produce the meniscus for the human knee. She has been a member of Theta Tau and IEEE (Institute of Electrical and Electronics Engineers) since 2007. 

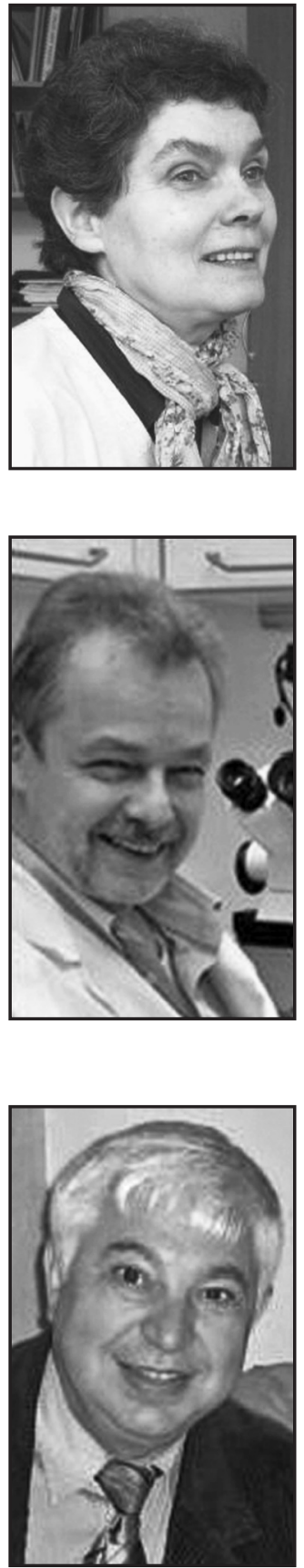

Halina Maria Kalinowska has worked at the Institute of Technical Biochemistry at the Technical University of Lodz since 1979. Since 1987, she has been employed as an academic teacher. She received her PhD in 1995 in the field of enzymology. Her scientific interests focus on the isolation, characterization, and application of enzymes from various sources including extremophilic microrganisms. She is the coauthor of numerous publications and poster presentations. Currently, she is involved in studies on enzymatic hydrolysis of lignocellulosic materials to be used in the synthesis of various bioproducts, including cellulose produced by Gluconacetobacter xylinus.

Raimund Kinne received his $\mathrm{PhD}$ for work on ceramic biomaterials for tooth replacement. He later focused on the role of inflammatory cells, in particular T-cells and macrophages, in the pathogenesis of inflammatory arthritis. Subsequent research topics included the contribution of resident synovial fibroblasts to joint inflammation and destruction in rheumatoid arthritis, and also the development of cartilage implants and calcium phosphate cement for the treatment of vertebral fractures. He became an associate professor in 2007 and is currently involved in the Interdisciplinary Center of Clinical Research as well as the Center for Sepsis Control and Care at the University Hospital, Jena.

Marek Kołodziejczyk graduated from the Faculty of Biology and Earth Sciences at Lodz University. He worked in the Laboratory of Experimental Surgery of the Endocrinological Clinic of Surgery at Lodz Medical University, where he received a PhD in medical biology. He has worked since 1994 at the Institute of Technical Biochemistry at the Technical University of Lodz as a researcher and lecturer. His current research interests concentrate on biomaterials for medical applications with a focus on novel bacterial cellulose-based products for external and internal use, including reconstructive surgery. $\mathrm{He}$ is the author or coauthor of numerous patents and publications. The achievements of the research group he joined have been awarded prizes and medals both in Poland and abroad. 


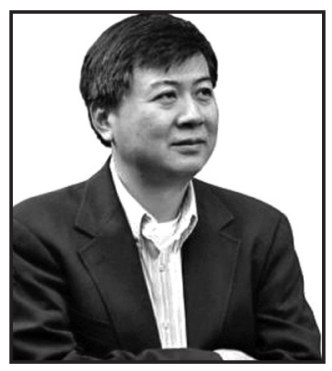

Tetsuo Kondo has contributed extensively to polymer physical chemistry as well as polysaccharide chemistry through basic and applied research using cellulose as a main subject. He has synthesized regioselectively substituted cellulose derivatives as cellulose models for understanding hydrogen bonding formation in cellulose homopolymer. His interests also include the supermolecular architecture of cellulose in native and artificial systems. Recently, he has established a new form of cellulose named nematic ordered cellulose (NOC), which exhibits noncrystalline yet ordered states. These findings create a new concept for understanding how glucan chains associate and crystallize and also indicate that the NOC may have expanded useful properties for commercial utilization. More recently, the NOC concept was extended to nano-patterned templates, including honeycomb-patterned cellulose films, in order to establish a hierarchical organizing design for three-dimensional nano/micro architecture of biobased materials using his original "bio-alchemy."

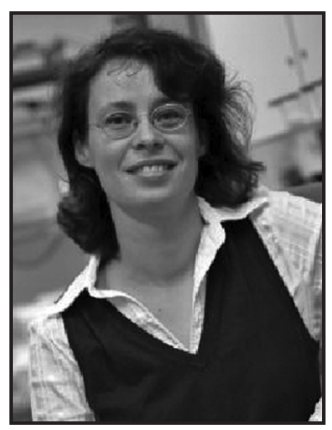

Dana Kralisch studied environmental chemistry at Friedrich Schiller University (FSU). After two years of work at the Agency for Agriculture of the Federal State of Thuringia, she moved to the Institute of Technical Chemistry and Environmental Chemistry at FSU. Since finishing her PhD in 2006, she has led the Green Process Engineering and Evaluation Research Group at FSU. Her current research activities are focused on the design and optimization of green processes, especially process development and scale-up of BNC generation in different forms as well as on microreaction technology.

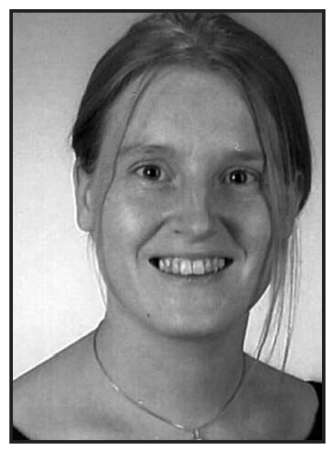

Friederike Kramer studied chemistry at Friedrich Schiller University. Since 2003, she has been working in the field of cellulose research and development in the group of D. Klemm. She graduated in 2004 with a diploma thesis on interpenetrating polymer networks of BNC and synthetic polymers. In 2008, she received her PhD from the University of Jena for research on nanocellulose and nanocellulose composites for the development of medical implants at the Polymet Jena Association. Since 2008, she has worked at Jenpolymer Materials Ltd. \& Co. KG and has been active in the field of flat and tubular medical implants based on BNC. 


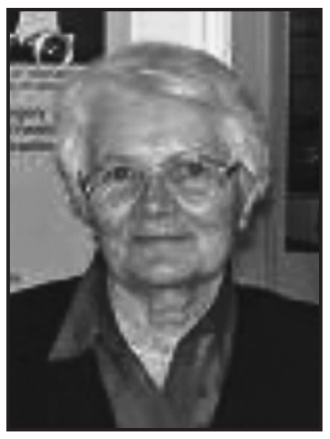

Alina Krystynowicz was employed in 1962 as an academic teacher at the Institute of Technical Biochemistry at the Technical University of Lodz, where in 1974 she received her $\mathrm{PhD}$. Although she retired in 2002, she has continued her research activities. Her scientific interests include the synthesis of enzymes from microbial sources (e.g., amylases and lipases) for large-scale applications in industry and environment protection. For the last twenty years she has been involved in studies on the synthesis of cellulose by strains of Gluconacetobacter xylinus. She is the coauthor of numerous patents and publications. The achievements of Dr. Krystynowicz and her coworkers in their research on bacterial cellulose production and it uses have been awarded prizes and medals both in Poland and abroad.

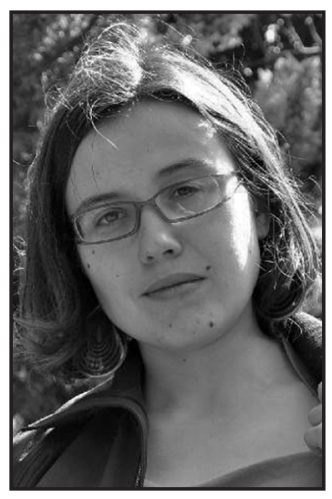

Katarzyna Agnieszka Kubiak has been employed as an academic teacher at the Institute of Technical Biochemistry at the Technical University of Lodz since 2008. She received her PhD in 2008 at the Centre of Molecular and Macromolecular Sciences, Polish Academy of Sciences, in the field of eukaryotic gene silencing with chemically obtained siRNAs. Her present scientific interests focus on regulatory RNAs in bacteria on the model of Gluconacetobacter xylinus. Her current research concerns molecular regulatory processes involved in the control of bacterial cellulose biosynthesis.

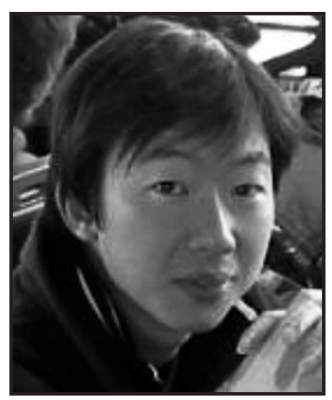

Takayuki Kurokawa graduated with a degree in polymer science from Hokkaido University in 2000. He received his $\mathrm{PhD}$ for his study on the effect of polymer dynamics on the friction of gels from Hokkaido University in 2005. He joined RIKEN, Japan, as a postdoctoral researcher. He has been an assistant professor at the Creative Research Institution at Hokkaido University since 2009. He focuses on the functions of polymer gels, such as mechanical properties, permeability, and biological properties. 

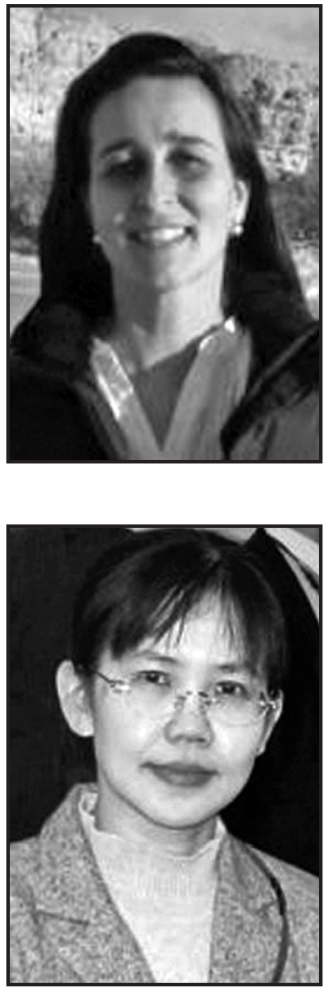

Daliana Muller graduated with a degree in chemistry from the Federal University of Santa Catarina, Brazil (2003). She has a master's degree in materials science and engineering from the Federal University of Santa Catarina (2008). Since 2009, she has been a PhD student at the Federal University of Santa Catarina studying conductive polymer blends based on bacterial cellulose and intrinsically conducting polymers.

Thi Thi Nge came from Myanmar, where she graduated from the University of Yangon with a major in chemistry in 1994. After receiving a master's degree in biotechnology from the Asian Institute of Technology, Thailand (1998), she came to Japan under a Japanese government fellowship program. In 2002, she received her $\mathrm{PhD}$ in biomaterials science from the University of Tokyo. She then worked as a JSPS postdoctoral fellow in the group of Junji Sugiyama (Research Institute for Humanosphere, Kyoto University), working on the development of bacterial cellulose-based biomimetic composites. Her research interest is novel utilization of naturally occurring biopolymers, chitin, chitosan, and cellulose for the development of socioecologically friendly materials across the spectrum of multidisciplines. Since April 2011, she has worked as a research fellow at the Institute of Scientific and Industrial Research at Osaka University.

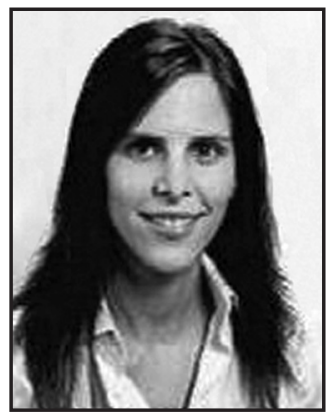

Catarina Nunes has a degree in applied chemistry and is a student in the master's program in micro/ nanotechnologies at the Department of Industrial Electronics at the University of Minho. She has been working in the FUNCARB (FUNctional CARBohydrates Nanobiotechnology) Group at the University of Minho on the development of bacterial cellulose (BC) composite films and the surface modification of BC nanofibers. 


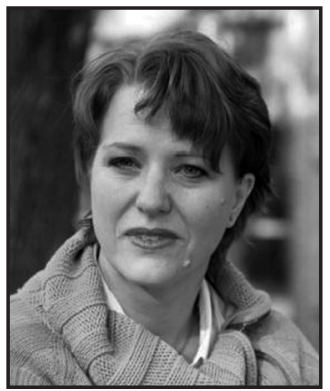

Lisbeth Olsson received her PhD at Lund University in applied microbiology in 1994. In 1994-2007 she worked at the Technical University of Denmark in the field of microbial cell factories and fermentation technology. Since 2008, she has been a professor in bioprocess technology at Chalmers University of Technology, heading the Industrial Biotechnology group. The Industrial Biotechnology group works on designing enzymes and microorganisms to be used in sustainable bioprocesses. Their primary interest is enzymes that act on plant cell wall materials with the aim to degrade, modify, or upgrade such materials. They work with the design of cell factories and development of fermentation processes producing chemicals, energy, and materials.

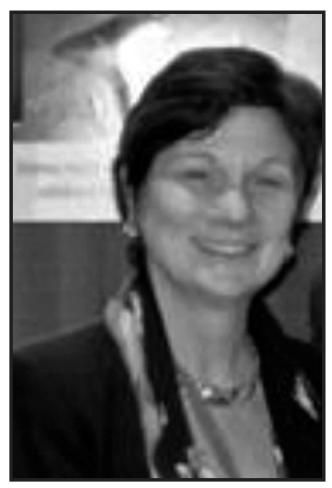

Laurie O'Rourke is a graduate of Purdue University's College of Veterinary Medicine (DVM) and the University of California, Davis (PhD). Her experience spans private practice, academics, diagnostics, research and development of pharmaceuticals, and recently, medical devices. She has a strong background in quality control and interpreting and implementing regulatory guidelines and is recognized as an expert in toxicologic clinical pathology. Her research interests center around the hematopoietic system and stem cells.

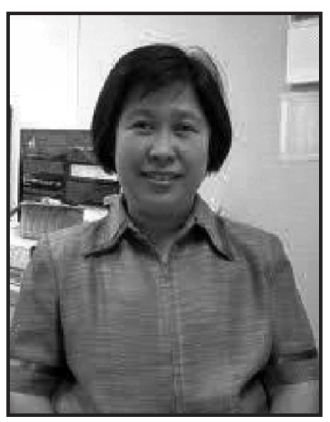

Muenduen Phisalaphong received her $\mathrm{PhD}$ from Colorado State University in 1999. At present, she is an associate professor in the Department of Chemical Engineering, Faculty of Engineering, Chulalongkorn University. She has served as head of the biochemical engineering research group since 2006. Her present research interests focus on biomaterials and bioprocess engineering. She has publications in international peer-reviewed journals and chapters on the development of new bacterial cellulose-based materials. 


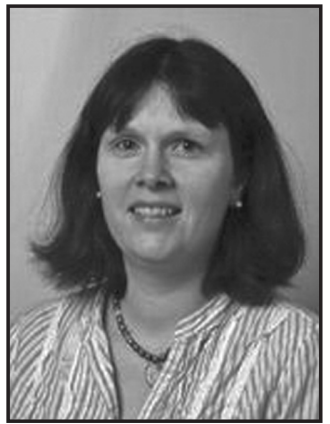

Maja Puchades received her DEA degree in 1993 at the University of Aix-Marseille III in biochemistry and her PhD in 2003 working with Davidsson and Blennow on the development of new proteomics methods to study biomarkers for Alzheimer's disease at the Department of Clinical Neurosciences, Sahlgrenska Academy, Gothenburg University, Sweden. After two years as a postdoctoral fellow with C. Nilsson, working on proteomics of glioblastoma tumors, she joined the laboratory of A. G. Ewing and worked with neuronal network cultures on microchips with electrochemical and fluorescence detection. In 2010, she was appointed as laboratory manager by Paul Gatenholm at Chalmers University of Technology and worked on the biosynthetic blood vessels project. In 2011, she moved with her family to Oslo, Norway. She is now studying neuro- and gliotransmission in mice models of Parkinson's disease with Gundersen and Storm-Mathisen at the Synaptic Neurochemistry Lab, Oslo University.

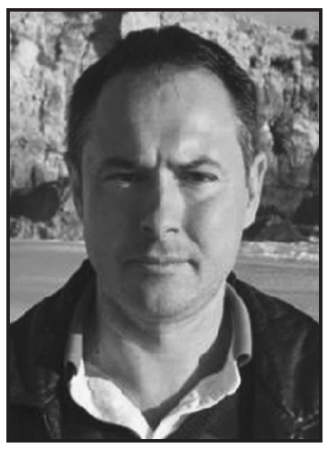

Carlos Renato Rambo has received a degree in physics from the University of São Paulo, Brazil (1994). He has a master's degree in materials science and engineering from the University of São Paulo, Brazil (1997), a PhD in materials science and engineering from the University of São Paulo, Brazil (2001), and is doing postdoctorate work at the University of Erlangen-Nurnberg, Germany (2005). He has worked since 2010 in the Electrical Engineering Department at the Federal University of Santa Catarina. He has been working on biomaterials, tissue engineering, and the development of highly porous ceramics and composites by biotemplating.

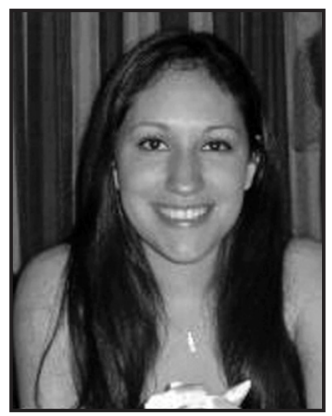

Andrea Rojas is a material science and engineering master student with a biomedical engineering minor at Virginia Tech. She did her undergraduate work in materials science and engineering at Virginia Tech. She works in the Bioelectromechanical Systems Laboratory applying her knowledge of electrokinetics, electrophoresis, and dielectrophoresis to manipulate particles and cells. Andrea is a recipient of the NIH Initiative to Maximize Student Diversity (IMSD) fellowship. Her research interests include fabricating biomaterials or biomedical devices for enhancing the quality of life for others. 

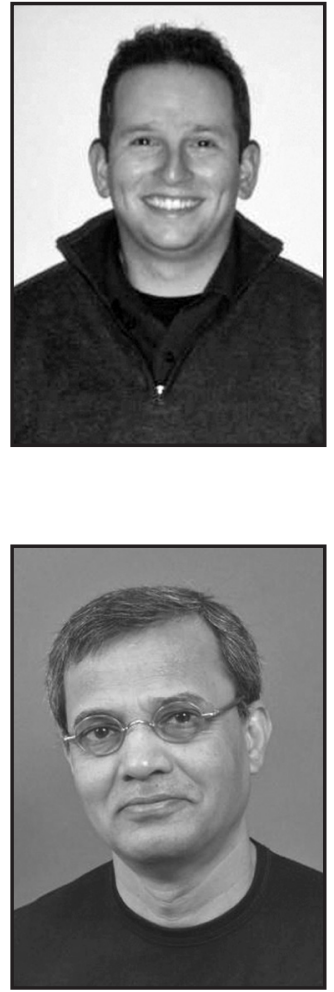

Inder Saxena received his $\mathrm{PhD}$ from Jawaharlal Nehru University in 1984. He is a lecturer at the University of Texas at Austin, where he came in 1986 and started work on the molecular biology of cellulose biosynthesis. He was one of the first to identify, isolate, and sequence the genes for cellulose synthesis from the bacterium Acetobacter xylinum (renamed Gluconacetobacter xylinus). His work on the sequence analysis of cellulose synthase and other proteins led to identification of conserved residues and a sequence motif in enzymes that synthesize not only cellulose but also a number of other polysaccharides. He is on the editorial board of the journal Cellulose and coedited the book Cellulose: Molecular and Structural Biology.

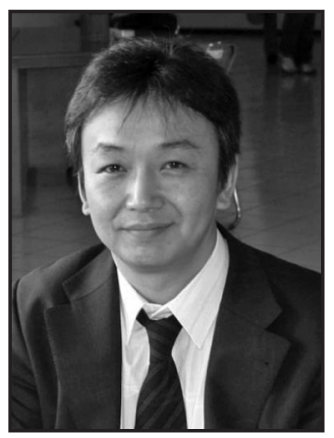

Junji Sugiyama obtained his BS and MS degrees in agriculture from Kyoto University and a PhD in agriculture from the University of Tokyo. The first visualization of cellulose crystal lattices by high-resolution electron microscopy was conducted during his MS thesis. He began his study of the crystallography of native cellulose that has continued to date in cooperation with Okano, at the University of Tokyo, and former research director Chanzy, at CERMAV-CNRS, Grenoble, France. One of his many good memories is that he and his colleagues succeeded in proposing the unit cells of cellulose allomorphs. More recently, as a professor at the Research Institute for Sustainable Humanosphere, Kyoto University, he is continuing his structural studies related to the biological or biochemical aspects of cellulose synthesis. He is also a curator at the Kyoto University Xylarium, promoting cooperative works in the multidisciplinary fields of wood sciences and humanity sciences. 


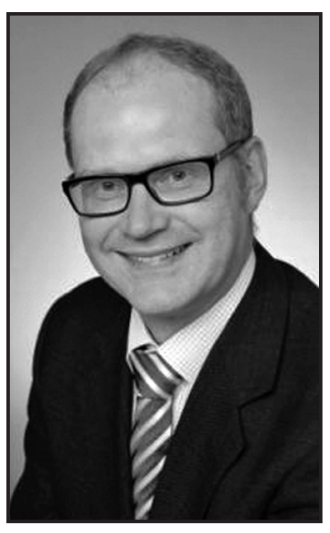

Jens Wippermann studied medicine at the Hannover Medical School. In 1993, he started his residency in cardiothoracic and vascular surgery, in which he became a specialist in 1999. During that time, he received his PhD. In 2000, he became senior surgeon in the Department of Cardiothoracic and Vascular Surgery at Friedrich Schiller University, where he joined the group of D. Klemm to investigate bacterial cellulose in large animals. The focus was the evaluation of cylindrical bacterial cellulose as a substitute for small arterial vessels. In 2006, he moved to the University of Cologne, becoming an assistant director for cardiothoracic surgery. There he headed the working group "Nanocellulose-A new biomaterial for cardiac and vascular surgery" in a close relationship with D. Klemm and fellows. 



\section{Biosynthesis of Bacterial Cellulose}

Inder M. Saxena and R. Malcolm Brown, Jr.

Section of Molecular Genetics and Microbiology,

University of Texas at Austin, Austin, Texas

\section{CONTENTS}

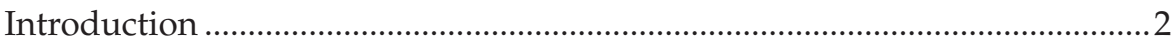

Gluconacetobacter xylinus is a Unique Cellulose-Producing Bacterium .............

Structure of Cellulose I is Determined by the Cell-Directed Assembly

of Glucan Chains

Cellulose Synthesis in Bacteria is Catalyzed by the

Membrane-Associated Cellulose Synthase

Genes for Cellulose Biosynthesis can be Classified into Three Groups

Based on the Function of the Codified Proteins

What is Known About the Proteins Involved in Cellulose Synthesis in

Bacteria?

"Guilt by Association" Model of the G. xylinus Cellulose-Synthesizing

Complex

Many Unanswered Questions Remain

Many Bacteria Have Genes for Cellulose Synthesis, but Only a

Few Produce Cellulose.

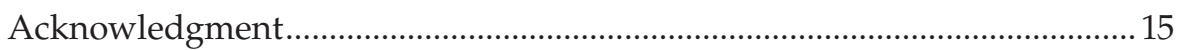

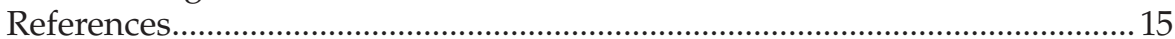

Since the first identification of genes for cellulose biosynthesis in Gluconacetobacter xylinus more than 20 years ago, homologous sequences have been identified in the genomes of a large number of bacterial species. The genes for cellulose biosynthesis are organized in an operon consisting of three to four genes and this organization is conserved in most bacteria. The first gene in the operon encodes for the enzyme cellulose synthase and so far this is the only protein with a known function in cellulose biosynthesis. Although genetic analysis demonstrated a role in cellulose biosynthesis for the other genes in the operon, the exact function of the proteins encoded by these genes remains to be determined. Genetic, biochemical, and structural data suggest that the proteins encoded by genes in the operon are assembled as a multiprotein 
complex and the organization of these complexes in the bacterial envelope determines the nature of the cellulose product. Interestingly, cellulose production has not been demonstrated for a majority of the bacteria where genes for cellulose biosynthesis have been identified, but with better assays it may be possible to determine cellulose production in these bacteria and discuss its evolutionary significance.

\section{Introduction}

The polysaccharide cellulose is considered to be the most abundant biomacromolecule, and one that is produced by almost all groups of living organisms. In nature, it is produced in large amounts by plants and algae, where it is an essential component of the cell walls. It provides not only mechanical strength to the cell wall surrounding the cells of these organisms, but also contributes to the direction of cell growth. In most other organisms it is secreted as an extracellular product, but it is not part of the cell envelope and so does not contribute to any known function in the synthesizing cells.

Cellulose is composed of $\beta$-1,4-glucan chains that assemble to form crystalline and noncrystalline forms depending on how the chains are assembled. The structure of cellulose produced by living cells is intimately linked to its synthesis, and the organization and arrangement of cellulose-synthesizing sites on the cell membrane are vital to the parallel assembly of glucan chains observed in the cellulose I allomorph found in nature (Brown 1996). Even though crystalline cellulose I is, in general, the form of cellulose that is assembled by living cells, the more stable form of cellulose is cellulose II, in which the glucan chains are assembled in an antiparallel arrangement. This form of cellulose is not commonly synthesized by cells and is mostly obtained by chemical treatment of native cellulose I.

Studies on the structure, synthesis, and applications of cellulose have mostly focused on plants, as they are the main producers of cellulose. At the same time, significant knowledge of cellulose synthesis has come from studies of bacteria, and bacterial nanocellulose (BNC) has found unique applications in various industries. Much of the discussion in this chapter will refer to cellulose synthesis in the bacterium Gluconacetobacter xylinus for reasons-the amount and form of cellulose produced, visualization of cellulose-synthesizing sites, and identification of genes and proteins involved in cellulose synthesis-that make this bacterium a unique organism for understanding the mechanism of biosynthesis of cellulose. In the last few years, interest in cellulose biosynthesis in other bacteria has also increased, mainly because of the identification of genes for cellulose biosynthesis from genome sequencing of these bacteria. Although many of these studies have provided interesting insights into the organization of genes for 
cellulose biosynthesis in these bacteria, in general they have not contributed much toward understanding the general mechanism of cellulose biosynthesis. One of the reasons is that it is not known whether a majority of these bacteria produce cellulose, if any at all, while others that do produce cellulose do it in very small amounts. However, cellulose biosynthesis has been studied in Escherichia coli and Salmonella to a considerable extent, as cellulose is a component of the biofilm in many strains of these bacteria, and many of these studies have provided details of the genes and proteins involved in the turnover of c-di-GMP, the activator of the polymerizing enzyme cellulose synthase (Römling 2007).

\section{Gluconacetobacter xylinus is a Unique Cellulose-Producing Bacterium}

G. xylinus is a Gram-negative, $\alpha$-proteobacteria that is grouped with the acetic acid bacteria. Known as Acetobacter xylinum for many years, this bacterium has undergone name changes in the last few years, so much so that the common laboratory strain ATCC 23769, a strain with a completely sequenced genome, is now classified as Gluconacetobacter hansenii (Iyer et al. 2010).

G. xylinus is found in nature on rotting fruits and a variety of niches that have fixed carbon in the form of sugars or alcohol. It is a strict aerobe, and so in many cases it is present at the air-medium interface where its presence can be detected readily if it produces a cellulose film or pellicle. The production of cellulose characterizes this bacterium both in liquid medium, where it produces a thick pellicle on the air-liquid interface, and isolated cells where a cellulose ribbon can be clearly seen attached to the long side of the cell. G. xylinus is a rod-shaped bacterium without a flagellum, and although it is nonmotile, cell movement can be observed microscopically in some strains during the formation of the cellulose ribbon.

In shaking cultures of G. xylinus, cellulose is produced as spherical or star-shaped structures in place of the matlike pellicle that is produced in stationary cultures. In addition, the amount of cellulose produced in shaking cultures is less than what is produced in stationary cultures. Another feature observed in shaking cultures is the presence, at a much higher frequency, of cells that do not produce a cellulose pellicle (Valla et al. 2009). The presence of these cells in large numbers makes the culture medium turbid. These cells produce smooth colonies on agar plates compared to rough colonies that are produced by cells that form a pellicle in stationary cultures. The smooth colony-producing cells have commonly been referred to as spontaneous $\mathrm{Cel}^{-}$mutants, although analysis of many of these spontaneous mutants has revealed that they produce relatively small amounts of cellulose II and have almost wild-type cellulose synthase activity (Roberts et al. 1989). 
The occurrence of these mutants is not necessarily due to a high rate of mutation as much as their ability to stay free and divide normally without being trapped in the cellulose product where cellulose-producing cells are trapped (Valla and Kjosbakken 1982). The cellulose-producing cells have a selective advantage in a static culture, as these cells are still attached to the cellulose product, and as the cellulose pellicle floats on the surface, these cells are brought to the air-liquid interface. In the same culture, $\mathrm{Cel}^{-}$cells are not attached to any cellulose microfibrils, and even though they may produce small amounts of cellulose, they do not get trapped in the cellulose pellicle and settle to the bottom of the container where their growth is constrained as the concentration of diffused oxygen becomes limiting.

G. xylinus strains have the ability to utilize a variety of sugars and other compounds for the synthesis of cellulose, and the pathways of carbon metabolism in this bacterium have been summarized very well by Ross et al. (1991). In laboratory cultures of G. xylinus, glucose is commonly provided in the medium, and essentially four enzymatic steps have been characterized in the pathway from glucose to cellulose. These are the phosphorylation of glucose by glucokinase, the isomerization of glucose-6-phosphate to glucose-1-phosphate by phosphoglucomutase, the synthesis of uridine diphosphate glucose (UDP-glucose) from glucose-1-phosphate by UDP-glucose pyrophosphorylase, and the synthesis of cellulose from UDP-glucose by cellulose synthase (Ross et al. 1991). Although a large fraction (almost 50\%) of the supplied glucose ends up in cellulose, a significant fraction of glucose in the medium is converted to gluconic acid in the early stages of culture of G. xylinum cells. Even though carbon from gluconic acid does end up in cellulose in the later stages of culture, one of the goals in the commercial production of BNC has been to isolate strains where the production of gluconic acid is reduced. However, such efforts have resulted in only a marginal increase in the yield of cellulose. A much more intriguing aspect of carbon metabolism that is linked to the aerobic lifestyle of G. xylinus and its inability to metabolize glucose anaerobically is the lack of phosphofructokinase activity in this bacterium (Ross et al. 1991). Whether this alone is the reason why G. xylinus is unable to metabolize glucose anaerobically can now be determined through analysis of the genome sequences available for two different strains of G. xylinus (Iyer et al. 2010; Ogino et al. 2011).

\section{Structure of Cellulose I is Determined by the Cell-Directed Assembly of Glucan Chains}

Microscopic studies of bacterial cells producing cellulose show the presence of cellulose ribbons attached to the longitudinal axis of the cell, and 
freeze-fracture analysis allowed the identification of a linear array of pores in the cell envelope of G. xylinus (Brown et al. 1976; Zaar 1979). The pores represent the cellulose-synthesizing sites and a number of glucan chains arise from a single pore forming a subelementary fibril. These subelementary fibrils assemble with adjoining subelementary fibrils giving rise to microfibrils that assemble into $20-50 \mathrm{~nm}$ wide ribbons in G. xylinus. The rather flat and twisted cellulose ribbon formed by G. xylinus cells is a fascinating structure that is made up of hundreds of glucan chains that associate with each other in a specific manner, giving rise to crystalline cellulose I.

The assembly of the cellulose microfibrils is visualized as a two-step process of polymerization and crystallization, with polymerization of glucose residues to form a glucan chain taking place in the membrane and final (second stage) crystallization of the glucan chains into cellulose I occurring in the extracellular space. This coupled process of polymerization and crystallization can be disturbed in the presence of certain compounds, such as calcofluor, and studies using these compounds have provided evidence that crystallization is the rate-limiting step in the synthesis of cellulose (Benziman et al. 1980). More importantly, assembly of the cellulose I crystallite is proposed to proceed in stages, with the formation of glucan chain sheets by van der Waals bonding in the first stage followed by stacking of the sheets by H-bonding to give rise to the crystalline structure (Cousins and Brown 1995, 1997a, 1997b).

A single G. xylinus cell produces a ribbon of cellulose with 10-100 microfibrils. Each microfibril assembles at a linear terminal complex (TC) that consists of three subunits (cellulose-synthesizing sites) and each subunit contains at least 16 cellulose synthase catalytic subunits (Brown 1996). Each catalytic subunit produces a single $\beta$-1,4-glucan chain, and 16 glucan chains in a single TC subunit form a minicrystal. Minicrystals from three adjacent TC subunits assemble to form a crystalline microfibril, and microfibrils originating from adjacent TCs assemble to form a cellulose ribbon. Assembly of cellulose synthase catalytic subunits with associated proteins to form a TC subunit (cellulose-synthesizing complex) is discussed in a later section.

\section{Cellulose Synthesis in Bacteria is Catalyzed by the Membrane-Associated Cellulose Synthase}

Soon after the identification of UDP-glucose as a sugar donor in glycosyl transfer reactions, it was shown to be the substrate for in vitro synthesis of cellulose in cell-free extracts of G. xylinus (Glaser 1958). However, a breakthrough in determining cellulose synthase activity in cell-free extracts from G. xylinus was not observed until the 1980s, when it was shown that a protein 
fraction in the presence of guanosine triphosphate (GTP) stimulated this activity (Aloni et al. 1982). The protein that activated cellulose synthase activity in the presence of GTP was shown to convert GTP to a cyclic nucleotide that was identified as cyclic diguanylate (c-di-GMP), the activator of cellulose synthase (Ross et al. 1987). The identification of c-di-GMP as an activator of cellulose synthase led to the synthesis of cellulose in sufficient amounts from cell-free extracts and this allowed characterization of the enzyme and the cellulose produced in vitro. The presence of c-di-GMP in the reaction mixture increased cellulose synthase activity 50-100-fold, and using this activator in the reaction mixture, it was clearly demonstrated that this activity resides in the inner membrane in G. xylinus (Bureau and Brown 1987). In addition, a significant quantity of the cellulose product was synthesized in vitro such that it could be characterized by X-ray diffraction, and it was shown to be cellulose II. A robust assay for cellulose synthase activity in vitro allowed further characterization of this enzyme and it was shown that this activity could be solubilized from the membrane fraction using various detergents. Solubilization of the cellulose synthase activity followed by product entrapment aided further in the purification of this enzyme, and in strain ATCC 53582 of G. xylinus two polypeptides of molecular weight (MW) $83 \mathrm{kD}$ and $93 \mathrm{kD}$ were identified in the purified fraction (Lin and Brown 1989). That the cellulose synthase activity was associated with these polypeptides was shown using 5-azido-UDP-glucose as a substrate, and it was found that the $83 \mathrm{kD}$ polypeptide bound to the substrate and therefore was the catalytic subunit of cellulose synthase (Lin et al. 1990). Purification of cellulose synthase activity in G. xylinus 1306-3 allowed identification of polypeptides of MW 90, 67 , and $54 \mathrm{kD}$ and the $67 \mathrm{kD}$ polypeptide was shown to bind c-di-GMP (Mayer et al. 1991). The sequence of this polypeptide was homologous to the $93 \mathrm{kD}$ polypeptide identified in G. xylinus ATCC 53582 and so the $93 \mathrm{kD}$ polypeptide was believed to be the c-di-GMP subunit of the cellulose synthase. However, analysis of c-di-GMP-binding proteins from a number of bacterial species now suggests the role of the PilZ domain in the binding of c-di-GMP by these proteins (Amikam and Galperin 2006). Interestingly, this domain is present in the cellulose synthase catalytic subunit and not in the $93 \mathrm{kD}$ protein that was originally proposed to be the c-di-GMP subunit.

\section{Genes for Cellulose Biosynthesis can be Classified into Three Groups Based on the Function of the Codified Proteins}

Genes for cellulose biosynthesis can be grouped into three main classes: those that are required for synthesis of the cellulose synthase substrate (UDP-glucose), those that are dedicated to cellulose biosynthesis only 
(cellulose synthase and associated proteins), and those that are required for turnover (synthesis and cleavage) of the cellulose synthase activator c-di-GMP. By chance, identification of these three groups of genes took place in the same order as listed here. Genes in the first group were identified by complementation of mutants that produced no pellicle in liquid culture. These mutants produced smooth colonies on agar plates and were considered to be cellulose negative $\left(\mathrm{Cel}^{-}\right)$. Using a genomic library of G. xylinus for complementation of these mutants, genes for UDP-glucose pyrophosphorylase (Valla et al. 1989) and phosphoglucomutase (Fjærvik et al. 1991) were identified and shown to be involved in cellulose biosynthesis because of their role in the synthesis of UDP-glucose. A more detailed analysis of $\mathrm{Cel}^{-}$mutants in G. xylinus strain 1306-3 and their complementation using a genomic library allowed isolation of a large DNA fragment that was shown to carry four genes that were organized as an operon, and these genes were suggested to code for the cellulose synthase and other proteins required for cellulose synthesis in bacteria (Wong et al. 1990). This operon was called the bacterial cellulose synthase (bcs) operon, and the genes were labeled as $b c s A, b c s B, b c s C$, and $b c s D$, with $b c s B$ suggested as the gene coding for cellulose synthase. Direct identification and isolation of the gene for cellulose synthase from G. xylinus strain ATCC 53582 was accomplished at the same time using oligonucleotide probes designed from the $\mathrm{N}$-terminal amino acid sequence of the $83 \mathrm{kD}$ polypeptide identified in the purified cellulose synthase (Saxena et al. 1990). More importantly, the amino acid sequence derived from the $83 \mathrm{kD}$ polypeptide coding gene was found to be homologous to the amino acid sequence derived from the $b c s A$ gene in the $b c s$ operon and correctly identified it to be the gene for cellulose synthase. Sequencing of a region adjacent to the $83 \mathrm{kD}$ polypeptide coding gene led to the identification of other genes, including that coding for the $93 \mathrm{kD}$ polypeptide that was also identified in the purified cellulose synthase (Saxena et al. 1991). Although the organization of genes for cellulose synthesis in G. xylinus strains ATCC 53582 and ATCC 23769 was similar to what was identified in the $b c s$ operon in strain 1306-3, the $b c s A$ and $b c s B$ genes in these strains were found to be fused as a single gene (acs $A B)$. Since this organization of genes for cellulose synthesis was first identified in G. xylinus (Acetobacter xylinum at that time), we named the operon the acetobacter cellulose-synthesizing operon (the acs operon) in which the genes were shown to be organized as $a \operatorname{cs} A B, a c s C$, and $a c s D$ (Saxena et al. 1994). Biochemical analysis revealed that no polypeptide of the size coded for by the $a \operatorname{cs} A B$ gene could be visualized in extracts of G. xylinus ATCC 53582; instead, two polypeptides of $83 \mathrm{kD}$ and $93 \mathrm{kD}$ were present in the purified cellulose synthase, suggesting that posttranslational processing of a large polypeptide results in these two polypeptides. Additionally these two polypeptides were present in a 1:1 ratio in the purified cellulose synthase (Chen and Brown 1996). Interestingly, a second fused cellulose synthase gene (acsAII) was identified in G. xylinus ATCC 23769, and although this gene was not required for cellulose synthesis, cellulose synthase activity determined by this gene was observed in mutants 
with an insertion in the $a c s A B$ gene (Saxena and Brown 1995). The role of the $a \operatorname{cs} A B, a c s C$, and $a c s D$ genes in in vivo and in vitro cellulose synthesis in G. xylinus ATCC 23769 was analyzed by mutant and biochemical analyses and it was found that while both $a \operatorname{cs} A B$ and $a c s C$ are essential for cellulose production in vivo, mutation of the acs $D$ gene affects both the amount and crystalline form of cellulose produced (Saxena et al. 1994).

Two additional genes (ORF1 and ORF2) present upstream of the cellulosesynthesizing operon in G. xylinus were identified during characterization of $\mathrm{Cel}^{-}$mutants (Standal et al. 1994), and both these genes have been suggested to have a role in cellulose biosynthesis. ORF1 codes for a CMCase (an endoglucanase) and the gene is also referred to as cmcax, while ORF2 codes for a protein that is suggested to play a role in both the production and crystallization of cellulose I (Nakai et al. 2002), and this gene is designated as ccp.

An organization of cellulose synthesis genes similar to those identified in G. xylinus strains ATCC 53582, ATCC 23769, and 1306-3 have been identified in a few other strains of this bacterium (Umeda et al. 1999). A representative organization of these genes from two different strains of G. xylinus is shown in Figure 1.1. After the identification of genes for cellulose synthesis in G. xylinus, homologous sequences and a similar organization of cellulose-synthesizing genes have been observed in a number of bacterial species (Nobles and Brown 2007; Römling 2007; Valla et al. 2009); the organization of genes from E. coli is shown in Figure 1.1. This bacterium has no homolog to the G. xylinus bcsD gene, and the endoglucanase gene (bcsZ) is present between $b c s B$ and $b c s C$. In addition, a $y h j Q$ gene is present upstream of $b c s A$ in E. coli. For the sake of uniformity, we suggest that the organization of genes containing the gene for cellulose synthase in bacteria be referred to as the bacterial cellulose synthesis operon (bcs operon) and the genes in this operon be labeled as $b c s A, b c s B$ (or $b c s A B$, where $b c s A$ and $b c s B$ are fused into a single gene), $b c s C$, and $b c s D$ (Figure 1.1).

\section{G. xylinus}

Strain ATCC 23769

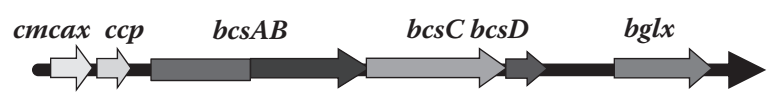

Strain JCM 7664
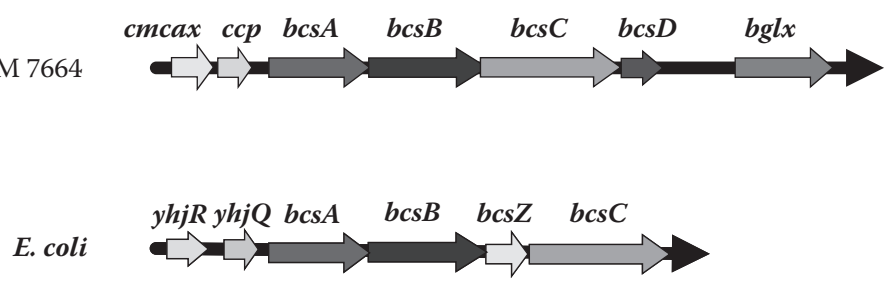

FIGURE 1.1

Organization of genes for cellulose synthase $(b c s A / b c s A B)$ and other proteins involved in cellulose biosynthesis in the bacterial cellulose synthesis (bcs) operon in G. xylinus and E. coli. 
Genes involved in the synthesis and cleavage of c-di-GMP were first identified using oligonucleotide primers designed from the partial amino acid sequence of a purified diguanylate cyclase (Tal et al. 1998). These primers were used to synthesize a short fragment by polymerase chain reaction (PCR), and through screening of a genomic library from G. xylinus strain 1306-11 and genetic analysis of mutants, three operons (cdg1, cdg2, cdg3) for turnover of c-di-GMP were identified in this strain. Each operon carries a diguanylate cyclase $(d g c)$ gene for synthesis of c-di-GMP and a phosphodiesterase-A ( $p d e A)$ gene for cleavage of c-di-GMP. The diguanylate cyclase and phosphodiesterase proteins coded for in these operons were found to have the GGDEF and EAL sequence motifs, and these motifs have since been identified in a large number of proteins in bacteria. Proteins containing these motifs are known to influence a variety of activities through synthesis and breakdown of c-di-GMP, which is now recognized as an important signaling molecule in bacteria.

\section{What is Known About the Proteins Involved in Cellulose Synthesis in Bacteria?}

So far, analyses of proteins encoded by genes in the bcs operon and by genes present adjacent to this operon in G. xylinus have provided only limited information as to their role in cellulose biosynthesis. Some of these proteins have been identified biochemically and a few of their features determined experimentally, while for others their known features are based on sequence analysis and structure determination. These proteins are discussed here briefly to highlight what is currently known about them and their possible role in cellulose synthesis in G. xylinus.

CMCase is a secreted protein coded by ORF1 (cmcax) that exhibits endo$\beta-1,4$-glucanase activity. The structure of this protein has been determined and it belongs to the glycoside hydrolase family 8 (Yasutake et al. 2006). Expression of the cmcax gene is induced by gentiobiose in the medium, and the presence of CMCase protein in the culture medium results in an increase in cellulose production. It is not very clear as to what role this protein may play in cellulose production, but it has been suggested to be partly attached to the cell surface where it affects organization of the glucan chains in the assembly of the cellulose ribbon.

The role of the protein coded by ORF2 ( $c c p)$ was identified through characterization of $\mathrm{Cel}^{-}$mutants of G. xylinus. Not much is known about this protein, although it has been suggested to have a role in both the production and crystallization of cellulose I (Nakai et al. 2002). 
The catalytic subunit of cellulose synthase coded by $b c s A$ or $b c s A B$ is a processive $\beta$-glycosyltransferase (Saxena et al. 1995) that belongs to glycosyltransferase family 2 (GT-2). It is the only protein coded for by a gene in the $b c s$ operon for which a defined role in cellulose biosynthesis is known. This protein catalyzes the synthesis of $\beta$-1,4-linked glucan chains using UDP-glucose as a substrate, and it is activated by c-di-GMP. It is a membrane protein that is localized in the cytoplasmic membrane. It has multiple transmembrane segments and a large globular region that is present in the cytosol. The cytosolic globular region contains the conserved sequence motif D,D,D,QXXRW. Residues in the conserved motif function in the catalytic activity. The structure of this protein has not been determined, although from sequence analysis it has been suggested to have the GT-A fold found in many glycosyltransferases. This protein contains the c-di-GMP-binding PilZ domain for activation by c-di-GMP (Ryjenkov et al. 2006).

The $\mathrm{Bcs} B$ protein is coded by $b c s B$ or $b c s A B$. It is a transmembrane protein, with most of the protein being exposed to the periplasmic space. This protein was originally thought to be the c-di-GMP-binding subunit of the cellulose synthase; however, identification of the c-di-GMP-binding PilZ domain in BcsA suggests that BcsB is not the c-di-GMP component of cellulose synthase. Using antibodies, this protein has been shown to be localized to the linear terminal complexes along the longitudinal axis of the cell (Kimura et al. 2001). This protein does not show significant homology to any other protein in the database. The $b c s B$ gene has been found to be present with bcsA in the bcs operon of almost all bacteria that carry the cellulose synthase gene.

$\mathrm{BcsC}$ is predicted to be localized to the outer membrane and the periplasmic space. Using modeling to determine the structure of this protein, it is predicted to fold into a $\beta$-barrel structure that is present in the outer membrane and a region with tetratricopeptide repeat (TPR) motifs that is present in the periplasmic space. Bioinformatics analysis suggests that $\mathrm{BcsC}$ is involved in the export of cellulose (Keiski et al. 2010). This protein is not required for in vitro cellulose synthase activity, but it is required for cellulose production in vivo. The gene for this protein is present in the $b c s$ operon of almost all bacteria that have this operon.

$\mathrm{BcsD}$ is a soluble protein coded by $b c s D$ in the $b c s$ operon. The structure of BcsD has been determined (Hu et al. 2010). The protein assembles into a cylinder-shaped structure with eight subunits and four inner passageways for glucan chains. The cellular localization of this protein is not confirmed, although it has been suggested to be present in the periplasmic space (Iyer et al. 2011). This protein is not required for in vitro cellulose synthase activity and is not essential for cellulose production in vivo. $b c s D$ mutants produce small amounts of cellulose. The $b c s D$ gene is found in G. xylinus and only a few other bacterial species. 


\section{"Guilt by Association" Model of the G. xylinus Cellulose-Synthesizing Complex}

What is the composition of a cellulose-synthesizing complex and how are these complexes assembled and localized along the longitudinal axis in a G. xylinus cell? In the absence of isolated complexes and because of a lack of information with respect to the nature and number of protein subunits in these complexes, any attempt to understand them will require some genetic, biochemical, and structural information. Fortunately, sufficient information is available from a variety of studies to make a beginning toward understanding the possible assembly of a cellulose-synthesizing complex. Although protein-protein interactions have not been demonstrated for any of the proteins coded for by genes in the bcs operon, the fact that they are coded in this operon makes them possible candidates for interaction (guilt by association in an operon). In addition, mutant analysis has clearly shown that all the proteins coded by genes in the bcs operon have a role in cellulose synthesis. At a minimum, each cellulose-synthesizing site (a TC subunit) could be comprised of a homomultimeric assembly of the cellulose synthase catalytic subunits (BcsAs). Considering the hierarchical assembly of sheets to minicrystals to microfibrils to a ribbon in G. xylinus (Brown 1996), each TC subunit could be an assembly of up to 16 closely packed BcsAs in the cytoplasmic membrane. Since $b c s B$ is present in almost all bacteria next to the $b \operatorname{cs} A$ coding region or as part of the $b \operatorname{cs} A B$ gene, $\mathrm{BcsB}$ must be part of the cellulose synthase complex, most likely in equal proportions to BcsA. Biochemical analysis of purified cellulose synthase always shows the presence of an $83 \mathrm{kD}$ polypeptide (corresponding to BcsA) and a $93 \mathrm{kD}$ polypeptide (corresponding to BcsB), suggesting a tight association of these two polypeptides in a 1:1 ratio (Chen and Brown 1996). Sequence analysis and TnPhoA mutagenesis experiments suggest that BcsB present in the cytoplasmic membrane as a transmembrane protein is exposed much more on the periplasmic side of the membrane (Saxena et al. 1994). The BcsC protein is present in the outer membrane through a $\beta$-barrel fold and extends into the periplasmic space where it has a number of TPR repeats that are suggested to function in protein-protein interactions (Keiski et al. 2010). The periplasmic region of $\mathrm{BcsC}$ most likely associates with the periplasmic portion of BcsB. The BcsD protein is most likely a cytosolic protein, and it forms a complex that associates with the cytosolic region of BcsA. The glucan chains synthesized in the cytosolic region of BcsA stay closely attached to the BcsA-BcsB-BcsC complex in a rigid and unassembled state until they reach the extracellular side, where they can associate with each other to form higher-order structures (Figure 1.2). 
Top view
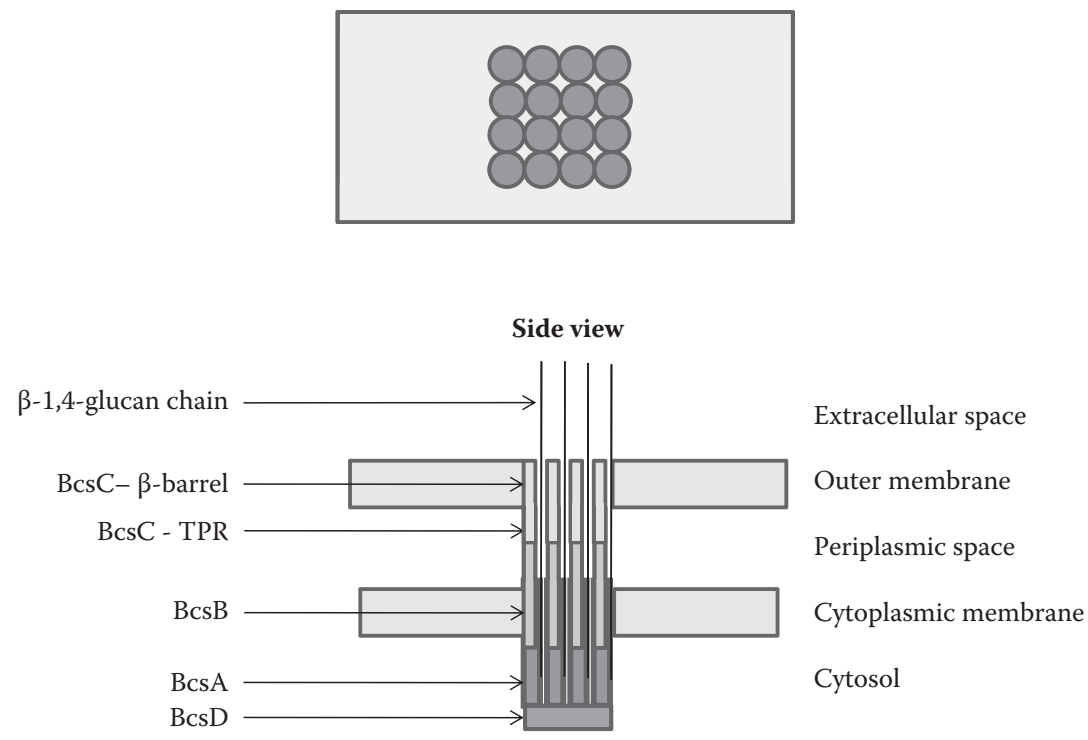

FIGURE 1.2

Organization of proteins in a cellulose-synthesizing complex (TC subunit) in G. xylinus. The top view shows the cytosolic side of 16 cellulose synthase catalytic subunits (BcsA) that are suggested to be present in a single complex in the cytoplasmic membrane. The side view shows four $\beta-1,4$-glucan chains being synthesized and translocated across the bacterial envelope in a region with an assembly of four $\mathrm{Bcs} A$ molecules in association with $\mathrm{B} \operatorname{cs} \mathrm{B}, \mathrm{B} \operatorname{cs} \mathrm{C}$, and $\mathrm{BcsD}$. Although BcsA is localized in the cytoplasmic membrane, a large globular region of this protein is present in the cytosol, and it is this region that contains the catalytic site. The BcsB protein is also localized in the cytoplasmic membrane, but it is exposed more toward the periplasmic space. This protein interacts with BcsA in the transmembrane region and with the TPR-containing region of $\mathrm{BcsC}$ in the periplasmic space. $\mathrm{BcsC}$ is present in the outer membrane where it has a $\beta$-barrel fold. It also extends into the periplasmic space and this region of BcsC has a number of TPR repeats that allow protein-protein interactions. The localization of BcsD is still uncertain, although there has been a suggestion of it being present in the periplasmic space. In the absence of any sequence features for it to be transported, we feel that this protein is localized in the cytosol where it may interact with the cytosolic region of BcsA molecules and keep them in an assembly. BcsD may also interact with glucan chains as they move from the cytosol into the BcsA membrane region. The glucan chains synthesized in the cytosolic region of BcsA are shown to stay closely attached to the BcsA-BcsB-BcsC complex in a rigid and unassembled state until they reach the extracellular side, where they can associate with each other to form higher-order structures.

\section{Many Unanswered Questions Remain}

The synthesis of a polysaccharide chain can be visualized as taking place in three stages: initiation, elongation, and termination, much like the synthesis of nucleic acids and proteins. However, unlike the requirement of a template for the synthesis of these polymers, no template is required for the 
synthesis of polysaccharide chains. At the same time, similar to these polymers, a single polymerizing enzyme is sufficient for the processive synthesis of homopolymers such as the $\beta$-1,4-glucan chains of cellulose. The mechanism by which $\beta$-1,4-glucan chain initiation occurs during cellulose synthesis in bacteria is not known, but it is evident from in vitro cellulose synthesis experiments that the enzyme cellulose synthase is sufficient for initiation and elongation of the glucan chains, and no primer is required for chain initiation. The glucan chains stay tightly associated with the cellulose synthase during cellulose synthesis and not much is known about the mechanism of chain termination. During cellulose synthesis by living cells, the cellulose microfibrils may either form a network, as in the case of G. xylinus, or associate with other components of the cell wall, as in the case of plants. In either case, the cellulose product gets fixed, and in the case of bacteria, the cells may move during cellulose synthesis until they get trapped in the cellulose network, whereas in the case of plants it is the cellulose synthases that move within the plane of the plasma membrane (Brown and Montezinos 1976). It is quite likely that chain termination may result due to a physical breakage of the microfibrils from the cells when either the bacterial cells or the cellulose synthases in plant cells are no longer able to move.

Although we can debate whether the repeating subunit in the $\beta-1,4$-glucan chains in cellulose is glucose or cellobiose, there is no doubt about the two-fold symmetry of these chains. The mechanism by which the adjacent glucose residues assume a $180^{\circ}$ rotation with respect to each other in the $\beta$-1,4-glucan chains is not clearly understood and a model proposing two UDP-glucose binding sites in the cellulose synthase suggested that this feature of the glucan chains is generated during cellulose synthesis (Saxena et al. 1995). Since then, other proposals have suggested that a dimer of cellulose synthases, each with a single UDP-glucose binding site, may be involved in generating the $180^{\circ}$ orientation of adjacent glucose residues, or that a single cellulose synthase with a single UDP-glucose binding site can add glucose residues to the growing end and these residues would then be able to rotate on their own once they have exited the catalytic site (Delmer 1999). Unfortunately the crystallographic structure of cellulose synthase remains to be determined, and in its absence this question remains unanswered.

\section{Many Bacteria Have Genes for Cellulose Synthesis, but Only a Few Produce Cellulose}

Cellulose production by Bacterium xylinum was described by A. J. Brown in 1886, almost 50 years after the first description of cellulose from plant tissues by Anselm Payen in 1838. G. xylinus has been the organism of choice for 
studying cellulose biosynthesis, for the reasons mentioned earlier, and until the 1990s only a few other bacteria were known to produce cellulose (Ross et al. 1991). In this group of other bacteria, cellulose production was analyzed in some detail in Agrobacterium tumefaciens, where cellulose was suggested to play a role in the infection of plants (Matthysse 1983). However, soon after the identification of the gene for cellulose synthase and other genes in the bacterial cellulose-synthesizing operon in G. xylinus, these genes were identified in other bacteria, suggesting that these bacteria have the potential to synthesize cellulose. Interestingly, one of the first organisms in which these genes were identified through analysis of the partial genome sequence was E. coli (Sofia et al. 1994). We were not completely surprised by this discovery, as we had detected c-di-GMP-activated in vitro cellulose synthase activity in permeabilized cells and crude membrane fraction from certain strains of E. coli (Saxena and Brown 2002) even before cellulose synthesis was demonstrated in this bacterium (Zogaj et al. 2001). Although genes for cellulose synthesis were identified in A. tumefaciens by complementation of $\mathrm{Cel}^{-}$mutants (Matthysse et al. 1995), in most other bacteria these genes have been identified from analysis of the genome sequences (Nobles and Brown 2007; Römling 2007; Valla et al. 2009). Using the BcsA sequence from G. xylinus, and the homologous sequences from A. tumefaciens and Nostoc, as the query in a BLAST search, homologous sequences were identified in cyanobacteria, Firmicutes, Planctomycetes, Aquificae, and proteobacteria belonging to the $\alpha, \beta, \gamma$, and $\delta$ subdivisions (Valla et al. 2009). The presence of the cellulose synthase sequence in all these groups suggests that this sequence likely predates the divergence of these groups (Nobles and Brown 2007). Although it has not been possible to study the evolution of cellulose synthesis in bacteria using the cellulose synthase sequences, relationships between different bacteria have been established by analyzing the organization of genes for cellulose synthesis and grouping them into four gene clusters (Nobles and Brown 2007). Group I gene clusters are present in the $\alpha, \beta$, and $\gamma$ subdivisions of proteobacteria, and this cluster represents the organization of cellulose-synthesizing genes associated with the cellulose synthase gene that is observed in G. xylinus and E. coli (Figure 1.1).

Even as the ability to synthesize cellulose is being recognized in many more bacterial species based on genome sequences, identification of celluloseproducing bacteria from natural habitats should be pursued more vigorously. An example of this endeavor is the identification of Asaia bogorensis as a novel cellulose producer (Kumagai et al. 2011). This bacterium produces a pellicle with ultrafine cellulose fibers and has an organization of genes for cellulose synthesis that is similar to that observed in G. xylinus, albeit with a $y h j Q$ gene upstream of the $b c s A B$ gene. Although the amount of cellulose produced by this bacterium is less than what is produced by strains of G. xylinus, it is much more than what is known to be produced by the other cellulose-producing bacteria, and so not only does this bacterium offer a new system to study cellulose biosynthesis, it is also a source of BNC 
with unique properties. Modification of cellulose synthesis in G. xylinus by genetic manipulations has not been very successful, and thus identification of new bacterial species with the ability to produce significant quantities of cellulose with novel and desirable properties may allow for a much wider range of applications of $\mathrm{BNC}$.

\section{Acknowledgment}

Supported in part by Welch Grant F1217 to R. Malcolm Brown, Jr.

\section{References}

Aloni, Y., D. P. Delmer, and M. Benziman. 1982. Achievement of high rates of in vitro synthesis of 1,4- $\beta$-glucan: activation by cooperative interaction of the Acetobacter xylinum enzyme system with GTP, polyethylene glycol, and a protein factor. Proc. Natl. Acad. Sci. USA 79:6448-6452.

Amikam, D., and M. Y. Galperin. 2006. PilZ domain is part of the bacterial c-di-GMP binding protein. Bioinformatics 22:3-6.

Benziman, M., C. H. Haigler, R. M. Brown, Jr., A. R. White, and K. M. Cooper. 1980. Cellulose biogenesis: polymerization and crystallization are coupled processes in Acetobacter xylinum. Proc. Natl. Acad. Sci. USA 77:6678-6682.

Brown, R. M., Jr.. 1996. The biosynthesis of cellulose. J. Macromol. Sci. - Pure Appl. Chem. A33:1345-1373.

Brown, R. M., Jr., and D. Montezinos. 1976. Cellulose microfibrils: visualization of biosynthetic and orienting complexes in association with the plasma membrane. Proc. Natl. Acad. Sci. USA 73:143-147.

Brown, R. M., Jr., J. H. M. Willison, and C. L. Richardson. 1976. Cellulose biosynthesis in Acetobacter xylinum: visualization of the site of synthesis and direct measurement of the in vivo process. Proc. Natl. Acad. Sci. USA 73:4565-4569.

Bureau, T. E., and R. M. Brown, Jr. 1987. In vitro synthesis of cellulose II from a cytoplasmic membrane fraction of Acetobacter xylinum. Proc. Natl. Acad. Sci. USA 84:6985-6989.

Chen, H. P., and R. M. Brown, Jr. 1996. Immunochemical studies of the cellulose synthase complex in Acetobacter xylinum. Cellulose 3:63-75.

Cousins, S. K., and R. M. Brown, Jr. 1995. Cellulose I microfibril assembly: computational molecular mechanics energy analysis favors bonding by van der Waals forces as the initial step in crystallization. Polymer 36:3885-3888.

Cousins, S. K., and R. M. Brown, Jr. 1997a. Photoisomerization of a dye-altered $\beta$-1,4-glucan sheet induces the crystallization of a cellulose composite. Polymer 38:903-912. 
Cousins, S. K., and R. M. Brown, Jr. 1997b. X-ray diffraction and ultrastructural analyses of dye-altered celluloses support van der Waals forces as the initial step in cellulose crystallization. Polymer 38:897-902.

Delmer, D. P. 1999. Cellulose biosynthesis: exciting times for a difficult field of study. Ann. Rev. Plant Physiol. Plant Mol. Biol. 50:245-276.

Fjærvik, E., K. Frydenlund, S. Valla, Y. Huggirat, and M. Benziman. 1991. Complementation of cellulose-negative mutants of Acetobacter xylinum by the cloned structural gene for phosphoglucomutase. FEMS Microbiol. Lett. 77:325-330.

Glaser, L. 1958. The synthesis of cellulose in cell-free extracts of Acetobacter xylinum. J. Biol. Chem. 232:627-636.

Hu, S.-Q., Y.-G. Gao, K. Tajima, N. Sunagawa, Y. Zhou, S. Kawano, T. Fujiwara, T. Yoda, D. Shimura, Y. Satoh, M. Munekata, I. Tanaka, and M. Yao. 2010. Structure of bacterial cellulose synthase subunit D octamer with four inner passageways. Proc. Natl. Acad. Sci. USA 107:17957-17961.

Iyer, P. R., J. Catchmark, N. R. Brown, and M. Tien. 2011. Biochemical localization of a protein involved in synthesis of Gluconacetobacter hansenii cellulose. Cellulose 18:739-747.

Iyer, P. R., S. M. Geib, J. Catchmark, T. Kao, and M. Tien. 2010. Genome sequence of a cellulose-producing bacterium, Gluconacetobacter hansenii ATCC 23769. J. Bacteriol. 192:4256-4257.

Keiski, C.-L., M. Harwich, S. Jain, A. M. Neculai, P. Yip, H. Robinson, J. C. Whitney, L. Riley, L. L. Burrows, D. E. Ohman, and P. L. Howell. 2010. AlgK is a TPR-containing protein and the periplasmic component of a novel exopolysaccharide secretin. Structure 18:265-273.

Kimura, S., H. P. Chen, I. M. Saxena, R. M. Brown, Jr., and T. Itoh. 2001. Localization of c-di-GMP-binding protein with the linear terminal complexes of Acetobacter xylinum. J. Bacteriol. 183:5668-5674.

Kumagai, A., M. Mizuno, N. Kato, K. Nozaki, E. Togawa, S. Yamanaka, K. Okuda, I. M. Saxena, and Y. Amano. 2011. Ultrafine cellulose fibers produced by Asaia bogorensis, an acetic acid bacterium. Biomacromolecules 12:2815-2821.

Lin, F. C., and R. M. Brown, Jr. 1989. Purification of cellulose synthase from Acetobacter xylinum. In: Cellulose and Wood-Chemistry and Technology, ed. C. Schuerch, 473-492. New York: John Wiley \& Sons.

Lin, F. C., R. M. Brown, Jr., R. R. Drake, Jr., and B. E. Haley. 1990. Identification of the uridine 5 '-diphosphoglucose (UDP-glc) binding subunit of cellulose synthase in Acetobacter xylinum using the photoaffinity probe 5-azido-UDP-glc. J. Biol. Chem. 265:4782-4784.

Matthysse, A. 1983. Role of bacterial cellulose fibrils in Agrobacterium tumefaciens infection. J. Bacteriol. 154:906-915.

Matthysse, A., S. White, and R. Lightfoot. 1995. Genes required for cellulose synthesis in Agrobacterium tumefaciens. J. Bacteriol. 177:1069-1075.

Mayer, R., P. Ross, H. Weinhouse, D. Amikam, G. Volman, P. Ohana, R. D. Calhoon, H. C. Wong, A. W. Emerick, and M. Benziman. 1991. Polypeptide composition of bacterial cyclic diguanylic acid-dependent cellulose synthase and the occurrence of immunologically crossreacting proteins in higher plants. Proc. Natl. Acad. Sci. USA 88:5472-5476. 
Nakai, T., Y. Nishiyama, S. Kuga, Y. Sugano, and M. Shoda. 2002. ORF2 gene involves in the construction of high-order structure of bacterial cellulose. Biochem. Biophys. Res. Commun. 295:458-462.

Nobles, D. R., Jr., and R. M. Brown, Jr. 2007. Many paths up the mountain: tracking the evolution of cellulose biosynthesis. In: Cellulose: Molecular and Structural Biology, eds. R. M. Brown, Jr. and I. M. Saxena, 1-15. Dordrecht: Springer.

Ogino, H., Y. Azuma, A. Hosoyama, H. Nakazawa, M. Matsutani, A. Hasegawa, K. Otsuyama, K. Matsushita, N. Fujita, and M. Shirai. 2011. Complete genome sequence of NBRC 3288, a unique cellulose-nonproducing strain of Gluconacetobacter xylinus isolated from vinegar. J. Bacteriol. 193:6997-6998.

Roberts, E. M., I. M. Saxena, and R. M. Brown, Jr. 1989. Biosynthesis of cellulose II in Acetobacter xylinum. In: Cellulose and Wood-Chemistry and Technology, ed. C. Schuerch, 689-704. New York: John Wiley \& Sons.

Römling, U. 2007. Cellulose biosynthesis in enterobacteriaceae. In: Cellulose: Molecular and Structural Biology, eds. R. M. Brown, Jr. and I. M. Saxena, 107-122. Dordrecht: Springer.

Ross, P., R. Mayer, and M. Benziman. 1991. Cellulose biosynthesis and function in bacteria. Microbiol. Rev. 55:35-58.

Ross, P., H. Weinhouse, Y. Aloni, D. Michaeli, P. Weinberger-Ohana, R. Mayer, S. Braun, E. de Vroon, G. A. van der Marel, J. H. van Boom, and M. Benziman. 1987. Regulation of cellulose synthesis in Acetobacter xylinum by cyclic diguanylic acid. Nature 325:279-281.

Ryjenkov, D. A., R. Simm, U. Römling, and M. Gomelsky. 2006. The PilZ domain is a receptor for the second messenger c-di-GMP: the PilZ domain protein YcgR controls motility in enterobacteria. J. Biol. Chem. 281:30310-30314.

Saxena, I. M., and R. M. Brown, Jr. 1995. Identification of a second cellulose synthase gene (acsAII) in Acetobacter xylinum. J. Bacteriol. 177:5276-5283.

Saxena, I. M., and R. M. Brown, Jr. 2002. Cellulose synthase activity in E. coli. Paper presented at the 1st International Cellulose Conference, Nov. 6-8, 2002, Kyoto, Japan.

Saxena, I. M., R. M. Brown, Jr., M. Fevre, R. A. Geremia, and B. Henrissat. 1995. Multidomain architecture of $\beta$-glycosyltransferases: implications for mechanism of action. J. Bacteriol. 177:1419-1424.

Saxena, I. M., K. Kudlicka, K. Okuda, and R. M. Brown, Jr. 1994. Characterization of genes in the cellulose-synthesizing operon (acs operon) of Acetobacter xylinum: implications for cellulose crystallization. J. Bacteriol. 176:5735-5752.

Saxena, I. M., F. C. Lin, and R. M. Brown, Jr. 1990. Cloning and sequencing of the cellulose synthase catalytic subunit gene of Acetobacter xylinum. Plant Mol. Biol. 15:5735-5752.

Saxena, I. M., F. C. Lin, and R. M. Brown, Jr. 1991. Identification of a new gene in an operon for cellulose biosynthesis in Acetobacter xylinum. Plant Mol. Biol. 16:947-954.

Sofia, H. J., V. Burland, D. L. Daniels, G. Plunkett III, and F. R. Blattner. 1994. Analysis of the Escherichia coli genome. V. DNA sequence of the region from 76.0 to 81.5 minutes. Nucleic Acids Res. 22:2576-2586.

Standal, R., T. G. Iversen, D. H. Coucheron, E. Fjærvik, J. M. Blatny, and S. Valla. 1994. A new gene required for cellulose production and a gene encoding cellulolytic activity in Acetobacter xylinum are colocalized with the bcs operon. J. Bacteriol. 176:665-672. 
Tal, R. H., H. C. Wong, R. Calhoon, D. Gelfand, A. L. Fear, G. Volman, R. Mayer, P. Ross, D. Amikam, H. Weinhouse, A. Cohen, S. Sapir, P. Ohana, and M. Benziman. 1998. Three $c d g$ operons control the turnover of cyclic di-GMP in Acetobacter xylinum: genetic organization and occurrence of conserved domains in isoenzymes. J. Bacteriol. 180:4416-4425.

Umeda, Y., A. Hirano, M. Ishibashi, H. Akiyama, T. Onizuka, M. Ikeuchi, and Y. Inoue. 1999. Cloning of cellulose synthase genes from Acetobacter xylinum JCM 7664: implication of a novel set of cellulose synthase genes. DNA Res. 6:109-115.

Valla, S., D. H. Coucheron, E. Fjærvik, J. Kjosbakken, H. Weinhouse, P. Ross, D. Amikam, and M. Benziman. 1989. Cloning of a gene involved in cellulose biosynthesis in Acetobacter xylinum: complementation of cellulose-negative mutants by the UDPG pyrophosphorylase structural gene. Mol. Gen. Genet. 217:26-30.

Valla, S., H. Ertesvåg, N. Tonouchi, and E. Fjærvik. 2009. Bacterial cellulose production: biosynthesis and applications. In: Microbial Production of Biopolymers and Polymer Precursors: Applications and Perspectives, ed. B. H. A. Rehm, 43-77. Norfolk, UK: Caister Academic Press.

Valla, S., and J. Kjosbakken. 1982. Cellulose-negative mutants of Acetobacter xylinum. J. Gen. Microbiol. 128:1401-1408.

Wong, H. C., A. L. Fear, R. D. Calhoon, G. H. Eichinger, R. Mayer, D. Amikam, M. Benziman, D. H. Gelfand, J. H. Meade, A. W. Emerick, R. Bruner, A. Ben-Bassat, and R. Tal. 1990. Genetic organization of the cellulose synthase operon in Acetobacter xylinum. Proc. Natl. Acad. Sci. USA 87:8130-8134.

Yasutake, Y., S. Kawano, K. Tajima, M. Yao, Y. Satoh, M. Munekata, and I. Tanaka. 2006. Structural characterization of the Acetobacter xylinum endo- $\beta$-1,4-glucanase CMCax required for cellulose biosynthesis. Proteins 64:1069-1077.

Zaar, K. 1979. Visualization of pores (export sites) correlated with cellulose production in the envelope of the Gram-negative bacterium Acetobacter xylinum. J. Cell Biol. 80:773-777.

Zogaj, X., M. Nimtz, M. Rohde, W. Bokranz, and U. Römling. 2001. The multicellular morphotypes of Salmonella typhimurium and Escherichia coli produce cellulose as the second component of the extracellular matrix. Mol. Microbiol. 39:1452-1463. 


\title{
Effect of Cultivation Conditions on the Structure and Morphological Properties of BNC Biomaterials with a Focus on Vascular Grafts
}

\author{
Paul Gatenholm, ${ }^{1}$ Kim Höglund, ${ }^{1}$ Sara Johannesson, ${ }^{1}$ Maja Puchades, ${ }^{1}$ \\ Christian Brackmann, ${ }^{2}$ Annika Enejder, ${ }^{2}$ and Lisbeth Olsson ${ }^{3}$ \\ ${ }^{1}$ Department of Chemical and Biological Engineering, \\ Biosynthetic Blood Vessel Laboratory, Biopolymer Technology, \\ Chalmers University of Technology, Gothenburg, Sweden \\ ${ }^{2}$ Department of Chemical and Biological Engineering, \\ Life Sciences, Molecular Microscopy, and Biotechnology, \\ Chalmers University of Technology, Gothenburg, Sweden \\ ${ }^{3}$ Department of Chemical and Biological Engineering, Industrial Biotechnology, \\ Chalmers University of Technology, Gothenburg, Sweden
}

\section{CONTENTS}

Introduction

Bacterial Cellulose Production: A Part of the Carbon Metabolism in

G. xylinus .22

Factors Involved during Nanocellulose Network Formation by G. xylinus ....24 Effect of Growth Medium Components on Bacterial Cellulose

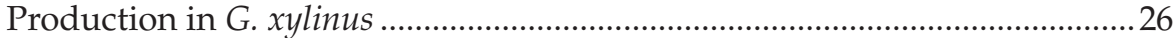

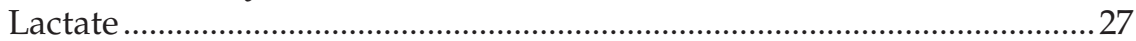

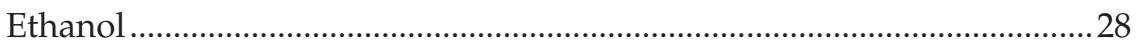

Carbon Substrate ................................................................................... 28

Corn Steep Liquor ..................................................................................... 28

Genetic Modifications of G. xylinus for Enhanced Production of

Bacterial Cellulose......................................................................................229

Transfection of G. xylinus with the pBla-VHb-122 Plasmid ......................29

Spontaneous Mutation in G. xylinus Can Lead to a Loss of the

Ability to Produce Cellulose...................................................................... 30

Production of Artificial Blood Vessels by G. xylinus in Bioreactors ................ 31

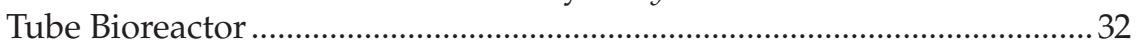

Cellulose Production Yield and Cellulose Content ......................................35

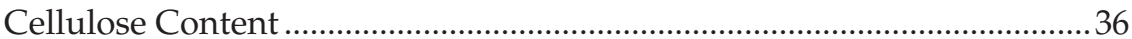


Influence of Oxygen Availability on Tube Formation................................36

Properties and Components of CSL .......................................................... 37

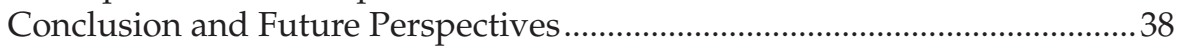

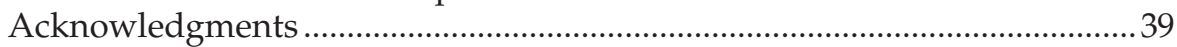

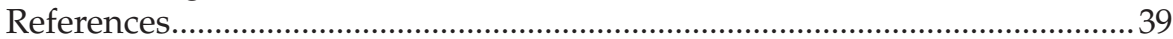

New materials that are not thrombogenic and have mechanical properties that mimic the native blood vessel are in very great demand. Nanocellulose produced by the bacteria Gluconacetobacter xylinus is a biomaterial that has gained interest in the field of tissue engineering because of its unique properties, such as great mechanical strength, high water content (around $99 \%$ ), and the ability to be shaped into three-dimensional structures during biosynthesis. The fabrication process of bacterial nanocellulose (BNC) vascular grafts is very unique because the material synthesis and product formation takes place simultaneously. The biomechanical performance, which includes rupture pressure and compliance along with biological response (endothelialization, blood compatibility, etc.), is dependent on the morphology of a fibrillar network. The network formation is affected by cellulose assembly and bacteria motion, proliferation rate, and other factors. An understanding of the effects of cultivation conditions on BNC network formation is therefore of great importance.

\section{Introduction}

The network of cellulose nanofibrils produced by the acetic acid bacteria Gluconacetobacter xylinus creates a biomaterial with great mechanical strength, high water content (around 99\%), and the ability to be shaped into three-dimensional (3D) structures during biosynthesis. Cultivation can be done in the presence of molds and porogens of different sizes and shapes, which makes it possible to design desirable biomaterial geometries. Bacterial nanocellulose (BNC) has been used in wound healing applications (Wei et al. 2011; Zhang et al. 2010), cartilage repair (Svensson et al. 2005), bone regeneration (Zaborowska et al. 2010), and most recently, for blood vessel replacements (Wippermann et al. 2009). The focus of this review will be on the development of tubular cellulose scaffolds for use as small-caliber artificial blood vessels (see Figure 2.1).

Cardiovascular disease is the major cause of death and morbidity in Western countries. Surgical bypass with autologous grafts remains the most used treatment. However, many patients in need of reconstruction of their vascular system, either in the heart, lower extremities, or other locations, do not have suitable vessels due to preexisting vascular disease or amputation. Approximately $5 \%-10 \%$ of all patients that need coronary bypass need a good 


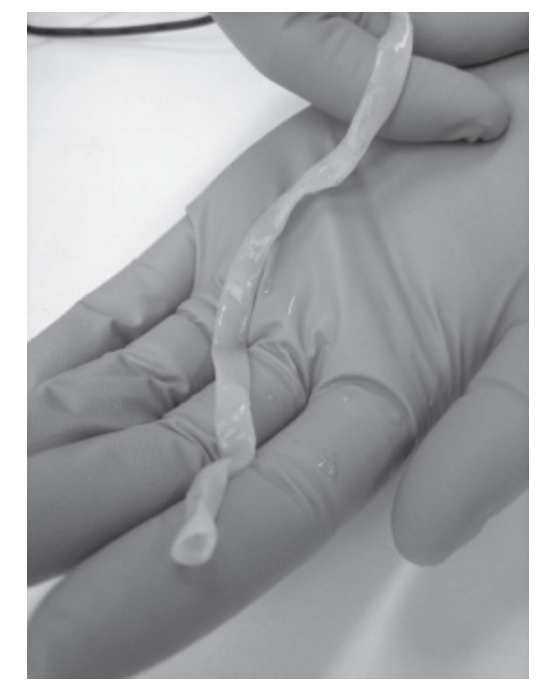

FIGURE 2.1

(See color insert.) An artificial blood vessel synthesized by the bacteria G. xylinus.

replacement for their own autologous grafts. Today it is possible to replace large arteries with synthetic grafts (e.g., Dacron, expanded polytetrafluoroethylene [ePTFE], and polyurethane). However, these materials give poor performance for small (less than $6 \mathrm{~mm}$ ) grafts due to the formation of thrombosis and occlusion (Hess et al. 1985). New materials that are not thrombogenic and have mechanical properties that mimic the native blood vessel are needed. Nanocellulose produced by G. xylinus is a biomaterial that has gained interest in the field of tissue engineering owing to its unique properties (i.e., great mechanical strength, high water content, and the ability to be shaped into 3D structures during biosynthesis). Cultivation can be done in the presence of molds and porogens of different sizes and shapes, making it possible to design desirable scaffold geometries (Bäckdahl et al. 2008; Bodin et al. 2007). Several generations of bioreactors have been designed and constructed to grow BNC tubes. Bioreactors have been designed and fermentation conditions developed that allow the growth of tubes in various diameters and lengths $(1-10 \mathrm{~mm}$ in diameter, $4-30 \mathrm{~cm}$ in length) as well as in various shapes. Several techniques to alter the porosity of BNC are currently under investigation.

The fabrication process of BNC vascular grafts is very unique because the material synthesis and product formation take place simultaneously. The biomechanical performance, including rupture pressure and compliance along with biological response (endothelialization, blood compatibility, etc.), is dependent on the morphology of the fibrillar network. The network formation is affected by cellulose assembly and bacteria motion, proliferation rate, and other factors. An understanding of the effects of cultivation conditions on BNC network formation is therefore of great importance. 


\section{Bacterial Cellulose Production: A Part of the Carbon Metabolism in G. xylinus}

The production of BNC in G. xylinus is a part of its carbon metabolism (Figure 2.2). Depending on the main carbon source used in the growth media, different initial pathways toward the central metabolites, $x$ and $y$, are used. The initial pathways for fructose and glucose are separate, but both lead to the central carbon metabolite glucose-6-phosphate (Figure 2.2).

G. xylinus can use carbon sources such as manitol, glycerol, sucrose, and galactose, in addition to glucose and fructose (Mikkelsen et al. 2009). When G. xylinus is cultivated in glucose- or sucrose-based media, 2- and 5-ketogluconate acids are produced by the conversion of glucose into gluconate by the enzyme glucose oxidase. The production of ketogluconate acids lowers the yield of BNC produced and contributes to a decrease in $\mathrm{pH}$. De Wulf et al. (1996) demonstrated that G. xylinus mutants, restricted in ketogluconic acids synthesis, are able to produce more BNC compared to wild-type G. xylinus.

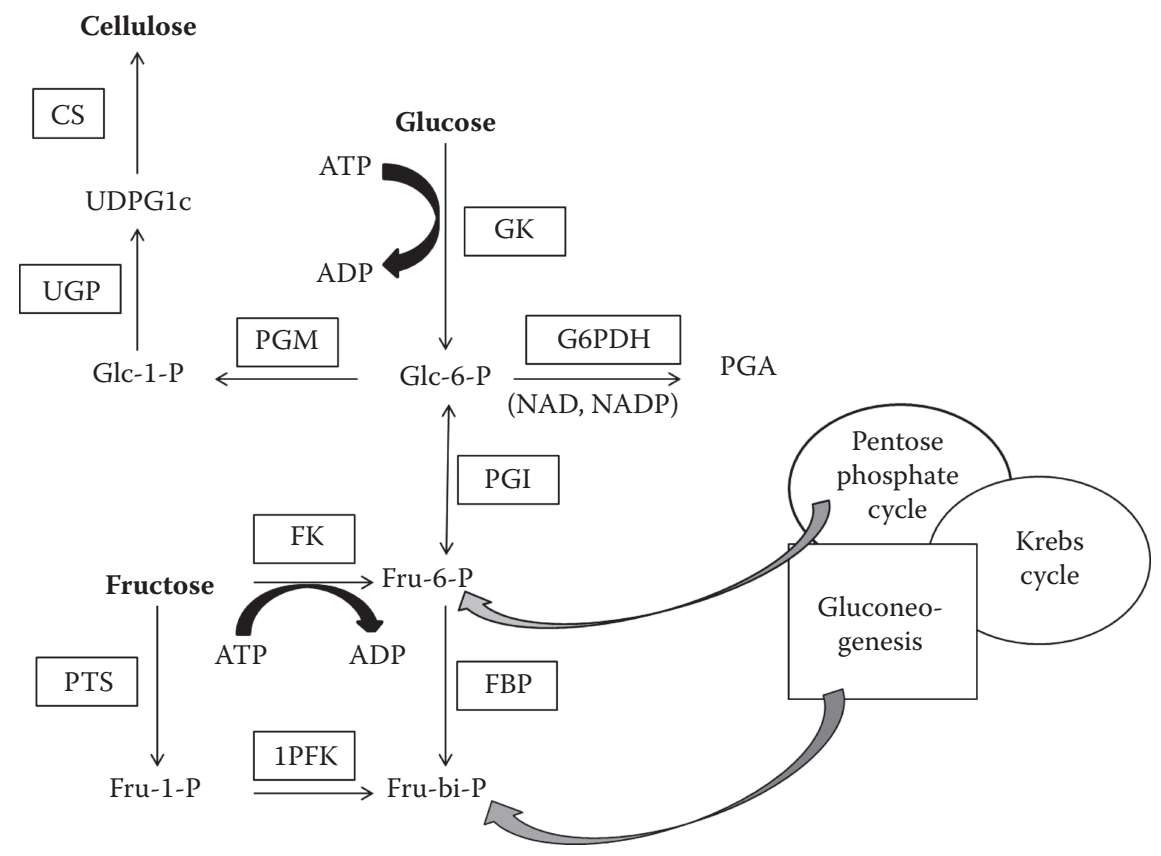

FIGURE 2.2

Illustration of the carbon metabolism in G. xylinus. Figure adapted from Ross et al. (1991). CS: cellulose synthase; FBP: fructose-1,6-biphosphate phosphatase; FK: glucokinase; G6PDH: glucose-6-phosphate dehydrogenase; 1PFK: fructose-1-phosphate kinase; PGI: phosphoglucoisomerase; PGM: phosphoglucomutase; PTS: system of phosphotransferases; UGP:pyrophosphorylase UDPGlc; Fru-bi-P: fructose-1,6-bi-phosphatase; Fru-6-P: fructose-6-phosphate; Glc-6(1)-P: glucose-6(1)phosphate; PGA: phosphogluconic acid; UDPGlc: uridine diphosphoglucose. 
This further supports the fact that the formation of ketogluconic acids is not optimal for BNC production. If fructose is used as the carbon source in growth media, only cellulose, acetan, and carbon dioxide are produced (Ross et al. 1991). Studies have shown that fructose utilization is suppressed when G. xylinus is cultivated in glucose-based growth media, which is not the case in sucrose-based media. This is probably because glucose has an inhibitory effect on fructokinase via the competition with fructose. Glucose is therefore physiologically significant, as it may serve as a sensitive regulatory mechanism (Benzamin and Rivertz 1972). On the other hand, glucose can be utilized as a substrate even if fructose or sucrose is added to the growth media as the main carbon source (Benzamin and Rivertz 1972).

The initial metabolic steps in the fructose and glucose utilization pathways are initially different, whereas, from glucose-6-phosphate and further, the same metabolic pathway is followed for BNC production. Glucose is taken up by the bacteria and transformed into glucose-6-phosphate by glucokinase. Fructose, on the other hand, is first phosphorylated into fructose- 6 phosphate by fructokinase and thereafter transformed into glucose-6-phosphate by phosphoglucoisomerase. Glucose-6-phosphate is transformed via isomerization into glucose-1-phosphate by phosphoglucomutase followed by transformation of glucose-1-phosphate into uridine diphosphate glucose (UDP-glucose) by pyrophosphorylase UDPGlc (Ross et al. 1991). G. xylinus mutants that are cellulose negative $\left(\mathrm{Cel}^{-}\right)$lack pyrophosphorylase UDPGlc and can therefore not produce UDP-glucose and BNC, since UDP-glucose is a crucial intermediate for cellulose synthesis (Krystynowicz et al. 2005). The organization of cellulose synthase in the membrane is important for regulating diverse cellular functions, including motility, biofilm formation, cell cycle progression, and virulence in bacteria. Cellulose synthase is regulated by the cyclic nucleotide bis- $\left(3^{\prime}, 5^{\prime}\right)$-cyclic dimeric guanosine monophosphate (c-di-GMP), which acts as a reversible allosteric activator for the enzyme; that is, when c-di-GMP binds cellulose synthase (at a site distant to the active site), it enhances the activity of the enzyme. The concentration of c-di-GMP is in turn regulated by diguanylate cyclase, which activates c-di-GMP and phosphodiesterase A and B (PDE-A and PDE-B) that degrade c-di-GMP. The degradation mechanism of c-di-GMP by PDE-A and PDE-B is still unclear (Ross et al. 1990). PDE-A is $\mathrm{Ca}^{2+}$ sensitive and studies have concluded that PDE-A is inhibited by $\mathrm{Ca}^{2+}$ since $\mathrm{Ca}^{2+}$ is suggested to compete for a binding site with $\mathrm{Mg}^{2+}$, which is required for the PDE-A activity (Ross et al. 1990). More recent studies have also demonstrated that PDE-A is negatively regulated by oxygen due to a heme-containing domain that binds oxygen reversibly (Chang et al. 2001). The regulating process is not fully understood and investigations are ongoing to unravel the full regulating process of cellulose synthase.

Many other enzymes and regulatory mechanisms are involved in the carbon metabolism of G. xylinus and only a fraction of them have been mentioned here since the focus of this review is BNC production and not the metabolism of bacteria in general. 


\section{Factors Involved during Nanocellulose Network Formation by G. xylinus}

There are different theories about the role of cellulose production by G. xylinus, which results in a nanocellulose network. The cellulose is believed to prevent the bacteria from dehydrating and also helps the bacteria to become floatable, via entrapment of carbon dioxide (produced from the tricarboxylic acid [TCA] cycle). This helps the bacteria to reach the air-liquid interface, an important issue since the bacteria is an obligate aerobe (Schramm and Hestrin 1954). The cellulose biofilm is also a natural way of immobilizing the bacteria and helps it attach to the surface (Cook and Colvin 1980). Moreover, the biofilm has been shown to protect the bacteria from ultraviolet (UV) light to some extent (Williams and Cannon 1989). Many external factors affecting the morphology of the nanocellulose network and the production rate of cellulose exist. Oxygen is one important factor, demonstrated by Watanabe and Yamanaka (1985) in their study, where they show that oxygen tension has a great influence on the cellulose network produced. Oxygen tension greater than $40 \%$ results in a higher growth rate of G. xylinus as compared to when the bacteria are cultured at oxygen tensions of less than 15\% (Watanabe and Yamanaka 1985).

The branching of the nanocellulose network is affected by the proliferation rate of G. xylinus (Figure 2.3A) (Yamanaka et al. 1989), which is in turn dependent on the oxygen tension (Figure 2.3B) (Watanabe and Yamanaka 1985) and affects the density of the cellulose network produced.

Prior to cell division, the catalytic sites for cellulose production are doubled and activated directly after division. No breakage of the cellulose ribbon occurs after division, just the creation of a branching point (Figure 2.3A) (Watanabe and Yamanaka 1985). When the dissolved oxygen tension is around 50\% of atmospheric pressure, a denser nanocellulose network is produced; if a lower oxygen tension is applied, the cellulose network becomes less dense (Figure 2.3B). Watanabe and Yamanaka found that the cellulose content increased as the oxygen tension increased. The opposite was found for lower oxygen tension, causing a decrease in the cellulose content. This phenomenon is probably due to the cell division rate of the bacteria, as a correlation between oxygen tension and cellulose network density was observed. Therefore the thickness of the cellulose membrane is only affected by the branching of the network, not by an increased width of the cellulose fibrils produced (Watanabe and Yamanaka 1985).

The degree of polymerization (DP) is the number of repeated units in a polymer, which in cellulose is D-glucose. The mechanism for development of the DP has been suggested to follow a Poisson distribution, which means that all molecules have the same probability to be added to the growing glucan chain. The DP in BNC is relatively high, around 2000-4000 (Marx-Figini and Pion 1974), whereas the cultivation conditions can have an impact on the DP of the produced cellulose. In an agitated culture of G. xylinus, the DP of the cellulose 


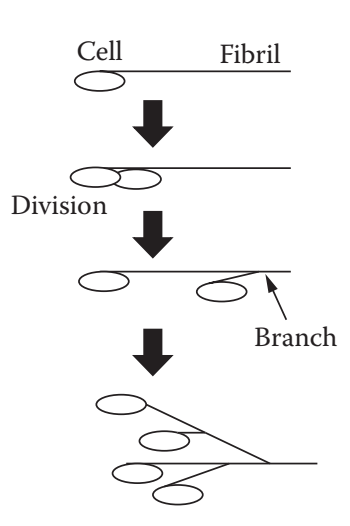

(a)
$10 \% \mathrm{O}_{2}$

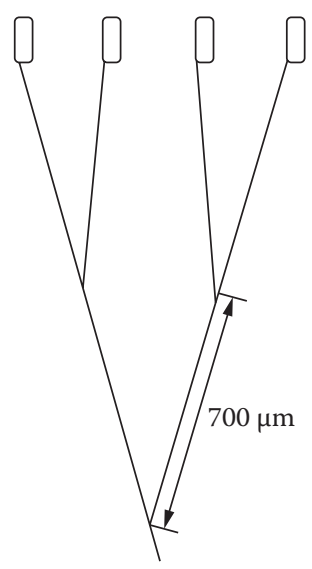

$50 \% \mathrm{O}_{2}$

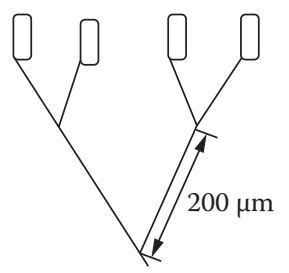

(b)

FIGURE 2.3

(a) The branching of cellulose formed by the cell division of G. xylinus. Adapted from Yamanaka et al. (1989). (b) The effect of oxygen tension on the branching of cellulose in the cellulose network formed during cell division. Adapted from Watanabe and Yamanaka (1985).

produced is lower than for cellulose produced in a static culture. The decrease in DP can be due to an increase in the activity of endoglucanases in agitated cultures (Czaja et al. 2004). Previous studies have shown that endoglucanases can have an effect on decreased DP in the long term (Tahara et al. 1997a, 1997b). Okajima et al. (1991) explained the phenomena of decreased DP as a function of cultivation time and generation time of the bacteria, not an effect of enzymes.

The $\mathrm{pH}$ is also an important factor for cellulose production and should be controlled and regulated during cultivation of G. xylinus. The $\mathrm{pH}$ outside the bacteria affects the structure and permeability of the bacteria cell membrane, which can have an effect on cellulose production and network formation. Verschuren et al. (2000) demonstrated that an optimal $\mathrm{pH}$ for BNC production is between pH 4 and 5, which is in accordance with Noro et al. (2004), who describe that $\mathrm{pH} 4.5$ is optimal for BNC production. A cultivation $\mathrm{pH}$ below 3.5 results in no production of cellulose and the proliferation of the bacteria is negatively affected (Embuscado et al. 1994). However, the bacteria has been shown to be able to recover from exposure to low $\mathrm{pH}$ following 8 days in optimal growth conditions (i.e., in this case a higher $\mathrm{pH} ; \mathrm{pH} 4-5)$ (Embuscado et al. 1994).

The cellulose network production is also affected by the production of watersoluble polysaccharides such as acetan (Figure 2.4A) and levan (Figure 2.4B). Depending on which carbon substrate is used-glucose, fructose, or sucrosedifferent amounts of water-soluble polysaccharides are produced. No levan is produced in fructose- and glucose-based media, and in a sucrose-based media, levan is produced in a smaller amount than acetan. The greatest amount of water-soluble polysaccharides produced has been observed when G. xylinus is 


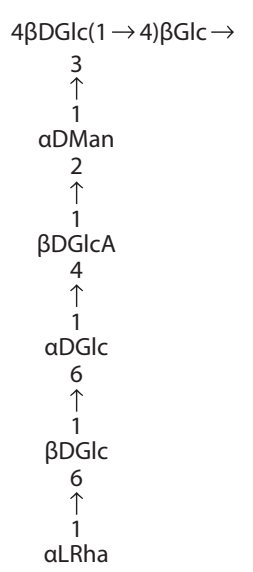

(a)

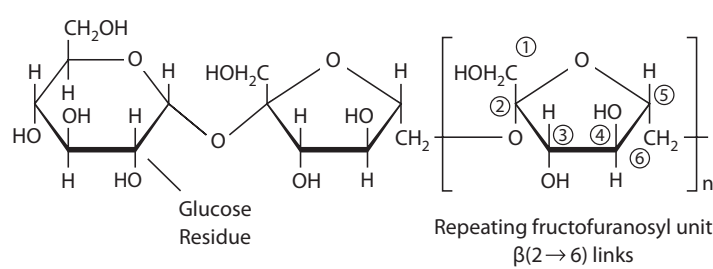

(b)

\section{FIGURE 2.4}

(a) Acetan: Glc = glucose; Man = mannose; GlcA = glucuronic acid; Rh = rhamnose. Adapted from Kirby et al. (1995). (b) Levan. Adapted from Arvidson et al. (2006).

grown in the presence of sucrose and the least when grown in the presence of glucose, depending on the formation of ketogluconate acids.

The production of these polysaccharides can differ, especially between mutants and wild-type strains of G. xylinus. The accumulated polysaccharides interfere with the cellulose crystallization process by preventing hydrogen bonding between the produced microfibrils (Watanabe et al. 1998).

Ishida et al. (2002) investigated a mutant of G. xylinus, EP1, that was not able to produce acetan. The hypothesis is that this mutant therefore should produce more BNC, since more of the carbon source can be used for BNC production. The outcome was the opposite, however; the mutant produced less BNC than the wild-type G. xylinus, which was used as a reference. When acetan was added to the cultivation medium for the EP1 mutant strain, BNC production was stimulated and the final yield was almost equal to the yield of the wild-type strain. The same phenomenon was seen when agar was added to the cultivation medium. The conclusion that can be drawn from these results is that the production of cellulose is dependent on how viscous the cultivation medium is during cultivation (Ishida et al. 2002).

\section{Effect of Growth Medium Components on Bacterial Cellulose Production in G. xylinus}

The growth media components have a great impact on BNC production in terms of cellulose production rate, yield, and network structure. The 


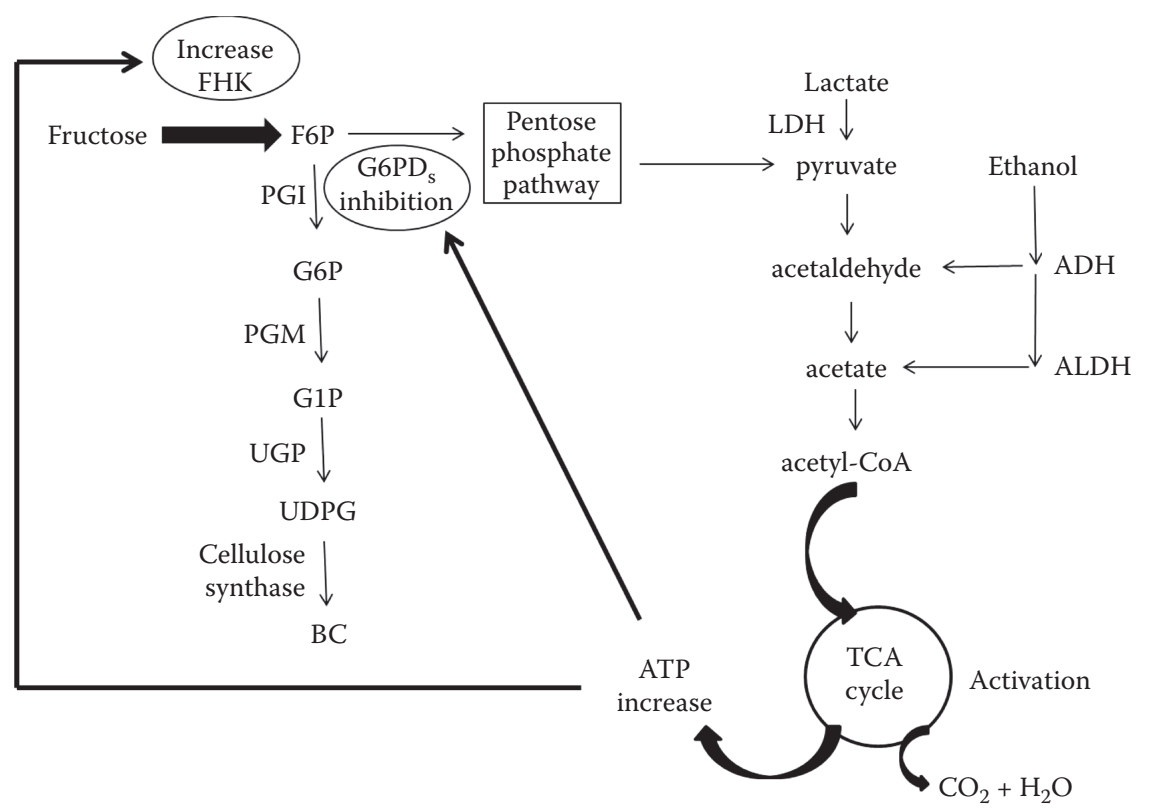

FIGURE 2.5

Effect of ethanol on bacterial cellulose production in continuous culture from fructose. Adapted from Naritomi et al. (1998).

components of the growth medium will increase the BNC production rate and yield and are often used by the bacteria as energy sources, not as a substrate for BNC production. Compounds that increase the production of water-soluble extracellular polysaccharides will probably affect the cellulose network, since the assembly of the cellulose chains is affected by the interference of water-soluble extracellular polysaccharides. Other compounds in the growth media can have an effect on the enzymes that are involved in the pathway where BNC is produced. The presence of a rapidly utilized carbon source in the growth media can lead to higher adenosine-5'-triphosphate (ATP) production, and ATP has an inhibitory effect on the enzyme glucose6-phosphate dehydrogenase (Figure 2.5).

\section{Lactate}

The addition of lactate to the growth media increases the cellulose production in G. xylinus since it is linked to the respiratory chain. Lactate generates energy and does not act as an extra substrate for cellulose production. This extra energy source provided by lactate increases the concentration of bacteria (i.e., biomass) during the early stages of cultivation, resulting in increased BNC production. Adding more lactate to the growth media will result in an increase in ATP production. A correlation between a high ATP level and a high specific 
BNC production rate has been found (Naritomi et al. 1998). This phenomenon is probably due to the activity of fructokinase being enhanced by ATP and the fact that ATP inhibits the enzyme glucose-6-phosphate dehydrogenase (Figure 2.5). This enzyme is a key enzyme that shuffles glucose-6-phosphate into the pentose phosphate pathway (Kornmann et al. 2003).

\section{Ethanol}

The oxidation of ethanol has the potential to produce extra energy for the bacteria in a similar way as lactate (Figure 2.5). However, the effect of adding ethanol to the growth medium is not as great as for the addition of lactate, which probably depends on the fact that the oxidation of ethanol produces higher levels of acetate. A high concentration of acetate has a negative effect on the bacteria, which is also true for acetic acid bacteria. The ethanol must be completely oxidized before the oxidation of acetate can start and an increase in biomass can be observed. The conclusion is that ethanol can increase BNC production via higher energy production, but not as efficiently as lactate due to the delay of biomass production (Kornmann et al. 2003; Matsuoka et al. 1996).

\section{Carbon Substrate}

The concentration of the carbon source has also been shown to affect the rate of cellulose synthesis, and when the substrate concentration increases, the utilization of the substrate decreases. Therefore an optimal level of substrate is needed to obtain high, consistent cellulose production throughout the cultivation (Embuscado et al. 1994). Regardless of the carbon source used, there are two different main phases according to Kornmann et al. (2003). In the first phase, biomass is produced and acetate oxidation occurs. The second phase begins when all the acetate is oxidized and only sugar is assimilated (Kornmann et al. 2003).

\section{Corn Steep Liquor}

Corn steep liquor (CSL) can be used as a component of complex microbial growth media. CSL contains different amino acids where methionine is the essential one (Matsuoka et al. 1996). Methionine stimulates growth of the bacteria during the early stages of cultivation and also reduces the lag time. An addition of different amino acids, including methionine, to the culture medium has been shown to induce an increase in cellulose production. The same increase in cellulose production has been observed when only methionine is added to the medium, clearly demonstrating that methionine is an important component of CSL. Methionine increases biomass production during the early stages of cultivation, which in turn has a positive effect on BNC production (Matsuoka et al. 1996). Amino acids together with ammonium in CSL account for more than 95\% of the total 
nitrogen concentration (Cardinal and Hedrick 1947) and can be used as a nitrogen source in culture media for microorganisms. Different CSL batches have been shown to differ in total nitrogen and lactic acid content (Noro et al. 2004), which can have a great impact on the production of BNC. According to Noro et al., $40 \mathrm{~mL}$ CSL/L growth media is the optimal concentration for optimal buffering capacity. With $40 \mathrm{~mL} / \mathrm{L} \mathrm{CSL}$, the $\mathrm{pH}$ was shown to stay constant at 4.5 during the whole cultivation time, which also seemed to be optimal for BNC production (Noro et al. 2004). This is in accordance with Verschuren et al. (2000) showing that the optimal pH for $\mathrm{BNC}$ production is between $\mathrm{pH} 4$ and 5. Moreover, Verschuren et al. used three different batches of CSL in their study and concluded that $\mathrm{pH}$ was the factor that affected cellulose production, since the amount of nitrogen, lactic acid, and fructose, respectively, were set to the same concentrations in all three CSL batches.

\section{Genetic Modifications of G. xylinus for Enhanced Production of Bacterial Cellulose}

Genetic modification of G. xylinus may be a way to increase BNC production. Environmental factors such as nutrient limitations and other factors such as stirring during cultivation and oxygenation can induce spontaneous mutations of the bacteria, which thereby may lose the ability to produce cellulose.

\section{Transfection of G. xylinus with the pBla-VHb-122 Plasmid}

The bacteria Vitreoscilla, discovered by E. G. Pringsheim in 1951, is a Gramnegative and obligate aerobe bacterium that lives in a microaerobic environment. During evolution these bacteria developed a way to utilize oxygen more efficiently. This occurs via the expression of bacterial hemoglobin, whose primary function is to enhance respiration in the bacteria. Vitreoscilla hemoglobin $(\mathrm{VHb})$ enhances respiration by capturing oxygen and facilitates the transfer of oxygen to the terminal oxidase (Tsai et al. 1996). The gene, vgb, encoding the hemoglobin in Vitreoscilla was isolated, sequenced, and characterized in 1988 by Dikshit and Webster, and has successfully been expressed in different heterologous hosts, such as Escherichia coli (Chien et al. 2006). The expression of the gene in E. coli enhances growth and product formation. The plasmid has successfully been amplified in E. coli and introduced into G. xylinus by electroporation (Setyawati et al. 2007). Chien et al. (2006) demonstrated that the expression of $\mathrm{VHb}$ enhances BNC production in G. xylinus. G. xylinus expressing $\mathrm{VHb}(\mathrm{VHb}+)$ have a higher specific growth rate under both aerobic and anaerobic conditions. The $\mathrm{VHb}+$ bacteria exhibited no obvious lag phase 
compared to $\mathrm{VHb}$ - bacteria (no $\mathrm{VHb}$ expression) with a lag time of 6 hours. This is probably due to an increased efficiency of oxygen uptake in the bacteria caused by the hemoglobin. Differences have also been observed between $\mathrm{VHb}+$ and $\mathrm{VHb}-$ in static cultures under normal oxygen conditions. Under microaerobic conditions, $\mathrm{VHb}$ expression was shown to enhance cellulose production two-fold. It is speculated that the increased production of BNC is dependent on increased production of ATP and that the glucose could be used for BNC production instead of ATP synthesis (Chien et al. 2006). This assumption is based on the findings, demonstrated in E. coli, where $\mathrm{VHb}$ expression enhances respiratory activity and increases ATP production.

\section{Spontaneous Mutation in G. xylinus Can Lead to a Loss of the Ability to Produce Cellulose}

G. xylinus is prone to spontaneous mutations sometimes resulting in a reduced or lost ability to produce cellulose $\left(\mathrm{Cel}^{-}\right)$. This type of mutation was first described by Schramm and Hestrin in 1954 and is more common in agitated and aerated cultures than in static cultures. Schramm and Hestrin found three different types of G. xylinus: the first type was the G. xylinus wild-type strain that produced cellulose (Cel+) and the other two were low producers of cellulose ( $\left.\mathrm{Cel}^{-}\right)$. To prevent mutation of G. xylinus in agitated cultures, ethanol can be added to the cultivation medium (Son et al. 2001). There are several other studies of G. xylinus mutations and different properties of the mutants have been discovered. Valla and Kjosbakken (1982) reported the probability of an indirect connection between cellulose production and the production of a soluble extracellular polysaccharide in $\mathrm{Cel}^{-}$mutants, since these produce large amounts of extracellular polysaccharides, such as acetan. In the same study it was also demonstrated that $\mathrm{Cel}^{-}$mutants grow faster than cellulose-producing cells. Furthermore, according to Valla et al. (1987), the plasmids by themselves can also be involved in cellulose production, since many mutants have demonstrated changes in their plasmid DNA profile in comparison with the wild-type G. xylinus strain. The mechanism is difficult to understand clearly since G. xylinus has a complex system of plasmids (Rezaee et al. 2005). One explanation may be that the $\mathrm{Cel}^{-}$bacteria lack two enzymes, phosphoglucomutase and glucose-1-phosphate uridylyltransferase (Krystynowicz et al. 2005), which are both key enzymes in cellulose production in G. xylinus. Moreover, spontaneous mutations of G. xylinus into $\mathrm{Cel}^{-}$can be caused by insertion sequences (ISs). Movement of the IS can lead to inactivation of genes involved in the cellulose synthesis. $\mathrm{Cel}^{-}$mutants have been shown to have two or more IS elements and their DNA appears to be rearranged within the IS element. This was not the case for $\mathrm{Cel}^{+}$G. xylinus (Coucheron 1991).

In $\mathrm{Cel}^{-}$mutants three enzymes were shown to be upregulated while two were downregulated, as demonstrated by Nguyen et al. (2010). One of the upregulated enzymes was deoxythymidine diphosphate (dTDP)-4-dehydrorhamnose 3,5-epimerase, which can be associated with a decrease in cellulose 
production in the mutant strain. This is probably due to the presence of dTDP rhamnose produced by the enzyme. The enzyme can activate acetan synthesis, which in turn reduces the production of cellulose. The same study also demonstrated that the enzyme dTDP-4-dehydrorhamnose 3,5-epimerase was absent in wild-type G. xylinus. Another mutant of G. xylinus, K3, has been shown to produce a cellulose network that is less dense than the one produced by wild-type G. xylinus. Investigations of the cellulose produced by wild-type G. xylinus and the K3 mutant were performed with nuclear magnetic resonance (NMR) and scanning electron microscopy (SEM), demonstrating the same crystalline structure. However, the difference between the wild-type G. xylinus and the mutant K3 appeared to be in their protein profiles (Nguyen et al. 2010).

Moreover, Nguyen et al. (2010) noticed that in some of their static cultures, the produced cellulose pellicle sank to the bottom of the culturing flask. After investigations of the pellicle with SEM, it was shown that the pellicle consisted of a looser cellulose network as compared with wild-type G. xylinus. Further investigations with NMR demonstrated that the cellulose network produced was cellulose I, which is the same type of cellulose that is produced by wild-type G. xylinus (Nguyen et al. 2010).

G. xylinus can be cultivated in static, agitated, or aerated conditions, and different strains of the bacteria are used depending on the cultivation conditions. The G. xylinus strain is prone to spontaneously mutate, whereas the special strain NQ5 of G. xylinus has a more stable genome and is therefore often preferable in agitated and aerated bioreactors (Brown and Lin 1990; Saxena et al. 1990).

As described earlier, there are differences between the wild-type G. xylinus and mutants of G. xylinus. In order to get a better understanding of the bacteria and its cellulose production these mutants need to be studied in more detail, since the mutants can give important information on regulatory mechanisms followed by changes in their environment. Even if spontaneous mutations easily occur in G. xylinus, natural changes in their metabolism, due to changes in the environment of the bacteria, can also be a reason for changing the appearance of the produced cellulose.

\section{Production of Artificial Blood Vessels by G. xylinus in Bioreactors}

Synthetic grafts used today, such as polyester (Dacron) and ePTFE, induce thrombosis and therefore are not suitable for the replacement of smaller blood vessels with a diameter of less than $6 \mathrm{~mm}$ (Nerem and Seliktar 2001). Research into new novel materials is thus needed. Nanocellulose produced by a microorganism can be used as a biomaterial in surgical applications, such as tissue 
implants in skin treatment. Furthermore, cellulose produced by microorganisms can be shaped for the particular application in the production process, for example, in the shape of cylinders (White and Brown 1989). One of the first production methods for forming BNC into various shapes with G. xylinus was described and patented by Roberts et al. (1986) (European patent number 0186495). In this method an oxygen-permeable polymer is used, such as polyvinyl chloride (PVC). This oxygen-permeable polymer is in contact with oxygen, whereas the other side is in contact with the liquid phase, including the bacteria. The growth is performed under static conditions; for the production of hollow tubes, the cultivation medium with bacteria is transferred into an oxygen-permeable polymer tube so that the inner surface is in contact with the liquid phase and the outer surface of the polymer tube is in contact with the oxygen. Since the polymer is oxygen permeable, the oxygen diffuses through the tube and becomes available to the bacteria in the liquid phase.

In 1990 Yamanaka et al. developed and patented a method, similar to that of Roberts et al. (1986), for producing long, hollow microbial cellulose by culturing Acetobacter xylinum (renamed G. xylinus by Yamanaka) in a hollow, oxygen-permeable container composed of silicon, cellophane, etc. (European patent application EP0396344). This method produced a cellulose membrane with a thickness of 0.01-20 mm. In $2001 \mathrm{Klemm}$ et al. described a method for production of tube-shaped cellulose, BASYC ${ }^{\circledR}$, for microsurgery with the use of G. xylinus (named in the article as A. xylinum). The method was specially designed for microsurgery applications for the replacement of blood vessels with a diameter of $1-3 \mathrm{~mm}$. The artificial graft of $\mathrm{BNC}$ is produced between cylindrical glass tubes, and the advantage of this method, compared with the Yamanaka model, is that the texture of the inner surface can be controlled. The cultivation is performed under static conditions with Hestrin-Schramm cultivation medium. The oxygen is supplied via a second opening to the air space in the bioreactor. The method is further described in their patent (U.S. patent WO2001061026).

\section{Tube Bioreactor}

Another type of bioreactor used for the production of artificial blood vessels as well as for the production of microporous scaffolds for bone regeneration (Bäckdahl et al. 2008; Zaborowska et al. 2010) was developed by Paul Gatenholm et al. (2010) (U.S. patent application 20100042197). In this bioreactor a silicone tube is used as a support and oxygen supply for G. xylinus. Since the bacteria are obligate aerobes, they are attracted to the silicon tube, where they start to grow and produce cellulose around the silicone tube, forming a tube-shaped cellulose vessel. The cellulose is produced at the outer surface of the silicone tube. The rate of oxygen supplied into the bioreactor can be controlled with the use of silicone tubing in a glass tube. The BNC tube produced has high mechanical resistance and high burst pressure (U.S. patent application 20100042197). This type of bioreactor is here referred to as "tube bioreactor" (Figure 2.6). 


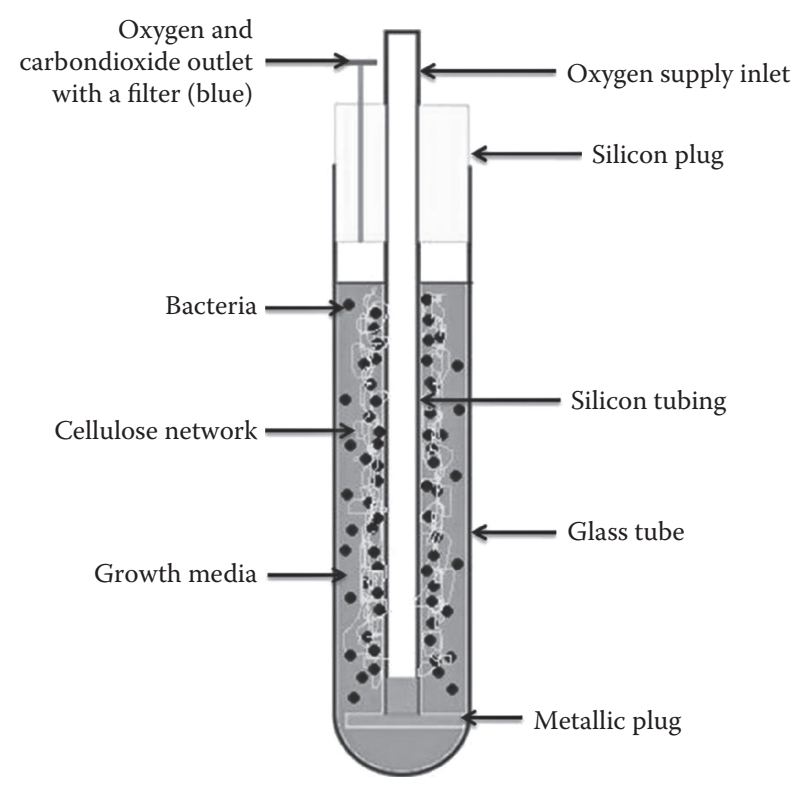

FIGURE 2.6

(See color insert.) Tube bioreactor setup for production of BNC vascular grafts. The bacteria, illustrated with black dots, produce the cellulose network, illustrated in beige, around the silicon tube.

$\mathrm{BNC}$ is an attractive material for vascular grafts due to its unique properties (great mechanical strength, high water content [approximately 99\%], good integration into host tissue, and flexibility of production in various shapes). The major challenge is to achieve the biomechanical properties required for long-term use as vascular grafts. The strain G. xylinus (ATCC 700178) is grown in a medium containing D-fructose, yeast extract, $\left(\mathrm{NH}_{4}\right)_{2} \mathrm{SO}_{4}, \mathrm{KH}_{2} \mathrm{PO}_{4}, \mathrm{MgSO}_{4} \cdot 7 \mathrm{H}_{2} \mathrm{O}, \mathrm{CSL}$, trace metal solution, and vitamin solution, and the $\mathrm{pH}$ is set to 5.5. Previous studies with the tube bioreactor have shown that G. xylinus synthesizes different cellulose layers with different densities, that is, a density gradient can be seen in the BNC tube produced (Bäckdahl et al. 2011). At the interface between these layers there is a tendency toward detachment (i.e., delamination). The production of two layers is desired, since they mimic two different types of extracellular matrix (ECM) in native connective tissue (Bäckdahl et al. 2011).

There is a clear difference between the two layers; the cellulose network closer to the lumen is denser and more ordered, whereas the layer far away from the lumen (i.e., closer to the cultivation medium) is less ordered and loose. The density gradient in the nanocellulose tube network produced can be clearly seen (Figure 2.7). Cross sections of cellulose vascular grafts produced after 4 days of cultivation (Figure 2.7A, magnification $\times 77$ ) and after 7 days of cultivation (Figure $2.7 \mathrm{~B}$, magnification $\times 130$ ) clearly show a density change in the cellulose network. The red ring (Figure 2.7A) represents a close-up (Figure 2.7C, magnification $\times 5000$ ) at the interface between the two layers. 


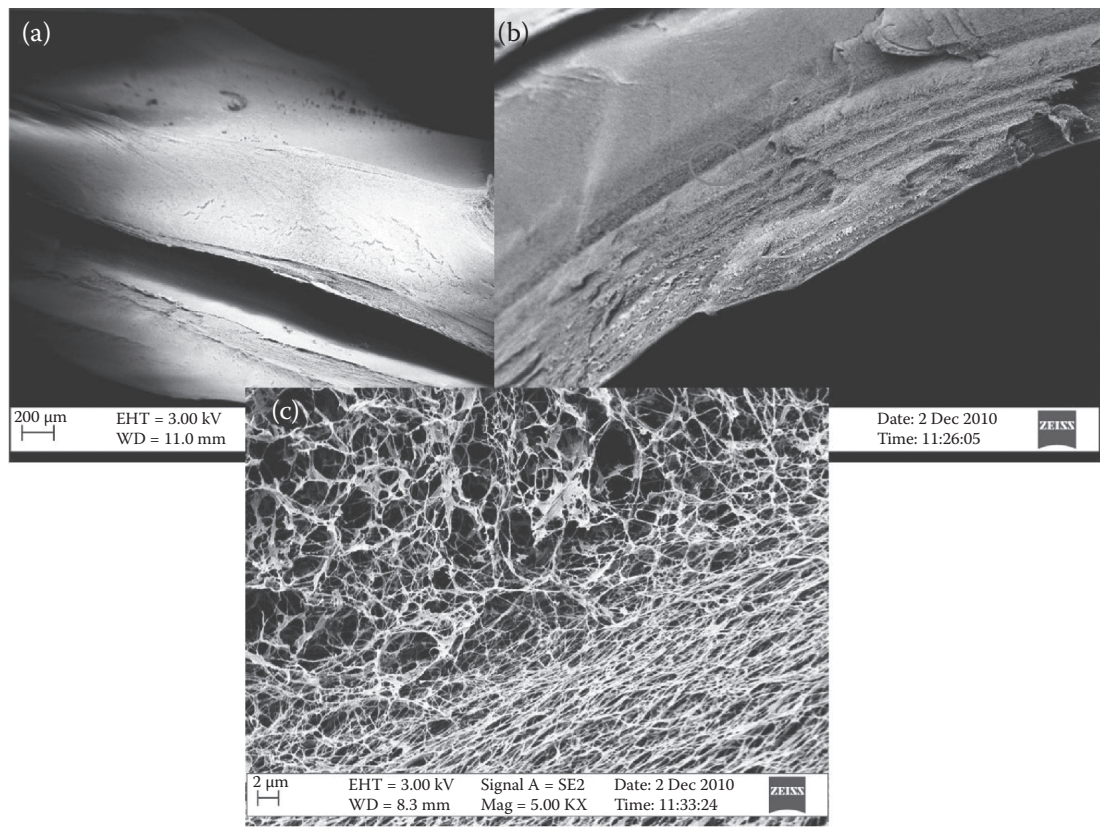

FIGURE 2.7

(a) Cross section of a cellulose tube after 4 days in culture (magnification $\times 77$ ). (b) Cross section of a cellulose tube after 7 days of cultivation (magnification $\times 130$ ). (c) Higher magnification of the red ring in (B) (magnification $\times 5000$ ) illustrating the change in density in the cellulose network.

Density changes can be monitored as early as 2 days after culture, when a relatively stable tube structure has been formed. The gradient in the fiber network can be monitored by means of second harmonic generation (SHG) microscopy, which enables visualization of spun fibers as the ordered arrangement of glucose units in the cellulose polymer chains induces frequency-doubling of laser light illuminating the sample (Brackmann et al. 2010; Brown et al. 2003). Since this technique allows for measurements on wet unmodified samples taken directly from cultivation, it could be confirmed that the arrangement of cellulose into layers is a result of the synthesis process and not an artifact of the subsequent material purification procedure. The SHG image in Figure 2.8A, measured on a 2-day-old tube, shows a dense cellulose region of strong SHG signal (light gray color) located toward the luminal side of the tube (left), whereas less compact regions of cellulose (darker gray) can be found on the outer side. Simultaneously with the SHG measurements on the unpurified cellulose, the bacterial distribution in the material was visualized using coherent anti-Stokes Raman scattering (CARS) microscopy (Cheng 2007) (Figure 2.8B). The profile in Figure 2.8B, corresponding to the cellulose profile of Figure 2.8A, shows dense regions of bacteria in the inner regions of the tube, in particular the region of high cellulose fiber 


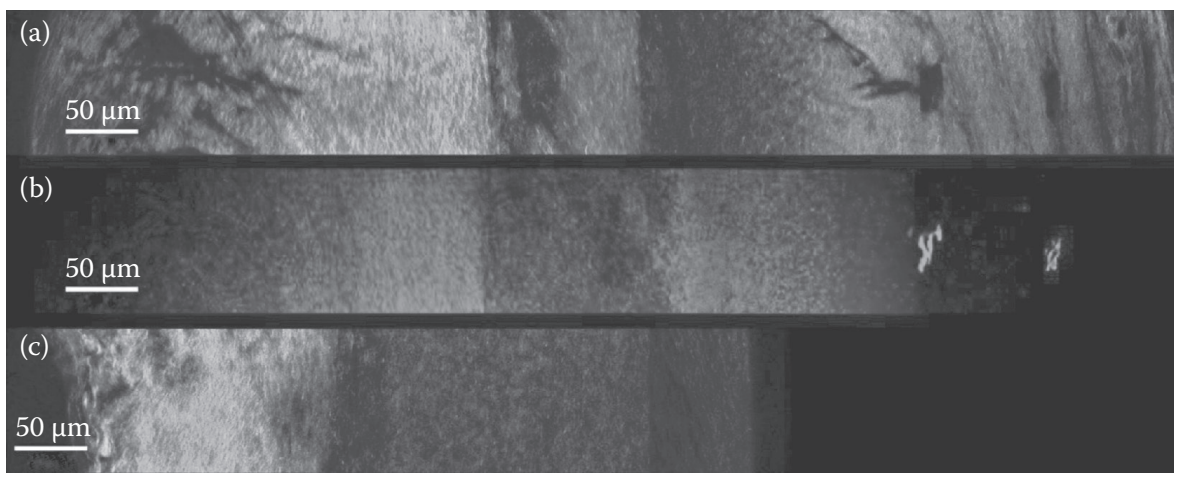

FIGURE 2.8

(See color insert.) Microscopy images showing radial distributions of cellulose fibers (blue, a and c) and G. xylinus (red, b) measured on 2-day-old tubes by SHG and CARS microscopy, respectively. Note the variations in cellulose density and SHG image intensity between regions on the inner luminal side (left) and outer side (right) observed for both a tube taken directly from cultivation (a) and a tube after purification (c).

density. A layered cellulose arrangement, with a dense region of high SHG signal at the luminal (left) side, can also be seen in the SHG image of Figure 2.8C, measured on a 2-day-old cellulose tube after material purification.

\section{Cellulose Production Yield and Cellulose Content}

The fructose level in the cultivation medium, before and after cultivation, and the cellulose dry weight were used to calculate the yield and production rate of bacterial cellulose (Table 2.1). The yield decreased during the 7 days of cultivation and the highest yield was obtained at day $1(2.402 \mathrm{~g} / \mathrm{g})$. This high yield is probably a result of the yield being calculated only for the fructose utilization and not the small amount of glucose present in the cultivation medium in the first 48 hours (Table 2.1).

\section{TABLE 2.1}

The Cellulose Production Yield is Presented in Both Grams of Bacterial Cellulose Obtained per Consumed Grams of Fructose and as Yield Obtained Per Day; pH 5.5.

\begin{tabular}{lcc}
\hline & $\begin{array}{c}\text { Yield p/s } \\
(\mathrm{g} / \mathrm{g})\end{array}$ & $\begin{array}{c}\text { Production Rate p/s } \\
\text { (g/g/day) }\end{array}$ \\
\hline Day 1 & 2.402 & 2.402 \\
Day 2 & 0.541 & 0.271 \\
Day 3 & 0.183 & 0.061 \\
Day 4 & 0.145 & 0.036 \\
Day 7 & 0.141 & 0.02 \\
\hline
\end{tabular}




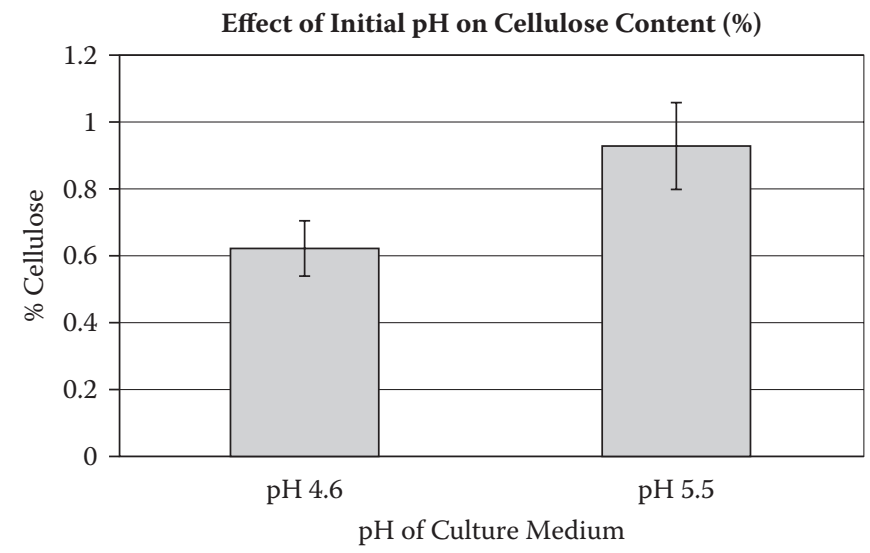

FIGURE 2.9

Cellulose content (\%) in BNC tubes cultured at $\mathrm{pH} 4.6$ and 5.5. Data are presented as the average \pm standard deviation. Four replicates in each group.

\section{Cellulose Content}

The initial $\mathrm{pH}$ of the culture medium affects the cellulose content in the BNC tubes. The cellulose content was calculated by dividing the dry weight of the BNC tube by the wet weight of the BNC tube:

Cellulose content $(\%)=($ dry weight $\mathrm{BNC}$ tube $/$ wet weight $\mathrm{BNC}$ tube $) \times 100$.

We investigated the effect of the initial $\mathrm{pH}$ of the growth media and found that the cellulose content increases during the cultivation period for both $\mathrm{pH}$ 4.6 and $\mathrm{pH}$ 5.5. At day 7, when the tubes were harvested, the highest cellulose content was shown to be in the BNC tubes cultured at pH 5.5 (see Figure 2.9).

\section{Influence of Oxygen Availability on Tube Formation}

Oxygen availability in the medium during culture in a tube bioreactor can be measured using an oxygen probe. This bioreactor is identical to the tube bioreactor with the exception of a side tube that allows for insertion of an oxygen probe. A needle oxygen probe and a Microx TX3 microfiber optic oxygen transmitter were used for the measurements. Oxygen measurements showed that oxygen passes through the silicon tubing and the level increases in the medium, but it drops rapidly to a few percent due to the consumption of oxygen by the bacteria (see Figure 2.10). The decrease was surprisingly fast (within 10 hours). During this time the number of bacteria increases, but no cellulose layer is yet visible. The oxygen level then remains around $2 \%$ for the rest of the culture time, which is usually 7 days. The cellulose tube is visible after 1 day and continues to be formed until day 4 , where no further increase in thickness can be observed. 


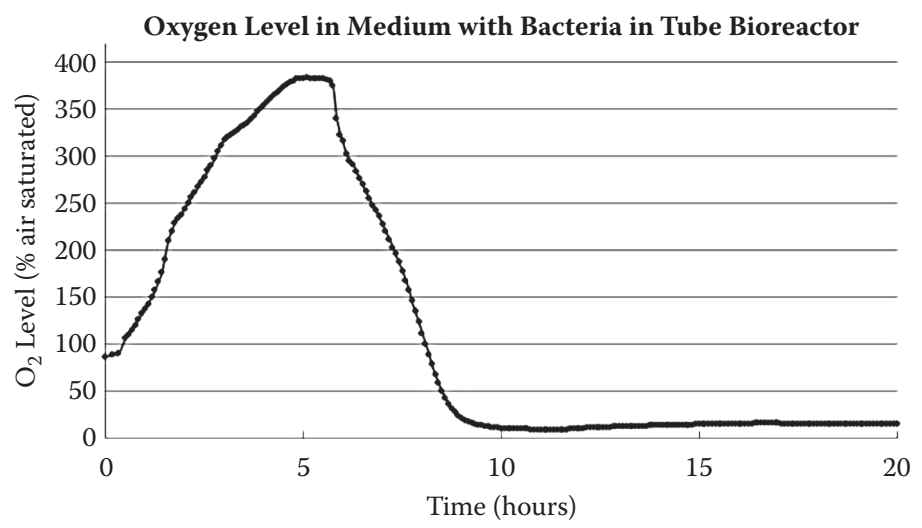

FIGURE 2.10

Oxygen level measurements during BNC tube culture using the tube bioreactor with $100 \% \mathrm{O}_{2}$ supply $(70 \mathrm{~mL} / \mathrm{min})$. Since pure oxygen is used, the oxygen level in the cultivation medium is saturated.

Close to the silicone surface, the bacteria have access to oxygen during cultivation; in the cultivation medium, however, the level of oxygen is very low, which might be due to the fact that a thin layer of cellulose was produced on the silicone surface and that this may act as an oxygen barrier. Thus the oxygen cannot diffuse out into the media. The oxygen limitation in the tube bioreactor may affect the metabolism, since the bacterium is an obligate aerobe. To avoid oxygen limitations and to get more evenly distributed oxygen in the cultivation medium, it would be preferable to use a continuous bioreactor. In a continuous bioreactor, an inflow of new cultivation medium, which is saturated with oxygen, can be used.

\section{Properties and Components of CSL}

The composition of CSL is not fully known and varies between different batches. This is most challenging, as it results in varying growth conditions and therefore a varying quality of the tubes produced. The $\mathrm{pH}$ of CSL is 3.5-4.5 (Liggett and Koffler 1948) and the variation depends on the composition of the CSL batch. Common for all the batches is that the color of CSL is light brown to brown and has a characteristic odor. The major components of CSL are crude proteins (47\%) and lactic acid (26\%) (White and Johnson 2003).

The amino acid composition of CSL has been analyzed and 16-20 different amino acids have been found. Saha and Racine (2010) compared the amino acid content of three different batches (see Table 2.2). In this table, it can be seen that the total amount of amino acids differs quite a lot between different batches (from 39.38 to $76.11 \mathrm{mg} / \mathrm{g}$ ). The highest amounts of amino acids are represented by glutamic acid, proline, alanine, leucine, and arginine (Saha 
TABLE 2.2

Amino Acid Composition of Three Different CSL Batches (Saha and Racine 2010).

\begin{tabular}{lrrr}
\hline Amino Acid & $\begin{array}{r}\text { CSL A } \\
(\mathbf{m g} / \mathbf{g})\end{array}$ & $\begin{array}{r}\text { CSL B } \\
(\mathbf{m g} / \mathbf{g})\end{array}$ & $\begin{array}{r}\text { CSL C } \\
(\mathbf{m g} / \mathbf{g})\end{array}$ \\
\hline Aspartic acid & 3.40 & 1.93 & 0.39 \\
Threonine & 2.40 & 1.23 & 2.42 \\
Serine & 3.07 & 1.59 & 3.11 \\
Aspargine & 3.77 & 1.84 & 2.60 \\
Glutamic acid & 5.45 & 2.44 & 4.49 \\
Glutamine & 1.05 & 0.60 & 0.04 \\
Proline & 9.16 & 4.76 & 7.37 \\
Glycine & 1.59 & 0.83 & 1.70 \\
Alanine & 7.12 & 3.57 & 8.52 \\
Valine & 4.48 & 2.37 & 4.35 \\
Cystine & 0.17 & 0.14 & 0.28 \\
Methionine & 2.29 & 1.24 & 2.39 \\
Isoleucine & 2.22 & 1.30 & 2.63 \\
Leucine & 9.65 & 5.15 & 8.84 \\
Tyrosine & 3.07 & 1.72 & 0.00 \\
Phenylalanine & 4.63 & 2.41 & 3.73 \\
Tryptophan & 0.37 & 0.00 & 0.00 \\
Lysine & 4.22 & 2.27 & 3.54 \\
Histidine & 1.66 & 0.84 & 0.04 \\
Arginine & 6.34 & 3.15 & 5.16 \\
Total & 76.11 & 39.38 & 61.60 \\
\hline & & &
\end{tabular}

and Racine 2010). In another study done in 1948, Cardinal and Hedrick found that amino acids together with ammonia account for more than $95 \%$ of the total nitrogen concentration in the CSL. The highest amino acids presented were alanine, glutamic acid, and arginine (Cardinal and Hedrick 1947). This is in accordance with Saha and Racine (2010), who also demonstrated large amounts of these amino acids.

\section{Conclusion and Future Perspectives}

Many attempts have been made to improve the compatibility and effectiveness of vascular grafts by modifying surface properties by incorporating biologically active substances. Several investigations focusing on increasing endothelial cell adhesion to nonadhesive polymeric biomaterials used for synthetic vascular grafts have been carried out. Precoating with the tripeptide Arg-Gly-Asp (RGD), an amino acid sequence found in many adhesive plasma 
and extracellular matrix proteins, has been used to enhance cell adherence. The binding of cells to the RGD sequence occurs via integrin receptors on the cell membrane.

A great challenge in the development of synthetic blood vessels is to set up a cyclic guanosine monophosphate (cGMP) facility for production of cellulose tubes, and this is relevant for all procedures in the manufacturing process. Regulatory approved product requires preclinical and clinical trials, since there is no "predicate" device on the market. The effectiveness of BNC as vascular grafts shown in preclinical trials is promising for the future use of $\mathrm{BNC}$ as vascular grafts.

\section{Acknowledgments}

We would like to thank the Swedish Foundation for Strategic Research, Vinnova, and the Swedish Research Council for funding the biosynthetic blood vessel program.

\section{References}

Arvidson, S. A., B. T. Rinehart, and F. Gadala-Maria. 2006. Concentration regimes of solutions of levan polysaccharide from Bacillus sp. Carbohyd. Polym. 65:144-149.

Bäckdahl, H., M. Esguerra, D. Delbro, B. Risberg, and P. Gatenholm. 2008. Engineering microporosity in bacterial cellulose scaffolds. J. Tissue Eng. Regen. Med. 2:320-330.

Bäckdahl, H., B. Risberg, and P. Gatenholm. 2011. Observations on bacterial cellulose tube formation for application as vascular graft. Mater. Sci. Eng. C 31:14-21.

Benzamin, M., and B. Rivertz. 1972. Factors affecting hexose phosphorylation in Gluconacetobacter xylinus. J. Bacteriol. 111:325-333.

Bodin, A., H. Bäckdahl, H. Fink, L. Gustafsson, B. Risberg, and P. Gatenholm. 2007. Influence of cultivation conditions on mechanical and morphological properties of bacterial cellulose tubes. Biotechnol. Bioeng. 97:425-434.

Brackmann, C., A. Bodin, M. Akeson, P. Gatenholm, and A. Enejder. 2010. Visualization of the cellulose biosynthesis and cell integration into cellulose scaffolds. Biomacromolecules 11:542-548.

Brown, R. M., Jr., and F. C. Lin. 1990. Multiribbon microbial cellulose. U.S. patent 4,954,439.

Brown, R. M., Jr., A. C. Millard, and P. J. Campagnola. 2003. Macromolecular structure of cellulose studied by second-harmonic generation imaging microscopy. Opt. Lett. 28:2207-2209.

Cardinal, E. V., and L. R. Hedrick. 1947. Microbiological assay of corn steep liquor for amino acid content. J. Biochem. 172:609-612. 
Chang, A. L., J. R. Tuckerman, G. Gonzalez, R. Mayer, H. Weinhouse, G. Volman, D. Amikam, M. Benziman, and M. A. Gilles-Gonzalez. 2001. Phosphodiesterase A1, a regulator of cellulose synthesis in Gluconacetobacter xylinus, is a heme-based sensor. Biochemistry 40:3420-3426.

Cheng, J. X. 2007. Coherent anti-Stokes Raman scattering microscopy. Appl. Spectrosc. 61:197-208.

Chien, L. J., H. T. Chen, P. F. Yang, and C. K. Lee. 2006. Enhancement of cellulose pellicle production by constitutively expressing Vitreoscilla hemoglobin in Acetobacter xylinum. Biotechnol. Prog. 22:1598-1603.

Cook, K., and R. J. Colvin. 1980. Evidence for a beneficial influence of cellulose production on growth of Acetobacter xylinum in liquid medium. Curr. Microbiol. 3:203-205.

Coucheron, D. H. 1991. An Acetobacter xylinum insertion sequence element associated with inactivation of cellulose production. J. Bacteriol. 173:5723-5731.

Czaja, W., D. Romanovvicz, and R. Malcolm Brown, Jr. 2004. Structural investigation of microbial cellulose produced in stationary and agitated cultures. Cellulose 11:403-411.

De Wulf, P., K. Joris, and E. J. Vandamme. 1996. Improved cellulose formation by Gluconacetobacter xylinus mutant limited in (keto)gluconate synthesis. J. Chem. Technol. Biotechnol. 67:376-380.

Dikshit, K. L., and D. A. Webster. 1988. Cloning, characterization and expression of the bacterial globin gene from Vitreoscilla in Escherichia coli. Gene 70:377-386.

Embuscado, M. E., J. S. Marks, and J. N. Bemiller. 1994. Bacterial cellulose I. Factors affecting the production of cellulose by Gluconacetobacter xylinus. Food Hydrocolloid 8:407-418.

Hess, F., C. Jerusalem, B. Braun, and P. Grande. 1985. Three years experience with experimental implantation of fibrous polyurethane microvascular prostheses in the rat aorta. Microsurgery 6:155-162.

Ishida, T., Y. Sugano, T. Nakai, and M. Shoda. 2002. Effects of acetan on production of bacterial cellulose by Acetobacter xylinum. Biosci. Biotechnol. Biochem. 66:1677-1681.

Kirby, A. R., A. P. Gunning, V. J. Morris, and M. J. Ridout. 1995. Observation of the helical structure of the bacterial polysaccharide acetan by atomic force microscopy. Biophys. J. 68:360-363.

Klemm, D., D. Schumann, U. Udhardt, and S. Marsch. 2001. Bacterial synthesized cellulose-artificial blood vessels for microsurgery. Progress in Polymer Science. 26:1561-1603.

Kornmann, H., P. Duboc, I. Marison, and U. von Stockar. 2003. Influence of nutritional factors on the nature, yield, and composition of exopolysaccharides produced by Gluconacetobacter xylinus I-2281. Appl. Environ. Microbiol. 69:6091-6098.

Krystynowicz, A., M. Koziolkiewicz, A. Wiktorowska-Jezierska, S. Bielecki, E. Klemenska, A. Masny, and A. Płucienniczak. 2005. Molecular basis of cellulose biosynthesis disappearance in submerged culture of Gluconacetobacter xylinus. Acta Biochim. Pol. 52:691-698.

Liggett, W. R., and H. Koffler. 1948. Corn steep liquor in microbiology. Bacteriol. Rev. 12:297-311.

Marx-Figini, M., and B. G. Pion. 1974. Kinetic investigation on biosynthesis of cellulose by Gluconacetobacter xylinus. Biochim. Biophys. Acta 338:382-393. 
Matsuoka, M., T. Tsuchida, K. Matsushita, O. Adachi, and F. Yoshinaga. 1996. A synthetic medium for bacterial cellulose production by Acetobacter xylinum subsp. sucrofermentans. Biosci., Biotechnol. Biochem. 60:575-579.

Mikkelsen, D., B. M. Flanagan, G. A. Dykes, and M. J. Gidley. 2009. Influence of different carbon sources on bacterial cellulose production by Gluconacetobacter xylinus strain ATCC 53524. J. Appl. Microbiol. 107:576-583.

Naritomi, T., T. Kouda, H. Yano, and F. Yoshinaga. 1998. Effect of ethanol on bacterial cellulose production in continuous culture from fructose. J. Ferment. Bioeng. 85:598-603.

Nerem, R. M., and D. Seliktar. 2001. Vascular tissue engineering. Annu. Rev. Biomed. Eng. 3:225-243.

Nguyen, V. T., B. Flanagan, D. Mikkelsen, S. Ramirez, L. Rivas, M. J. Gidley, and G. A. Dykes. 2010. Spontaneous mutation results in lower cellulose production by a Gluconacetobacter xylinus strain from Kombucha. Carbohyd. Polym. 80:337-343.

Noro, N., Y. Sugano, and M. Shoda. 2004. Utilization of the buffering capacity of corn steep liquor in bacterial cellulose production by Acetobacter xylinum. Appl. Microbiol. Biotechnol. 64:199-205.

Okajima, K., Y. Matsuda, and K. Kamide. 1991. Study on changes in the degree of polymerization of bacterial cellulose produced by Gluconacetobacter xylinus during its cultivation. Polym. Int. 25:145-151.

Pringsheim, E. G. 1951. The Vitreoscillaceae: a family of colorless, gliding, filamentous organisms. J. Gen. Microbiol. 5:124-149.

Rezaee, A., S. Solimani, and M. Forozandemogadam. 2005. Role of plasmid in the production of Acetobacter xylinum biofilms. Am. J. Biochem. Biotechnol. 1:121-124.

Roberts, E. M., L. K. Hardison, and R. M. Brown, Jr. 1986. Production of microbial cellulose, 1986-07-02. European patent number 0186495.

Ross, P., R. Mayer, and M. Benziman. 1991. Cellulose biosynthesis and function in bacteria. Microbiol. Rev. 55:35-58.

Ross, P., R. Mayer, H. Weinhouse, D. Amikam, Y. Huggirat, and Y. Benziman. 1990. The cyclic diguanylic acid regulatory system of cellulose synthesis in Acetobacter xylinus. J. Biol. Chem. 265:18933-18943.

Saha, B. C., and F. M. Racine. 2010. Effects of pH and corn steep liquor variability on mannitol production by Lactobacillus intermedius NRRL B-3693. Appl. Microbiol. Biotechnol. 87:553-560.

Saxena, I. M., E. M. Roberts, and R. M. Brown, Jr. 1990. Modification of cellulose normally synthesized by cellulose-producing microorganisms. U.S. patent 4,950,597.

Schramm, M., and S. Hestrin. 1954. Factors affecting production of cellulose at the air/liquid interface of a culture of Acetobacter xylinum. J. Gen. Microbiol. 11:123-129.

Setyawati, M. I., L. J. Chien, and C. K. Lee. 2007. Expressing Vitreoscilla hemoglobin in statically cultured Acetobacter xylinum with reduced $\mathrm{O}_{2}$ tension maximizes bacterial cellulose pellicle production. J. Biotechnol. 132:38-43.

Son, H. J., M. S. Heo, Y. G. Kim, and S. J. Lee. 2001. Optimization of fermentation conditions for the production of bacterial cellulose by a newly isolated Acetobacter sp. A9 in shaking cultures. Biotechnol. Appl. Biochem. 33:1-5.

Svensson, A., E. Nicklasson, T. Harrah, B. Panilaitis, D. L. Kaplan, M. Brittberg, and P. Gatenholm. 2005. Bacterial cellulose as a potential scaffold for tissue engineering of cartilage. Biomaterials 26:419-431. 
Tahara, N., M. Tabuchi, K. Watanabe, H. Yano, Y. Morinaga, and F. Yoshinaga. 1997a. Degree of polymerization of cellulose from Gluconacetobacter xylinus BPR2001 decreased by cellulose produced by the strain. Biosci. Biotechnol. Biochem. 61:1862-1865.

Tahara, N., H. Yano, and F. Yoshinaga. 1997b. Two types of cellulose activity produced by a cellulose producing Acetobacter strain. J. Ferm. Bioeng. 83:389-392.

Tsai, P. S., M. Nägeli, and J. E. Bailey. 1996. Intracellular expression of Vitreoscilla hemoglobin modifies microaerobic Escherichia coli metabolism through elevated concentration and specific activity of cytochrome o. Biotechnol Bioeng. 49:151-160.

Valla, S., D. H. Coucheron, and J. Kjosbakken. 1987. The plasmids of Acetobacter xylinum and their interaction with the host chromosome. Mol. Gen. Genet. 208:76-83.

Valla, S., and J. Kjosbakken. 1982. Cellulose-negative mutants of Gluconacetobacter xylinus. J. Gen. Microbiol. 128:1401-1408.

Verschuren, P. G., T. D. Cardona, M. J. Nout, K. D. De Gooijer, and J. C. Van den Heuvel. 2000. Location and limitation of cellulose production by Acetobacter xylinum established from oxygen profiles. J. Biosci. Bioeng. 89:414-419.

Watanabe, K., M. Tabuchi, Y. Morinaga, and F. Yoshinaga. 1998. Structural features and properties of bacterial cellulose produced in agitated culture. Cellulose 5:187-200.

Watanabe, K., and S. Yamanaka. 1985. Effects of oxygen tension in the gaseous phase on production and physical properties of bacterial cellulose formed under static culture conditions. Biosci. Biotechnol. Biochem. 59:65-68.

Wei, B., G. Yang, and F. Hong. 2011. Preparation and evaluation of a kind of bacterial cellulose dry films with antibacterial properties. Carbohyd. Polym. 84:533-538.

White, D. G., and R. M. Brown, Jr. 1989. Prospects for the commercialization of the biosynthesis of microbial cellulose. In: Cellulose and Wood-Chemistry and Technology, ed. C. Schuerch, 573-590. New York: John Wiley \& Sons.

White, P., and L. A. Johnson. 2003. Corn: chemistry and technology, 2nd ed. St. Paul, $\mathrm{MN}$ : American Association of Cereal Chemists.

Williams, W. S., and R. E. Cannon. 1989. Alternative environmental roles for cellulose produced by Acetobacter xylinum. Appl. Environ. Microbiol. 55:2448-2452.

Wippermann, J., D. Schumann, D. Klemm, H. Kosmehl, S. Salehi-Gelani, and T. Wahlers. 2009. Preliminary results of small arterial substitute performed with a new cylindrical biomaterial composed of bacterial cellulose. Eur. J. Vasc. Endovasc. Surg. 37:592-596.

Yamanaka, S., K. Watanabe, N. Kitamura, M. Iguchi, S. Mitsuhashi, Y. Nishi, and M. Uryu. 1989. The structure and mechanical properties of sheets prepared from bacterial cellulose. J. Mater. Sci. 24:3141-3145.

Zaborowska, M., A. Bodin, H. Bäckdahl, J. Popp, A. Goldstein, and P. Gatenholm. 2010. Microporous bacterial cellulose as a potential scaffold for bone regeneration. Acta Biomater. 6:2540-2547.

Zhang, H. Y., X. J. Yan, Y. Jiang, and J. Cong. 2010. Development and characteristic of bacterial cellulose for antimicrobial wound dressing. Adv. Mater. Res. 978:152-153. 


\section{Large-Scale Production of BNC: State and Challenges}

\section{Dana Kralisch and Nadine Hessler}

Friedrich Schiller University, Institute of Technical Chemistry and

Environmental Chemistry, Jena, Germany

\section{CONTENTS}

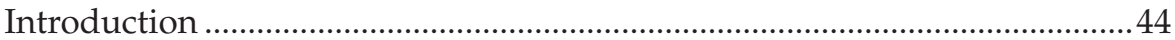

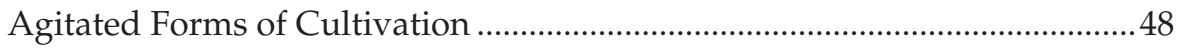

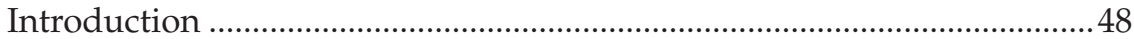

Benefits and Limits of Agitated Cultivation Forms .................................48

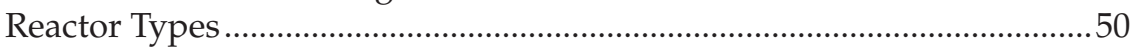

Agitated Cultivation by Shaking …...................................................... 51

Agitated Cultivation by Stirring ........................................................... 52

Batch Fermentation under Stirring Using Plastic Composite Supports... 53

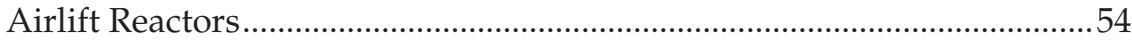

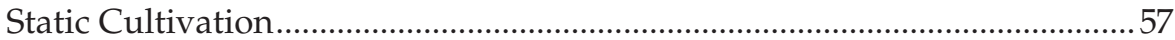

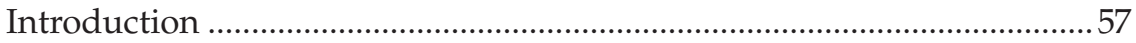

Benefits and Limitations of Static Cultivation Forms in Batch Vessels ....58

Fed-Batch Cultivation ............................................................................... 58

Combination of Static and Moving Cultivation ............................................6 60

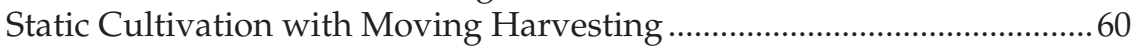

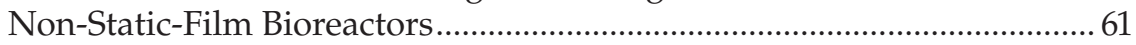

(Semi)continuous Generation of Flat BNC ...............................................63

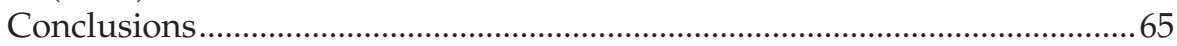

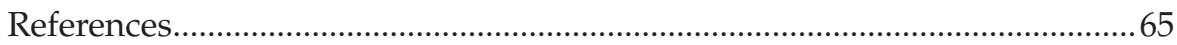

This chapter provides an overview of the methods and reactor devices used for the generation of bacterial nanocellulose (BNC). Cultivation approaches ranging from batch, to fed-batch, to continuous operation under agitated, static, and nonstatic conditions are described in detail and the advantages as well as the limitations of these methods are discussed. Typically utilized types of reactors are introduced and reviewed against the current state of BNC generation within these devices. 


\section{Introduction}

A variety of approaches are available for the generation of bacterial nanocellulose (BNC), ranging from batch, to fed-batch, to continuous cultivation methods applying typical bioreactors as well as several specific devices. The choice of a cultivation technique depends on the application of the BNC material, since the cellulose supramolecular structure and its physical and mechanical properties are strictly influenced by the production method.

In 1886 A. J. Brown observed the formation of a gelatinous mass on the solution surface during vinegar fermentation (Brown 1886). The responsible microorganism is called Gluconacetobacter xylinus according to today's nomenclature. In addition, in the mid-20th century Hestrin and Schramm (1954) began with the description of basic metabolic processes of this bacteria strain and ended with the development of a special culture medium for optimized production of BNC on a laboratory scale, whereas D-glucose is used as a carbon source and bacto peptone as well as yeast extract as sources of nitrogen and vitamins. Both discoveries are important fundamentals for the variety of all BNC production methods available today.

After elucidation of the formation of $\mathrm{BNC}$ and its underlying metabolic processes, this Gram-negative bacterium was initially applied as a model organism for studying cellulose synthesis and crystallization as well as its degradation on a laboratory scale (Chen and Brown 1999; Hirai et al. 1997; Ross et al. 1991; Saxena and Brown 2001). Based on this, versatile knowledge was gained about the biosynthesis and the resulting structure (Colvin et al. 1957; Correns et al. 1972; Gagnaire and Taravel 1975; Takai et al. 1975; Zaar 1979). The systematic and comprehensive research during the last decades, aimed at a detailed understanding of the bioprocess of BNC generation, provides an essential contribution to the integration of white biotechnological methods in polysaccharide chemistry and product development based on nanocellulose, with its special properties. According to these findings, a broad range of commercial application possibilities and the immense economic potential of BNC have become visible (Czaja et al. 2006).

In order to extend the range of commercial BNC applications, taking also into account the ongoing development of novel materials based on BNC with groundbreaking new features, access to various types of BNC materials with varying supramolecular structure and diverse material properties became indispensable. For this purpose, and in respect to a potential scale-up to a future commercial production process, different types of cultivation (see Table 3.1) partly combined with in situ modifications by additives or supports have been established during the last 20 years. At this time, the in situ formability of BNC displays one important and specific advantage compared with other natural or synthetic polymers such as plant cellulose, polylactic acid, or polyethylene.

Depending on the type of cultivation, as well as on the cultivation conditions, BNC can be produced in different forms and sizes (e.g., flat BNC fleeces 
TABLE 3.1

Summary of BNC Generation Processes

\begin{tabular}{|c|c|c|c|c|}
\hline $\begin{array}{l}\text { Type of } \\
\text { Cultivation }\end{array}$ & $\begin{array}{l}\text { Type of } \\
\text { Agitation }\end{array}$ & Form of BNC & $\begin{array}{c}\text { Type of } \\
\text { Bioreactor }\end{array}$ & Examples \\
\hline \multirow[t]{4}{*}{ Agitated } & \multirow[t]{3}{*}{$\begin{array}{l}\text { Stirring, } \\
\text { shaking, or } \\
\text { air bubbling }\end{array}$} & \multirow[t]{3}{*}{$\begin{array}{l}\text { Pulp, pellets, } \\
\text { spherelike or } \\
\text { fibrous } \\
\text { (diameter }<10 \mathrm{~mm} \text { ) }\end{array}$} & Batch & $\begin{array}{l}\text { Chao et al. 1997; Choi } \\
\text { et al. 2009; Jung et al. } \\
\text { 2005; Lee and Zhao } \\
\text { 1999; Son et al. 2003; } \\
\text { Song et al. 2009; } \\
\text { Vandamme et al. } 1998\end{array}$ \\
\hline & & & Fed batch & Bae and Shoda 2004 \\
\hline & & & Continuous & Naritomi et al. 1998 \\
\hline & \multirow[t]{8}{*}{$\begin{array}{l}\text { Stirring and } \\
\text { support }\end{array}$} & $\begin{array}{l}\text { Irregular masses } \\
\text { (diameter }>10 \mathrm{~mm} \text { ) }\end{array}$ & Batch & Cheng et al. 2009 \\
\hline \multirow[t]{2}{*}{ Static } & & \multirow[t]{2}{*}{ Flat } & Batch & $\begin{array}{l}\text { Farah et al. 2006; } \\
\text { Ishihara et al. 2002; } \\
\text { Jonas and Farah 1998; } \\
\text { Levy et al. 2004; } \\
\text { Onodera et al. 2002; } \\
\text { Parvulescu et al. 2008 }\end{array}$ \\
\hline & & & Fed batch & Weuster-Botz et al. 2011 \\
\hline \multirow[t]{5}{*}{ Nonstatic } & & $\begin{array}{l}\text { Pellicles } \\
\text { (diameter >10 mm) }\end{array}$ & Fed batch & $\begin{array}{l}\text { Krystynowicz et al. } \\
\text { 2002; Shezad et al. } 2009\end{array}$ \\
\hline & & $\begin{array}{l}\text { Three-dimensional } \\
\text { bioprintings }\end{array}$ & Fed batch & Gatenholm et al. 2011 \\
\hline & & Fibrous & Batch & $\begin{array}{l}\text { Sakairi et al. 1998; } \\
\text { Tokura et al. } 1998\end{array}$ \\
\hline & & Tubes & Batch & $\begin{array}{l}\text { Ciechanska et al. 2010; } \\
\text { Hessler and Klemm } \\
2008\end{array}$ \\
\hline & & Flat & $\begin{array}{l}\text { (Semi) } \\
\text { continuous }\end{array}$ & Kralisch et al. 2010b \\
\hline
\end{tabular}

or foils, thin pellicles, spheres, fibrous aggregates, tubes, or even irregular masses such as pulp). Up to now, flat BNC materials have gained commercial importance due to simple nontechnical access by static cultivation in diverse vessels (e.g., nata de coco generation in plain plastic reservoirs; see Chapter 10) and due to the great application potential of BNC fleeces and foils in medical (e.g., as a wound dressing) (Czaja et al. 2004a; Legeza et al. 2004) and technical applications (e.g., as filter material or membranes) (Sokolnicki et al. 2006).

Flat BNC is usually produced by conventional static cultivation or in thin layer cultivation on solid phases such as agar (Brown et al. 1992), silicone rubber (Onodera et al. 2002), or on different porous membranes (Putra et al. 2008). On these supports, the BNC can be accumulated on the interface of the air and culture medium. The resulting fleeces or foils are typically characterized by a homogeneous, three-dimensional (3D) network of nanofibers and a uniform surface. The properties of the resulting BNC can be controlled 


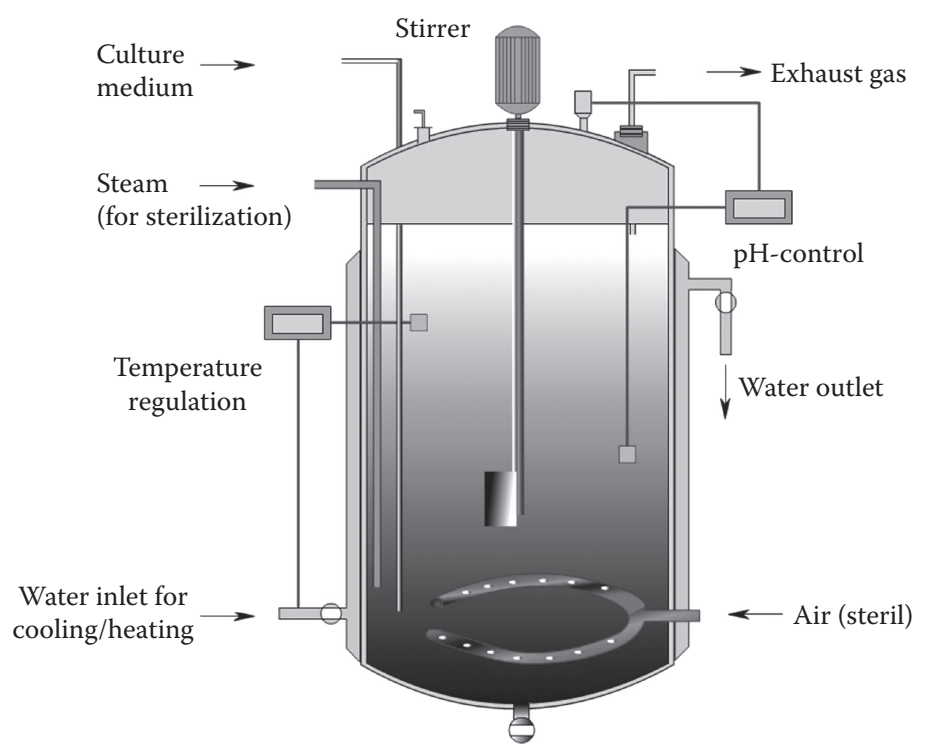

FIGURE 3.1

(See color insert.) Example for a typical deep-tank fermenter based on the stirred tank reactor (STR) concept. Figure adopted from http://www.chemgapedia.de.

by several process parameters. In 1989 Williams and Cannon discovered a change in thickness of the flat BNC by the use of different Gluconacetobacter strains. Further control of the thickness and size of BNC can be achieved by variation of the volume of the culture medium (Udhardt 2004), the cultivation time (Borzani and De Souza 1995), and the cultivation vessel (Levy et al. 2004; Tamura et al. 1999). Static cultivation is described in detail later in this chapter.

Besides BNC production under static conditions, there are a large number of other cultivation types. Cultivation under shaking and stirring (Watanabe and Yamanaka 1995), as well as several modified production procedures based on these techniques (Chao et al. 2001b; Wu et al. 2001), are the most important. In Figure 3.1, an example of a typical deep-tank fermenter for aerated cultivation is shown. The reactor consists of a vessel typically made from stainless steel or glass, a stirrer, inlets and outlets for culture medium as well as air or oxygen, a cooling or heating jacket, and several control systems for sensible process parameters such as temperature and $\mathrm{pH}$.

Agitation during the biosynthesis results in the synthesis of BNC in the form of a fibrous suspension, irregular masses, pellets, or spheres (Krystynowicz et al. 2002). The production of individualized (isolated) BNC in defined sizes and different surface characteristics can be controlled by the choice of specific cultivation conditions (Hessler and Klemm 2008).

Several working groups have seen the possibility of producing commercial quantities of BNC by agitated forms of cultivation (Czaja et al. 2004b). They argue that static cultivation delivers only restricted BNC yields with the 
concurrent use of large surfaces and space demands. By means of an agitated cultivation in fermenters, the formation limit should be avoided and continuous production of BNC should be feasible (Ben-Bassat et al. 1987; Kouda et al. 2000; Tokura et al. 1993). Indeed, these cultivation conditions can lead to an increase in BNC formation (Krystynowicz et al. 2002). But only a few Gluconacetobacter strains, such as ATCC 53582, have been found to be suitable for agitated cultivation (Toyosaki et al. 1995). This can be seen in shear forces that cause a reduction in BNC biosynthesis or disruption of the bacteria cells. In addition, a spontaneous formation of mutations from active bacterial cells to non-cellulose producing is favored (Park et al. 2004). In spite of the restrictions discussed earlier, these agitated cultivation methods possess their own unique potential for future industrial use, because other areas of application become possible. The resulting BNC pulps, filaments, or spheres are suitable as, for example, an additive for papermaking (Xiu et al. 2005) and for fiber reinforcement of synthetic or biopolymers (Tomé et al., 2011). Agitated types of cultivation are described in detail later in this chapter.

Other cultivation types between static and agitated cultivation have been developed as well. Well-known examples are the nonstatic rotating disk and linear conveyor film bioreactors used by Bungay and Serafica (2000) for BNC generation for the first time, or the generation of BNC in tubular form developed by Klemm et al. (1999).

Independent from the cultivation method or the bacteria strain, the process of BNC generation can also be influenced by a large number of other variables, including the composition of the culture media (Heo and Son 2002; Ishihara et al. 2002; Seto et al. 1997) and its $\mathrm{pH}$ value (Strobin et al. 2003), the temperature (Coban and Biyik 2011) and oxygen tension (Nakajima et al. 2001), as well as by several additives, such as water-soluble polymers (Astley et al. 2001), organic or inorganic substances (Hessler and Klemm 2009; Ishihara et al. 2002; Yamanaka et al. 2000), and metal or metal oxide nanoparticles (Serafica et al. 2002). In all cases, the optimization of the cultivation conditions plays a specific role during the scale-up of a cultivation process from experimental (grams per day) to pilot (kilograms per day) or industrial scale (tons per day).

In addition to biological conditions, various technical parameters such as sterilization of the equipment, robustness and stability against corrosion of the reactor material, equally distributed supply of air and culture medium, and protection against contaminants must be investigated and adapted.

Although a variety of approaches for the production of BNC in various forms have been suggested over the past 20 years, in industrial practice BNC is still almost exclusively produced using static cultivation in shallow containers using minimally automated work processes. Thus extensive commercialization of BNC has been hindered by the lack of efficient methods and high costs for the generation of high-quality BNC on an industrial scale.

However, the testing and scale-up of ideas for efficient BNC generation continues unabated to overcome these hurdles. For instance, Song et al. 
(2009) used a modified airlift-type bubble column bioreactor to transfer an agitated cultivation process of BNC into pilot scale. Furthermore, recently a novel bioreactor, called a horizontal lift reactor (HoLiR), was developed by Kralisch et al. (2010a, 2010b). It allows for semicontinuous cultivation of flat fleeces and foils of BNC with undisturbed nanostructure comparable to those gained from static cultivation, but in a more efficient manner. The HoLiR approach has been transferred to pilot-scale processing combined with continuous downstream processing and extensively tested under variations of the bacteria strain. In this way the transferability of the concept as well as the robustness and scalability of the process could be demonstrated.

\section{Agitated Forms of Cultivation}

\section{Introduction}

Because of many years of experience in the use of microorganisms for food production and in the manufacture of tobacco, the first suggested manufacturing methods for large-scale production of BNC were based on fermentation processes. The nutrient medium within a typical fermenter relies on agitation, thus diffusion of nutrient components is enhanced by mixing of the culture medium. Also the oxygen solubility is increased by this mixing compared to static cultivation. Because of this, early approaches for the bioprocess intensification and for the production of BNC on a larger scale were dominated by agitated cultivation forms.

As one of the first companies, Weyerhaeuser tested the production of bacterial cellulose called "Cellulon," produced by stirred fermenters (1988; U.S. Patent 4863965).

Since then several kinds of culture devices, such as shake flasks, stirred tanks, airlift reactors, and modified airlift or gas-lift bioreactors have been tested for BNC production under agitated conditions. At this time, stirred-tank and air- or oxygen-supplying reactors have been used most often (Sani and Dahman 2009). Spherical particles or fiberlike structures are usually formed within such devices.

\section{Benefits and Limits of Agitated Cultivation Forms}

Applying agitated cultivation techniques including submerged cultures in shaken flasks or in stirred aerated fermenters, the cultivation time can be reduced up to 10 times in comparison with static fermenters (Dudman 1960), whereas the space-time yield increases in a significant manner. However, the idea of enhancing the BNC yield by higher air flow rates was not successful (Ross et al. 1991). It led to a decrease in the BNC yield, since 
the aeration of cultures of most G. xylinus strains by stirring or shaking gives rise to spontaneous non-cellulose-producing mutants, a phenotype gains an advantage when oxygen is available in abundance (Krystynowicz et al. 2002; Ross et al. 1991). The transformation from cellulose-producing to non-cellulose-producing bacteria cells was also reported by Park et al. (2004) for Gluconacetobacter hansenii PJK. They reasoned this was because of shear forces, resulting in the inhibition of BNC formation.

Hestrin and Schramm (1954) reported and described in detail a spontaneous occurrence of $\mathrm{Cel}^{-}$mutants in agitated cultures. They isolated morphologically different types of Gluconacetobacter colonies, showing different abilities to synthesize cellulose. They were mentioned by Yamada (2000) as $\mathrm{Cel}^{+}$, which are fully able to synthesize cellulose (round, gelatinous, and markedly convex colonies), whereas $\mathrm{Cel}^{-}$describes non-cellulose-producing mutants (generally flat and dull colonies) with or without the ability to revert to $\mathrm{Cel}^{+}$forms.

The optimal culture conditions for effective BNC production were investigated in detail by Krystynowicz et al. (2002). They argued that $\mathrm{Cel}^{+}$colonies dominate under stationary culture conditions, not allowing for uniform medium aeration, and explained the phenomenon by Gluconacetobacter cell behavior upon culturing under different conditions. In static conditions, the cellulose-synthesizing cells move toward the oxygen-rich medium-air interface, where they form a BNC membrane that limits access of oxygen into the lower parts of the culture. In agitated cultures, cell growth is preferred instead of polymer synthesis due to the uniform aeration, which results in $\mathrm{Cel}^{-}$domination in the culture. In order to prove this hypothesis, they compared BNC production under stationary culture conditions and under intensive agitation and aeration. They found out that "the major obstacle encountered in agitated and highly aerated cultures of G. xylinus is the tendency of cellulose producing strains to revert to non-cellulose producing mutants (Cel- mutants), which contributes to a decline in BNC production."

Moreover, Krystynowicz et al. (2002) examined the influence of different medium compositions on the occurrence of G. xylinus E25 mutants both in stationary and agitated culture conditions. In experiments with stationary cultures, the type of carbon source and presence or absence of ethanol had no significant impact on $\mathrm{Cel}^{-}$mutant occurrence. In contrast, in experiments with agitated cultures, $\mathrm{Cel}^{-}$mutants were observed immediately after the first culture transfer, and their numbers increased continuously during subsequent transfers, reaching a maximum value of $80 \%$ after the third transfer. Because of this, only a few G. xylinus strains have been found to be suitable for agitated cultivation of BNC (e.g., ATCC 53582; Czaja et al. 2004b; Toyosaki et al. 1995). In this context, it was also discussed by Jung et al. (2005) as well as Park et al. (2003) that the addition of ethanol to the culture medium may prevent the transformation process.

In contrast to this, Ben-Bassat et al. (1992) determined that agitated cultures exhibit substantially reduced byproducts such as gluconic and 
ketogluconic acids, which may be a reason for the reduction in the resulting BNC. Further, Hu and Catchmark (2010a) investigated the influence of the inhibitor 1-methylcyclopropene to either exogenous or endogenous ethylene during the biological senescence of plants on the growth of G. xylinus and its BNC production. A higher concentration of biomass and lower BNC formation was observed in cultures excluding the additive, while the cellulose yield was enhanced up to $25 \%$ in media containing the inhibitor.

\section{Reactor Types}

As mentioned earlier, shake flasks, stirred tanks, bubble columns, airlift reactors, modified airlift reactors, and gaslift bioreactors have been used for BNC production under agitated conditions. The stirred-tank and airlift reactors are the most commonly used bioreactors (Figure 3.2).

In contrast to a bubble-column reactor, the airlift bioreactor contains of a draft tube, which improves circulation as well as oxygen transfer, and equalizes shear forces in the reactor. Depending on the position of the draft tube, the reactor is called an airlift bioreactor with an internal or external loop (Sakairi et al. 1998).

An important process parameter in such reactors is the oxygen transfer. High BNC production rates require a high oxygen transfer rate associated with a high oxygen transfer coefficient $\left(K_{L} a\right)$ (Kouda et al. 1997). However, this may result in high energy costs. To minimize these costs, detailed investigations of agitator configurations have been made. Since in an agitated and aerated culture BNC production increases the viscosity and reduces the homogeneity of the broth, the oxygen level in the broth and following the production rate of BNC is reduced. As one of the first, Kouda et al. (1997) investigated the effect of the agitator configuration on the resulting BNC production. They reported the level of mixing and oxygen transfer capacity for various types of impellers in a stirred-tank bioreactor (Figure 3.3).

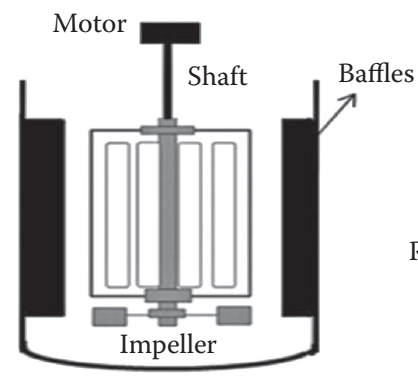

(a) Stirred-tank bioreactor

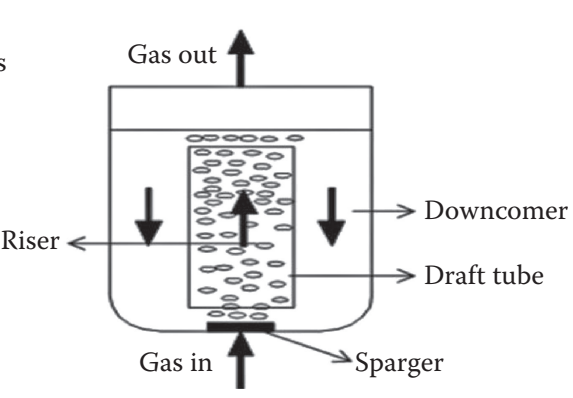

(b) Airlift bioreactor

FIGURE 3.2

Bioreactors typically used to produce BNC (Sani and Daham 2009). 


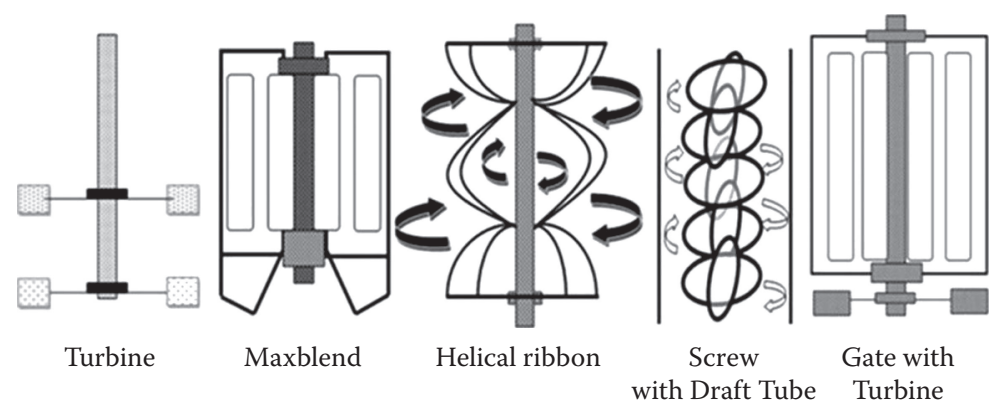

FIGURE 3.3

Agitator configurations including turbine and maxblend impellers used in BNC production (Kouda et al 1997).

They found that the Maxblend and gate with turbine impellers mix culture broths more effectively than the other agitators investigated. In consequence, larger $K_{L} a$ values were achieved. They recommended smaller distances between the area of the culture broth and the impeller in order to further increase the $K_{L} a$. They argue that this characteristic should reduce the high viscosity of the culture broth, where the shear speed is low. Moreover, the impeller should induce a high shear region around the sparger in order to increase the dispersion of bubbles and $K_{L} a$.

\section{Agitated Cultivation by Shaking}

As very simple bioreactors, shaken flasks can be used for BNC generation in the form of spheres. Czaja et al. (2004b) focused their investigations on the structural influence of BNC formed in agitated culture conditions. Furthermore, they were interested in bacteria behavior and BNC product accumulation during the biosynthesis. They found that the G. strain ATCC 53582 effectively synthesizes BNC in the form of unique, large spheres and in large amounts similar to stationary culture after 7 days. Other authors have investigated the influence of the bioreactor vessel size on the production of BNC spheres. By utilizing 150 and $100 \mathrm{ml}$ Erlenmeyer flasks under the same conditions (medium volume and rotation speed), the size of the spheres was decreased by one order of magnitude (Hu and Catchmark 2010b).

Most of the cells have been found to be distributed at the sphere surface and only a few of them are randomly scattered inside. Based on the hypothetical formation process, cells are assumed to become attached around the surface of air bubbles existing in the agitated liquid. The bacteria then start to proliferate and synthesize cellulose ribbons, resulting in a compact structure. Close observation by Czaja et al. (2004b) revealed that cellulose characterized by uniaxially oriented ribbons is formed in stationary culture, whereas cellulose synthesized under agitated conditions possesses a structure of disordered, curved, and overlapping ribbons with a smaller 
fiber diameter. In contrast to other strains, no mutation to non-cellulose-producing cells of this bacterium occurred under agitation, thus this strain is characterized by its great genetic stability. This fact, and the good production yield, might have a great impact on its future applicability in large-scale fermentation. Joseph et al. (2003) studied shaken flask cultures with added polyacrylamide-co-acrylic acid. Using G. xylinus BPR2001 (ATCC 700178), an increase in BNC production was observed. Depending on the rotation speed, the cellulose production increased from $2.7 \pm 0.8$ to $6.5 \pm 0.5 \mathrm{~g} / 1$ at a shaker speed of $175 \mathrm{rpm}$ and from $1.7 \pm 0.01$ to $3.7 \pm 0.5 \mathrm{~g} / 1$ at a speed of $375 \mathrm{rpm}$ for polyacrylamide-co-acrylic acid concentrations up to $3 \mathrm{~g} / \mathrm{l}$ and a 7 day incubation period. Also, a change in the BNC morphology from amorphous, stringy forms to spheroidal particles with diameters $\leq 2 \mathrm{~mm}$ was observed by the authors.

\section{Agitated Cultivation by Stirring}

In contrast to shaking, agitation by stirring can be produced by impellers or gas transfer. Stirred-tank fermenters and bubble columns use this effect; stirred-tank fermenters provide superior oxygen transfer by manipulating the aeration and agitation speed.

Using G. xylinus NUST4.2, Zhou et al. (2007a) examined the structure of BNC produced in stationary culture in comparison with that generated in a stirred-tank reactor. They found a BNC yield of $7.5 \mathrm{~g} / 1$ in stationary culture after 6 days and $3.13 \mathrm{~g} / \mathrm{l}$ in the stirred-tank reactor after $72 \mathrm{~h}$. With regard to productivity, a decrease from $0.052 \mathrm{~g} / \mathrm{l} / \mathrm{h}$ to $0.043 \mathrm{~g} / \mathrm{l} / \mathrm{h}$ could be estimated for the latter cultivation. Furthermore, the cellulose ribbons produced in static culture were more entangled and built a denser network with curved and overlapping fibers compared with the BNC materials produced in the stirred-tank reactor. Nevertheless, BNC synthesized in static culture was characterized by higher crystallinity, higher $I_{\alpha}$ content, and larger crystallite size than cellulose produced in the stirred-tank reactor. The authors argued that all of these results revealed that stirring in the reactor strongly interferes with the process of nascent microfibril crystallization, favoring the formation of smaller microfibrils and increased $I_{\beta}$.

In relation to this, Watanabe et al. (1998) compared BNCs produced in two cultures and found no difference between reticulated structures of bacterial cellulose fibrils produced in agitated and static cultures. Nevertheless, the BNC produced in agitated culture exhibited microstructural changes, namely, a lower degree of polymerization, with a $D P_{\mathrm{w}}$ of 10,900 (static cultivation $\left.D P_{\mathrm{w}} 14,400\right)$ and a crystallinity index of $63 \%$, in contrast to $71 \%$ for static cultivation, as well as a decrease of cellulose $I_{\alpha}$ content, with a value of $61 \%$ for agitated culture (static cultivation $73^{\circ} \%$ ). Furthermore, a lower Young's modulus of the sheet, a higher water-holding capacity, and a higher dispersion viscosity in the disintegrated form than that produced in static culture have been observed. 
Based on these results, higher shear stresses, which are necessary for high oxygen transfer, seem to be unsuited for cultivation of mostcellulose-producing bacteria strains. In comparison, bubble columns have low shear stresses, but they are also characterized by low oxygen transfer and poor mixing. Because of the sensitivity of some bacteria cells to shear stress and the necessity for high oxygen consumption rates, both the stirred-tank fermenter and the bubble column seem to be unfavorable. To solve this problem, Wu et al. (2001) developed a modified bubble column, whereby several wire mesh draft plates were installed inside the column. Thus the air from the sparger entered the column between two wire mesh draft plates, resulting in a 201 column with three modules of draft plates. In the proposed modified bubble column, higher values of gas holdup and of the volumetric mass transfer coefficient, as well as lower mixing times than those in the bubble column without wire mesh draft plates, were estimated. Experimental testing of the proposed modified column to cultivate sensitive G. xylinus confirmed the improvement with better production yields.

Further investigation from Zhou et al. (2007b) dealt with BNC production by G. xylinus strain NUST4.1 in shake flasks and in a stirred-tank reactor by adding sodium alginate into the medium. They found that an addition of $0.04 \%(\mathrm{w} / \mathrm{v})$ sodium alginate into a shake flask produced a BNC yield of $6.0 \mathrm{~g} / \mathrm{l}$. The conversion rate from sugar to cellulose was $27 \%$, compared with $3.7 \mathrm{~g} / \mathrm{l}$ and $24 \%$ under static conditions. Using a stirred-tank reactor, the addition of sodium alginate influenced the BNC morphology from irregular clumps and fibrous masses entangled in the internals to discrete masses dispersed in the broth. The authors assumed that sodium alginate hinders the formation of large clumps of BNC and enhances the cellulose yield. As a result, some differences in reticulated structures and ribbon width as well as lower crystallinity (decreased from 78\% to 59\%) and a smaller crystallization size of BNC produced with sodium alginate added were observed.

\section{Batch Fermentation under Stirring Using Plastic Composite Supports}

Cheng et al. (2009) recently described the implementation of different types of plastic composite supports (PCSs) in a submerged fermentation process to enhance the resulting yield and material properties of BNC. The general idea followed was the generation of BNC biofilms growing on a solid support where microorganisms become attached as a natural kind of cell immobilization. The PCSs were an extrusion product of a mixture between polypropylene and nutritious compounds (Pometto et al. 1997). The polypropylene was used as a matrix integrating agricultural mixtures (e.g., ground soybean hulls, soybean flour, and microbial nutrients such as bovine albumin, red blood cells, yeast extract, and peptone) as well as mineral salts and released these nutrients slowly during fermentation. The fermentations were conducted at $30^{\circ} \mathrm{C}$ in a 1.251 Bioflo 3000 fermenter (New Brunswick Scientific, Edison, NJ) using a working volume of 11 and an agitation rate of $100 \mathrm{rpm}$. 
Within this bioreactor, 12 PCS tubes were bound to the agitator shaft in a gridlike fashion, with six rows of two parallel tubes. Batch fermentation in this reactor for $120 \mathrm{~h}$ using the best suited type of PCS tubes resulted in higher yields and improved material properties of the BNC generated compared to suspended-cell fermentation used as control. For example, the BNC tested from the PCS biofilm reactor showed an increase in the stress at break from 2.8 to $34.2 \mathrm{MPa}$ (PCS-grown BNC) and of the Young's modulus from 286 to $2401 \mathrm{MPa}$ (PCS-grown BNC).

\section{Airlift Reactors}

Instead of a stirred bioreactor, Chao et al. (2000) successfully used a $50 \mathrm{~L}$ internal-loop airlift bioreactor (Figure 3.4) to improve the oxygen transfer rate and achieved a high BNC yield of $3.8 \mathrm{~g} / 1$ after $67 \mathrm{~h}$ (corresponding productivity $0.0567 \mathrm{~g} / \mathrm{l} / \mathrm{h}$ ) using G. xylinus subsp. sucrofermentans (BPR2001). However, the production rate of BNC was significantly increased by the utilization of an oxygen-enriched gas in the system. The BNC amount rose to $0.116 \mathrm{~g} / \mathrm{l} / \mathrm{h}$. Chao et al. pointed out that the value is twice that of a typical air-supplied culture and comparable to that of a mechanically agitated

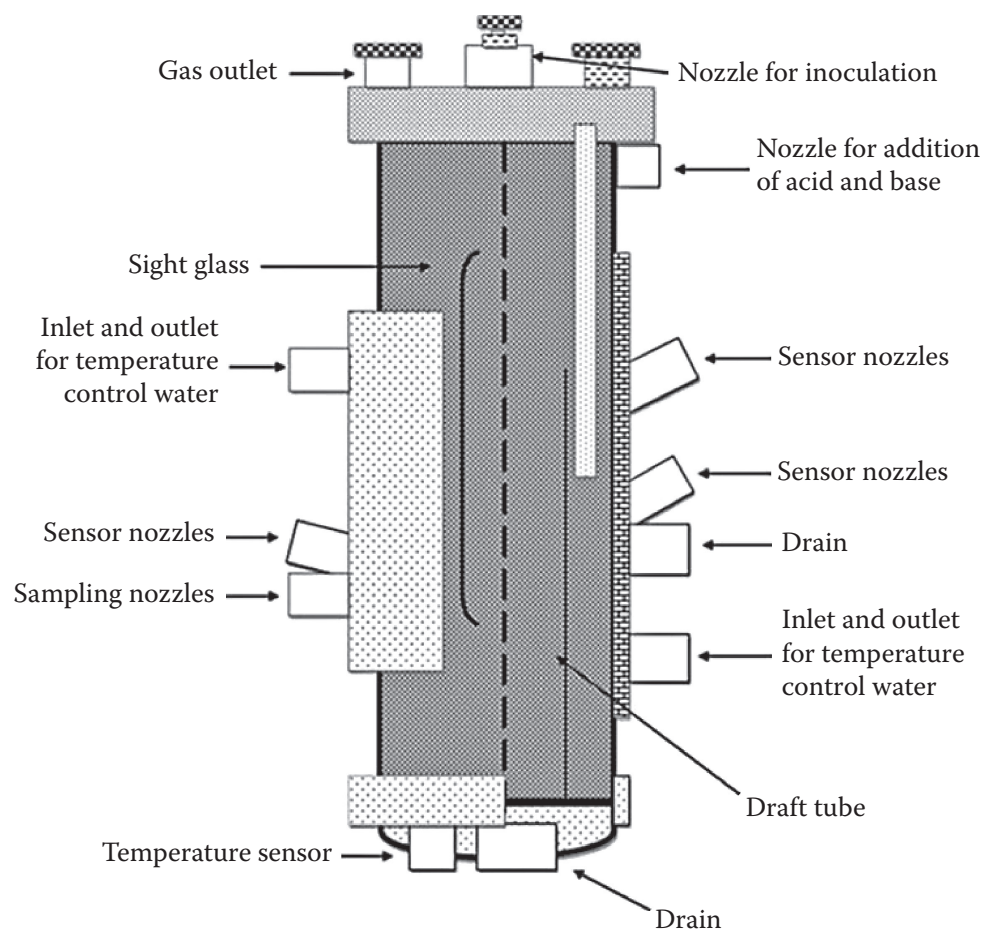

FIGURE 3.4

Schematic representation of the $50 \mathrm{~L}$ internal-loop airlift reactor (Chao et al. 2000). 
stirred-tank fermenter. They explained this outcome by the higher oxygen concentration dissolved and the lower shear force environment of the airlift bioreactor. In previous investigations they clarified that higher shear caused by the impeller in the stirred-tank bioreactor results in a negative effect on BNC production. The effect increases with increasing stirring speed (Joseph et al. 2003; Kouda et al. 1996).

In further investigations, Chao et al. (2000) realized a BNC production of $8.7 \mathrm{~g} / \mathrm{l}$ compared with $6.3 \mathrm{~g} / \mathrm{l}$ in the control medium using the addition of $0.1 \%(w / w)$ agar (water-soluble polysaccharide). Also, the length of the cultivation period until the BNC maximum was significantly shortened, to approximately half of the outcome without adding polysaccharide. They assumed that the agar addition hindered the formation of large BNC clumps, which resulted in the increased BNC production rate. Since smaller pellets are advantageous for transferring nutrients and oxygen into bacterial cells located inside and on the surface of the cellulose matrix, faster growth rates were achieved. They noted that in a stirred-tank reactor, where turbines and baffle plates destroy the cellulose clumps, the addition of polysaccharide would have no influence on the BNC production rate.

The main parts of such an airlift reactor include a nozzle for inoculation, a gas outlet, nozzles for the addition of acid and base, sensor nozzles, an inlet and outlet for temperature-controlled water, a sampling nozzle, a temperature sensor, a drain, a sight glass, and a draft tube (Figure 3.4).

The application of airlift bioreactors results in BNC arranged in elliptical pellets instead of the fibrous forms produced by stirred-tank reactors. The pellets are also more compact and dense. The cellulose nanofibers within the pellets are characterized by a higher degree of polymerization and improved physicochemical properties than fibrous forms. The pellet sizes have been found to decrease with an increase in the amount of substrate in the feed, but also with a decrease in the dissolved oxygen concentration in the medium (Chao et al. 2001a, 2001b). In contrast, the density of the pellets increased with the increase in the amount of substrate used.

Wu et al. (1990) were the first to combine a bubble column and a conventional internal-loop airlift reactor. The resulting proposed reactor system included a circular, wire mesh draft tube. It was found that the $K_{L} a$ depends on the gas velocity. If the gas velocity exceeded $2 \mathrm{~cm} / \mathrm{s}$, the $K_{L} a$ of the proposed reactor would be higher than that of the original bubble-column or airlift reactor (Wu 1992; $\mathrm{Wu}$ and $\mathrm{Wu}$ 1991). At a superficial gas velocity of $5.02 \mathrm{~cm} / \mathrm{s}$, the $K_{L} a$ value of the proposed reactor with wire mesh draft tube was found to be double that of the airlift reactor. In the case where the proposed reactor was used with a fed-batch culture, an increase in cell mass was obtained.

Furthermore, Cheng et al. (2002) developed a rectangular wire mesh draft tube that allowed bubble slicing and subdivision. The modified airlift reactor was found to possess a higher volumetric oxygen transfer coefficient and mixing capability. As a result, the final BNC concentrations produced after $72 \mathrm{~h}$ of cultivation were three times higher than those produced in conventional 
bubble-column reactors. Within this modified airlift reactor, elliptical BNC pellets are formed. Their size depended strongly on the amount of dissolved oxygen in the broth. One additional possibility of agitated culture for producing food-use gelatinous BNC is applying a two-stage fermentation process: a cell-growth stage in an airlift fermenter (agitated culture) was followed by the BNC formation stage in production incubators (static culture) (Okiyama 1992).

Recently Song et al. (2009) used a modified airlift-type bubble-column bioreactor to transfer an agitated cultivation process of BNC into pilot scale. For this they constructed a $50 \mathrm{~L}$ spherical-type bubble-column bioreactor with low shear stress and high oxygen transfer rates (see Figure 3.5). Saccharified food waste was used as culture medium due to its low cost.

BNC produced in agitated culture exhibits microstructural changes compared to material produced by static cultivation, namely, a lower degree of polymerization and lower crystallinity index, allowing for easier chemical modification. Despite a reduction in the ultimate BNC yield and the conversion rate of sugar to cellulose as a result of the spontaneous transformation of the bacterial cells, several agitated cultivation methods produce high space-time yields and produce various shapes and structures. Accordingly, this kind of cultivation constitutes an important basis for the diversity of the high-tech biopolymer BNC and opens up novel potential application fields.

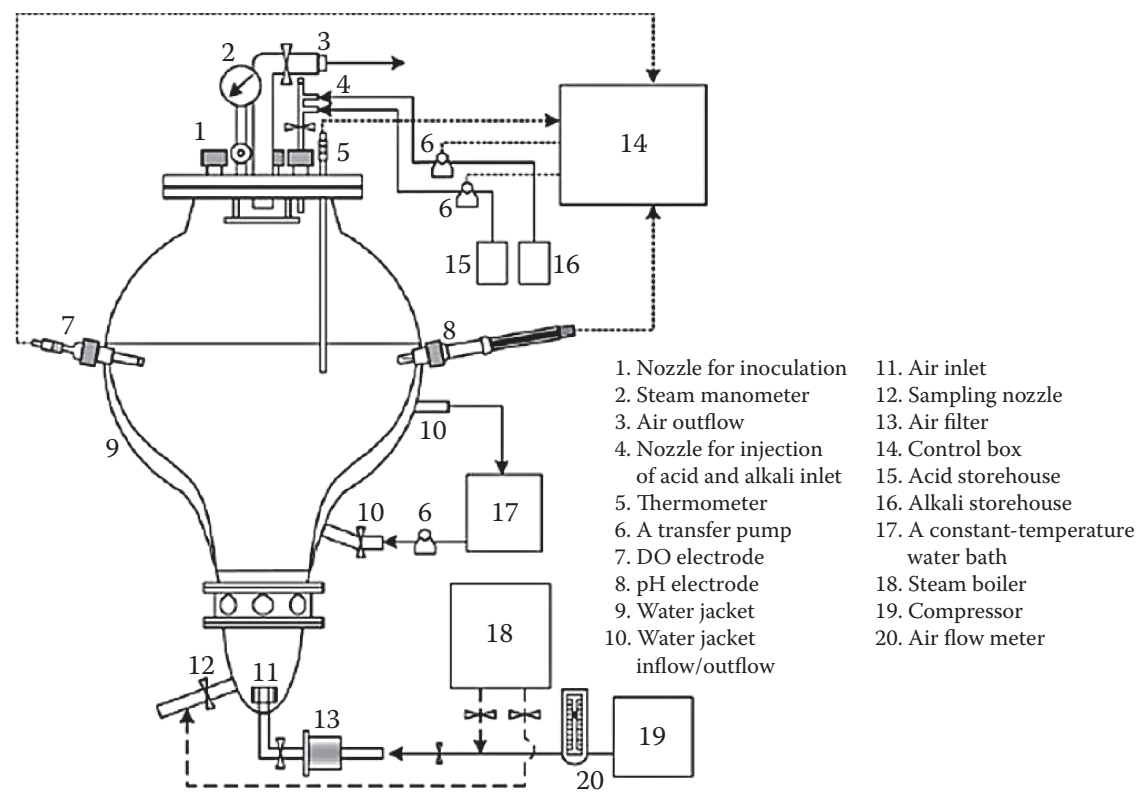

FIGURE 3.5

L spherical-type bubble-column bioreactor (Song et al. 2009). 


\section{Static Cultivation}

\section{Introduction}

Since the beginning of intensive BNC research activities in the mid-1990s, static cultivation has been considered the standard type of cultivation for BNC. Based on various studies, it has been shown that the most homogeneous supramolecular BNC structure, resulting in highly stable material, can be produced with this method. If the culture conditions are changed, it leads to drastic and often negative changes in the resulting BNC properties. Accordingly, this form of cultivation is still regarded as the preferred manufacturing method to synthesize flat BNC with excellent and reproducible structures and properties for commercial applications.

As one of the first purchasable products based on BNC, the dessert nata de coco is well established in the Asian food market. It is usually synthesized by static cultivation, where the product is skimmed in the form of sheets from the culture medium.

In nata de coco home industries, plastic vessels are used (see Figure 3.6). After inoculation, the vessels are covered and kept in a storehouse for 8-10 days. For purification, the bacteria in the BNC are removed by immersing it in dilute alkaline solution and washing with water (Budhiono et al. 1999; Iguchi 2000; Kouda et al. 1998).

Scientific investigations in the context of static cultivation forms of BNC dealt with the optimization of the cultivation conditions, such as the culture medium,

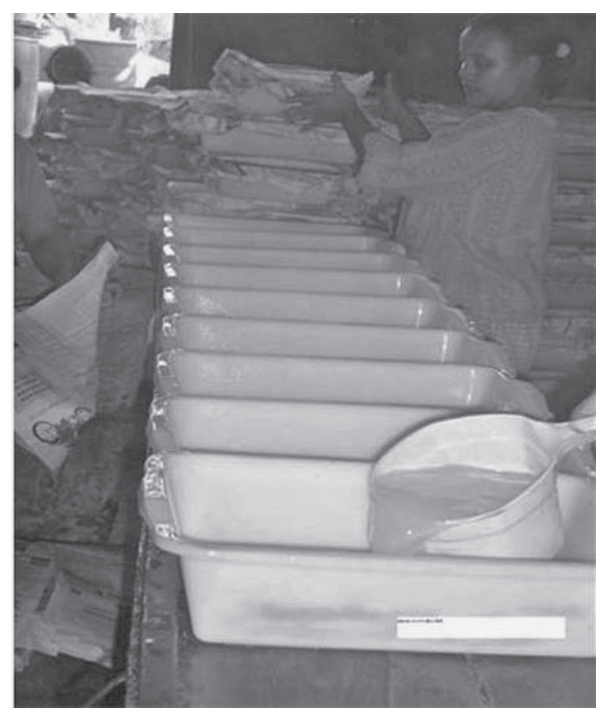

FIGURE 3.6

(See color insert.) Production process of nata de coco (Source: http://www.bi.go.id/sipuk/en/). 
oxygen content, as well as the $\mathrm{pH}$ value and the temperature. For example, Watanabe and Yamanaka (1995) examined the effect of oxygen tension in the gaseous phase on the production and physical properties of BNC formed under static cultivation conditions. They stated that the cellulose production was much higher at oxygen tensions of $10 \%$ or $15 \%$ than under atmospheric conditions.

Likewise, Verschuren et al. (2000) investigated the development of oxygen concentration gradients and the effective diffusion coefficient, $D_{\mathrm{e}}$, of oxygen in a cellulose pellicle generated under static conditions. They obtained an oxygen flux of $9.1 \times 10^{-6} \mathrm{~mol} / \mathrm{m}^{2} / \mathrm{s}$ at a $\mathrm{pH}$ value of 4.0 . The effective diffusion coefficient of oxygen was equal to $1.4 \times 10^{-9} \mathrm{~m}^{2} / \mathrm{s}$ under all conditions investigated.

Levy et al. (2004) suggested producing sheets and membranes of BNC using a tray with culture medium that is prepared in a mechanical mixer. The reactor is furnished with a jacket for temperature control and heat exchange, whereas the temperature is controlled and maintained through the circulation of hot or cold water. The BNC sheets produced are collected from the surface of the culture medium and are then submitted to a process of purification, passing through a semicontinuous system of rollers and belt conveyors made of adsorbent material. The approach has been improved by Farah et al. (2006) so that replacement of the culture medium in the tray is not necessary. The resulting BNC fleece is drained via the box and the cultivation is complete. This procedure can be repeated until a volume of only $15 \%-25 \%$ of the initial volume occurs. Then new growth medium had to feed in.

\section{Benefits and Limitations of Static Cultivation Forms in Batch Vessels}

Because the BNC is formed at the air-liquid interface, any commercial production process based on this type of cultivation is area intensive. Often the process is also less automated than agitated cultivation in deep tank fermenters and therefore demands more manual work and higher personnel costs. Moreover, the synthesized flat BNC material depends on the reaction vessels used, and thus is limited in size and not variable. Under batch conditions, the concentration of nutrients changes during cultivation, limiting the maximum cultivation time and requiring regular costly holding times.

\section{Fed-Batch Cultivation}

The cultivation time of static cultivation can be prolonged by the feeding of new culture medium during the bioprocess. Toda et al. (1994) reported a yield increase of BNC using a culture with a continuous or periodic supply of carbon sources from underneath the cellulose pellicles.

In 2007 Hornung et al. presented the aerosol bioreactor. They reported that earlier experiments with a self-constructed rotating disk system resulted in nonhomogeneous BNC with low quality and BNC sections that were mechanically weaker than others. Furthermore, unwanted cellulose grew in the substrate reservoir, interfering with the movement of the rotating disk 


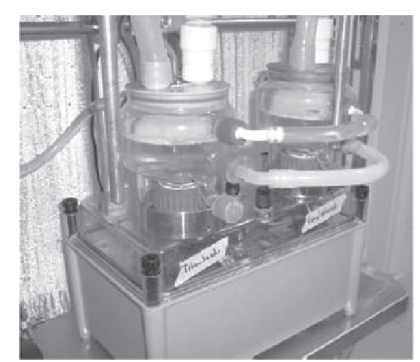

(a)

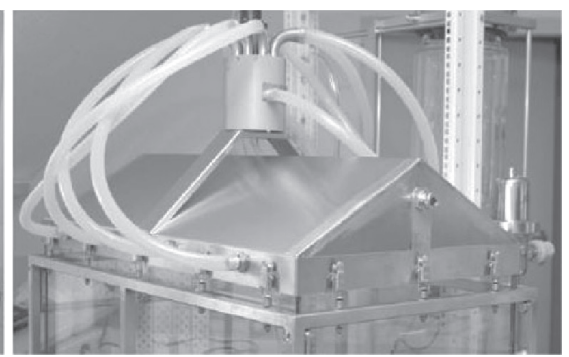

(b)

FIGURE 3.7

The substrate aerosol generator (left) and aerosol spreading (right) (Hornung et al. 2007).

and the BNC tended to separate from the metallic mesh on which it was supposed to form when the cellulose layer became thicker. The authors stated that these problems were overcome by the aerosol bioreactor.

The aerosol generator (see Figure 3.7, left panel) produces a spray of the nutrient broth by ultrasound using two removable and sterilizable chambers. These are filled by gravity from a feed tank above the chambers, and sterile air is introduced. Then the spray is transported into the aerosol distribution box (see Figure 3.7, right panel). Piezoelectric crystals are used as a vibration source.

The spray is fed to a rotating disk device ( $4 \mathrm{rpm}$ ) that feeds two of the eight exit channels at any time to ensure a homogeneous distribution. Flexible tubes lead the spray to a special roof-shaped distribution box situated on top of the culture box. The two streams come at the same time, but on opposite sides, horizontally into the vessel in order to cause turbulence. The culture box, with dimensions of $500 \mathrm{~mm} \times 900 \mathrm{~mm} \times 500 \mathrm{~mm}$ is made of temperatureresistant security glass (see Figure 3.8). The whole construction is operated under a slightly positive pressure to avoid contamination from the outside. The average growth of BNC observed in this apparatus was $2 \mathrm{~mm}$ /day or approximately $9 \mathrm{~g}$ cellulose dry mass/day. The runs were stopped when contamination was observed.

To harvest the cellulose layer from the aerosol bioreactor, the distributor box is removed and the block of product is lifted out by hand. In order to get a number of slices rather than a block of BNC material, the supply of substrate is switched off for a period of time.

The best results were found by the authors when a $6 \mathrm{~h}$ interruption was used, resulting in 3-4 cm thick layers. One drawback to the bioreactor is the ingress of contaminating organisms, which usually occurs between 6 and 10 weeks after the start of the fermentation process. The location of the problem is usually the aerosol generator or the distribution box, which cannot be disinfected during a production run. The authors were unsure as to whether the contaminating species come from outside the apparatus or arise from insufficient sterilization of the nutrient components at the start of the process. 


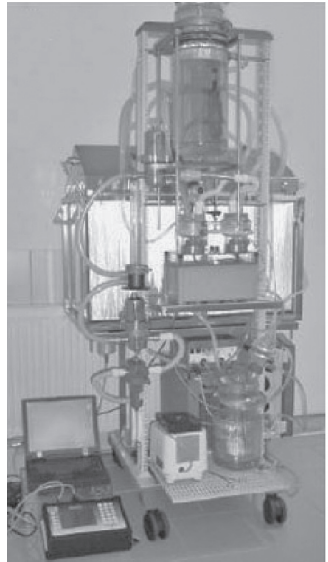

(a)

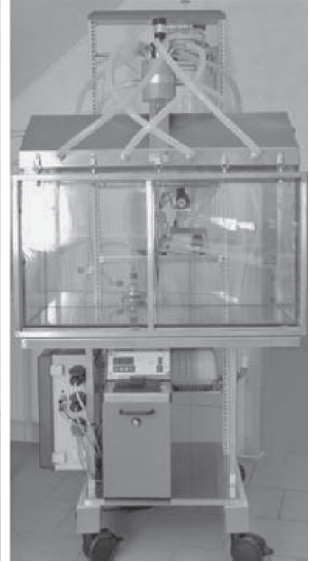

(b)

FIGURE 3.8

The aerosol bioreactor (left hand: back; right hand: front). (Hornung et al. 2007).

Shezad et al. (2009) recently described fed-batch cultivation for the generation of BNC sheets by G. hansenii PJK under static cultivation conditions using a chemically defined medium. They used waste from beer fermentation broth to decrease the material costs. For the fed-batch cultivation, $500 \mathrm{ml}$ of a culture medium were initially inoculated in a 31 jar fermenter (Kobiotech Co., Korea) without impeller. Fresh medium was fed in periodically. The cultivation was carried out at $3^{\circ} \mathrm{C}$ applying an aeration rate of $1 \mathrm{vvm}$. Compared to a batch cultivation after 30 days of cultivation, the authors described an increase of two to three times the BNC production compared to fed-batch cultivation.

Weuster-Botz et al. (2011) recently presented a lab-scale membrane bioreactor prototype that can be used to produce a BNC pellicle with a homogeneous layered structure. They used a hydrophilic microfiltration polyethersulfone membrane to separate the bacteria from the cultivation medium and to provide mechanical support for the formation of the BNC layer.

\section{Combination of Static and Moving Cultivation}

Other concepts investigated during the last couple of years include a combination of static and moving cultivation principles.

\section{Static Cultivation with Moving Harvesting}

One of these approaches was suggested by Saikiri et al. (1998) as a method for direct harvesting of BNC filaments during the cultivation of G. xylinus. 


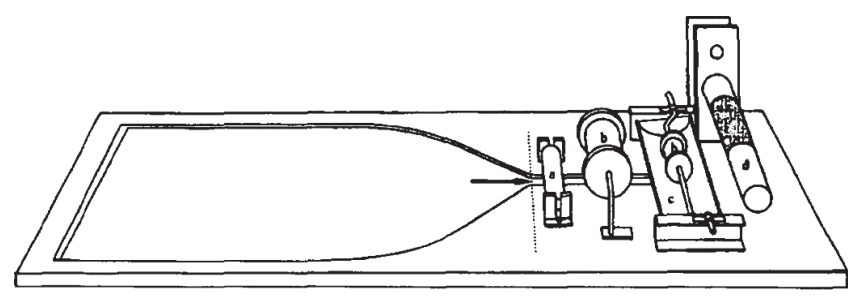

FIGURE 3.9

Outline of culture pan for the direct filamentation of BNC. a, sinker; b, roller; c, washing pan; d, wind up roller developed by Sakairi (Sakairi et al. 1998).

They devised a shallow pan, conserving the culture medium under static conditions, for continuous filamentation of a thin BNC gel (see Figure 3.9). One side of the shallow pan was curved gently to permit harvesting of the BNC pellicle through a narrow mouth. The filament obtained was wound slowly on an attached basket, whereas the BNC gel was stretched in a slightly twisted mode on the in situ formability of BNC, Klemm et al. (1999) used the G. xylinus strain DSM 14666 as a "cellulose factory" to produce BNC in the form of tubes. With this patented matrix technology it is possible to synthesize cellulose in the shape of hollow bodies of different length, wall thickness, and inner diameter directly in the culture medium. The resulting tube, also called BASYC ${ }^{\circledR}$, has an inner diameter of $1-3 \mathrm{~mm}$, a length of about $5 \mathrm{~mm}$, and a wall thickness of $0.7 \mathrm{~mm}$. These parameters make the resulting tubes well suited for microsurgical applications.

\section{Non-Static-Film Bioreactors}

Non-static-film bioreactors without agitation by a stirrer or shaker, but with movement by discs, belt conveyors, or others methods were used in the production of BNC by Bungay and Serifica (2000). BNC pellicles were obtained by culturing cellulose-producing organisms for long periods of time under nonstatic conditions under application of linear conveyors or rotating disks operated in fed-batch mode. In the first, a linear conveyor passes through and exits the culture medium contained in vessels coated by a BNC gel film, which is partially dehydrated (see Figure 3.10, right panel). The gel film is abraded afterward. In the latter reactor, the same effect is obtained using rotating disks instead of the conveyor (see Figure 3.10, left panel). These bioreactors provide highly hydrated pellicular and film forms of BNC and may lead to nonuniform BNC material due to the bundling and aggregation of thin layers or filaments. During cultivation, sugar is added in order to maintain a certain level of sugar concentration in the reactor during the course of the fermentation.

Later, the generation of $\mathrm{BNC}$ on rotating rollers or discs was investigated by Krystynowicz et al. (2002). A specific biofilm of cells and polymer 

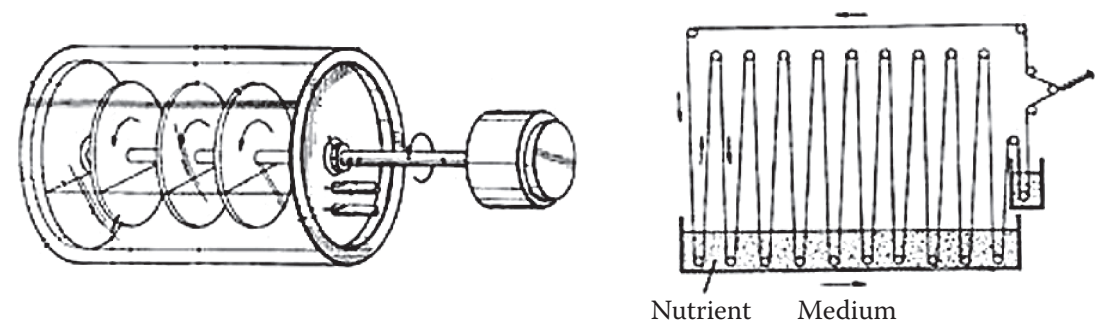

FIGURE 3.10

Rotating disk and linear conveyors reactors (adopted from U.S. 15,955,326).

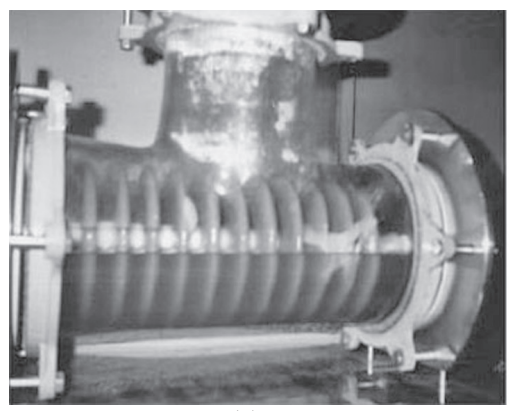

(a)

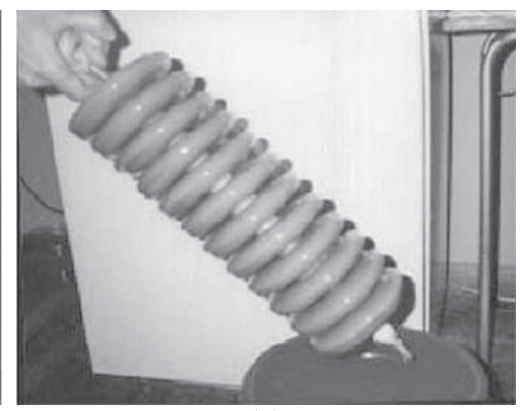

(b)

\section{FIGURE 3.11}

Biosynthesis of BC in the RDF. (a) Cultivation in RDF (b) BC attached to the discs after 7 days of culture or with rollers (Krystynowicz et al. 2002).

is temporarily dipped in the liquid medium during cultivation. The BNC attaches and accumulates on the rough surface of the rollers or discs, which rotate along the long axis and are temporarily dipped in the liquid nutrient medium. In their study, they used two different types of horizontal fermenters equipped with plastic discs or rollers (see Figure 3.11). They found many similarities between the time course of the BNC synthesis in the rotating disk fermenter and in a typical stationary culture. Differences were related to the membrane thickness, which was slightly greater in the case of culture in the rotating disk fermenter, whereas other parameters, such as cell numbers, $\mathrm{pH}$, and glucose utilization, were similar.

They found that "at a higher rotation speed, cellulose membranes that accumulated on the surface of the discs were much thicker, despite the fact that the total mass of synthesized cellulose was lower" as a result of a greater degree of cellulose hydration. The liquid layer on the surface of the disk is dependent on disk diameter and the material used, as well as on the depth of the disk immersion in the culture medium.

Also BNC biomaterial in several forms suggested for tissue engineering has been developed in a combination of static and agitated cultivations. 
By this process the production of 3D BNC bodies can be realized using the two-step method for cultivation of G. hansenii (Porto et al. 2010).

\section{(Semi)continuous Generation of Flat BNC}

Inspired by the increasing demand for a commercial production process for planar BNC material for medical as well as technical applications and supported by the approaches discussed earlier, Kralisch et al. (2010a) designed a new, efficient process for the (semi)continuous cultivation of planar BNC fleeces and foils. By means of the so-called horizontal-lift reactor concept, a continuous tape of BNC material with freely selectable length and adjustable thickness can be produced. The planar BNC material generated has the same homogeneous, undisturbed 3D network structure as when cultured under static conditions. This beneficial combination of the advantages of continuous processing and nearly static cultivation results in smooth extraction of the $\mathrm{BNC}$, which is produced in this apparatus and has reached the size requested. It is lifted up and transported out of the culture apparatus in a continuous manner using an extractor device at the opposite side of the apparatus without disrupting the surface of the culture medium and without disturbing the BNC sheet, which is under cultivation. At the front side of the apparatus, new BNC starts to grow again in the vacant part of the interface between the air and culture medium.

In order to test and optimize this continuous production process for planar BNC fleeces or foils, two plants (Schmidt et al. 2010) based on this concept were constructed. First, an experimental HoLiR plant was designed as a plane culture apparatus $(V=20 \mathrm{l})$ made from stainless steel. Two inlet and two outlet tubes, arranged at the front and the back of the apparatus, allow for continuous dosage and regulation of the culture medium. The cultivation temperature can be maintained using an electrical heating pad. The whole apparatus is enclosed by a housing and filtered air is passed through this housing in order to ensure an appropriate oxygen content in the air. After an initial cultivation phase, the BNC, which has been produced in this apparatus and has reached the size requested, is lifted up and transported out of the culture apparatus in a continuous or gradual manner using an extractor device. The output, still not cut off from the rest, is immediately treated with a bacteria-deadening and cleaning solution in order to inhibit ongoing growth of the BNC. This is followed by a cleaning procedure using a (semi)continuous system of rollers, belt conveyors, and baths filled with cleaning agents.

After a successful proof of concept by means of the experimental plant, a pilot plant was constructed by transferal of the plant characteristics described earlier. For this, the dimensions of the culture apparatus were scaled up to a volume of $180 \mathrm{l}$. 

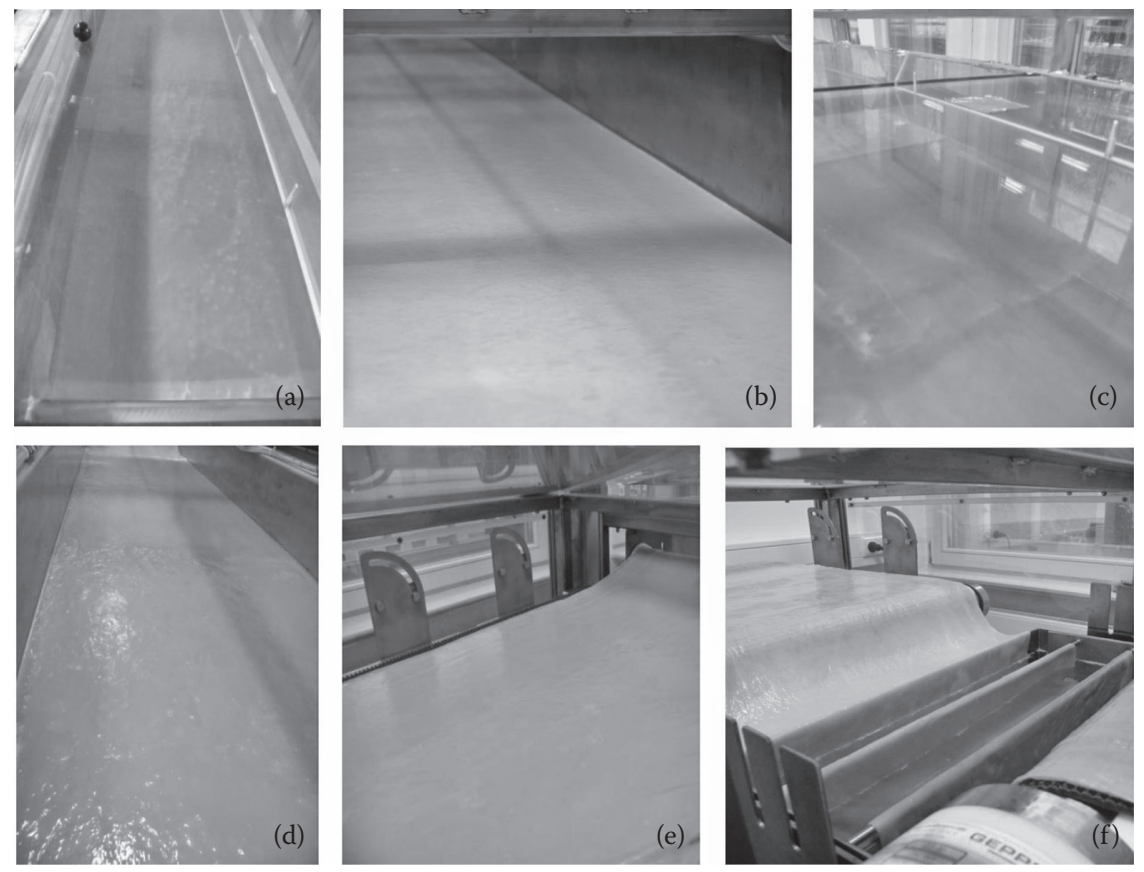

FIGURE 3.12

Stages of BNC cultivation within the HoLiR pilot-plant; G. xylinus strain DSM 14666, a) beginning of BNC generation, b) completed BNC fleece, c) start of continuous harvesting and generation of new BNC at the vacant surface of cultivation medium, d) growing of the BNC fleece during continuous transportation, e) harvesting via belt conveyor, f) continuous cleaning.

The BNC generation process within this pilot plant can be subdivided into the following stages (see Figure 3.12) (Kralisch et al. 2011).

1. After inoculation of the culture medium, the BNC generation becomes visible in the form of a mechanically unstable prepolymer with a wide meshed fiber network.

2. A plane BNC material with a uniform surface is generated with a growth thickness of the material of $0.5-1.5 \mathrm{~mm} /$ day after the surface of the prepolymer is completely closed. Depleted culture medium is led out. The initial fed-batch cultivation is switched to a slow-moving cultivation and continuous harvesting between day 7 and 10 .

3. At the vacant surface of the cultivation medium, new $\mathrm{BNC}$ is generated.

4. During its growth, the BNC fleece is continuously transported through the bioreactor. The sizes of the fleeces can be adjusted by the speed of transportation.

5. The material is harvested via several belt conveyors.

6. The material is then treated in cleaning baths, integrated in the process. 
Once the process has been started (stages 1 and 2), the bioprocess can be kept stable over several months, without any need for disrupting, manual intervention, or shutdown. Since air and culture medium are continuously fed into the apparatus, steady-state cultivation conditions are maintained.

The BNC material called JeNaCell ${ }^{\circledR}$, cultivated within the HoLiR, is characterized by a high crystallinity of $82 \%$ (G. xylinus ATCC 11142) to $86 \%$ (G. xylinus DSM 14666) and a homogeneous, undisturbed network structure. The costs for BNC generation as well as the time for starting, shutdown, and cleaning of the reactor could be significantly reduced by this novel approach compared to conventional static cultivation.

\section{Conclusions}

Although several BNC-based products are commercially available today, no efficient automated process for mass production of this biopolymer on an industrial scale has been realized so far. Thus BNC is currently not economically competitive compared with other more established polymers in most applications. Exceptions are the hand-made production of nata de coco in Asian countries, since the product is produced from the cheap raw material coconut water, and with low salaries and simple techniques.

However, the design and optimization of technical processes for BNC generation are ongoing for the different realizable forms of this biopolymer addressing various future applications. Aiming for BNC in the form of pellets, fibrous material, or sphere-like particles, agitated culture conditions utilizing airlift or stirred-tank reactors have been used. Airlift and adapted bubble-column-type reactors have been successfully tested on a pilot scale using a reactor volume of 50 l. Even for the generation of flat BNC in the form of fleeces and foils, novel reactor types have been developed to overcome the most important drawbacks of static cultivation: area and workforce intensity. The (semi)continuous HoLiR process has been recently transferred to pilot scale as well.

These developments strengthen the hope for future large-scale production processes, which are urgently needed to bring the multitude of promising product developments based on BNC from the laboratory to the market.

\section{References}

Astley, O. M., E. Chanliaud, A. M. Donald, and M. J. Gidley. 2001. Structure of Acetobacter cellulose composites in the hydrated state. Int. J. Biol. Macromol. 29:193-202. 
Bae, S., and M. Shoda. 2004. Bacterial cellulose production by fed-batch fermentation in molasses medium. Biotechnol. Prog. 20:1366-1371.

Ben-Bassat, A., R. Bruner, S. P. Shoemaker, Y. Aloni, H. Wong, D. C. Johnson, and A. N. Neogi. 1987. Reticulated cellulose product, sheets formed therefrom, methods and microorganisms for their production. European patent application EP 228779.

Ben-Bassat, A., R. Bruner, S. Shoemaker, Z. Aloni, H. Wong, D. C. Johnson, and A. N. Neogi. 1992. Reticulated cellulose and methods and microorganisms for the production thereof. U.S. Patent 5,079,162.

Borzani, W., and S. J. De Souza. 1995. Mechanism of the film thickness increasing during the bacterial production of cellulose on non-agitated liquid media. Biotechnol. Lett. 17(11):1271-1272.

Brown, A. J. 1886. An acetic ferment which forms cellulose. J. Chem. Soc. 49:432-439.

Brown R. M., Jr., K. Kudlicka, S. K. Cousins, and R. Nagy. 1992. Gravity effects on cellulose assembly. Am. J. Bot. 79(11):1247-58.

Budhiono, A., B. Rosidi, H. Taher, and M. Iguchi. 1999. Kinetic aspects of bacterial cellulose formation in nata de coco culture system. Carbohydr. Polym. 40:137-143.

Bungay, H. R., and G. C. Serafica. 2000. Production of microbial cellulose. Contributionin-part of U.S. Patent 15,955,326.

Chao, Y., T. Ishida, Y. Sugano, and M. Shoda. 2000. Bacterial cellulose production by Acetobacter xylinum in a 50-L internal-loop airlift reactor. Biotechnol. Bioeng. 68:345-352.

Chao, Y., M. Mitarai, Y. Sugano, and M. Shoda. 2001a. Effect of addition of water soluble polysaccharides on bacterial cellulose production in a 50-L airlift reactor. Biotechnol. Prog. 17:781-785.

Chao, Y., Y. Sugano, and M. Shoda. 2001b. Bacterial cellulose production under oxygen-enriched air at different fructose concentrations in a 50-liter, internal-loop airlift reactor. Appl. Microbiol. Biotechnol. 55:673-679.

Chao, Y. P., Y. Sugano, T. Kouda, F. Yoshinaga, and M. Shoda. 1997. Production of bacterial cellulose by Acetobacter xylinum with an air-lift reactor. Biotechnol. Tech. 11:829-832.

Chen, H. P., and R. M. Brown, Jr. 1999. Thermal stability of the cellulose synthase complex of Acetobacter xylinum. Cellulose 6:137-152.

Cheng, H., P. Wang, J. Chen, and W. Wu. 2002. Cultivation of Acetobacter xylinum for bacterial cellulose production in a modified airlift reactor. Biotechnol. Appl. Biochem. 35:125-132.

Cheng, K.-C., J. M. Catchmark, and A. Demirci. 2009. Enhanced production of bacterial cellulose by using a biofilm reactor and its material property analysis. J. Biol. Eng. 3: .

Choi, C. N., H. J. Song, M. J. Kim, M. H. Chang, and S. J. Kim. 2009. Properties of bacterial cellulose produced in a pilot-scale spherical type bubble column bioreactor. Korean J. Chem. Eng. 26:136-140.

Ciechanska, D., J. Wietecha, D. Kazmierczak, and J. Kazimierczak. 2010. Biosynthesis of modified bacterial cellulose in a tubular form. Fibres Text. East. Eur. 18:98-104.

Coban, E. P., and H. Biyik. 2011. Evaluation of different $\mathrm{pH}$ and temperatures for bacterial cellulose production in HS (Hestrin-Scharmm) medium and beet molasses medium. Afr. J. Microbiol. Res. 5(9):1037-1045.

Colvin, J. R., S. T. Bayley, and M. Beer. 1957. The growth of cellulose microfibrils from Acetobacter xylinum. Biochim. Biophys. Acta 23:652-653. 
Correns, E., A. Groebe, H. J. Purz, H. H. Schwarz, and I. Hagen. 1972. Verfahren zur Herstellung von Membranen und Flächengebilden aus Bakteriencellulose. DD 92136.

Czaja, W., M. Kawecki, A. Krystynowicz, K. Wysota, S. Sakiel, P. Wroblewski, J. Glik, and S. Bielecki. 2004a. Application of bacterial cellulose in treatment of second and third degree burns. Presented at the 227th American Chemical Society National Meeting, Anaheim, CA, USA.

Czaja, W., D. Romanovicz, and R. M. Brown, Jr. 2004b. Structural investigations of microbial cellulose produced in stationary and agitated culture. Cellulose 11:403-411.

Czaja, W., A. Krystynowicz, S. Bielecki, and R. M. Brown, Jr. 2006. Microbial cellulosethe natural power to heal wounds. Biomaterials 27:145-151.

Dudman, W. F. 1960. Cellulose production by Acetobacter strains in submerged culture. J. Gen. Microbiol. 22:25-39.

Farah, L. F. X., P. A. S. Podlech, C. D. R. Archanjo, and L. A. Coral. 2006. Continuous fermentation process to produce bacterial cellulosic sheets. WO 2006066377.

Gagnaire, D., and F. R. Taravel, 1975. Biosynthesis of bacterial cellulose from glucose selectively deuterated in position 6: NMR study. FEBS letters, 60/2:317-21.

Gatenholm, P., H. Backdahl, T. J. Tzavaras, R. V. Davalos, and M. B. Sano. 2011. Threedimensional bioprinting of biosynthetic cellulose implants and scaffolds for tissue engineering. Virginia Tech Intellectual Properties, Inc., USA.

Heo, M.-S., and H.-J. Son. 2002. Development of an optimized, simple chemically defined medium for bacterial cellulose production by Acetobacter sp. A9 in shaking cultures. Biotechnol. Appl. Biochem. 36:41-45.

Hessler, N., and D. Klemm. 2008. Control of structure and shape of bacterial synthesized nanocellulose. Presented at the 235th American Chemical Society National Meeting, New Orleans, LA, USA.

Hessler, N., and D. Klemm. 2009. Alteration of bacterial nanocellulose structure by in situ modification using polyethylene glycol and carbohydrate additives. Cellulose 16:899-910.

Hestrin, S., and M. Schramm. 1954. Synthesis of cellulose by Acetobacter xylinum: II. Preparation of freeze-dried cells capable of polymerizing glucose to cellulose. Biochem. J. 58:345-352.

Hirai, A., M. Tsuji, and F. Horii. 1997. Culture conditions producing structure entities composed of cellulose I and II in bacterial cellulose. Cellulose 4:239-245.

Hornung, M., M. Ludwig, and H. P. Schmauder. 2007. Optimizing the production of bacterial cellulose in surface culture: a novel aerosol bioreactor working on a fed batch principle. Part 3. Eng. Life Sci. 7:35-41.

$\mathrm{Hu}, \mathrm{Y}$. , and J. M. Catchmark. 2010a. Formation and characterization of sphere like bacterial cellulose particles produced by Acetobacter xylinum JCM 9730 strain. Biomacromolecules 11(7):1727-1734.

$\mathrm{Hu}$, Y., and J. M. Catchmark. 2010b. Influence of 1-methylcyclopropene (1-MCP) on the production of bacterial cellulose biosynthesized by Acetobacter xylinum under the agitated culture. Lett. Appl. Microbiol. 51(1):109-13.

Iguchi, M. 2000. Bacterial cellulose—a masterpiece of nature's arts. J. Mater. Sci. 35:261-270.

Ishihara, M., M. Matsunaga, N. Hayashi, and V. Tisler. 2002. Utilization of D-xylose as carbon source for production of bacterial cellulose. Enzyme Microbial Technol. 31(1):986-91. 
Jonas, R., and L. F. Farah. 1998. Production and application of microbial cellulose. Polym. Degrad. Stabil. 59:101-106.

Joseph, G., E. Rowe, A. Margaritis, and W. Wan. 2003. Effects of polyacrylamide co-acrylic acid on cellulose production by Acetobacter xylinum. J. Chem. Technol. Biotechnol. 78:964-970.

Jung, J. Y., J. K. Park, and H. N. Chang. 2005. Bacterial cellulose production by Gluconacetobacter hansenii in an agitated culture without living non-cellulose producing cells. Enzyme Microbial Technol. 37:347-354.

Klemm, D., U. Udhardt, S. Marsch, and D. Schumann. 1999. Cellulose. BASYC, bacterially synthesized cellulose. Miniaturized tubes for microsurgery. Polymer News 24(11):377-378.

Kouda, T., Y. Nagata, H. Yano, and F. Yoshinaga. 2000. Method for cultivating apparatus for the production of bacterial cellulose in an aerated and agitated culture. U.S. Patent application 6,013,490.

Kouda, T., T. Naritomi, H. Yano, and F. Yoshinaga. 1998. Inhibitory effect of carbon dioxide on bacterial cellulose production by Acetobacter in agitated culture. J. Ferment. Bioeng. 85(3):318-321.

Kouda, T., H. Yano, and F. Yoshinaga. 1997. Effect of agitator configuration on bacterial cellulose productivity in aerated and agitated culture. J. Ferment. Bioeng. 83:371-376.

Kouda, T., H. Yano, F. Yoshinaga, M. Kaminoyama, and M. Kamiwano. 1996. Characterization of non-Newtonian behavior during mixing of bacterial cellulose in a bioreactor. J. Ferment. Bioeng. 82:382-386.

Kralisch, D., N. Hessler, and D. Klemm. 2010a. Method for the production of bacterial cellulose or cellulose-containing material in a planar form. Friedrich-SchillerUniversity, Jena, Germany. Chemical indexing equivalent to 152:309986 (DE).

Kralisch, D., N. Hessler, D. Klemm, R. Erdmann, and W. Schmidt. 2010b. White biotechnology for cellulose manufacturing — the HoLiR concept. Biotechnol. Bioeng. 105:740-747.

Kralisch, D., N. Hessler, M. Munteanu, B. Sultanova, D. Klemm, R. Erdmann, W. Schmidt, F. Wesarg, and F. A. Mueller. 2011. HoLiR: continuous generation of planar bacteria-produced nanocellulose. Abstr. Pap. Am. Chem. Soc. 241.

Krystynowicz, A., W. Czaja, A. Wiktorowska-Jezierska, M. Goncalves-Miskiewicz, M. Turkiewicz, and S. Bielecki. 2002. Factors affecting the yield and properties of bacterial cellulose. J. Ind. Microbiol. Biotechnol. 29:189-195.

Lee, H. C., and X. Zhao. 1999. Effects of mixing conditions on the production of microbial cellulose by Acetobacter xylinum. Biotechnol. Bioproc. E 4:41-45.

Legeza, V., V.P.Galenko-Yaroshevskii,E. V.Zinov'ev, B. A.Paramonov, G. S. Kreichman, I. I. Turkovskii, E. S. Gumenyuk, A. G. Karnovich, and A. K. Khripunov. 2004. Effects of new wound dressings on healing of thermal burns of the skin in acute radiation disease. Exp. Biol. Med.138:311-315.

Levy, N. L. F., E. C. Kurokawa, and P. A. S. Podlech. 2004. A process for obtaining a cellulosic wet sheet and a membrane and equipment for their manufacture. International application WO 2004050986.

Nakajima, M., L. Pang, and T. Yoshida. 2001. Kinetic study of mass transfer on bacterial cellulose production. Biotechnol. Sustain. Util. Biol. Resourc. Trop. 15:438-442.

Naritomi, T., T. Kouda, H. Yano, and F. Yoshinaga. 1998. Effect of ethanol on bacterial cellulose production from fructose in continuous culture. J. Ferment. Bioeng. 85:598-603. 
Okiyama, A., H. Shirae, H. Kano, and S. Yamanaka. 1992. Two-stage fermentation process for cellulose production by Acetobacter aceti. Food Hydrocolloid 6:471-477.

Onodera, M., I. Harashima, K. Toda, and T. Asakura. 2002. Silicone rubber membrane bioreactors for bacterial cellulose production. Biotechnol. Bioproc. E 7(5):289-294.

Park, J. K., S. H. Hyun, and J. Y. Jung. 2004. Conversion of G. hansenii PJK into non-cellulose-producing mutants according to the culture condition. Biotechnol. Bioproc. E 9:383-388.

Park, J. K., J. Y. Jung, and Y. H. Park. 2003. Bacterial cellulose production by Gluconacetobacter hansenii in an agitated culture without living non-cellulose producing cells. Biotechnol. Lett. 25:2055-2059.

Parvulescu, O. C., T. Dobre, A. Stoica, M. Stroescu, and G. Iavorschi. 2008. Factors influence on bacterial cellulose growth in static reactors. Rev. Chim. Bucharest 59:591-594.

Pometto, A. L., III, A. Demirci, and K. E. Johnson. 1997. Immobilization of microorganisms on a support made of synthetic polymer and plant material. U.S. Patent 5,595,893.

Porto, L. M., D. O. S. Recouvreux, R. V. Antonio, C. R. Rambo, C. A. Carmanatti, and C. M. Costa. 2010. Process and method for the production of three-dimensional bodies of bacterial cellulose. BR 2008003173.

Putra, A., A. Kakugo, H. Furukawa, J. P. Gong, Y. Osada, T. Uemura, and M. Yamamoto. 2008. Production of bacterial cellulose with well oriented fibril on PDMS substrate. Polym. J. (Tokyo) 40(2):137-142.

Ross, P., R. Mayer, and M. Benziman. 1991. Cellulose biosynthesis and function in bacteria. Microbiol. Rev. 55:35-58.

Sakairi N., H. Asano, M. Ogawa, N. Nishi, and S. Tokura. 1998. A method for direct harvest of bacterial cellulose filaments during continuous cultivation of Acetobacter xylinum. Carbohyd. Polym. 35(3-4):233-237.

Sani, A., and Y. Dahman. 2010. Improvements in the production of bacterial synthesized biocellulose nanofibres using different culture methods. J. Chem. Technol. Biotechnol. 85:151-164.

Saxena, I. M., and R. M. Brown, Jr. 2001. Biosynthesis of cellulose. Prog. Biotechnol. 18:69-76.

Schmidt, W., N. Henkel, R. Erdmann, D. Kralisch, and N. Hessler. 2010. Fermentation system for the production of bacterial cellulose or cellulosic surface materials. EPC Engineering Consulting $\mathrm{GmbH}$, Germany. Chemical indexing equivalent to $152: 310014(\mathrm{DE})$.

Serafica, G., R. Mormino, and H. Bungay. 2002. Inclusion of solid particles in bacterial cellulose. Appl. Microbiol. Biotechnol. 58:756-760.

Seto, A., Y. Kojima, N. Tonouchi, T. Tsuchida, and F. Yoshinaga. 1997. Screening of bacterial cellulose-producing Acetobacter strains suitable for sucrose as a carbon source. Biosci. Biotechnol. Biochem. 61:735-736.

Shezad, O., S. Khan, T. Khan, and J. K. Park. 2009. Production of bacterial cellulose in static conditions by a simple fed-batch cultivation strategy. Korean J. Chem. Eng. 26:1689-1692.

Sokolnicki, A. M., R. J. Fisher, T. P. Harrah, and D. L. Kaplan. 2006. Permeability of bacterial cellulose membranes. J. Membr. Sci. 272(1-2):15-27.

Son, H.-J., H.-G. Kim, K.-K. Kim, H.-S. Kim, Y.-G. Kim, and S.-J. Lee. 2003. Increased production of bacterial cellulose by Acetobacter sp. V6 in synthetic media under shaking culture conditions. Bioresourc. Technol. 86:215-219. 
Song, H.-J., H. Li, J.-H. Seo, M.-J. Kim, and S.-J. Kim. 2009. Pilot-scale production of bacterial cellulose by a spherical type bubble column bioreactor using saccharified food wastes. Korean J. Chem. Eng. 26:141-146.

Strobin, G., A. Wlochowicz, D. Ciechanska, S. Boryniec, H. Struszczyk, and S. Sobcza. 2003. Molecular parameters of bacterial cellulose. Effect of temperature and $\mathrm{pH}$ of the biosynthesis medium. Polimery 48(11/12):779-783.

Takai, M., Y. Tsuta, and S. Watanabe. 1975. Biosynthesis of cellulose by Actetobacter xylinum. Characterization of bacterial cellulose. Polym. J. (Tokyo) 7(2):137-146.

Tamura, H., Y. Tsuruta, and S. Tokura. 1999. Shallow pan cultivation to enhance the yield of bacterial cellulose, Preprints of Symposia-American Chemical Society, Division of Fuel Chemistry, 44/2:320-323.

Toda, K., J. Koizumi, and T. Asakura. 1994. Continuous manufacture of cellulose materials with Acetobacter. JP 06253877.

Tokura, M., M. Fukaya, H. Okumura, and K. Kawamura. 1993. Permselective membranes for gases and their manufacture. Japan Kokai Tokkyo Koho JP 05293343.

Tokura, S., H. Asano, N. Sakairi, and N. Nishi. 1998. Direct filature of bacterial cellulose from culture medium. Macromol. Symp. 127:23-30.

Tomé, L. C., R. J. B. Pinto, E. Trovatti, C. S. R. Freire, A. J. D. Silvestre, C. P. Neto, and A. Gandini. 2011. Transparent bionanocomposites with improved properties prepared from acetylated bacterial cellulose and poly(lactic acid) through a simple approach. Green Chem. 13:419.

Toyosaki, H., T. Naritomi, A. Seto, M. Matsuka, T. Tsuchida, and F. Yoshinaga. 1995. Screening of bacterial cellulose-producing Acetobacter strains suitable for agitated culture. Biosci. Biotechnol. Biochem. 59:1498-1502.

Udhardt, U. 2004. Synthese, Eigenschaften und Strukturdesign von Bakteriencellulose mit speziellem Anwendungspotential von BASYC ${ }^{\circledR}$ - Implantaten in der Mikrochirurgie. Dissertation, Friedrich Schiller University, Jena, Germany.

Vandamme, E. J., S. De Baets, A. Vanbaelen, K. Joris, and P. De Wulf. 1998. Improved production of bacterial cellulose and its application potential. Polym. Degrad. Stabil. 59:93-99.

Verschuren, P., T. Cardona, M. Nout, K. De Gooijer, and J. Van den Heuvel. 2000. Location and limitation of cellulose production by Acetobacter xylinum established from oxygen profiles. J. Biosci. Bioeng. 89(5):414-419.

Watanabe, K., M. Tabuchi, M. Yasushi, and F. Yoshinaga. 1998. Structural features and properties of bacterial cellulose produced in agitated culture. Cellulose. 5:187-200.

Watanabe, K., and S. Yamanaka. 1995. Effects of oxygen tension in the gaseous phase on production and physical properties of bacterial cellulose formed static culture conditions. Biosci. Biotechnol. Biochem. 59(1):65-68.

Weuster-Botz, D., M. Hofinger, and G. Bertholdt. 2011. Microbial production of homogenously layered cellulose pellicles in a membrane bioreactor. Biotechnol. Bioeng. 108:2237-2240.

Williams, W. S., and R. E. Cannon. 1989. Alternative environmental roles for cellulose produced by Acetobacter xylinum. Appl. Environ. Microbiol. 55:2448-2452.

Wu, J. Y., and W. T. Wu. 1991. Fed-batch culture of Saccharomyces cerevisiae in an airlift reactor with net draft tube. Biotechnol. Progr. 7:230-233.

Wu, W. 1992. Mass transfer in an airlift reactor with a net draft tube. Biotechnol. Prog. 8:465-468. 
Wu, W., and J. Wu. 1990. Airlift reactor with net draught tube. J. Ferment. Bioeng. 70:359-361.

Wu, W.-T., P.-M. Wang, H.-P. Cheng, and J.-W. Chen. 2001. Tower-type fermenter with high mass transfer capability. Abstracts of papers from the 221st American Chemical Society National Meeting, San Diego, CA, USA, April 1-5.

Xiu, H., Z. Wang, and J. Li. 2005. Effect of bacterial cellulose on paper property. Zhongguo Zaozhi 24(3):14-17.

Yamada, Y. 2000. Transfer Acetobacter oboediens and A. intermedius to the genus Gluconacetobacter as G. oboediens comb. nov. and G. intermedius comb. nov. Int. J. Syst. Evol. Microbiol. 50:225-227.

Yamanaka, S., M. Ishihara, and J. Sugiyama. 2000. Structural modification of bacterial cellulose. Cellulose 7:213.

Zaar, K. 1979. Visualization of pores (export sites) correlated with cellulose production in the envelope of the Gram-negative bacterium Acetobacter xylinum. J. Cell Biol. 80(3):773-777.

Zhou, L. L., D. P. Sun, L. Y. Hu, Y. W. Li, and J. Z. Yang. 2007a. Effect of addition of sodium alginate on bacterial cellulose production by Acetobacter xylinum. J. Ind. Microbiol. Biotechnol. 34(7):483-489.

Zhou, L. L., D. P. Sun, Q. H. Wu, J. Z. Yang, and S. L. Yang. 2007b. Influence of culture mode on bacterial cellulose production and its structure and property. Weishengwu Xuebao 47(5):914-917. 



\title{
Tough Bacterial Nanocellulose Hydrogels Based on the Double-Network Technique
}

\author{
Anamul Haque, ${ }^{1}$ Takayuki Kurokawa, ${ }^{2,3}$ and Jian Ping Gong ${ }^{2}$ \\ ${ }^{1}$ Division of Biological Sciences, Graduate School of Science, \\ Hokkaido University, Sapporo, Japan \\ ${ }^{2}$ Faculty of Advanced Life Science, Hokkaido University, Sapporo, Japan \\ ${ }^{3}$ Creative Research Institution, Hokkaido University, Sapporo, Japan
}

\section{CONTENTS}

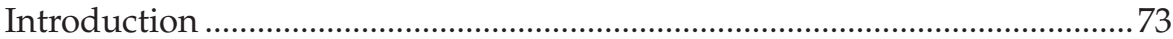

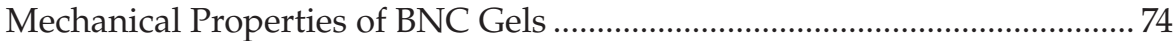

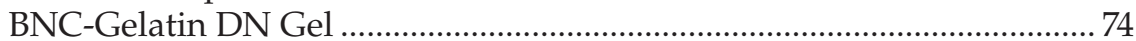

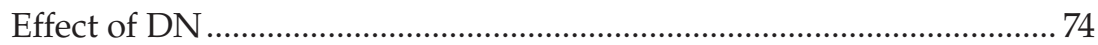

Effect of Cross-Linking .............................................................................

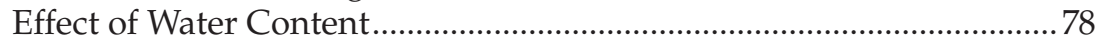

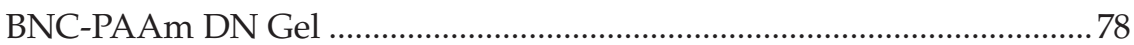

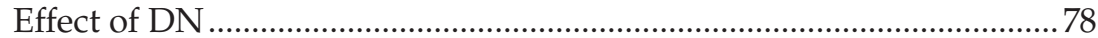

Effect of AAm Monomer Concentration ................................................79

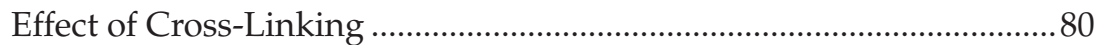

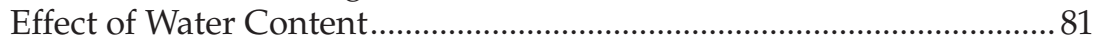

Application of BNC-Based DN Gels as a Replacement of Biomaterials .......82

Wear Property of DN Gels in the Pin-On Flat Test......................................83

Biodegradation of DN Gels after Implantation into Subcutaneous Tissue ... 85

Biological Responses of DN Gels after Implantation into Muscle

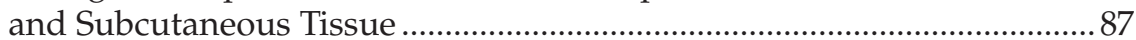

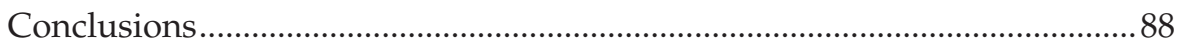

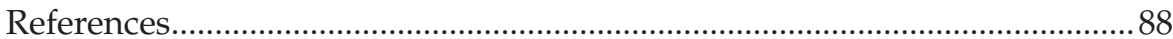

\section{Introduction}

The design and fabrication of materials suitable for soft tissue replacement is a demanding task. These materials should not only display mechanical properties similar to those of the tissues they replace, but they should also display an improved life span, biocompatibility, durability, a low degree of 
calcification, resistance to biodegradation, and low friction. Hydrogelsthree-dimensional hydrophilic polymer networks in which a large amount of water is interposed-may be suitable for this purpose. Due to their unique properties, a wide range of medical, pharmaceutical, and prosthetic applications have been proposed (Gong et al. 2001, 2003; Kakugo et al. 2002; Osada and Matsuda 1995; Osada et al. 1992). However, most of them lack mechanical toughness, and only a few applications have been accomplished.

Bacterial nanocellulose (BNC) is one such hydrogel. BNC is an extracellular cellulose, produced by Acetobacter, consisting of a hydrophobic ultrafine fiber network stacked in a stratified structure (Hestrin and Schramm 1954). Due to this unique structure, it is mechanically anisotropic, with a high tensile modulus along the fiber layer direction, but a low compressive modulus in perpendicular to the stratified direction. Water is easily squeezed out of BNC gel through slight compression and it does not recover swelling because of hydrogen-bond formation between the cellulose fibrils.

In this chapter, a BNC-based biocompatible hydrogel with high mechanical strength and reversible swelling after repeated compression cycles is reported, developed by combining BNC with natural polymers such as gelatin, as well as synthetic ones such as polyacrylamide, using the technique known as the double network (Gong et al. 2003). These gels exhibit high compressive fracture stress comparable to cartilage and high tensile strength comparable with ligament or tendon. In addition, some biological properties of these gels, such as resistance to wear, biodegradation, and biological interactions within the living body, are discussed.

The detailed procedure for the preparation of BNC gel (Takai et al. 1975), gelatin gel and BNC-gelatin double-network (DN) gel (Nakayama et al. 2004), BNC-PAAm and BNC-PDMAAm DN gel (Hagiwara et al. 2010), basic biomechanical and wear properties (Baily 2003; Gong et al. 2003; McKellop et al. 1981; Ueno et al. 2002; Yasuda et al. 2005), and implantation of DN gel specimens into subcutaneous tissue (Azuma et al. 2007; Ministry of Health, Labour and Welfare, Japan 2003; Tanabe et al. 2008) can be found in articles referenced here.

\section{Mechanical Properties of BNC Gels}

\section{BNC-Gelatin DN Gel}

\section{Effect of $D N$}

Due to this unique structure, BNC shows mechanical anisotropy, with a high tensile modulus (2.9 MPa) along the fiber layer direction, but a low compressive modulus $(0.007 \mathrm{MPa})$ perpendicular to the stratified direction. BNC contains $90 \%$ water; however, the water is easily squeezed out and hydrogen-bond formation between cellulose fibers avoids reswelling (Figure 4.1, column 1). 
(a)

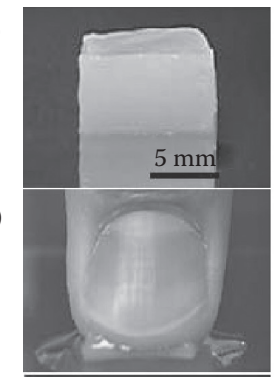

(c)
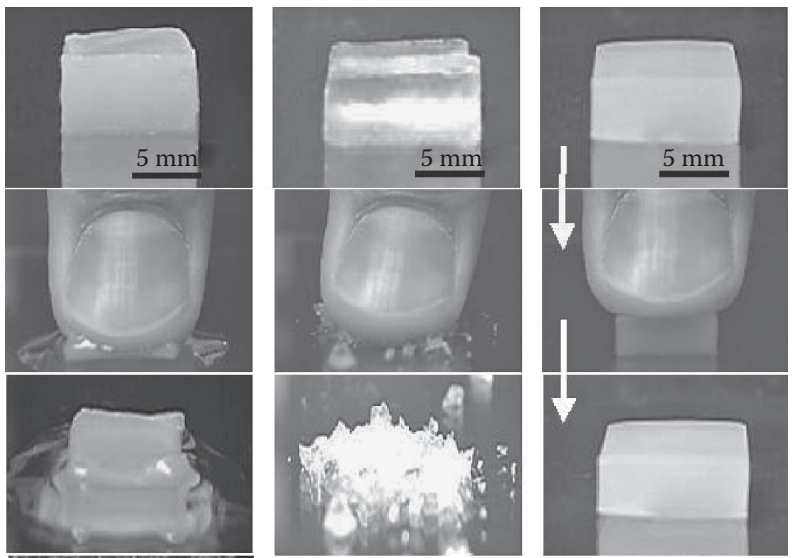

(d)
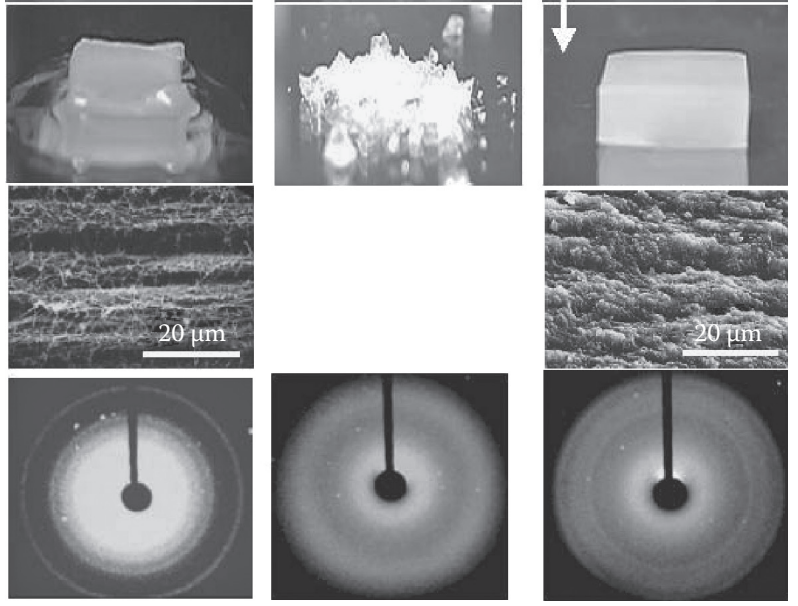

(e)

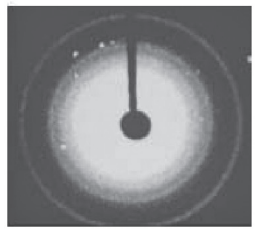

BNC

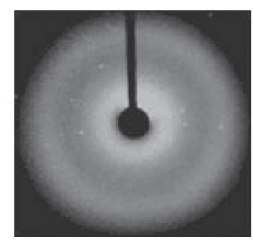

Gelatin

BNC-gelatin

\section{FIGURE 4.1}

Pictures show the gel images $(a, b, c)$, scanning electron microscopy (SEM) images (d) and wide-angle X-ray diffraction (WAXD) patterns (e) of BNC, gelatin, and BNC-gelatin DN gels. (a) Before compression. (b) During compression. (c) After 10 min. of compression. (d) SEM images of the stratified structure of EC and BNC-gelatin DN gel. (Reproduced with permission of Nakayama et al. 2004.)

Gelatin is a polypeptide derived from an extracellular matrix-collagen. Gelatin gel can keep water and recovers from repeated compression. Gelatin gel is brittle and is easily broken into fragments under modest compression of $0.1 \mathrm{MPa}$ (Figure 4.1, column 2). In contrast, BNC-gelatin DN gel can hold water even under pressure as high as $3.7 \mathrm{MPa}$ and completely recovers its original shape after repeated compression (Figure 4.1, column 3).

The typical compressive stress-strain curves of BNC, gelatin, and their composite BNC-gelatin DN gels are shown in Figure 4.2a. The compression is applied in a direction vertical to the stratified structure of BNC and BNC-gelatin gels. The gelatin gel and BNC-gelatin DN gel were prepared from a gelatin solution of $30 \%$ concentration in the presence of 1 M 1-ethyl-3-(3-dimethylaminopropyl) carbodiimide hydrochloride (EDC).

The BNC-gelatin DN gel shows a different stress-strain profile compared with those of individual BNC and gelatin gels. The composite gel shows a 


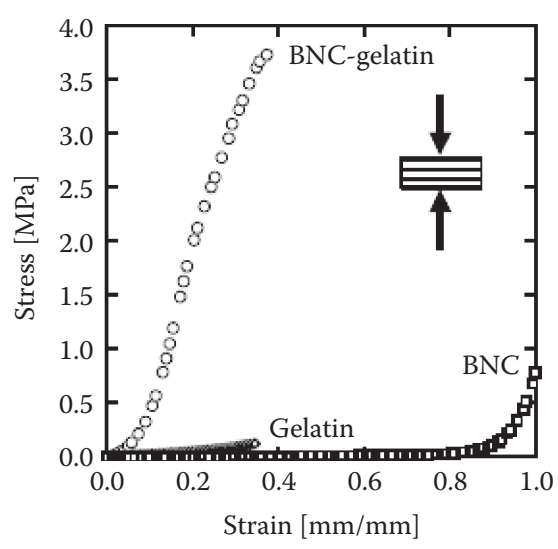

(a)

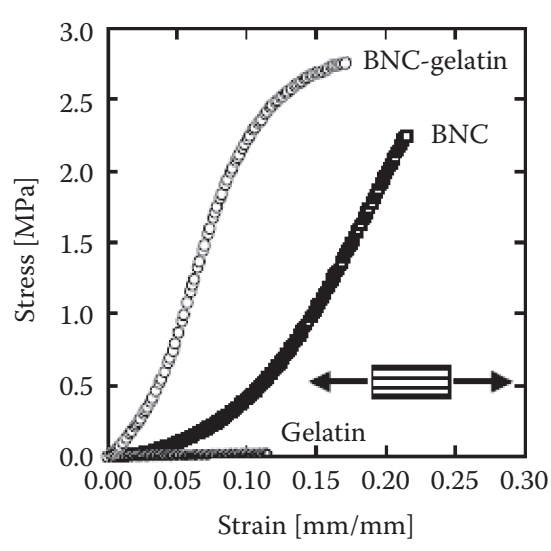

(b)

FIGURE 4.2

Compressive (a) and elongational (b) stress-strain curves of BNC-gelatin DN, gelatin, and BNC gels. The compression and elongation were performed perpendicular to and parallel to the stratified direction of the BNC and BNC-gelatin DN gels, respectively. Concentration of gelatin in feed: $30 \mathrm{wt} \%$. EDC concentration: $1 \mathrm{M}$. (Reproduced with permission of Nakayama et al. 2004.)

compressive elastic modulus of $1.7 \mathrm{MPa}$ in a direction perpendicular to the stratified structure, which is more than 240 times greater than that of BNC $(0.007 \mathrm{MPa})$ and 11 times that of gelatin gel (0.16 MPa) (Table 4.1). The fracture strength of BNC-gelatin DN gel against compression reaches $3.7 \mathrm{MPa}$, which is about 31 times greater than that of gelatin gel (0.12 MPa) (Table 4.1). The tensile stress-strain curves of gels along the direction of the stratified layer are demonstrated in Figure 4.2b. BNC-gelatin DN gel can sustain nearly $3 \mathrm{MPa}$ and has an elastic modulus of $23 \mathrm{MPa}$, which is 112 times greater than that of gelatin gel. From the cyclic compressive test, it can be seen that BNC does not show its original stress-strain profile, while the BNC-gelatin DN gel recovers well from compressive deformation after second testing under a repeated compressive stress of up to $30 \%$ strain. Thus the DN structure led to a substantial improvement of the mechanical properties in tensile strength as well. Similar improvement in the mechanical strength was also observed for the combination with polysaccharides, such as sodium alginate, gellan gum, and i-carrageenan (Nakayama et al. 2004).

For a material composed of a layered structure with two layers of elastic modulus $E_{s}$ and $E_{h}\left(E_{s} \ll E_{h}\right)^{\prime}$, the macroscopic modulus $E$ of the material perpendicular to the stratified direction depends only on the modulus of the soft component $E_{s}^{\prime}$, not on the hard component $E_{h}$ (Okumura and de Gennes 2001):

$$
E=\frac{d_{s}+d_{h}}{d_{s}} E_{s},
$$




\section{TABLE 4.1}

Effect of Gelatin Concentration in Feed on the Degree of Swelling and Mechanical Properties of BNC-Gelatin and Gelatin Gels. The EDC Concentration was $1 \mathrm{M}$. (Reproduced with permission of Nakayama et al. 2004.)

\begin{tabular}{|c|c|c|c|c|c|c|c|}
\hline \multirow[b]{2}{*}{ Sample } & \multirow[b]{2}{*}{$\begin{array}{l}\text { Degree of } \\
\text { Swelling, } q\end{array}$} & \multicolumn{3}{|c|}{ Compression } & \multicolumn{3}{|c|}{ Elongation } \\
\hline & & $\begin{array}{c}\text { Elastic } \\
\text { Modulus, } \\
\text { E (MPa) }\end{array}$ & $\begin{array}{l}\text { Fracture } \\
\text { Stress, } \sigma_{\mathrm{F}} \\
(\mathrm{MPa})\end{array}$ & $\begin{array}{l}\text { Fracture } \\
\text { Strain, } \varepsilon_{\mathrm{F}} \\
(\mathrm{mm} / \mathrm{mm})\end{array}$ & $\begin{array}{c}\text { Elastic } \\
\text { Modulus, } \\
\text { E (MPa) }\end{array}$ & $\begin{array}{l}\text { Fracture } \\
\text { Stress, } \sigma_{\mathrm{F}} \\
(\mathrm{MPa})\end{array}$ & $\begin{array}{l}\text { Fracture } \\
\text { Strain, } \varepsilon_{\mathrm{F}} \\
(\mathrm{mm} / \mathrm{mm})\end{array}$ \\
\hline \multicolumn{8}{|c|}{ BNC-Gelatin } \\
\hline $15 \mathrm{wt} \%$ & 11 & 1.3 & 2.1 & 0.41 & 5.0 & 1.1 & 0.22 \\
\hline $30 \mathrm{wt} \%$ & 5.8 & 1.7 & 3.7 & 0.37 & 23 & 2.8 & 0.17 \\
\hline $40 \mathrm{wt} \%$ & 3.6 & 2.8 & 5.2 & 0.36 & 19 & 3.7 & 0.23 \\
\hline $50 \mathrm{wt} \%$ & 3.1 & 3.9 & 5.3 & 0.44 & 21 & 3.8 & 0.28 \\
\hline \multicolumn{8}{|l|}{ Gelatin } \\
\hline $15 \mathrm{wt} \%$ & 41 & 0.047 & 0.029 & 0.34 & 0.040 & 0.006 & 0.11 \\
\hline $30 \mathrm{wt} \%$ & 11 & 0.16 & 0.12 & 0.35 & 0.23 & 0.025 & 0.11 \\
\hline $40 \mathrm{wt} \%$ & 5.2 & 0.93 & 0.44 & 0.29 & 0.78 & 0.070 & 0.10 \\
\hline $50 \mathrm{wt} \%$ & 3.8 & 1.2 & 1.2 & 0.37 & 1.8 & 0.18 & 0.10 \\
\hline $\mathrm{BNC}$ & 120 & 0.007 & - & - & 2.9 & 2.2 & 0.21 \\
\hline
\end{tabular}

where $d_{s}$ and $d_{h}$ are the thickness of the soft and hard component, respectively. Therefore, if one assumes that the cellulose-rich part behaves as a hard layer and the gelatin part as a soft layer, the overall macroscopic modulus should largely depend on the modulus of the soft component-gelatinsince $d_{s} \approx d_{h}$.

\section{Effect of Cross-Linking}

The mechanical property of gelatin can be modulated by the extent of cross-linking with EDC. Therefore the effect of the degree of cross-linking on the elastic modulus was investigated by changing the EDC concentration. Without EDC, gelatin at a concentration of $30 \mathrm{wt} \%$ cannot form a gel that is strong enough for mechanical measurement. In the presence of EDC, gelatin forms a chemically cross-linked gel with a compressive elastic modulus of about $0.1 \mathrm{MPa}$, as shown in Figure 4.3. As shown in Figure 4.3a, a substantial increase in the compressive elastic modulus of BNC-gelatin DN gel was observed with an increase in cross-linker, while only a modest enhancement of the elastic modulus was observed in gelatin gel. It was also found that the compressive fracture stress of BNC-gelatin DN gel increases with an increase in EDC concentration, which is similar to the behavior observed in gelatin gel (Figure 4.3b). Thus the mechanical property of DN gel is predominantly enhanced by the cross-linkage of gelatin. 


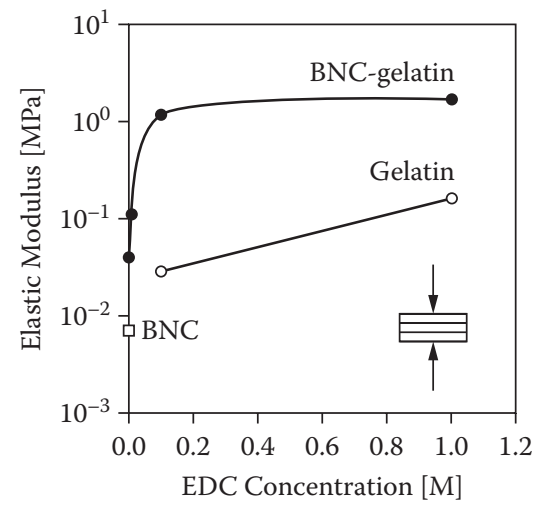

(a)

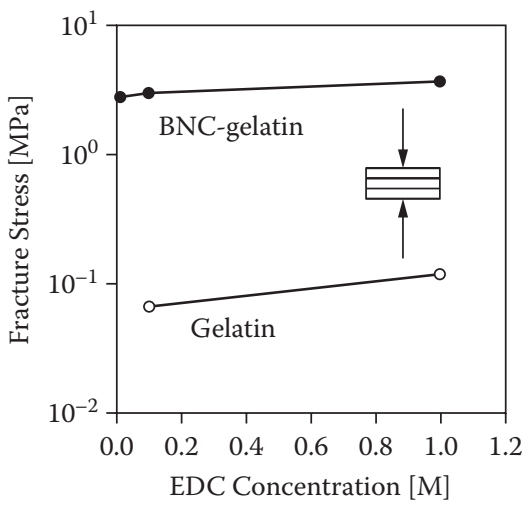

(b)

\section{FIGURE 4.3}

Effect of cross-linker EDC concentration on the compressive elastic modulus (a) and the compressive fracture stress (b) of BNC-gelatin DN gel and gelatin gel. The compression was performed perpendicular to the stratified structure of the BNC-gelatin gel. Concentration of gelatin in feed: $30 \mathrm{wt} \%$. (Reproduced with permission of Nakayama et al. 2004.)

\section{Effect of Water Content}

Since the mechanical property of hydrogel is changed by its water content (i.e., swelling degree), the effect of the degree of swelling, $q$, on the elastic modulus and fracture stress of BNC-gelatin DN gel was investigated (Figures 4.4a and 4.4b). As shown in the figures, both the elastic modulus and fracture stress decrease with an increase in the degree of swelling, $q$, following a power law relation. The fracture stress of BNC-gelatin DN gel is 4-18 times greater than that of gelatin gel over the same degree of swelling range. Also, the elastic modulus of the BNC-gelatin DN gel is 2-8 times greater than that of gelatin gel. Both values decrease less sensitively with increasing swelling for the DN gels. Thus, despite its high water content of up to $70 \mathrm{wt} \%$, BNC-gelatin DN gel exhibits a high mechanical strength of $5 \mathrm{MPa}$ in fracture stress and $4 \mathrm{MPa}$ in elastic modulus; both are almost equivalent to those of articular cartilage (McCutchen 1978).

\section{BNC-PAAm DN Gel}

\section{Effect of $D N$}

A great improvement in the mechanical properties of BNC gel is obtained through combination with poly(acrylamide) (PAAm) (BNC-PAAm) using the DN technique, which sustains not only high compression, but also high elongation. By controlling the water content of the BNC gel prior to polymerization of the second (PAAm) network, a ligament-like, tough BNC-PAAm DN gel with a tensile strength of $40 \mathrm{MPa}$ was successfully obtained. 


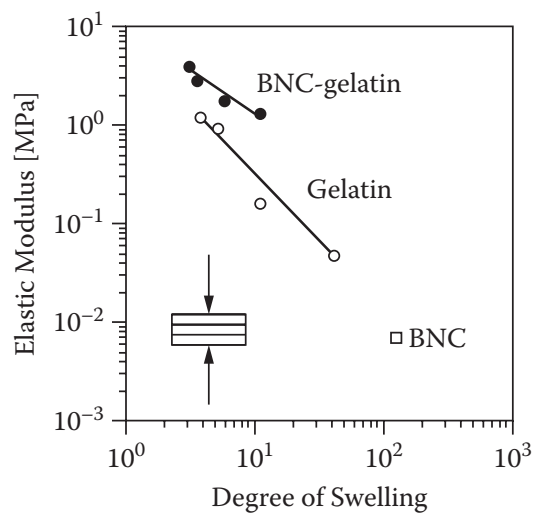

(a)

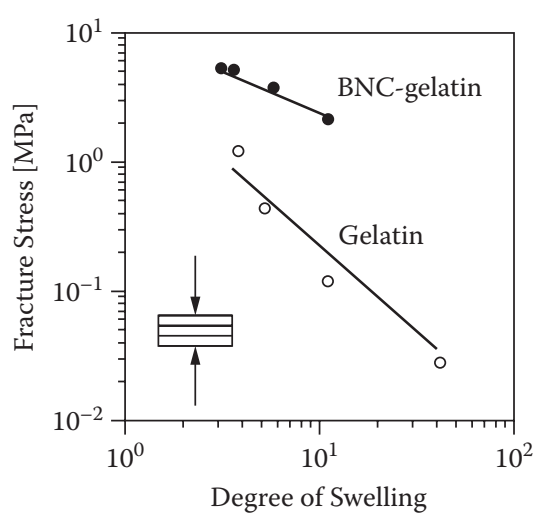

(b)

FIGURE 4.4

Dependences of compressive elastic modulus (a) and compressive fracture stress (b) on swelling degree for BNC-gelatin DN $(\bullet)$, gelatin $(\bigcirc)$, and BNC $(\square)$ gels. The compression was performed perpendicular to the stratified structure of the BNC and BNC-gelatin gels. (Reproduced with permission of Nakayama et al. 2004.)

\section{Effect of AAm Monomer Concentration}

The effect of AAm concentration on the mechanical properties of BNC-PAAm $\mathrm{DN}$ gel is shown in Figure 4.5. For comparison, the swelling degree, $q$, and mechanical properties of ligament are also shown (Woo et al. 2004). To measure the mechanical properties of BNC-PAAm DN gel, tensile tests were carried out on samples in directions parallel to the stratified structure of the BNC gel. BNC gel, having an ultrafine fibril network stacked in a stratified structure, shows mechanically anisotropic properties, with a high tensile modulus $(E=2.9 \mathrm{MPa})$ along the fibril layer direction, but a low compressive modulus $(E=0.007 \mathrm{MPa}$ ) perpendicular to the stratified direction (Hagiwara et al. 2010; Nakayama et al. 2004). Figure 4.5 shows the effect of the concentration of AAm on the tensile strength of BNC-PAAm DN gels.

The swelling of the BNC gel as a single network drastically decreased (by about 10 times) after the BNC gel was combined with AAm (1 M) to produce BNC-PAAm DN gel. This indicates that the water in the BNC gel network was replaced by the PAAm gel. The swelling of the BNC-PAAm DN gel continued to decrease with increasing concentrations of AAm (Figure 4.5a). Up to an AAm concentration of $4 \mathrm{M}$, the swelling of BNC-PAAm DN gel was 4.5. This swelling was still greater than that of ligament $(q=2.86)$. Meanwhile, the tensile modulus, E, of BNC-PAAm DN gel increased linearly with increasing AAm concentration, but was still much lower (more than 100 times) than the tensile modulus of ligament $(E=332 \mathrm{MPa})$. The tensile fracture stress, $\sigma_{\mathrm{F}}$, of BNC-PAAm DN gel tended to increase with increasing AAm concentration and was more than 20 times slower than that of ligament $\left(\varepsilon_{\mathrm{F}}=38.6 \mathrm{MPa}\right.$; Figure $4.5 \mathrm{~b})$. However, the tensile fracture strain of BNC-PAAm DN gel 


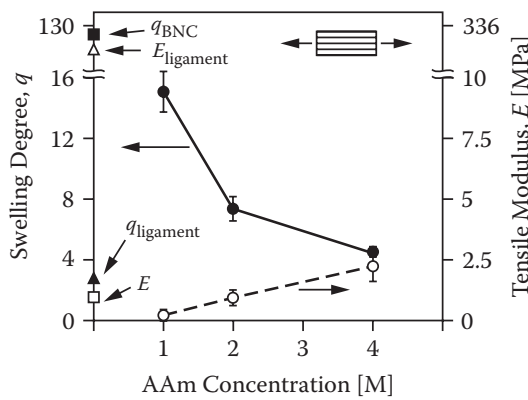

(a)

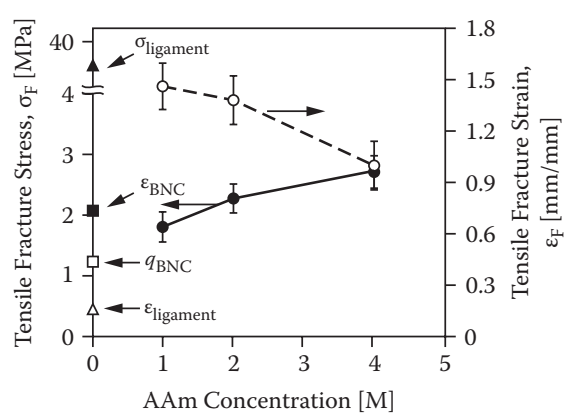

(b)

\section{FIGURE 4.5}

Effect of AAm concentration on the tensile strength performance of BNC-PAAm gel under the conditions of $0.1 \mathrm{~mol} \% \mathrm{MBAA}$ and $0.1 \mathrm{~mol} \% \mathrm{KPS}$ : (a) swelling degree, $q$, and tensile modulus, $E$, as functions of the concentration of AAm (M) in the BNC-PAAm gel. (b) Tensile fracture stress, $\sigma_{\mathrm{F}}$ and tensile fracture strain, $\varepsilon_{\mathrm{F}}$, as functions of the concentration of AAm $(\mathrm{M})$ in the BNC-PAAm gel. The symbols are as follows: (a) $(\mathbf{O}) q_{\mathrm{BNC}-\mathrm{PAAm} ;}(\boldsymbol{\square}) q_{\mathrm{BNC}},(\boldsymbol{\Lambda}) q_{\text {ligament }} ;(\bigcirc) E_{\mathrm{BNC}-\mathrm{AAm}} ;$ $(\square) E_{\mathrm{BNC}},\left(E_{\text {ligament }} ;(\mathrm{b})(\mathbf{\bigcirc}) \sigma_{\mathrm{F}} \mathrm{BNC}-\mathrm{PAAm} ;(\mathbf{\square}) \sigma_{\mathrm{F}} \mathrm{BNC} ;(\mathbf{\Lambda}) \sigma_{\mathrm{F}}\right.$ ligament; $(\bigcirc) \varepsilon_{\mathrm{F}} \mathrm{BNC}$-PAAm; $(\square) \varepsilon_{\mathrm{F}} \mathrm{BNC} ;\left(\backslash \varepsilon_{\mathrm{F}}\right.$ ligament. (Reproduced with permission of Hagiwara et al. 2010.)

decreased with increasing AAm concentration and was more than 10 times greater than that of ligament $\left(\varepsilon_{\mathrm{F}}=0.17 \mathrm{~m} / \mathrm{m}\right)$. The tendency for the compressive strength was similar to that of the tensile strength for the BNC-PAAm DN gel (Hagiwara et al. 2010). These results indicate that the concentration of AAm affected the mechanical performance in terms of both the tensile and compressive strengths. A higher concentration of AAm (4 M) led to high tensile and compressive strength of the BNC-PAAm DN gels.

\section{Effect of Cross-Linking}

Since the mechanical properties of the gel also depend on the cross-linker concentration of the second network, the effect of the degree of cross-linking on the tensile and compressive strength was investigated by changing the $\mathrm{N}, \mathrm{N}^{\prime}$-methylenebis acrylamide (MBAA) concentration while keeping the in-feed AAm concentration constant at $4 \mathrm{M}$. The concentration of potassium peroxydisulfate $\left(\mathrm{K}_{2} \mathrm{~S}_{2} \mathrm{O}_{8}\right)$ (KPS) was also kept constant at $0.1 \mathrm{~mol} \%$. Figure 4.6 shows the effect of MBAA concentration on the tensile properties of BNC-PAAm DN gel. Tensile moduli of BNC-PAAm DN gel increased with increasing MBAA concentration (Figure 4.6a). Meanwhile, as shown in Figure 4.6b, a drastic increase in the tensile fracture stress of the BNC-PAAm DN gel was observed for MBAA concentrations below $1 \mathrm{~mol} \%$. In contrast, the tensile fracture strain of the BNC-PAAm gels drastically decreased up to an MBAA concentration of $1 \mathrm{~mol} \%$. Above $1 \mathrm{~mol} \%$ MBAA concentration, there was no significant improvement of either tensile fracture stress or strain. This indicates that $1 \mathrm{~mol} \%$ was an effective MBAA concentration 


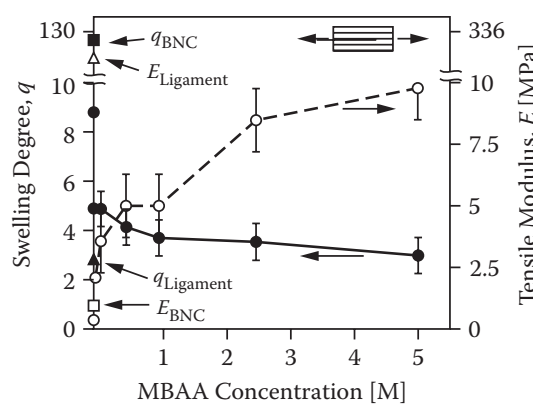

(a)

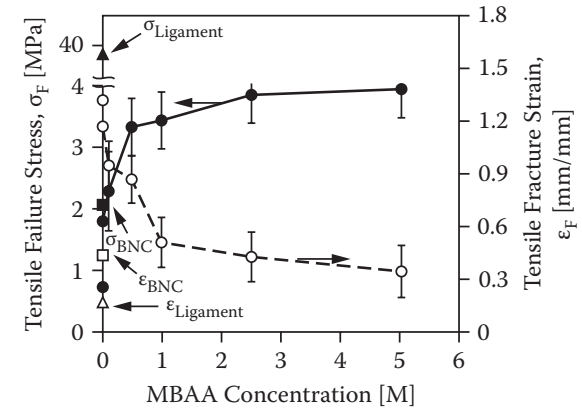

(b)

\section{FIGURE 4.6}

The effect of MBAA cross-linker concentration on the tensile strength performance of BNC-PAAm gel under the conditions of $4 \mathrm{M}$ AAm and $0.1 \mathrm{~mol} \% \mathrm{KPS}$ : (a) swelling degree, $q$, and tensile modulus, $E$, as functions of the concentration of MBAA ( $\mathrm{mol} \%)$ in the BNC-PAAm gel. (b) Tensile fracture stress, $\sigma_{\mathrm{F}}$, and tensile fracture strain, $\varepsilon_{\mathrm{F}}$ as functions of the concentration of MBAA (mol\%) in the BNC-PAAm gel. The symbols are as follows: (a) () $q_{\text {BNC-PAAm; }}$; $(\square) q_{\mathrm{BNC}},(\boldsymbol{\Lambda}) q_{\text {ligament; }}(\bigcirc) E_{\mathrm{BNC}-\mathrm{AAm}} ;(\square) E_{\mathrm{BNC}},\left(\backslash E_{\text {ligament }} ;(\mathrm{b})(\mathbf{\bullet}) \sigma_{\mathrm{F}} \mathrm{BNC} / \mathrm{PAAm} ;(\boldsymbol{\square}) \sigma_{\mathrm{F}} \mathrm{BNC}\right.$; $(\boldsymbol{\Lambda}) \sigma_{\mathrm{F}}$ ligament; $(\bigcirc) \varepsilon_{\mathrm{F}} \mathrm{BNC}-\mathrm{PAAm} ;(\square) \varepsilon_{\mathrm{F}} \mathrm{BNC} ;\left(\backslash \varepsilon_{\mathrm{F}}\right.$ ligament. (Reproduced with permission of Hagiwara et al. 2010.)

for the cross-linker to improve the tensile strength performance of the BNC-PAAm DN gel. This value is in agreement with the concentration of cross-linker for any DN gel synthesized by the DN method (Gong et al. 2003). The compressive moduli of BNC-PAAm DN gels increased with increasing MBAA concentration (Hagiwara et al. 2010). The compressive fracture stress, $\sigma_{\mathrm{F}}$, increased substantially in the BNC-PAAm DN gels with increasing MBAA concentration. The maximum tensile and compressive fracture stress was observed to be about 3.5 and $6.5 \mathrm{MPa}$, respectively, at $5 \mathrm{~mol} \%$ MBAA concentration, similar to the compressive and tensile fracture stress of the BNC-gelatin DN gel system discussed earlier (Nakayama et al. 2004). It should be noted that these values are still lower than the tensile fracture stress of ligament $\left(\sigma_{\mathrm{F}}=39 \mathrm{MPa}\right.$; Woo et al. 2004) and the compressive fracture stress of articular cartilage (McCutchen 1978).

\section{Effect of Water Content}

In order to investigate whether swelling is a universal parameter, the relationship between swelling and the mechanical properties of BNC-PAAm DN gels prepared at different concentrations of AAm (1-4 M) and MBAA (0-5 mol\%) were investigated. The relationships of tensile and compressive modulus, and between tensile and compressive fracture stress and strain, are plotted against swelling on a logarithmic graph, which is useful to elucidate the correlation over a wide range of these parameters (Figure 4.7). In Figures 4.7a and $4.7 \mathrm{~b}$, it can be seen that the tensile and compressive modulus of the DN gel 


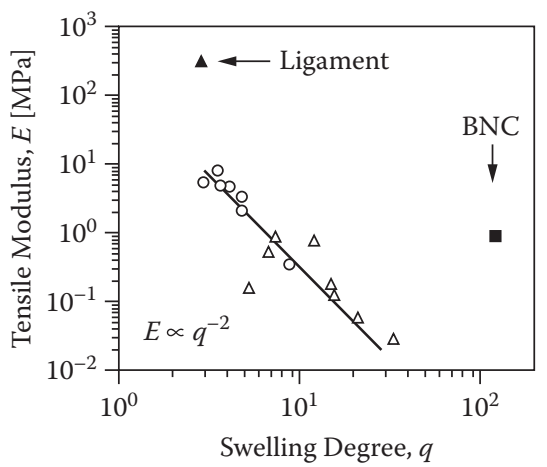

(a)

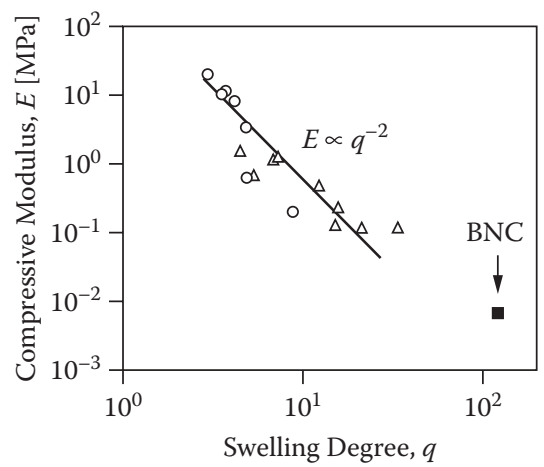

(b)

FIGURE 4.7

Dependence on swelling degree, $q$, of (a) tensile modulus and (b) compressive modulus. The symbols are as follows: (ם) BNC; (O) ligament; and BNC-PAAm DN gel with various concentrations of $(\bigwedge$ AAm and $(\bigcirc)$ MBAA. (Reproduced with permission of Hagiwara et al. 2010.)

increased with decreasing swelling, following a power law of $E \propto q^{-2}$, regardless of the structural parameter changes of either AAm or MBAA concentration. This relation is close to that of a hydrogel swollen in good solvent $\left(E \propto q^{-2.25}\right)$ (Obukhov et al. 1994), indicating that the contribution to the initial modulus from the fine stratified structure of the BNC gel was negligible.

\section{Application of BNC-Based DN Gels as a Replacement of Biomaterials}

The normal cartilage tissue highly contributes to the normal joint functions involving ultralow friction, distribution of loads, and absorption of impact energy. The cartilage tissue is occasionally injured in athletic activities, and frequently becomes degenerated by aging. Partial or complete loss of cartilage tissues leads to future problems of the knee joint, such as osteoarthrosis. When the normal cartilage tissue is damaged, it is extremely difficult to regenerate these tissues with currently available therapeutic treatments. Therefore it is important to develop substitutes for the normal cartilage tissue as a potential therapeutic option. Potential materials for the artificial cartilage are required to be viscoelastic, strong, durable to repetitive stress, low in friction, resistant to wear, and resistant to biodegradation within the living body. The unique cellulose-based DN gel materials have great potential for application as an artificial cartilage because they are viscoelastic, strong, durable to repetitive stress, and have low friction. In 
the following section, the resistance of these gels against wear and biodegradation within the living body is discussed to understand the applicability of these gels as a potential cartilage material.

\section{Wear Property of DN Gels in the Pin-On Flat Test}

The maximum wear depth of the BNC-PDMAAm gel and BNC-gelatin gel along with some other DN gels such as PAMPS-PAAm and PAMPS-PDMAAm gel using the pin-on flat-type wear test is evaluated. In addition, these properties were compared with the maximum wear depth of ultrahigh molecular weight polyethylene (UHMWPE), which is currently used in artificial joints. During wear testing, the apparent friction coefficient varied. There were no significant differences in the friction coefficient among PAMPS-PAAm, PAMPS-PDMAAm, BNC-PDMAA, and UHMWPE. On the other hand, the friction coefficient of the BNC-gelatin gel was significantly higher than that of the others $(p=0.001)$ (Yasuda et al. 2005).

In gross observations after the wear tests (Figure 4.8), a mildly concave lesion appeared on the surface of each specimen of the PAMPS-PAAm gel and the BNC-PDMAA gel, while a severely concave lesion appeared on the surface of each BNC-gelatin gel specimen. On the surface of the concave lesion there were a number of scratches that were aligned parallel to the sliding direction. The PAMPS-PDMAAm gel specimens and UHMWPE specimens did not show an obviously concave lesion on the surface, although the UHMWPE specimens showed a lesion that appeared to shine.

In the evaluation of the maximum wear depth with the confocal laser microscope (Table 4.2), the mean value was minimal in the PAMPS-PDMAAm gel $(3.20 \mu \mathrm{m})$ and in the UHMWPE $(3.33 \mu \mathrm{m})$. There was no significant difference between the two materials. The PAMPS-PAAm gel $(9.50 \mu \mathrm{m})$ and the BNC-PDMAAm gel $(7.80 \mu \mathrm{m})$ showed two to three times the depth of the UHMWPE. The BNC-gelatin gel showed a tremendous amount of wear $(1302.40 \mu \mathrm{m})$. There were significant differences between one of these three gels and UHMWPE. The surface of each gel material was extremely smooth
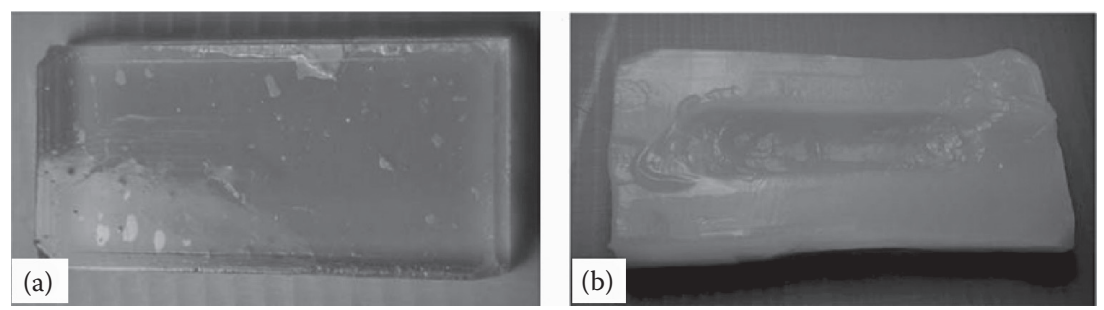

FIGURE 4.8

Gross observations of representative samples after the wear tests. (a) BNC-PDMAAm DN gel, and (b) BNC-gelatin DN gel. (Reproduced with permission of Yasuda et al. 2005.) 


\section{TABLE 4.2}

The Maximum Wear Depth and the Roughness of the BNC-PDMAAm DN Gel, BNC-Gelatin DN Gel, and the UHMWPE after Pin-On Flat Testing. (Reproduced with permission of Yasuda et al. 2005.)

\begin{tabular}{|c|c|c|c|}
\hline & BNC-PDMAAm & BNC-Gelatin & UHMWPE \\
\hline Maximum depth $(\mu \mathrm{m})$ & $7.80 \pm 8.67$ & $1302.40 \pm 867.52$ & $3.33 \pm 5.77$ \\
\hline Roughness $\left(R_{\mathrm{a}}\right)(\mu \mathrm{m})$ & $0.09 \pm 0.03$ & Not measurable & $10.0 \pm 1.0$ \\
\hline
\end{tabular}

before testing. In the observation on the concave surface after testing, the roughness, $R_{\mathrm{a}}$, averaged $0.008 \mu \mathrm{m}$ in the PAMPS-PDMAAm gel, $0.23 \mu \mathrm{m}$ in the PAMPS-PAAm gel, and $0.34 \mu \mathrm{m}$ in the BNC-PDMAAm gel (Table 4.2). In the BNC-gelatin gel, the roughness was not measurable because the wear surface was extremely fluffy. On the other hand, in the UHMWPE, the $R_{\mathrm{a}}$ was $14.1 \mu \mathrm{m}$ before testing, while this value decreased to $10.8 \mu \mathrm{m}$ after testing. It meant that the roughness of the surface decreased due to wear.

The above results demonstrated that the BNC-PDMAAm DN gel could bear one million times of cyclic friction, although the maximum wear depth was two to three times that of the UHMWPE. These gels are also resistant to wear to greater degrees than conventionally reported hydrogels. This is an amazing property as a hydrogel. On the other hand, this study showed that the BNC-gelatin DN gel was not resistant to wear compared to the BNC-PDMAAm gel. The maximum wear depth of other DN gels such as PAMPS-PDMAAm gel was similar to that of UHMWPE under one million times of cyclic friction, which was equivalent to $50 \mathrm{~km}$ of friction $\left(50 \mathrm{~mm} \times 10^{6}\right)$. In addition, the results showed that the PAMPS-PAAm DN gel could bear the one million times of cyclic friction, although the maximum wear depth was two to three times that of UHMWPE. In terms of the pin-on flat testing, it should be noted that the PAMPS-PDMAAm DN gel has a unique ability as a potential material for artificial cartilage. The cellulose-PDMAAm gel and the BNC-gelatin gel include natural materials, cellulose and gelatin, however, the wear property of the BNC-gelatin gel was extremely inferior to the other DN gels, although that of the BNC-PDMAAm gel was comparable to that of the PAMPS-PAAm gel. This is important information to consider in applying this material.

These DN gel materials have the potential to be applied to various types of arthroplasty, including not only the substitution of UHMWPE in total joint replacement, but also substitutes for part of the articular cartilage and the meniscus, which are contacted with the normal articular cartilage. In particular, the latter application-development of artificial cartilage and meniscus-is a main goal of our study. We expect that these DN gels, especially the PAMPS-PDMAAm DN gel, may have an effective lubrication system against normal cartilage. 


\section{Biodegradation of DN Gels after Implantation into Subcutaneous Tissue}

Materials for artificial cartilage are required to be not only tough, but also resistant to biodegradation, which is a phenomenon whereby a material implanted in a living body deteriorates over time by biological mechanisms. The biodegradation properties of the BNC-PDMAAm and BNC-gelatin gels, along with some other DN gels such as PAMPS-PAAm gel and PAMPS-PDMAAm gel, were tested by implanting them in the subcutaneous tissue of mature female Japanese white rabbits. In each rabbit, the skin incision was well healed without any infection, and the body weight was not reduced over the 6-week period. In each rabbit, no sign of inflammation or infection, including redness of the skin, effusion, and abscess, was observed at the implantation site at the time of sacrifice. The surface of all BNC-PDMAAm DN gel specimens became rough, although they were smooth before implantation (Figure 4.9a). All BNC-gelatin DN gel specimens were mildly deformed, and a part of the specimen was absorbed (Figure 4.9b). Concerning PAMPS-PAAm and PAMPS-PDMAAm DN gel specimens, no obvious changes were found on the gel specimens (Azuma et al. 2007).

The BNC-PDMAAm DN gel specimens did not show any significant changes in their mechanical properties or water content after implantation (Table 4.3). In the BNC-gelatin DN gel specimens (Table 4.3), the ultimate stress was dramatically reduced from 4.30 to $1.98 \mathrm{MPa}$ after implantation $(p<0.0001)$. The tangent modulus was also reduced, from 2.50 to $1.18 \mathrm{MPa}$, on average, after implantation. There were no significant differences in the strain at failure. The water content was significantly increased from $78.00 \%$ to $86.33 \%$ after implantation $(p=0.0002)$.

Regarding the mechanical properties of other DN gels without $\mathrm{BNC}$ - for example, in PAMPS-PAAm DN gel specimens (Azuma et al. 2007), the ultimate stress was extremely high (average $11.40 \mathrm{MPa}$ ) and the water content was $90 \%$ before implantation, which is the strongest in the ultimate stress

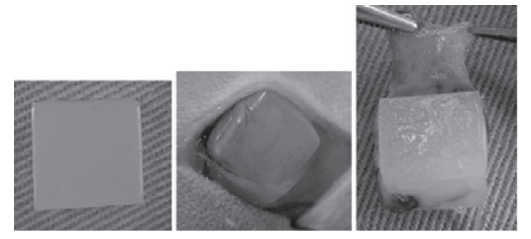

(a)

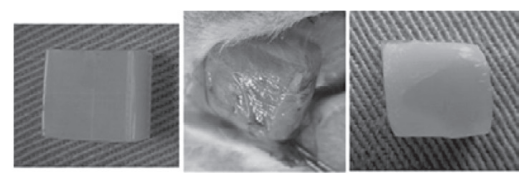

(b)

FIGURE 4.9

(a) Gross observations of the BNC-PDMAAm DN gel specimens before implantation (left) and 6 weeks after implantation: immediately before harvest (middle) and after harvest (right). The surface of all specimens became rough, although they were smooth before implantation. (b) Gross observations of the BNC-gelatin DN gel specimens before implantation (left) and 6 weeks after implantation immediately before harvest (middle) and after harvest (right). All specimens were mildly deformed and a part of the specimen was absorbed. (Reproduced with permission of Azuma et al. 2007.) 


\section{TABLE 4.3}

Changes in the Mechanical Properties and the Water Content of Each DN Hydrogel Due to Implantation. Before: Specimens before Implantation; After: Specimens Harvested 6 Weeks after Implantation. (Reproduced with permission of Azuma et al. 2007.)

\begin{tabular}{llll}
\hline & & BNC-PDMAAm & BNC-Gelatin \\
\hline Tangent modulus $(\mathrm{MPa})$ & Before & $1.70(0.70)$ & $2.50(1.10)$ \\
& After & $1.00(0.60)$ & $1.18(1.10)$ \\
& $p$-value & N.S. & N.S. \\
Ultimate stress $(\mathrm{MPa})$ & Before & $1.90(0.28)$ & $4.30(0.35)$ \\
& After & $2.17(0.35)$ & $1.98(0.52)$ \\
& $p$-value & N.S. & $<0.0001$ \\
Strain at failure $(\mathrm{m} / \mathrm{m})$ & Before & $0.37(0.03)$ & $0.33(0.04)$ \\
& After & $0.40(0.06)$ & $0.40(0.11)$ \\
& $p$-value & N.S. & N.S. \\
Water content $(\%)$ & Before & $85.00(0.71)$ & $78.00(2.30)$ \\
& After & $85.17(0.75)$ & $86.33(0.75)$ \\
& $p$-value & N.S. & 0.0002 \\
\hline
\end{tabular}

among the four DN gels-the mechanical parameters except for the tangent modulus and the water content did not change after implantation, although the modulus was significantly reduced. In contrast, the PAMPS-PDMAAm DN gel, which is highly resistant to wear in pin-on flat wear testing (Yasuda et al. 2005), the ultimate stress and the tangent modulus were significantly increased with a significant reduction of the water content after implantation (Azuma et al. 2007).

These results suggest that degradation properties of BNC-based DN hydrogels and some other conventional DN hydrogels within the living body were extremely different, depending on the properties of the polymers contained in each hydrogel. Concerning the mechanical deterioration observed in each DN hydrogel, two mechanisms are considered: biodegradation and simple degradation of the polymer network that would occur over time. In the BNC-gelatin DN gel, the ultimate stress dramatically decreased after implantation with the obvious deformation of the gel specimens, while obvious deterioration was not observed in the BNC-PDMAAm DN gel. These facts indicate that the structure composed of gelatin was greatly deteriorated by biological mechanisms, although the increase in the water content may also be a possible cause of the mechanical reduction. In the BNC-PDMAAm DN gel, however, the surface of all specimens became rough, although it was smooth before implantation. Therefore the structure of the cellulose appeared to deteriorate more slowly than gelatin. It can be speculated that the structure of PDMAAm in this gel material might compensate for the deterioration of the second structure of cellulose, at least during the 6-week period. It is important to clarify these mechanisms in the future. 
The present study suggested that each unique DN gel tested in this study has its own degradation properties within the living body. It is important to know precisely the characteristics of each material when considering its mechanical application. In addition, if the reasons for each characteristic in these materials could be clarified in the near future, this will be useful to improve DN gels not only for the development of artificial cartilage, but also to apply them to other implants. Therefore further studies should be conducted to fully clarify the biodegradation properties of the four unique DN gels.

Finally, the development of artificial cartilage is one main goal of this study. According to Eisenberg and Grodzinsky (1985), the compressive stress and the tangent modulus of the knee joint cartilage are $0.06-0.21 \mathrm{MPa}$ and 0.26-1.37 MPa, respectively, depending on the sodium chloride $(\mathrm{NaCl})$ concentration of the water. Concerning the human knee cartilage, Athanasiou et al. (1991) reported that the aggregate modulus averaged 0.71 MPa. The ultimate compressive stress of the BNC-based DN hydrogels tested in the present study is greater than that of the previously described natural cartilage. The tangent modulus of the BNC-PDMAA DN gel is comparable with that of the previously described natural cartilage. This study implied that the tested BNC-PDMAA gel along with other DN gels, except for the BNC-gelatin $\mathrm{DN}$ gel, are potential materials that may meet the requirements of artificial cartilage. However, potential materials for artificial cartilage also have to be durable to endure repetitive stress, resistant to wear, and resistant to biodegradation within the living body.

\section{Biological Responses of DN Gels after Implantation into Muscle and Subcutaneous Tissue}

Although BNC-PDMAAm and BNC-gelatin DN are unique gels that include natural polymers, it is important to evaluate what kind of tissue reaction is induced by implantation of these gel materials in the living body and how strong the reaction is in order to consider application of these DN gels for clinical implants as a replacement for natural cartilage. For this purpose, the pellet implantation test into the muscle and the subcutaneous implantation test with massive material, which can evaluate local reaction in the muscle or subcutaneous tissue around the implanted material, were investigated (Iyakushin-Hatsu 2003). This study, composed of macroscopic observations, was conducted on rabbits using the subcutaneous implantation test with massive material and histological examinations using the pellet implantation test into the para-vertebral muscle and the subcutaneous tissue, according to the international guideline for biological evaluation of the safety of biomaterials (Iyakushin-Hatsu 2003; Tanabe et al. 2008).

The implanted BNC-PDMAAm DN gels induced strong inflammation at each period (Tanabe et al. 2008). Currently these materials are difficult to apply as clinical implants. Around the BNC-gelatin DN gel, which was composed of two natural polymers, inflammation was very mild at 1 week, but significantly 
increased with numerous lymphocytes, monocyte/macrophage-like cells, plasma cells, and eosinophils at 4 and 6 weeks. In addition, the surface of the gel blocks implanted into the subcutaneous tissue was absorbed (Tanabe et al. 2008). As was discussed previously, the ultimate stress of the BNC-gelatin gel block dramatically decreased after implantation, while obvious deterioration was not observed in the BNC-PDMAAm DN gel (Azuma et al. 2007). These facts show that the structure composed of gelatin was absorbed by the previously described cell activity. This fact indicates that the BNC-gelatin DN gel has a unique absorbable property in the living body. This material has the potential to be applied as an absorbable implant, such as a material for drug delivery systems, although some improvement may be needed to reduce the inflammatory reaction.

\section{Conclusions}

The BNC-based DN hydrogel has been successfully developed from natural as well as synthetic polymers, having not only a mechanical strength as great as several megapascals, on the order of that for cartilage, ligament, and tendon, but also a low frictional coefficient, on the order of $10^{-3}$, high resistance against wear, durability to endure repetitive stress, and resistant to biodegradation within the living body. These hydrogels could open a new era of soft and wet materials as substitutes for biotissue such as articular cartilage, ligament, tendon, etc., and could be a potential candidate for drug delivery systems in the living body.

\section{References}

Athanasiou, K. A., M. P. Rosenwasser, J. A. Buckwalter, T. I. Malinin, and Y. C. Mow. 1991. Interspecies comparisons of in situ intrinsic mechanical properties of distal femoral cartilage. J. Orthop. Res. 9:330-340.

Azuma, C., K. Yasuda, and Y. Tanabe. 2007. Biodegradation of high-toughness double network hydrogels as potential materials for artificial cartilage. J. Biomed. Mater. Res. A 81:373-380.

Baily, S. J., ed. 2003. Annual Book of ASTM Standards. West Conshohocken, PA: American Society for Testing and Materials, 269-278.

Eisenberg, S. R., and A. J. Grodzinsky. 1985. Swelling of articular cartilage and other connective tissues: electromechanochemical forces. J. Orthop. Res. 3:148-159.

Gong, J. P., Y. Katsuyama, T. Kurokawa, and Y. Osada. 2003. Double network hydrogels with extremely high mechanical strength. Adv. Mater. 15:1155-1158. 
Gong, J. P., T. Kurokawa, and T. Narita. 2001. Synthesis of hydrogels with extremely low surface friction. J. Am. Chem. Soc. 123:5582-5583.

Hagiwara, A., A. Putra, A. Kakugo, H. Furukawa, and J. P. Gong. 2010. Ligament-like tough double-network hydrogel based on bacterial cellulose. Cellulose 17:93-101.

Hestrin, S., and M. Schramm. 1954. Synthesis of cellulose by Acetobacter xylinum. II. Preparation of freeze-dried cells capable of polymerizing glucose to cellulose. Biochem. J. 58:345-352.

Iyakushin-Hatsu. 2003. Basic concept on biological evaluation of the safety for therapeutic devices (including transcription of document ISO 10993-6: Biological evaluation of medical devices. Part 6: Tests for local effects after implantation). No. 0213001 (official document) from the Ministry of Health, Labor and Welfare, Japan.

Kakugo, A., S. Sugimoto, J. P. Gong, and Y. Osada. 2002. Gel machines constructed from chemically cross-linked actins and myosins. Adv. Mater. 14:1124-1126.

McCutchen, C. W. 1978. Lubrication of Joints, the Joints and Synovial Fluid. New York: Academic Press.

McKellop, H., I. Clarke, K. Markolf, and H. Amstutz. 1981. Friction and wear properties of polymer, metal, and ceramic prosthetic joint materials evaluated on a multichannel screening device. J. Biomed. Mater. Res. 15:619-653.

Ministry of Health, Labour, and Welfare, Japan. 2003. Basic concept on biological evaluation of the safety for therapeutic devices. Iyakushin-Hatsu no. 0213001, Tokyo, Japan.

Nakayama, A., A. Kakugo, and J. P. Gong. 2004. High mechanical strength doublenetwork hydrogel with bacterial cellulose. Adv. Funct. Mater. 14:1124-1128.

Obukhov, S. P., M. Rubinstein, and R. H. Colby. 1994. Network modulus and superelasticity. Macromolecules 27:3191-3198.

Okumura, K., and P. G. de Gennes. 2001. Why is nacre strong? Elastic theory and fracture mechanics for biocomposites with stratified structures. Eur. Phys. J. E 4:121-127.

Osada, Y., and A. Matsuda. 1995. Shape memory in hydrogels. Nature 376:219.

Osada, Y., H. Okuzaki, and H. Hori.1992. A polymer gel with electrically driven motility. Nature 355:242-244.

Takai, M., Y. Tsuta, and S. Watanabe. 1975. Biosynthesis of cellulose by Acetobacter xylinum. I. Characterizations of bacterial cellulose. Polym. J. 7:137-146.

Tanabe, Y., K. Yasuda, and C. Azuma. 2008. Biological responses of novel high-toughness double network hydrogels in muscle and the subcutaneous tissues. J. Mater. Sci. Mater. Med. 19:1379-1387.

Ueno, M., K. Ikeuchi, T. Nakamura, and M. Akagi. 2002. Comparison of the wear property of polyethylene plate in total knee prostheses (TKP) using different femoral component materials. Bioceramics 15:801-804.

Woo, S., S. D. Abramowitch, J. C. Loh, V. Musahl, and J. C. Wang. 2004. Ligament healing: present status and the future of functional tissue engineering. In: F. Guilak, D. L. Butler, S. A. Goldstein, and D. J. Mooney, eds., Functional tissue engineering. Dordrecht: Springer, 17-21.

Yasuda, K., J. P. Gong, and Y. Katsuyama 2005. Biomechanical properties of hightoughness double network hydrogels. Biomaterials 26:4468-4475. 



\title{
Bacterial Cellulose Surface Modifications
}

\author{
João P. Silva, Fábia K. Andrade, and Francisco Miguel Gama \\ Center of Biological Engineering, Institute for Biotechnology and \\ Bioengineering, University of Minho, Braga, Portugal
}

\section{CONTENTS}

Cell Adhesion ............................................................................................. 91

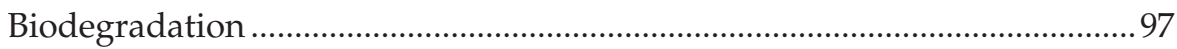

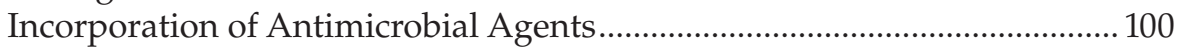

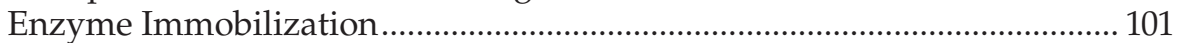

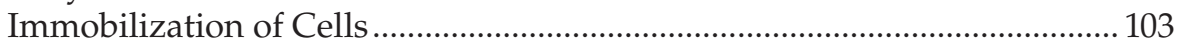

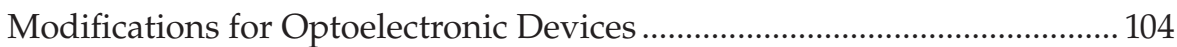

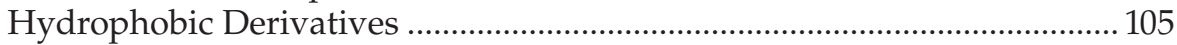

Precipitation of Metal Ions......................................................................... 106

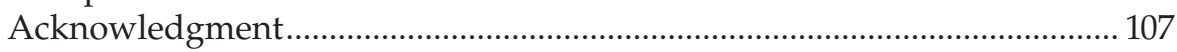

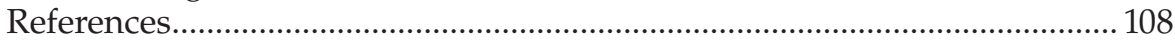

The unique properties of bacterial nanocellulose (BNC) provide the basis for a wide range of applications in human and veterinary medicine, odontology, pharmaceuticals, acoustic and filter membranes, biotechnological devices, and in the food and paper industry. In this chapter, an overview of surface modifications of bacterial cellulose is presented. Depending on the envisaged applications, chemical modifications, incorporation of bioactive molecules, modification of the porosity, crystallinity, and biodegradability may be obtained, further enlarging the potential of BNC.

\section{Cell Adhesion}

Surface characteristics play a vital role in the in vivo performance of biomaterials. The fate of implants is determined by the interactions, to a large extent cell specific, between the biomaterial and tissues (Chu et al. 2002). Polymeric materials do not always possess the specific bioactivity required to promote 
suitable interaction with cells, thus methods to enhance biocompatibility are required (Ma et al. 2007; Wang et al. 2004).

The surface properties of a scaffold, such as wettability, topography, chemistry, surface charge, the presence of hydrophobic and hydrophilic domains, density, and the conformation of functional groups, all play a crucial role in the cell-material interaction (Vesel et al. 2008). The control of cell adhesion on the polymer substrate, and therefore the ability to guide proliferation, migration, and differentiation, is highly desirable and a central issue in the development of scaffolds for tissue engineering (Lucchesi et al. 2008).

The attachment of cells to biomedical materials can be improved by using adhesion molecules, present in the extracellular matrix substances, such as fibronectin, vitronectin, or laminin. A binding site present in many adhesive proteins is the three amino acid sequence Arg-Gly-Asp (RGD), which binds integrin receptors on the cell surface (Hersel et al. 2003). Proteins (such as those with RGD sequences) that induce desirable cell behaviors (e.g., cell adhesion, spreading, and other functions) have been incorporated into biomaterials to control tissue reactions.

Bacterial nanocellulose (BNC) is a new promising material as a replacement for small blood vessels. For the reconstruction of arteries with large caliber, currently available synthetic grafts offer a reasonable solution and proven clinical efficacy. However, for small-size $(<6 \mathrm{~mm})$ grafts these materials generally give poor performance, due to anastomotic intimal hyperplasia and surface thrombogenicity (Bos et al. 1998; Conte 1998; Zilla et al. 2007). BNC is highly biocompatible in vivo (Helenius et al. 2006), but shows poor cell adhesion. In the native blood vessel, the endothelium creates a smooth nonthrombogenic surface. In order to sustain cell adhesion to improve blood compatibility, adhesive peptides have been introduced on the BNC surface through different techniques. Bodin et al. (2007) reported a novel method to activate the bacterial cellulose (BC) surface with the RGD peptide using xyloglucan-Gly-Arg-Gly-Asp-Ser (XG-GRGDS) conjugates. The surface modification of BNC with XG, in water, did not alter the morphology of the fibers, but increased the wettability, which might explain the negligible amount of protein adsorbed on the modified material. Cell adsorption studies showed that adhesion of human endothelial cells is enhanced when the BNC hydrogel is modified with XG-GRGDS (Bodin et al. 2007). Moreover, endothelial cells on RGD-modified BNC showed enhanced spreading and displayed more organized stress fibers (thus allowing cellular tension to be maintained and modified in response to mechanical stress), while developing a more mature phenotype (Fink et al. 2011).

The RGD peptide was also used by Andrade et al. (2010); in this case the adhesion peptides (RGD or Gly-Arg-Gly-Asp-Tyr [GRGDY]) were fused to a carbohydrate-binding module with affinity to cellulose (CBM3). For artificial grafts based on cellulose, the use of a CBM exhibiting specific high affinity for cellulose surfaces may be a facile and smart strategy to avoid losing the biological agents coating the graft. BNC was treated with recombinant 

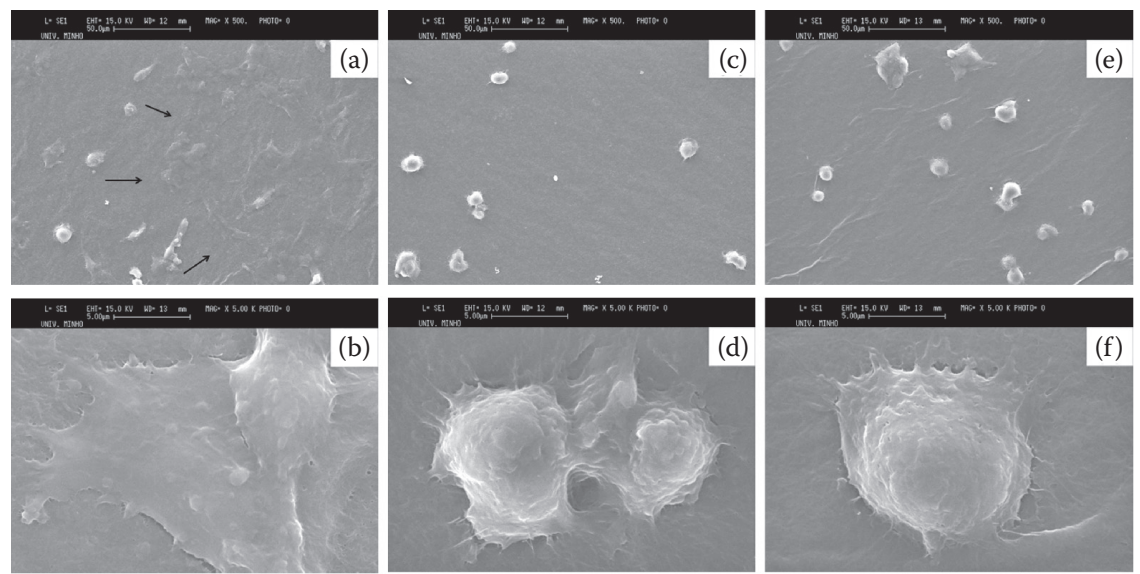

\section{FIGURE 5.1}

SEM micrographs of BC treated with (a, b) RGD-CBM, (c, d) CBM, and (e, f) buffer. The arrows mark cells with an elongated morphology. (a, c, e) Scale $50 \mu \mathrm{m}$; (b, d, f) scale $5 \mu \mathrm{m}$. (Reproduced from Andrade et al. 2010.)

proteins (different constructs containing CBM-RGD) and tested in fibroblast cell cultures. The results showed that only the proteins with the RGD sequence significantly improved fibroblast adhesion as compared to the unmodified membrane (Figure 5.1).

The results also demonstrated that the protein containing a single RGD copy had, surprisingly, a stronger effect than the protein containing two copies of RGD. Another work from the same group showed that the presence of chimeric peptides (RGD and GRGDY) significantly increases the adhesion of human microvascular endothelial cells (HMECs) on BNC, with RGD showing a stronger effect than GRGDY (Figure 5.2).

Moreover, the presence of RGD stimulated elongation of the cells and the early formation of cordlike structures, however, it decreased the ingrowth of these endothelial cells through the BNC (Andrade et al. 2010). In a recent work, Andrade et al. (2011) studied the blood compatibility of BNC treated with the RGD-CBM protein. The presence of RGD increased platelet adhesion, probably because the RGD was recognized by the platelets' integrins. However, when endothelial cells were cultured on RGD-treated BNC, a confluent cell layer was formed and almost no platelets adhered to the material (Figure 5.3). Thus the improvement of BNC blood compatibility through modification with adhesion peptides seems to be an interesting strategy for the development of BNC vascular grafts.

Regarding the potential use of BNC for vascular tissue engineering scaffolds, Wan et al. (2011) developed heparinized membranes. Although a common way to perform heparinization is the coating process where heparin is immobilized on the surface of the materials by ionic binding, the early depletion of heparin (leaving a heparin-free material behind) is a major 


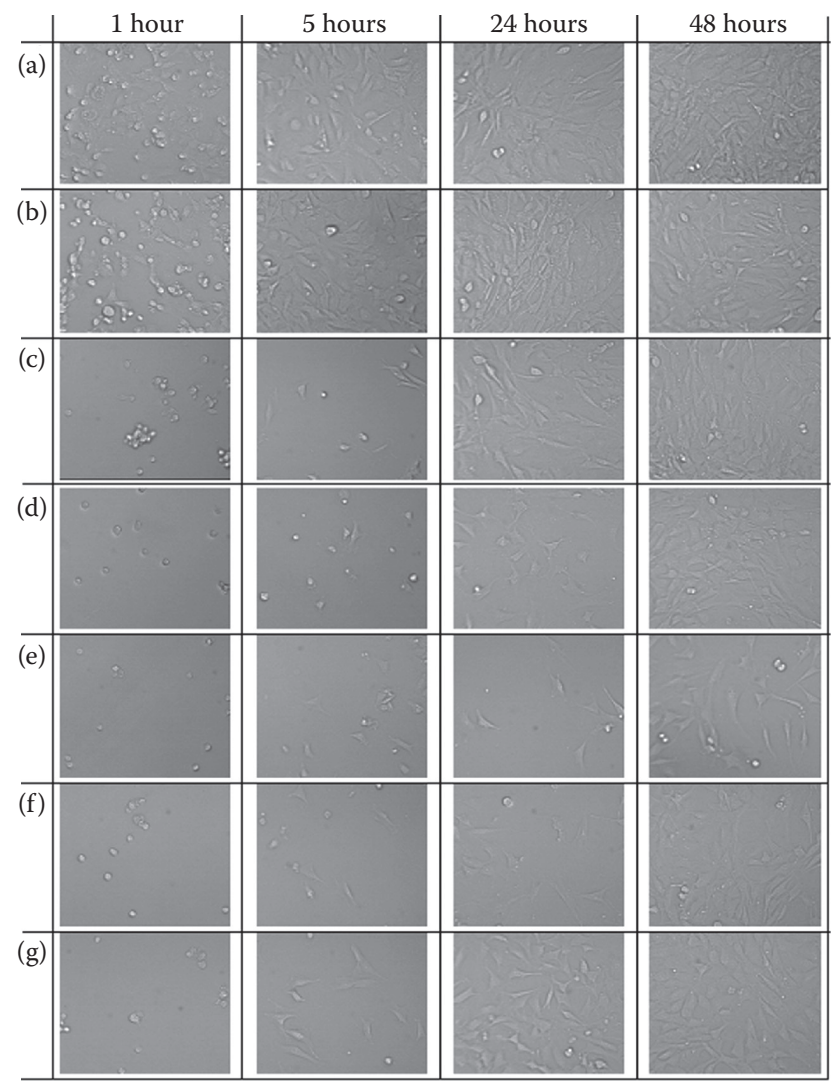

FIGURE 5.2

Photographs showing the effect of the recombinant proteins on cell (fibroblasts 3T3) attachment to polystyrene plate. (a) RGD/CBM, (b) RGD/CBM/RGD, (c) GRGDY/CBM, (d) GRGDY/ CBM/GRGDY, (e) CBM, (f) buffer, and (g) control. The photographs were taken at 1, 5, 24, and $48 \mathrm{~h}$ after the addition of cells. (Reproduced from Andrade et al. 2009.)

limitation. Therefore, in the mentioned work, heparin was combined with BNC during the growth of Gluconacetobacter xylinus by adding heparin to the culture medium. Results revealed no differences in morphology between Hep-BNC and BNC, although significant changes were observed in the crystallographic structure when heparin was incorporated into the BNC's flask cultures. In addition, Fourier transform infrared spectroscopy (FTIR) and X-ray photoelectron spectroscopy (XPS) analysis demonstrated the presence of the anticoagulant sulfate groups in the Hep-BC hybrid nanofibers, suggesting their potential use for blood-related products.

In order to improve cell adhesion, Watanabe et al. (1993) chemically modified $\mathrm{BNC}$, aiming the introduction of electric charge. In this context, membranes of trimethyl ammonium betahydroxy propyl-BC (TMAHP-BNC), diethyl aminoethyl-BNC (DEAE-BNC), aminoethyl-BC (AE-BNC), and carboxymethyl-BC 


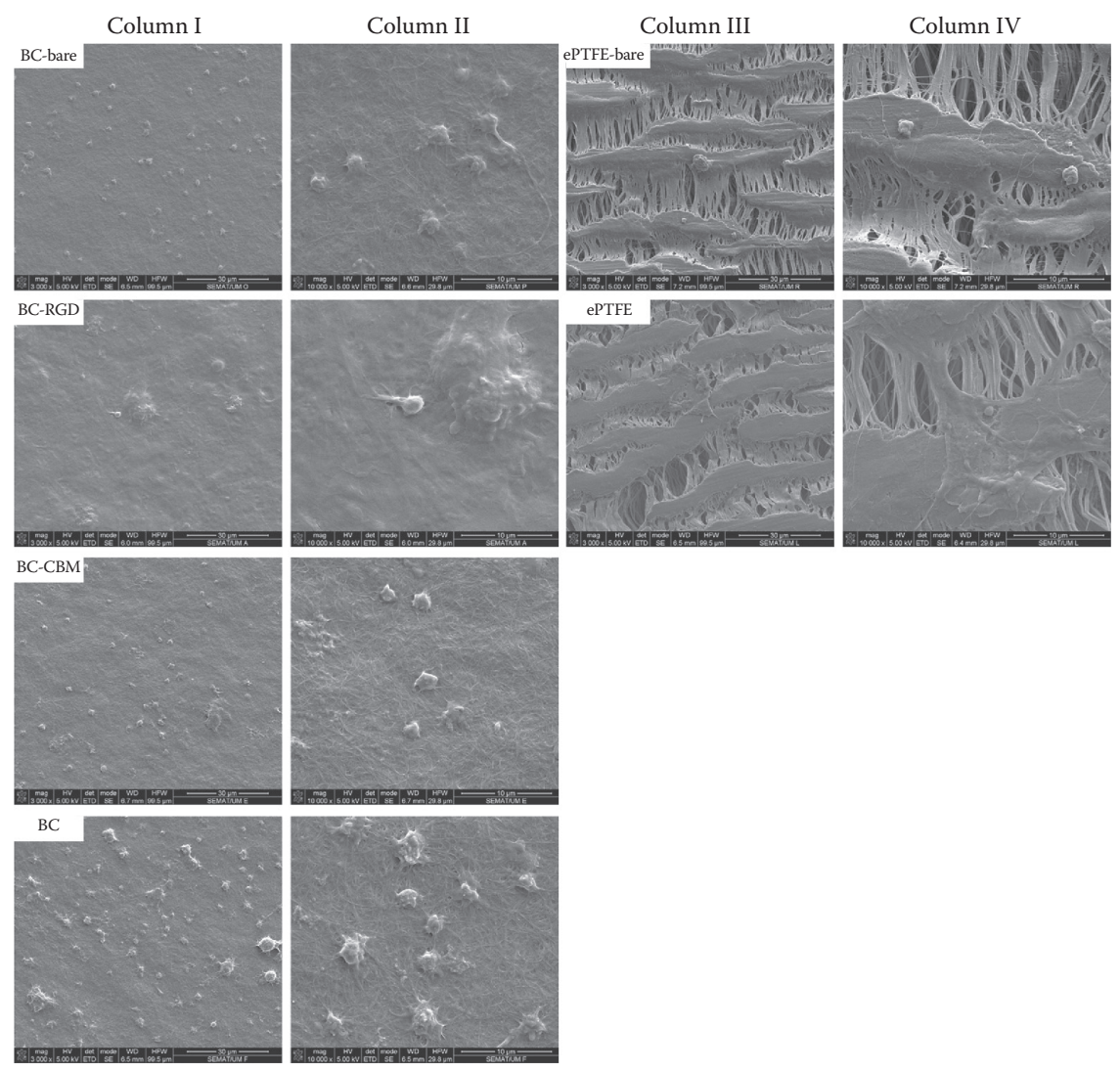

FIGURE 5.3

SEM images of the adhered platelets on endothelialized BC untreated or treated with recombinant proteins and expanded polytetrafluoroethylene (ePTFE). The bare BC and ePTFE were used as controls. The captions without "bare" indicate the cultured HMEC surface. Columns II and IV (scale bar $10 \mu \mathrm{m}$ ) are the magnified images of columns I and II (scale bar $30 \mu \mathrm{m}$ ), respectively. (Reproduced from Andrade et al. 2011.)

(CM-BNC) were produced. No morphological changes were observed in either kind of membrane produced. The study showed that L929 fibroblast cells grew better on the modified membranes in comparison with the native ones, with TMAHP-BNC membrane being the most suitable scaffold for cell growth. Further, the TMAHP-BNC was coated with adhesive protein (collagen type I) and tested as a support for the production of recombinant erythroid differentiation factor (EDF)/activin A, using genetically engineered Chinese hamster ovary cells. Both the viability of the cells and production of EDF/activin A were maintained for about 1 month, while cultures on plastic dishes lasted only 12 days.

Trauma and disease of bone and joints, frequently damaging both the articular cartilage and the subchondral bone, result in severe pain and disability 
for millions of people worldwide, posing a major health problem. The support structures in tissue engineering of cartilage should fulfill some requirements in order to comply with potential clinical applications. They should be biocompatible, able to aid in cell development and differentiation, have the porosity necessary to support cell ingrowth, allow for efficient nutrition and gas exchange, and have a suitable degradation rate, able to cope with the formation of new tissue, in order to maintain the mechanical stability of the system (Hutmacher 2000; Svensson et al. 2005).

In order to mimic the glucosaminoglycans of cartilage tissue in vivo, Svensson et al. (2005) added surface charges through phosphorylation and sulfation on BNC matrices. The materials were analyzed for mechanical properties, microstructure, and cell-material interactions in order to assess the potential of this matrix as a scaffold for cartilage tissue engineering. The compressive modulus of the phosphorylated samples increased with the reaction time and improved with regard to native BNC. This result was probably due to the more compact structure of the three-dimensional (3D) network in the modified material. An even more compact network structure was found in sulfated BNC, which showed a greater resistance to compressive forces. Sulfated-BNC had a significantly lower Young's modulus than the unmodified one, resulting in a reduction of the mechanical integrity. The lower strength of sulfated-BNC may be due to the prevention of hydrogen bonding between the cellulose chains by the covalently bonded sulfate groups, chain scission by acid hydrolysis, or a combination of these. Native and chemically modified BNC materials were evaluated for cell interaction studies using bovine chondrocytes. The unmodified BNC supports chondrocyte proliferation of up to $50 \%$ of the levels obtained using the native tissue substrate, collagen type II. However, in comparison with tissue culture plastic and calcium alginate, unmodified BC showed significantly higher levels of chondrocyte growth. Chemical sulfation and phosphorylation of the BNC, performed to mimic the glucosaminoglycans of native cartilage, did not enhance chondrocyte growth. It was verified that $\mathrm{BNC}$ supports cell ingrowth, and chondrocytes preserve the differentiated phenotype. In general, BNC appeared to have potential as a scaffold for tissue engineering of cartilage.

The modification of surfaces using plasma techniques is becoming increasingly common in biomaterials engineering. The most important advantage of plasma surface modifications is the ability to selectively change the surface properties, improving biocompatibility and mimicking the local tissue environment without altering the bulk attributes. Furthermore, it is an easy way to introduce the desired groups or polymer chains onto the surface of materials with complex shapes. Plasma thus provides a versatile and effective means to modify surfaces, enhancing the physicochemical properties and optimizing the biofunctionality (Chu et al. 2002). Pertile et al. (2010) modified $\mathrm{BNC}$ membranes with nitrogen plasma in order to enhance the cell-material interactions through the incorporation of N-groups on the surface. The results showed that the nitrogen plasma treatment did not increase the wettability 

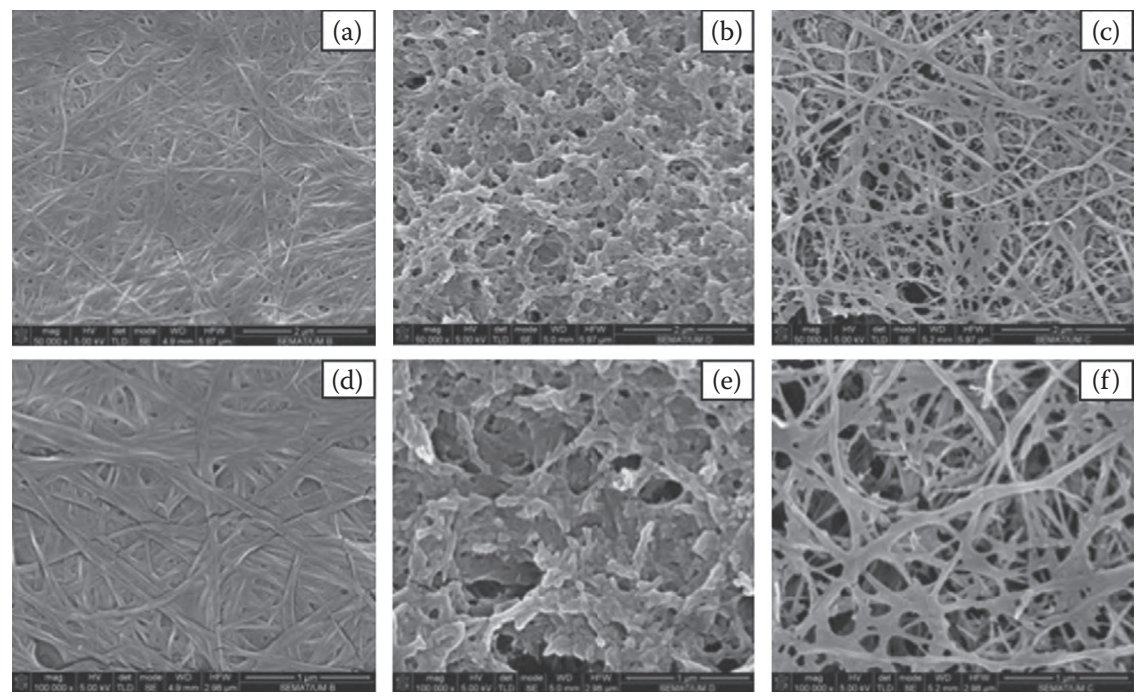

FIGURE 5.4

SEM micrographs of BC $(a, b), B C P(c, d)$, and autoclaved BCP $(e, f)$. (Reproduced from Pertile et al. 2010.)

of the material, but increased the porosity and surface chemistry, as noticed by the presence of nitrogen. The scanning electron microscopy (SEM) observations revealed that plasma changes BNC morphology (Figure 5.4). While BNC presents relatively low porosity and a tight interfiber contact, the plasma treatment seems to disrupt the fibers to some extent, leading to a more porous and rough material. The results also showed that different cell lines display a different behavior in contact with modified biopolymers, once the modification by nitrogen plasma efficiently improves the adhesion of N1E-115 and HMEC-1 cells, although in the case of the 3T3 fibroblasts, no effect was observed.

\section{Biodegradation}

Tissue engineering scaffolds are, following implantation, subsequently replaced by native tissue as they are gradually removed from the implant site through biodegradation. The scaffold degradation rate must be appropriate to the regrowth of the new tissue in order to maintain the graft structure and mechanical properties and to avoid failure (Isenberg et al. 2006; Karande and Agrawal 2008).

Cellulose and its derivatives are degradable by several bacteria and fungi that synthesize cellulose-specific enzymes (i.e., cellulases) (Tomsic et al. 
2007). The biodegradation of cellulose has been widely investigated (Sannino et al. 2009). BNC is not enzymatically degradable in the animal and human body because of the absence of hydrolases that attack the $\beta(1-4)$ linkage; as a consequence, biodegradability has become an essential limiting factor for some BNC applications as a scaffold in tissue engineering (Li et al. 2009).

Aiming at the production of an in vivo degradable polysaccharide, while exhibiting both chitin- and cellulose-like properties, attempts have been made to incorporate $\mathrm{N}$-acetylglucosamine (GlcNAc) residues into BC. Ogawa et al. (1992) characterized the enzymatic susceptibility of BNC containing $\mathrm{N}$-acetylglucosamine residues for cellulase, lysozyme, and chitinase hydrolysis. The results showed that modified BNC possesses high susceptibility for lysozyme (proportional to the GlcNAc content) and cellulase, but only slight susceptibility for chitinase. The random distribution of GlcNAc residues on modified BNC is responsible for the higher lysozyme reactivity. This approach was also studied by Shirai et al. (1994), who described a G. xylinus strain adapted to a medium containing GlcNAc that was used to prepare a novel cellulosic polysaccharide containing residual GlcNAc. The amino sugar content in the pellicles was measured after cultivation of the bacteria in the presence of various ammonium salts. Ammonium chloride seems to be the best additive to enhance GlcNAc incorporation, under rotatory and aerobic conditions. The maximum amount of $\mathrm{N}$-acetylglucosamine (GlcNAc) incorporation was found to be $4 \%$ (on a molar basis) and the resulting polysaccharide was lysozyme susceptible. The acceleration of GlcNAc incorporation in the presence of ammonium salts seems to be due to a shift in the aminotransferase equilibrium in the presence of a high concentration of ammonium ion.

The production of similar polysaccharides was obtained by incubation of G. xylinus in a modified Hestrin-Schramm medium containing lysozymesusceptible phosphoryl chitin (P-chitin) and D-glucose (Shirai et al. 1997). Analysis of the culture medium by high-performance liquid chromatography (HPLC) showed that the P-chitin is depolymerized to monomeric and oligomeric residues during the incubation, and these were utilized as a carbon source by the bacteria. Furthermore, monomeric GlcNAc 6-phosphate was also found to enhance the incorporation of GlcNAc residues into the polysaccharide. Also, Ciechanska (2004) obtained a modified BNC by adding two different chitosan forms (chitosan acetate and chitosan lactate) to the culture medium during bacterial growth. The authors showed that glucosamine and $\mathrm{N}$-acetylglucosamine units were incorporated into the cellulose chain following degradation of chitosan modifiers present in the nutrient medium within the biosynthesis process. The modified BNC obtained presented good mechanical properties in the wet state and high moisture-holding properties. Enzymatic degradation assays demonstrated that modified BNC is susceptible to lysozyme degradation. Furthermore, modified BNC possesses bacteriostatic activity against Gram-negative and Gram-positive bacteria and bactericidal activity against Gram-positive bacteria. Lee et al. (2001) 
investigated the ability of an Acetobacter xylinum ATCC 10245 strain to incorporate glucosamine and $\mathrm{N}$-acetylglucosamine into exopolymers. The study showed that glucose resulted in the highest production of exopolymer among the carbon sources used. The exopolymers synthesized with glucosamine or $\mathrm{N}$-acetylglucosamine as the main carbon source were found to contain glucose and glucosamine or N-acetylglucosamine. The author suggested that, probably, cellulose synthase and other enzymes involved in cellulose synthesis have broad specificities. Preliminary analysis of the fibers obtained (cellulose and the new copolymers) by environmental SEM suggested similar gross morphology (e.g., diameter and surface smoothness). Kobayashi et al. (2006) produced a cellulose-chitin hybrid polysaccharide by enzymatic polymerization, using a chitinase and a cellulase from Trichoderma viride. The molecular weight (MW) values of the cellulose-chitin hybrid polysaccharides reached 4030 and $2840 \mathrm{Da}$, which correspond to 22 and 16 saccharide units, respectively. These are rather low values as compared with naturally occurring chitin and cellulose. The obtained cellulose-chitin hybrid polysaccharide did not exhibit a crystalline structure and was hydrolyzed in vitro by lysozyme. Also, Phisalaphong and Jatupaiboon (2008) obtained a nanostructured BNC-chitosan composite by supplementing the BNC culture medium with low molecular weight chitosan during biosynthesis by $A$. xylinum. The BNC-chitosan films presented improved mechanical properties and water absorption capacity, significantly denser fibril structure, smaller pore diameter, and greater surface area compared with those of native BNC films. However, no significant influence in the water vapor transmission rates (indicative of the water vapor permeability), crystallinity, and antimicrobial activity were observed.

The use of BNC is currently expanding as an excellent biomaterial with a 3D nanonetwork for scaffold preparation in the tissue engineering field. However, BNC is not enzymatically degradable in the human body. As a consequence, biodegradability has become an essential limiting factor in BNC applications as a scaffold material in tissue engineering.

A limited degree (typically $1 \%-20 \%$ ) of periodate oxidation of polysaccharides may give rise to derivatives with altered chemical and physical properties, namely concerning chain flexibility and extension, crystallinity, physical interactions with other biopolymers, and, most importantly, hydrolytic lability, which may provide a basis for biomaterials with increased biodegradability. Periodate oxidation is characterized by the specific cleavage of the C2-C3 bond of the glucopyranoside ring, and this cleavage results in the formation of two aldehyde groups per glucose unit. 2,3-dialdehyde cellulose degrades at physiological $\mathrm{pH}$ in vivo, and in vitro it can be degraded into glycolic acid and 2,4-dihydroxybutyric acid. For a review of the fundamental aspects of periodate oxidation, see Kristiansen et al. (2010) and Perlin (2006).

Li et al. (2009) produced biodegradable 2,3-dialdehyde cellulose BNC from $B C$ by periodate oxidization. While this chemical treatment maintained the original 3D nanonetwork structure, the obtained scaffold could 
degrade rapidly in water, phosphate-buffered saline (PBS), and simulated body fluids. In another study, Hutchens et al. (2009) demonstrated that periodate oxidized BNC retains its original structure and ability to initiate the mineralization of calcium-deficient hydroxyapatite, similar to natural bone apatite. These studies suggest that the improved biodegradability provided by periodate oxidization of BNC can increase its potential applications in tissue engineering.

\section{Incorporation of Antimicrobial Agents}

For some biomedical applications and in the food industry, where BC is widely used, control of microorganisms is of great importance. Although BNC itself has no antimicrobial activity, its structure allows the incorporation of antimicrobial agents, which may vary according to the final application.

Mainly due to its ability to maintain a moist environment within wounds, significantly reduce pain, accelerate reepithelialization and formation of granulation tissue, and reduce scar formation, $\mathrm{BNC}$ is considered an effective wound dressing material (Czaja et al. 2006). In fact, BNC-based commercial products, such as BioFill ${ }^{\circledR}$ (BioFill Produtos Biotecnológicos, Brazil), Bionext ${ }^{\circledR}$ (Bionext Produtos Biotecnológicos, Brazil), and $\mathrm{XCell}^{\circledR}(\mathrm{Xylos}$ Corporation, PA, USA) have been shown to effectively accelerate the wound healing process (Alvarez et al. 2004; Fontana et al. 1990; Osman et al. 2007). Maneerung et al. (2008) used silver nanoparticles to achieve antimicrobial activity in BNC membranes for wound healing applications, thus preventing wound infection. For this purpose, BNC was immersed in a silver nitrate solution, then sodium borohydride was used to reduce the absorbed silver ion $\left(\mathrm{Ag}^{+}\right)$inside the BNC network to metallic silver nanoparticles $\left(\mathrm{Ag}^{0}\right)$. Results obtained showed that the freeze-dried silver nanoparticle-impregnated BC exhibited strong antimicrobial activity against Escherichia coli (Gram negative) and Staphylococcus aureus (Gram positive), bacteria commonly found in infected wounds. In fact, the unique structure and high oxygen density (ether and hydroxyl groups) of BNC fibers was shown to be an effective nanoreactor for in situ synthesis of silver nanoparticles. These properties are essential for the introduction of silver ions since they allow the tight anchoring of these ions onto BNC fibers via ion-dipole interactions, as well as they help stabilize silver nanoparticles by strong interactions with their surface metal atoms.

In 2009 Cai et al. developed a BC-chitosan blend by immersing wet BNC pellicles in a chitosan solution, followed by freeze-drying. The final product presented a foamy structure with an interconnected porous network. Incorporation of chitosan did not affect the BNC structure, although crystallinity decreased from $82 \%$ to $61 \%$ as chitosan content increased from $12 \%$ to $41 \%$, and thermal stability was improved. Moreover, the authors 
demonstrated that the mechanical and antibacterial properties of the BNC-chitosan pellicle were appropriate for biomedical applications, such as tissue engineering scaffold and wound dressing material.

More recently, a new, functional, dry BNC film containing benzalkonium chloride was developed by Wei et al. (2011). Benzalkonium chloride is an antimicrobial cationic surfactant widely used in commercial wound dressings, especially effective against Gram-positive bacteria. The antimicrobial BNC dry films were prepared by immersing freeze-dried BNC films in a benzalkonium chloride solution for $24 \mathrm{~h}$, followed by removal of nonabsorbed antimicrobial agent and another $24 \mathrm{~h}$ of freeze-drying. The final dry BNC film possessed good portability, high water absorption capability, and strong antibacterial properties, being able to sustain a stable and lasting release of the antimicrobial agent for at least $24 \mathrm{~h}$ (Figure 5.5). Therefore there is potential for these films as a commercial product for the treatment of acute traumas.

Bacterial nanocellulose has also attracted interest in the food industry as a material for active packaging, which is defined as packaging able to perform roles other than that of a barrier to the outside environment. This is mostly due to BNC's edibility and potential as a good carrier for a wide range of antimicrobial agents (Cagri et al. 2004). This potential has been exploited by Nguyen et al. (2008), who developed BNC films incorporating nisin as a proof-of-concept study to control Listeria monocytogenes and total aerobic bacteria on the surface of vacuum-packaged frankfurters. For that purpose, BNC films were soaked in a nisin solution under static conditions, followed by drying at $50^{\circ} \mathrm{C}$. Nisin-containing BNC films showed effectiveness in controlling L. monocytogenes and reducing total plate counts on the surface of frankfurters, thus opening interesting prospects as a new method to improve the safety and extend the shelf life of processed meats.

\section{Enzyme Immobilization}

Immobilization of biologically active proteins is of great relevance in different fields, from the food industry to biomedical engineering and biosensors, among others (Shpigel et al. 1999). Enzymes, in particular, may be immobilized by adsorption or covalent bonding to solid matrices, as well as by entrapment or encapsulation in polymeric substances. Immobilization allows the improvement of enzyme stability under specific process conditions (Wu and Lia 2008).

Cellulose is an attractive matrix for enzyme immobilization because of its highly suitable physical properties and low cost. Therefore cellulose-binding domains (CBDs) constitute an excellent tool to immobilize proteins on cellulose, as previously demonstrated by Shpigel et al. (1999), by fusing heparinase I to CBDs. 

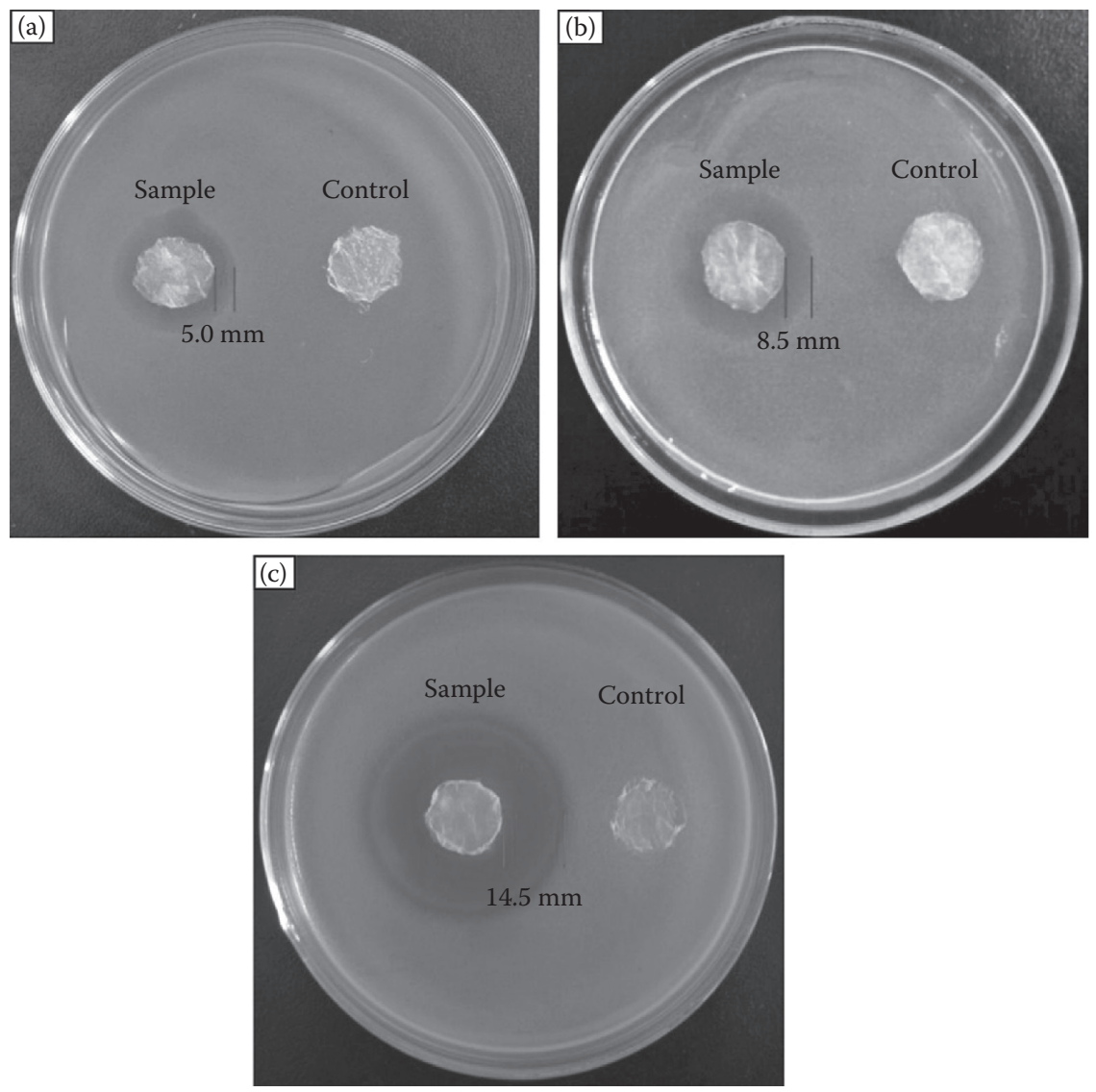

FIGURE 5.5

(See color insert.) Comparisons of antibacterial activity between benzalkonium chloridecontaining BC dry films and BC without drug as a control against (a) Escherichia coli, (b) Staphylococcus aureus, and (c) Bacillus subtilis. The BC film was prepared in a $0.102 \%$ drug solution. (Reproduced from Wei et al. 2011.)

Wu and Lia (2008) successfully immobilized glucoamylase (an enzyme widely used in the food industry) on BNC beads via an epoxy method with glutaraldehyde coupling. In this method, the BC beads are first produced via an epoxy method, involving the beads' suspension in sodium hydroxide $(\mathrm{NaOH})$ followed by the addition 1,4-butanediol diglycidyl ether and stirring at $60^{\circ} \mathrm{C}$ for $2 \mathrm{~h}$. The beads resulting from this reaction are then mixed with distilled water and $30 \%$ ammonia, again at $60^{\circ} \mathrm{C}$ for $2 \mathrm{~h}$. Finally, the beads are washed in distilled water and mixed with $25 \%(\mathrm{w} / \mathrm{v})$ aqueous glutaraldehyde for $2 \mathrm{~h}$, yielding glutaraldehyde-activated BNC beads. Glucoamylase immobilization is then carried out by adding the BNC beads to a suspension of glucoamylase in sodium phosphate at $25^{\circ} \mathrm{C}, \mathrm{pH} 4.5$, for $60 \mathrm{~min}$. The immobilization enhanced the enzyme's ability to withstand changes in $\mathrm{pH}$ 
and temperature. The relative activity of the immobilized glucoamylase was still greater than $77 \%$ at $\mathrm{pH} 2.0$, the highest value reported in the literature.

Recently Hu and Catchmark (2011a) developed a bioresorbable BNC material integrating one or more cellulose degrading enzymes (cellulases) in order to achieve higher in vivo degradation for in vivo tissue regeneration applications. A double lyophilization process was used to preserve the BNC microstructure, as well as the activity of embedded enzymes, allowing for long-term storage of the material. For the immobilization of cellulases, enzyme solution was added to small pieces of lyophilized BC pellicles. In vitro studies showed that acidic cellulases from $T$. viride presented reasonable activity for $\mathrm{pH}$ values between 4.5 and 6 . This bioresorbable BNC could be ideal for many wound care and tissue engineering applications. Nevertheless, changes in wound or tissue $\mathrm{pH}$ may occur, thus decreasing the activity of some enzymes. So the same authors, in a later work (Hu and Catchmark 2011b) also incorporated buffer ingredients into the BC along with the cellulases in order to provide an optimal $\mathrm{pH}$ microenvironment for the preferred acid cellulases, whose activity is significantly decreased at physiological $\mathrm{pH}$.

Also in a recent work, Wang et al. (2011) developed a novel matrix for enzyme immobilization and biosensor manufacturing consisting of a gold nanoparticles-BC nanofibers (Au-BNC) nanocomposite, prepared through a one-step biotemplated method in aqueous suspension using poly(ethyleneimine) as a reducing and linking agent. The authors were able to successfully immobilize heme proteins with different sizes, like horseradish peroxidase, hemoglobin, and myoglobin, by embedding them on the surface of the Au-BNC nanocomposite, while maintaining the bioactivity of the protein. In this way, this nanocomposite material proved to have vast applications in the field of biosensors and biocatalysis.

\section{Immobilization of Cells}

The investigation of immobilized microbial cells with applications in wastewater treatment has gained great interest. This technique accounts for high cell concentration in the bioreactor tank, and thus increased efficiency, as well as it facilitates the separation of liquids and solids in the settling tank. In addition, biological denitrification has proved to be one of the most feasible, advanced, selective, and cost-effective processes for nitrate removal via dissimilatory reduction. Exploiting this concept, Rezaee et al. (2008b) investigated the use of BNC as a matrix for the immobilization of denitrifying bacteria, namely Pseudomonas stutzeri. After BNC production, small pieces $(5-10 \mathrm{~mm})$ were shaken together with a cell suspension of $P$. stutzeri for $96 \mathrm{~h}$ on a rotating shaker (to promote physical surface immobilization of the bacterium) and subsequently washed twice to remove free cells. The 
immobilization of P. stutzeri on BNC increased the nitrate adsorption capacity, decreased the cell leakage, resulted in higher activity of immobilized cells, and allowed better operational control (Rezaee et al. 2008a, 2008b).

In a similar work, Nguyen et al. (2009) used BNC as a carrier for the immobilization of Saccharomyces cerevisiae. The immobilization method included two steps: adsorption and incubation. First, the initial cell concentration of the yeast suspension and immobilization time were optimized for maximization of cell immobilization yield. Then, the BNC pieces were isolated and incubated in a sterile empty vessel at a suitable temperature so that cell growth occurs. This method led to an increase in the metabolic activities of the immobilized yeast in BNC and proved useful during wine fermentation.

\section{Modifications for Optoelectronic Devices}

Transmitting and presenting information is very important in today's society. Advances in technology, applied to computer and television screens, has resulted in lightweight and flexible display devices such as liquid crystal displays (LCDs), cathode ray tube displays, organic light emitting diode (OLED) displays, and plasma screens. Nevertheless, mostly due to its optical properties, low cost, and flexibility, the printed page on paper (i.e., cellulose) is still the most popular display medium.

In recent years, efforts have concentrated on the development of an electronic display screen that combines the desired optical properties of paper with the dynamic capability of conventional digital screens, a technology commonly referred to as "electronic paper." This concept was first put into practice by Shah and Brown (2005), who created a dynamic display on BC. After production of a BNC sheet, ions are deposited around the BNC microfibrils, thus making the cellulose an electrically conducting (or semiconducting) sheet. Electrochromic dyes are then immobilized within the microstructure. The system is then encased between transparent electrodes, allowing reversible color changes upon application of switching potentials. The device obtained has several advantages, such as high paperlike reflectivity, flexibility, contrast, and biodegradability, with the potential to be used in e-book tablets, e-newspapers, and other applications.

More recently, Legnani et al. (2008) used a BNC membrane as an OLED substrate. In this work, thin films of indium tin oxide (an attractive material for optoelectronic devices due to its high transmittance in the visible region of the spectrum and high conductivity) were deposited onto dried BNC membranes at room temperature using radio frequency sputtering. The functionalized membrane obtained presented full biocompatibility and demonstrated the potential for use in optoelectronic devices, including in the biomedical field for photodynamic therapy. 
In addition to conductivity, transparency is also a useful characteristic for optoelectronic devices. In 2005 Yano et al. discovered the potential of BC nanofibers as a reinforcement material for optical functional composites. As a result of the size effect, the composites were optically transparent even at high fiber contents such as $70 \%$. Moreover, the composites presented low thermal expansion and high strength, due to BNC nanofiber reinforcement, without modifying the ease of bending of pure polymers. However, because of the several hydroxyl groups on the cellulose nanofiber, the composite is quite hygroscopic, which may lead to moisture absorption that may result in the deformation of the composite, thus hindering the advantage of dimensional stability against temperature. For this reason, Nogi et al. (2006) prepared a nanocomposite reinforced with acetylated BNC nanofibers with a low degree of substitution (DS), which was determined to be 0.17 . Even at a low DS, acetylation effectively reduced the hygroscopy of BC nanocomposites, while not changing transparency, since hydrophilic hydroxyl groups on the nanofiber surface were replaced by less hydrophilic acetyl groups. In the follow-up of that work, Ifuku et al. (2007) studied the effect of different degrees of substitution on BNC acetates by characterizing their hygroscopy and other properties. The authors found that acetylation improved various properties of BNC and their composites until an optimum DS was reached, after which excessive acetylation reduced those properties. Acetylation decreased the refractive index of $\mathrm{BNC}$ and the loss in regular transmittance of transparent nanocomposite at $580 \mathrm{~nm}$ from neat acrylic resin was reduced. In addition, it effectively reduced the hygroscopy of $\mathrm{BC}$ nanocomposites, water absorption at $20^{\circ} \mathrm{C}$ becoming inferior to $0.5 \%$, and leading to a decrease in thermal expansion. These results demonstrate that surface acetylation of $\mathrm{BC}$ nanofibers without collapsing the crystalline structure may be desirable for the enhancement of various properties of optically transparent composites.

\section{Hydrophobic Derivatives}

Recent research has also focused on increasing the hydrophobic surface character of BNC in order to improve its barrier properties, while keeping its stability and good mechanical ability. Acylation of cellulose fibers with fatty acids may result in an attractive way to produce biodegradable plastics, as well as to reinforce materials for composites with common polymeric matrices. The reactions leading to these modifications can be performed in homogeneous (cellulose fibers are previously dissolved in an appropriate medium) or heterogeneous (modifications take place in reaction media, where the formed cellulose derivatives are gradually dissolved) conditions, being the supramolecular structure of cellulose destroyed and the original physicomechanical properties of fibers lost, in both systems. However, under 
specific heterogeneous conditions, when not all hydroxyl groups are accessible for reaction or if the reaction is stopped at an early stage, modifications may occur mainly at the fiber surface or in more accessible regions of the inner layers (Freire et al. 2006).

A more hydrophobic BNC was first developed by Cunha et al. (2007) through pentafluorobenzoylation. This is a common derivatization procedure used for the chromatographic analysis of amines and alcohols, since it provides high rates and yields, in addition to the stability of the ensuing products. In that work, BNC membranes were mixed with pentafluorobenzoyl chloride in the presence of dry toluene (a nonswelling medium, chosen to limit the modification to the fibers' surface) and pyridine. This procedure resulted in esterified membranes more resistant to hydrolysis. Moreover, the possibility to easily remove the perfluorinated moieties in an aqueous alkaline medium constituted an additional advantage, since it ensured the straightforward reaggregation of the BNC fibers. In a later work, Tomé et al. (2010) produced and characterized BNC membranes esterified with hexanoyl chloride using the same cellulose/fatty acyl chloride/pyridine system. The controlled heterogeneous modification of $\mathrm{BNC}$ resulted in more hydrophobic membranes with preserved bulk structure, although with decreased thermal stability. A significant decrease in water and gas (namely carbon dioxide $\left[\mathrm{CO}_{2}\right]$, oxygen $\left[\mathrm{O}_{2}\right]$, and nitrogen $\left[\mathrm{N}_{2}\right]$ ) permeation, indicative of the improvement of BNC barrier properties, was also observed.

In another work, the effect of surface functionalization of BC nanofibrils to be used in the reinforcement of polylactide (PLLA) nanocomposites for the improvement of compatibility (interfacial adhesion) between BNC and PLLA was investigated by Lee et al. (2009). These researchers functionalized BNC with different organic acids (acetic, hexanoic, and dodecanoic acid) via esterification. It was concluded that the degree of hydrophobicity could be tailored by the length of the organic acid $\left(C_{2}-C_{12}\right)$ used. In addition, as expected, incorporation of the functionalized BNC into PLLA resulted in reinforced composites showing improved tensile modulus and tensile strength.

Thus surface modification of BNC via esterification reactions is a useful approach to produce more hydrophobic membranes, with improved surface and barrier properties, and with promising applications, namely in the packaging industry.

\section{Precipitation of Metal Ions}

Bacterial cellulose can be used as a hydrophilic matrix for the incorporation of metals into thin, flexible, thermally stable membranes. In fact, in contrast to plant cellulose, BNC is capable of catalyzing the deposition of metals within its structure to generate a finely divided homogeneous catalyst layer. 
This is mostly due to the presence of reducing groups, which are able to initiate the precipitation of palladium, gold, and silver from aqueous solution. In 2003 Evans et al. successfully precipitated palladium ions into BNC pellicles by soaking these membranes in an ammonium hexachloropalladate solution. The obtained material, termed palladium-cellulose, was further explored as a catalyst, which was prepared by inserting two platinum wires into a hydrated cube of palladium-cellulose.

In recent years palladized BNC was studied by another group of researchers as a versatile and bio-organic catalyst to mediate the reductive decolorization of a reactive textile dye, Drimarene Red, in a rotating catalyst contact reactor (Vyjayanthi and Suresh 2010). In this work, nano-size zero valent form palladium $\left(\mathrm{Pd}^{0}\right)$ deposits were shown to be irreversibly impregnated into BNC fibrils, and allowing approximately $90 \%$ efficiency regarding dye decolorization.

The potential of BNC to act as an adsorbent and an ion exchange membrane has also been explored to recover heavy metals from industrial wastewater. Choi et al. (2004) modified a BNC membrane with cation exchangeable acrylic acid using ultraviolet (UV) graft polymerization in order to provide BNC with ion exchange capacity and with greater structural density. Briefly, BNC was immersed in a benzophenone/methanol solution and, after drying, irradiated with UV light for a few minutes, under a nitrogen atmosphere, in order to activate the BNC membrane. Acrylic acid was added to the resulting material, which was then irradiated again with UV for a slightly longer period of time. Acrylic-modified BNC membrane showed reasonable mechanical properties, mostly due to the high crystallinity, and its electrochemical characteristics were comparable to those of the commercial membrane CMX. Moreover, it should be noted that the production of this acrylic-modified BNC was an environmentally compatible procedure, while it allows the easy disposal of the waste ion exchange membrane, as a result of BNC's biodegradable nature.

In a recent work, BNC was biosynthesized and used as a substrate for the production of BNC-titanium oxide $\left(\mathrm{TiO}_{2}\right)$ hybrid nanofibers (Sun et al. 2010). This was attained by implanting the anatase $\mathrm{TiO}_{2}$ nanoparticle arrays on $\mathrm{BNC}$ fibers using a molecular imprinting technique. The hybrid nanofibers were mesoporous, consisted of partially cemented anatase particles with small diameters $(4.3-8.5 \mathrm{~nm})$, and showed high photocatalytic activity (even greater than that of the commercial photocatalyst P25). Moreover, it was observed that doping the hybrid nanofibers with nitrogen greatly increased their photocatalytic activity.

\section{Acknowledgment}

The authors thank the Portuguese Foundation for Science and Technology (FCT) for funding through PTDC and COMPETE funding programs. The 
authors also acknowledge the publishers of Elsevier and John Wiley \& Sons for kindly authorizing the reproduction of images published in their journals.

\section{References}

Alvarez, O. M., M. Patel, J. Booker, and L. Markowitz. 2004. Effectiveness of a biocellulose wound dressing for the treatment of chronic venous leg ulcers: results of a single center randomized study involving 24 patients. Wounds 16:224-233.

Andrade, F. K., R. Costa, L. Domingues, R. Soares, and M. Gama. 2010. Improving bacterial cellulose for blood vessel replacement: functionalization with a chimeric protein containing a cellulose-binding module and an adhesion peptide. Acta Biomater. 6:4034-4041.

Andrade, F. K., S. M. Moreira, L. Domingues, and F. M. Gama. 2009. Improving the affinity of fibroblasts for bacterial cellulose using carbohydrate-binding modules fused to RGD. J. Biomed. Mater. Res. A 92A:9-17.

Andrade, F. K., J. P. Silva, M. Carvalho, E. M. S. Coutinho, R. Soares, and F. M. Gama. 2011. Studies on the hemocompatibility of bacterial cellulose. J. Biomed. Mater. Res. A 98:554-566.

Bodin, A., L. Ahrenstedt, H. Fink, H. Brumer, B. Risberg, and P. Gatenholm. 2007. Modification of nanocellulose with a xyloglucan-RGD conjugate enhances adhesion and proliferation of endothelial cells: implications for tissue engineering. Biomacromolecules 8:3697-3704.

Bos, G. W., A. A. Poot, T. Beugeling, W. G. van Aken, and J. Feijen. 1998. Small-diameter vascular graft prostheses: current status. Arch. Physiol. Biochem. 106:100-115.

Cagri, A., Z. Ustunol, and E. T. Ryser. 2004. Antimicrobial edible films and coatings. J. Food Protect. 67:833-848.

Cai, Z., H.-J. Jin, and J. Kim. 2009. Chitosan blended bacterial cellulose as a smart material for biomedical application. Proc. SPIE 7291:72910U.1-72.

Choi, Y.-J., Y. Ahn, M.-S. Kang, H.-K. Jun, I. S. Kim, and S.-H. Moon. 2004. Preparation and characterization of acrylic acid-treated bacterial cellulose cation-exchange membrane. J. Chem. Technol. Biotechnol. 79:79-84.

Chu, P., J. Chen, L. Wang, and N. Huang. 2002. Plasma-surface modification of biomaterials. Mater. Sci. Eng. Rep. 36:143-206.

Ciechanska, D. 2004. Multifunctional bacterial cellulose/chitosan composite materials for medical applications. Fibres Text. East. Eur. 12:69-72.

Conte, M. S. 1998. The ideal small arterial substitute: a search for the Holy Grail? FASEB J. 12:43-45.

Cunha, A. G., C. S. R. Freire, A. J. D. Silvestre, C. P. Neto, A. Gandini, E. Orblin, and P. Fardim. 2007. Highly hydrophobic biopolymers prepared by the surface pentafluorobenzoylation of cellulose substrates. Biomacromolecules 8:1347-1352.

Czaja, W. K., D. J. Young, M. Kawecki, and R. M. Brown. 2006. The future prospects of microbial cellulose in biomedical applications. Biomacromolecules 8:1-12.

Evans, B. R., H. M. O'Neill, V. P. Malyvanh, I. Lee, and J. Woodward. 2003. Palladiumbacterial cellulose membranes for fuel cells. Biosens. Bioelectron. 18:917-923. 
Fink, H., L. Ahrenstedt, A. Bodin, H. Brumer, P. Gatenholm, A. Krettek, and B. Risberg. 2011. Bacterial cellulose modified with xyloglucan bearing the adhesion peptide RGD promotes endothelial cell adhesion and metabolism-a promising modification for vascular grafts. J. Tissue Eng. Regen. Med. 5:454-463.

Fontana, J. D., A. M. Desouza, C. K. Fontana, I. L. Torriani, J. C. Moreschi, B. J. Gallotti, S. J. Desouza, G. P. Narcisco, J. A. Bichara, and L. F. X. Farah. 1990. Acetobacter cellulose pellicle as a temporary skin substitute. Appl. Biochem. Biotechnol. 24:253-264.

Freire, C. S. R., A. J. D. Silvestre, C. P. Neto, M. N. Belgacem, and A. Gandini. 2006. Controlled heterogeneous modification of cellulose fibers with fatty acids: effect of reaction conditions on the extent of esterification and fiber properties. J. Appl. Polym. Sci. 100:1093-1102.

Helenius, G., H. Backdahl, A. Bodin, U. Nannmark, P. Gatenholm, and B. Risberg. 2006. In vivo biocompatibility of bacterial cellulose. J. Biomed. Mater. Res. A 76:431-438.

Hersel, U., C. Dahmen, and H. Kessler. 2003. RGD modified polymers: biomaterials for stimulated cell adhesion and beyond. Biomaterials 24:4385-4415.

$\mathrm{Hu}, \mathrm{Y}$., and J. M. Catchmark. 2011a. In vitro biodegradability and mechanical properties of bioabsorbable bacterial cellulose incorporating cellulases. Acta Biomater. 7:2835-2845.

$\mathrm{Hu}, \mathrm{Y}$. , and J. M. Catchmark. 2011b. Integration of cellulases into bacterial cellulose: toward bioabsorbable cellulose composites. J. Biomed. Mater. Res. B 97B:114-123.

Hutchens, S. A., R. S. Benson, B. R. Evans, C. J. Rawn, and H. O’Neil. 2009. A resorbable calcium-deficient hydroxyapatite hydrogel composite for osseous regeneration. Cellulose 16:887-898.

Hutmacher, D. W. 2000. Scaffolds in tissue engineering bone and cartilage. Biomaterials 21:2529-2543.

Ifuku, S., M. Nogi, K. Abe, K. Handa, F. Nakatsubo, and H. Yano. 2007. Surface modification of bacterial cellulose nanofibers for property enhancement of optically transparent composites: dependence on acetyl-group DS. Biomacromolecules 8:1973-1978.

Isenberg, B. C., C. Williams, and R. T. Tranquillo. 2006. Small-diameter artificial arteries engineered in vitro. Circ. Res. 98:25-35.

Karande, T., and M. Agrawal. 2008. Functions and requirements of synthetic scaffolds in tissue engineering. In: Nanotechnology and Tissue Engineering: The Scaffold, ed. L. S. Nair and C. T. Laurencin, 53-88. Boca Raton, FL: CRC Press.

Kobayashi, S., A. Makino, H. Matsumoto, S. Kunii, M. Ohmae, T. Kiyosada, K. Makiguchi, A. Matsumoto, M. Horie, and S. Shoda. 2006. Enzymatic polymerization to novel polysaccharides having a glucose- $\mathrm{N}$-acetylglucosamine repeating unit, a cellulose-chitin hybrid polysaccharide. Biomacromolecules 7:1644-1656.

Kristiansen, K. A., A. Potthast, and B. E. Christensen. 2010. Periodate oxidation of polysaccharides for modification of chemical and physical properties. Carbohydr. Res. 345:1264-1271.

Lee, J. W., F. Deng, W. G. Yeomans, A. L. Allen, R. A. Gross, and D. L. Kaplan. 2001. Direct incorporation of glucosamine and $\mathrm{N}$-acetylglucosamine into exopolymers by Gluconacetobacter xylinus (= Acetobacter xylinum) ATCC 10245: production of chitosan-cellulose and chitin-cellulose exopolymers. Appl. Environ. Microbiol. 67:3970-3975. 
Lee, K.-Y., J. J. Blaker, and A. Bismarck. 2009. Surface functionalisation of bacterial cellulose as the route to produce green polylactide nanocomposites with improved properties. Compos. Sci. Technol. 69:2724-2733.

Legnani, C., C. Vilani, V. L. Calil, H. S. Barud, W. G. Quirino, C. A. Achete, S. J. L. Ribeiro, and M. Cremona. 2008. Bacterial cellulose membrane as flexible substrate for organic light emitting devices. Thin Solid Films 517:1016-1020.

Li, J., Y. Wan, L. Li, H. Liang, and J. Wang. 2009. Preparation and characterization of 2,3-dialdehyde bacterial cellulose for potential biodegradable tissue engineering scaffolds. Mater. Sci. Eng. C 29:1635-1642.

Lucchesi, C., B. M. Ferreira, E. A. Duek, A. R. Santos, Jr., and P. P. Joazeiro. 2008. Increased response of Vero cells to PHBV matrices treated by plasma. J. Mater. Sci. Mater. Med. 19:635-643.

Ma, Z., Z. Mao, and C. Gao. 2007. Surface modification and property analysis of biomedical polymers used for tissue engineering. Colloids Surfaces B 60:137-157.

Maneerung, T., S. Tokura, and R. Rujiravanit. 2008. Impregnation of silver nanoparticles into bacterial cellulose for antimicrobial wound dressing. Carbohyd. Polym. 72:43-51.

Nguyen, D. N., N. M. N. Ton, and V. V. M. Le. 2009. Optimization of Saccharomyces cerevisiae immobilization in bacterial cellulose by "adsorption-incubation" method. Int. Food Res. J. 15:59-64.

Nguyen, V. T., M. J. Gidley, and G. A. Dykes. 2008. Potential of a nisin-containing bacterial cellulose film to inhibit Listeria monocytogenes on processed meats. Food Microbiol. 25:471-478.

Nogi, M., K. Abe, K. Handa, F. Nakatsubo, S. Ifuku, and H. Yano. 2006. Property enhancement of optically transparent bionanofiber composites by acetylation. Appl. Phys. Lett. 89:233123.

Ogawa, R., Y. Miura, S. Tokura, and T. Koriyama. 1992. Susceptibilities of bacterial cellulose containing $\mathrm{N}$-acetylglucosamine residues for cellulolytic and chitinolytic enzymes. Int. J. Biol. Macromol. 14:343-347.

Osman, S. A., F. C. Souza, and J. E. Dolci. 2007. Estudo experimental sobre a aplicação de película de celulose bacteriana (bionext) em área cruenta de ressecção de concha nasal de coelhos. Acta ORL/Técnicas de Otorrinolaringologia 25:304-311.

Perlin, A. S. 2006. Glycol-cleavage oxidation. Adv. Carbohydr. Chem. Biochem. 60:183-250.

Pertile, R. A. N., F. K. Andrade, C. Alves, Jr., and M. Gama. 2010. Surface modification of bacterial cellulose by nitrogen-containing plasma for improved interaction with cells. Carbohyd. Polym. 82:692-698.

Phisalaphong, M., and N. Jatupaiboon. 2008. Biosynthesis and characterization of bacteria cellulose-chitosan film. Carbohyd. Polym. 74:482-488.

Rezaee, A., H. Godini, and H. Bakhtou. 2008a. Microbial cellulose as support material for the immobilization of denitrifying bacteria. Environ. Eng. Manag. J. 7:589-594.

Rezaee, A., H. Godini, S. Dehestani, A. Reza Yazdanbakhsh, G. Mosavi, and A. Kazemnejad. 2008b. Biological denitrification by Pseudomonas stutzeri immobilized on microbial cellulose. World J. Microbiol. Biotechnol. 24:2397-2402.

Sannino, A., C. Demitri, and M. Madaghiele. 2009. Biodegradable cellulose-based hydrogels: design and applications. Materials 2:353-373.

Shah, J., and R. M. Brown. 2005. Towards electronic paper displays made from microbial cellulose. Appl. Microbiol. Biotechnol. 66:352-355. 
Shirai, A., M. Takahashi, H. Kaneko, S. Nishimura, M. Ogawa, N. Nishi, and S. Tokura. 1994. Biosynthesis of a novel polysaccharide by Acetobacter xylinum. Int. J. Biol. Macromol. 16:297-300.

Shirai, A., N. Sakairi, N. Nishi, and S. Tokura. 1997. Preparation of a novel (1 $\rightarrow 4)-\beta-D-$ glycan by Acetobacter xylinum-a proposed mechanism for incorporation of a N-acetylglucosamine residue into bacterial cellulose. Carbohyd. Polym. 32:223-227.

Shpigel, E., A. Goldlust, G. Efroni, A. Avraham, A. Eshel, M. Dekel, and O. Shoseyov. 1999. Immobilization of recombinant heparinase I fused to cellulose-binding domain. Biotechnol. Bioeng. 65:17-23.

Sun, D., J. Yang, and X. Wang. 2010. Bacterial cellulose $/ \mathrm{TiO}_{2}$ hybrid nanofibers prepared by the surface hydrolysis method with molecular precision. Nanoscale 2:287-292.

Svensson, A., E. Nicklasson, T. Harrah, B. Panilaitis, D. L. Kaplan, M. Brittberg, and P. Gatenholm. 2005. Bacterial cellulose as a potential scaffold for tissue engineering of cartilage. Biomaterials 26:419-431.

Tomé, L., L. Brandão, A. Mendes, A. Silvestre, C. Neto, A. Gandini, C. Freire, and I. Marrucho. 2010. Preparation and characterization of bacterial cellulose membranes with tailored surface and barrier properties. Cellulose 17:1203-1211.

Tomsic, B., B. Simoncic, B. Orel, A. Vilcnik, and H. Spreizer. 2007. Biodegradability of cellulose fabric modified by imidazolidinone. Carbohyd. Polym. 69:478-488.

Vesel, A., I. Junkar, U. Cvelbar, J. Kovac, and M. Mozetic. 2008. Surface modification of polyester by oxygen- and nitrogen-plasma treatment. Surf. Interface Anal. 40:1444-1453.

Vyjayanthi, J. P., and S. Suresh. 2010. Decolorization of drimarene red dye using palladized bacterial cellulose in a reactor. Water Environ. Res. 82:601-609.

Wan, Y., C. Gao, M. Han, H. Liang, K. Ren, Y. Wang, and H. Luo. 2011. Preparation and characterization of bacterial cellulose/heparin hybrid nanofiber for potential vascular tissue engineering scaffolds. Polym. Adv. Technol. 22:2643-2648.

Wang, W., T.-J. Zhang, D.-W. Zhang, H.-Y. Li, Y.-R. Ma, L.-M. Qi, Y.-L. Zhou, and X.-X. Zhang. 2011. Amperometric hydrogen peroxide biosensor based on the immobilization of heme proteins on gold nanoparticles-bacteria cellulose nanofibers nanocomposite. Talanta 84:71-77.

Wang, Y. X., J. L. Robertson, W. B. Spillman, Jr., and R. O. Claus. 2004. Effects of the chemical structure and the surface properties of polymeric biomaterials on their biocompatibility. Pharm. Res. 21:1362-1373.

Watanabe, K., Y. Eto, S. Takano, S. Nakamori, H. Shibai, and S. Yamanaka. 1993. A new bacterial cellulose substrate for mammalian cell culture. A new bacterial cellulose substrate. Cytotechnology 13:107-114.

Wei, B., G. Yang, and F. Hong. 2011. Preparation and evaluation of a kind of bacterial cellulose dry films with antibacterial properties. Carbohyd. Polym. 84:533-538.

Wu, S.-C., and Y.-K. Lia. 2008. Application of bacterial cellulose pellets in enzyme immobilization. J. Mol. Catal. B Enzym. 54:103-108.

Yano, H., J. Sugiyama, A. N. Nakagaito, M. Nogi, T. Matsuura, M. Hikita, and K. Handa. 2005. Optically transparent composites reinforced with networks of bacterial nanofibers. Adv. Mater. 17:153-155.

Zilla, P., D. Bezuidenhout, and P. Human. 2007. Prosthetic vascular grafts: wrong models, wrong questions and no healing. Biomaterials 28:5009-5027. 



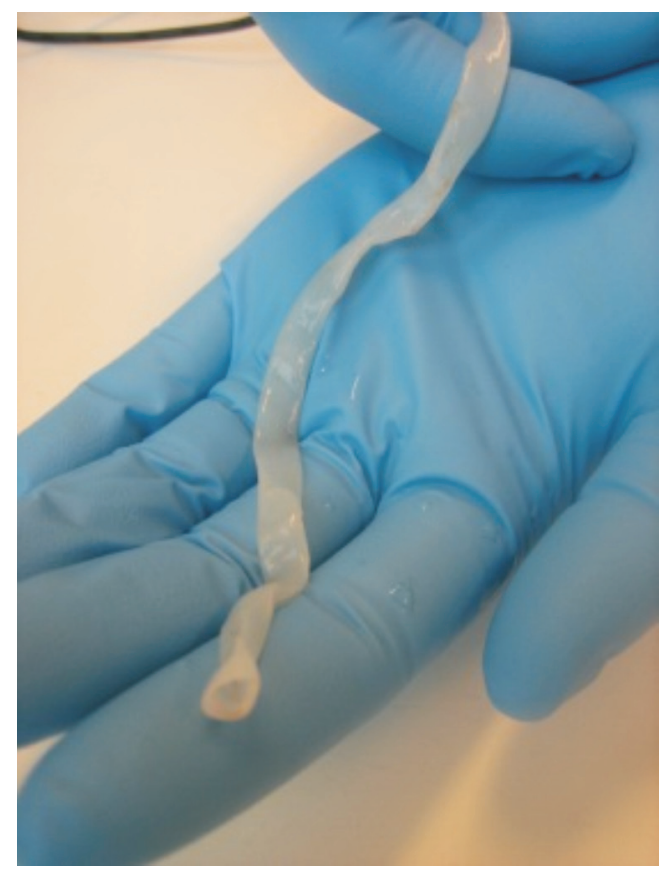

\section{FIGURE 2.1}

An artificial blood vessel synthesized by the bacteria G. xylinus.

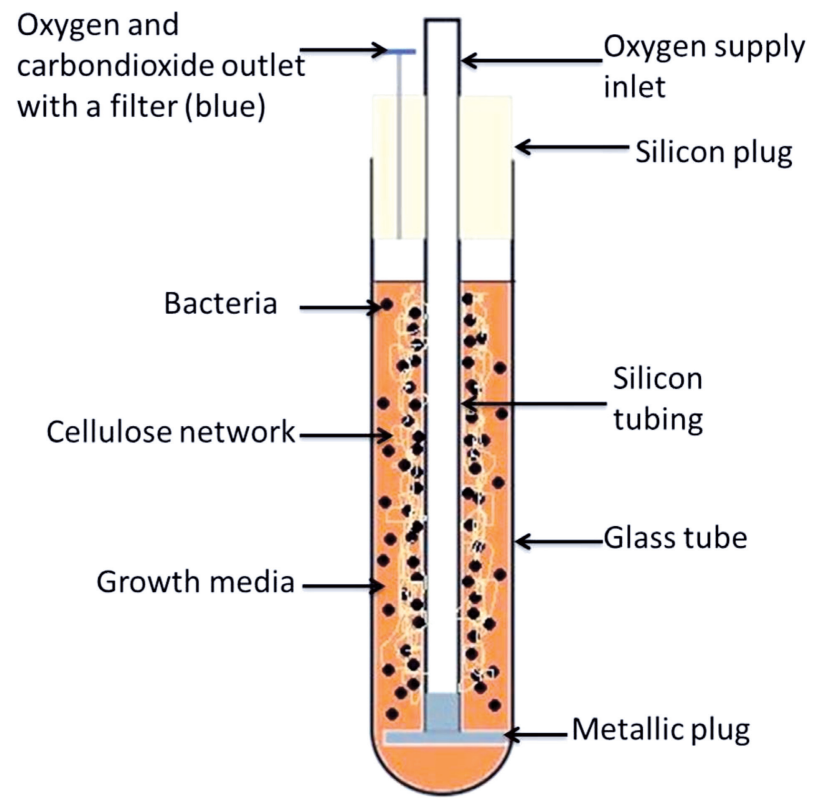

FIGURE 2.6

Tube bioreactor setup for production of BNC vascular grafts. The bacteria, illustrated with black dots, produce the cellulose network, illustrated in beige, around the silicon tube. 


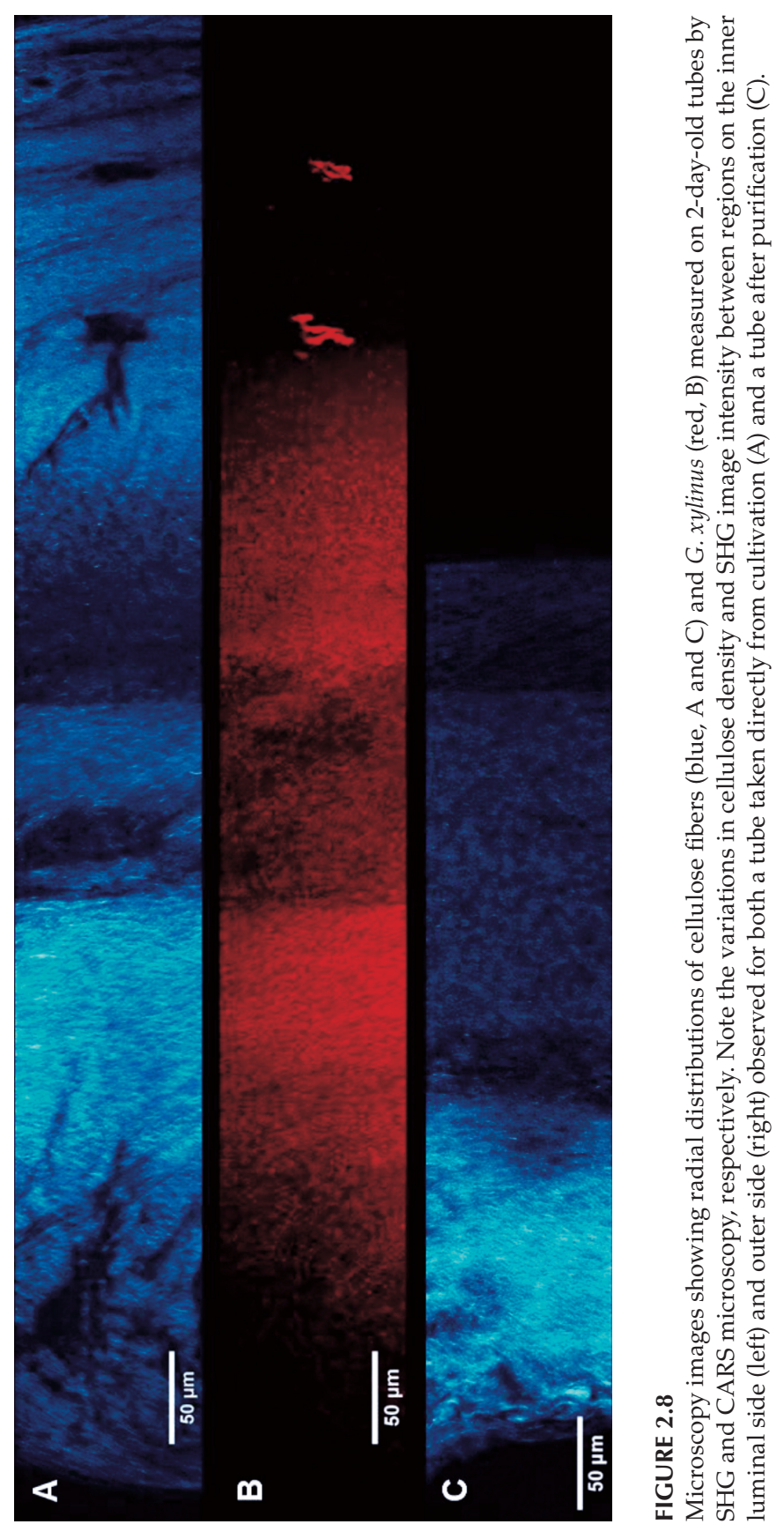




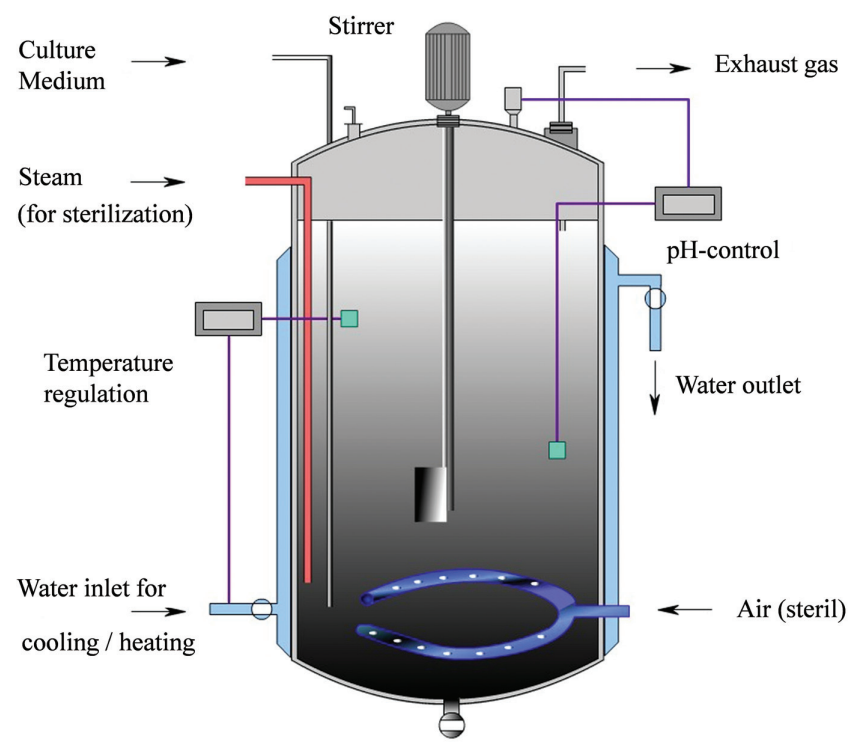

FIGURE 3.1

Example for a typical deep-tank fermenter based on the stirred tank reactor (STR) concept. Figure adopted from http://www.chemgapedia.de.

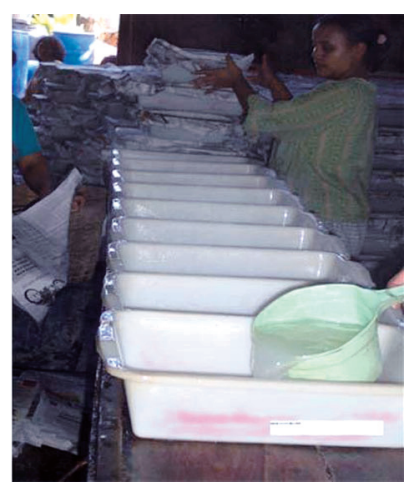

FIGURE 3.6

Production process of nata de coco (Source: http://www.bi.go.id/sipuk/en/). 

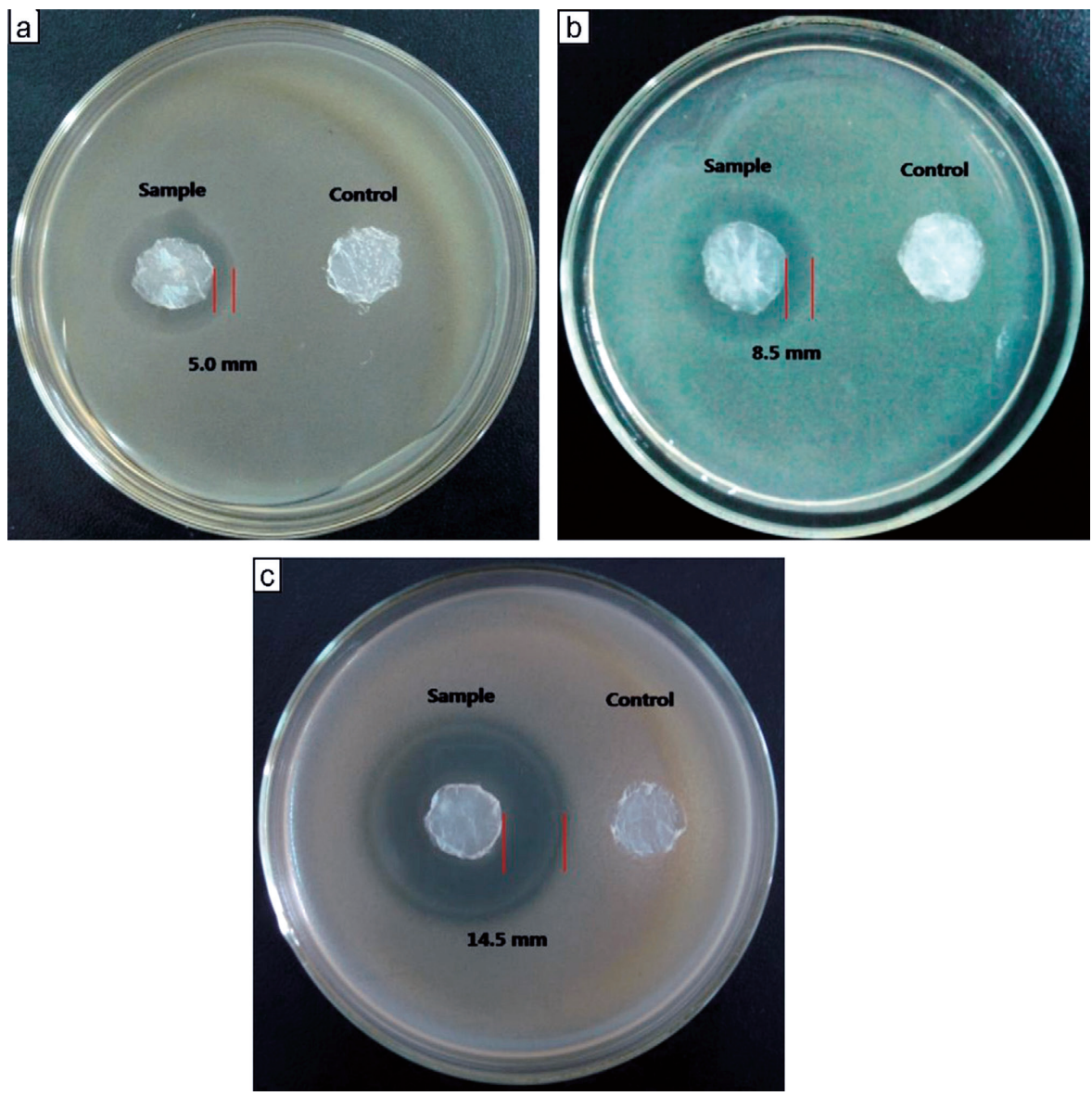

FIGURE 5.5

Comparisons of antibacterial activity between benzalkonium chloride-containing BC dry films and BC without drug as a control against (a) Escherichia coli, (b) Staphylococcus aureus, and (c) Bacillus subtilis. The BC film was prepared in a $0.102 \%$ drug solution. (Reproduced from Wei et al. 2011.) 


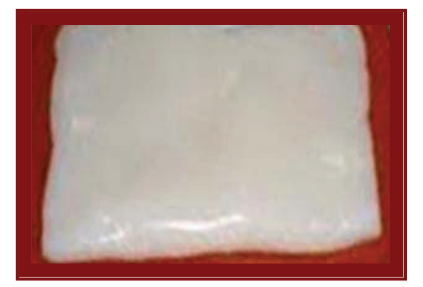

(A)

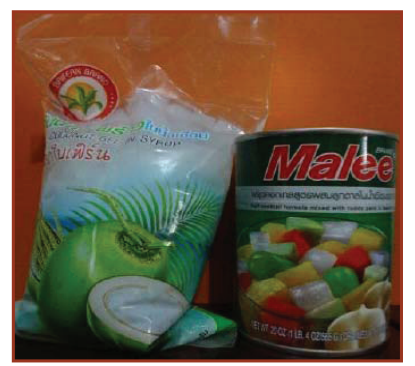

(B)

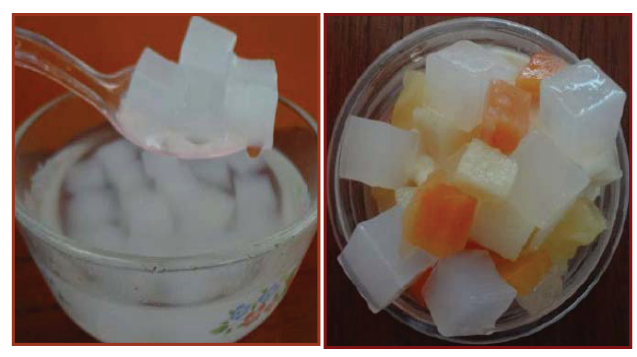

(C)

FIGURE 7.3

Nata de coco products: (a) harvested nata de coco sheet, (b) nata de coco products packed in a plastic bag and a can, and (c) nata de coco in low-calorie desserts and fruit salads.

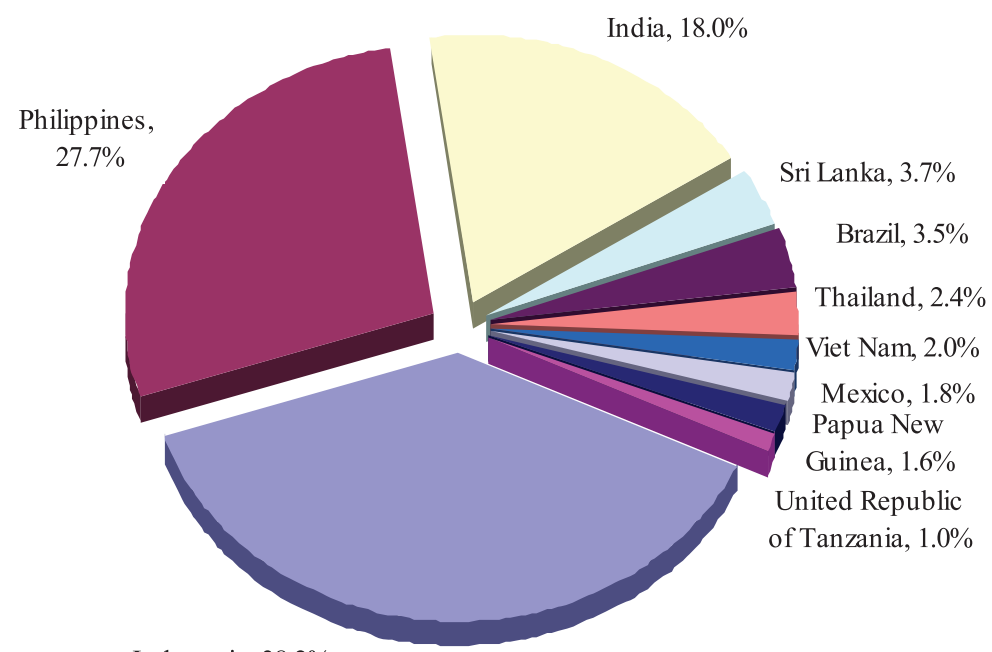

Indonesia, $38.2 \%$

FIGURE 7.4

Percentage of world coconut production by country in 2009. 


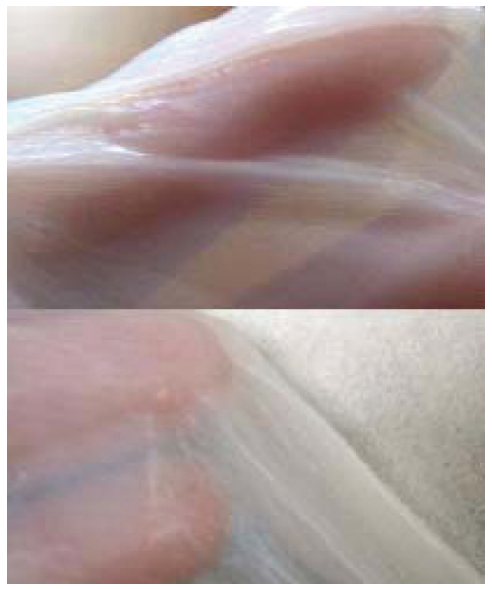

FIGURE 7.5

Comparison of the texture of facial masks made from nata de coco (top) and paper (bottom).

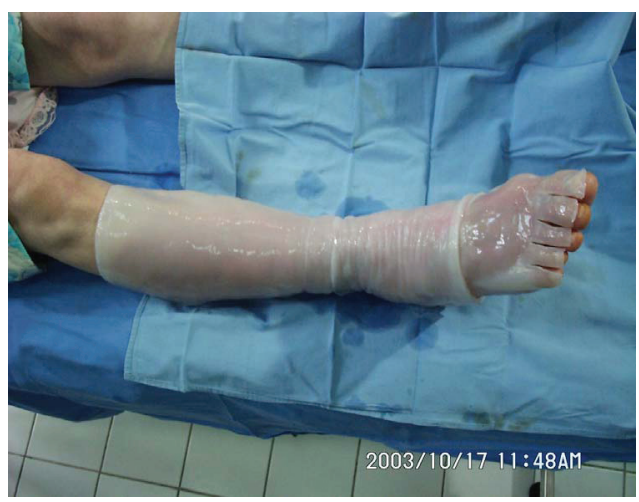

FIGURE 8.1

BNC dressing.

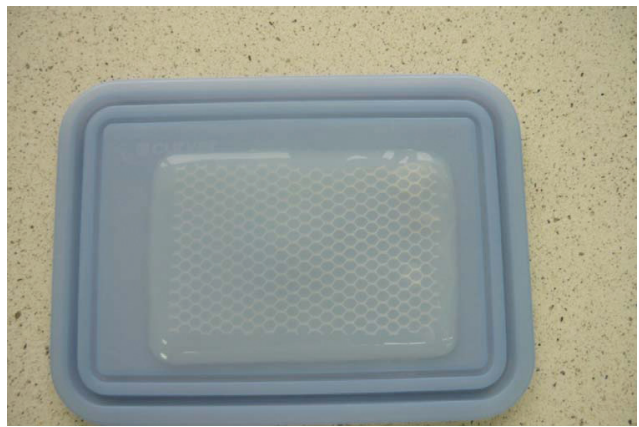

FIGURE 8.2

BNC-coated hernia mesh. 

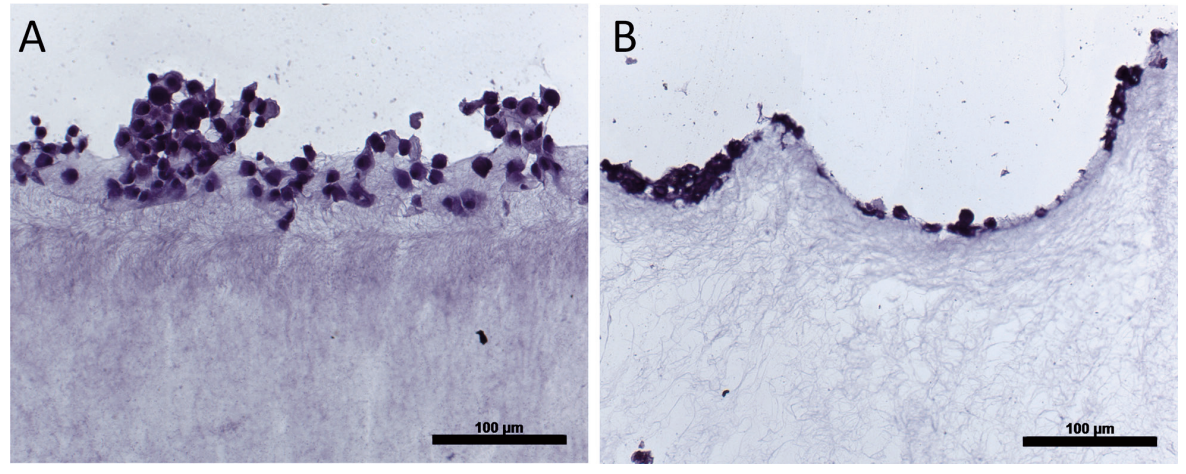

\section{FIGURE 9.1}

Histological cross-section of the lower (a) and upper (b) surface of a BNC hydrogel seeded with human chondrocytes in a transwell system with serum gradient for $24 \mathrm{~h}$. While the more porous lower surface enables cell ingrowth up to $70 \mu \mathrm{m}$, the upper surface restricts cell migration into the bulk staining with hematoxylin and eosin.

A

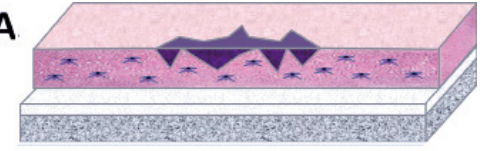

B

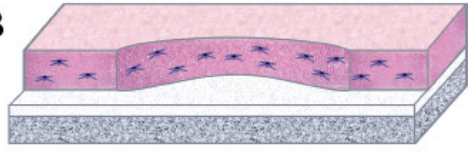

C

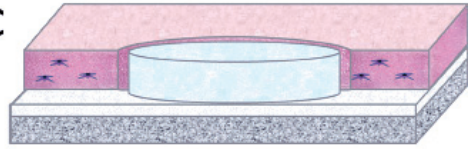

D

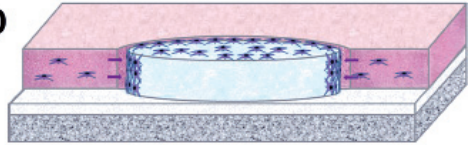

$\mathbf{E}$

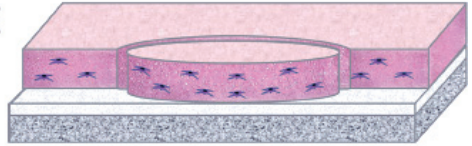

$\mathbf{F}$

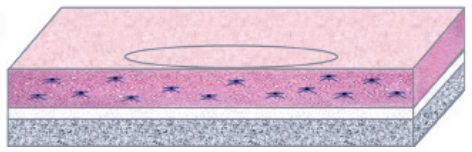

FIGURE 9.4

Principle of cartilage repair by use of a bioactive implant. Schematic representation of a chondral defect in the joint cartilage (a). After removing the damaged areas, the cartilage defect is filled with the preformed BNC implant. $(b, c)$ Cartilage and cartilage precursor cells migrate into this implant $(\mathrm{d}, \mathrm{e})$. The result of a complete colonization of the BNC inlay is $(\mathrm{f})$ a regenerated cartilage layer. 


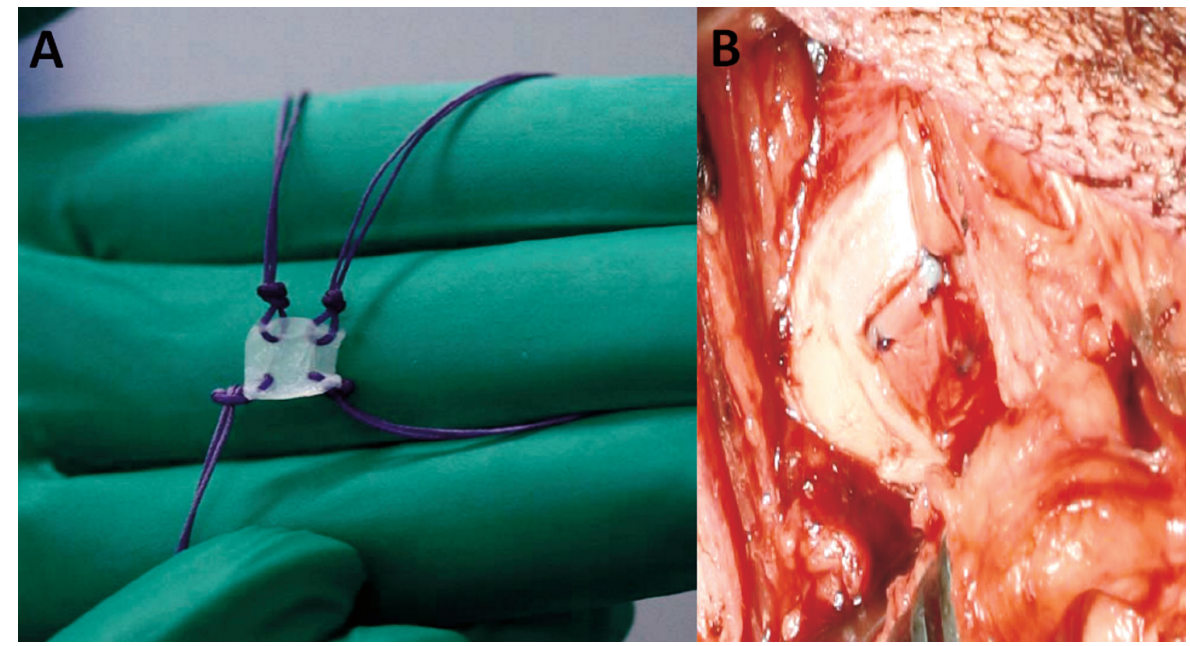

FIGURE 9.5

Photographs of BNC hydrogels designed for implantation in joint cartilage. (a) The implant after manual suturing to fix it in defined defects in the knee of a sheep (b).
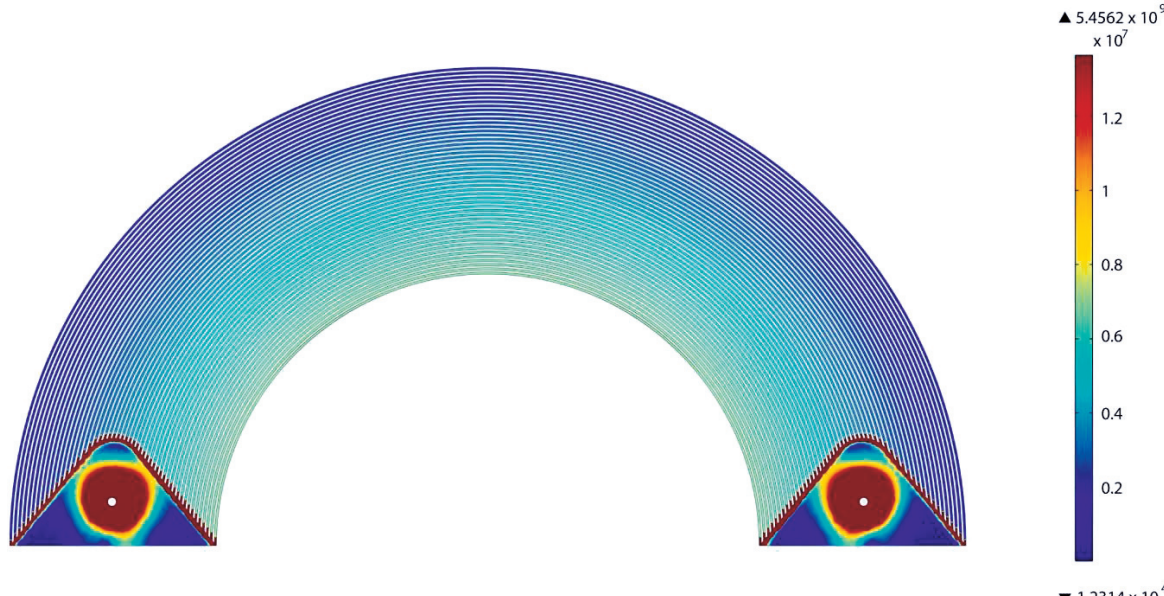

\section{FIGURE 10.4}

Meniscus design with channels $100 \mu \mathrm{m}$ wide separated by PDMS barriers that are $100 \mu \mathrm{m}$ thick. Gradient of the inner product of the electric field modeled at $5 \mathrm{~V}$ and $1 \mathrm{~Hz}$.
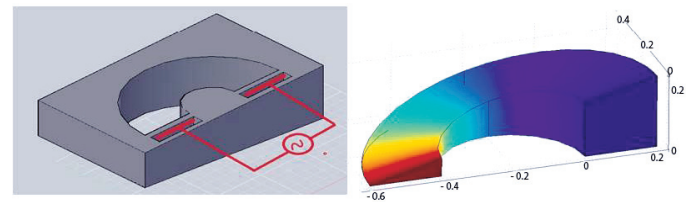

\section{FIGURE 10.5}

(Left) AutoCAD drawing of a prototype. (Right) COMSOL model of potential distribution fashioned using an exaggerated slope. 

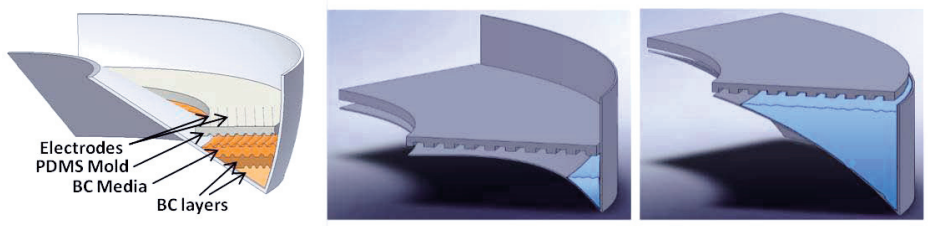

\section{FIGURE 10.7}

Three schematics illustrating the concept of depressing the curved portion of the silastic meniscus form overlaying the bacteria-rich media with the thin PDMS nanoconduit mold. Eventually enough media and growth will occur to be fully covered by the overlay.
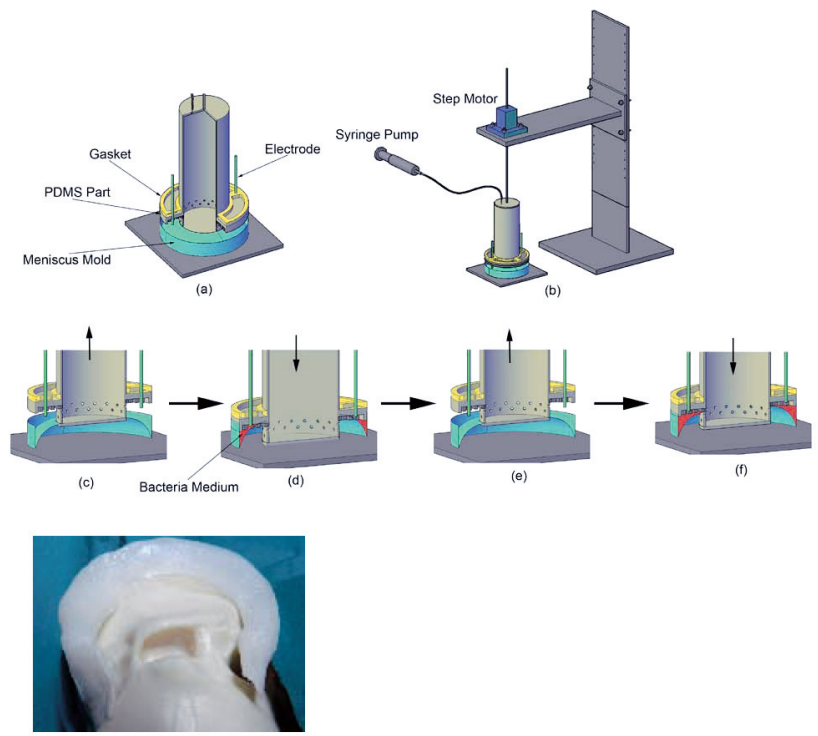

(g)

FIGURE 10.12

Microweaver bioreactor for biofabrication of meniscus implant and a prototype of a human BNC meniscus implant. Source: BC Genesis.

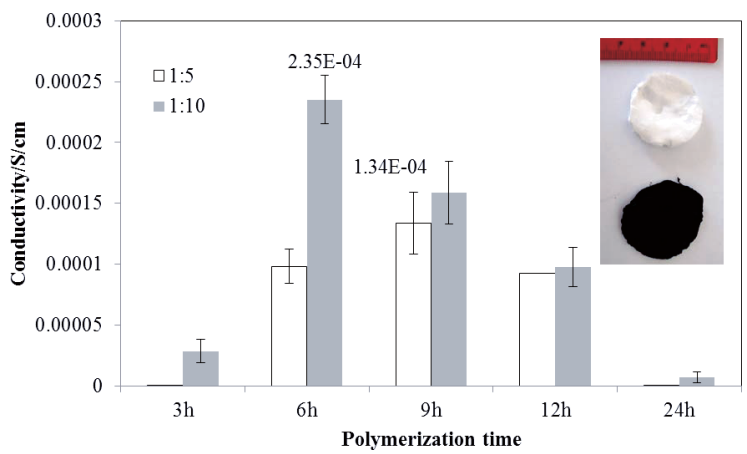

FIGURE 12.6

Effects of the reaction time on the electrical conductivity of BNC/PAni. $\mathrm{HCl}$ composites. 

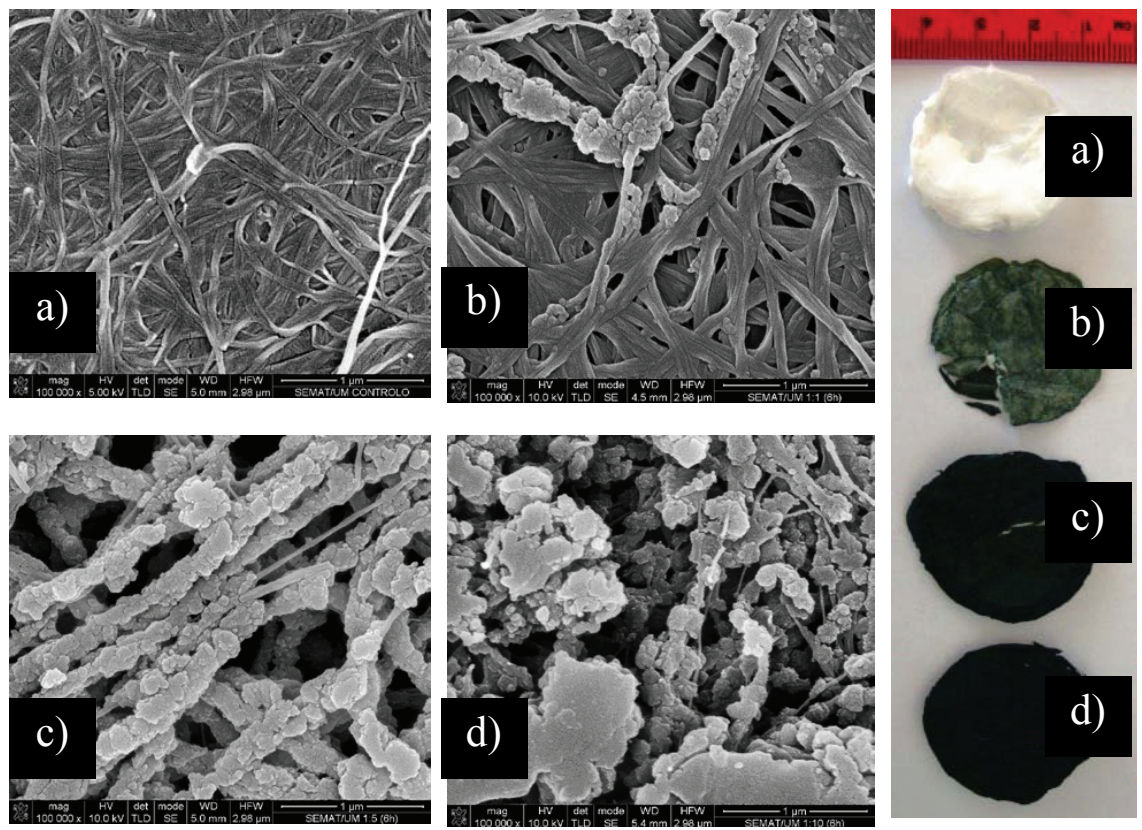

\section{FIGURE 12.8}

Scanning Electron Microscopy (SEM) with EDS X-ray analysis of BNC and BNC/PAni.HCl composites at different BNC:Ani molar proportions. (a) - BNC (Control); (b) - 1:1; (c) - 1:5; (d) $-1: 10$. All micrographs correspond to a polymerization time of $6 \mathrm{hr}$. Samples were coated with $\mathrm{Au}$. 


\section{Nematic Ordered Cellulose Templates}

\section{Tetsuo Kondo}

Graduate School of Bioresource and Bioenvironmental Sciences, Kyushu University, Hakozaki, Higashi-ku, Fukuoka, Japan

\section{CONTENTS}

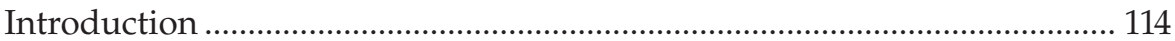

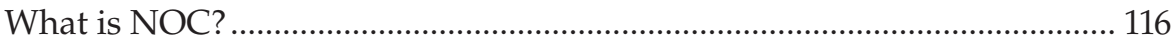

Various Nematic Ordered Templates and Modified NOC.............................. 124

Nematic Ordered Chitin and Chitin/Cellulose Blends .............................. 124

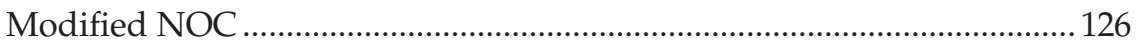

Exclusive Surface Properties of NOC and Its Unique Applications............ 128

Biodirected Epitaxial Nanodeposition on Molecular Tracks of

NOC Template

Critical Factors in Biodirected Epitaxial Nanodeposition on

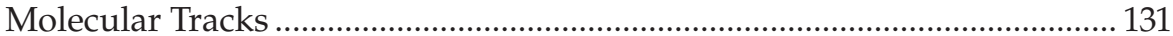

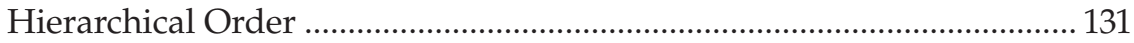

Epitaxial Deposition.................................................................................... 131

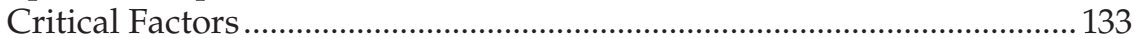

Regulated Patterns of Bacterial Movements Based on Their Secreted

Cellulose Nanofibers Interacting Interfacially with Ordered Chitin

Templates....

NOC Templates Mediating Order Patterned Deposition Accompanied by Synthesis of Calcium Phosphates as Biomimic Mineralization ............... 138

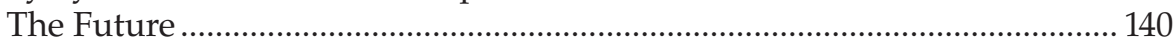

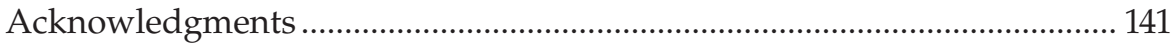

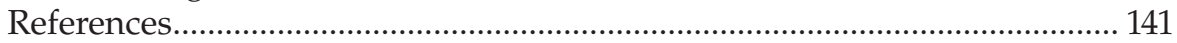

A unique form of $\beta$-glucan association, nematic ordered cellulose (NOC), has been developed that is molecularly ordered, yet noncrystalline. NOC has unique characteristics, in particular, its surface properties provide tracks or scaffolds for regulated movements and fiber production of Gluconacetobacter xylinus (formerly Acetobacter xylinum), which produces cellulose ribbon-like nanofibers $40-60 \mathrm{~nm}$ in width and moves due to the inverse force of the secretion of the fibers. Since the interaction 
between the produced microbial cellulose nanofibers and specific sites of the oriented molecules on the unique surface of NOC is very strong, such ordered cellulose can be used as a template for the construction of nanocomposites, and the growth direction of the secreted cellulose is controlled by the epitaxial deposition of the microfibrils. Accordingly, the template as a scaffold could regulate and establish deposition of the NOC-patterned three-dimensional (3D) architecture of cellulose ribbon-like nanofibers when the bacteria are incubated on it. This review attempts to reveal the exclusive structure-property relationship in order to extend the use of NOC film as a functional template for bacterium culture and biomimic mineralization. In addition, the other carbohydrate polymers with a variety of hierarchical nematic ordered states on various scales, so called nano-/microstructures, are described, which would allow development of new functional ordered scaffolds. The regulating factors for the rate of the microbial 3D construction are also discussed.

\section{Introduction}

Attempts to understand interfacial surface structures and the interaction of materials at the nanoscale have brought us into the field of nanotechnology (Drexler 1992). Microbiological systems combined with nanotechnology have become bionanotechnology (Taton 2002). Recent studies showing the unique interaction of biological systems with entirely synthetic molecular assemblies have prompted consideration of a new generation of approaches for controlled nanoassembly. More recently, we found a unique phenomenon that the molecular tracks of nematic ordered cellulose (NOC) (Kondo 2007; Kondo et al. 2001) substrate regulate the direction of fiber secretion of Gluconacetobacter xylinus (formerly Acetobacter xylinum), a Gram-negative bacterium that synthesizes and extrudes a cellulose nanofiber, resulting also in regulating the direction of the bacterial movement caused by the inverse force of the secretion. An epitaxial deposition of the nanofibers was induced due to the strong interaction between the nascent nanofibrils and influenced the order of molecules of the template (Kondo 2007; Kondo et al. 2002). This indicated that the conjunction of directed biosynthesis and the ordered fabrication from the nano to the micro scales could lead to new methodologies for the design of functional materials with desired nanostructures.

In the unique phenomenon, the most influential factor for the regulated bacterial movement was the surface of the nano-/microstructure of the template. The NOC substrate, where cellulose molecules are highly ordered but not assembled to form the crystalline state, was prepared by uniaxial stretching of water-swollen and fixed cellulose gel from dimethyl acetamide $/ \mathrm{LiCl}$ solution. Under wet conditions, the cellulose molecular chains tend to be oriented toward the stretching axis. Simultaneously the hydroxyl $(\mathrm{OH})$ groups 
at the C6 position that are equatorially bonded to the anhydroglucose unit in the cellulose molecules are automatically oriented at a certain angle against the surface and also aligned along the same axis as the tracks (Kondo 2007). On the contrary, the lateral order of the $\mathrm{OH}$ groups is not well coordinated because of the slipping molecular chains with each other in the NOC that was caused by the uniaxial stretching. Therefore the $\mathrm{OH}$ groups tend to be oriented as molecular tracks only in one direction across the entire NOC surface (Kondo 2007; Kondo et al. 2001). These ordering tracks of the $\mathrm{OH}$ groups on the NOC surface appear to induce epitaxial deposition of the biosynthesized cellulose nanofibers as they are secreted from G. xylinus along the same axis of the tracks in NOC. In addition, the regulated movement of the bacterium was not observed in the synthetic polymers having $\mathrm{OH}$ groups such as polyvinyl alcohol (Kondo et al. 2002). Therefore, when the nematic ordered state is prepared using other natural polymers, it is of interest and importance to know whether similar regulated bacterial movement may occur in order to extend this phenomenon into a methodology for fabrication of new types of functional bio-based materials.

In this chapter, after properties of NOC are described, the behavior of cellulose production of two types of G. xylinus bacteria (NQ-5, ATCC 53582; and AY-201, ATCC 23769) is at first shown in Hestrin-Schramm media on NOC templates (Hesse and Kondo 2005; Kondo 2007; Kondo et al. 2002). In addition, a modified distribution of carbon isotopes ( $\beta$-D-glucose- $-{ }^{13} \mathrm{C}_{6}$ ) as a precursor for the biological synthesis did not have any influence on the microgravitative effects of the building of cellulose.

Second, we employed chitin and cellulose-chitin blends as the components for the above templates instead of NOC (Kondo et al. 2012). Chitin is a repeating carbohydrate polymer of $(1,4)$-linked 2 -deoxy-2-acetamide- $\beta$-D-glucose. That is, as the primary structure, chitin has only a replacement of $\mathrm{OH}$ groups into acetamide groups at the $\mathrm{C} 2$ position of the anhydroglucose unit when compared with cellulose. However, when treated in the same manner as with NOC, the chitin exhibited a variety of different micro/nano hierarchical nematic ordered states (Kondo et al. 2004). Furthermore, the NOC-like ordered templates of cellulose/cellulose derivative blends were also employed for the bacterial cultures.

Third, as an extended concept for the NOC, attempts are discussed to propose a sort of biomimic mineralization of calcium phosphate using modified NOC templates. This process was initially mediated by the modified NOC template having a pair of roles in the ion supply sources and scaffolds for three-dimensional (3D) ordering architecture of the calcium phosphate as a biomineral (Higashi and Kondo 2012).

This chapter will provide unique surface properties of NOC templates for patterning of the bacterial movement and deposition of their secreted cellulose nanofibers, and patterned deposition of biominerals. Furthermore, the regulated fabrication could lead to the design of $3 \mathrm{D}$ architecture from the nano to the micro scales of functional bio-based materials with desired 
patterned nanostructures, which also opens pathways toward preparation of potentially versatile organic-inorganic as well as organic-organic order-patterned composites with less energy consumption.

\section{What is NOC?}

Prior to NOC, one needs to know the characteristic feature of a cellulose molecule (Figure 6.1): cellulose has an extended structure with a 2 screw axis composed of $\beta-1,4$ glucosidic linkages between anhydroglucose units. Thus it would be natural to accept the dimer called "cellobiose" as a repeating unit. The three kinds of $\mathrm{OH}$ groups within an anhydroglucose unit exhibit different polarities, which contribute to formation of various kinds of inter- and intramolecular hydrogen bonds, including secondary $\mathrm{OH}$ at the $\mathrm{C} 2$, secondary $\mathrm{OH}$ at the $\mathrm{C} 3$, and primary $\mathrm{OH}$ at the $\mathrm{C} 6$ position. In addition, all the $\mathrm{OH}$ groups are bonded to a glucopyranose ring equatorially. This causes the appearance of a hydrophilic site parallel to the ring plane. In contrast, the $\mathrm{CH}$ groups are bonded to a glucopyranose ring axially, causing a hydrophobic site perpendicular to the ring, as shown in the upper image of Figure 6.1. These effects lead to the formation of hydrogen bonds parallel to the glucopyranose ring, and to van der Waals interactions perpendicular to the ring.

Side view

Cellulose molecule $(=1,4-\beta$-D-glucan $)$

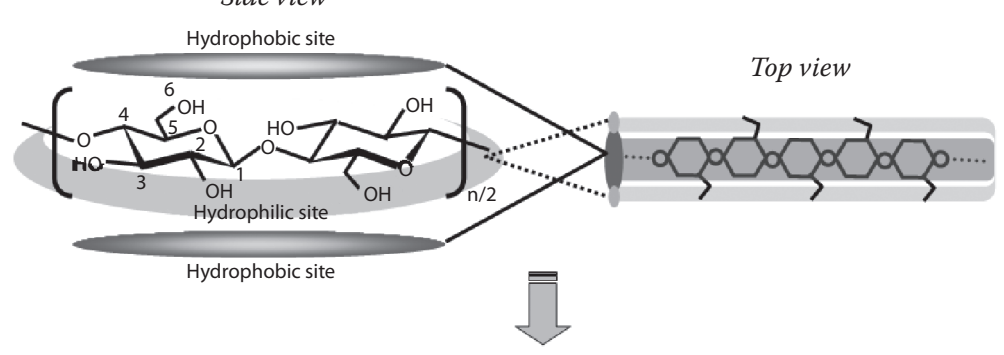

Surface properties of cellulose films depend on the tilted angle of anhydroglucose planes when the glucan chains are uniaxially aligned.
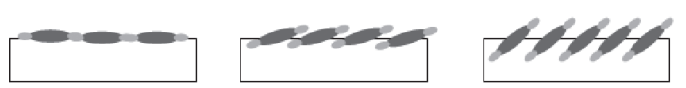

thith

Hinimiti

Hydrophobicity

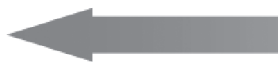

Hydrophilicity

\section{FIGURE 6.1}

The site-specific, amphiphilic nature of cellulose chemical structure leading to unique surface properties of cellulose films, depending on the tilted angle of the anhydroglucose planes when the glucan chains are uniaxially aligned. 
Another important feature of the $\mathrm{OH}$ groups is the type of hydroxymethyl conformation at the $\mathrm{C} 6$ position, because the conformation of $\mathrm{C} 5-\mathrm{C} 6$ and the resulting interactions including inter- and intramolecular hydrogen bonds in the cellulose structure may differ from that in crystallites, and also it is assumed to make up the extent of crystallization, as well as the final morphology of cellulose. In the noncrystalline regions, the rotational position of hydroxymethyl groups at the C6 position may be considered as indeterminate or totally nonoriented, which are not identical with those in the crystallites. Therefore it was important to confirm the type of $\mathrm{O} 6$ rotational position with respect to the $\mathrm{O} 5$ and $\mathrm{C} 4$ in a $\beta$-glucan chain by employing $\mathrm{CP} / \mathrm{MAS}$ ${ }^{13} \mathrm{C}$ NMR (Horii et al. 1983). The type of hydroxymethyl conformations is gauche-trans $(g t)$, trans-gauche $(t g)$, or gauche-gauche $(g g)$ at the C6 position in carbohydrates. As for the noncrystalline states, they are considered to be the $g g$ conformation (see Figure 6.2).

$\mathrm{NOC}$ is prepared by uniaxial stretching of water-swollen cellulose from the $\mathrm{N}, \mathrm{N}$-dimethylacetamide (DMAc)/ $\mathrm{LiCl}$ solution at a draw ratio of 2.0 to provide highly oriented $\beta$-1,4-glucan molecular chains of cellulose toward the stretching axis (Togawa and Kondo 1999). When the dissolved cellulose molecules in the $\mathrm{DMAc} / \mathrm{LiCl}$ system are self-aggregated in water vapor to form a gel, presumably with a minimum amount of restricted engagements among the hydrophobic sites of the ring previously described, it is stretched

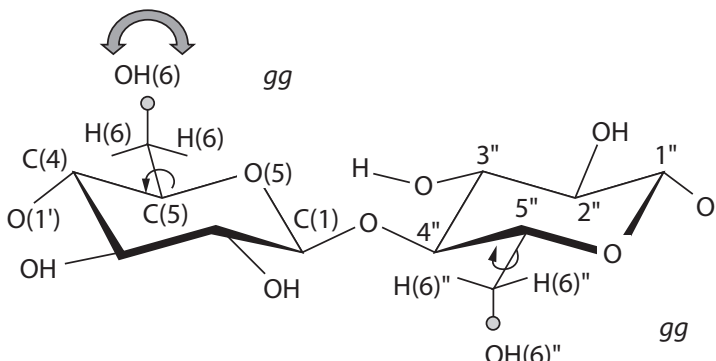

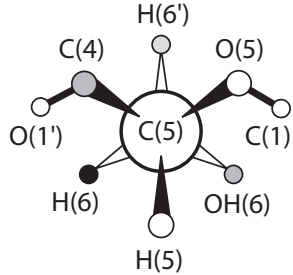

gt

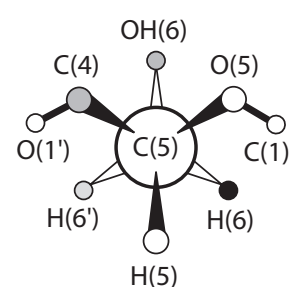

$g 9$

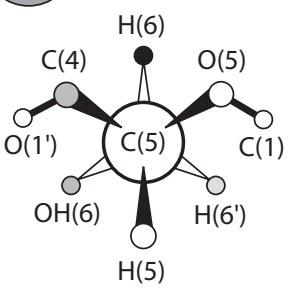

tg

\section{FIGURE 6.2}

Schematic diagram of the hydroxymethyl conformations at the C6 position, that is, the orientation of the C6-O6 bond, gauche-trans $(g t)$, trans-gauche $(t g)$, or gauche-gauche $(g g)$ with a cellobiose unit. 
to reach NOC. It should be noted that the drawability requires entanglements such as cross-linking, but they are supposed to be minimized. In the present DMAc/LiCl system for NOC preparation, however, slower coagulation using water vapor yielded stable noncrystalline cellulose. The key to maintaining the amorphous or noncrystalline state should be formation of small amounts of the 3D cross-linking network. When the cellulose solution spread on the petri dish is exposed to a saturated water vapor, it turns out to be a gel state with shrinking. The shrinkage is probably due to the interchain hydrogen bonds formed during the coagulation process of cellulose molecules into a sort of network structures. After the solvent was exchanged into water, a transparent water-swollen fixed gel of cellulose (WSC) is obtained. The WSC is composed of approximately $93 \mathrm{wt} \%$ water and $7 \mathrm{wt} \%$ cellulose. During drawing of the WSC, water retained inside the WSC appeared to bleed out from its surface. The exclusion of excess water from the drawn WSC gel may be attributed to adjoining the cellulose chains between the junction zones, as shown in Figures 6.3a and 6.3b. The junction zone in Figure 6.3a represents the strongly hydrogen-bonded area where even $\mathrm{D}_{2} \mathrm{O}$ vapor could not penetrate (Hishikawa et al. 1999). The zone probably plays a role as a cross-linking point in the WSC fixed gel of an initial state for NOC. After drying of the drawn WSC under a stressed state, it showed a decrease in thickness of more than $80 \%$ and shrinking in width of more than $60 \%$ when compared with the starting sizes of the WSC. The NOC films thus obtained exhibited almost perfect transparency, as much as the coagulated cellulose solution under water vapor atmosphere. This transparency suggests that the NOC should consists of noncrystalline regions.

Figure 6.3 also illustrates structural characteristics of NOC in relation to the situation of $\mathrm{OH}$ groups. When NOC is prepared by uniaxial stretching of WSC, the cellulose molecular chains tend to be oriented toward the stretching axis, as described already. Further, the hydroxymethyl groups at the $\mathrm{C} 6$ position of the anhydroglucose unit are vertically stuck against the surface, which indicates that the neighboring anhydroglucose ring planes are facing each other. Simultaneously the stuck $\mathrm{OH}$ groups in the individual molecular chains are also aligned like tracks along the stretching axis. In contrast, the lateral order of the $\mathrm{OH}$ groups among the neighboring chains is not well coordinated because of the slipped molecular chain situation with each other. Therefore the hydrophilic and polarized $\mathrm{OH}$ groups are totally oriented as molecular tracks only in the stretching direction across the entire NOC surface. Between the hydrophilic molecular tracks, the hydrophobic phase due to the anhydroglucose plane also appeared alternately, resulting in both hydrophilic and hydrophobic tracks next to each other across the NOC surface. The amphiphilic molecular tracks enhance the unique surface properties of NOC, as described later.

The NOC structure was shown by the high-resolution transmission electron microscopy (TEM) image. The NOC template with molecular ordering is shown in Figure 6.4 (Kondo 2007; Kondo et al., 2001). The high-resolution 
(a)

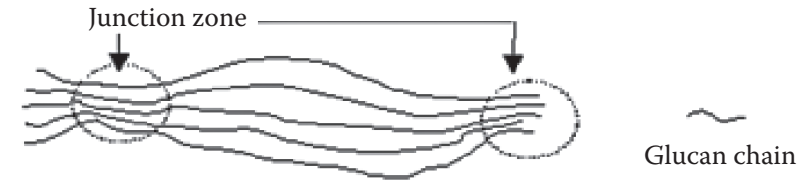

(b)

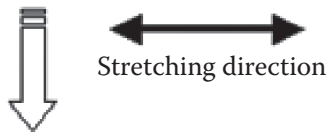

$$
\text { Junction zone }
$$

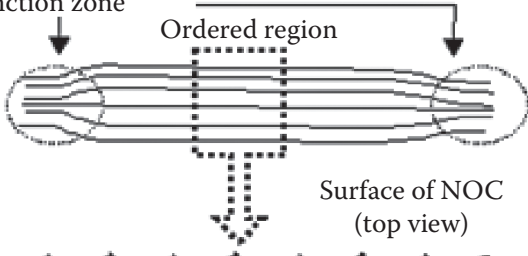

Glucan chain
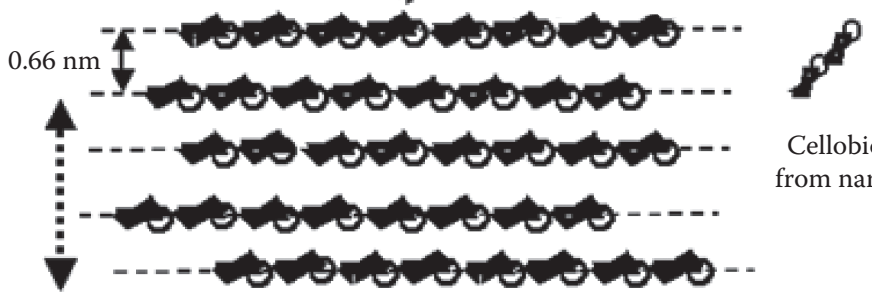

Cellobiose unit from narrow axis

Lateral direction

Glucan chain orientation

(=Stretching direction)

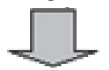

Site-specific amphiphilic NOC

OH hydropilic tracks ( =stretching direction)

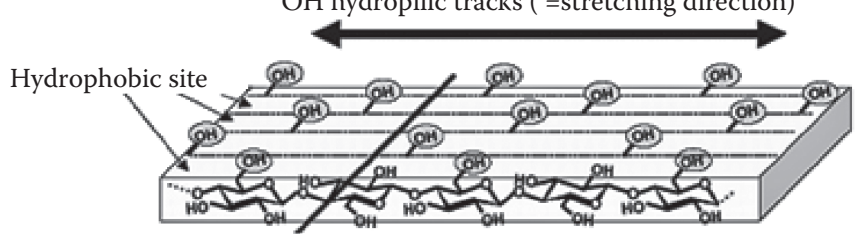

FIGURE 6.3

Schematics of NOC: (a) the initial state before stretching, (b) after stretching, the arrangement of cellulose molecules on the NOC surface.

TEM image shows a preferentially oriented direction on the surface. These observations confirmed the mean width of a single glucan chain corresponding to its known dimensions as viewed from the narrow axis of the anhydroglucose ring. The average chain width was $0.462 \mathrm{~nm}$ (standard deviation $\pm 0.0517 \mathrm{~nm}$ ). Thus the true width seems to be approximately $0.4-0.5 \mathrm{~nm}$ when taking into account the negative stain. The average distance between two parallel chains was $0.660 \mathrm{~nm}$ (standard deviation $\pm 0.068 \mathrm{~nm}$ ), which is larger 

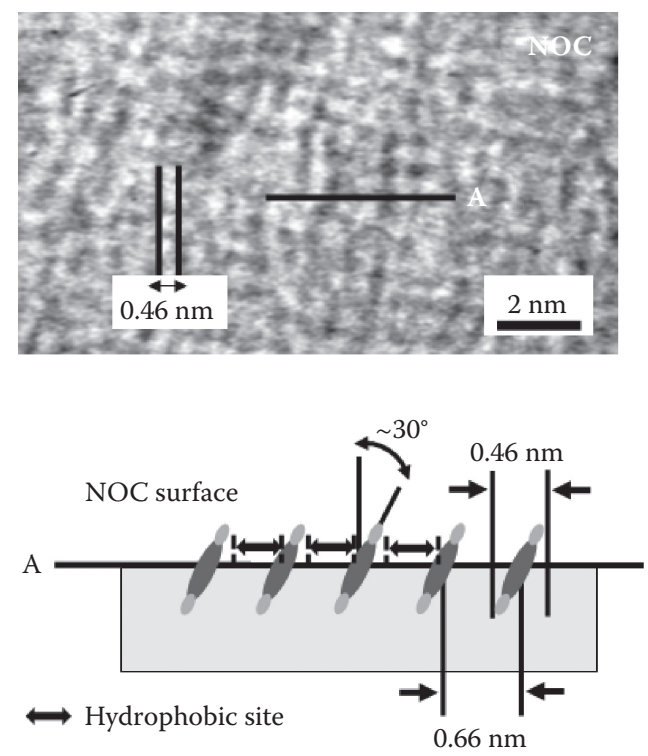

Tilted glucose ring planes

\section{FIGURE 6.4}

High-resolution TEM image of NOC, the molecular ordering template, together with the schematic cross-section image perpendicular (lateral direction) to the stretching direction indicating the tilted arrangement of glucan chains. The film was negatively stained by uranyl acetate and spans a region of the copper support grid. Note the individual glucan chains that are separated by an average distance of $0.66 \mathrm{~nm}$ (arrows). The arrangement of glucan chains is clearly resolved with a width of $0.46 \mathrm{~nm}$.

than values for any crystalline cellulose. The average width of $0.462 \mathrm{~nm}$ from the top view is wider than that of $0.45 \mathrm{~nm}$ of the narrow axis in the anhydroglucose ring predicted from the space-filling model. This suggests tilting of the glucose planes of a cellulose molecule with an angle of $29.3^{\circ}$ to the vertical axis against the surface of NOC, as shown in the bottom image of Figure 6.4. It should be noted that the static sessile contact angle of a drop of water on the NOC surface is approximately $72^{\circ}$, indicating it is fairly hydrophobic (E. Togawa and T. Kondo, unpublished data). Prior to stretching for NOC preparation, the dried WSC exhibits approximately $50^{\circ}$ as the contact angle by the sessile drop method. Thus the surface condition of it is totally altered by stretching to provide tilting of the glucose planes for exposure of the specific hydrophobic site, as shown in Figure 6.4. The hydrophobic phases due to the anhydroglucose plane appear alternately next to the hydrophilic $\mathrm{OH}$ tracks, resulting in unique amphiphilic molecular tracks.

Infrared spectroscopic analyses combined with other methods revealed characteristic features of NOC film templates (Hishikawa et al. 2010). The vapor-phase deuteration of the NOC film indicated that the amount of unexchangeable $\mathrm{OH}$ groups, $10.6 \%$, of the total $\mathrm{OH}$ groups corresponded to the 
highly engaged intermolecular hydrogen-bonded domains or the junction zone, as indicated in Figure 6.3a. In addition, polarized Fourier transform infrared spectroscopy (FTIR) combined with the vapor-phase deuteration was enabled to reveal orientation of the cellulose main chains to the stretching direction in both the crystalline domains and the noncrystalline regions of NOC films. The NOC film was also considered to have both the oriented main chains and the nonoriented $\mathrm{OH}$ groups as the side chain. This flexible state of the $\mathrm{OH}$ groups might further hinder the oriented crystallization in the NOC preparation process and yet induce the unique properties in the surface of the NOC film (Higashi and Kondo 2012; Kondo 2007; Kondo et al. 2002, 2012). In Figure 6.5a, the CP/MAS ${ }^{13}$ C NMR spectra for NOC samples, as well as amorphous (a noncrystalline state without any preferred orientation) cellulose prepared from cellulose- $\mathrm{SO}_{2}$-dimethylamine-dimethyl sulfoxide solution (Isogai and Atalla 1991) and CF11 cellulose powder (Whatman International Ltd.) are shown in the range from 50 to $80 \mathrm{ppm}$ where chemical shifts at the C6 position appear. The chemical shift of CF11 appears at $65 \mathrm{ppm}$, corresponding to $\mathrm{tg}$ conformation, indicating that CF11 is a native cellulose. Our NOC sample exhibits a broader signal similar to amorphous cellulose within the range of the type of the hydroxymethyl conformation, $g g$, which also supports our suggestion that NOC is noncrystalline even though it is well ordered.

The orientation parameter calculated from wide-angle X-ray diffraction (WAXD) photographs (Figure 6.5b) became 0.88, which indicated a high degree of orientation. However, the crystallinity did not significantly follow the increase of the orientation by stretching (Kondo et al. 2001). Simultaneous orientation and crystallization did not occur as is often seen with crystalline polymers (Ward 1997), which agrees with the infrared (IR) analyses of deuterated NOC samples based on the ratio of the remaining $\mathrm{OH}$ groups in the drawn films corresponding to the crystallinity index. The crystallinity was approximately $14.8 \%$ and $16.8 \%$ before and after stretching, respectively (Kondo et al. 2001; Togawa and Kondo 1999). IR spectra of deuterated samples also supported a low crystallinity of the film based on the ratio of the remaining $\mathrm{OH}$ groups in the drawn films that corresponds to the crystallinity index. Figure $6.5 \mathrm{~b}$ also shows WAXD intensity curves in both the equatorial and meridional directions of NOC (B) together with cellulose II fibers (A). In the equatorial diffraction of NOC, typical crystalline diffraction patterns representing cellulose II were not observed. The diffuseintensity equatorial profile for NOC indicated that it contains considerably more "amorphous" regions. The meridional scan of WAXD indicates the order along the stretching direction for NOC. Meridional intensities of cellulose are affected by the disorder of the neighboring chains, which is symmetrical for the chain axis. In general, cellulose polymorphs provide almost the same meridional patterns; that is, two strong distinct reflections of the (002) and (004) planes $\left(2 \theta=17.2^{\circ}, d=0.516 \mathrm{~nm} ; 2 \theta=34.7^{\circ}, d=0.259 \mathrm{~nm}\right.$, respectively). The present results demonstrate that NOC, which is highly ordered 

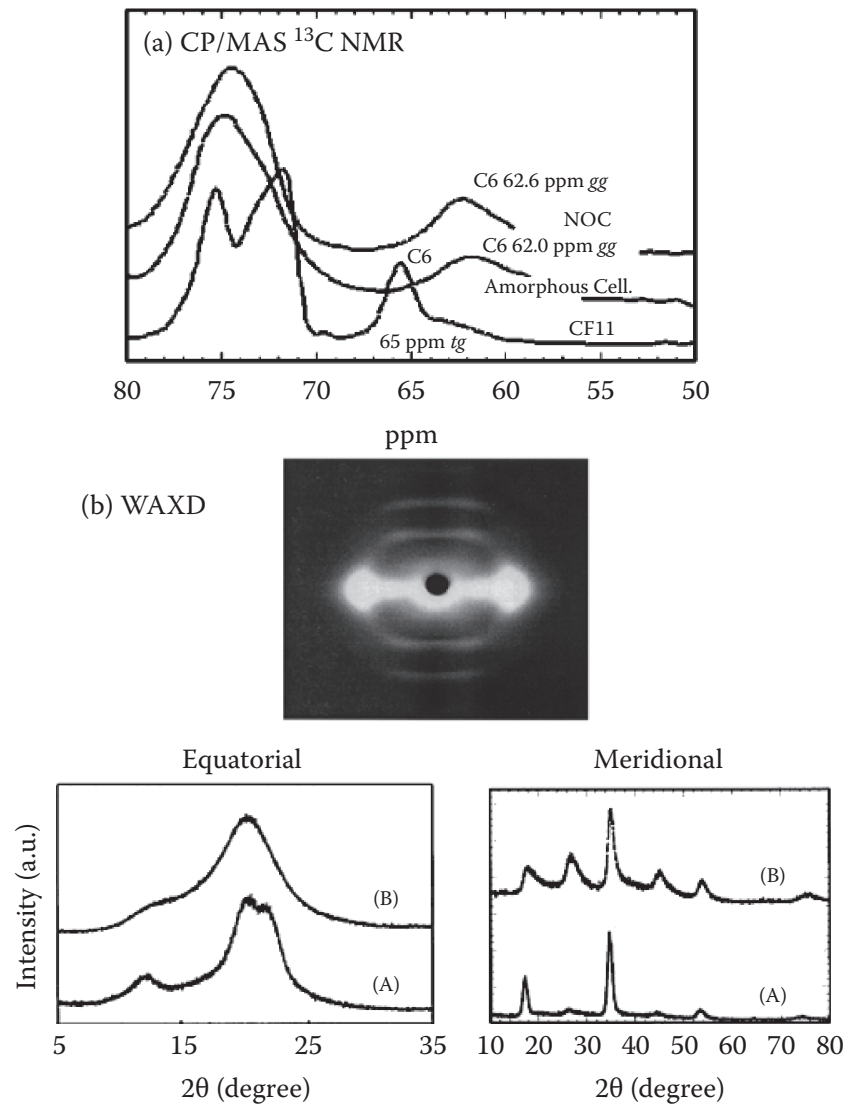

\section{FIGURE 6.5}

Characterization of NOC: (a) CP/MAS ${ }^{13} \mathrm{C}$ NMR spectra of NOC, amorphous cellulose, and native cellulose powder (Whatman CF11); (b) WAXD photograph of NOC together with equatorial (left) and meridional (right) intensity curves of the WAXD for cellulose II fibers (A) and NOC (B). The term "a.u." indicates arbitrary unit.

but noncrystalline, gives a totally different profile for the meridional scan, as shown in Figure 6.5b. More reflections were found in the meridional direction when compared with cellulose II crystals. The characteristics are considerable line broadening of the individual meridional reflection peaks in the WAXD profile of NOC. This indicates that the structure of the NOC film along the chain direction may have a certain disorder that causes the ordered, but noncrystalline regions (for more detail, see Kondo 2007). Thus we should consider the states of the structure for NOC to be ordered states that are neither crystalline nor amorphous.

The structural stability of NOC in water was examined. So far, most conventional noncrystalline cellulosics have no orientation in their supermolecular structure, and it is difficult to avoid recrystallization in water, even at 

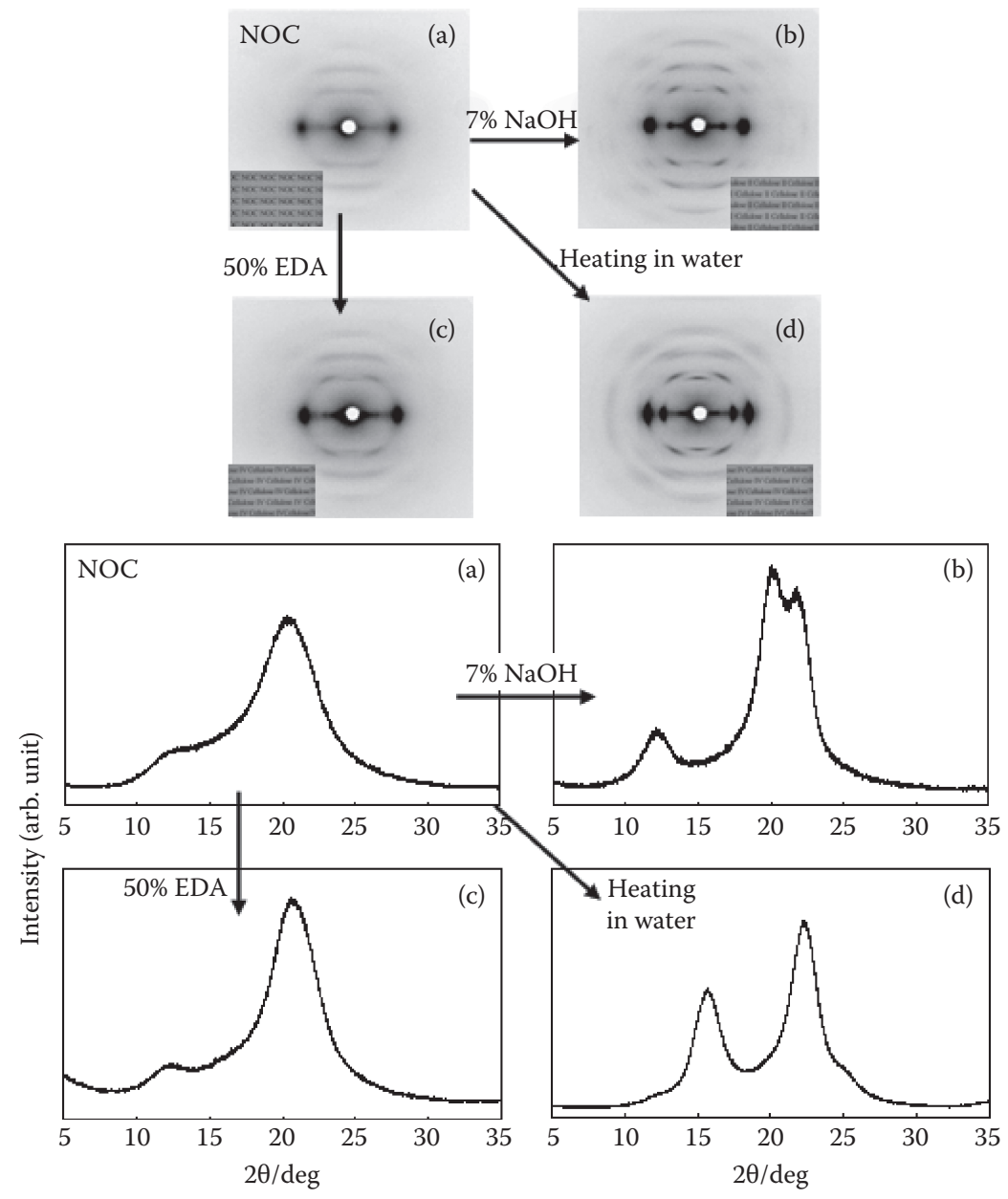

FIGURE 6.6

WAXD images (showing transparent films inserted) and profiles recorded on the imaging plate for NOC film and transformed NOC films: (a) NOC, (b) NOC treated with $7 \% \mathrm{NaOH}$, (c) NOC treated with $50 \%$ ethylenediamine (EDA), and (d) NOC heated in water at $180^{\circ} \mathrm{C}$ for $3 \mathrm{~h}$.

room temperature (Isogai and Atalla 1991). Interestingly, the noncrystalline character based on the specific chain orientation of NOC was retained after the treatment in water, even at $50^{\circ} \mathrm{C}$ for long periods.

Nematic ordered regions, as shown in Figure 6.3b, of the NOC films facilitate transformation into polymorphs. Figure 6.6 shows the X-ray fiber diagrams and photographs of cellulose polymorphs prepared from NOC films, together with the respective equatorial WAXD profiles of the X-ray photographs. Figure 6.6a shows the initial NOC structure as the starting material prior to the crystallization process. Each crystallized structure transformed from NOC (Figures 6.6b-6.6d) had an orientation of crystallites along the 
molecular orientation axis of the NOC. Moreover, the obtained films were all transparent, as the photographs in Figure 6.6 indicate. Thus the orientation of molecular chains and the transparency of NOC was maintained, even after crystallization took place. This indicates that the domain sizes of the crystallites were quite small even though they were well oriented along the same molecular orientation direction of the initial NOC film.

The equatorial WAXD patterns of the profiles in Figures $6.6 \mathrm{~b}-6.6 \mathrm{~d}$ were easily identified as cellulose II, III, and IV, respectively. The results suggest that transformation into each allomorph was completed. It should be noted that all of the transformations were accomplished under milder conditions than the conventional methods reported for the preparation of each allomorphs (Chidambareswaran et al. 1978; Fengel et al., 1995). For example, $16.5 \% \mathrm{NaOH}$ aqueous solution has thus far been required for transformation from cellulose I to cellulose II when cotton fibers are used as the starting cellulose (Fengel et al. 1995). Using NOC as the starting cellulose assembled state, $7 \% \mathrm{NaOH}$ was enough for the transformation. In particular, NOC yielded cellulose IV directly by a hydrothermal treatment at more than $100^{\circ} \mathrm{C}$ without the formation of cellulose III as an intermediate. In a similar manner, we employed cotton cellulose and a commercially available cellophane film as a reference of the starting cellulose to transform into polymorphs under the same conditions used for NOC. However, both cotton and cellophane could never be transformed into each allomorph. The facile transformations are attributed only to the supramolecular structure of NOC, which is well ordered but composed mainly of noncrystalline regions. In fact, the degrees of crystallinity of the three crystallized films from NOC were around $45 \%$. Moreover, our results have demonstrated that such NOC structure in a film material provides, under milder treatments, a facile direct transformation into the oriented films having highly aligned domains of cellulose II, III, and IV allomorphs along the initial orientation in the NOC.

\section{Various Nematic Ordered Templates and Modified NOC Nematic Ordered Chitin and Chitin/Cellulose Blends}

Figure 6.7 shows an image of the chitin template prepared in the same manner as the NOC template and observed with high-resolution TEM as well as atomic force microscopy (AFM) (Kondo et al. 2004, 2012). The two images exhibited the orientation of molecular chains, but they were not clear enough to be estimated. To obtain the distance between individual molecular chains, selected oriented areas (shown in the rectangles of Figure 6.7) of both high-resolution images were at first Fourier transformed to obtain the diffraction images. Then the manipulated diffraction images, as seen in the 


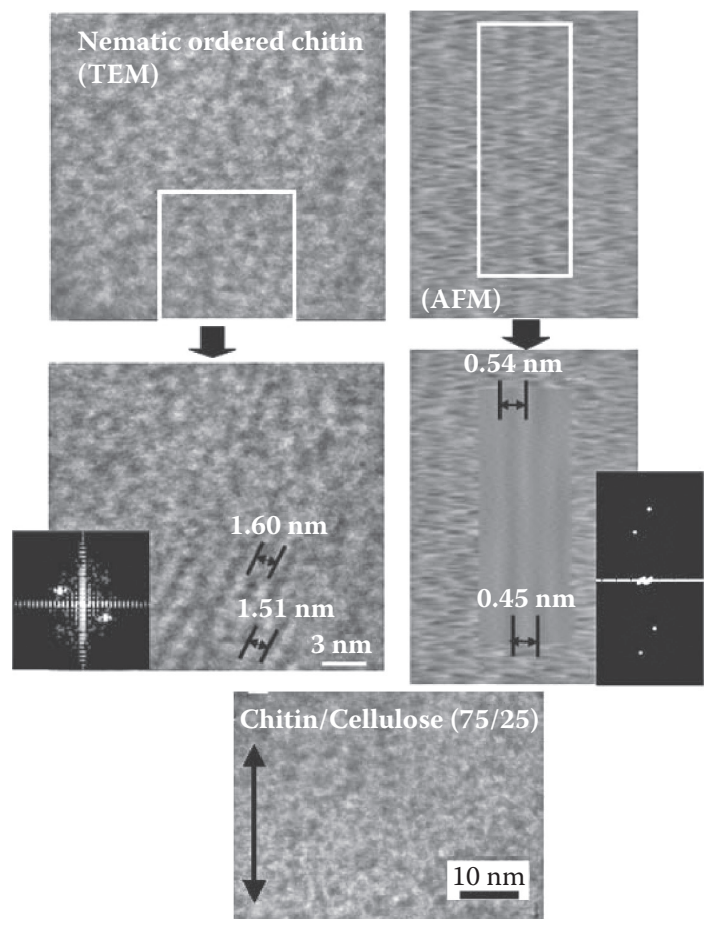

FIGURE 6.7

An image of the chitin template prepared in the same manner as the NOC template: (left upper) observed with high-resolution TEM; (left bottom) an image manipulated by inverse Fourier transformation; (right upper) observed with AFM; (right bottom) an image manipulated by inverse Fourier transformation. The inserted images (both the left and right) are the manipulated diffraction images prior to inverse Fourier transformation. The bottom image is a TEM image of the order structure of the stretched water-swollen gel films from a chitin-cellulose (75-25) blend at a drawing ratio of 2.0. The double arrows indicate the stretching direction. The sample specimens were negatively stained with $2 \%$ uranium acetate aqueous solution.

inserted images of Figure 6.7, were inversely Fourier transformed back to the noise-canceled images shown in the bottom images of Figure 6.7. In this way, the average distance between two parallel molecular chains in each image were provided for interpretation. Both observations indicate that the distance between individual chains ( $1.6 \mathrm{~nm}$ for TEM, $0.54 \mathrm{~nm}$ for AFM) is larger than the crystalline lattice constant of the $a$ axis of $\alpha$-chitin ([a] $0.474 \mathrm{~nm}$, [b] $1.886 \mathrm{~nm}$, [c] $1.032 \mathrm{~nm}, \alpha=\beta=\gamma=90^{\circ}$ ) (Minke and Blackwell 1978). Similar to NOC, in which the glucan chain dimensions are $0.4-0.5 \mathrm{~nm}$ viewed from its narrow axis, AFM images with a chain width of $0.45 \mathrm{~nm}$ (see Figure 6.7, right) clearly exhibit single molecular chains in the narrow axis on the surface of the chitin template. This means that nematic ordered chitin was achieved, similar to our previous work with cellulose (Kondo et al. 2001). High-resolution TEM images of molecular assembly in the stretched samples 
of a chitin-cellulose blend with a composition of 75-25, which was prepared in the same manner as NOC, also exhibit orientation of molecular aggregation, but are not resolved at the individual molecular chain scale (Kondo et al. 2004). The white dots or lines in the chitin-cellulose blend of Figure 6.7 indicate molecular chains or molecular aggregates. Some parts are well oriented parallel to the stretching direction and the molecular aggregates are entirely ordered along the stretching axis.

By image analyses of the TEM image in this figure, the average width and distance between two parallel lines were obtained as $1.38 \pm 0.18 \mathrm{~nm}$ and $1.65 \pm 0.27 \mathrm{~nm}$, respectively. The average line width is narrower than that from the TEM image $(1.51 \pm 0.27 \mathrm{~nm})$ of nematic ordered pure chitin, indicating that the intermolecular interaction between cellulose and chitin may be engaged. Possibly each molecular chain is facing each other against the surface by a hydrophobic interaction such as van der Waals forces. On the other hand, the average distance between two parallel lines was not significantly different between the two stretched films from pure chitin $(1.62 \pm 0.21 \mathrm{~nm})$ and the cellulose-chitin blend $(1.65 \pm 0.27 \mathrm{~nm})$. Therefore it is thought that the cellulose-chitin molecular aggregates in the stretched film are aligned similarly to nematic ordered pure chitin. In addition, these results indicate the presence of another nematic ordered state with a different scale (e.g., nano/micro hierarchical structures) in chitin and the blends with cellulose. (See the more detailed study using WAXD on nematic ordered pure chitin and the blends with cellulose in Kondo et al. [2004] and Kondo [2007].)

\section{Modified NOC}

The author proposes in the following sections a novel type of biomineralization process by developing a bifunctional cellulose template (Higashi and Kondo 2012). Namely, the NOC template has been modified into a bifunctional template having a pair of the functions required for biomineralization, resulting in oriented deposition of the biominerals as well as supplying anions in the synthesis. In this case, the new NOC template that is prepared to contain phosphate anions (termed p-NOC) is expected to mediate a unique order patterned deposition of calcium phosphates on the surface by reaction with calcium cations during immersion in a buffer solution.

The modification of NOC is performed as follows: A transparent waterswollen fixed gel of cellulose (WSC) is followed by a procedure using a $\mathrm{DMAc} / \mathrm{LiCl}$ solvent system, as previously described (Kondo et al. 2001; Togawa and Kondo 1999). The WSC is immersed in phosphate buffered saline (PBS; Sigma-Aldrich) for 1 week to prepare a WSC gel containing phosphate anions. Starting with the gel-like films containing phosphate anions, $\mathrm{p}-\mathrm{NOC}$ is obtained by uniaxial elongation at a draw ratio of more than 2.0 at room temperature. In the drawing process, the specimen is kept in a wet state by pouring the PBS on the surface of the film. The specimen is then air dried for $24 \mathrm{~h}$ 

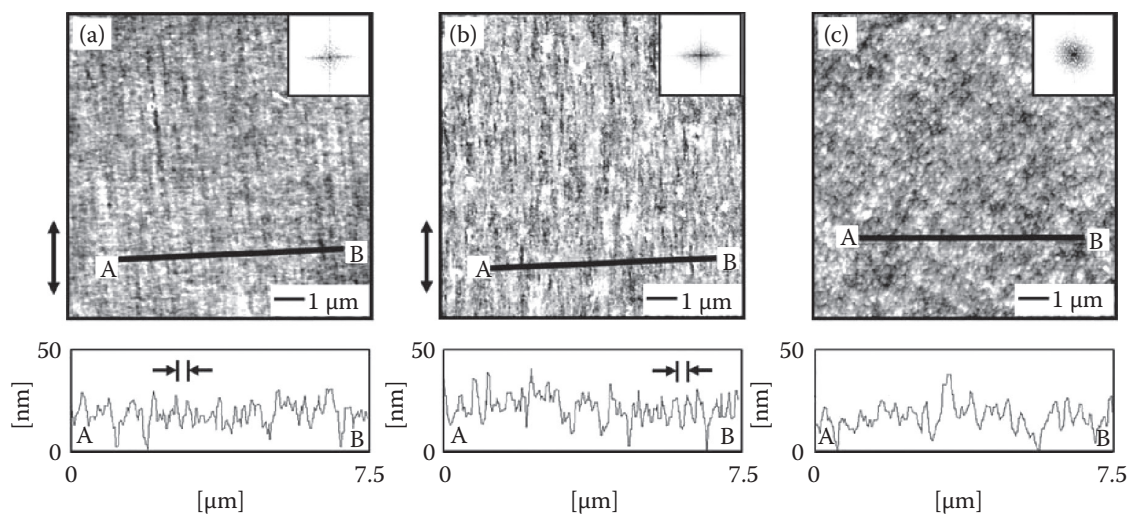

FIGURE 6.8

AFM images of (a) NOC template, (b) p-NOC template, and (c) nonordered cellulose template as a reference for the $\mathrm{p}-\mathrm{NOC}$ before the reaction with calcium cations. Insets in the upper right indicate the FFT images. The cross-section profiles along the perpendicular (A-B) are shown in the bottom of each figure. In ( $a$ and $b$ ), the average distances between two parallel lines were (a) approximately $0.17 \mu \mathrm{m}$ and (b) approximately $0.14 \mu \mathrm{m}$. The double arrows indicate the stretching direction.

before being vacuum dried at $25^{\circ} \mathrm{C}$ for more than $48 \mathrm{~h}$. The AFM images of the p-NOC surface containing phosphate anions together with the original NOC substrate are shown in Figure 6.8. As a reference, a nonordered cellulose template containing phosphate anions is also added (Higashi and Kondo 2012).

In a comparison of the images in Figures 6.8a and 6.8b, the AFM images of the height mode demonstrate that $\mathrm{p}-\mathrm{NOC}$ containing phosphate anions possesses a well-ordered surface morphology along the stretching direction, similar to the original NOC substrate. Furthermore, the polarized distribution of spots in the two-dimensional fast Fourier transform (2D-FFT) image in Figure $6.8 \mathrm{~b}$ confirms a high degree of uniaxial orientation on the surface of p-NOC. The average line width was calculated from the cross-section analyses of the height along the A-B line perpendicular to the stretching direction (see the bottom figures in Figure 6.8). The average distance between two parallel cellulose chains was $0.14 \pm 0.03 \mu \mathrm{m}$ in the $\mathrm{p}-\mathrm{NOC}$ template surface (Figure 6.8b). The line width was similar to that of the original NOC template, $0.17 \pm 0.06 \mu \mathrm{m}$, as shown in Figure 6.8a, indicating that a physical association of cellulose chains is not interfered even if phosphate anions existed in the $\mathrm{p}-\mathrm{NOC}$ template. In addition, the negatively polarized surface due to the tracks of $\mathrm{OH}$ groups along the molecular orientation is supposed to appear to some extent on the surface of the original NOC (Kondo 2007; Kondo et al. 2001, 2002). Thus, if phosphate anions are contained in NOC templates, then they are likely to be immobilized on the glucose planes only between the $\mathrm{OH}$ tracks in the surface that are negatively charged (see Figure 6.9). This means that anions with negative charges could be introduced as the second minus charge into an initially negatively charged film surface. Accordingly, 


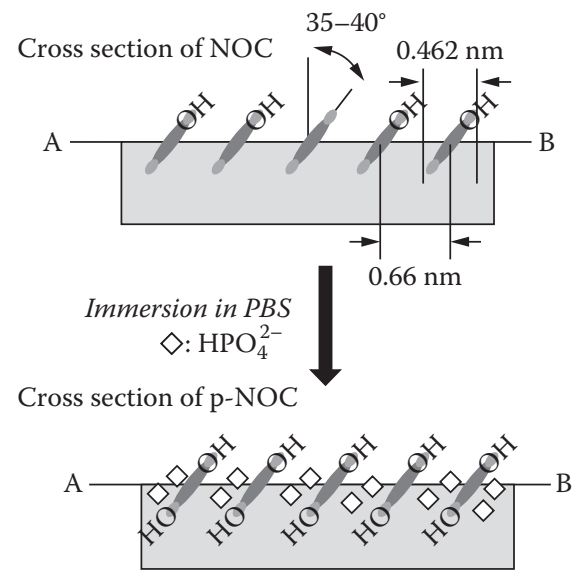

FIGURE 6.9

Schematics of NOC and p-NOC.

the phosphate anions are also supposed to be aligned along the molecular tracks in the newly prepared p-NOC template.

Without stretching of the gel containing phosphate anions, a nonordered structure was observed on the surface, which is confirmed by 2D-FFT images, as shown in Figure 6.8c. By uniaxial stretching of the cellulose gel, it is thought that the p-NOC will have a unique surface morphology similar to NOC that is totally different from the nonordered cellulose template containing phosphate anions. The root mean square (RMS) volumes were calculated from the obtained AFM height images to compare the surface roughness of each template. The RMS values for $\mathrm{p}-\mathrm{NOC}$ and nonstretched cellulose corresponded to $8.3 \mathrm{~nm}$ and $6.2 \mathrm{~nm}$, respectively. Thus p-NOC exhibited a rougher surface than cellulose without stretching.

\section{Exclusive Surface Properties of NOC and Its Unique Applications}

\section{Biodirected Epitaxial Nanodeposition on Molecular Tracks of NOC Template}

As already described, NOC exhibits several unique properties as a template having the exclusive surface of a nano-/microstructure. That is, the $\mathrm{OH}$ groups tend to be oriented as molecular tracks in only one direction across the entire NOC surface. In addition, as shown in Figure 6.4, the hydrophobic sites are supposed to appear alternately next to the $\mathrm{OH}$ tracks, resulting in amphiphilic tracks in one direction. 
The ordered amphiphilic tracks of hydrophilic $\mathrm{OH}$ groups and hydrophobic polarity in NOC can induce an epitaxial deposition of biosynthesized cellulose nanofibers secreted from the Gram-negative bacterium, G. xylinus, along the same axis of the tracks in NOC (Kondo et al. 2002). When active G. xylinus cells are transferred to the oriented surface, they synthesize cellulose nanofibers parallel to the molecular orientation of the substrate. This is evidenced by direct video imaging of the motion of the bacteria as they synthesize the cellulose nanofiber. The movement of G. xylinus in relation to cellulose biosynthesis was reported in 1976 by Brown et al. The cell movement (at a constant rate of $4.5 \mu \mathrm{m} / \mathrm{min}$ at $24^{\circ} \mathrm{C}$ ) is the result of an inverse force imposed by the directed polymerization and crystallization of the cellulose. It is also well known that the bacterium rotates on its own axis. The biodirected epitaxial nanodeposition of secreted fibers was studied over time and under varied conditions, including different substrates. Typically a time course analysis of an NOC template shows a bacterium moving in contact with a stretched NOC substrate, as shown in Figure 6.10. Basically the cells on the NOC template move straight along the molecular tracks. Midway through this series the cell "jumps off" of the oriented substrate and continues to secrete its cellulose nanofiber, but now in the form of a spiral or tunnel structure that is the normal pattern of formation when not in contact with any organized substrate (Thompson et al. 1988). When the interaction between the bacterium and

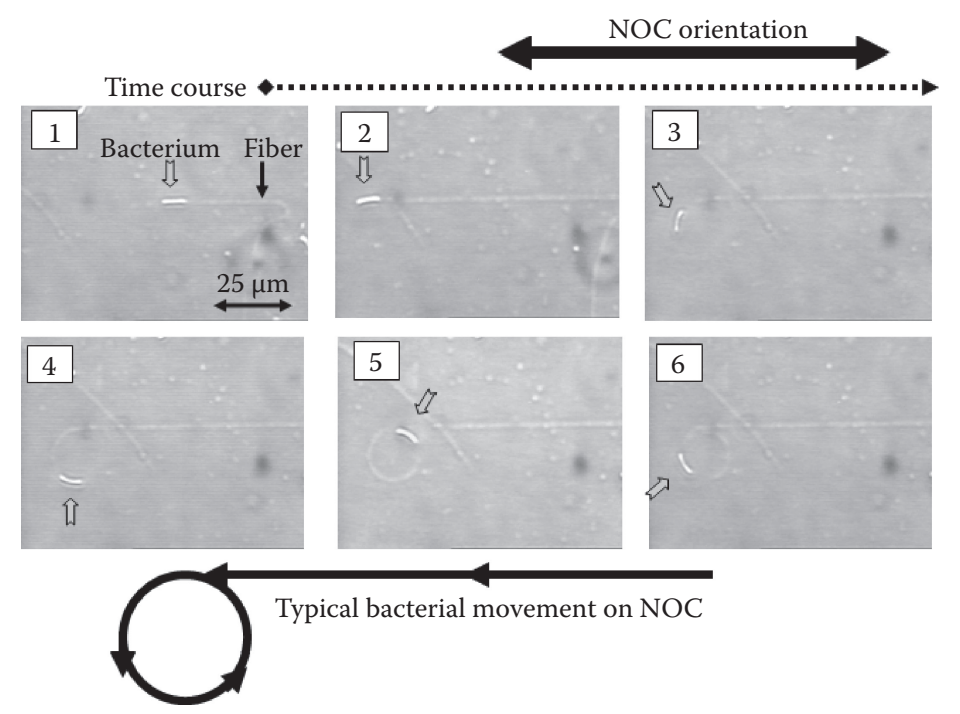

FIGURE 6.10

Successive images showing the motion of a bacterium as it secretes a cellulose ribbon using real-time video analysis. In (1), the bacterium is attached to and synthesizing its cellulose on the monomolecular rail track. In (2), the bacterium has jumped the track and is beginning to change its orientation. In (3-5), the bacterium is generating the first complete spiral. In (6), the bacterium is on the second rotation of a spiral. 
the surface of the NOC is strong enough, the bacterium follows the track of the molecular template. As the polymer orientation of the NOC is not necessarily perfect, the bacterium may jump off the track at a structural defect (Figure 6.10-3). After the bacterium leaves the track (Figures 6.10-3-10-6), two forces, the inverse force of the secretion and the close interaction of adjacent microfibrils, affect the bacterium, possibly resulting in the synthesis of a spiral ribbon of microfibrils. In addition, the bacterium begins to rotate on its own axis. This rotation is the visible result of ribbon twisting, which occurs to relieve strain induced by the absence of interaction with the substrate. When the ribbon is assembled in direct contact with the oriented molecular NOC substrate, ribbon twisting is prevented, thus suggesting a control of this oriented solid surface over the final physical interaction of polymer chains immediately after synthesis and during the early stages of crystallization.

Ribbon interaction is more clearly shown by field emission scanning electron microscopy (FE-SEM) in Figures 6.11A and 6.11B. In this experiment, the motion of the same sample is observed using light microscopy and then the sample is prepared for FE-SEM observations. In Figures 6.10a and 6.10b the time course sequence demonstrates an intense, directed nanodeposition that is exclusively linear and without cell rotation as the bacterium follows the track. This is confirmed by the ribbon structure shown by the FE-SEM images in Figures 6.11A and 6.11B, which reveal no twisting and a more perfect alignment with the substrate. Likewise, when the bacterium "jumps off" the track, the cellulose ribbon is twisted (data not shown). Figures 6.11c-6.11f indicate the active Gluconacetobacter cells cultured on the oriented surface
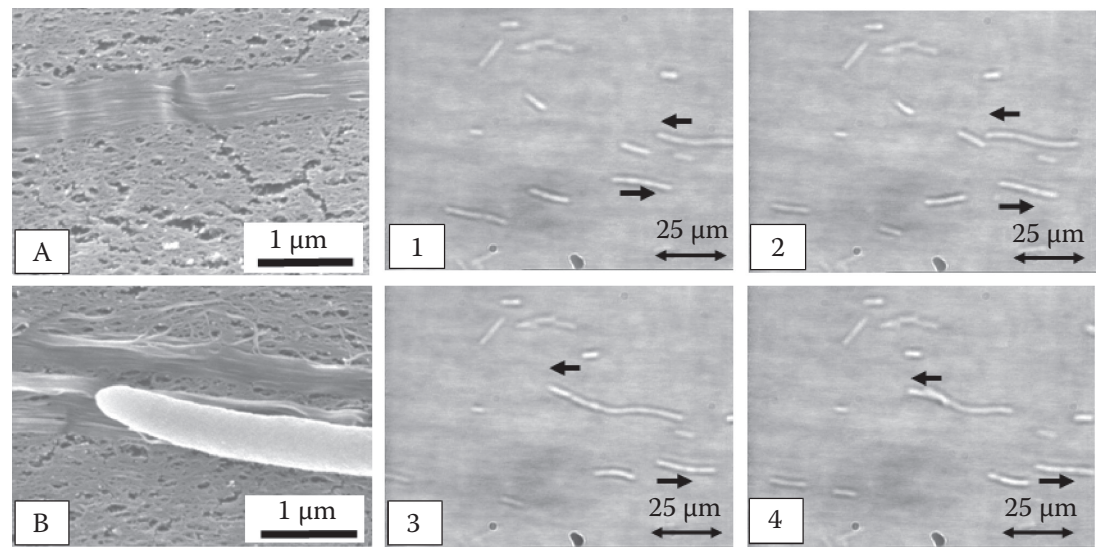

FIGURE 6.11

FE-SEM images of the cellulose nanofiber deposition process. (A, B) Examples of bacteria synthesizing cellulose fibers on the oriented molecular track of NOC. (A) Demonstrates the tight association between the molecular track and the cellulose fiber. In (B), a bacterium shows a flat fiber immediately behind its site of synthesis. (1-4) Successive images showing the motion of a bacterium on a nematic ordered blended film of cellulose and cellulose acetate that has been unixially stretched in the same manner as NOC. 
of a blend of cellulose and O-methylcellulose with DS $1.6(50 / 50 \mathrm{w} / \mathrm{w})$ that has been unixially stretched in the same manner for NOC. The bacteria also tend to follow the molecular tracks on the surface of the oriented cellulosic blend template, similar to the NOC case. In addition, the bacteria exhibit similar linear movements along the molecular tracks on NOC/cellulose acetate (DS 1.7) templates as the NOC. That is, the NOC blends as well as NOC are supposed to show similar unique properties as templates that direct the bacterial movement. When Gluconacetobacter cells are placed on an agar surface as a control, their interaction seems to be reduced, and twisted nanofibers are always deposited, also in a random fashion. It should be noted that this regulated movement of the bacterium is not observed in the synthetic polymers having $\mathrm{OH}$ groups, such as polyvinyl alcohol, but only in the nematic ordered states of carbohydrate polymers described earlier (Kondo et al. 2002).

\section{Critical Factors in Biodirected Epitaxial Nanodeposition on Molecular Tracks}

\section{Hierarchical Order}

Figures 6.12a-b show AFMimages beforeand afterincubation of Gluconacetobacter cells showing fabrication of a 3D architecture along the molecular tracks of NOC as a scaffold. After culturing for $10 \mathrm{~h}$ (Figure 6.12b), the height increased by 10 times, to approximately $350 \mathrm{~nm}$, selectively in the stripes on the NOC. This is evidence of the directed close contact between the secreted nanofibers and the molecular tracks on NOC templates. Moreover, the elastic modulus increased slightly from $6.2 \pm 0.1$ GPa to $6.9 \pm 0.0$ GPa by patterned fabrication with the secreted cellulose nanofibers on the NOC surface. Presumably the change is mostly influenced by the sufficient strength of NOC as a scaffold when compared with the deposited nanofibers on the above. Here one can observe that the additional layers secreted over time continue to mimic the original orientation initiated in the first deposited layer (e.g., the epitaxial deposition).

\section{Epitaxial Deposition}

The adhesion occurs between biosynthesized cellulose microfibrils and the $\mathrm{C} 6$ primary $\mathrm{OH}$ groups and/or hydrophobic tracks described previously. The important factor for controlling bacterial movements may be close contact and strong interaction between the molecular tracks on NOC and the newly synthesized cellulose microfibrils. A schematic explaining this concept is shown in Figure 6.13. Normally G. xylinus secretes a cellulose nanofiber that comprises a microfibril approximately $3.5 \mathrm{~nm}$ in width, as shown in Figure 6.13 (left). The microfibrils from the subunit of terminal complexes 

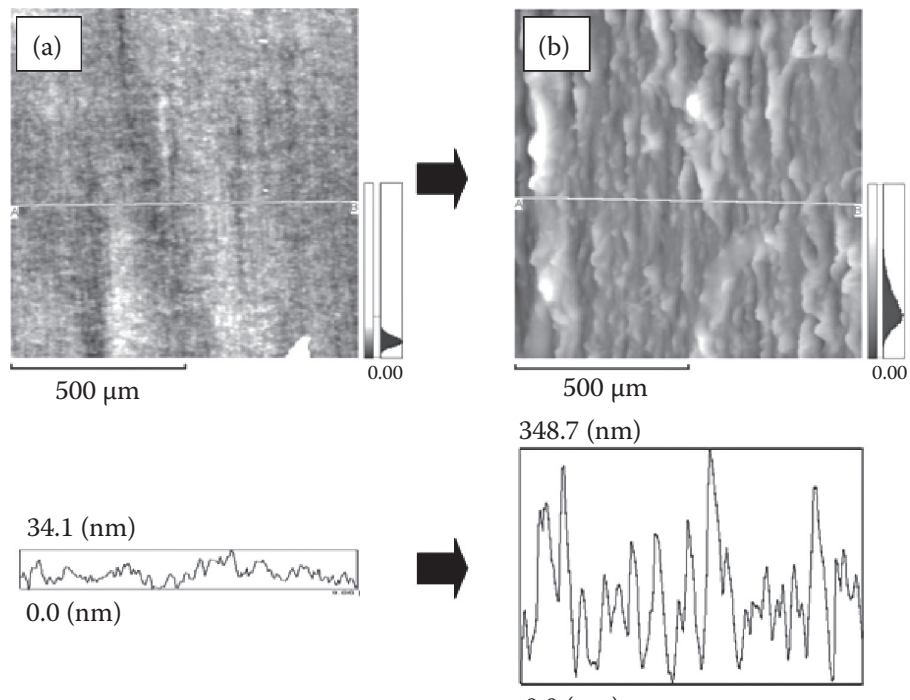

$0.0(\mathrm{~nm})$

\section{FIGURE 6.12}

AFM images (a) before and (b) after incubation of G. xylinus on NOC for $10 \mathrm{~h}$ in Hestrin-Schramm media.

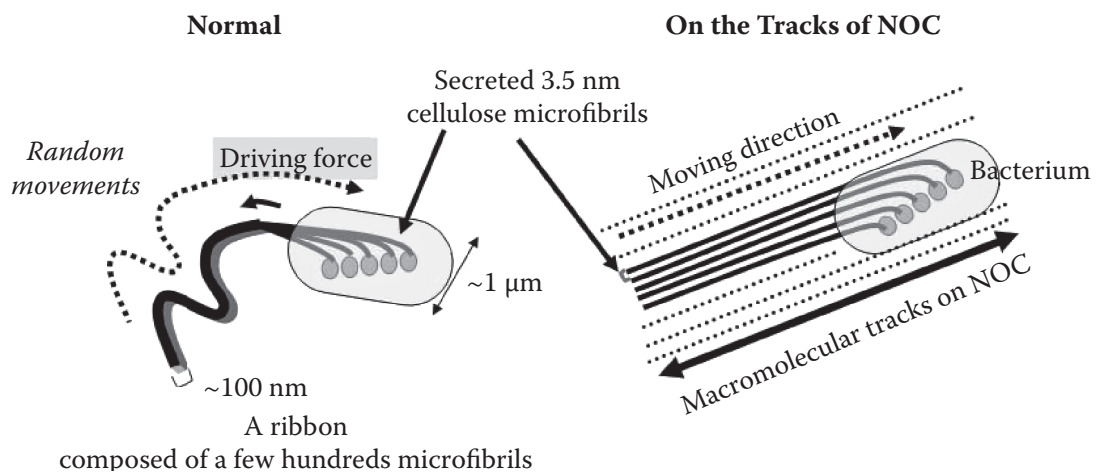

\section{FIGURE 6.13}

Schematics of self-assembly of cellulose microfibrils secreted from G. xylinus in normal fashion (left) and on the tracks of NOC templates (right). The circles indicate cellulose-synthesizing enzyme subunits linearly arranged across the cell body.

are assembled by interfacial interactions, including hydrogen bonding and van der Waals forces, between the component molecules, resulting in the formation of a ribbon-like cellulose nanofiber approximately $50 \mathrm{~nm}$ in width and $10 \mathrm{~nm}$ in thickness (Tokoh et al. 1998). In contrast, a cellulose microfibril would be synthesized along the molecular track on the NOC by the strong adhesive effect (Figure 6.13, right). This epitaxial deposition is clearly confirmed by the FE-SEM image of the NOC-altered cellulose ribbons with a 
width of $500 \mathrm{~nm}$ with splayed microfibrils $7-8 \mathrm{~nm}$ apart (see Figure 6.11A and 6.11B) (Kondo et al. 2002).

The amphiphilic molecular tracks on NOC allow close contact with the glucan chains of microfibrils as they are being secreted and crystallized. Thus, in theory, this interaction is predicted to alter the self-assembly of glucan chains into the crystalline microfibril (Benziman et al. 1980; Haigler et al. 1980, 1982; Tokoh et al. 1998; Yamamoto et al. 1996). In fact, alteration of the aggregation of cellulose microfibrils on NOC was demonstrated as described earlier. Such interactions with the substrate alter production of the cellulose ribbon and also the motion of the bacterium, and provide support for the epitaxial nature of cellulose deposition on NOC. Thus nanoscale interactions induce microscale structural changes. Such interactions could be used on a larger scale to selectively modify biopolymers for many applications.

\section{Critical Factors}

The adhesion for epitaxial growth of microfibril deposition requires several different critical factors relating to molecular "order" of the substrate. This order is represented by three factors: molecular chain orientation, specific polymer chain conformation, and O6 rotational position with respect to O5 and $C 4$ in a $\beta$-glucan chain. The importance of molecular chain orientation is supported by the observation that unstretched NOC does not cause epitaxial deposition. As already described, we believe the C6 hydroxymethyl conformation of NOC is also of importance. Its role is like an anchor for molecular tracks to direct the epitaxial deposition of cellulose nanofibers. The conformation of $\mathrm{C} 6 \mathrm{OH}$ groups is believed to contribute to the crystalline forms, depending on either $g t$ (cellulose II) or $t g$ (native cellulose). When an NOC with the $g g$ hydroxymethyl conformation is crystallized, it is converted to ordered films containing cellulose II crystalline forms with $g t$ hydroxymethyl conformation (Togawa and Kondo 2007). When Gluconacetobacter is incubated on the crystallized NOC, there is no adhesion of the secreted microbial cellulose nanofibers. The crystallized films do not exhibit the molecular track effect to direct bacterial movements any longer. The same lack of adhesion is observed with substrates prepared from native crystalline cellulose I from Valonia (data not shown). In the crystalline samples, the specific polymer chain conformation is changed, and the rotational conformation at $\mathrm{C} 6$ is gt in crystallized NOC and $t g$ in Valonia cellulose. Considering this, we conclude that the role of primary $\mathrm{OH}$ groups at the $\mathrm{C} 6$ position is greater than those of $\mathrm{OH}$ groups at the $\mathrm{C}(2)$ and $\mathrm{C}(3)$ positions. In addition, it is also noted that not only the $\mathrm{OH}$ tracks but also the hydrophobic tracks are supposed to appear on the NOC surface, and thus the amphiphilic molecular tracks are critical for regulating the epitaxial deposition of the cellulose nanofibers.

Ordered noncrystalline cellulose (NOC) and related blends have been proven to work very well as templates. Surfaces of nematic ordered chitin also show the adhesive effect, but in an unusual fashion. 
The rate and direction of the movement correspond to those of the fiber production. The fibers were reported to be produced at a rate of $2 \mu \mathrm{m} / \mathrm{min}$ at $25^{\circ} \mathrm{C}$ (Brown et al. 1976). However, G. xylinus produced the fiber faster on NOC templates, at a rate of $4.5 \mu \mathrm{m} / \mathrm{min}$ at $24^{\circ} \mathrm{C}$. This gap is thought to be due to the strength of the interaction between the biosynthesized fiber and the NOC surface. It is also indicated that the NOC surface promoted the secretion rate of the fibers.

The optimal condition on the templates containing the additives for the culture medium was studied in order to understand the factors influencing the rate of movement of G. xylinus accompanied with the secretion of a nanofiber (Tomita and Kondo 2009). The template having a molecular orientation at a higher draw ratio was more effective at enhancing the moving rate. Further, the moving rate may depend on the constituents of the template. The additive, CMC, with a lower DP, was effective at preventing secreted cellulose microfibrils from self-assembling, which resulted in an increase in the rate of movement on the agar substrate. In fact, the Hestrin-Schramm (1954) medium containing 1\% CMC with a low DP enhanced the moving rate of the bacterium cultured on the template without orientation. Furthermore, on an NOC template, the Hestrin-Schramm medium containing 1\% CMC with a high DP was more effective at increasing in the moving rate. Presumably alignment of the CMC molecules onto the oriented cellulose molecules in the NOC by a strong interaction caused the increase in the moving rate. From the results, the conditions for increasing the moving rate are proposed to employ a flat drawn template with an orientation that can engage with other materials by a strong interaction. Further, supplying an additive to the medium, which prevents self-assembly of secreted cellulose microfibrils, is also effective. Thus understanding how to prevent self-assembly of the secreted microfibrils on the oriented templates could be the easiest way to find an optimal condition for enhancing the fiber production. More importantly, the regulated movement of the bacteria due to the ordered surface of the NOC may trigger the development of a 3D structure of hierarchical architecture from the nano to the micro levels. Therefore, we could regulate the 3D architecture of materials using nanofiber secreted by G. xylinus as a building block.

\section{Regulated Patterns of Bacterial Movements Based on Their Secreted Cellulose Nanofibers Interacting Interfacially with Ordered Chitin Templates}

When active G. xylinus cells are transferred to the nematic ordered chitin surface, they synthesize cellulose nanofibers (i.e., cellulose ribbons) that are not parallel to the molecular orientation of the substrate, unlike the secreted 

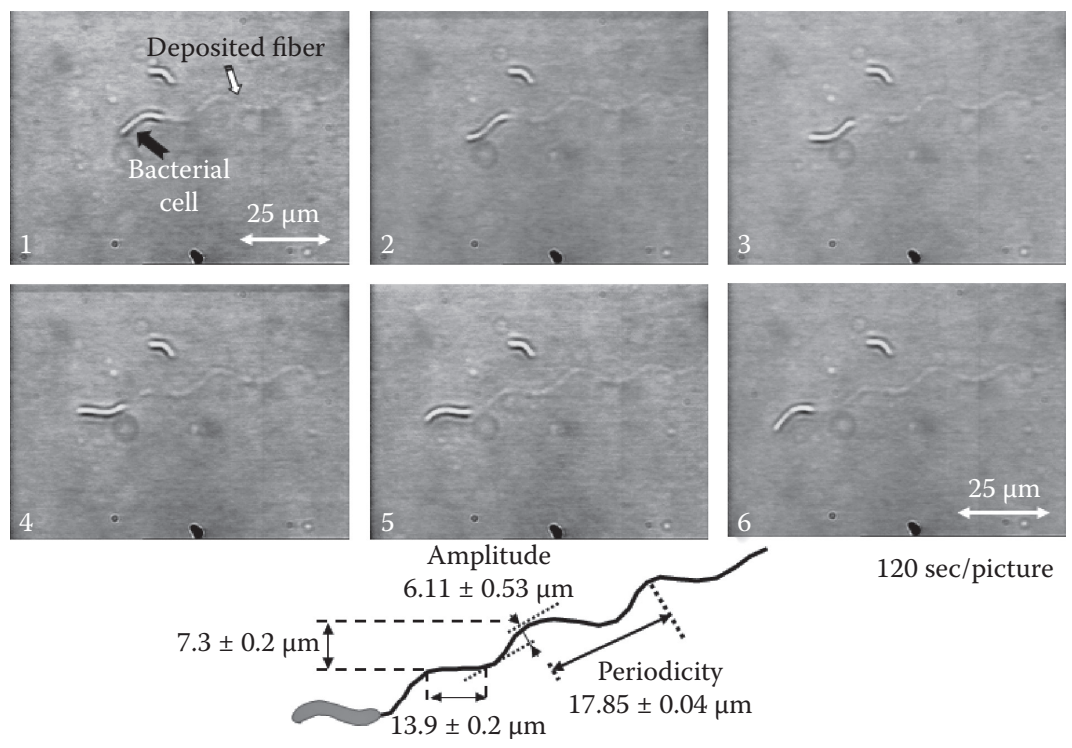

$120 \mathrm{sec} /$ picture

\section{FIGURE 6.14}

Successive images showing the motion using real-time video analysis of a bacterium as it secretes a cellulose nanofiber on the nematic ordered chitin template. The bacterium is attached and thereafter is synthesizing the fiber on the molecular tracks in the template showing a "waving" motion pattern.

fibers deposited on NOC templates. Instead, the bacteria begin to follow the molecular tracks, but soon they jump off the track and synthesize their cellulose parallel to neighboring tracks, once again soon jumping off. This "waving" pattern was repeated across the template, as evidenced by direct video imaging of the motion of the bacteria as they secrete the cellulose nanofiber, as shown in Figure 6.14 (Kondo et al. 2012).

Figure 6.14 shows the time course (120 s between each frame) of the waving pattern of movement for a G. xylinus cell having a width of $1 \mu \mathrm{m}$ and a length of $10 \mu \mathrm{m}$ in length, together with the deposited fiber in the same waving pattern. The time-lapse observation also showed that the cell movement was at a constant rate of $2.05 \mu \mathrm{m} / \mathrm{min}$ (standard deviation $\pm 0.14 \mu \mathrm{m} / \mathrm{min}$ ) at $24^{\circ} \mathrm{C}$, which is thought to be the result of an inverse force imposed by the directed polymerization and crystallization of the cellulose in the bacterium. The amplitude and periodicity of the waving as indicated in Figure 6.14 was $6.11 \pm 0.53 \mu \mathrm{m}$ and $17.85 \pm 0.04 \mu \mathrm{m}$, respectively. As seen in the minimum standard deviation in the periodicity of the waving pattern, the movement of the bacterium is fairly periodic and regular. However, the rate of motion on the nematic ordered chitin template was the same as that for cell movements without templates. Furthermore, there was no difference in the rate of movement on the track and off the track, indicating that the interaction of the 
nascent cellulose with the nematic ordered chitin template was not as strong as that with the NOC template (Kondo et al. 2002).

In nematic ordered chitin templates, hydrophilic $\mathrm{OH}$ groups at the $\mathrm{C} 6$ position of the glucopyranose ring and relatively hydrophobic acetamide groups at the $\mathrm{C} 2$ position appeared alternately on the surface because of the $2{ }_{1}$ screw axis of the molecular chain, whereas the NOC template provides only $\mathrm{OH}$ groups at the C6 position on the surface. The balance of the two substituents strongly affects the strength of the interaction between the surface and secreted cellulose microfibrils. When the contact with the chitin template is not as strong as that for NOC, the biosynthesized individual subelementary fibrils (a few nanometers in width) usually tend to be self-assembled to form a ribbon-type nanofiber with a width of 40-60 nm. The situation when the bacteria secrete cellulose nanofibers cannot be changed without such a strong interaction between the interfaces. Accordingly, the rate of deposition of the fibrils on the nematic ordered chitin template that corresponds to the rate of the bacterial movement did not change from the initial rate without the template (both the moving rates were $2.05 \mu \mathrm{m} \pm 5 \%$ at $24^{\circ} \mathrm{C}$ ). In contrast, when the interaction was strong enough, as in the case of NOC, the rate of movement was much higher when compared with movements on the templates composed of chitin.

To explain this phenomenon one needs to determine the rate-determining step for both the production of fibers and bacterial movements. In a previous chapter (Tomita and Kondo 2009), it was assumed to be the self-assembly process of biosynthesized subelementary fibrils for forming a cellulose nanofiber. That is, the strong interfacial contact with the template prevented the assembly of the individual subelementary fibrils soon after biosynthesis, resulting in an increase in the rate. This could also cause the release of stress for the enzymatic synthesis of cellulose. As a result, the production of fibers on the NOC template increases, as does the rate of bacterial movement. In fact, we previously found by FE-SEM observations that the bacterial subelementary fibrils extruded by the synthesizing enzymes could not self-assemble on the NOC template to form cellulose nanofibers, later becoming flattened by strongly attaching to the molecular tracks of $\mathrm{OH}$ groups on the template surface. This shows the prevention of self-assembly of the subelementary fibrils, and, simultaneously, epitaxial nanodeposition along the molecular track direction on the surface of the template.

The unique patterning of the biodirected nanodeposition process can be also observed by imaging the deposited cellulose nanofibers using FE-SEM correlated with real-time video analysis using light microscopy.

At first, light microscopic observations of the deposited fiber clarified that adherence to the molecular tracks occurred every $13.9 \mu \mathrm{m}$ (standard deviation $\pm 0.2 \mu \mathrm{m}$ ) as a mean distance along the orientation of the template, as shown in Figure 6.14. Alternating with this parallel movement, the cell "jumped off" the tracks and detoured every $7.3 \mu \mathrm{m}$ (standard deviation $\pm 0.2 \mu \mathrm{m}$ ) until it became parallel to neighboring tracks, as also indicated in 

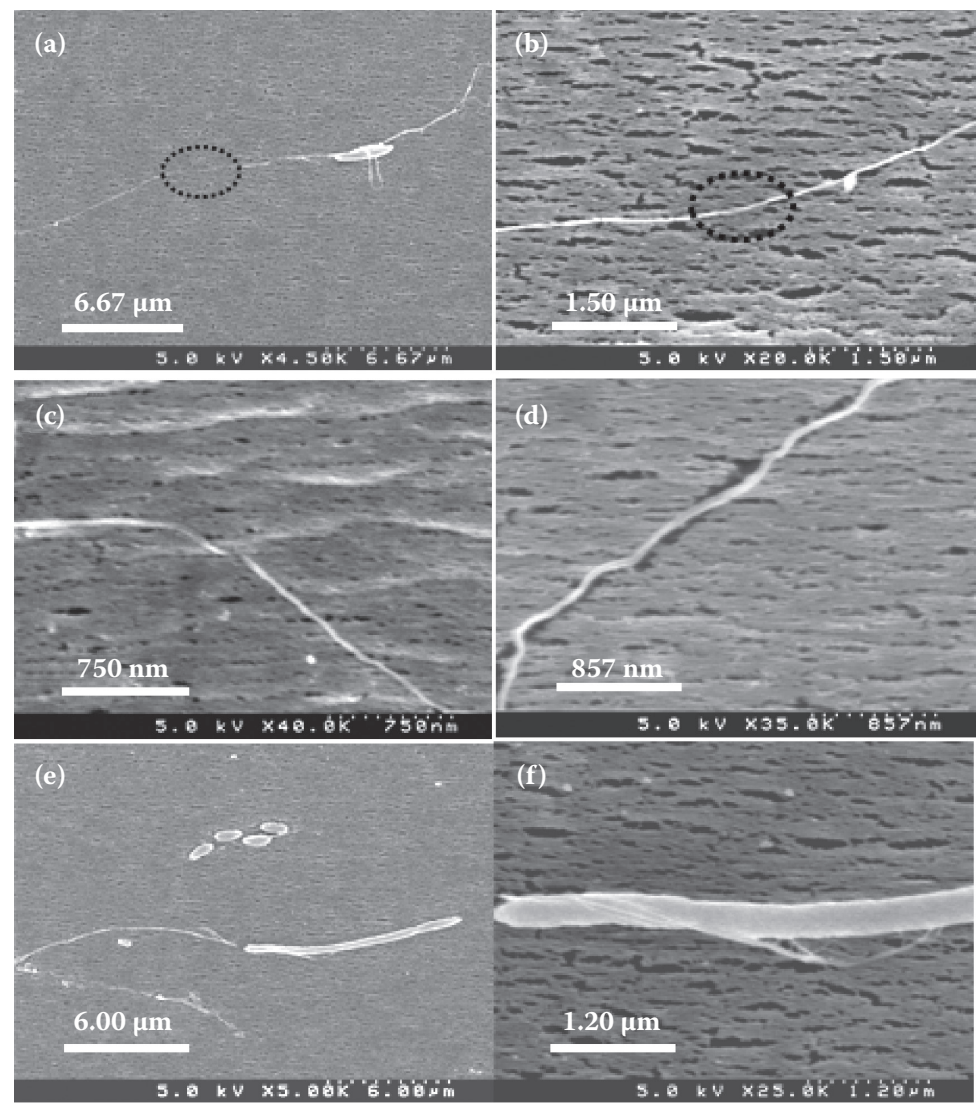

\section{FIGURE 6.15}

FE-SEM images of the cellulose nanofiber deposition process. (a-d) Examples of bacteria synthesizing cellulose nanofibers on the oriented molecular tracks of nematic ordered chitin template. (e, f) Examples indicate self-rotation of a G. xylinus cell.

Figure 6.14. Once again, soon jumping off, it repeated in the same manner until it stopped moving.

Figure 6.15a shows a bacterium in contact with a nematic ordered chitin template that was moving in a "waving" fashion. During the moving pattern, it deposited a cellulose nanofiber that relatively flattened due to interaction with the nematic ordered chitin prior to the curving pattern in the movement. In the FE-SEM images of Figures 6.15a and 6.15b, the dotted circle indicates the area where the interaction between the fiber and template occurred because the contrast of the image was not clear when compared with other areas of the fiber as assimilated. Thus such an interaction may be a trigger for a change in the direction of the bacterial cell.

After the cell "jumped off" the tracks on the oriented chitin template, it continued to secrete its cellulose ribbon, but now in the form of a twisted 
ribbon (Figures 6.15c and 6.15d), which is the normal morphology when not in contact with an ordered substrate. This alternating pattern was observed to repeat as many as 20 times across the entire template.

G. xylinus usually rotates around the self-axis with secretion of a cellulose nanofiber, resulting in twisted cellulose nanofibers. In fact, Figures $6.15 \mathrm{c}$ and $6.15 \mathrm{~d}$ show this rotating movement that was an indication of no interaction of the secreted fiber with the chitin template. Thus Figures 6.15e and 6.15f, which show winding fibers around the bacterium body, confirm self-rotation of the bacterium cell during the detour following jumping off the tracks. Figure $6.15 \mathrm{f}$ is an enlarged view of the cell in Figure 6.15e. It is clear that the cell extruded the fiber with twisting, attributed to self-rotation of the cell. These results suggest that the magnitude of interfacial interaction can regulate the waving pattern of bacterial movements and the deposition of nanofibers, which leads to autonomous fabrication of 3D structures with a unique pattern and a controlled growth direction.

With the addition of cellulose as a component for nematic ordered chitin template, the molecular ordering was altered (Kondo et al. 2012). It was found that application of the preparative method for NOC to chitin and cellulosechitin blends did not permit the formation of nematic ordered states like NOC on the molecular scale, but instead induced a variety of hierarchical nematic ordered states on various scales (Kondo et al. 2004). It was expected that the magnitude of interfacial interactions that occurred with the microfibrils by addition of cellulose as a component would be altered. In the two templates of nematic ordered chitin-cellulose blends of 50-50 and 25-75, the moving patterns were still "waving." However, a remarkable difference appeared depending on the cellulose component ratio. The more cellulose that was added, the smaller the amplitude became-getting close to a linear pattern. This indicates that more cellulose reduces the moving amplitudes into a linear pattern. The moving rate for the individual blend ratios seemed to be within a nonsignificant range.

\section{NOC Templates Mediating Order Patterned Deposition Accompanied by Synthesis of Calcium Phosphates as Biomimic Mineralization}

This section briefly introduces an advanced possibility of NOC templates having bifunction ( $\mathrm{p}-\mathrm{NOC}$ ) in terms of a novel biomineralization (Higashi and Kondo 2012). As described previously, NOC exhibits a unique property as a scaffold for fabrication of organic hierarchical structures as seen in living things. Recently this concept was extended to fabrication of organic-inorganic hierarchical structures using less energy consumption. The NOC was at first modified into a bifunctional template containing 

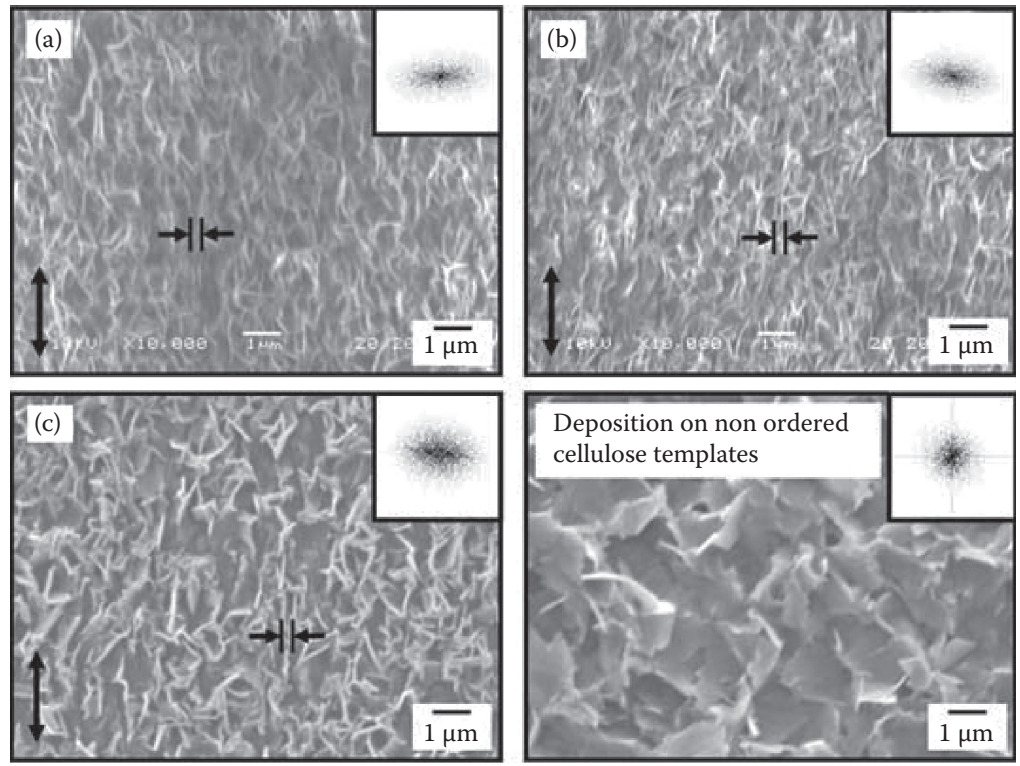

FIGURE 6.16

SEM images of the surface of p-NOC templates after reactions with calcium cations for (a) 0.5, (b) 5.0, and (c) 10 min with the FFT images as insets on the right. The morphology of calcium phosphate deposition on the nonordered cellulose template after the same reaction for $10 \mathrm{~min}$ is also displayed as a reference for the $\mathrm{p}-\mathrm{NOC}$ template. In $(\mathrm{a}-\mathrm{c})$, the line width of calcium phosphate was $0.23 \pm 0.07 \mu \mathrm{m}, 0.19 \pm 0.04 \mu \mathrm{m}$, and $0.30 \pm 0.08 \mu \mathrm{m}$, respectively. The double arrows indicate the stretching direction.

phosphate anions for biomineralization, resulting in induction of oriented deposition of calcium phosphates as well as supplying phosphate anions.

The surfaces of both p-NOC and nonordered p-cellulose templates as a reference for the p-NOC after reaction with the calcium cations in the buffer solution were analyzed using SEM observation. Figures 6.16a-6.16c show the surface morphological change of the p-NOC with calcium cations. Large amounts of calcium phosphates were formed over the surface of the p-NOC template after the reaction with calcium cations. In addition, the assembled pattern of the deposited minerals was well arranged along the molecular orientation of the p-NOC surface. The FFT images corresponding to each SEM image in Figures 6.16a-6.16c indicate that the longer the reaction time, the poorer the orientation of the deposition of calcium phosphate aggregates became. The average distance between the oriented mineral lines $(n=50)$ was approximately $0.23 \pm 0.07 \mu \mathrm{m}(\mathrm{a}), 0.19 \pm 0.04 \mu \mathrm{m}(\mathrm{b})$, and $0.30 \pm 0.08 \mu \mathrm{m}$ (c). These values were larger than those between the apparent tracks due to cellulose molecular chains on the p-NOC surface (see Figure 6.8b; approximately $0.14 \pm 0.03 \mu \mathrm{m}[n=50])$. The FFT image with a polar distribution confirms better orientation of the lines as shown in Figures 6.16a-6.16c, when compared with the nonordered cellulose template in Figure 6.16. 
The calcium phosphate did not take any ordered shape on the surface of the nonordered p-cellulose template as a reference for $\mathrm{p}$-NOC, although large amounts of calcium phosphate were also formed over the surface (see the SEM image in the bottom right of Figure 6.16). In addition, the FFT image of the nonordered cellulose template exhibited randomly distributed spots, indicating that the deposition pattern of the calcium phosphate on the nonordered template was not induced in a preferable manner. This result strongly suggests that the p-NOC surface encourages uniaxially oriented deposition accompanied by synthesis of the calcium phosphate. It was obvious that the surface orientation of the p-NOC template mediated the orientation of the calcium phosphate, because of the correspondence with each other. However, the orientation of the inorganic deposits on the p-NOC surface tended to be reduced with the longer reaction time (Figure 6.16a-6.16c).

Furthermore, the particle size of the calcium phosphate on the p-NOC template was smaller than that on the nonordered p-cellulose template. It was presumably because 3D mineral deposition and its growth tended to be inhibited by the close contact of the uniaxially oriented cellulose molecular chains on p-NOC. The deposition of calcium phosphate was mostly limited to the orientation direction of the cellulose molecular chain, and thereby the calcium phosphate was supposed to be arranged along the stretching direction of p-NOC. It was a similar mechanism to biomineralization with inhibition of the 3D crystalline growth and the arrangement of the mineral shape mediated by oriented scaffolds such as collagen or chitin (Kato et al. 2002; Rho et al. 1998). Thus the p-NOC template is a biomimic template in terms of inducing the orientation of calcium phosphate, as well as a phosphate anion supplier (Higashi and Kondo 2012).

\section{The Future}

In biological systems, skeletal materials such as cell walls, bones, and shells are made primarily of a nanoscale building block of polysaccharides, proteins, and inorganic salts. The assembly of these building blocks facilitates the production of a hierarchical framework structure. The formation dynamics observed in this NOC could be applicable to the design of nanoscale, controlled, hierarchically structured materials with specific properties. We have employed a biological system combined with a polymer platform having an NOC-like surface in order to directly fabricate hierarchically ordered materials from the nano level up to the micron level. Such a surface property would greatly extend the possibilities of cellulose use to new areas. Thus, if a nano-/micropatterned film having similar surface characteristics as an NOC can be fabricated, the 3D-patterned materials will be built up by mediation of the cellulose or related scaffolds, and thereby widely appreciated and used. 


\section{Acknowledgments}

The author thanks R. M. Brown, Jr., at the University of Texas, Austin, for research collaboration. W. Kasai and Y. Tomita, in my laboratory at Kyushu University, are also acknowledged. This research was supported partly by a Grant-in-Aid for Scientific Research (nos. 22380097 and 23658146) from the Japan Society for the Promotion of Science (JSPS).

\section{References}

Benziman, M., C. H. Haigler, R. M. Brown, Jr., A. R. White, and K. M. Cooper. 1980. Cellulose biogenesis: polymerization and crystallization a coupled processes in Acetobacter xylinum. Proc. Natl. Acad. Sci. USA 77:6678-6682.

Brown, R. M., Jr., J. H. M. Willison, and C. L. Richardson. 1976. Cellulose biosynthesis in Acetobacter xylinum: visualization of the site synthesis and direct measurement of the in vivo process. Proc. Natl. Acad. Sci. USA 73:4565-4569.

Chidambareswaran, P. K., S. Sreenivasan, N. B. Patil, H. T. Lokhande, and S. R. Shukla. 1978. Fine structural changes in native and mercerized fibrous cellulose brought about by ethylenediamine and methyl alcohol. J. Appl. Polym. Sci. 22:3089-3099.

Drexler, K. E. 1992. Nanosystems: molecular machinery, manufacturing, and computation. New York: Wiley Interscience.

Fengel, D., H. Jakob, and C. Strobel. 1995. Influence of the alkali concentration on the formation of cellulose II. Study by X-ray diffraction and FTIR spectroscopy. Holzforschung 49:505-511.

Haigler, C. H., R. M. Brown, Jr., and M. Benziman. 1980. Calcofluor white alters the in vivo assembly of cellulose microfibrils. Science 210:903-906.

Haigler, C. H, A. R. White, R. M. Brown, Jr., and K. M. Cooper. 1982. Alteration of in vivo cellulose ribbon assembly by carboxymethylcellulose and other cellulose derivatives. J. Cell. Biol. 94:64-69.

Hesse, S., and T. Kondo. 2005. Behavior of cellulose production of Acetobacter xylinum in 13C-enriched cultivation media including movements on nematic ordered cellulose templates. Carbohyd. Polym. 60:457-465.

Hestrin, S., and M. Schramm. 1954. Synthesis of cellulose by Acetobacter xylinum. 2. preparation of freeze dried cells capable of polymerizing glucose to cellulose. Biochem. J. 58:345-352.

Higashi, K., T. and Kondo. 2012. Nematic ordered cellulose templates mediating order-patterned deposition accompanied with synthesis of calcium phosphates. Cellulose 19:81-90.

Hishikawa, Y., E. Togawa, Y. Kataoka, and T. Kondo. 1999. Characterization of amorphous domains in cellulosic materials using a deuteration-FTIR monitoring analysis. Polymer 40:7117-7124.

Hishikawa, Y., E. Togawa, and T. Kondo. 2010. Molecular orientation in the nematic ordered cellulose film using polarized FTIR accompanied with a vapor-phase deuteration method. Cellulose 17:539-545. 
Horii, F., A. Hirai, and R. Kitamaru. 1983. Solid-state ${ }^{13} \mathrm{C}-\mathrm{NMR}$ study of conformations of oligosaccharides and cellulose conformation of $\mathrm{CH}_{2} \mathrm{OH}$ group about the exo-cyclic C-C bond. Polym. Bull. 10:357-361.

Isogai, A., R. H. and Atalla. 1991. Amorphous celluloses stable in aqueous media: regeneration from $\mathrm{SO}_{2}$-amine solvent systems. J. Polym. Sci. Polym. Chem. 29:113-119.

Kato, T., A. Sugawara, and N. Hosoda. 2002. Calcium carbonate-organic hybrid materials. Adv. Mater. 14:869-877.

Kondo, T. 2007. Nematic ordered cellulose: its structure and properties. In: Cellulose: Molecular and Structural Biology, ed. R. M. Brown, Jr. and I. M. Saxena, 285-306. Dordrecht: Springer.

Kondo, T., W. Kasai, and R. M. Brown, Jr. 2004. Formation of nematic ordered cellulose and chitin. Cellulose 11:463-474.

Kondo, T., W. Kasai, M. Nojiri, Y. Hishikawa, E. Togawa, D. Romanovicz, and R. M. Brown, Jr. 2012. Regulated patterns of bacterial movements based on their secreted cellulose nanofibers interacting interfacially with ordered chitin templates. J. Biosci. Bioeng. 114:113-120.

Kondo, T., M. Nojiri, Y. Hishikawa, E. Togawa, D. Romanovicz, and R. M. Brown, Jr. 2002. Biodirected epitaxial nanodeposition of polymers on oriented macromolecular templates. Proc. Natl. Acad. Sci. USA 99:14008-14013.

Kondo, T., E. Togawa, and R. M. Brown, Jr. 2001. "Nematic ordered cellulose": a concept of glucan chain association. Biomacromolecules 2:1324-1330.

Minke, R., and J. Blackwell. 1978. The structure of $\alpha$-chitin. J. Mol. Biol. 120:167-181.

Rho, J. Y., L. Kuhn-Spearing, and P. Zioupos. 1998. Mechanical properties and the hierarchical structure of bone. Med. Eng. Phys. 20:92-102.

Taton, T. A. 2002. Bio-nanotechnology: two-way traffic. Nature Mater. 2:73-74.

Thompson, N. S., H. M. Kaustinen, J. A. Carlson, and K. I. Uhlin. 1988. Tunnel structures in Acetobacter xylinum. Int. J. Biol. Macromol. 10:126-127.

Togawa, E., and T. Kondo. 1999. Change of morphological properties in drawing water-swollen cellulose films prepared from organic solutions: a view of molecular orientation in the drawing process. J. Polym. Sci. B Polym. Phys. 37:451-459.

Togawa, E., and T. Kondo. 2007. Unique structural characteristics of nematic ordered cellulose: stability in water and its facile transformation. J. Polym. Sci. B Polym. Phys. 45:2850-2859.

Tokoh, C., K. Takabe, M. Fujita, and H. Saiki. 1998. Cellulose synthesized by Acetobacter xylinum in the presence of acetyl glucomannan. Cellulose 5:249-261.

Tomita, Y., and T. Kondo. 2009. Influential factors to enhance the moving rate of Gluconacetobacter xylinus due to its nanofiber secretion on oriented templates. Carbohydr. Polym. 77:754-759.

Ward, I. M. 1997. Introduction. In: Structure and Properties of Oriented Polymers, 2nd ed., ed. I. M. Ward, 1-15. London: Chapman \& Hall.

Yamamoto, H., F. Horii, and A. Hirai. 1996. In situ crystallization of bacterial cellulose II. Influences of different polymeric additives on the formation of celluloses I $\alpha$ and I $\beta$ at the early stage of incubation. Cellulose 3:229-242. 


\title{
Applications and Products-Nata de Coco
}

\author{
Muenduen Phisalaphong and Nadda Chiaoprakobkij \\ Department of Chemical Engineering, Faculty of Engineering, \\ Chulalongkorn University, Bangkok, Thailand
}

\section{CONTENTS}

Nata de Coco Food Products............................................................................... 144

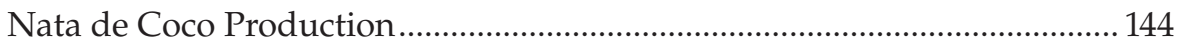

Markets, Producers, and Companies .............................................................. 149

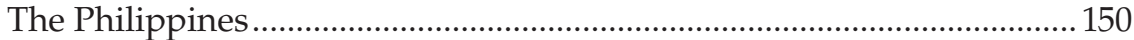

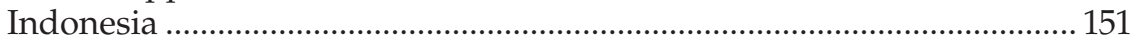

Other Commercial Producers ..................................................................... 152

Nata de Coco Facial Mask............................................................................. 152

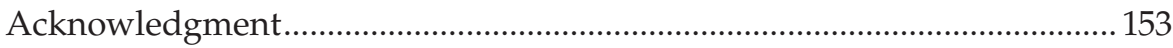

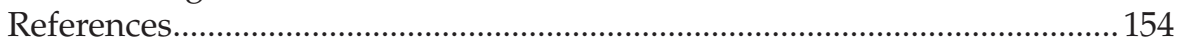

One of the first commercially available products of bacterial nanocellulose (BNC) was nata de coco. Nata de coco is a white, gelatinous and chewy material used for the production of sweets and desserts. It is produced from coconut water or coconut milk by the microorganism Acetobacter aceti subsp. xylinum through a fermentation process under static conditions. Nata de coco production originated in the Philippines during the 1990s and spread to nearby countries. In 1991 nata de coco was introduced to Japan through its use in diet drinks and became very popular, especially among young girls. Production of nata de coco has increased in recent years for not only domestic markets, but also for export markets, especially Europe, Japan, the United States, and the Middle East. Nata de coco is usually produced in the form of thick sheets of pure cellulose that are then cut into small uniform cubes, washed, and boiled in water before cooking in sugar syrup for food applications. Nata de coco is used in food products such as low-calorie desserts, salads, and high-fiber foods. It is regarded for its high content of dietary fiber and its low fat and cholesterol content. Nata de coco also promotes a healthy digestive system. Commercial nata de coco is made by large and mid-size companies and small or home industries in East Asian countries such as the Philippines, Indonesia, China, Vietnam, Malaysia, and Thailand. 


\section{Nata de Coco Food Products}

One of the first commercially available products of bacterial nanocellulose (BNC) was nata de coco. Nata de coco is a white, smooth, jelly-like, chewy material that is usually used for making sweets and desserts. It is produced from coconut water or coconut milk by the microorganism Acetobacter aceti subsp. xylinum through a fermentation process. Nata de coco originated in the Philippines (Lapuz et al. 1969). The growth of nata de coco pellicle by Gluconacetobacter xylinus was first described in the 1880s by Brown (1886a, 1886b), who identified this jelly-like product as chemically equivalent to cellulose (Budhiono et al. 1999). In 1991 nata de coco was introduced to Japan through its use in diet drinks, and it became very popular, especially among young girls. Nata de coco can be consumed in many types of food items, such as desserts and candies, salads, fruit cocktails, ice cream, juices, dairy products, ketchup, and sauces. Nata de coco can be used as a texture modifier for frozen foods, baked products, sauces, and other food products. Nata de coco in syrup form has a fresh taste, translucent color, and high nutritive value because of its high fiber content. In addition to its natural flavor, the taste can be modified by adding various fruit or flower juices like mango, lychee, pineapple, kiwi, orange, maple syrup, and rose. Articles published by Okiyama et al. in 1992 and 1993 discussed the many applications of BNC and nata de coco in the food industry. In its undried state, BNC is quite tough to chew; however, it becomes softer and has a texture similar to fruit and other foods after being treated with ethanol and calcium chloride. Applications of BNC in food products are mainly as low-calorie desserts, salads, and prepared foods. This material can be used as a food additive in place of xanthan gum or as a food thickener (Okiyama et al. 1993). A health-food product derived from $6 \%$ unpolished rice, $18 \%$ mung bean, $36 \%$ sweet corn, and $40 \%$ nata de coco significantly lowered the serum triglyceride level in rats (Mesomya et al. 2002). Additional studies of the effects of food prepared from cereal and nata de coco on the serum lipids of patients with hyperlipidemia showed that health foods made from unpolished rice, hulled mung bean, sweet corn, and nata de coco could reduce the serum total triglyceride and total cholesterol levels (Mesomya et al. 2006).

\section{Nata de Coco Production}

The most commonly used bacteria for BNC production is Gram-negative Acetobacter xylinium (Bielecki et al. 2004). A stable cellulose-producing strain can be maintained over many generations and hence many years (Andrade 
et al. 2010). There are a number of operating factors that affect the production of nata de coco. A culture medium with appropriate concentrations and types of nitrogen and carbon is required for the growth of Acetobacter bacteria and $\mathrm{BNC}$ biosynthesis. Other important factors are $\mathrm{pH}$, temperature, and dissolved oxygen (DO). During the first stage of fermentation, the process of cellulose formation and the consumption of glucose are controlled by oxygen concentration; however, in the later stages, sucrose becomes a limiting substrate (Budhiono et al. 1999; Verschuren et al. 2000). Although glucose and sucrose are generally used as carbon sources for BNC production, other carbon sources such as fructose, mannitol, glycerol, ethanol, and many residues from agroindustries can also be used (Carreira et al. 2011; Jung et al. 2010; Masaoka et al. 1993; Mikkelsen et al. 2009; Takaaki et al. 1998; White and Brown 1989). Sugar concentration, $\mathrm{pH}$, and ammonium sulfate concentration are important factors that affect moisture content, hardness, and other physical properties of nata de coco (Jagannath et al. 2008). The $\mathrm{pH}$ of the fermentation medium strongly affects growth and cellulose formation. The optimum fermentation medium pH has been reported to be 4.0-6.0 (Masaoka et al. 1993; Phunsri et al. 2003).

The common process for the production of nata de coco uses coconut water or coconut milk as a fermentation medium with supplements of sucrose and ammonium sulfate $\left(\left(\mathrm{NH}_{4}\right)_{2} \mathrm{SO}_{4}\right)$ at concentrations of $5 \%-10 \%$ and $0.5 \%-0.7 \%$, respectively (Jagannath et al. 2008; Phisalaphong et al. 2010). The initial pH of the fermentation medium is normally adjusted to $4.0-5.0$ with diluted acetic acid (Figures 7.1 and 7.2).

The medium is statically cultivated with $5 \%-10 \%$ of a stock culture in a plastic tray (or jar) at a level of approximately $2-4 \mathrm{~cm}$ in height and covered with a piece of clean newspaper or similar material. The optimum temperature range for nata production is between $23^{\circ} \mathrm{C}$ and $32^{\circ} \mathrm{C}$. Under suitable conditions, good quality nata de coco that has a smooth surface and a soft, chewy texture is obtained after 7-14 days of incubation. The quality aspects of nata de coco that should be considered are shape, thickness, color, purity, and acidity. Nata de coco is usually produced in the form of rectangular sheets with a thickness of approximately 1-2 cm (Figure 7.3a). The sheet is harvested by separating the nata from the spent liquor, scraping thin bacterial films off the gel surface, and soaking the nata in water. To make nata de coco in syrup, the gel is cut into small, uniform cubes (approximately $1.5 \mathrm{~cm} \times 1.5 \mathrm{~cm} \times 1.5 \mathrm{~cm}$ ), washed by soaking the cubes (nata) for 1 or more days in several changes of water to remove the sour (acid) taste and smell, then boiled in water for 5-10 minutes. After that, it is cooked in sugar syrup for food applications. The sweetened nata product is packed in sterilized preserving jars, cans, or plastic bags (Figure 7.3b) before using it in low-calorie desserts, salads, and high-fiber foods (Figure 7.3c). Nata de coco is highly regarded for its high content of dietary fiber and its low fat and cholesterol content. It promotes a healthy digestive system. 


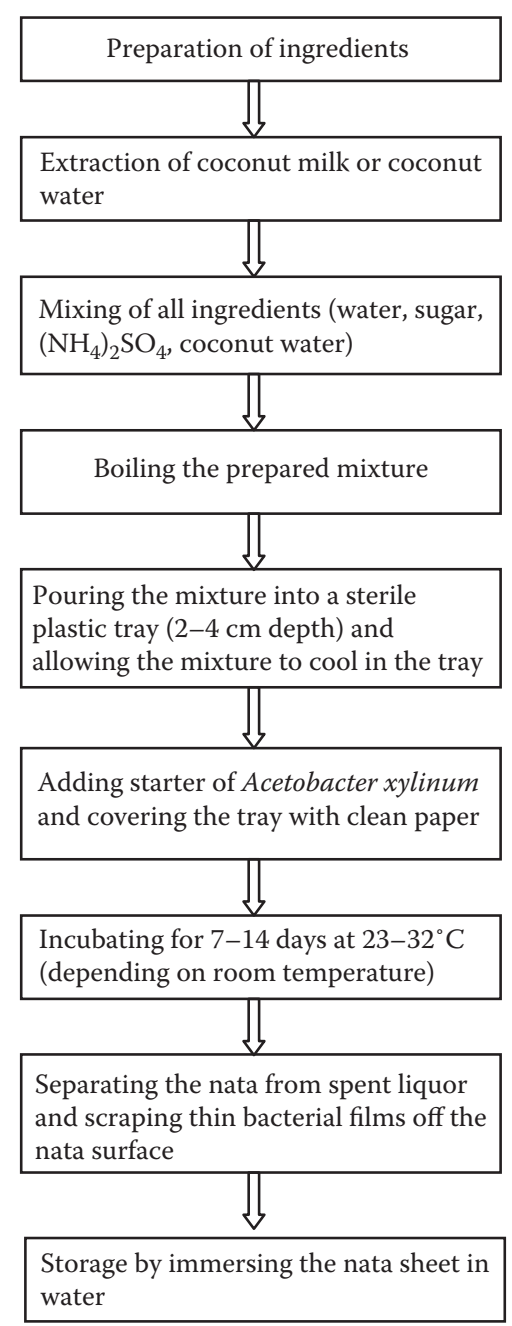

\section{FIGURE 7.1}

Diagram for the primary production process of nata de coco.

Apart from coconut water, some tropical countries use pineapple juice, a by-product from agroindustries as commercial raw materials in BNC production. In Indonesia, the nata processing industry previously used pineapple extract as its raw material, and the product was called nata de pina. However, because pineapples are seasonal fruits, nata de pina cannot be produced throughout the year. It has also been reported that coconut juice is a better substrate for the growth of Acetobacter bacteria than pineapple juice (Kongruang 2008). Several other fruit juices, including orange, apple, Japanese pear, and grape, have also been used to produce BNC 


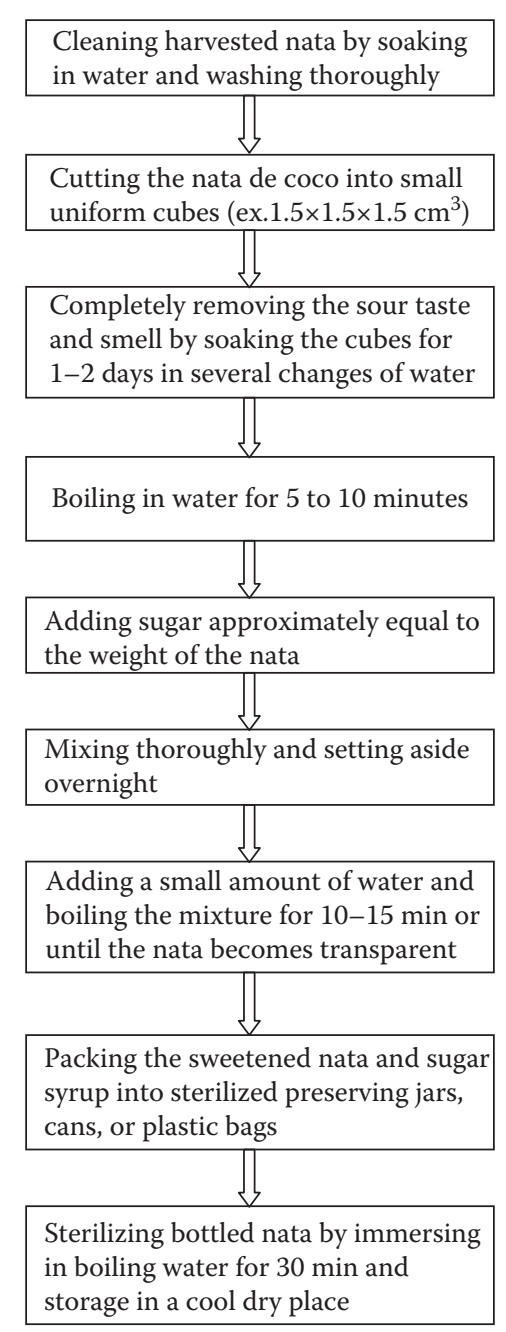

\section{FIGURE 7.2}

Diagram of the process for preparation of nata de coco in sugar syrup.

(Akihiro et al. 2009). The main sugars in fruit juice are glucose, sucrose, and fructose. Because the sugar compositions and $\mathrm{pH}$ values are different according to the fruit type, the initial $\mathrm{pH}$ and total sugar concentration of these fruit juice media should be adjusted to optimal levels before use as culture media for BNC production. The addition of nitrogen sources, such as yeast extract or bacto peptone, to the fruit juice media may be used to improve the yield of BNC, as nitrogen sources contain amino acids and proteins that promote cell growth. Some methodologies that have been reported for enhanced BNC productivity include the addition of 

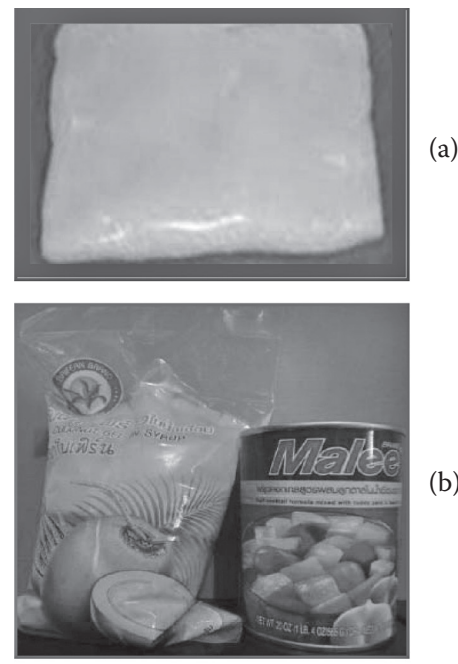

(b)

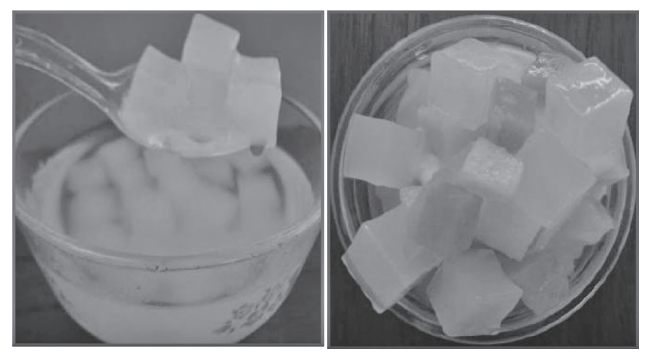

(c)

\section{FIGURE 7.3}

(See color insert.) Nata de coco products: (a) harvested nata de coco sheet, (b) nata de coco products packed in a plastic bag and a can, and (c) nata de coco in low-calorie desserts and fruit salads.

water-soluble polysaccharides such as agar into the culture media (Sangok et al. 2004), or the use of konjac powder as a carbon source (Feng and Kaiyan 2008). Maple syrup was reported as an alternative carbon source for BNC production. Maple syrup contains $67 \%$ (w/w) total sugar, and $89 \%$ of the sugar content is sucrose. In a study that used shake flask culture, the $\mathrm{BNC}$ production rate of maple syrup was comparable to that of using pure fructose as the carbon source (Zeng et al. 2011).

The major waste from nata de coco production is liquid waste from the fermentation and cleaning processes. This liquid waste $(\mathrm{pH}$ ranging from 4.0 to 5.9) contains high concentrations of acetic acid (Green Productivity Demonstration Program 2003), thus direct discharges of the wastewater into soil could lead to soil acidification. Therefore proper management of the wastewater is required. In some areas of Indonesia the waste treatment process is carried out in a simple way by making a collection chamber in the ground and storing this wastewater for a period of time; later the waste can be used on coconut plantations. 


\section{Markets, Producers, and Companies}

The world's leading producers of coconut are tropical countries, including (in order of production) Indonesia, the Philippines, India, Sri Lanka, Brazil, and Thailand. Table 7.1 shows coconut production in Indonesia, the Philippines, India, and the worldwide total for 1995-1999 as reported by the Agricultural Statistics Bureau of the Philippines.

The top-producing countries for coconut and the percentage of world production of coconut by country in 2009 are shown in Table 7.2 and Figure 7.4, respectively. Exports of coconut and processed coconut products are an important source of income for these countries. Aside from copra and coconut oil, other exports that have a significant volume are desiccated coconut, copra meal, coco chemicals (fatty acids, fatty alcohol, and methyl ether), shell

TABLE 7.1

World Coconut Production (in 1000 Metric Tons) in 1995-1999

\begin{tabular}{lrrrrr}
\hline Area & $\mathbf{1 9 9 5}$ & $\mathbf{1 9 9 6}$ & $\mathbf{1 9 9 7}$ & $\mathbf{1 9 9 8}$ & $\mathbf{1 9 9 9}$ \\
\hline Indonesia & 13,868 & 14,138 & 14,710 & 14,710 & 13,000 \\
Philippines & 10,300 & 11,318 & 12,053 & 10,493 & 11,000 \\
India & 8,000 & 9,649 & 9,800 & 10,000 & 11,000 \\
World total & 45,068 & 47,733 & 49,354 & 47,696 & 47,480 \\
\hline
\end{tabular}

Source: Agricultural Statistic Bureau - Philippines, http:// www.da.gov.ph/agribiz/coconut1.html

TABLE 7.2

Top Coconut-Producing Countries in 2009

\begin{tabular}{clrc}
\hline Rank & \multicolumn{1}{c}{ Country } & $\begin{array}{c}\text { Production } \\
\text { (Int\$1000) }\end{array}$ & Production (t) \\
\hline 1 & Indonesia & $2,384,584$ & $21,565,700$ \\
2 & Philippines & $1,709,892$ & $15,667,600$ \\
3 & India & $1,122,094$ & $10,148,000$ \\
4 & Sri Lanka & 232,092 & $2,099,000$ \\
5 & Brazil & 218,201 & $1,973,370$ \\
6 & Thailand & 152,699 & $1,380,980$ \\
7 & Viet Nam & 124,781 & $1,128,500$ \\
8 & Mexico & 111,093 & $1,004,710$ \\
9 & Papua New Guinea & 102,832 & 930,000 \\
10 & Tanzania & 63,811 & 577,099 \\
\hline
\end{tabular}

$\mathrm{t}=$ metric ton.

Int $\$ 1000$, calculated based on 2000-2005 international prices.

Source: Food and Agriculture Organization of the United Nations, http:/ / faostat.fao.org/site/339/default.aspx 


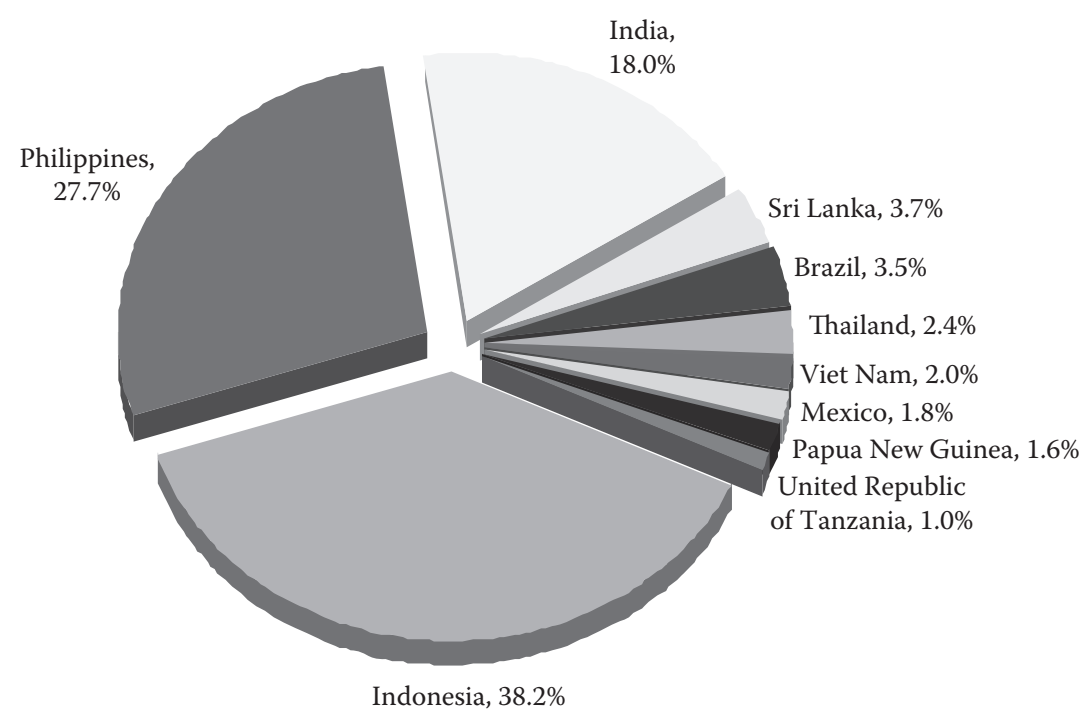

FIGURE 7.4

(See color insert.) Percentage of world coconut production by country in 2009.

charcoal and activated carbon, fiber products, coconut cream, coconut milk, coconut powder, and nata de coco. Nata de coco is one of the value-added products from the coconut industry. Nata de coco is highly regarded for its high dietary fiber, which is beneficial for the digestive system. Moreover, it contains no fat or cholesterol. Therefore it is considered a diet and health food product. Production of nata de coco has been increasing in recent years, not only for domestic markets, but also for export markets, especially Europe, Japan, the United States, and the Middle East. Commercial nata de coco is produced in East Asian countries such as the Philippines, Indonesia, Thailand, China, Vietnam, and Malaysia. The Philippines and Indonesia are the top exporting countries of nata de coco products. Nata de coco production is carried out by large and mid-size companies and also small and home industries that have their own market segments. The large and mid-size companies have relatively large markets, including domestic and export markets. Small and home industries have local markets and surrounding areas. Small and home industry production of nata de coco can employ many local workers.

\section{The Philippines}

The Philippines is one of the top exporters of nata de coco. Sixty-eight of the 79 provinces in the Philippines are coconut areas, with $3.6 \mathrm{M}$ hectares or $26 \%$ of total agricultural land planted with coconut trees. More than $50 \%$ of the total Philippine supply of coconuts is sourced from Mindanao (Philippine Trade Training Center 2011). BNC was first discovered in the Philippines through the observation of floating slimy substances on pineapple juice 
waste. In the past, coconut milk and water were low economic value by-products of coconut processing from the copra or cooking oil industries (Pecoraro et al. 2008). The successful nata de coco industry, using coconut milk or water as a medium for fermentation, was first established in Laguna and then expanded to Batangas, Quezon, Iloilo, Cagayan de Oro, Cavite, and many other places in the Philippines. From an initial demand of approximately US $\$ 4960$ in 1987, there was a sudden increased demand for exports to Japan of approximately US\$170,320 in 1991 and approximately US\$2.1 million to Taiwan in 1992. Nata de coco exports to Japan dramatically increased to US\$20.39 million in 1994. The rapidly increasing demand in Japan was due to the belief that nata de coco can protect the body from cancer and can be used as a diet food (SIPUK-Bank Sentral Republik Indonesia 1993). According to the United Coconut Associations of the Philippines (UCAP) Newsbrief (2010), among nontraditional coconut export products in 2009, nata de coco was the second largest, earning US\$6.034 million from the sale of 6051 metric tons (t). The greatest market for nata de coco was Japan $(77.8 \%)$, the second largest market was the United States.

The intensive promotion of nata de coco in Japan by Philippine companies has also benefited other processed fruit products, whose exports to Japan have likewise grown. The export of coconut products has been consistently increasing, with an average growth rate of approximately $8 \%$ over the period of 2007-2010. In 2010, total exports of coconut reached US\$1.6 billion and accounted for $20 \%$ of world market share, the highest among key sectors identified in the Philippine Export Development Plan (PEDP). From a recent report, based on Philippine Authority data, nata de coco was the sixth top export of coconut products, with an income of US\$273,184 from the export of $172 \mathrm{t}$ in February 2010. Japan remained the largest buyer, with approximately $34.0 \%$ of total sales. Other import countries were the Dominican Republic, the United States, Canada, Hong Kong, and the United Arab Emirates (Asian and Pacific Coconut Community Cocommunity Newsletter 2010). According to the results of a survey, the Japanese consider nata de coco a healthy food for a number of reasons: anticancer properties (39\%), good for pregnant women $(30 \%)$, diet food $(15 \%)$, good for growing children $(1 \%)$, and a good source of dietary fiber $(1 \%)$.

\section{Indonesia}

Indonesia has great potential for nata de coco production because Indonesia is the top coconut producer in the world. The amount of coconut milk produced in Indonesia is approximately $900 \mathrm{Ml}$ /year (SIPUK - Bank Sentral Republik Indonesia 2011; Irnia and Nur 2001). Indonesia has many nata de coco producers at business scales ranging from household and small to middle and large scale. Companies that can reach an economy of scale will produce continuously. Household businesses are usually sporadic; such businesses produce when there is excess local demand, such as during the holidays. However, the 
nata de coco supply does not rely on a season because coconuts are available all year long. Based on a feasibility analysis by the Central Bank of Indonesia, the price of nata de coco rounds up to $460 € / t$ (dry weight) (Andrade et al. 2010).

Nata de coco is one of the most reliable export commodities from the South Lampung Regency of Indonesia. Coconut water and coconut milk can be used as raw materials for nata de coco. One of the biggest companies is PT Keong Nusantara Abadi. This company obtains 32,000 l of coconut milk every day (from 96,000 coconuts) for the production of nata de coco. Another large company is PT Sari Segar Husada, which concentrates on the canned coconut industry. This company uses $85,000-100,000$ coconuts per day to produce $12.2 \mathrm{t}$ of dried coconuts per day and $20 \mathrm{t}$ of nata de coco per month, which are then exported to Britain and the Netherlands. Other coconut production areas in Indonesia are North Sulawesi, Riau, Jambi, Lampung, Daerah Istimewa Aceh, West Sumatra, North Sumatra, Central, West Java, Central Java, East Java, West Kalimantan, South Kalimantan, East Nusa Tenggara, and West Nusa Tenggara. As much as $90.86 \%$ of the total coconut production in Indonesia comes from these areas (Ministry of Agriculture of Republic of Indonesia, September 2008).

\section{Other Commercial Producers}

Thailand, Vietnam, and Malaysia are also large commercial producers of nata de coco food products. Coconut water from mature nuts, which are abundantly available at coconut factories, can be used as a medium for nata de coco production. Previously coconut water from mature nuts was an agricultural waste that caused many disposal problems in coconut factories. Its conversion into nata de coco thus presents an attractive option for reducing waste and producing a valuable product. Typical commercial food products using nata de coco include fruit jellies and jams, dairy products, yogurt, ice cream, baked products, canned beverages, milk, tea, sausage and other meat products, and seasonings. High quality standards of these products must be maintained to compete in the global market.

\section{Nata de Coco Facial Mask}

Aside from its food uses, nata de coco has other possible nonfood applications. Nata de coco can be applied in a number of ways due to its unique properties: high purity, high water-holding capacity, and great mechanical strength. One of the interesting applications of nata de coco is facial mask products. Nata de coco facial masks are made of BNC film derived from nata de coco. BNC films have been developed for medical purposes such as healing wounds and burns. In addition, it can be applied as a treatment mask for 


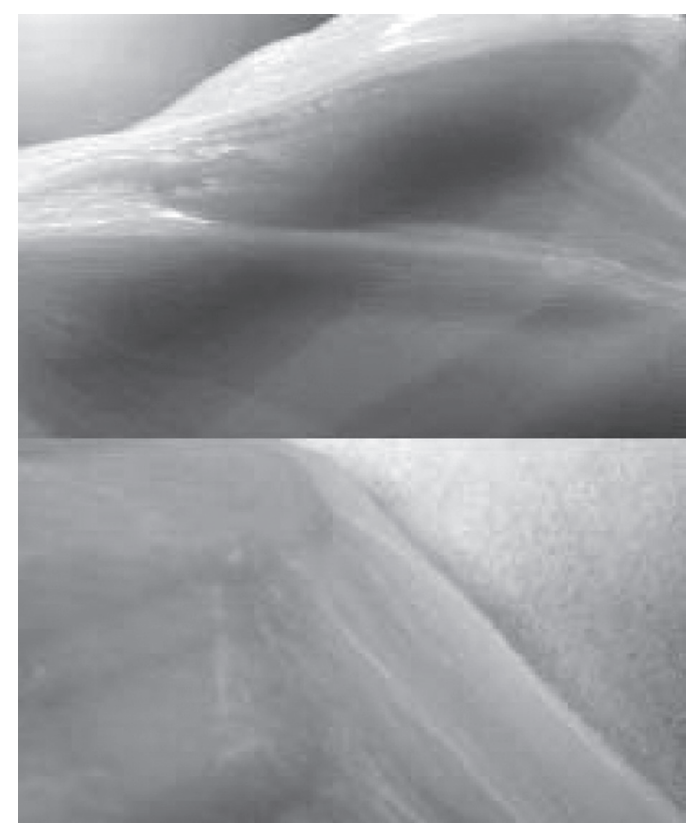

FIGURE 7.5

(See color insert.) Comparison of the texture of facial masks made from nata de coco (top) and paper (bottom).

cosmetic applications. A nata de coco facial mask or BNC mask is a crystalline, flexible, thin but strong film. Due to its high water-holding capacity, BNC is an excellent carrier for active cosmetic ingredients. BNC films are nontoxic to human skin cells and support human skin cell growth (Sanchavanakit et al. 2006). In a study of the effects of BNC mask on facial skin characteristics and user satisfaction, a single application of a trial BNC mask enhanced moisture uptake by facial skin more than moist towels. Users also reported being satisfied with the trial product (Amnuaikit et al. 2011). Many commercial BNC facial masks are now available worldwide. Compared with a general facial mask made from paper sheets, the texture of the BNC mask is finer, smoother, and more transparent (Figure 7.5). This product is expected to be highly popular among women, and perhaps even some men.

\section{Acknowledgment}

The authors are grateful for financial support from the Thailand Research Fund (TRF), the Commission on Higher Education, Chulalongkorn University (contract grant no. RMU 5380024). 


\section{References}

Akihiro, K., S. Chizuru, Y. Yuya, and N. Yoshitoshi. 2009. Utilization of various fruit juices as carbon source for production of bacterial cellulose by Acetobacter xylinum NBRC 13693. Carbohyd. Polym. 76:333-335.

Amnuaikit, T., T. Chusuit, P. Raknam, and P. Boonme. 2011. Effects of a cellulose mask synthesized by a bacterium on facial skin characteristics and user satisfaction. Med. Dev. Evid. Res. 4(1):77-81.

Andrade, F. K., R. A. N. Pertile, F. Dourado, and F. M. Gama. 2010. Bacterial cellulose properties, production and applications. In: Cellulose: Structure and Properties, ed. A. Lejeune and T. Deprez, 427-458. New York: Nova Science.

Bielecki, S., A. Krystynowicz, M. Turkiewicz, and H. Kalinowska. 2004. Bacterial cellulose. In: Polysaccharides I: polysaccharides from prokaryotes, Vol. 1, Biopolymers, ed. E. J. Vandamme, S. De Baets, and A. Steinbüchel, 37-45. Weinheim: Wiley-VCH.

Brown, A. J. 1886a. The chemical action of pure cultivation of Bacterium aceti. J. Chem. Soc. 49:432-439.

Brown, A. J. 1886b. On an acetic ferment that forms cellulose. J. Chem. Soc. 49:172-186.

Budhiono, A., B. Rosidia, H. Tahera, and M. Iguchib. 1999. Kinetic aspects of bacterial cellulose formation in nata-de-coco culture system. Carbohyd. Polym. 40:137-143.

Carreira, P., J. Mendes, E. Trovatti, L. Serafim, C. Freire, A. Silvestre, and C. Neto. 2011. Utilization of residues from agro-forest industries in the production of high value bacterial cellulose. Bioresource Technol. 102:7354-7360.

Feng, H., and Q. Kaiyan. 2008. An alternative carbon source from konjac powder for enhancing production of bacterial cellulose in static cultures by a model strain Acetobacter aceti subsp. xylinus ATCC 23770. Carbohyd. Polym. 72:545-549.

Jagannath, A., S. Kalaiselvan, S. Manjunatha, P. Raju, and A. Bawa. 2008. The effect of $\mathrm{pH}$, sucrose and ammonium sulphate concentrations on the production of bacterial cellulose (nata-de-coco) by Acetobacter xylinum. World J. Microbiol. Biotechnol. 24:2593-2599.

Jung, H., J. Jeong, O. Lee, G. Park, K. Kim, H. Park, S. Lee, Y. Kim, and H. Son. 2010. Influence of glycerol on production and structural-physical properties of cellulose from Acetobacter sp. V6 cultured in shake flasks. Bioresource Technol. 101:3602-3608.

Kongruang, S. 2008. Bacterial cellulose production by Acetobacter xylinum strains from agricultural waste products. Appl. Biochem. Biotechnol. 148(1-3):245-256.

Lapuz, M. M., E. G. Gallardo, and M. A. Palo. 1969. The nata organism-cultural requirements, characteristics, and identity. Philipp. J. Sci. 96:91-109.

Masaoka, S., T. Ohe, and K. Sakota. 1993. Production of cellulose from glucose by Acetobacter xylinum. J. Ferm. Bioeng. 75:18-22.

Mesomya, W., Y. Cuptapun, D. Hengsawadi, P. Tangkanakul, P. Jittanoonta, and R. Pakpeankitvatana. 2002. Serum lipid lowering in rats fed with high dietary fiber from cereal and nata de coco. Kasetsart J. (Nat. Sci.) 36:187-192.

Mesomya, W., V. Pakpeankitvatana, S. Komindr, P. Leelahakul, Y. Cuptapun, D. Hengsawadi, P. Tammarate, and P. Tangkanakul. 2006. Effects of health food from cereal and nata de coco on serum lipids in human. Songklanakarin J. Sci. Technol. 28:23-28. 
Mikkelsen, D., B. M. Flanagan, G. A. Dykes, and M. J. Gidley. 2009. Influence of different carbon sources on bacterial cellulose production by Gluconacetobacter xylinus strain ATCC 53524. J. Appl. Microbiol. 107(2):576-583.

Okiyama, A., M. Motoki, and S. Yamanaka. 1992. Bacterial cellulose II. Processing of the gelatinous cellulose for food materials. Food Hydrocolloid. 6:479-487.

Okiyama, A., M. Motoki, and S. Yamanaka. 1993. Bacterial cellulose IV. Application to processed foods. Food Hydrocolloid. 6:503-511.

Pecoraro, E., D. Manzani, Y. Messaddeq, and S. J. L. Ribeiro. 2008. Bacterial cellulose from Glucanacetobacter xylinus: preparation, properties and applications. In: Monomers, Polymers and Composites from Renewable Resources, ed. M. N. Belgacem and A. Gandini, 369-383. Oxford: Elsevier.

Phisalaphong, M., N. Jatupaiboon, and I. Kingkaew. 2010. Biosynthesis of cellulose chitosan composite. In: Chitin, Chitosan, Oligosaccharides and Their Derivatives, ed. S. K. Kim, 53-65. New York: CRC Press.

Phunsri, A., P. Tammarate, W. Krusong, and S. Tantratian. 2003. The liquid/air interface area and depth of liquid medium suitable for cellulose production from Acetobacter TISTR 975. J. Sci. Res. Chulalongkorn Univ. 28(1):35-43.

Sanchavanakit, N., W. Sangrungraungroj, R. Kaomongkolgit, T. Banaprasert, P. Pavasant, and M. Phisalaphong. 2006. Growth of human keratinocytes and fibroblasts on bacterial cellulose film. Biotechnol. Prog. 22:1194-1199.

Sangok, B., S. Yasushi, and S. Makoto. 2004. Improvement of bacterial cellulose production by addition of agar in a jar fermenter. J. Biosci. Bioeng. 97:33-38.

Takaaki, N., K. Tohru, Y. Hisato, and Y. Fumihiro. 1998. Effect of ethanol on bacterial cellulose production in continuous culture from fructose. J. Ferm. Bioeng. 85:598-603.

Verschuren, P. G., T. D. Cardona, M. J. R. Nout, K. D. De Gooijer, and J. C. Van den Heuvel. 2000. Location and limitation of cellulose production by Acetobacter xylinum established from oxygen profiles. J. Biosci. Bioeng. 89(5):414-419.

White, D. G., and R. M. Brown, Jr. 1989. Prospects for the commercialization of the biosynthesis of microbial cellulose. In: Cellulose and Wood-Chemistry and Technology, ed. C. Schuerch, 573-590. New York: John Wiley \& Sons.

Zeng, X., D. Small, and W. Wan. 2011. Statistical optimization of culture conditions for bacterial cellulose production by Acetobacter xylinum BPR 2001 from maple syrup. Carbohyd. Polym. 85:506-513.

Agricultural Statistic Bureau - Philippine, http:/ / www.da.gov.ph/agribiz/coconut1. html (accessed 10-2011).

Food and Agriculture Organization of the United Nations, http://faostat.fao.org/ site/339/default.aspx (accessed 10-2011).

Philippine Trade Training Center (PTTC), August 18, 2011, http:/ /www.pttc.gov.ph/ component/content/article/12-latest-news /221-coco-sector-posts-gains-fromphilippine-fta-markets.html (accessed 10-2011).

United Coconut Associations of the Philippines (UCAP) Newsbrief, March 2010, http:/ / www.ucap.org.ph/031810.htm\#wk11nw1 (accessed 10-2011).

Coconut Market Information Center (CMIC), http://coconutmic.com/en/marketinformation/export-and-import/102-performance-of-philippines-top-nontraditional-coco-exports-in-february-2010 (accessed 10-2011).

SIPUK - Bank Sentral Republik Indonesia, http:/ / www.bi.go.id/sipuk/en/?id=4\&n $\mathrm{o}=52301 \& \mathrm{idrb}=46501$ (accessed 10-2011). 



\title{
Wound Dressings and Cosmetic Materials from Bacterial Nanocellulose
}

\author{
Stanislaw Bielecki, Halina Kalinowska, Alina Krystynowicz, \\ Katarzyna Kubiak, Marek Kołodziejczyk, and Manu de Groeve \\ Institute of Technical Biochemistry, Technical University of Lodz, Lodz, Poland
}

\section{CONTENTS}

Performance Requirements of Wound Dressings.......................................... 158

Properties of BNC Dressings ...................................................................... 160

BNC Dressings with Antimicrobial Properties ………………………….... 164

BNC Dressings with Antioxidant and Protease-Binding Activities......... 166

Bioresorbable BNC Wound Dressings....................................................... 167

Nanocomposite BNC Dressings................................................................ 167

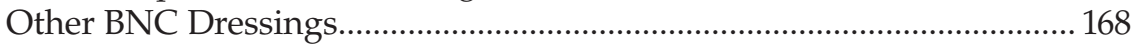

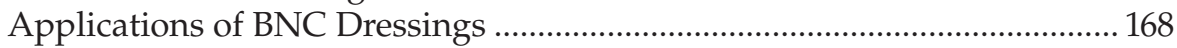

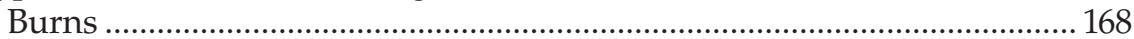

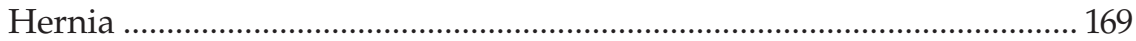

Venous Leg Ulcers .................................................................................... 170

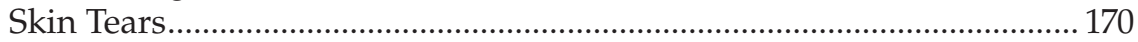

Cosmetic Applications of Bacterial Cellulose ………................................... 170

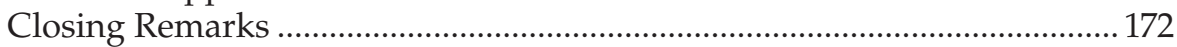

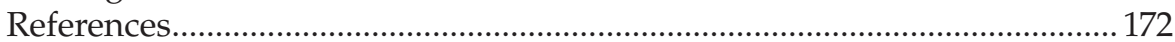

Bacterial nanocellulose (BNC) has proven to be a versatile biomaterial that can be used to fabricate excellent wound dressings that promote rapid and virtually painless wound healing as well as cosmetics with moisturizing and antiaging activities. It is a natural, nontoxic, and stable hydrogel with excellent affinity to connective tissue. Properties of microbial nanocellulose are the same as those of ideal wound care dressings: (1) it is biocompatible and causes neither toxic nor allergic side effects when remaining in long-term contact with living tissues; (b) it displays high water-holding capacity (up to 100 times its dry mass) and maintains a moist environment at the wound surface, which in turn prevents additional tissue loss from dehydration, stimulates the action of lytic enzymes that remove residual debris in the early stages of wound 
healing, speeds up restoration of the wounded tissue to its normal state, and significantly reduces pain; (3) it is highly porous, absorbable, and capable of donating moisture while simultaneously absorbing exudates; (4) it conforms to any shape of wound and is very light and transparent, but forms a mechanically durable and tight physical barrier to microbial contamination and additional injuries while providing gaseous exchange; (5) it allows easy and painless removal of wound coverings; (6) it is sterilizable, nondegradable in mammalian systems, flexible, elastic, and available in any size and shape; (7) it can be infused with other therapeutic substances without deterioration of its inherent features; (8) its properties can be tailored to individual needs through deliberate changes in culture conditions of bacterial producers, their genetic modifications, or postculture treatments of the polymer; and (9) it forms homogeneous blends with other biocompatible polymers like alginate, hyaluronic acid, or soluble cellulose derivatives. BNC wound dressings have been successfully used in the treatment of acute and chronic wounds. BNC has also been applied as a scaffold material in skin grafts and as a protective membrane in end-to-end nerve repair. Cosmetic uses of BNC include manufacturing of moisturizing facial masks and creams, peeling agents, fingernail polish, and as a base for artificial nails.

\section{Performance Requirements of Wound Dressings}

The basic function of wound dressings is promotion of rapid wound healing with a focus not only on functional, but also cosmetic results. Modern wound dressings are designed to accelerate the major outcome of treatment, which is a fully reepithelialized wound, and reduce the related trauma and pain in patients.

A wound is defined as breakage in the continuity of skin (Enoch and Price 2004). It can affect the epithelial layer of skin or also the underlying dermis, subcutaneous fat, and even the muscle, nerves, and bone. Wounds are classified according to etiology (caused by burns, cuts, friction or shear force, pressure, disease, etc.), anatomical location, or duration of healing (acute and chronic wounds). Wound healing is a complex process involving various types of cells (connective tissue and immune system components) and controlled by a variety of signal molecules, such as growth factors, proteinases and their inhibitors, cytokines, and other inflammatory response mediators. Acute wound healing is a systematic cascade of overlapping but carefully regulated processes that requires the coordinated completion of a variety of cellular activities. These processes, which are triggered by tissue injury, involve four phases: hemostasis, inflammation, proliferation (repair), and remodeling. The healing of acute wounds usually takes a few weeks or months, while chronic wounds fail to heal within this period because of the 
permanent inflammatory state caused by high activity of destructive proteolytic enzymes such as metalloproteinases and elastase, and excessive amount of exudates. The latter can cause maceration of healthy tissue surrounding the wound and increase its surface area. Examples of chronic wounds are decubitus wounds (pressure sores); venous, arterial, and diabetic foot ulcers; and wounds caused by autoimmune diseases.

In general, modern dressings create and maintain a moist environment around wounds, which in the mid-1900s was found to be superior to keeping wounds dry and uncovered. Selection of wound dressings by clinicians is usually based on their in-use characteristics, including wear time, number of dressings used, time required to change dressings, absorbency, leakage, ease of application, conformability to the wound, and ease of removal (Davies and Rippon 2010). In 2007 the World Union of Wound Healing Societies defined performance requirements of an ideal wound dressing (shown in Table 8.1), taking into consideration outcomes that are of concern to patients, such as minimizing dressing-related trauma, pain, malodor, and discomfort (Thomas 2008).

Based on the impact on the wound healing process, the dressings were categorized as (1) passive (gauze or tulle), which just cover the wound; (2)

\section{TABLE 8.1}

Performance Requirements of the Ideal Wound Dressing

\section{Primary Requirements}

1. Free of toxic/irritant extractables.

2. Does not release particles/nonbiodegradable fibers into the wound.

3. Forms an effective bacterial barrier (effectively contains exudates or cellular debris to prevent the transmission of microorganisms into or out of the wound.

4. If self-adhesive, forms an effective water-resistant seal to the periwound skin, but is easily removed without causing trauma or skin stripping.

5. Maintains the wound and the surrounding skin in an optimum state of hydration (this implies the ability to function effectively under compression).

6. Requires minimal disturbance or replacement.

7. Provides protection to the periwound skin from potentially irritating wound exudates and excess moisture.

8. Produces minimal pain during application and removal as a result of adherence to the wound surface.

9. Maintains the wound at the optimum temperature and $\mathrm{pH}$.

\section{Secondary Requirements}

1. Possesses antimicrobial activity capable of containing localized infection.

2. Has odor absorbing/combating properties.

3. Has the ability to remove or inactivate proteolytic enzymes in chronic wound fluid.

4. Possesses hemostatic activity.

5. Exhibits wound cleansing (debriding) activity.

Reprinted with permission from Steven Thomas, "The role of dressings in the treatment of moisture-related skin damage." World Wide Wounds, http:/ / www.worldwidewounds.com/2008/ march/Thomas/Maceration-and-the-role-of-dressings.html (2008). 
interactive, which are moist and efficiently absorb exudates; and (3) bioactive, which are also moist and promote wound healing by decreasing the activity of destructive proteases (e.g., collagen-based dressings), releasing bioactive agents like antimicrobials, antibiotics, and growth factors (Vasconcelos and Cavaco-Paulo 2011). These bioactive wound dressings, which are used in the cleansing of necrotic tissues, contain immobilized proteolytic enzymes like trypsin or collagenase (Yudanova and Reshetov 2006). They may also carry antimicrobial agents.

Passive wound coverings require frequent changes that are usually painful and cause scab and skin stripping. Interactive and bioactive dressings are more likely to prevent dressing-related pain and discomfort in patients with exuding wounds. They are fabricated from natural polymers (e.g., chitin and chitosan, alginate, cellulose and its derivatives, keratin, elastin, fibrin, silk fibroin, collagen or its denatured form, gelatin, etc.) and synthetics (e.g., polyurethane, polypropylene, polytetrafluoroethylene [PTFE] or silicone-based) (Davies and Rippon 2010; Gupta et al. 2010; Vasconcelos and Cavaco-Paulo 2011). Recently the tendency to replace synthetic dressings with natural ones or to use their blends has been observed.

Carbohydrate polymers like cellulose are frequently used in the form of hydrogels, which have a three-dimensional (3D) network structure and can swell in aqueous media without dissolution. Plant cellulose hydrogels are obtained by using complex and environmentally unfriendly solvent systems (Chang and Zhang 2011), while bacterial nanocellulose (BNC) hydrogels are natural products derived through environmentally friendly stationary or agitated cultures of certain bacteria, among which Gluconacetobacter xylinus strains are considered to be the most efficient producers (Bielecki et al. 2002). BNC, which can be easily subjected to patient-oriented modifications, is an excellent matrix for fabrication of interactive and bioactive wound dressings.

\section{Properties of BNC Dressings}

BNC membranes have been used as natural polymeric wound care materials since the 1980s. They have been shown to meet the majority of performance requirements of an ideal wound dressing and to substantially accelerate the healing process in various types of wounds. In-use characteristics, patient-centered outcomes of BNC wound dressings, and their potential utilization methods are collected in Table 8.2. Sterile BNC membranes completely deprived of microbial cells, their fragments, and culture medium residues have been successfully used in the treatment of various acute and chronic wounds (Alvarez et al. 2004; Petersen and Gatenholm 2011; Solway et al. 2010). The suitability of BNC wound dressings for the treatment of hard-to-heal wounds is also claimed in patents and patent applications (Table 8.3). 


\section{TABLE 8.2}

The In-Use Characteristics, Patient-Centered Outcomes, and Potential Utilization Methods of BNC-Based Wound Dressings

In-Use Characteristics

1. Versatile, sterilizable, mechanically durable, available in any size and shape, can be used for treatment of various types of wounds.

2. Biocompatible, nonpyrogenic, cause neither toxic nor allergenic side effects.

3. Can be used as carriers of antimicrobial agents, debriding enzymes, or other agents, increasing the rate of wound healing.

4. Provides a moist environment around wounds, accelerating their healing.

5. Relatively long dressing wear time caused by high wound exudate holding capacity.

6. Easy to change or remove-saves nursing time and labor costs.

7. Translucent-allows monitoring of the process of wound healing between dressing changes.

8. Excellent hemostatic properties.

9. Attenuation of thrombogenicity.

10. Nonresorbable (however, specific modifications make them resorbable).

11. Conform to the contour of a wound irrespective of its shape and depth and thereby reduces the threat of harmful microbial contamination.

\section{Patient-Centered Outcomes}

1. Pain relief and patient comfort.

2. Reduces dressing-related trauma because of painless and easy application and removal.

3. Dressing changes are not frequent and do not cause stripping of the wound surface.

4. Eliminates maceration and stripping of the periwound skin.

5. Very light and flexible.

6. Reduces scarring and provides excellent cosmetic results.

\section{End of Life Cycle - Potential Utilization Methods}

1. BNC is fully biodegradable, so after the sell-by date the unused dressings can be subjected to enzymatic, microbial, or chemical saccharification.

2. Used wound dressings can be incinerated to produce heat or subjected to acid hydrolysis and resulting hydrolysates can be used to produce biogas.

Manufacturers of cellulose dressings recommend them for use as a temporary covering for the treatment of wounds, including pressure sores, skin tears, venous stasis, ischemic and diabetic wounds, second-degree burns, skin graft donor sites, traumatic abrasions and lacerations, and biopsy sites. Examples of over-the-counter BNC-based wound dressings are the products manufactured and marketed by the Brazilian multinational company Fibrocel under a number of trademarks such as Dermafill, BioFill, BioProcess, and Nexfill. They are said to be identical in performance, structure, and manufacturing process. Another manufacturer of microbial cellulose dressings is Xylos Corporation, which marketed them under trademarks such as XCell and Prima Cel ${ }^{\mathrm{TM}}$. Dressings that have been manufactured in Poland according to the technology developed by researchers from the Technical University of Lodz by using a highly efficient strain G. xylinus E25 have been marketed under the trademarks CelMatC and CelMatMG. 


\section{TABLE 8.3}

Selection of Patents and Patent Applications Related to the Use of Bacterial Cellulose in Fabrication of Wound Dressings

\begin{tabular}{|c|c|c|c|}
\hline Patent Description & Assignee & $\begin{array}{c}\text { Patent/Application } \\
\text { Number }\end{array}$ & Filed \\
\hline $\begin{array}{l}\text { Cellulose membrane and } \\
\text { method for manufacture } \\
\text { thereof }\end{array}$ & $\begin{array}{l}\text { University of } \\
\text { Western Ontario, } \\
\text { Canada }\end{array}$ & U.S. Patent 876,400 & June 1997 \\
\hline \multirow{3}{*}{$\begin{array}{l}\text { Microbial cellulose wound } \\
\text { dressing for treating } \\
\text { chronic wounds }\end{array}$} & \multirow{3}{*}{$\begin{array}{l}\text { Lohmann \& } \\
\text { Rauscher GmbH }\end{array}$} & U.S. Patent 7,390,499 & April 2002 \\
\hline & & U.S. Patent $7,704,523$ & April 2003 \\
\hline & & U.S. Patent $7,709,021$ & December 2003 \\
\hline $\begin{array}{l}\text { Microbial-derived cellulose } \\
\text { amorphous hydrogel } \\
\text { wound dressing }\end{array}$ & Xylos Corporation & $\begin{array}{l}\text { U.S. Patent } \\
\text { application } \\
10,345,394\end{array}$ & January 2003 \\
\hline $\begin{array}{l}\text { Microbial cellulose wound } \\
\text { dressing for treating } \\
\text { chronic wounds }\end{array}$ & Xylos Corporation & $\begin{array}{l}\text { U.S. Patent } \\
\text { application } \\
10,864,804\end{array}$ & June 2004 \\
\hline $\begin{array}{l}\text { Nanosilver-coated bacterial } \\
\text { cellulose }\end{array}$ & $\begin{array}{l}\text { Axcelon } \\
\text { Biopolymers } \\
\text { Corporation }\end{array}$ & $\begin{array}{l}\text { U.S. Patent } \\
\text { application } \\
12,226,669\end{array}$ & April 2007 \\
\hline $\begin{array}{l}\text { Photoactivated antimicrobial } \\
\text { wound dressing and } \\
\text { method relating thereto }\end{array}$ & $\begin{array}{l}\text { Lotec, Inc., DBA } \\
\text { Vesta Sciences Inc., } \\
\text { UT-Batelle, LLC }\end{array}$ & $\begin{array}{l}\text { U.S. Patent } \\
\text { application } \\
12,034,629\end{array}$ & February 2008 \\
\hline $\begin{array}{l}\text { Oxidized microbial cellulose } \\
\text { and use thereof }\end{array}$ & Xylos Corporation & U.S. Patent $7,709,631$ & January 2007 \\
\hline $\begin{array}{l}\text { A method of modification of } \\
\text { bacterial cellulose } \\
\text { membranes }\end{array}$ & $\begin{array}{l}\text { Technical University } \\
\text { of Lodz, Poland }\end{array}$ & $\begin{array}{l}\text { PL Patent } \\
\text { application 392,480 }\end{array}$ & June 2010 \\
\hline
\end{tabular}

The dominant advantages of BNC, such as biocompatibility, are discussed elsewhere in this book. Because BNC is not digested by body fluids, neither cellulose fibers nor products of their degradation are released into the wound; this has also been proven by microscopic observations (Mendes et al. 2009). Furthermore, when bacterial cellulose is brought into contact with blood, it attenuates thrombogenicity (Fink et al. 2010). This advantageous feature is rather unique, and therefore the development of nonthrombogenic biomaterials has been an objective of intensive research in recent decades.

The high water-holding capacity of BNC dressings enables maintenance of a moist environment at the wound surface. This in turn prevents additional tissue loss from dehydration. Humidity is known to stimulate the action of lytic enzymes, which remove residual debris in the early stages of wound healing, and speeds up restoration of the wounded tissue to its normal state.

BNC dressings are absorbable and capable of donating moisture while simultaneously absorbing exudates. It is of particular importance in the treatment of chronic wounds, as their exudates are known to display relatively high activity for proteolytic enzymes, which are responsible for 


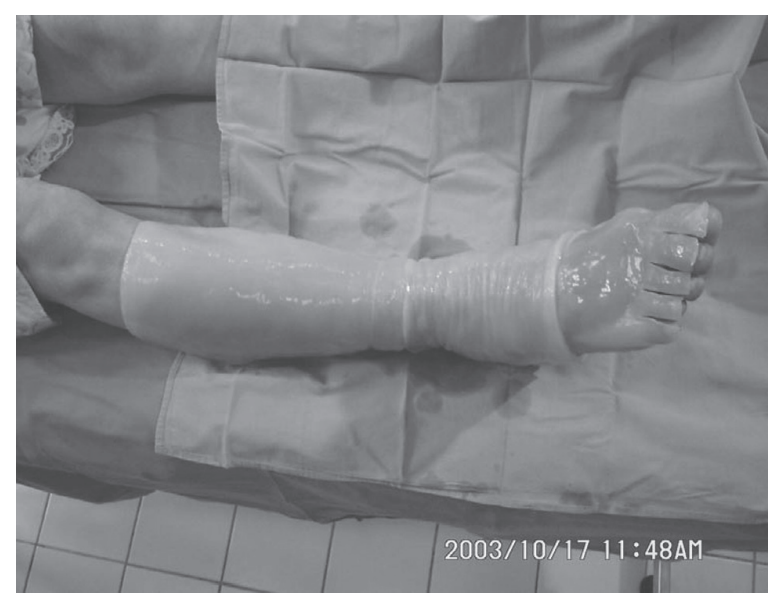

FIGURE 8.1

(See color insert.) BNC dressing.

detrimental degradation of proteins contained in the wound and healthy tissue around the wound. The activity of these proteases is not reduced by bacterial cellulose unless it is modified through incorporating collagen fibers (Wiegand et al. 2006).

BNC wound dressings are flexible and conform to the contour of a wound irrespective of its shape (Figure 8.1) and depth and thereby reduce the threat of harmful microbial contamination. They are available in any size and shape, and even large wounds can be covered by a single membrane. BNC membranes are very light and transparent, but form a mechanically durable and tight physical barrier to microbial contamination and additional injuries while providing gaseous exchange.

An interesting property of BNC membranes is that they interact with wounded and intact skin differently (Alvarez et al. 2004). The perimeter, being in contact with intact skin, desiccates and forms a thin cellophane-like sheet that adheres to the outermost layer of the epidermis (stratum corneum) while the central part of the dressing, which interacts with the wound, remains moist. This behavior prevents maceration of periwound skin caused by long-term exposure to moist dressings.

Application of BNC coverings significantly reduces wound-related pain. They do not adhere to the wound bed, so their changes and removal are very easy and painless. Furthermore, these dressings can be infused with other therapeutic substances without deterioration of their inherent features. Their characteristics can be tailored to a patient's needs through selection of appropriate bacterial producers (properties of BNC are strain dependent); their genetic modifications; changes in the culture conditions of these strains, enabling synthesis of diverse nanocellulose pellicles, beads, or dispersions; and various postculture treatments of nanocellulose. 
Microbial cellulose hydrogels can play not only the role of wound dressings, but they can also be used for transdermal delivering of biologically active agents penetrating through the stratum corneum (U.S. Patent application 11,406,528, assignee: Xylos Corporation, filed in April 2006). Controlled local drug release systems are known to offer many advantages over traditional delivery methods such as ingestion, injection, or inhalation, since they provide a constant drug concentration at the delivery site, lower drug levels in the patient, and reduced risk of harmful side effects. One of the most important parameters characterizing such systems is the rate of drug diffusion, which was found to be changed by $\gamma$-irradiation (Stoica-Guzun et al. 2007). Investigation of tetracycline permeation through irradiated (doses of either 5 or $15 \mathrm{kGy}$ ) and nonirradiated bacterial cellulose membranes showed that the diffusion was faster through the latter. The decrease in bacterial cellulose membrane permeability and adsorption capacity was caused by its shrinkage, and probably by formation of radiolysis products. Regardless of this subtle change in properties, $\gamma$-irradiated BNC hydrogels can be used as transdermal drug delivery systems.

\section{BNC Dressings with Antimicrobial Properties}

Antimicrobial properties of BNC-based wound dressings can be achieved by incorporation of appropriate compounds. Additives that are used for this purpose in wound management include silver-based compounds, chlorhexidine gluconate, benzalkonium chloride, parabens, and polyhexamethylene biguanide hydrochloride (PHMB). Some of these substances have been embedded in BNC hydrogels to achieve antimicrobial activity.

Bacterial cellulose-silver nanocomposites that are potentially applicable in wound treatment were obtained by various methods. To produce silver nanoparticles uniformly distributed throughout bacterial cellulose membranes, Maneerung et al. (2008) immersed them in silver nitrate solution and then the silver ions $\left(\mathrm{Ag}^{+}\right)$were reduced by sodium borohydride to $\mathrm{Ag}^{0}$. This process was controlled by measurements of absorbance at approximately $420 \mathrm{~nm}$, which is characteristic of silver nanoparticles, the size of which is affected by the $\mathrm{NaBH}_{4}: \mathrm{AgNO}_{3}$ ratio. Also, X-ray diffraction provided evidence of silver nanoparticle formation. The impregnated BNC membranes were then freeze-dried. They showed strong activity against Escherichia coli and Staphylococcus aureus. Although dried BNC membranes are known to display a much lower water absorption performance and swelling ratio than the never-dried ones, they are superior in terms of convenience, stability, and portability. The freeze-dried silver nanoparticle-impregnated membranes fabricated by Maneerung et al. (2008) displayed high swelling ability, as they absorbed 62.25 times their own weight in water after immersion in deionized water for $4 \mathrm{~h}$. This high swelling ratio provides evidence that the freeze-dried bacterial cellulose maintains its porous 3D structure and can suck the molecules of water into capillaries within the network of nanofibers more easily. 
To impregnate colloidal silver submicron particles into bacterial cellulose, Maria et al. (2009) soaked them in $\mathrm{AgNO}_{3}$ solution and then treated them with reducing agents (hydrazine, hydroxylamine, or ascorbic acid) in solutions of either polyvinylpyrrolidone or gelatin used as colloid protectors. The resulting composites were dried and subjected to ash content assay and thermogravimetric analysis to determine the level of silver loading. However, neither the swelling capacity nor antibacterial activity of these membranes was tested.

Pinto et al. (2009), who fabricated nanocomposites of silver and bacterial or plant cellulose, applied two different methods of reduction of $\mathrm{Ag}^{+}$ions. Bacterial cellulose membranes were immersed in $\mathrm{AgNO}_{3}$ solution and then treated with either an excess of sodium borohydride or ultraviolet (UV) radiation. The resulting nanocomposites displayed bacteriostatic effect against Bacillus subtilis, S. aureus, and Klebsiella pneumonia at silver nanoparticle concentrations of $5.0 \times 10^{-4} \mathrm{wt} \%$. Their antimicrobial activity increased with the concentration of $\mathrm{Ag}^{0}$ nanoparticles.

Fabrication of nanosilver-coated bacterial cellulose displaying antimicrobial activity is also described in U.S. Patent application 12,226,669 (Table 8.3). To obtain the composite material, a suspension of cellulose fibers was first oxidized to form dialdehyde cellulose fibers, after which a functional group (e.g., thio-) was incorporated. The functionalized cellulose fibers were then exposed to an aqueous solution of silver compound (e.g., silver proteinate), causing the formation of nanosilver particles that were bound to the functionalized cellulose fibers. These nanosilver particles were further grown by exposing them to a solution containing a second silver compound (e.g., silver ammonia). The silver-coated bacterial cellulose displayed antimicrobial activity towards E. coli and S. aureus.

Another U.S. Patent application (12,034,629; Table 8.3) describes a photoactivated BNC wound dressing with antimicrobial activity made of bacterial cellulose hydrogel membrane containing immobilized photocatalytic particles. The membrane displays antimicrobial activity when the photocatalytic particles are exposed to light, which triggers the generation of reactive oxygen species capable of reacting with microbes and killing them. The method is based on photocatalytic oxidation using metal oxide catalysts like $\mathrm{TiO}_{2}$ that generate species like hydroxyl radical, hydrogen peroxide, and superoxide. The $\mathrm{TiO}_{2}$-impregnated $\mathrm{BNC}$ membrane was made by adding a $\mathrm{TiO}_{2}$ suspension to a cleaned bacterial cellulose membrane in a vacuum filtration unit.

Wei et al. (2011) used benzalkonium chloride to fabricate freeze-dried BNC membranes with antimicrobial properties destined for certain practical applications such as the treatment of acute traumas. For this purpose, BNC membranes synthesized by a G. xylinus strain were thoroughly purified to eliminate bacterial cells and other impurities, cut into disks (a diameter of $15 \mathrm{~mm}$ ), freeze-dried for $24 \mathrm{~h}$, and soaked for $24 \mathrm{~h}$ in benzalkonium chloride solutions (concentrations ranging from $0.003 \%$ to $0.102 \%$ ). The nonabsorbed benzalkonium chloride was removed from the BNC disks by a $10 \mathrm{~s}$ immersion in distilled water and wiping with filter paper. The resulting films 
were freeze-dried for $24 \mathrm{~h}$ and then analyzed for swelling ratio and antibacterial activity against strains of E. coli, S. aureus, and Bacillus subtilis. They were found to absorb at least 26.2 and 37.3 times their own weight of water and saline, respectively, within $24 \mathrm{~h}$. Drug upload capacities ranged from 0.0291 to $0.145 \mathrm{mg} / \mathrm{cm}^{2}$ and stable antimicrobial effect was observed against Gram-positive bacteria (S. aureus and B. subtilis) for at least $24 \mathrm{~h}$.

Described in U.S. Patent 7,704,523 (Table 8.3), microbial cellulose wound dressings containing 2700 ppm of PHMB displayed antimicrobial activity against bacteria like E. coli and S. aureus and fungi like Aspergillus niger and Candida albicans. PHMB is a disinfectant commonly used in hospitals, with a broad spectrum of activities against Gram-negative and Gram-positive bacteria, fungi, and protozoa. It ionically binds to cellulose nanofibers. The resulting dressings are effective in the treatment of chronic wounds that are particularly prone to microbial infections.

Good antibacterial and barrier properties as well as high mechanical durability and moisture retention were achieved when bacterial cellulose was modified with chitosan (Gupta et al. 2010). The obtained composite material was found to be an excellent wound dressing, displaying very good bioactivity, biocompatibility, and biodegradability. It was applicable in the management of burns, bedsores, skin ulcers, and other chronic wounds, and accelerated wound healing without any symptoms of irritation or allergy.

Another method of manufacturing BNC dressings with bactericidal activity is proposed in PL Patent application 392,480 (Table 8.3). The purified BNC membranes are oxidized with sodium periodate to generate aldehyde groups between $\mathrm{C} 2$ and $\mathrm{C} 3$ of glucose residues that enable covalent binding of lysozyme, which degrades bacterial cell wall peptidoglycan.

A potential method of modification of bacterial cellulose wound dressings, making them resistant to microbial adhesion, consists of covalent immobilization of antimicrobial peptides (Costa et al. 2011). Such peptides were linked to many polymers, including plant cellulose.

\section{BNC Dressings with Antioxidant and Protease-Binding Activities}

As was mentioned earlier, bacterial cellulose has no impact on the activity of proteolytic enzymes, which are particularly high in exudates from chronic wounds such as venous, pressure, and diabetic ulcers (Wiegand et al. 2006). Such wounds persist in the inflammatory state and fail to heal because of elevated activity of destructive proteases, proinflammatory cytokines, and reactive oxygen species. One of the methods used in the treatment of such chronic wounds is the application of dressings containing collagen fibrils, which display antioxidant activity and decrease the activity of enzymes such as elastase from polymorphonuclear granulocytes (PMN elastase) and matrix metalloproteases (MMPs) produced by macrophages. The first enzyme participates in wound debridement and combating microbial infections, however, its elevated activity is harmful to healthy tissue surrounding the wound. 
Incorporation of insoluble fibrous proteins like collagen into the more porous underside of growing microbial cellulose pellicles is a difficult task, but it is achievable during G. xylinus stationary cultures. Their integration into the underside of these pellicles was achieved only when the distance between collagen fibers at the flask bottom and the air-liquid interface (i.e., the site of active cellulose synthesis) was as small as possible. The freeze-dried pellicles (called collagen BC) were $5 \mathrm{~mm}$ thick and displayed antioxidant capacity. They scavenged reactive oxygen species such as peroxynitrite anion $\left(\mathrm{ONOO}^{-}\right)$ and superoxide anion $\left(\mathrm{O}_{2}^{-}\right)$. Furthermore, they reduced the concentration of interleukin 6 and 8 in a time-dependent manner, and bound matrix metalloprotease MMP-2, but had no effect on the concentration of MMP-13.

One of the chemical modifications of BNC that has the potential to be used in the fabrication of protein-containing wound dressings consists of its phosphorylation, which proceeds more efficiently than phosphorylation of plant cellulose (Oshima et al. 2011). Due to the nanofibrillar structure and large specific surface area, the phosphorylated BNC exhibited larger adsorption capacity for certain enzymes (e.g., lysozyme) compared with its phosphorylated plant counterpart. Although phosphorylated BNC has not yet been used in the manufacture of wound dressings, it seems to be a promising carrier of enzymes or other proteins, like collagen.

\section{Bioresorbable BNC Wound Dressings}

The resistance of BNC to digestion by human body fluids is thought to be a disadvantage in certain applications, including wound healing and tissue regeneration, and, in particular, in subcutaneous uses (Hu and Catchmark 2011). One of approaches to production of bioresorbable BNC wound dressings consists of the binding of one or more cellulose degrading enzymes. Before adsorption of tested commercial enzymes on bacterial cellulose it was lyophilized to retain its nanostructure. The product containing immobilized cellulases was freeze-dried again to ensure its long-term stability during the storage. Hydration of this product caused activation of BC-embedded enzymes. It occurred in the contact with wound exudates and body fluids. To achieve complete $\mathrm{BC}$ hydrolysis to glucose it was necessary to immobilize a cellulase optimally active at slightly acidic $\mathrm{pH}$ and $\beta$-glucosidase.

Another method of obtaining bioresorbable microbial cellulose suitable for medical and surgical applications is based on its oxidation by sodium periodate followed by air drying as described in U.S. Patent 7,709,631 (Table 8.3). Properties of this material, such as mechanical stability and degradation rate, can be easily changed depending on the desired application.

\section{Nanocomposite BNC Dressings}

Nanocomposite materials reinforced with bacterial cellulose became very popular because of their attractive mechanical properties. Some of them are 
used as effective temporary wound coverings. For instance, bacterial cellulose was used to fabricate biocompatible, biodegradable, strongly hydrated and transparent nanocomposites containing poly(L-lactic acid) (Kim et al. 2009) or poly(3-hydroxybutyrate) (Zhijiang and Guang 2011). One of potential medical applications of these nanocomposites is manufacturing of wound dressings. Also, bacterial cellulose-alginate composite sponges have the potential to be used as wound dressings (Chiaoprakobkij et al. 2011). These biocompatible sponges, which are used as mucosal flaps in oral tissue regeneration, were fabricated using a freeze-drying process. Because of good tear resistance for sewing, great mechanical strength, stability in water, and excellent water uptake ability, these nonadherent bacterial cellulose-alginate hydrogels can be used as wound dressings.

\section{Other BNC Dressings}

According to U.S. Patent 876,400 (Table 8.3), strong and elastic cellulose films, having mechanical properties superior to plant derived cellulose membranes and useful as wound and burn dressings, can be prepared from a solution of cellulose produced by Acetobacter xylinum in a stirred tank. The polymer is then dissolved in a solvent system of dimethylacetamide and lithium chloride. The resulting solution is cast onto a flat surface and a film is regenerated in a gelation bath. After incorporation of a humectant by solvent exchange into the film, it is sterilized and packaged for long-term storage.

An amorphous bacterial cellulose hydrogel useful for treating chronic burns and wounds is described in U.S. Patent application 10,345,394 (Table 8.3). This hydrogel has similar fluid-handling capability as the wound dressing described in the patents mentioned earlier. Its flowable nature allows the dressing to fill areas that an ordinary pad cannot effectively treat and, as a result, wound healing is improved.

\section{Applications of BNC Dressings}

Microbial cellulose wound dressings are versatile and accelerate the repair of various kinds of wounds. The most intensively investigated uses are briefly described here.

\section{Burns}

The classification of burns, which are caused by too high temperature or chemical agents, is based on their depth. The deepest burns (third degree) often result not only in destruction of the epidermis and dermis, but also the subcutaneous tissue, and usually require the application of skin grafts. 
The most important aims of the treatment of burns are pain alleviation, prevention of wound desiccation and body fluids loss, prevention of microbial infection, and minimizing or preventing excessive scarring.

Nanostructured microbial cellulose is particularly well suited for the treatment of burns, irrespective of their degree and wound surface area (Bielecki et al. 2002). It not only substantially reduces pain by heat absorption and maintains moisture around the injury, but also stimulates the processes of necrotic tissue removal and granulation tissue development. Clinical trials have revealed that BNC dressings considerably shorten scab demarcation and burn healing processes, give excellent cosmetic outcomes, and are very well accepted by patients.

\section{Hernia}

Hernia is defined as the protrusion of an organ or the fascia of an organ through the wall of the cavity that normally contains it. Most commonly it develops in the abdomen. Postherniation abdominal wall repair was found to have better outcomes when mesh scaffolds were used (Melman et al. 2011). These scaffolds are synthetic (polypropylene, polyester, or expanded PTFE), composite (synthetic meshes covered by a protective membrane against adhesion to bowels), or biological (grafts harvested from human or animal dermis, pericardium, or intestinal submucosa). Studies on animal models have shown that bacterial cellulose membranes with incorporated conventional polypropylene hernia mesh (Figure 8.2) act as biocompatible scaffolds, protecting the site from postsurgery complications (Kołodziejczyk et al. 2010). The sterilizable prosthetic meshes with incorporated BNC neither provoke inflammation nor necrosis. They stimulate angiogenesis and are rapidly overgrown with host tissue, thereby accelerating the patient's recovery. Moreover, they significantly limit the level of adhesions of internal organs, thus reducing the feeling of internal discomfort and pain.

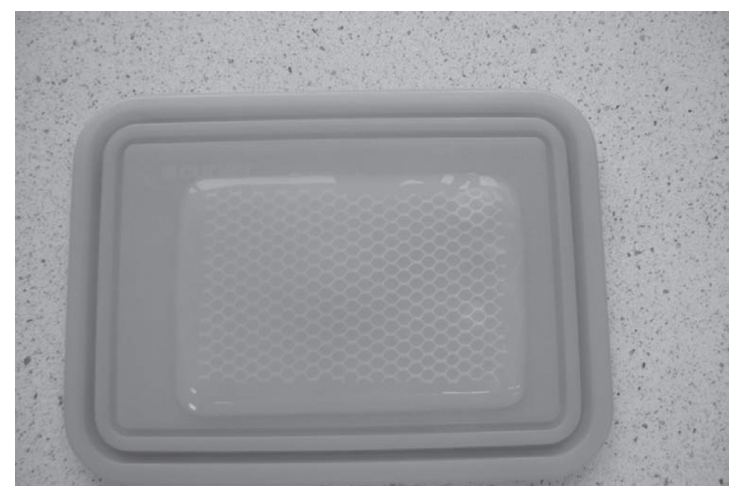

FIGURE 8.2

(See color insert.) BNC-coated hernia mesh. 


\section{Venous Leg Ulcers}

Venous leg ulcers, caused by chronic venous insufficiency and venous hypertension, rank high among chronic skin abnormalities (Alvarez et al. 2004). Their treatment with never-dried BNC dressing was more effective as compared with standard care in terms of faster autolytic debridement and a decrease in wound area and wound pain. The odor of microbial cellulose dressing with absorbed wound exudates was mild and disappeared when the dressing was removed.

\section{Skin Tears}

Skin tears are partial-thickness wounds resulting from fragile dermal and epidermal attachment (Solway et al. 2010). They are particularly frequently encountered in elderly nursing home residents. Treatment of these painful wounds in patients, who are usually characterized not only by their advanced age, but also sensory and cognitive impairment, immobility, and chronic diseases, requires the application of nonadherent dressings to minimize pain during removal and ensure rapid wound closure. A randomized study involving 27 residents of a nursing home suffering from category II and III skin tears treated with Dermafill bacterial cellulose membrane, which was covered with a protective stockinette, revealed the excellent properties of this dressing. The control group ( 24 residents) was treated with standard dressings (gauze secured with a secondary dressing) that required changing every 3 days, while in most of the 27 patients only one application of a bacterial cellulose pad was necessary. It should be noted that the latter did not adhere to the wound and underwent biodegradation with epithelialization. This study proved that in terms of pain control, ease of use, and satisfaction of patients, the BNC dressing was superior to the standard dressing. The healing time of skin tears was the same for both types of dressings.

\section{Cosmetic Applications of Bacterial Cellulose}

Nanofibrillated bacterial cellulose, with its high degree of hydration (approximately 99\%), is used as an ingredient in moistening cream capable of penetrating skin pores and carrying other active agents (Ioelovich 2008). BNC also acts as an efficient peeling agent in clarifying scrubs. Dispersions of BNC are known to modify rheology, stabilize suspensions, and act as reinforcing agents in nanocomposites. Therefore these dispersions are used as a component of a fingernail polish and a base for artificial nails. Cosmetic applications of bacterial cellulose also include nanofiber facial masks. 


\section{TABLE 8.4}

Selection of Patents and Patent Applications Related to the Use of Bacterial Cellulose in Fabrication of Cosmetic Materials

\begin{tabular}{|c|c|c|c|}
\hline Patent Description & Assignee & $\begin{array}{l}\text { Patent/ } \\
\text { Application } \\
\text { Number }\end{array}$ & Filed \\
\hline $\begin{array}{l}\text { Composition in the form of an oil-in-water } \\
\text { emulsion containing cellulose fibrils, and its } \\
\text { uses, especially cosmetic uses }\end{array}$ & L'Oreal & $\begin{array}{l}\text { U.S. Patent } \\
6,535,071\end{array}$ & May 2000 \\
\hline $\begin{array}{l}\text { Assembly comprising a substrate comprising } \\
\text { biocellulose, and a powdered cosmetic } \\
\text { composition to be brought into contact with the } \\
\text { substrate }\end{array}$ & L'Oreal & $\begin{array}{l}\text { U.S. Patent } \\
\text { application } \\
12,165,924\end{array}$ & July 2008 \\
\hline $\begin{array}{l}\text { Personal cleansing compositions comprising a } \\
\text { bacterial cellulose network and cationic polymer }\end{array}$ & $\begin{array}{c}\text { Procter \& } \\
\text { Gamble }\end{array}$ & $\begin{array}{l}\text { U.S. Patent } \\
\text { application } \\
12,854,340\end{array}$ & August 2010 \\
\hline
\end{tabular}

Because they easily fit to the skin, BNC facial masks can act as a carriers of substances that nourish, moisturize, revitalize, and bleach the skin, providing elasticity, softness, and smoothness. An example of BC-based facial masks is a product known as Bio Nano Cellulose Mask, produced by Green Mountain Co., Ltd., from Taiwan.

Patents and patent applications related to cosmetic uses of BNC are collected in Table 8.4. Two of them were filed by L'Oreal. U.S. Patent 6,534,071 describes the use of commercially available bacterial cellulose (CELLULON ${ }^{\circledR}$, Kelco) to make oil-in-water emulsions for cosmetic applications. The advantages of cellulose fibrils used for this purpose are much greater stability and better cosmetic and physicochemical properties of the resultant oil-in-water emulsions. Furthermore, BNC irritates neither skin nor eyes.

U.S. Patent application 12,165,924 (Table 8.4) describes the use of bacterial cellulose for facial treatment masks consisting of hydrated cellulose produced by A. xylinum on which powdered cosmetic compositions are homogeneously distributed and dissolved. Interestingly, bacterial cellulose was able to dissolve the powders much faster than an acrylic hydrogel. This patent application describes the use of facial masks for exfoliant and brightening effects (when the powder contains salicylic acid, ascorbic acid, citric acid, and sodium bicarbonate), for purifying effects (hot clay masks are obtained when the powder contains green clay, kaolin, and magnesium sulfate), and for antiwrinkle patches (containing lyophilized thermal plankton powder). In each case, powders containing active ingredients for specific applications are spread onto the hydrated cellulose film and immediately applied to the face.

U.S. Patent application 12,854,340 (Table 8.4), filed in 2010 by Procter \& Gamble, describes a personal cleansing composition (e.g., facial scrub) containing a bacterial cellulose network. Cellulose, which was obtained from 
Kelco (AxCEL CG-PX or CELLULON®), was used as an external structurant together with a cationic polymer such as derivatized quaternized hydroxyethyl cellulose esters, guar gum, and cationic polysaccharide polymers. The cationic polymer is believed to improve the cellulose network structure so that larger amounts and more dense particulates can be suspended. The benefit of such a composition is that it provides good lathering and can be easily rinsed off without producing an undesirable slimy or filmy feel.

\section{Closing Remarks}

Microbial cellulose is a natural nanostructured polymer characterized by numerous advantageous attributes that make it applicable in many areas, including health care and cosmetics. This biocompatible, nontoxic, nonallergenic, and biodegradable polysaccharide that can be easily produced from inexpensive nutrient sources by G. xylinus and some other bacteria has been shown to be one of the most patient-friendly wound dressings as well as an efficient moisturizing and suspension stabilizing agent in cosmetics production.

\section{References}

Alvarez, O. M., M. Patel, J. Booker, and L. Markowitz. 2004. Effectiveness of a biocellulose wound dressing for the treatment of chronic venous leg ulcers: results of a single center randomized study involving 24 patients. Wounds 16:224-233.

Bielecki, S., A. Krystynowicz, M. Turkiewicz, and H. Kalinowska. 2002. Bacterial cellulose. In: Biopolymers, vol.5, ed. A. Steinbuchel, 37-90. Weinheim: Wiley-VCH.

Chang, C., and L. Zhang. 2011. Cellulose-based hydrogels: present status and application prospects. Carbohyd. Polym. 84:40-53.

Chiaoprakobkij, N., N. Sanchavanakit, K. Subbalekha, P. Pavasant, and M. Phisalaphong. 2011. Characterization and biocompatibility of bacterial cellulose/alginate composite sponges with human keratinocytes and gingival fibroblasts. Carbohyd. Polym. 85:548-553.

Costa, F., I. F. Carvalho, R. C. Montelaro, P. Gomes, M. C. L. Martins. 2011. Covalent immobilization of antimicrobial peptides (AMPs) onto biomaterial surfaces. Acta Biomater. 7:1431-1440.

Davies, P., and M. Rippon. 2010. Comparison of foam and hydrocolloid dressings in the management of wounds: a review of the published literature. World Wide Wounds, http:/ / www.worldwidewounds.com/2010/July/DaviesRippon/DaviesRippon. html 
Enoch, S., and P. Price. 2004. Cellular, molecular and biochemical differences in the pathophysiology of healing between acute wounds, chronic wounds and wounds in the aged. World Wide Wounds, http://www.worldwidewounds. com/2004/august/Enoch/Pathophysiology-Of-Healing.html

Fink, H., L. Faxalv, G. F. Molnar, K. Drotz, B. Risberg, and T. L. Lindahl. 2010. Real-time measurements of coagulation on bacterial cellulose and conventional vascular graft materials. Acta Biomater. 6:1125-1130.

Gupta, B., R. Agarwal, and M. S. Alam. 2010. Textile-based smart wound dressings. Indian J. Fibre Text. 35:174-187.

$\mathrm{Hu}$, Y., and J. M. Catchmark. 2011. Integration of cellulases into bacterial cellulose: toward bioabsorbable cellulose composites. J. Biomed. Mater. Res. B Appl. Biomater. 79B:114-123.

Ioelovich, M. 2008. Cellulose as a nanostructured polymer: a short review. BioResources 3:1403-1418.

Kim, Y., R. Jung, H.-S. Kim, and H.-J. Jin. 2009. Transparent nanocomposites prepared by incorporating microbial nanofibrils into poly(L-lactic acid). Curr. Appl. Phys. 9:569-571.

Kołodziejczyk, M., A. Krystynowicz, H. Kalinowska, T. Pankiewicz, Z. Pasieka, B. Grobelski, K. Kowalska, P. Lawniczak, and S. Bielecki. 2010. The application of microbial cellulose in reconstructive surgery. Mil. Pharm. Med. 3:62-65.

Maneerung, T., S. Tokura, and R. Rujiravanit. 2008. Impregnation of silver nanoparticles into bacterial cellulose for antimicrobial wound dressing. Carbohyd. Polym. 72:43-51.

Maria, I. C. D., A. L. C. Santos, P. C. Oliveira, H. S. Barud, Y. Messadeq, and S. J. I. Ribeiro. 2009. Synthesis and characterization of silver nanoparticles impregnated into bacterial cellulose. Mater. Lett. 63:797-799.

Melman, L., E. D. Jenkins, N. A. Hamilton, L. C. Bender, M. D. Brodt, C. R. Deeken, S. C. Greco, M. M. Frisella, and B. D. Matthews. 2011. Early biocompatibility of crosslinked and non-crosslinked biologic meshes in a porcine model of ventral hernia repair. Hernia 15:157-164.

Mendes, P. N., S. C. Rahal, O. C. M. Pereira, Jr., V. E. Fabris, S. L. R. Lenharo, J. F. de Lima-Neto, and F. da Cruz Landim-Alvarenga. 2009. In vivo and in vitro evaluation of an Acetobacter xylinum synthesized microbial cellulose membrane intended for guided tissue repair. Acta Vet. Scand. 51:12-19.

Oshima, T., S. Taguchi, K. Ohe, and Y. Baba. 2011. Phosphorylated bacterial cellulose for adsorption of proteins. Carbohyd. Polym. 83:953-958.

Petersen, N., and P. Gatenholm. 2011. Bacterial cellulose-based materials and medicinal devices: current state and perspectives. Appl. Microbiol. Biotechnol. 91:1277-1286.

Pinto, R. J. B., P. A. A. P. Marques, C. P. Neto, T. Trindade, S. Daina, and P. Sadocco. 2009. Antibacterial activity of nanocomposites of silver and bacterial or vegetable cellulosic fibers. Acta Biomater. 5:2279-2289.

PLPatent application 392,480. A method of modification of bacterial cellulose membranes.

Solway, D. R., M. Consalter, and D. J. Levinson. 2010. Microbial cellulose wound dressing in the treatment of skin tears in the frail elderly. Wounds 22:17-19.

Stoica-Guzun, A., M. Stroescu, F. Tache, T. Zaharescu, and E. Grosu. 2007. Effect of electron beam irradiation on bacterial cellulose membranes used as transdermal drug delivery systems. Nucl. Instrum. Meth. B 265:434-438.

Thomas, S. 2008. The role of dressings in the treatment of moisture-related skin damage. World Wide Wounds, http:/ / www.worldwidewounds.com/2008/march/Thomas/ Maceration-and-the-role-of-dressings.html 
U.S. Patent 876,400. Cellulose membrane and method for manufacture thereof.

U.S. Patent 6,534,071. Composition in the form of an oil-in-water emulsion containing cellulose fibrils, and its uses, especially cosmetic uses.

U.S. Patent 7,390,499. Microbial cellulose wound dressing for treating chronic wounds.

U.S. Patent 7,704,523. Microbial cellulose wound dressing for treating chronic wounds.

U.S. Patent 7,709,021. Microbial cellulose wound dressing for treating chronic wounds.

U.S. Patent 7,709,631. Oxidized microbial cellulose and use thereof.

U.S. Patent application 10,345,394. Microbial-derived cellulose amorphous hydrogel wound dressing.

U.S. Patent application 10,864,804. Microbial cellulose wound dressing for treating chronic wounds.

U.S. Patent application 11,406,528. Microbial cellulose materials for use in transdermal drug delivery systems, method of manufacturing thereof.

U.S. Patent application 12,034,629. Photoactivated antimicrobial wound dressing and method relating thereto.

U.S. Patent application 12,165,924. Assembly comprising a substrate comprising biocellulose, and a powdered cosmetic composition to be brought into contact with the substrate.

U.S. Patent application 12,226,669. Nanosilver-coated bacterial cellulose.

U.S. Patent application 12,854,340. Personal cleansing compositions comprising a bacterial cellulose network and cationic polymer.

Vasconcelos, A., and A. Cavaco-Paulo. 2011. Wound dressings for a proteolytic-rich environment. Appl. Microbiol. Biotechnol. 90:445-460.

Wei, B., G. Yang, and F. Hong. 2011. Preparation and evaluation of a kind of bacterial cellulose dry films with antibacterial properties. Carbohyd. Polym. 84:533-538.

Wiegand, C., P. Elsner, U.-C. Hipler, and D. Klemm. 2006. Protease and ROS activities influenced by a composite of bacterial cellulose and collagen type I in vitro. Cellulose 13:689-696.

World Union of Wound Healing Societies. 2007. Principles of Best Practice: Minimizing Pain at Wound Dressing-Related Procedures. A Consensus Document. Toronto: WoundPedia.

Yudanova, T. N., and I. V. Reshetov. 2006. Modern wound dressings: making and properties. II Wound dressings containing immobilized proteolytic enzymes (a review). Pharm. Chem. J. 40:430-434.

Zhijiang, C., and Y. Guang. 2011. Optical nanocomposites prepared by incorporating bacterial cellulose nanofibrils into poly(3-hydroxybutyrate). Mater. Lett. 65:182-184. 


\title{
Bacterial Nanocellulose Hydrogels Designed as Bioartificial Medical Implants
}

\author{
Dieter Klemm, ${ }^{1}$ Hannes Ahrem, ${ }^{2}$ Friederike Kramer, ${ }^{2}$ \\ Wolfgang Fried, ${ }^{1}$ Jens Wippermann, ${ }^{3}$ and Raimund W. Kinne ${ }^{4}$ \\ ${ }^{1}$ Polymet Jena e.V., Jena, Germany \\ ${ }^{2} J e n p o l y m e r$ Materials Ltd. \& Co. KG, Jena, Germany \\ ${ }^{3}$ Klinik für Herz- und Thoraxchirurgie - Universitätsklinik zu Köln, Köln, Germany \\ ${ }^{4}$ AG Experimentelle Rheumatologie, Lehrstuhl für Orthopädie, Universitätsklinikum Jena, \\ Waldkrankenhaus "Rudolf-Elle" GmbH, Eisenberg, Germany
}

\section{CONTENTS}

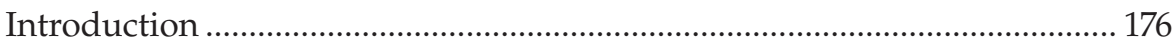

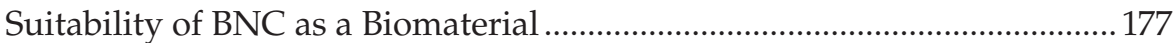

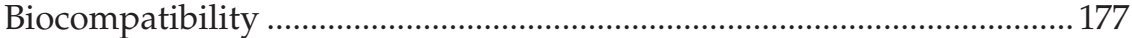

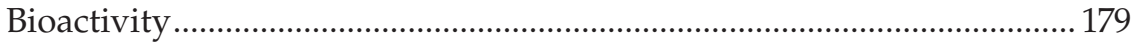

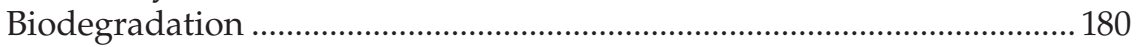

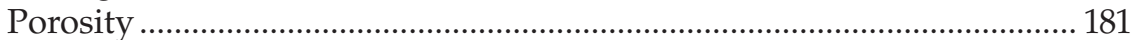

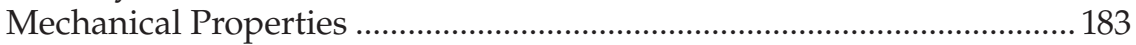

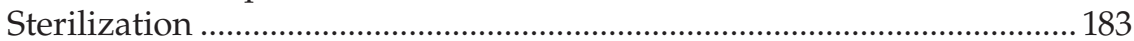

Controlled Biotechnological Production of BNC.................................... 184

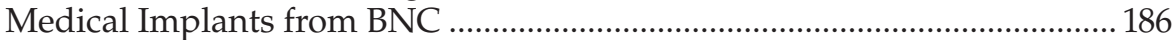

BNC as an Implant Material for the Regeneration of Chondral

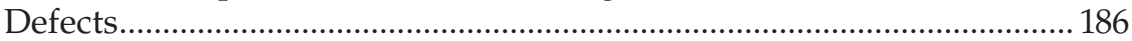

BNC as a Vascular Graft Material ............................................................. 189

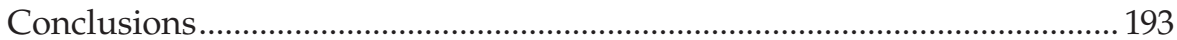

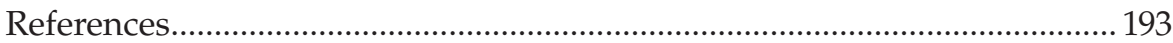

The outstanding structure and properties of biofabricated bacterial nanocellulose (BNC) hydrogels allow the design of novel types of bioactive medical implants in an effective way. In particular, the hierarchical three-dimensional nanofiber network, which is similar to collagen, the high water content, the control of biotechnological production, and 
proven biocompatibility provide an excellent basis. This chapter assembles current knowledge in the research, development, and application of BNC implants regarding cardiovascular and joint diseases. The topics include the design of flat inlays for cartilage repair and tubular vascular grafts. Shaping, structure control, and composite formation directly during biosynthesis will be discussed in detail. The comprehensive characterization of the implants, including interaction with living cells and animal studies, are presented.

\section{Introduction}

The natural origin and hierarchical three-dimensional (3D) nanofiber network structure of bacterial nanocellulose (BNC), the unique state of a highly form-stable and nondegradable hydrogel (at least in mammalians), together with good mechanical properties and easy handling turn this special cellulose type into an exceptional polymer for applications in medicine, cosmetics, pharmaceutics, and cell cultivation. Particularly for use as medical implants, the high purity of BNC, a water content up to $99 \%$, and the analogy of its network architecture to that of the extracellular collagen matrix of the human body are exciting features. Furthermore, the work of recent years has highlighted that BNC fulfills the general requirements of an innovative biomaterial, including biocompatibility, bioactivity, porosity, stability, sterilizability, and excellent surgical handling.

The biotechnological production not only allows control of the network structure, but also above all, the 3D shaping and surface design of BNC implants. In postprocessing steps, a partial or total drying of the implants in a vacuum, by heating or hot pressing, but also by freeze-drying or solvent exchange, can be realized effectively. In addition, specific perforation of the material by channels with diameters up to $300 \mu \mathrm{m}$ can be reliably carried out. Larger diameters are not required for cell migration, but can be realized. By combining BNC with other polymers or inorganic compounds (such as hydroxyapatite)—during biosynthesis or after treatment-nanocomposites are formed or material surfaces can be coated with BNC. In addition, metals and metal oxides can be easily precipitated on the BNC nanofiber network and active agents of various kinds, such as pharmaceuticals and dyes, can be incorporated reversibly or irreversibly.

In this chapter the suitability of BNC as a biomaterial will be presented in detail. This should be of interest to readers from the polymer sciences and their applications, medicine, pharmaceutics, galenics, and cosmetics. Examples of flat BNC implants for cartilage repair and tubular BNC blood vessel grafts are described. 


\section{Suitability of BNC as a Biomaterial}

Biomaterials must fulfill certain properties to be suitable for use in medicine. Generally these requirements can be classified into the following broad features: biocompatibility, functionality, and sterilizability. These features will be considered for BNC with respect to applications in regenerative medicine. In particular, the functionality of BNC will be thoroughly discussed concerning the interdisciplinary aspects of bioactivity, biodegradation, porosity, and mechanical properties.

\section{Biocompatibility}

The interaction of a material with cells, tissue, or whole organisms without toxic or immunological effects may be regarded as biocompatibility (Bumgardner et al. 2008). The diversity of biomaterials used for medical devices is broad and the choice of a given biomaterial is driven by its desired function, anatomical location, and application time. The contact of biomaterials with body tissue may be only for a short time, up to 10-15 years, or even for the lifetime of a patient. This variance in biomaterial use led to the general definition that biocompatibility is "the ability of a material to perform with an appropriate host reaction in specific application" (Williams 1999). Evaluation of the biocompatibility of a material includes characterization of the material's properties (chemical composition, biodegradability, porosity, mechanical properties, and surface properties) as well as biological testing. In vitro biocompatibility tests offer reproducible and standardized conditions and are relatively cheap in comparison to in vivo tests. However, the in vitro performance of a biomaterial does not necessarily reflect its characteristics in vivo, because environmental factors such as complex interactions between the biomaterial and surrounding cells or tissues, biomechanical strain, as well as hormonal factors may be not considered in these assays.

For BNC, both in vitro and in vivo tests have been performed. Before the interest in $\mathrm{BNC}$ as a biomaterial in regenerative medicine and tissue engineering, either cellulose of plant origin, regenerated cellulose, or cellulose derivatives (e.g., cellulose acetate, cellulose phosphate) showed high biocompatibility with negligible foreign body reactions and immunological responses (Martson et al. 1998; Miyamoto et al. 1989). Because of the diverse properties of BNC, the in vivo interaction between the material and the adjacent tissues had to be characterized in detail and compared to that of cellulose materials of plant origin. For example, BNC membranes do not cause skin irritation and are therefore suitable as an artificial skin for temporary covering of wounds in human and veterinary medicine (Fontana et al. 1990; Jonas and Farah 1998; Klemm et al. 2001b). This is particularly true in view of their great mechanical strength in the wet state and substantial permeability for 
liquids and gases. In addition, Mello et al. (1997) pointed out the low foreign body reaction of BNC as a substitute material for the dura mater after implantation in dogs. Good biocompatibility was also described by Klemm et al. (2001b) for tubular-shaped hollow BNC grafts applied as prostheses for blood vessels. After replacement of the carotid artery in rats, there was a complete incorporation into the surrounding tissue without any macroscopically observable rejection reaction. Histological investigations indicate that the whole BNC tube was coated with connective tissue. No signs of "inappropriate inflammation" were detectable after 4 weeks of implantation (Klemm et al. 2001b). In addition, the in vivo biocompatibility of BNC was assessed by implanting flat-shaped pieces of BNC subcutaneously into rats for 1, 4, and 12 weeks (Helenius et al. 2006). Because of the absence of any chronic inflammatory response, multinuclear giant cells, fibrosis, or encapsulation, the authors considered BNC as a biomaterial with good biocompatibility for tissue engineering. Similarly, implantation of BNC in the lumbar subcutaneous tissue of Swiss albino mice for a maximum time of 90 days resulted in a low inflammatory response until 30 days postsurgery, but induced no chronic foreign body reaction, as indicated by the absence of multinuclear giant cells (Mendes et al. 2009). Finally, BNC was applied for nasal reconstruction in rabbits by elevating the dorsum of the nose (Lopes et al. 2009). $\mathrm{BNC}$ was used as a cartilaginous implant for 3 months and 6 months in 10 rabbits each. At both time points, a tendency for fragmentation of the BNC grafts was observed, accompanied with intense, acute inflammation and partially necrotic skin in some of the tested animals. Despite these limited complications, however, the authors considered BNC as biocompatible and a good candidate for rhinoplasty surgery.

The results from in vivo studies were supported by biological tests in vitro. Cytotoxicity tests (e.g., MTT assay, a colorimetric assay with 3-(4,5-dimethylthiazol-2-yl)-2,5-diphenyltertrazolium bromide) are mainly used to screen biomaterials for their potential of promoting death or damage at the cellular level by direct lysis or by fatally altering cellular metabolism (Bumgardner et al. 2008). Most studies regarding BNC as biocompatible have used standardized cell lines like L929 cells (Chen et al. 2009) and 3T3 cells (Cai and Kim 2010; Moreira et al. 2009), or cell lines isolated from native target tissues for regeneration purposes (e.g., mesenchymal stem cells [Gao et al. 2011], urine-derived stem cells [Bodin et al. 2010], smooth muscle cells [Backdahl et al. 2006], human umbilical vein endothelial cells [Recouvreux et al. 2011], osteoblast cells [Chen et al. 2009], or chondrocytes [Svensson et al. 2005]). The interaction of BNC with cells clearly depends on specific cell-type aspects such as their adhesion and proliferation capability. For example, Chen et al. (2009) observed that osteoblasts are more susceptible for cytotoxicity than standardized and commercially available L929 cells. The authors pointed out that tissue-derived functional cells are more suitable than cell lines to evaluate the cytotoxicity of a biomaterial with respect to its application site and its suitability for the regeneration of specific tissues or organs. 
Besides its cytocompatibility, the lack of contaminating bacterial or medium components derived from the biotechnological production process is an important criterion for the biocompatibility of BNC. An effective cleaning process for BNC is essential to remove substances that are associated with the biosynthesis and to prepare the hydrogels for implantation as a scaffold for tissue engineering. Impurities of the material may cause immunological responses and rejection reactions. In particular, lipopolysaccharides (LPS) from the outer membrane of Gluconacetobacter species induce systemic reactions by activation of monocytes (Langstein et al. 2000) and have to be removed from the material. The most common cleaning process after cultivation of BNC referred to in the literature is a treatment with aqueous sodium hydroxide $\left(0.1-1 \mathrm{M}\right.$ at $\left.60^{\circ} \mathrm{C}-120^{\circ} \mathrm{C}\right)$ (Grande et al. 2009; Helenius et al. 2006; Watanabe et al. 1993). A flat implant of defined size (e.g., $8 \mathrm{~mm} \times 8 \mathrm{~mm} \times 2 \mathrm{~mm}$ ) has to be cleaned under such conditions for 20-60 min. An additional treatment with the detergent sodium dodecyl sulfate has also been described (Svensson et al. 2005). Also, boiling BNC hydrogels in alkali sodium hydroxide solution leads to depyrogenation and effectively reduces the biological activity of LPS (Williams 2007).

\section{Bioactivity}

Bioactivity means that a biomaterial has a direct influence on the physiology and morphology of living cells by controlling their adhesion, migration, proliferation, differentiation, and release of extracellular matrix (ECM) molecules leading to the formation of new tissue. Several factors, such as structural and surface properties, the inclusion of composite partners, and/or the ability to release incorporated substances (e.g., growth factors, cytokines) contribute to the bioactivity of biomaterials. BNC shows a texture of irregular, randomly, and three-dimensionally oriented nanofibers that is very similar to that of the collagen network. This biomimetic structure of BNC - mimicking a body's own tissue-offers a good matrix for in vitro cell seeding prior to tissue engineering applications. Due to increased surface area and the possibility of 3D seeding, BNC both supports efficient adhesion of the cells and prevents dedifferentiation (Bodin et al. 2010). In addition, the BNC structure allows the mass transport of nutrients and oxygen to support the survival of cells. Finally, the BNC nanofibers may participate in guiding the orientation of the ECM molecules deposited by the cells.

Besides the structural aspects, the surface chemistry plays an important role in the bioactivity of BNC. Several authors have shown that chemical modification of the surface leads to augmented adhesion of cells. Modification of the BNC surface by phosphorylation and sulfation in order to mimic glucosaminoglycans of native cartilage, for example, increased the adhesion of bovine chondrocytes to the material, but did not augment their proliferation (Svensson et al. 2005). The finding that very strong adhesion prevents proliferation has also been confirmed by others (Sieminski and Gooch 2000). 
Another possibility to enhance the adhesion of cells is the coating of BNC surfaces with arginine-glycine-aspartic acid (RGD) peptides (Bodin et al. 2007a). To mimic ECM binding sites, xyloglycan bearing a peptide containing the RGD sequence was bound to BNC pellicles. Initial cell adhesion studies showed that the adhesion of endothelial cells was significantly higher on the RGD-modified samples. The RGD sequence has been found in proteins of the extracellular matrix, especially in fibronectin and vitronectin, and acts as a binding site of specific cell receptors like integrins. The RGD-related cell adhesion leads to a mechanical fixation of cells and generates cell-specific signals. This result was confirmed by Andrade et al. (2010), who showed that an RGD peptide, but not a glycine-arginine-glycine-aspartic acid-tyrosine (GRGDY) control peptide enhanced the adhesion of cultured fibroblasts on the BNC surface.

As an example for a composite structure of the biomaterial, nanocomposites of BNC and hydroxyapatite (HA) have been designed to mimic the natural composition of organic and inorganic compounds of bone (Jiang et al. 2007; Wan et al. 2007), which showed excellent biocompatibility and a complete lack of cytotoxicity (Grande et al. 2009).

\section{Biodegradation}

Hydrolytic degradation of cellulose can be catalyzed either chemically (e.g., by the action of acids) or enzymatically. Cellulose-degrading enzymes, called cellulases, are common in certain species of fungi and bacteria and enable them to convert cellulose to glucose monomers. The human body does not contain enzymes capable of catalyzing the hydrolysis of the $\beta-1,4$ linkages of cellulose chains, rendering plant cellulose or BNC biostable and nonresorbable, at least inside the human body. In vivo and in vitro investigations have been performed to evaluate the degradability of BNC with respect to medical applications. Since glucose as the potential degradation product is not detectable in vivo, recent studies have investigated the macroscopic and physical composition of the material after implantation in animals. No changes in the structure of BNC membranes were detected after implantation into Swiss albino mice for 90 days (Mendes et al. 2009). The same was reported by Helenius et al. (2006) after subcutaneous implantation of BNC membranes in rats for 12 weeks. In this study, no evidence for in vivo degradation was observed, but the authors pointed out that the implantation time may have been too short for detectable structural changes caused by degradation. In addition to the action of cellulases, cellulose could be degraded on the basis of an alteration of the highly ordered structure of the cellulose. The loss of high crystallinity and the accompanying formation of amorphous structures may cause interactions with chemically reactive substances and cleavage of the $\beta-1,4$ glycosidic bonds. Such degradation has been verified by measuring the concentration of reducing sugars in the supernatant of BNC by in vitro testing (Chen et al. 2009). BNC samples were 
stored in phosphate buffered saline (PBS) at $37^{\circ} \mathrm{C}$ for a period of 12 weeks, during which the structure built fuzzy aggregates or formed fragments via slow degradation. In contrast, Li et al. (2009) measured the mass loss of the samples and observed no degradation of BNC in vitro. Indeed, in contrast to biodegradable materials like polyglycolic acid, polylactic acid or copolymers of the two, BNC showed only negligible degradation.

It is presently unclear, whether bioimplants or scaffolds for tissue engineering should be degradable or not. Potential problems of degradable materials include nonsynchronized resorption and regeneration of new extracellular matrix resulting in compromised biofunctionality of the implant, and induction of subchronic inflammation in the surrounding tissue by an altered $\mathrm{pH}$ value or the release of solid particles. In contrast, nondegradable materials such as BNC may support adequate regeneration of defects by guiding cellular migration and providing mechanical stability. The result should be a composite of nonresorbable, highly porous $\mathrm{BNC}$ and the newly formed extracellular matrix. On the other hand, the degradation properties of BNC can be changed by oxidation to so-called 2,3-dialdehyde cellulose ( $\mathrm{Li}$ et al. 2009), leading to its rapid degradation in water, PBS, and simulated body fluid (SBF) at $37^{\circ} \mathrm{C}$, subsequent collapse of its porous structure, and loss of its mechanical stability.

\section{Porosity}

Often the migration of cells and the 3D distribution inside a tissue engineering scaffold or a cell-free implant are essential for regenerative aspects. Cells must have the possibility to enter the biomaterial structure in order to build a homogeneous extracellular matrix. Especially for nondegradable biomaterials, porosity is an important feature, because it allows cell immigration and offers a 3D scaffold during the entire regeneration process. In addition, nutrition and oxygen delivery are essential for growth, proliferation, and matrix biosynthesis. Particularly at implantation sites with no vascularization or angiogenesis during the regeneration process (e.g., cartilage), the porous structure must support diffusion of nutrients over distances of more than $1 \mathrm{~mm}$.

In its native hydrated state, BNC consists of more than $99 \%$ water and less than $1 \%$ solid cellulose (Klemm et al. 2011). Due to the extremely small volume fraction of the cellulose nanofibers, the porosity of BNC ranges from $92 \%$ to $94 \%$ (void volume in relation to total volume) (Sokolnicki et al. 2006; Tang et al. 2010). Nevertheless, the meshes of the texture vary between 1 and $20 \mu \mathrm{m}$ and are therefore mostly smaller than most of the cells. The dimensions of the meshes vary within the BNC hydrogel because of its layered, nonhomogeneous structure, preconditioned by the BNC biosynthesis. The middle layer of the BNC hydrogel body generated by static culture is relatively homogeneous and the dimensions of the meshes range from 1 to $10 \mu \mathrm{m}$. In contrast, the bottom part of the BNC hydrogel shows a wide-meshed structure 
(diameter up to $20 \mu \mathrm{m}$ ) with lower mechanical properties, whereas the structure on the upper surface is denser (mesh diameter $<1 \mu \mathrm{m}$ ) and carries aggregated fibers (Helenius et al. 2006; Tang et al. 2010). These structural features are strongly influenced by the bacterial strain, the composition of the culture medium, and the culture conditions. In addition, the features can be modified by adding solid or solubilized components during biosynthesis or by applying postprocessing steps (Andersson et al. 2010; Baeckdahl et al. 2008; Bodin et al. 2010; Klemm et al. 2011; Tang et al. 2010; Wan et al. 2007).

The layered structure of the BNC hydrogel body causes a location-dependent differential ingrowth of cells upon in vitro cell culture or in vivo after implantation. Indeed, Helenius et al. (2006) observed an immigration of cells on the wide-meshed layer on the bottom of the BNC fleece, whereas no cells infiltrated the dense network on the upper surface after implantation of BNC into rats for 12 weeks.

Our own in vitro results confirmed these findings, in that human chondrocytes seeded on the upper surface of the BNC hydrogels in serum-free Dulbecco's Modified Eagle Medium (DMEM) and subjected to a chemotactic gradient by $10 \%$ fetal calf serum (FCS) in transwell inserts for $24 \mathrm{~h}$ entered the $\mathrm{BNC}$, but only migrated to the border region between the bottom and the middle layer of the hydrogel (Figure 9.1a). In contrast, chondrocytes were not able to enter the material at the upper surface, even during cultivation up to 7 days (Figure 9.1b). The precise reasons for this differential behavior and the influence of the specific cell type investigated are currently the subject of intense discussion (Backdahl et al. 2006; Helenius et al. 2006; Wan et al. 2007).

Current efforts are directed at improving the network structure of BNC and thereby optimizing the immigration and distribution of the cells within the bulk material. One approach described an opening of the BNC structure by incorporation of solid porogen particles during biosynthesis (Baeckdahl et al. 2008) and subsequent leaching of the particles in order to obtain a
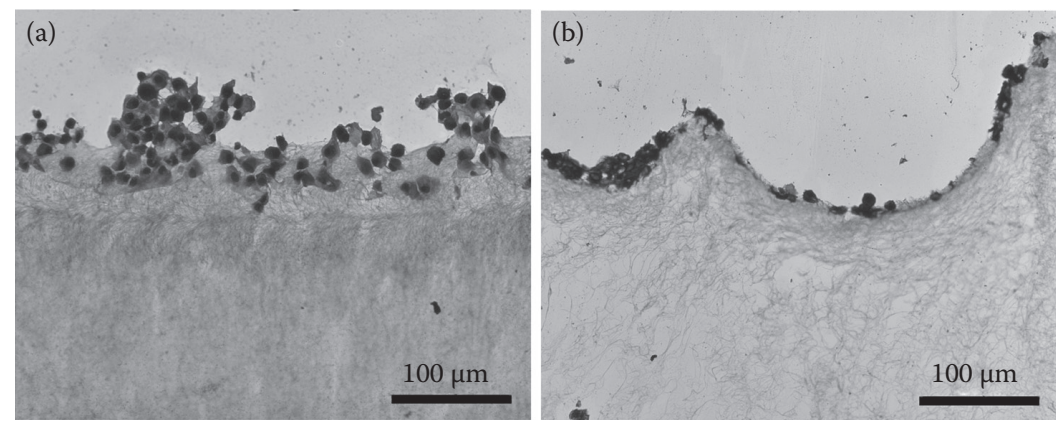

\section{FIGURE 9.1}

(See color insert.) Histological cross-section of the lower (a) and upper (b) surface of a BNC hydrogel seeded with human chondrocytes in a transwell system with serum gradient for $24 \mathrm{~h}$. While the more porous lower surface enables cell ingrowth up to $70 \mu \mathrm{m}$, the upper surface restricts cell migration into the bulk staining with hematoxylin and eosin. 
microporous and interconnected scaffold adequate for 3D migration of cells. This material allowed a homogeneous distribution of smooth muscle cells (Baeckdahl et al. 2008), osteoprogenitor cells (Zaborowska et al. 2010), human chondrocytes (Andersson et al. 2010), and urine-derived stem cells (Bodin et al. 2010) in the porous structure. Alternatively, a porous foam of BNC fiber aggregates was generated by disintegration of the BNC bodies and addition of a composite substance (e.g., polyethylene glycol [PEG] 400) (Gao et al. 2011). However, future studies have to address the delicate balance between enlarged pore size and sufficient mechanical strength.

\section{Mechanical Properties}

The modulus of elasticity, ultimate tensile strength, elongation to failure, and fracture toughness are important features describing the mechanical properties of biomaterials (Teoh 2004). In comparison to stiff materials like metals or ceramics, BNC in its native, hydrated state is a hydrogel with low modulus of elasticity and fracture toughness. While the single BNC fibers have the mechanical strength of steel or Kevlar (Klemm et al. 2011), the mechanical properties of the whole hydrogel are partially defined by its water content and the discharge of water during compression. The water retention capacity of a BNC hydrogel is influenced by the structural composition of the fiber network. Because of the anisotropic architecture of the fleeces, there are regions with different mechanical properties. The wide-meshed network of the bottom layer leads to lower water binding and reduced mechanical properties. Sufficient mechanical properties of the BNC biomaterial are pivotal for surgical fixation and the compressive and tribological stress experienced by the biomaterial at the site of implantation. Current studies therefore focus on comparing the biomechanical properties of $\mathrm{BNC}$ and body tissues or the formation of composite (e.g., double-network) gels.

When comparing the mechanical characteristics of BNC with those of pig meniscus and a collagen implant, BNC had a higher Young's modulus and compression modulus than the collagen matrix, but the meniscus material was stronger than BNC (Bodin et al. 2007b).

By soaking BNC hydrogels in gelatinous solution for an adapted period, the water is replaced by the composite substance and an additional cross-linking leads to a double-network gel with improved compression and tensile strength (Nakayama et al. 2004). Other network components, such as the polysaccharides sodium alginate or i-Carrageenan, also lead to the formation of double-network gels. A prominent feature of such double-network gels is their considerably improved mechanical stability.

\section{Sterilization}

Cellulose is thermostable up to at least $200^{\circ} \mathrm{C}$. Similar to cellulose of plant origin, the thermal degradation of $\mathrm{BNC}$ starts in the range of $200^{\circ} \mathrm{C}-400^{\circ} \mathrm{C}$ 

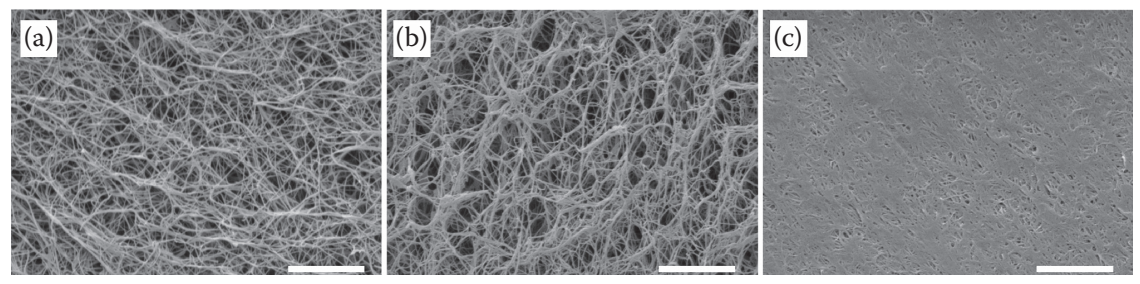

FIGURE 9.2

Scanning electron micrographs showing the effect of sodium hydroxide concentration on the network morphology of BNC during autoclaving conditions. Treating BNC hydrogels with (a) $1 \mathrm{M}$ and (b) $2 \mathrm{M}$ sodium hydroxide solution has no detectable effects on the network structure, while (c) treatment with $4 \mathrm{M}$ sodium hydroxide solution leads to shrinking of hydrogels and a dense structure. Pictures showing the cross-section of BNC after freeze-drying.

(Barud et al. 2007). BNC thus withstands the heat treatment necessary (scale bar $=5 \mu \mathrm{m}$ ) for sterilization techniques such as dry heat sterilization $\left(>140^{\circ} \mathrm{C}\right)$ or autoclaving $\left(121^{\circ} \mathrm{C}\right)$, the latter representing the most important and practical technique. It is possible to sterilize hydrogels in the initial wet or freeze-dried state (aerogels) without reswelling of the network structure. The latter aspect seems to be very important for the long-term storage and stability of BNC materials. In addition, it is important to note that steam sterilization has no detectable effect on the integrity and network structure of $\mathrm{BNC}$ in the wet or dry state.

Instead of water, aqueous sodium hydroxide can also be used as the fluid phase for sterilization of BNC hydrogels, thereby combining sterilization of the material with purification and removal of pyrogens. BNC can be treated with aqueous sodium hydroxide solution under autoclaving conditions up to a concentration of $3 \mathrm{M}$ without any shrinking and structural changing of the material (Figure 9.2).

Alternatively, BNC can theoretically be sterilized by treatment with ethylene oxide. To ensure contact of the complete BNC structure with the toxic gas, the material must be in a dry state and, for releasing toxic substances, an adapted gas emission time must follow. However, there are currently no studies on the effects of ethylene oxide treatment on BNC (e.g., chemical modification) and thus potential adverse effects of the highly reactive gas on the BNC structure cannot be completely excluded.

In contrast, gamma sterilization performed under standardized conditions ( $\geq 25 \mathrm{kGy}$ ) does not cause any macroscopically detectable changes of the hydrogel integrity, although systematic investigations concerning the effects of gamma sterilization on the features of BNC are currently unavailable.

\section{Controlled Biotechnological Production of BNC}

As already mentioned, BNC is biotechnologically produced from low molecular weight sugars such as glucose. For its application as a biomaterial, it is 
TABLE 9.1

Selected Material Characteristics of the Different BNC Samples (Wesarg et al. 2010)

\begin{tabular}{lcccc}
\hline & DSM 14666 & ATCC 53582 & ATCC 10245 & ATCC 23769 \\
\hline Degree of polymerization & 2761 & 1966 & 920 & 775 \\
Crystallinity (\%) & 90 & 88 & 86 & 84 \\
Tensile strength (MPa) & $0.340 \pm 0.020$ & $0.314 \pm 0.009$ & $0.184 \pm 0.028$ & $0.035 \pm 0.009$ \\
Compressive stress (MPa) & $2.658 \pm 0.092$ & $2.408 \pm 0.067$ & $0.434 \pm 0.102$ & $0.029 \pm 0.002$ \\
Water retention value (\%) & 642 & 626 & 235 & 250 \\
Storage modulus $\left(\mathrm{G}^{\prime}\right)(\mathrm{Pa})$ & $30,926 \pm 858$ & $26,286 \pm 2898$ & $15,282 \pm 1701$ & $1495 \pm 355$ \\
\hline
\end{tabular}

pivotal to exactly determine the relative importance of the different experimental factors on the structure and properties of BNC. This concerns both the reproducibility of the biofabrication and the tightly controlled fine tuning of specific features of the product. There are several relevant modules of the process chain: (1) composition of the culture medium; (2) design and mode of action of the bioreactor; (3) process parameters (e.g., temperature, time, gas concentration); and (4) postprocessing steps (e.g., drying, pressing, perforation, inclusion of composite partners).

Most importantly, the structure and properties of the BNC strongly depend on the choice of the cellulose-forming bacterial strain. The differences in the structure and properties of BNC fleeces are exemplified using four selected BNC-producing Gluconacetobacter strains: DSM 14666, ATCC 10245, ATCC 53582, and ATCC 23769 (DSM, Deutsche Sammlung von Mikroorganismen und Zellkulturen; ATCC, American Type Culture Collection) (Wesarg et al. 2010). The strains DSM 14666 and ATCC 53582, for example, produce stable hydrogel bodies. In contrast, the fleeces generated by ATCC 53582 and ATCC 23769 are significantly less stable. Such structural differences may significantly affect the material characteristics.

In Table 9.1 typical parameters of the BNC materials produced by the four different above-mentioned bacterial strains are compiled. Selected characteristics (tensile strength, compressive stress, water retention value, and storage modulus) are presented and compared to the degree of polymerization and the crystallinity of the respective products. BNC samples produced by DSM 14666 and ATCC 53582 are characterized by considerably higher values of tensile strength and compressive stress in comparison to those of the more labile samples produced by ATCC 10245 and ATCC 23769. Concordantly, the two stable BNC samples show a higher water retention value and a slightly higher crystallinity than the labile ones. The concurrently increased storage modulus $\mathrm{G}^{\prime}$ of the two stable BNC samples was interpreted by the authors as an indication of a correlation between augmented crystallinity and elasticity.

Since the determination of further material characteristics, and especially the Certification Experts (CE) certification of hydrogels such as BNC by conventional methods shows severe technical limitations, specific characterization techniques for hydrogels have been developed by the American Society 
for Testing and Materials (ASTM) International, Subcommittee F04.42 and have been published as a "Guide for Characterization of Hydrogels Used in Regenerative Medicine" (Industry Codes 2011).

\section{Medical Implants from BNC}

Its specific structure and properties render $\mathrm{BNC}$ highly suitable as a biomaterial for a wide spectrum of medical implants, comprising fleeces and films for inlays, patches and hollow cylinders for cardiovascular and other tubular grafts, and complex shapes for the replacement of complicated 3D organ structures. In the following two sections, examples are described for the development of flat BNC implants for joint cartilage repair, and tubular BNC materials for application as a blood vessel substitute. The examples include current work ranging from initial in vitro experiments to preclinical studies in large animals.

\section{BNC as an Implant Material for the Regeneration of Chondral Defects}

The specific properties of BNC discussed previously make this special cellulose hydrogel a promising candidate for the regeneration of joint cartilage. The nanofiber network of BNC can be colonized by cartilage cells without problems. Figure 9.3 shows cartilage cells on a BNC implant. The focus of the last few years has been to develop bioactive biomaterials for the repair and

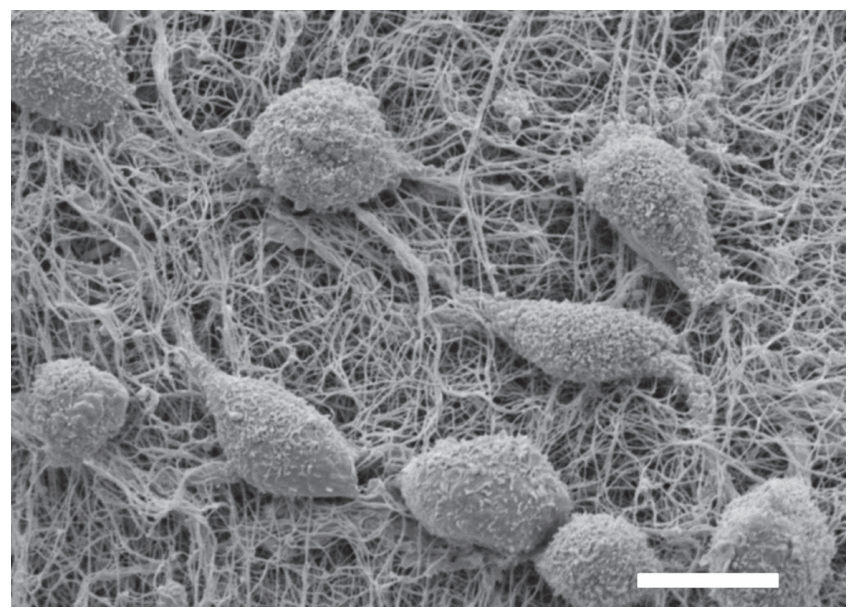

FIGURE 9.3

Scanning electron micrograph of human chondrocytes on the surface of BNC after $24 \mathrm{~h}$ cell seeding. (Scale bar $=10 \mu \mathrm{m}$.) 
regeneration of soft tissue that are designed to ensure mechanical stability immediately following implantation, the presence of attractants for the recruitment and migration of cells from the target tissue into the implant, and inclusion of differentiation factors promoting tissue maturation and integration.

To fulfill the requirements that are suitable to overcome problems associated with implants for cartilage regeneration, recent investigations have been aimed at developing either implants containing primary cells for tissue engineering approaches (Andersson et al. 2010; Svensson et al. 2005) or cell-free solutions (Endres et al. 2011). The primary aim of cell-free grafts is to overcome the disadvantages of established cell-containing implants used for matrix-associated autologous chondrocyte transplantation (MACT), such as multiple surgical procedures for cell harvesting and implantation, injury to healthy cartilage regions, high costs (in vitro expansion), and potential cell dedifferentiation or hypertrophy. In our own research, the cell-free implants are therefore focused on generating flat BNC scaffolds with cell-recruiting factors to attract cartilage cells and/or progenitor cells into the inlays and thereby induce the in situ formation of repair tissue for improved integration. Figure 9.4 shows the principle of cartilage repair by use of such a bioactive implant.

Using suitable bioreactor development and postprocessing techniques, the production of standardized BNC inlays with specific shape designs was established on a laboratory scale to produce cartilage regeneration materials. By modifying the pore size of the BNC, a colonization of the implants with cartilage cells was accomplished (bovine model) (Ahrem et al. 2010). In vitro studies resulted in the identification of bioactive substances able to stimulate directed migration (chemotaxis) and proliferation of cartilage cells. The biocompatibility of the BNC implant materials was confirmed by extensive in vitro and in vivo (sheep) experiments, including documentation of the first stages of cartilage regeneration in the in vitro tests. Prerequisites of a universally applicable BNC network are a permanent, scaffold-forming stability and quick and homogeneous seeding with chondrocytes in order to promote the regeneration of cartilage tissue.

Present prototypes of BNC implants consist of a wide-meshed phase and a narrow-meshed phase, both firmly connected (Klemm et al. 2009). Colonization of this BNC and transmigration was investigated using isolated human and bovine cartilage cells and the transwell system. Colonization of the wide-meshed BNC phase with the isolated chondrocytes was already observed after $24 \mathrm{~h}$ of the cultivation process, and chondrocyte migration into deeper layers of the BNC network was confirmed histologically. These layers showed a wide-meshed network with pore sizes well adapted to the size of the chondrocytes. In addition, cartilage defects in bovine cartilage discs were filled with preformed BNC implants to investigate the cell migration in vitro. After 4-8 weeks of culture, early stages of cartilage matrix regeneration were observed in the defect-filling BNC network. The integrity of the cartilage was preserved despite extended culture periods and immunohistochemical evidence for the deposition of intact aggrecan, and 
(a)

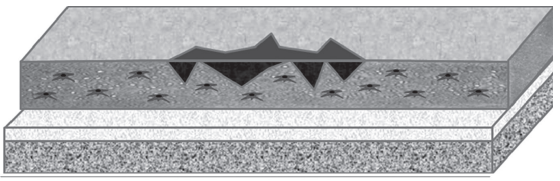

(b)

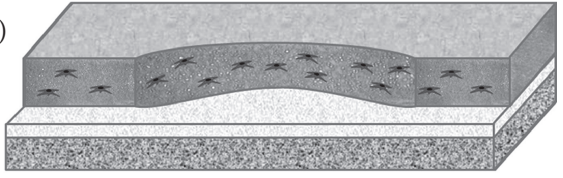

(c)

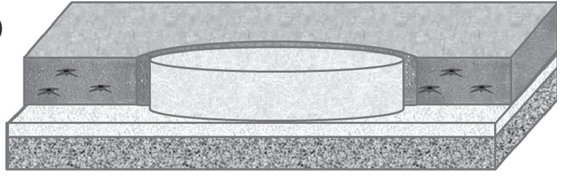

(d)

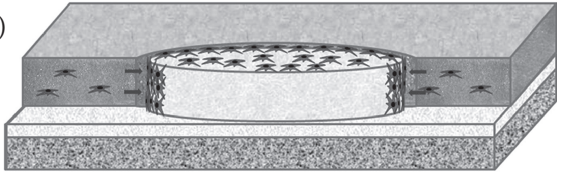

(e)

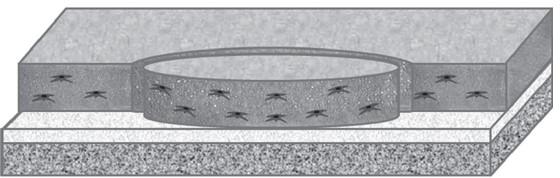

(f)

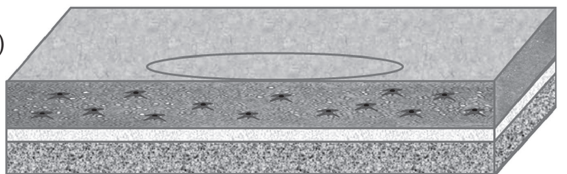

\section{FIGURE 9.4}

(See color insert.) Principle of cartilage repair by use of a bioactive implant. Schematic representation of a chondral defect in the joint cartilage (a). After removing the damaged areas, the cartilage defect is filled with the preformed BNC implant. (b,c) Cartilage and cartilage precursor cells migrate into this implant $(\mathrm{d}, \mathrm{e})$. The result of a complete colonization of the BNC inlay is (f) a regenerated cartilage layer.

collagen of type II in the BNC network was obtained in both nonstimulated and transforming growth factor $\beta 1$ (TGF- $\beta 1$ )-stimulated samples. In addition, chondrocytes migrated out of the cartilage and adhered to the surface of the BNC implant.

These results underline the principal biocompatibility and suitability of cell-free BNC implants for in situ cartilage regeneration and call for in vivo proof-of-concept studies in large animals. Preliminary results from animal experimental studies (sheep) can be seen in Figure 9.5. 


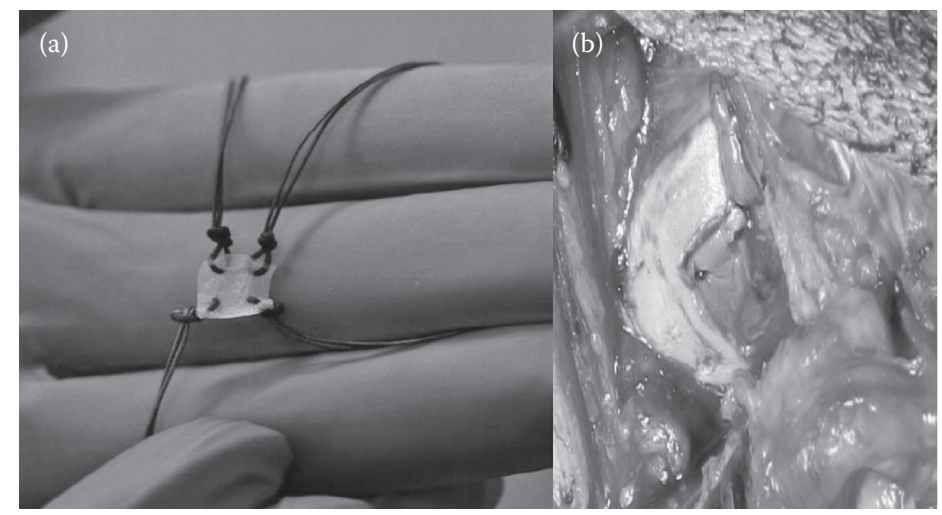

FIGURE 9.5

(See color insert.) (a) Photographs of BNC hydrogels designed for implantation in joint cartilage. (b) The implant after manual suturing to fix it in defined defects in the knee of a sheep.

\section{BNC as a Vascular Graft Material}

The suitability of BNC combined with the versatility of the biofabrication process make BNC a strong candidate for a bioactive implant material with a large scope of applications. The natural origin, hydrogel character, and bioactivity of BNC lead to some peculiarities. As already mentioned in the previous section, hydrogel implants, in particular BNC vascular implants, require new techniques for characterization. The standard International Organization for Standardization (ISO) techniques used for the characterization of common synthetic polymer implants made from polytetrafluoroethylene (PTFE) or polyesters (Dacron) cannot be transferred directly to the analysis of BNC. Also, since the in vivo activity of BNC indicates a strong interaction with the components of the living body and a distinct alteration of the prosthesis after implantation, characterization of ex vivo explants will have to be performed instead of or in combination with in vitro tests. Considerable research and development efforts by our interdisciplinary team of chemists, biologists, polymer and materials scientists, and surgeons have been directed at the design of blood vessel implants of different inner diameters and lengths, from microvessels up to vascular grafts with the potential to be used for coronary bypass.

Atherosclerotic vascular diseases, such as coronary artery disease and peripheral vascular disease, are still the leading cause of mortality in industrialized Western societies. Despite the widespread use of synthetic grafts in cardiovascular surgery, PTFE and Dacron may still induce a low-level foreign body reaction and chronic inflammation, and as artificial materials, they carry an increased risk for microbial infections. In addition, the development of BNC tubular implants represents an innovative approach to overcome reconstructive problems associated with extended vascular diseases, especially by providing small-caliber vascular grafts. 
It is a key advantage of BNC that the tubular implants can be produced directly during biofabrication. The type and construction of the bioreactor produce a specific design of nanofiber network structure, shape, and surface quality of the implant. The corresponding patented matrix technology (Klemm et al. 2001a) operates by forming the tubular implant on a matrix in contact with the culture medium. After removal from the bioreactor, and subsequent purification and sterilization, the tubes are ready to be used as stable vascular conduits for animal surgery. In addition, these tubes are suitable as training material for surgeons (e.g., in microsurgical techniques).

In a first microsurgical study, small BNC implants were attached to artificial defects of the carotid arteries of a rat for 1 year. These long-term results showed the incorporation of BNC tubes under a formation of neointima (the inner lining of an artery or vein or lymphatic vessel) and ingrowth of active fibroblasts (Klemm et al. 2001b, 2005, 2006). In a second study (Schumann et al. 2009), tubes of BNC were generated with a length of $10 \mathrm{~mm}$, an inner diameter of 3.0-3.7 mm, and a wall thickness of 0.6-1.0 mm. Shaping of the tube wall, as well as calibration of the inner diameter, is performed by the matrix technology using a glass mandril of the respective size. Whereas BNC tubes produced by this technology (BActerial SYnthesized Cellulose [BASYC]) are characterized by a smooth inner surface, common vascular grafts (Dacron and expanded PTFE [ePTFE]) show an increased roughness of the inner surfaces, as demonstrated by confocal laser scanning microscopy (LSM) (Figure 9.6). In the case of Dacron, single polyester filaments are visible (Figure 9.6a), while ePTFE shows channels caused by the expansion during manufacturing (Figure 9.6b). With its smooth surface (Figure 9.6e), BASYC closely resembles the inner wall of human arteries and veins (Figures 9.6c and $9.6 \mathrm{~d})$.

BASYC vascular grafts were used to replace the carotid arteries of eight pigs. After a follow-up period of 3 months, the grafts were removed and analyzed both macroscopically and microscopically. The aim of this study was to evaluate the new cylindrical BNC biomaterial as a potential bioactive scaffold for small arterial substitutes.

In detail, the animals were heparinized systemically with an intravenous bolus injection of 200 units $/ \mathrm{kg}$ body weight and the right carotid artery was carefully prepared. The target artery was then resected and the BNC graft was inserted by interrupted suture using 7-0 monofilament polypropylene (Prolene; Ethicon, Hamburg, Germany). Complete hemostasis occurred rapidly following a short period of oozing through the graft after declamping. To assess the patency of the grafts, flow measurement (MediStim AS, Oslo, Norway) was carried out at a mean blood pressure of $70 \mathrm{mmHg}$. After wound closure, all animals were allowed to grow for 3 months in a stable with free access to food and water. After 3 months the carotid artery with the implanted graft was harvested en bloc and the implants were evaluated for chronic inflammation, foreign body responses, cell ingrowth, and diameter using light and electron microscopy (LEO 1450 VP/REM, Oberkochen-Zeiss, Germany). 

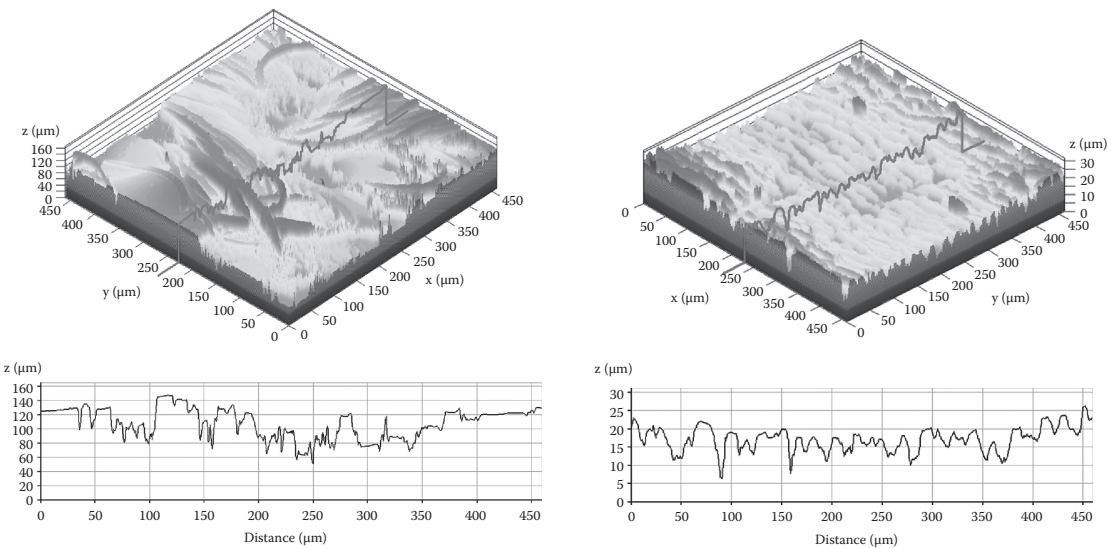

(a)

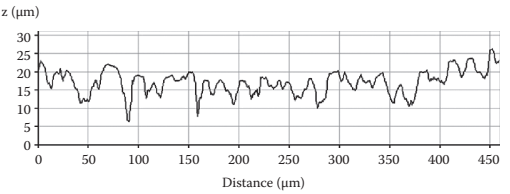

(b)
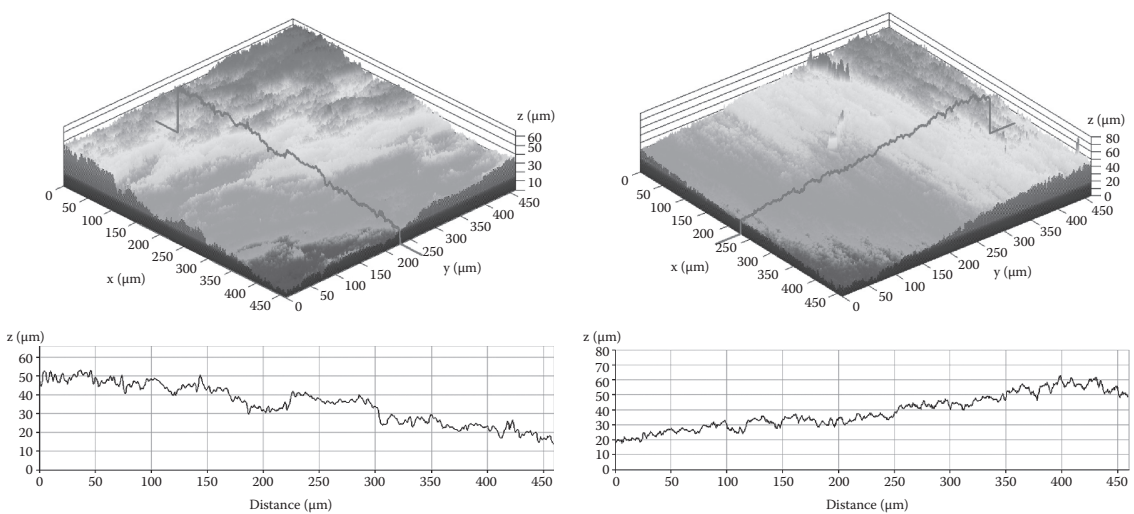

(c)

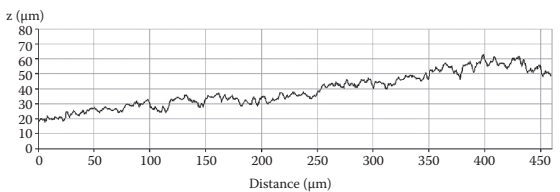

(d)
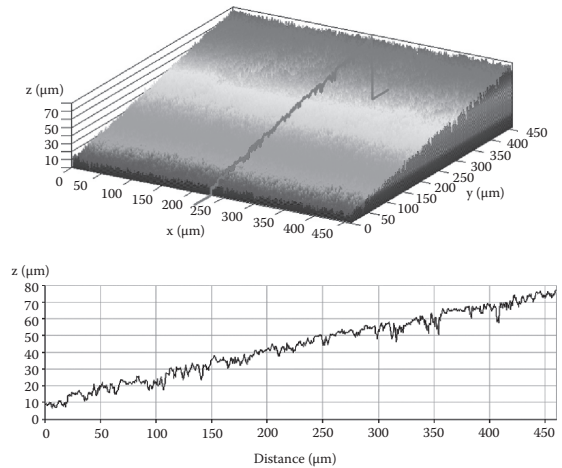

(e)

\section{FIGURE 9.6}

Confocal laser scanning microscopy images (magnification $\times 20$ ): (a) Dacron, (b) ePTFE, (c) human artery, (d) human vein, and (e) BASYC implants produced by matrix reservoir technology. Reprinted with permission from Dieter Schumann et al., Artificial vascular implants from bacterial cellulose: preliminary results of small arterial substitutes, Cellulose 16:877-885. 


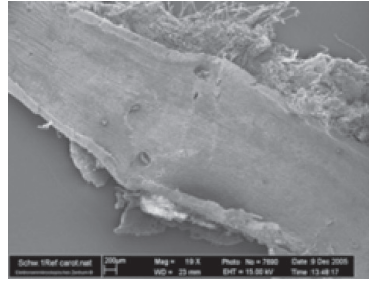

(a)

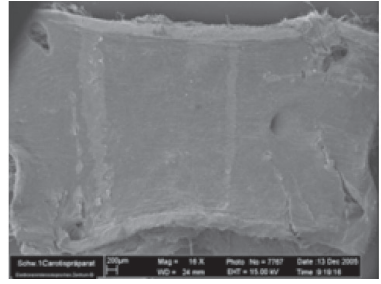

(b)

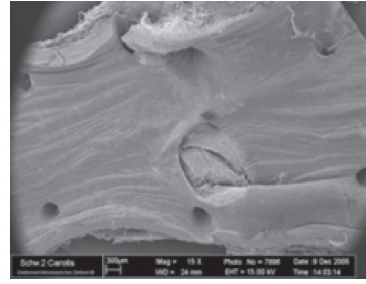

(c)

\section{FIGURE 9.7}

Scanning electron microscopy (magnification $\times 16-\times 19$ ): (a) untreated segment of the carotid artery, (b, c) good endothelialization of bacterial cellulose grafts. Reprinted with permission from Dieter Schumann et al., Artificial vascular implants from bacterial cellulose: preliminary results of small arterial substitutes, Cellulose 16:877-878.

After 3 months, seven grafts (87.5\%) were patent, whereas one graft was occluded. Scanning electron microscopy revealed complete recellularization of the inner surface by recipient endothelial cells (Figure 9.7). Light microscopy showed a three-layered wall structure of the BNC segments with the cellulose still being present. These data demonstrate the potential of engineered BNC grafts for small-caliber vessel substitution. As the innovative BNC engineering technique can provide stable small-caliber vascular conduits of any desired size and length, it offers a highly attractive approach in cardiovascular surgery. All the pigs survived after surgery. In summary, the following results were obtained:

- Paraplegia was not observed in any animal during the postoperative period.

- BNC demonstrated sufficient surgical properties for suturing and handling.

- Flow was restored with no leaks or oozing through the polymer after the anastomoses were completed in all animals.

- There were no significant differences in blood flow at the proximal and distal anastomoses after the initial implantation $(53.6 \pm 10 \mathrm{ml}$ and $53.7 \pm 8.5 \mathrm{ml})$.

- After 3 months a capsule-like tissue formation was observed in all animals.

- One graft was found collapsed and occluded, perhaps owing to the surrounding tissue.

- Seven grafts were patent (patency rate $87.5 \%$ ). There was no stenosis observed histologically at the proximal or distal anastomoses in any animal.

- No apparent changes in the graft, including dilatation, dehiscence, or aneurysmatic enlargement were detected. 
- There were no statistical differences in the diameter of the graft before and 90 days after surgery.

- There were no arteriovenous fistulae formations or signs of hematoma indicating bleeding from the grafts in the chronic course.

This study served as an evaluation of a novel bioengineered synthetic small-caliber cellulose graft using short grafts. Longer grafts need to be verified concerning stress resistance of the wall and shear resistance of the neointimal layer. Therefore, and as a work in progress, long segments of BNC tubes are under investigation as vascular grafts in the carotid artery of sheep (graft length approximately $10 \mathrm{~cm}$, bursting strength up to $1000 \mathrm{mmHg}$ ). Thus far the results demonstrate very good mechanical stability of the BNC grafts in the high blood pressure system under nonrestricted animal activities over 3 months.

\section{Conclusions}

The goal of this chapter was to deepen understanding and discuss recent knowledge in the field of BNC materials for applications as medical implants. The contribution is based on the cooperative work of a truly interdisciplinary team of polymer chemists, materials scientists, biologists, and physicians. The natural hydrogel BNC appears to provide substantial combinations of properties essential for a biomaterial applicable as an implant for crucial fields of medical care. Under proper control of the biotechnological formation of BNC, prototypes of inlays for the regeneration of articular cartilage and implants for blood vessels have been successfully produced. Detailed in vivo characterization of the performance of BNC implants (large animal studies) will be required, addressing in particular their long-term stability and their suitability to replace load-bearing cartilage or small-caliber blood vessels without significant thrombogenicity.

\section{References}

Ahrem, H., D. Pretzel, U. Udhart, and D. Klemm. 2010. Structure design for enhanced cell ingrowth in bacterial nanocellulose implants. Paper presented at the 239th American Chemical Society National Meeting, March 23-25, San Francisco, CA. Andersson, J., H. Stenhamre, H. Baeckdahl, and P. Gatenholm. 2010. Behaviour of human chondrocytes in engineered porous bacterial cellulose scaffolds. J. Biomed. Mater. Res. A 94A:1124-32. 
Andrade, F. K., S. M. G. Moreira, L. Domingues, and F. M. P. Gama. 2010. Improving the affinity of fibroblasts for bacterial cellulose using carbohydrate-binding modules fused to RGD. J. Biomed. Mater. Res. A 92A:9-17.

Backdahl, H., G. Helenius, A. Bodin, U. Nannmark, B. R. Johansson, B. Risberg, and P. Gatenholm. 2006. Mechanical properties of bacterial cellulose and interactions with smooth muscle cells. Biomaterials 27:2141-2149.

Baeckdahl, H., M. Esguerra, D. Delbro, B. Risberg, and P. Gatenholm. 2008. Engineering microporosity in bacterial cellulose scaffolds. J. Tissue Eng. Regen. Med. 2:320-330.

Barud, H. S., C. A. Ribeiro, M. S. Crespi, M. A. U. Martines, J. Dexpert-Ghys, R. F. C. Marques, Y. Messaddeq, and S. J. L. Ribeiro. 2007. Thermal characterization of bacterial cellulose-phosphate composite membranes. J. Therm. Anal. Calorim. 87:815-818.

Bodin, A., L. Ahrenstedt, H. Fink, H. Brumer, B. Risberg, and P. Gatenholm. 2007a. Modification of nanocellulose with a xyloglucan-RGD conjugate enhances adhesion and proliferation of endothelial cells: implications for tissue engineering. Biomacromolecules 8:3697-3704.

Bodin, A., S. Bharadwaj, S. Wu, P. Gatenholm, A. Atala, and Y. Zhang. 2010. Tissueengineered conduit using urine-derived stem cell seeded bacterial cellulose polymer in urinary reconstruction and diversion. Biomaterials 31:8889-8901.

Bodin, A., S. Concaro, M. Brittberg, and P. Gatenholm. 2007b. Bacterial cellulose as a potential meniscus implant. J. Tissue Eng. Regen. Med. 1:406-408.

Bumgardner J. D., M. Vasquez-Lee, K. S. Fulzele, D. H. Smith, K. D. Branch, S. I. Christian, and D. L. Williams. 2008. Biocompatibility testing. In: Encyclopedia of Biomaterials and Biomedical Engineering, 2nd ed., ed. G. E. Wnek and G. L. Bowlin, 169-178. New York: Informa Healthcare.

Cai, Z., and J. Kim. 2010. Bacterial cellulose/poly(ethylene glycol) composite: characterization and first evaluation of biocompatibility. Cellulose 17:83-91.

Chen, Y. M., T. Xi, Y. Zheng, T. Guo, J. Hou, Y. Wan, and C. Gao. 2009. In vitro cytotoxicity of bacterial cellulose scaffolds used for tissue-engineered bone. J. Bioact. Compat. Pol. 24:137-145.

Endres, M., T. Rüdiger, R. W. Kinne, H. Ahrem, D. Klemm, A. A. Hegewald, C. Thomé, A. Arshi, U. Merklein, U. Klinge, A. Fiebeler, S. Schneemelcher, and C. Kaps. 2011. Bioactive implants for the repair and regeneration of soft tissue. Project numbers 13N9827-13N9833, Federal Ministry for Education and Research, Berlin, Germany.

Fontana, J. D., A. M. Desouza, C. K. Fontana, I. L. Torriani, J. C. Moreschi, B. J. Gallotti, S. J. Desouza, G. P. Narcisco, J. A. Bichara, and L. F. X. Farah. 1990. Acetobacter cellulose pellicle as a temporary skin substitute. Appl. Biochem. Biotechnol. 24-25:253-264.

Gao, C., Y. Wan, C. Yang, K. Dai, T. Tang, H. Luo, and J. Wang. 2011. Preparation and characterization of bacterial cellulose sponge with hierarchical pore structure as tissue engineering scaffold. J. Porous Mater. 18:139-145.

Grande, C. J., F. G. Torres, C. M. Gomez, and M. C. Bano. 2009. Nanocomposites of bacterial cellulose/hydroxyapatite for biomedical applications. Acta Biomater. 5:1605-1615.

Helenius, G., H. Bäckdahl, A. Bodin, U. Nannmark, P. Gatenholm, and B. Risberg. 2006. In vivo biocompatibility of bacterial cellulose. J. Biomed. Mater. Res. A 76:431-438. 
Industry Codes. 2011. ASTM F2900-Standard guide for characterization of hydrogels used in regenerative medicine. http://industrycodes.com/products/edc44f/ standard-guide-characterization-hydrogels-used (accessed December 2011).

Jiang, H., Y. Wang, S. Jia, Y. Huang, F. He, and Y. Wan. 2007. Preparation and characterization of hydroxyapatite/bacterial cellulose nanocomposite scaffolds for bone tissue engineering. Key Eng. Mater. 330-332:923-926.

Jonas, R., and L. F. Farah. 1998. Production and application of microbial cellulose. Polym. Degrad. Stabil. 59:101-106.

Klemm, D., B. Heublein, H. P. Fink, and A. Bohn. 2005. Cellulose: fascinating biopolymer and sustainable raw material. Angew Chem. Int. Ed. 44:3358-3393.

Klemm, D., R. W. Kinne, F. Kramer, D. Pretzel, and U. Udhardt. 2009. Bakterielle Nanocellulose zur Knorpelneubildung. DE102009024133 (A1).

Klemm, D., F. Kramer, S. Moritz, T. Lindstroem, M. Ankerfors, D. Gray, and A. Dorris. 2011. Nanocelluloses: a new family of nature-based materials. Angew Chem. Int. Ed. 50:5438-5466.

Klemm, D., S. Marsch, D. Schumann, and U. Udhardt. 2001a. Method and device for producing shaped microbial cellulose for use as biomaterial, especially for microsurgery. WO 2001061026 (A1).

Klemm, D., D. Schumann, F. Kramer, N. Heßler, M. Hornig, H. P. Schmauder, and S. Marsch. 2006. Nanocelluloses as innovative polymers in research and application. Adv. Polym. Sci. 205:49-96.

Klemm, D., D. Schumann, U. Udhardt, and S. Marsch. 2001b. Bacterial synthesized cellulose—artificial blood vessels for microsurgery. Prog. Polym. Sci. 26:1561-1603.

Langstein, J., F. M. Becke, L. Sollner, G. Krause, G. Brockhoff, M. Kreutz, R. Andreesen, and H. Schwarz. 2000. Comparative analysis of CD137 and LPS effects on monocyte activation, survival, and proliferation. Biochem. Biophys. Res. Commun. 273:117-122.

Li, J., Y. Wan, L. Li, H. Liang, and J. Wang. 2009. Preparation and characterization of 2,3-dialdehyde bacterial cellulose for potential biodegradable tissue engineering scaffolds. Mater. Sci. Eng. C 29:1635-1642.

Lopes, A. W., C. H. Olival, S. F. de Coelho, C. M. de Germanos, and S. da Leonardo. 2009. Experimental study of the tissue reaction caused by the presence of cellulose produced by Acetobacter xylinum in the nasal dorsum of rabbits. Braz. J. Otorhinolaryngol. 75:200-207.

Martson, M., J. Viljanto, T. Hurme, and P. Saukko. 1998. Biocompatibility of cellulose sponge with bone. Eur. Surg. Res. 30:426-432.

Mello, L. R., L. T. Feltrin, P. T. Fontes Neto, and F. A. Ferraz. 1997. Duraplasty with biosynthetic cellulose: an experimental study. J. Neurosurg. 86:143-150.

Mendes, P. N., S. C. Rahal, O. C. M. Pereira-Junior, V. E. Fabris, S. L. R. Lenharo, J. Ferreira de Lima-Neto, and L. A. Fernanda da Cruz. 2009. In vivo and in vitro evaluation of an Acetobacter xylinum synthesized microbial cellulose membrane intended for guided tissue repair. Acta Vet. Scand. 51:12.

Miyamoto, T., S. Takahashi, H. Ito, H. Inagaki, and Y. Noishiki. 1989. Tissue biocompatibility of cellulose and its derivatives. J. Biomed. Mater. Res. 23:125-133.

Moreira, S., N. B. Silva, J. Almeida-Lima, H. A. O. Rocha, S. R. B. Medeiros, C. Alves, and F. M. Gama. 2009. BC nanofibers: in vitro study of genotoxicity and cell proliferation. Toxicol. Lett. 189:235-241. 
Nakayama, A., A. Kakugo, J. P. Gong, Y. Osada, M. Takai, T. Erata, and S. Kawano. 2004. High mechanical strength double-network hydrogel with bacterial cellulose. Adv. Funct. Mater. 14:1124-1128.

Recouvreux, D. O. S., C. R. Rambo, F. V. Berti, C. A. Carminatti, R. V. Antonio, and L. M. Porto. 2011. Novel three-dimensional cocoon-like hydrogels for soft tissue regeneration. Mater. Sci. Eng. C 31:151-157.

Schumann, D. A., J. Wippermann, D. O. Klemm, F. Kramer, D. Koth, H. Kosmehl, T. Wahlers, and S. Salehi-Gelani. 2009. Artificial vascular implants from bacterial cellulose: preliminary results of small arterial substitutes. Cellulose 16:877-85.

Sieminski, A. L., and K. J. Gooch. 2000. Biomaterial-microvasculature interactions. Biomaterials 21:2233-2241.

Sokolnicki, A. M., R. J. Fisher, T. P. Harrah, and D. L. Kaplan. 2006. Permeability of bacterial cellulose membranes. J. Membrane Sci. 272:15-27.

Svensson, A., E. Nicklasson, T. Harrah, B. Panilaitis, D. L. Kaplan, M. Brittberg, and P. Gatenholm. 2005. Bacterial cellulose as a potential scaffold for tissue engineering of cartilage. Biomaterials 26:419-431.

Tang, W., S. Jia, Y. Jia, and H. Yang. 2010. The influence of fermentation conditions and post-treatment methods on porosity of bacterial cellulose membrane. World J. Microbiol. Biotechnol. 26:125-131.

Teoh S. H. 2004. Introduction to biomaterials engineering and processing-an overview. In: Engineering Materials for Biomedical Applications, ed. S. H. Teoh, 1-16. Singapore: World Scientific Publishing.

Wan, Y. Z., Y. Huang, C. D. Yuan, S. Raman, Y. Zhu, H. J. Jiang, F. He, and C. Gao. 2007. Biomimetic synthesis of hydroxyapatite/bacterial cellulose nanocomposites for biomedical applications. Mater. Sci. Eng. C 27:855-864.

Watanabe, K., Y. Eto, S. Takano, S. Nakamori, H. Shibai, and S. Yamanaka. 1993. A new bacterial cellulose substrate for mammalian cell culture. Cytotechnology 13:107-114.

Wesarg, F., N. Heßler, D. Kralisch, D. Klemm, W. Fried, and F. A. Müller. 2010. Structural and mechanical properties of bacterial nanocellulose produced by different Gluconacetobacter strains. Paper presented at the 239th American Chemical Society National Meeting, March 23-29, San Francisco, CA.

Williams, D. F. 1999. The Williams Dictionary of Biomaterials. Liverpool: Liverpool University Press.

Williams, K. L. 2007. Depyrogenation validation, pyroburden, and endotoxin removal. Drugs Pharm. Sci. 167:301-327.

Zaborowska, M., A. Bodin, H. Baeckdahl, J. Popp, A. Goldstein, and P. Gatenholm. 2010. Microporous bacterial cellulose as a potential scaffold for bone regeneration. Acta Biomater. 6:2540-2547. 


\title{
Bacterial Nanocellulose Biomaterials with Controlled Architecture for Tissue Engineering Scaffolds and Customizable Implants
}

\author{
Paul Gatenholm, ${ }^{1,3,4}$ Joel Berry, ${ }^{2}$ Andrea Rojas, ${ }^{1}$ Michael B. Sano, ${ }^{1}$ \\ Rafael V. Davalos, ${ }^{1}$ Kara Johnson, ${ }^{3}$ and Laurie O’Rourke ${ }^{3}$ \\ ${ }^{1}$ School of Biomedical Engineering and Sciences, \\ Virginia Tech, Blacksburg, Virginia, USA \\ ${ }^{2}$ Department of Biomedical Engineering, \\ University of Alabama at Birmingham, Birmingham, Alabama, USA \\ ${ }^{3}$ BC Genesis, Blacksburg, Virginia, USA \\ ${ }^{4}$ Department of Chemical and Biological Engineering, Biopolymer Technology, \\ Chalmers University of Technology, Gothenburg, Sweden
}

\section{CONTENTS}

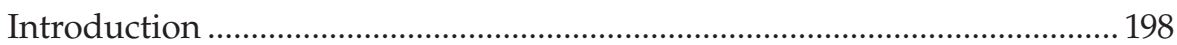

BNC as a Viable Candidate for Tissue Engineering Scaffolds ....................... 199

Scale-Dependent Properties of BNC ………………..................................... 202

Design of a Mask and the Production of a PDMS Conduit Mold.................. 207

Testing PDMS Conduits Using Charged Fluorescent Beads or

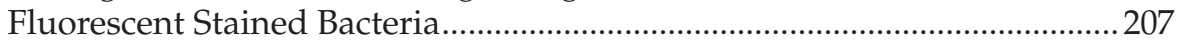

Description of a Prototype BNC Meniscus Microweaver ................................. 210

BNC Scaffolds with Microvessels .................................................................... 212

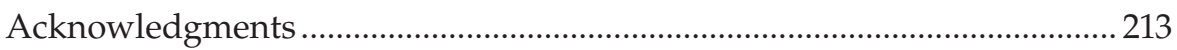

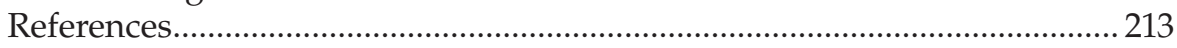

The bacterium Gluconacetobacter xylinus (previously known as Acetobacter xylinum) has been shown to produce cellulose fibrils of $10-30 \mathrm{~nm}$ in diameter. Through careful control of their motion, it is possible to direct them to produce well-defined three-dimensional (3D) scaffolds for tissue reconstruction. This chapter reviews the state of the art in fabrication of tissue engineered structures and biomedical implants using bacterial nanocellulose (BNC). Of particular focus is the use of electric fields to produce highly oriented cellulose networks and also the development 
of a microvascular network within BNC structures. The movement of G. xylinus at the nanoscale can be controlled by electric fields to create custom cellulose networks for engineered tissues and biomedical implants. This is the first attempt to control a bottom-up biofabrication process in three dimensions. The manipulation of electrokinetic forces acting upon a bacterial cell can produce complex cellulose patterns on the nanoscale not achievable in static culture. The ability to control the direction of fiber orientation could be readily expanded to weave structures of multiple fiber layers by changing the orientation of the applied electric field for each layer. Using this method, these structures could be tailored to have desired mechanical properties for a variety of applications, including tissue engineering, microelectromechanical systems (MEMS), textiles, and electronics.

\section{Introduction}

Tissue engineering holds great promise for treating some of the most devastating diseases of our time. Because engineered tissue and organ replacements can be developed in a laboratory, therapies can potentially be delivered on a large scale for multiple disease states with dramatic reductions in waiting times for patients. The concept of engineering tissue using selective cell transplantation has been applied experimentally and clinically for a variety of disorders, including the successful use of engineered skin for burn patients (Fontana 1990), engineered cartilage for knee replacement procedures (Solchaga et al. 1999), and engineered bladder tissue for bladder reconstruction (Atala 2002). The process for engineering tissues involves in vitro seeding and attachment of human cells onto a scaffold (Temenoff and Mikos 2000). These cells then proliferate, migrate, and differentiate within the scaffold while secreting (or resorbing) the extracellular matrix components of the specific tissue type of interest. Therefore the choice of scaffold material is crucial to enable the cells to produce tissues and organs of the desired shape, size, and mechanical properties. Scaffolding for tissue engineering usually consists of polymers, both natural and synthetic. Some methods of forming polymer scaffolds for tissue engineering are decellularization of animal tissue (Badylak et al. 1989), solvent casting, particulate leaching, gas foaming of polymers (Nam et al. 2000), phase separation (Lo et al. 1995), and solution casting (Zhou et al. 2005). Electrospinning is another popular method for creating fibrous scaffolds for engineered tissues and organs (Stitzel et al. 2006).

Currently scaffold fabrication techniques suffer from fundamental manufacturing limitations that have, to date, prevented their clinical translation. Specifically, there is a distinct lack of processes capable of reproducibly creating structures on the nano-, micro-, and millimeter scales that predictably promote cell growth and function. 
Biofabrication of the natural polymer bacterial nanocellulose (BNC) has great potential to overcome the limitations of scaffold production for regenerative medicine. Some key advantages of BNC are that it has unique biocompatibility, mechanical integrity, and hydroexpansivity, and is structurally stable under a wide range of conditions (Bäckdahl et al. 2006; Helenius et al. 2006). Moreover, the movement of living bacteria can be guided to leave behind a network of cellulose nanofibrils of almost any architecture. It is thus ideal as a scaffolding material on which the cells can be seeded for regenerative medicine applications.

\section{BNC as a Viable Candidate for Tissue Engineering Scaffolds}

$\mathrm{BNC}$ is an emerging biomaterial in the important and quickly expanding fields of personal care, medicine, and life sciences (Gatenholm and Klemm 2010; Klemm et al. 2006; Petersen and Gatenholm 2011). The nanostructured network and morphological similarities with collagen make BNC very attractive for cell attachment, cell migration, and the production of additional extracellular matrices. In vitro and in vivo studies have shown that BNC implants do not elicit any foreign body reaction, fibrosis, or capsule formation. Inflammatory giant cells have not been detected around BNC networks and connective tissue integrates well with BNC structures (Helenius et al. 2006). $\mathrm{BNC}$ is neither cytotoxic nor genotoxic; however, only a limited number of studies have evaluated the interactions between BNC fibrils and surrounding cells and tissues (Bodin et al. 2010; Esguerra et al. 2010). Worldwide, activities have intensified in the research, development, and application of hydrated and partially dehydrated BNC materials in biomedical applications such as wound care and novel bioactive implants (Lohmann \& Rauscher 2009). Other applications include materials for bone and cartilage repair and tubular prototypes as grafts for vascular surgery (Bäckdahl et al. 2011). In all cases, BNC functions as a three-dimensional (3D) template for in vitro and in vivo tissue growth (Czaja et al. 2006; Klemm et al. 2006; Webster 2007; Yano et al. 2005). There is also growing interest in the potential of BNC as an integral participant in the healing of chronic wounds through reduction of proteolytic enzymes, cytokines, and reactive oxygen species. BNC-collagen composites formed in situ have been shown to reduce selected proteases and interleukin production and show distinct antioxidant capacity (Wiegand et al. 2006). Silver chloride-impregnated BNC films have been produced as antibacterial dressings from the never-dried material by postprocessing immersion steps. Silver chloride nanoparticles were present on the BNC fibers in quantities up to $21 \% \mathrm{w} / \mathrm{w}$ (Wang et al. 2008). Silver nanoparticles were prepared in situ by the hydrolytic decomposition of silver triethanolamine complexes. Scanning electron microscopy (SEM) images and X-ray diffraction patterns showed 
that the spherical metallic silver particles with a mean diameter of $8 \mathrm{~nm}$ were well adsorbed on the BNC fibrils (Barud et al. 2008).

Broad patenting has been a hallmark of the recent developments in BNC biomedical implants. However, these patent claims are frequently based on insufficient background investigations to determine the manufacture-dependent structure of the material and its function and stability in the body. The same is true for BNC scaffolds for tissue engineering. In particular, the in-growth of living cells requires further investigation. In this context, the fabrication of BNC scaffolds and implants with a porosity and pore size relevant to the particular application is important.

BNC hydrogel is very attractive for use as a scaffold for tissue engineering. Novel processes using porous wax particles in the fermentation broth have recently led to macroporous BNC materials with good interconnectivity (Bäckdahl et al. 2008). Such macroporous BNC has been used to support human smooth muscle cell migration, proliferation, and differentiation (Bäckdahl et al. 2008). Figure 10.1 shows the morphology of macroporous BNC. Several research groups are evaluating macroporous BNC scaffolds for regeneration of cartilage, bone, urethra, and bladder (Andersson et al. 2010; Bodin et al. 2010; Zaborowska et al. 2010). Examples of approaches used to create pores of different sizes include the use of porogens, the use of different cultivation times and inoculation volumes, and posttreatment with different alkaline solutions and by freeze-drying (Hu and Catchmark 2009). Moreover, results on the behavior of freezable water in BNC and thermoporosity investigations have been reported (Tang et al. 2010). In the case of the formation of pores by porogens and leaching of the porogens, additional information on the purity and integrity of the implants and their essential structure is desirable. Further insight is required with respect to the pore parameters in the never-dried state and after different drying methods.

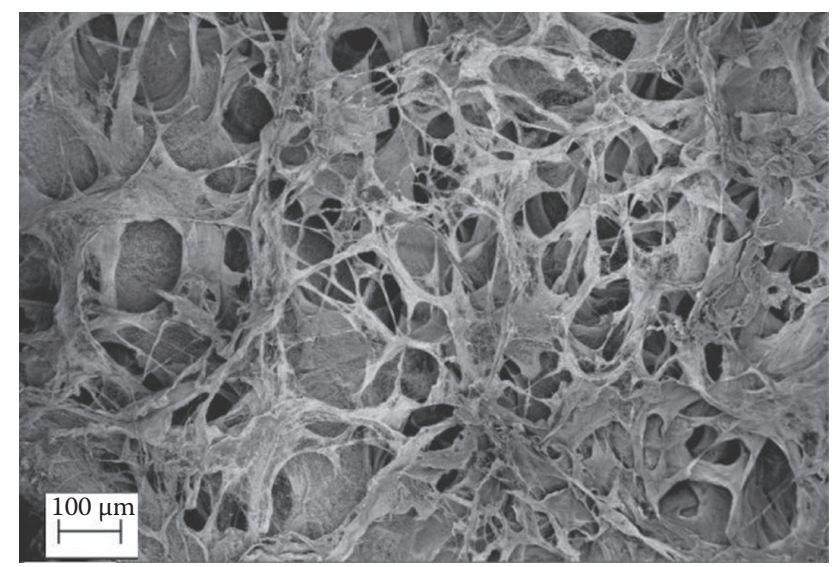

FIGURE 10.1

Microporous bacterial cellulose scaffolds biofabricated using porogens. 
Several research groups have developed prototypes of BNC tubes in the required diameter range and with a length of $5-25 \mathrm{~cm}$ or more for vascular bypass surgery. The material properties of these vessels depend strongly on the cultivation conditions. The wall of the tubes is formed into the typical transparent BNC hydrogel and is also characterized by a stable inner lumen, good stability of sutures, essential mechanical strength, and the important feature of being permeable to water, other liquids, ions, and small molecules. Moreover, the tubes show very good surgical handling and can be sterilized in standard ways (Klemm et al. 2001). In animal experiments with rats, pigs, and sheep (implantation in the carotid artery) good biocompatibility and performance for at least 13 months were demonstrated (Malm et al. 2012). Histological examination of rats after 1 week, 1 month, and 1 year showed that the tubular BNC implants were integrated by endothelization of the inner surface, colonized on the outer wall by connective tissue, and characterized by the in-growth of vital collagen-forming fibroblasts (Klemm et al. 2001, 2006; Wippermann et al. 2009). Confocal laser scanning microscopy showed that the inner surface of the BNC tubes was smooth and not structured from its preparation (Brackmann et al. 2010). The low surface roughness appears to support good endothelization and to be at low risk of thrombosis or aneurysm (Schumann et al. 2009). Commonly used artificial vascular grafts formed from synthetic polymers such as polytetrafluoroethylene (PTFE) or polyesters are prone to thrombosis when used as small-diameter vessels, which are essential for coronary artery bypass grafts. A study in which BNC tubes were compared with synthetic grafts over a period of 2 weeks also demonstrated good biocompatibility of BNC and its incorporation in the body. Evaluation of stents (endoluminal vascular prostheses) covered with BNC in a rabbit iliac artery model demonstrated that the implantation was safe and no acute or late vessel occlusion due to stent thrombosis occurred (Bueno et al. 2009).

BNC, hydrogels with a uniaxially oriented fibril structure and excellent mechanical properties, can be prepared by the cultivation of Gluconacetobacter xylinus strains on ridged oxygen-permeable silicon substrates. Cultivation inside silicone tubes produced BNC tubes with uniaxially oriented fibrils along the longitudinal axis of the silicon template (Putra et al. 2008). The inability of BNC to degrade in vivo has been suggested as a limitation to its use. There are no enzymes in mammalian cells or the extracellular matrix that use cellulose as a substrate. Limited periodate oxidation of BNC leads to a modified BNC (containing 2,3-dialdehyde cellulose structural units) that can break down at physiological $\mathrm{pH}$ values, although the $\mathrm{BNC}$ retains its hydrogel structure, crystallinity, and, for example, its ability to form biomimetically calcium-deficient hydroxyapatite nanocrystallites within the oxidized matrix (Evans et al. 2008). The resorbable BNC hydrogel has a great capacity for bone tissue repair (Hutchens et al. 2009).

The large amount of water inside BNC hydrogels acts as a spacer and stabilizing element for the nanofibril network. In the case of slow dewatering 
(i.e., under mild drying conditions), the fibril network is stable, and aerogels are formed. Such aerogels are characterized by a high reswelling capacity (i.e., the aerogels can absorb nearly the same large amount of water as was removed by the drying step). By drying under pressure, very flat foils (loss of $99 \%$ water) of high density and high mechanical stability can be obtained. In such materials, the nanofibril network collapses, resulting in a dense physical cross-linking of the cellulose chains ("hornification"). Such foils are of interest for technical (e.g., membranes for audio technologies) and medical (wound dressings) applications. In the latter context, partially dewatered materials are also important because they combine the hydrophilic properties of the native BNC gels with the better mechanical stability of the compressed materials. The water uptake of the compressed and completely dried BNC materials is low. Before drying, dissolved or suspended substances, such as proteins, pharmaceutics, colorants, and nanosilica, can be incorporated into BNC by simple dipping and insertion technologies. After drying, these additives are fixed inside the nanocellulose materials.

The placement of different agents into the BNC also can be carried out by impregnating freeze-dried BNC under adsorption conditions in the fibers and/or by filling the network meshes. That is, the nanofibers can act as templates for nanoparticles or organic polymer layers. A very important part of the development of $\mathrm{BNC}$ materials concerns the design of $\mathrm{BNC}$ composites by either in situ or later modification. In the case of additives with hydroxyl or similar groups, the in situ insertion is affected by hydrogen bridge formation. In the case of nanoparticles, the BNC fibers can act as nanofiber templates. A recent example is the impregnation of $\mathrm{BNC}$ with silver nitrate and the subsequent reduction with water-soluble sodium borohydride (a technical reducing agent), which forms silver metal deposited as nanoparticles on the supporting BNC nanofibers. Nanofibers of BNC material, when modified with carboxylic functionalities, have shown the unique ability to induce biomimetic crystallization of calcium-deficient hydroxyapatite (Bodin et al. 2006). The size of the crystals can be controlled by selecting the fibril density of the BNC network. The presence of nanosize hydroxyapatite crystals on the surfaces of cellulose nanofibrils promotes osteoprogenitor cell adhesion and differentiation (Zimmermann et al. 2011). The ability to control macroporosity combined with surface decoration with hydroxyapatite makes BNC materials promising candidates for bone grafts.

\section{Scale-Dependent Properties of BNC}

Control of the 3D morphology of cellulose networks from the nanoscale through the mesoscale to the macroscale is a critical aspect in developing 
customizable implants and scaffolds for tissue engineering, especially since cell response to a material directly correlates to nanoscale topography (Stevens and George 2005). This can be attained by using electric fields to control the movement of G. xylinus at the nanoscale level to create custom cellulose networks (Sano et al. 2010). By carefully controlling the electrokinetic forces, bacterial motion can be directed while the organisms extrude cellulose networks. The manipulation of electrokinetic forces acting upon a bacterial cell can produce complex cellulose patterns on the nanoscale not achievable in static culture. This nanoscale control provides the ability to develop and affect the macroscale tissue properties by controlling the direction of fiber orientation. Using electrokinetic forces, structures can be tailored to have the desired mechanical properties for a variety of tissue engineering and implantable device applications.

Electrokinetics is the result of several effects that occur once a particle is introduced to a fluid. In theory, an object in a fluid will form an electrical double layer composed of ions. The first layer, or surface charge, is composed of either all positive or all negative ions. The second layer, or diffuse layer, forms in the fluid by surface charge attraction. When an external force, such as an electric field, is applied on a particle in a fluid, the motion of the particle moves toward a region of opposite charge as a result of the diffuse layer combined with the Coulombic force. The net forces on the particle are known as electrokinetic forces and can be identified as electroosmosis and electrophoresis. Electroosmosis is the response of the fluid to an electric field and typically can affect the motion of a particle within the fluid, while electrophoresis is the motion of charged particle in a uniform electric field. The electrokinetic velocity can be calculated using Huckel's equation, where the $\mu_{\mathrm{eo}}$ and $\mu_{\mathrm{ep}}$ are the electroosmotic and electrophoretic mobilites and $\vec{E}$ is the magnitude of the applied electric field (Tandon et al. 2008):

$$
V_{e k}=\left(\mu_{e o}+\mu_{e p}\right) \vec{E} .
$$

The weavers of BNC, G. xylinus, are 1-2 $\mu \mathrm{m}$ in length and therefore can feel the effects of electrokinetics. Furthermore, their size assists with electrokinetics having a stronger effect than Brownian motion and negligible effects from gravity (Pohl 1978). With electrokinetics, the phenomena of electrophoresis can play a dominant role in precise control over bacteria cells and has been utilized to align bacterial cellulose fiber orientation, as seen in Figure 10.2. Using low direct current (DC) fields in the range of $0.25-1 \mathrm{~V} / \mathrm{cm}$, bacterial cells continue to secrete cellulose nanofibrils and result in fiber alignment, with their electrophoretic mobility estimated to be $4.68 \times 10^{-9} \mathrm{~m}^{2} \cdot \mathrm{V} / \mathrm{s}$. As electric fields are increased above this threshold, cellulose production slows down or halts due to rapid velocities that impede metabolic processes. 


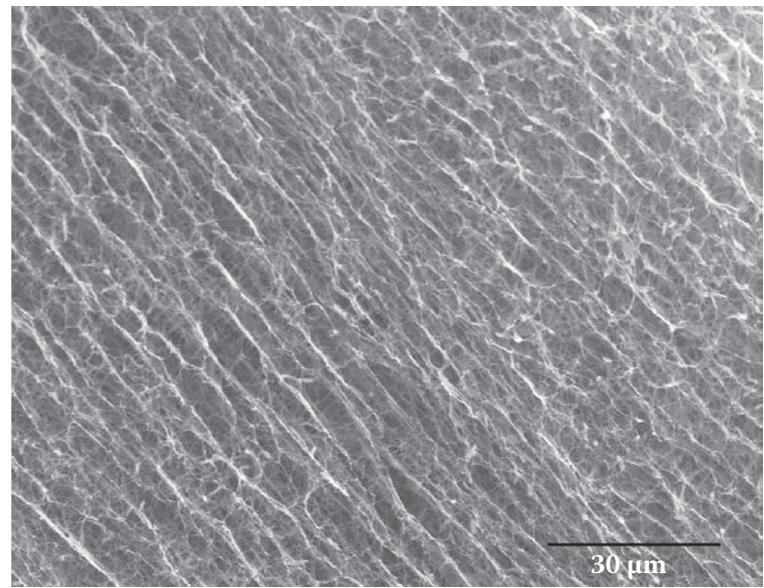

\section{FIGURE 10.2}

Aligned bacterial cellulose nanofibers.

Furthermore, direct control of bacteria can also be applied using dielectrophoresis, where nonuniform electric fields are utilized. A dielectrophoretic force on a particle, neutral or charged, drives the particle toward regions of maximal field gradient. The extent of the force is dependent on the particle's size and shape, electrical properties of the particle relative to the medium, the frequency applied, and the geometric shape in coordination with where the field is applied. The dielectrophoretic mobility term, $\mu_{\mathrm{DEP}}$, encompasses these attributes with the exception of the gradient of the inner product of the electric field, $\nabla(E \mathrm{n} E)$ :

$$
F_{\mathrm{DEP}}=\mu_{\mathrm{DEP}} \nabla(E \mathrm{n} E)
$$

The capability of aligning fiber direction using electrophoresis and dielectrophoresis is a large step for creating whole complex engineered structures such as a meniscus. The need for controlling the 3D morphology of a meniscus, for example, results from the collagen fiber structure influencing the mechanical integrity. This fiber structure is composed of radial fibers integrated into an arrangement of circumferential fibers, as seen in Figure 10.3.

With electrophoresis, dielectrophoresis, or both, G. xylinus can be manipulated to create circumferential fibers and radial fibers as needed. Since the cellulose grows at the air-liquid interface, one layer can be made at a time with a focus on circumferential fibers or radial fibers. A two-dimensional (2D) meniscus structure was designed and modeled using the Laplace equation to determine regions where the gradient of the electric field is highest. At these locations, the dielectrophoretic force attracts bacteria 


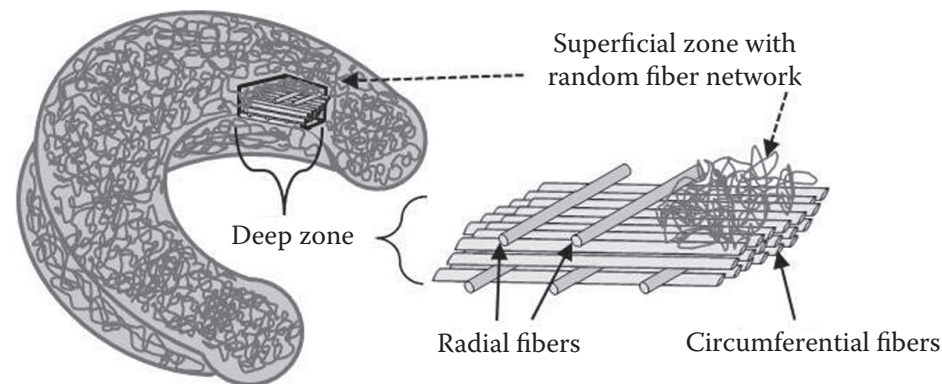

FIGURE 10.3

Meniscus fiber collagen network showing radial fibers integrated with circumferential fibers.

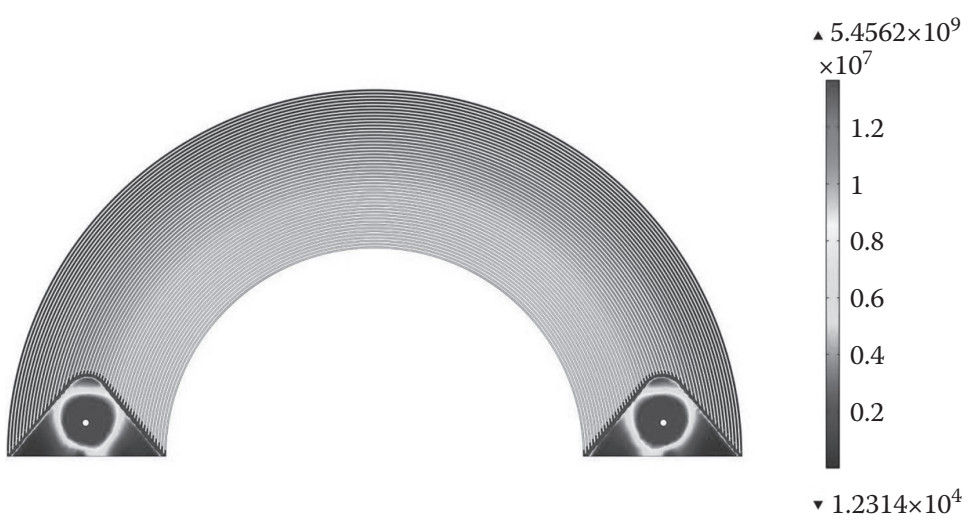

\section{FIGURE 10.4}

(See color insert.) Meniscus design with channels $100 \mu \mathrm{m}$ wide separated by PDMS barriers that are $100 \mu \mathrm{m}$ thick. Gradient of the inner product of the electric field modeled at $5 \mathrm{~V}$ and $1 \mathrm{~Hz}$.

movement. According to the model in Figure 10.4, the highest gradients of the electric field are expected to be where the horns of the meniscus should be located, resulting in uniform movement across the entire region instead of at certain locations within the meniscus. Experimentally, the bacteria movement was seen using $20 \mathrm{~V}$ peak to peak at $1 \mathrm{~Hz}$ and can be optimized by adjusting the amplitude. Adjusting the amplitude will fine tune the bacteria oscillation distance, which can affect the density of each layer and the surface morphology.

Dielectrophoresis can be used to move bacteria in a radial pattern by applying a field between the inner and outer radius of the meniscus mold. This produces a gradient with an exact analytical solution. Moving bacteria in a circumferential pattern, however, is a challenge if dielectrophoresis is used alone. We found that by slightly increasing the height of the medium from one end of the meniscus form to the other, we create the nonuniform 

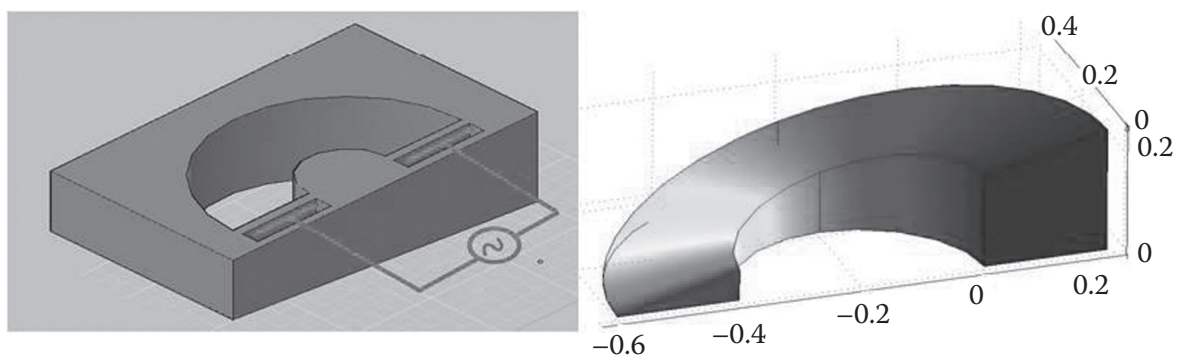

FIGURE 10.5

(See color insert.) (Left) AutoCAD drawing of a prototype. (Right) COMSOL model of potential distribution fashioned using an exaggerated slope.

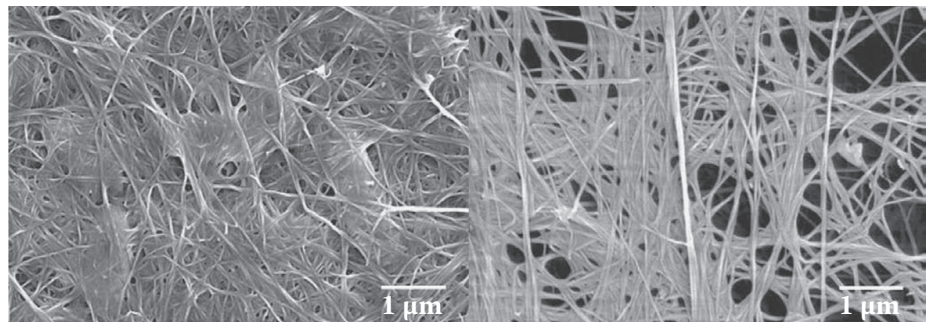

\section{FIGURE 10.6}

SEM images. (Left) Cuvette CONTROL example (magnification $\times 50,000$ ). (Right) Cuvette slanted at a small angle, AC $1 \mathrm{~V} 0.01 \mathrm{~Hz}$ (magnification $\times 50,000)$.

field needed to drive dielectrophoresis. To simulate this effect, the electric field distribution and its gradient $\nabla E=\nabla(\nabla \varphi)$ were modeled numerically in COMSOL Multiphysics 3.5 using the AC/DC module (COMSOL Inc., Burlington, MA, USA). This is done by solving for the potential distribution, $\varphi$, using the governing equation, $\nabla\left(\sigma^{*} \nabla \varphi\right)=0$, where $\sigma^{*}$ is the complex conductivity $\left(\sigma^{*}=\sigma+j \omega \varepsilon\right)$ of the subdomains in the meniscus mold. We imposed electrically insulating boundary conditions on the inner and outer radius and an applied potential from either end of the meniscus. Figure 10.5 shows a computer-aided design (CAD) drawing of our prototype and a numerical model of the prototype showing the voltage distribution.

SEM images, shown in Figure 10.6, illustrate that fibers are laid down in a more linear pattern with alternating current $(\mathrm{AC})$ and when the mold is slanted.

An alternative design to capture the unique geometry of the meniscus was created by combining nanoimprinting with biofabrication knowledge (see Figure 10.7). The individual layers using a mold reminiscent of a vinyl record containing curved nanoconduits for the bacteria to traverse were defined. The meniscal mold was inverted, flattened, and placed within a custom bioreactor. Initially a small amount of bacteria-rich media was added to the bioreactor and allowed to establish the nanocellulose layer (approximately 3 days). This was then overlaid with the thin polydimethylsiloxane (PDMS) 

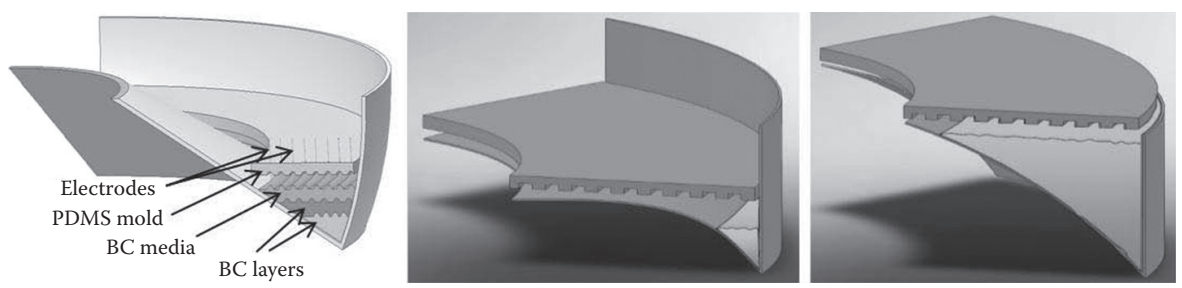

\section{FIGURE 10.7}

(See color insert.) Three schematics illustrating the concept of depressing the curved portion of the silastic meniscus form overlaying the bacteria-rich media with the thin PDMS nanoconduit mold. Eventually enough media and growth will occur to be fully covered by the overlay.

nanoconduit mold, and the silastic meniscus form was mechanically raised in concert with a slow infusion of media for approximately 5 days, reaching a height of $8 \mathrm{~mm}$ along the lateral wall.

\section{Design of a Mask and the Production of a PDMS Conduit Mold}

The microfluidic features and channels were designed using AutoCAD and COMSOL Multiphysics numerical modeling. Designs for a meniscal shape (semicircle) and for a full circle, generated using AutoCAD, were used to produce masks for our lithography fabrication process. Silicon master stamps of the conduit mask design were fabricated using deep reactive ion etching (photolithography fabrication) followed by Teflon deposition based on techniques developed by Davalos et al. (2008). PDMS monomers and the curing agent were mixed in a 10:1 ratio (Sylgard 184, Dow Corning, USA). Liquid PDMS was poured onto the silicon master and cured at room temperature or for $15 \mathrm{~min}$ at $115^{\circ} \mathrm{C}$ (Figure 10.8). Test cultures were set up to confirm that the PDMS conduit mold would produce the desired pattern in the bacteria cellulose.

\section{Testing PDMS Conduits Using Charged Fluorescent Beads or Fluorescent Stained Bacteria}

The objective of the first set of experiments was to evaluate the movement of charged beads and bacteria along the PDMS conduits. The purpose was to determine if the beads/bacteria moved smoothly along the track and to establish an ideal voltage and frequency range for bacteria movement. 


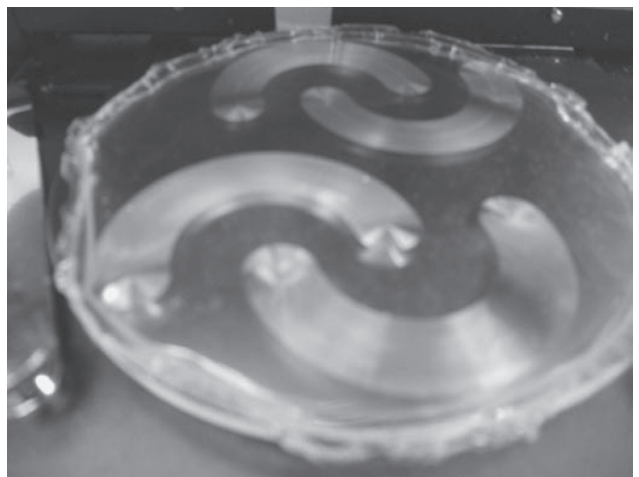

FIGURE 10.8

Example of a PDMS mold produced from the silicon master.
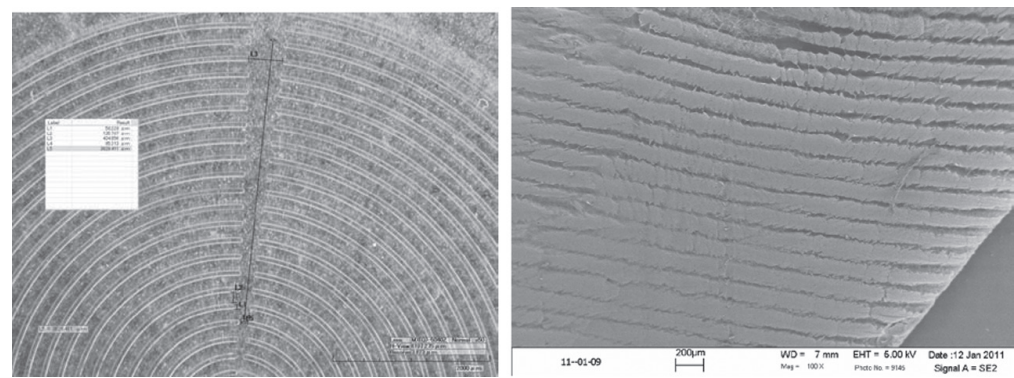

FIGURE 10.9

(Left) Microscopic image of the meniscus PDMS conduit mold showing channels converging into a narrow "V," where the electrode is applied. (Right). SEM image of bacteria cellulose showing channeled pattern (magnification $\times 100$ ).

Thick PDMS conduit molds of the semicircle design, with holes punctured through the " $\mathrm{V}$ " area of the tracks, were bonded to glass using a plasma and vacuum device, effectively enclosing the tracks and allowing the beads/bacteria to be trapped within the grooves (see Figure 10.9). Charged fluorescent beads, $2 \mu \mathrm{m}$ in size, were suspended in water and injected through the holes into the tracks; the electrodes were then placed in the holes. An Elenco GF8046 was used for power and a Temma 72-8395 oscilloscope was used for recording and observing the wave. The bacteria were stained with BacLight, a fluorescent stain, and injected into the tracks in the same manner as the beads. The lighting and camera settings on the microscope were changed to allow observation of the bacteria. With the electrodes held in place, the movement of the beads was very clear (Figure 10.10). The normal settings for the AC generator were a $20 \mathrm{Vp}-\mathrm{p}$ square wave at $0.05 \mathrm{~Hz}$ (the highest voltage and the lowest frequency available). Likewise, it was easy to visualize the movement of the bacteria at the normal settings. 

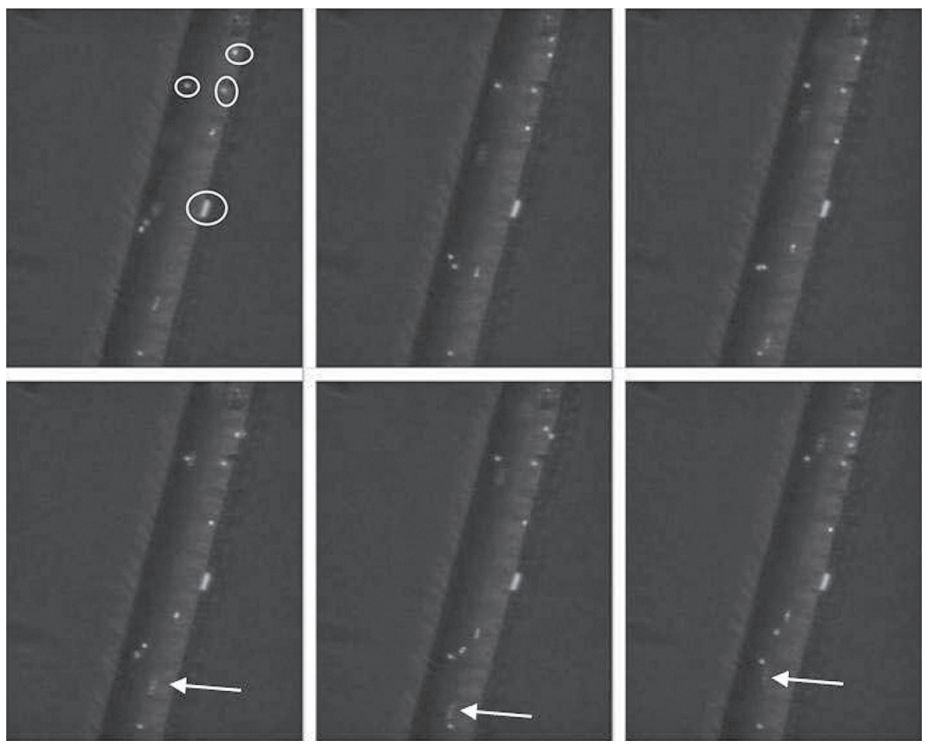

\section{FIGURE 10.10}

Images captured by video show the movement of beads controlled by an electric field. White circles in upper left pinpoint beads that remained in position. White arrows in the lower three images target a specific bead moving back and forth.

Using the same test system described previously, but with the full circle design, bacteria movement was tested to determine the relationships between the speed of the bacteria's movement and the frequency, and between the speed of the bacteria's movement and the voltage amplitude. The distance of one cycle for each change in the variable was measured by determining the forward distance ("To") and the return distance traveled ("Back"). Only bacteria with the most significant movement were measured, and the distance traveled was directly affected by the traffic (number of bacteria) within the channel. The true distance traveled, determined by using the ratio of the screen's actual and displayed size, was $0.3659 \mu \mathrm{m}$ for every centimeter on the display screen.

First, the speed was compared with the frequency with the voltage set to maximum (20 Vp-p or $10 \mathrm{Vac})$ and the function set to a sine wave. Next, the same experiment was repeated, changing the function to a square wave. Finally, the voltage effect on the speed of the bacteria was determined, setting the frequency to $0.25 \mathrm{~Hz}$ and using a square wave. The last experiment measured voltage versus frequency to determine the lowest frequency/voltage that would control bacteria movement. For every voltage, the frequency was lowered until the bacteria no longer moved in a controlled pattern within the channels. The lowest frequency associated with controlled movement was recorded and is graphically presented in Figure 10.11. 


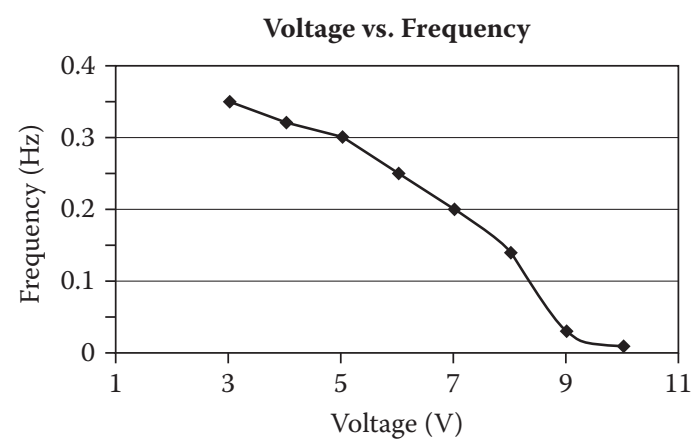

FIGURE 10.11

Graphic illustration of voltage versus minimum frequency required to achieve control over bacteria movement within the PDMS conduit mold.

These experiments demonstrated that a sine wave with the AC generator is superior to the square wave, which caused sudden changes in the bacteria's direction, causing collisions within the channels. The sine wave allows a smoother flow since it gradually rises to the maximum voltage. The lower amount of stress on the bacteria may prove useful for better cellulose production. We also determined that $0.60 \mathrm{~Hz}$ with a sine wave and a voltage range between 4 and $6 \mathrm{Vac}$ is optimum when using AC to control bacteria movement. Based on these results, we concluded that our design would be more successful using the full circle design rather than the semicircle that mimics the shape of the native meniscus.

\section{Description of a Prototype BNC Meniscus Microweaver}

The meniscus microweaver is a bioreactor designed to biofabricate meniscus implants (see Figures 10.12a-f). It is placed in a closed plexiglass system that maintains a sterile environment and minimizes water loss. Parts include a circular silastic meniscus mold, a PDMS conduit mold attached to a gasket to keep the PDMS mold in place with holes to guide the electrodes (see Figure 10.12a), and a central perforated cylinder connected to a stepping motor through which medium is delivered (see Figure 10.12b). Production begins with the silastic center of the meniscus mold in a depressed position, held in place by a cylinder. Once a pellicle of bacteria cellulose is established (static incubation of 3 days), the microweaver operation switches to a dynamic mode (Figures 10.12c-f). The stepping motor gradually draws up the cylinder $(1 \mathrm{~mm} / 24 \mathrm{~h})$, eventually allowing the meniscus mold to recover its original shape (Figures 10.12c and 10.12e). As this is happening, fresh culture medium is dispensed through the center of the cylinder using a syringe 


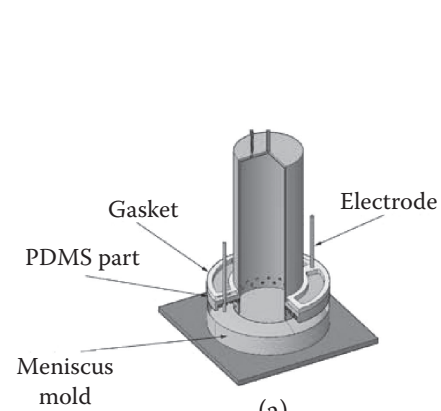

(a)

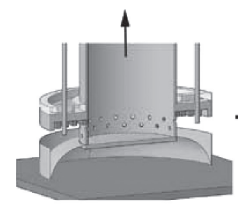

(c)

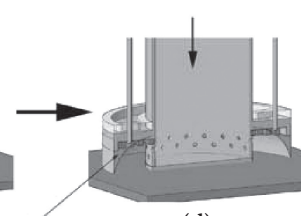

(d)

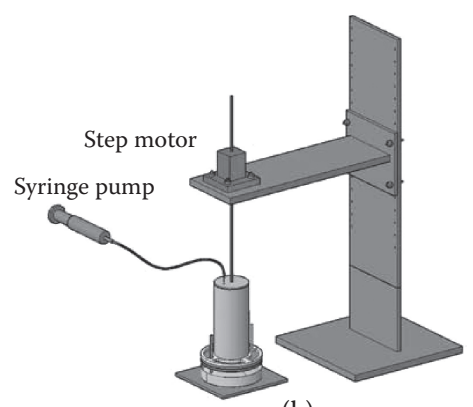

(b)

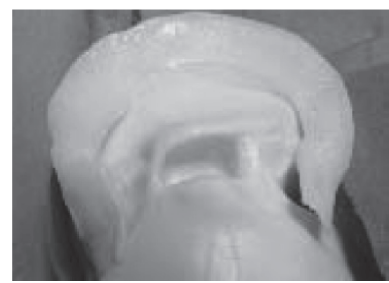

(g)

\section{FIGURE 10.12}

(See color insert.) Microweaver bioreactor for biofabrication of meniscus implant and a prototype of a human BNC meniscus implant. Source: BC Genesis.

pump that delivers the amount required to fill the volume created as the cylinder lifts. The AC electric field is applied to electrodes that are set into the medium through holes in opposing spokes of the gasket. Bacteria move forward and backward, producing dense cellulose fibers that are circumferentially oriented. The process repeats until the coil moves up and the meniscus form is filled with BNC.

The electromagnetic field is powered using a computer with a soundcard oscilloscope and a universal serial bus (USB) function generator. The frequency, amplitude, and trigger functions of the oscilloscope are computer controlled. Figure $10.12 \mathrm{~g}$ shows the prototype of a human meniscus.

We have demonstrated that this can be attained by controlling the movement of G. xylinus at the nanoscale by electric fields to create custom cellulose networks. To the best of our knowledge, this study demonstrates the first direct control over a bottom-up biofabrication process in three dimensions. By carefully controlling the electrokinetic forces, we can direct the bacteria 
while they extrude cellulose networks. The manipulation of electrokinetic forces acting upon a bacterial cell can produce complex cellulose patterns on the nanoscale not achievable in static culture. The ability to control the direction of fiber orientation could be readily expanded to weave structures of multiple fiber layers by changing the orientation of the applied electric field for each layer. Using this method, these structures could be tailored to have the desired mechanical properties for a variety of applications, including tissue engineering, microelectromechanical systems (MEMS), textiles, and electronics.

\section{BNC Scaffolds with Microvessels}

Of fundamental importance to the survival of most engineered tissue structures is gas and nutrient exchange. In nature, this is accomplished by virtue of microcirculation feeding oxygen and nutrients to tissues and removing waste at the capillary level. Gas exchange in most engineered tissue scaffolds is accomplished by passive diffusion ( $<1 \mathrm{~mm}$ distances) or actively by elution of oxygen from specific material types of fibers (Harrison et al. 2007). The architecture for microcirculation is difficult to fabricate, particularly since the cross-sectional dimension of a capillary is only about 5-10 $\mu \mathrm{m}$ in diameter. The existing manufacturing processes were not developed to create a network of microscopic blood vessels. Currently there are no known tissue engineering scaffolds with microcirculation designed into the structure for gas exchange. As a result, the scaffolding for tissues and organs is limited in size and shape. In addition to gas exchange, engineered tissue scaffolds must exhibit mechanical properties comparable to the native tissues they are intended to replace. This is true because the cells that populate native tissues sense physiologic strains, which can help to control tissue growth and function.

We created a BNC scaffold with 200-300 $\mu$ m cylindrical channels for the purpose of endothelializing the walls of the microchannels (Figure 10.13). The channels were created by growing BNC around an array of $300 \mu \mathrm{m}$ silica capillaries. It was shown that we could endothelialize these channels deep within a thick scaffold using human umbilical vein endothelial cells (HUVEC). For this purpose, HUVECs were seeded statically onto purified $\mathrm{BNC}$ and incubated for $24 \mathrm{~h}$ at $37^{\circ} \mathrm{C}$ and $5 \%$ carbon dioxide and then exposed to pulsatile media flow for $36 \mathrm{~h}$. After harvest and fixation of the material, one hundred $5 \mu \mathrm{m}$ sections were sliced in a microtome. Cross-sectional hematoxylin and eosin staining revealed a confluent layer of endothelial cells at each section. This result has implications in the development of larger volumes of $\mathrm{BNC}$ for engineered tissues since those volumes can now be perfused with oxygenated media. 


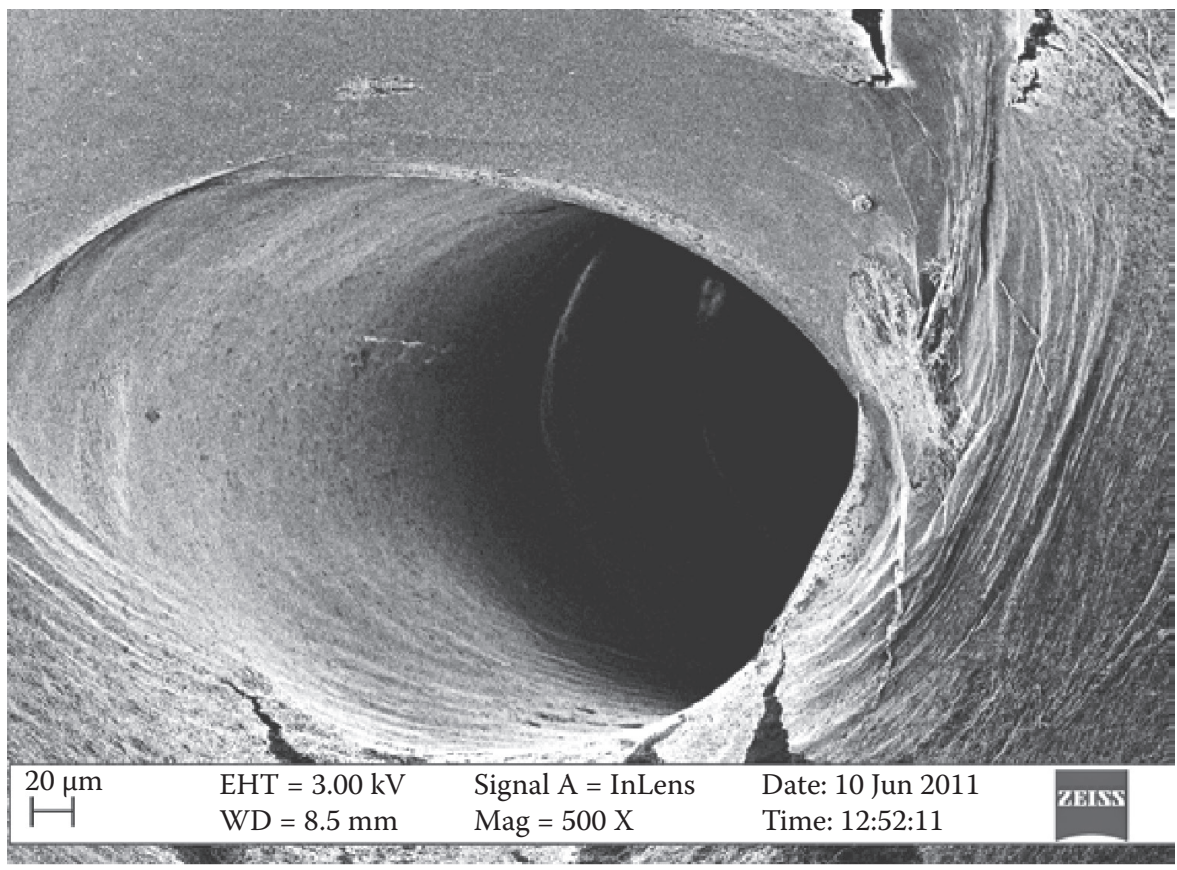

FIGURE 10.13

SEM showing a microchannel in a BNC scaffold with a diameter ranging from 200 to $350 \mu \mathrm{m}$.

\section{Acknowledgments}

The National Science Foundation is greatly acknowledged for their financial support of project NSFIIP-102642. We also acknowledge the excellent technical support provided by Ms. Isabel Löwstedt for the creation of microvascularized BNC.

\section{References}

Andersson, J., H. Stenhamre, H. Bäckdahl, and P. Gatenholm. 2010. Behavior of human articular chondrocytes on microporous bacterial cellulose scaffolds. J. Biomed. Mater. Res. A 94:1124-1132.

Atala, A. 2002. Experimental and clinical experience with tissue engineering techniques for urethral reconstruction. Urol. Clin North Am. 29:485-492, ix.

Bäckdahl, H., M. Esguerra, D. Delbro, B. Risberg, and P. Gatenholm. 2008. Engineering tissue-bacterial cellulose as a potential biomaterial. J. Tissue Eng. Regen. Med. 2(6):320-330. 
Bäckdahl, H., G. Helenius, A. Bodin, B. Johansson, U. Nanmark, B. Risberg, and P. Gatenholm. 2006. Bacterial cellulose as potential scaffold for tissue engineered blood vessels: mechanical properties and cell interactions. Biomaterials 27:2141-2149.

Bäckdahl, H., B. Risberg, and P. Gatenholm. 2011. Observation on bacterial cellulose tube formation for application as vascular graft. Mater. Sci. Eng. C 31(1):14-21.

Badylak, S. F., G. C. Lantz, A. Coffey, and L. A. Geddes. 1989. Small intestinal submucosa as a large diameter vascular graft in the dog. J. Surg. Res. 47:74-80.

Barud, H. S., C. Barrios, T. Regiani, R. F. C. Marques, M. Verelst, J. Dexpert-Ghys, Y. Messaddeq, and S. J. L. Ribeiro. 2008. Self-supported silver nanoparticles containing bacterial cellulose membranes. Mater. Sci. Eng. C 28:515-518.

Bodin, A., L. Gustafsson, and P. Gatenholm. 2006. Surface-engineered bacterial cellulose as template for crystallization of calcium phosphate. J. Biomater. Sci. Polym. Ed. 17(4):435-447.

Bodin, A., S. Wu, S. Bharadwaj, P. Gatenholm, A. Atala, and Y. Zhang. 2010. Tissue engineered conduit using urine-derived stem cells seeded bacterial cellulose polymer in urinary reconstruction and diversion. Biomaterials 31: 8889-8901.

Brackmann, C., A. Bodin, M. Åkeson, P. Gatenholm, and A. Enejder. 2010. Imaging of real-time bacterial cellulose scaffold biosynthesis and in-vivo tissue integration. Biomacromolecules 11:542-548.

Bueno, R. R., J. F. Tanguay, F. S. Brito, Jr., E. E. Guerios, J. E. Tarastchuk, P. A. Sanches, P. M. Andrade, P. F. Rossi, and F. L. Bueno. 2009. Evaluation of the efficacy and safety of a stent covered with biosynthetic cellulose in a rabbit iliac artery model. J. Invasive Cardiol. 21:392-396.

Czaja, W., A. Krystynowicz, S. Bielecki, and R. M. Brown, Jr. 2006. Microbial cellulose-the natural power to heal wounds. Biomaterials 27:145-151.

Davalos, R. V., G. J. McGraw, T. I., Wallow, A. M. Morales, K. L. Krafcik, G. J. Fiechtner, Y. Fintschenko, E. B. Cummings, and B. A. Simmons. 2008. The application of insulator-based dielectrophoresis polymer microfluidic devices as particle separators and the impact of dynamic surface coatings on performance. Anal. Bioanal. Chem. 390:847-855.

Esguerra, M., H. Fink, M. W. Laschke, D. Delbro, A. Jeppsson, P. Gatenholm, M. G. Menger, and B. Risberg. 2010. Intravital fluorescent microscopic evaluation of bacterial cellulose as scaffold for vascular grafts. J. Biomed. Mater. Res. A. 93:140-149.

Evans, B. R., H. O'Neill, S. A. Hutchens, R. S. Benson, and C. J. Rawn. 2008. A degradable bacterial cellulose composite for bone replacement. Paper presented at the 235th American Chemical Society National Meeting, April 6-10, New Orleans, LA.

Fontana, J. D. 1990. Acetobacter cellulose pellicle as a temporary skin substitute. Appl. Biochem. Biotechnol. 24:253-264.

Gatenholm, P., and D. Klemm. 2010. Bacterial nanocellulose as a renewable material for biomedical applications. MRS Bull. 35:208-213.

Harrison, B. S., D. Eberli, S. J. Lee, A. Atala, and J. J. Yoo. 2007. Oxygen producing biomaterials for tissue regeneration. Biomaterials 28:4628-4634.

Helenius, G., H. Bäckdahl, A. Bodin, U. Nanmark, P. Gatenholm, and B. Risberg. 2006. In vivo biocompatibility of bacterial cellulose. J. Biomed. Mater. Res. A. 76:431-438. 
Hu, J., and M. Catchmark. 2009. Effect of freeze-drying behavior on the density and structure of bacterial cellulosic films by different acidic and alkaline treatments. Paper presented at the ASABE Annual International Meeting, June 21-24, Reno, NV. Paper no. 09677.

Hutchens, S. A., R. S. Benson, B. R. Evans, C. J. Rawn, and H. O’Neill. 2009. A resorbable calcium-deficient hydroxyapatite hydrogel composite for osseous regeneration. Cellulose 16:887-898.

Klemm, D., D. Schumann, F. Kramer, N. Hessler, M. Hornung, H. P. Schmauder, and S. Marsch. 2006. Nanocelluloses as innovative polymers in research and application. In: Advances in Polymer Science, Vol. 205, Polysaccharides II, ed. D. Klemm, 49-96. Heidelberg: Springer.

Klemm, D., D. Schumann, U. Udhardt, and S. Marsch. 2001. Bacterial synthesized cellulose - artificial blood vessels for microsurgery. Prog. Polym. Sci. 26:1561-1603.

Lo, H., M. S. Ponticiello, and K. W. Leong. Fabrication of controlled release biodegradable foams by phase separation. Tissue Eng. 1995 Spring; 1(1):15-28.

Lohmann \& Rauscher GmbH \& Co. KG, Suprasorb X + PHMB. http://www.activahealthcare.co.uk/casestudies-files/SXP016-Nielsen_-HB-cellulose-basedwound-dressing-with-po.pdf.

Malm, C. J., B. Risberg, A. Bodin, H. Bäckdahl, B. R. Johansson, P. Gatenholm, and A. Jeppsson. 2012. Small calibre biosynthetic bacterial cellulose blood vessels: 13-months patency in a sheep model. Scand. Cardiovasc. J. 46:57-62.

Nam, Y. S., I. J. Yoon, and T. G. Park. 2000. A novel fabrication method of macroporous biodegradable polymer scaffolds using gas foaming salt as a porogen additive. J. Biomed. Mater. Res. 53:1-7.

Petersen, N., and P. Gatenholm. 2011. Bacterial cellulose-based materials and medical devices: current state and perspectives. Appl. Microbiol. Biotechnol. 91:1277-1286.

Pohl, H. A. 1978. Dielectrphoresis-The Behavior of Neutral Matter in Non-Uniform Electric Fields. Cambridge: Cambridge University Press.

Putra, A., F. Furukawa, A. Kakugo, and J. P. Gong. 2008. Novel oriented bacterial cellulose gels on oxygen-permeable substrates. Cellulose Commun. 15:73-78.

Sano, M., A. Rojas, P. Gatenholm, and R. Davalos. 2010. Electromagnetically controlled biological assembly of aligned bacterial cellulose nanofibers. Ann. Biomed. Eng. 38:2475-2484.

Schumann, D. A., J. Wippermann, D. O. Klemm, F. Kramer, D. Koth, H. Kosmehl, T. Wahlers, and S. Salehi-Gelani. 2009. Artificial vascular implants from bacterial cellulose: preliminary results of small arterial substitutes. Cellulose 16:877-885.

Solchaga, L. A., J. E. Dennis, V. M. Goldberg, and A. Caplan. 1999. Hyaluronic acid-based polymers as cell carriers for tissue-engineered repair of bone and cartilage. J. Orthop. Res. 17:205-213.

Stevens, M. M., and J. H. George. 2005. Exploring and engineering the cell surface interface. Science 310:1135-1138.

Stitzel, J., J. Liu, S. J. Lee, M. Komura, N. Levi, J. Berry, S. Soker, G. Lim, M. Van Dyke, R. Czerw, J. Yoo, and A. Atala. 2006. Controlled fabrication of a biological vascular substitute. Biomaterials 27:1088-1094.

Tandon, V., S. K. Bhagavatula, W. C. Nelson, and B. J. Kirby. 2008. Zeta potential and electroosmotic mobility in microfluidic devices fabricated from hydrophobic polymers: 1. The origins of charge. Electrophoresis 29(5):1092-1101. 
Tang, W., S. Jia, Y. Jia, and H. Yang. 2010. The influence of fermentation conditions and post-treatment methods on porosity of bacterial cellulose membrane. World J. Microbiol. Biotechnol. 26:125-131.

Temenoff, J. S., and A. G. Mikos. 2000. Review: Tissue engineering for regeneration of articular cartilage. Biomaterials 21:431-440.

Wang, H., W. Hu, S. Chen, S. Shi, X. Zhang, W. Shen, and X. Li. 2008. Bacteria cellulose membrane containing silver chloride nanoparticle and preparation and application thereof, Donghua University Sep, 17 2008: CN 200810037074.

Webster, T. 2007. Nanotechnology: better materials for all implants. Mater. Sci. Forum 539-543:511-516.

Wiegand, C., P. Elsner, U. C. Hipler, and D. Klemm, 2006. Protease and ROS activities influenced by a composite of bacterial cellulose and collagen type I in vitro. Cellulose 13:689-696.

Wippermann, J., D. Schumann, D. Klemm, H. Kosmehl, S. Salehi-Gelani, and T. Wahlers. 2009. Preliminary results of small arterial substitute performed with a new cylindrical biomaterial composed of bacterial cellulose. Eur. J. Vasc. Endovasc. 37:592-596.

Yano, H., J. Sugiyama, A. N. Nakagaito, M. Nogi, T. Matsuura, M. Hikita, and K. Handa. 2005. Optically transparent composites reinforced with networks of bacterial nanofibers. Adv. Mater. 17:153-155.

Zaborowska, M., A. Bodin, H. Bäckdahl, J. Popp, A. Goldstein, and P. Gatenholm. 2010. Microporous bacterial cellulose as a potential scaffold for bone regeneration. Acta Biomater. 6:2540-2547.

Zhou, Q., Y. Gong, and C. Gao. 2005. Microstructure and mechanical properties of poly(L-lactide) scaffolds fabricated by gelatin particle leaching method. J. Appl. Polym. Sci. 98:1373-1379.

Zimmermann, K. A., K. T. Sheets, R. Fox, J. M. LeBlanc, and P. Gatenholm. 2011. Biomimetic design of a bacterial cellulose/hydroxyapatite nanocomposite for bone healing applications. Mater. Sci. Eng. C 31(1):43-49. 


\section{1}

\section{Biomimetic Mineralization of Apatite on Bacterial Cellulose}

\section{Thi Thi Nge $\mathrm{N}^{1,2}$ and Junji Sugiyama ${ }^{2}$}

${ }^{1}$ Institute of Scientific and Industrial Research (ISIR),

Osaka University, Ibaraki, Osaka, Japan

${ }^{2}$ Research Institute for Sustainable Humanosphere (RISH),

Kyoto University, Gokasho, Uji, Kyoto, Japan

\section{CONTENTS}

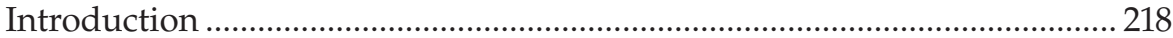

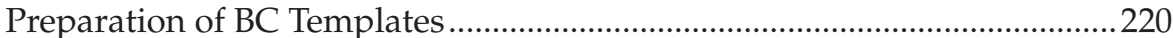

Microbial Synthesis of BC from Standard Culture Medium

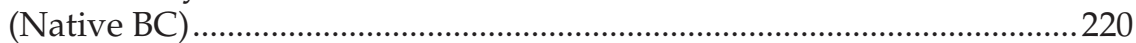

Surface Modification by TEMPO-Mediated Oxidation (BC-TEMPO)....221

Microbial Synthesis of BC from Modified Culture Medium

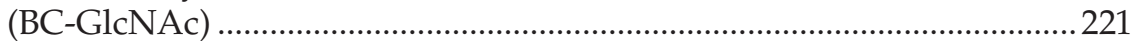

Morphology of BC Templates...................................................................225

Biomimetic Mineralization of Apatite on BC Templates ................................222

Soaking BC Templates in an SBF (1.5 SBF) ..............................................222

Soaking BC Templates in Calcium and Phosphate Solutions ...................222

Morphology and Structural Analyses of the Mineralized Ca-P Phase.........228

BC Templates Soaked in 1.5 SBF ..............................................................228

BC Templates Alternatively Soaked in Calcium and Phosphate

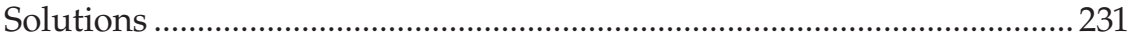

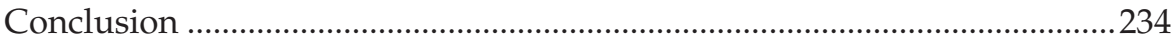

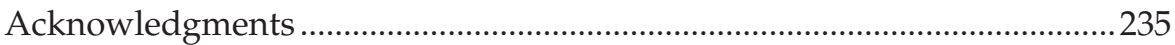

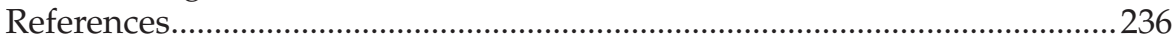

Biomimetic mineralization of apatite on bacterial cellulose (BC) was investigated by soaking different modified $\mathrm{BC}$ templates in a simulated body fluid (1.5 SBF) and/or alternate soaking in $0.05 \mathrm{M}$ calcium chloride and $0.03 \mathrm{M}$ sodium dihydrogen phosphate solutions prepared with a calcium:phosphorus molar ratio of 1.67 at a physiological temperature of $37^{\circ} \mathrm{C}$. Modification of $\mathrm{BC}$ includes surface modification by 
2,2,6,6-tetramethylpyperidine-1-oxyl (TEMPO)-mediated oxidation and medium modification by addition of amino sugar $\mathrm{N}$-acetylglucosamine (GlcNAc) during cellulose biosynthesis. The carboxylate content of TEMPO-oxidized BC (BC-TEMPO) measured by conductimetric titration was $0.25 \mathrm{mmol} / \mathrm{g}$ cellulose. The amount of GlcNAc incorporated in BC determined by the radiolabeling method was $0.03-0.36$ mole $\%$, depending on the amount of nonradioactive GlcNAc present in the culture medium and the type of starter culture used. Attenuated total reflectance-Fourier transform infrared spectroscopy (ATR-FTIR) and inductively coupled plasma atomic emission spectroscopy (ICP-AES) analysis indicated that the crystalline phase nucleated on the $\mathrm{BC}$ microfibrils surface was calcium-deficient carbonated apatite through the initial formation of octacalcium phosphate (OCP) or OCP-like calcium phosphate phase. From field emission scanning electron microscopy (FE-SEM) and transmission electron microscopy (TEM) observations, the apatite mineral was in the form of globules or clusters of thin film plates, which varied in size from less than $1 \mu \mathrm{m}$ to approximately $6 \mu \mathrm{m}$ with respect to different BC templates and the soaking method. The BC-TEMPO having active carboxyl functional groups showed a faster nucleation rate than native BC and GlcNAc-incorporated BC. It was found that the ability of $\mathrm{BC}$ templates to induce mineral nucleation differed according to the surface structure of the $\mathrm{BC}$, which strongly influenced the growth behavior of the apatite crystals.

\section{Introduction}

Bacterial cellulose (BC) produced by the bacterium (Hestrin and Schramm 1954) Gluconacetobacter xylinus - previously named Acetobacter xylinum - has unique properties, including an ultrafine network with higher-order architecture, great mechanical strength and crystallinity, high water-holding capacity, in situ moldability, and chemical purity (free of lignin and hemicellulose), that distinguish it from other forms of plant cellulose (Jonas and Farah 1998; Yamanaka et al. 1989). The unique physical and mechanical properties of BC as well as its purity have been exploited for multiple applications that range from high-quality audio membranes, electronic paper, and fuel cells to biomedical materials (Czaja et al. 2006; Klemm et al. 2001). Moreover, the in vivo biocompatibility evaluation of $\mathrm{BC}$ in rats has demonstrated that it is well integrated into the host tissues and does not elicit any chronic inflammatory reaction, making it a potentially interesting scaffolding material for tissue engineering (Helenius et al. 2006). Because of its high water-holding capacity, the hydrophilic network of BC enables particulates to infiltrate into its network. This unique property allows BC to be used as a template for 
mineralization of inorganic particles in the form of small apatite crystals (Nge and Sugiyama 2007), which will be discussed in this chapter.

The growth and form of biologically relevant minerals, such as calcium phosphate (in bone tissue) and calcium carbonate (in mollusk shell) involve the deposition of these solids in the presence of organic molecules. Bone tissue is a type I collagen fiber-apatite composite in which hierarchical structure collagen fibrils are calcified by deposition of calcium and phosphate in the form of small apatite crystals. The bone cells integrating within the mineralized collagen matrix are biochemically active and communicate with each other as well as with cells outside the tissues. In this way, the mineral becomes an integrated system capable of functional adaptation, accommodating the mechanical force encountered in bone tissues (Gilmcher 1998).

Therefore bioceramic composites based on calcium phosphates are important materials for bone substitution, repair, and tissue engineering applications. It was reported that bioactive materials such as Bioglass ${ }^{\circledR}$, sintered hydroxyapatite, and glass-ceramic apatite-wollastonite (A-W) have the ability to form a carbonate-containing hydroxyapatite (bonelike apatite) layer on their surface in the living body and bond to host living bony tissue through this apatite layer (Kokubo 1991). Formation of bonelike apatite on the surface of the artificial implant is thus essential for bonding the implant to the living bone without intervention of the fibrous tissues (Kokubo 1993). A biomimetic route for the formation of bonelike apatite on various materials such as metals (Uchida et al. 2003) and polymers (Bigi et al. 2002; Maeda et al. 2002; Takeuchi et al. 2003) was developed to improve bonding abilities.

Fabrication of matrix-assisted bioceramic composites by in situ precipitation or mineralization on biocompatible and/or biodegradable polymers using a biomimetic approach has become an attractive method because the polymer matrices serve as not only templates, but also binders to prevent migration of the mineral particles, as well as improve mechanical integrity of the developed materials owing to their toughness and flexibility. The biomimetic approach involves soaking the template materials either in a simulated body fluid (SBF) having equal ion concentrations to that of human blood plasma at physiological pH and temperature (Kokubo et al. 1990), or alternatively in calcium and phosphate solutions prepared with a calcium (Ca):phosphorus $(\mathrm{P})$ ratio of 1.67, similar to the Ca:P ratio of synthetic hydroxyapatite $\left(\mathrm{HA} ; \mathrm{Ca}_{10}\left(\mathrm{PO}_{4}\right)_{6}(\mathrm{OH})_{2}\right)$. The concept of mineralization includes the heterogeneous nucleation of apatite crystals in the presence of surface functional groups of polymer matrices and the increased supersaturation of the surrounding fluid to accelerate the nucleation process and growth.

Since surface chemical structures exert a significant influence on the formation of the apatite layer on materials surfaces, activation of the BC surface appears to be a prerequisite for biomimetic mineralization. Granja et al. (2001a) reported that the derivatization of the cellulose with anionic phosphate groups or pretreatment of cellulose with calcium dichloride (Granja et al. 2001b) activated the deposition of apatite on cellulose from SBF under 
physiological conditions. In this work, chemical modification of $\mathrm{BC}$ by 2,2,6,6-tetramethylpyperidine-1-oxyl (TEMPO)-mediated oxidation to introduce carboxyl functional groups, and modification of the culture medium G. xylinus by addition of amino sugar (N-acetylglucosamine) were performed to render the $\mathrm{BC}$ materials biomimetic. TEMPO, a water-soluble and stable nitroxyl radical, is well known for its catalytic and selective oxidation of primary hydroxyl groups of polysaccharides under aqueous conditions (Montanari et al. 2005; Saito and Isogai 2004). On the other hand, the amino sugar $\mathrm{N}$-acetylglucosamine (GlcNAc) is the monomer unit of chitin, the second most abundant natural biopolymer, which is commonly found in the shells of marine crustaceans and cell walls of fungi (Roberts 1992). GlcNAc also shares the structural feature of glycosaminoglycan (GAGs), which are unbranched heteropolysaccharides consisting of the repeating unit [uronic acid-amino sugar $]_{\mathrm{n}}$. GAGs are extracellular matrix (ECM) constituents of skeletal tissues and are considered to play an important role in stimulating chondrogenesis by modulating chondrocyte morphology, differentiation, and function (Di Martino et al. 2005; Suh and Matthew 2000). Considering the function of GAGs in the ECM of skeletal tissues, our strategy was to attempt incorporating an amino sugar in the $\mathrm{BC}$ to prepare a biomimetic ECM. The overall objective is to fabricate a new generation of BC-based biomimetic composites for potential osteological applications.

\section{Preparation of BC Templates}

\section{Microbial Synthesis of BC from Standard Culture Medium (Native BC)}

The bacterial strain of cellulose-producing species, Acetobacter aceti (AJ12368), closely related to G. xylinus, and the Schramm-Hestrin (SH) medium (Hestrin and Schramm 1954) were used for BC production (Nge and Sugiyama 2007). The $\mathrm{pH}$ of the medium was adjusted to 5 with $1 \mathrm{~N}$ hydrochloric acid $(\mathrm{HCl})$ and autoclaved at $120^{\circ} \mathrm{C}$ for $20 \mathrm{~min}$. The cells used as the inoculum were statically precultured in test tubes at $27^{\circ} \mathrm{C}$ for 2 weeks. The thick gelatinous membrane was squeezed aseptically to remove cells embedded inside the pellicle. A $25 \mathrm{~mL}$ cell suspension $\left(1.3 \times 10^{7}\right.$ cells $\left./ \mathrm{ml}\right)$ was then transferred to $500 \mathrm{ml}$ fresh medium and successively distributed $(9 \mathrm{ml} /$ well) into six-well culture plates (Iwaki-Asahi Techno Glass, Japan). The BC pellicles (approximately $35 \mathrm{~mm} \Phi$ ) recovered after static culture at $27^{\circ} \mathrm{C}$ for 9 days were purified in two steps. First, the pellicles were placed in boiling distilled water for $1 \mathrm{~h}$, followed by incubation in $0.1 \mathrm{M}$ sodium hydroxide $(\mathrm{NaOH})$ at $80^{\circ} \mathrm{C}$ for $2 \mathrm{~h}$. The pellicles were then washed with distilled water until neutral $\mathrm{pH}$ was reached. Some of the purified pellicles were subjected to freeze-drying after 
having been flash frozen in liquid nitrogen (namely native $\mathrm{BC}$ ) and some were used for TEMPO-mediated oxidation.

\section{Surface Modification by TEMPO-Mediated Oxidation (BC-TEMPO)}

TEMPO-mediated oxidation was performed to introduce surface carboxyl groups at the C6 primary hydroxyl groups of the cellulose chains. Briefly, the never-dried BC pellicles (dry weight of $0.15 \pm 0.003 \mathrm{~g}$ ) were suspended in distilled water $(80 \mathrm{ml})$ containing TEMPO $(0.0075 \mathrm{~g})$ and sodium bromide $(0.075 \mathrm{~g})$ under continuous stirring. An appropriate amount of sodium hypochlorite solution corresponding to $2.42 \mathrm{mmole} / \mathrm{g}$ cellulose was added slowly and the $\mathrm{pH}$ of the reaction was maintained at 10.5 by addition of $0.5 \mathrm{M} \mathrm{NaOH}$. The oxidation was carried out for approximately $2 \mathrm{~h}$, when no more $\mathrm{NaOH}$ consumption was observed. Then, $2 \mathrm{ml}$ of ethanol was added to quench the oxidation, followed by the addition of $0.1 \mathrm{~N} \mathrm{HCl}$ to adjust the reaction mixture to reach neutral $\mathrm{pH}$. The oxidized $\mathrm{BC}$ pellicles were washed with a large amount of distilled water to remove residual reagents and subjected to freeze-drying, as mentioned above (namely BC-TEMPO). The carboxylate content measured by conductimetric titration was $0.25 \mathrm{mmol} / \mathrm{g}$ cellulose.

Some of the TEMPO-oxidized BC pellicles recovered after washing with distilled water were immersed in $0.1 \mathrm{M}$ calcium chloride $\left(\mathrm{CaCl}_{2}\right)$ solution with stirring at room temperature for $6 \mathrm{~h}$ to exchange the sodium counterion of the carboxylate groups to calcium. The ion-exchanged TEMPO-oxidized $\mathrm{BC}$ pellicles were subjected to freeze-drying (BC-TEMPO-Ca) after washing with distilled water. The amount of calcium ions in BC-TEMPO-Ca measured by X-ray fluorescence analysis (MESA-500, Horiba Co., Japan) was $0.22 \mathrm{mmol} / \mathrm{g}$ cellulose. With a carboxylate content of $0.25 \mathrm{mmol} / \mathrm{g}$ cellulose, the ion exchange ratio was approximately 1:1. Therefore the formation of $\mathrm{BC}-\mathrm{COOCa}{ }^{+}$was predominant over (BC-COO) ${ }_{2} \mathrm{Ca}$ (Saito and Isogai 2005).

\section{Microbial Synthesis of BC from Modified Culture Medium (BC-GIcNAc)}

The standard culture medium was modified by the addition of amino sugar $\mathrm{N}$-acetylglucosamine (GlcNAc) as a source of sugar together with glucose in different compositions (Table 11.1) (Nge et al. 2010a). As shown in Figure 11.1, adaptation of Acetobacter cells to the presence of the amino sugar was performed by five repeated transfers of 3-day-old inoculum into the medium containing different GlcNAc concentrations. The 3-day-old inoculum was selected based on cell density estimated by measuring the optical density at $600 \mathrm{~nm}$. The volume ratio of inoculum to medium was 1:10 for all compositions and the static culture fermentation was carried out as described previously.

The effect of sugar composition on the rate of cellulose production is shown in Figure 11.2a. The cellulose dried mass produced in the presence of glucose (AJ2000) increased over 14 days and reached $6.41 \mathrm{mg} / \mathrm{ml}$, with an exponential mass production from days 3-7 followed by a slower production rate 


\section{TABLE 11.1}

Composition of Glucose and N-Acetylglucosamine (GlcNAc) at a Fixed Carbon Source of $2 \%$

\begin{tabular}{lccc}
\hline & Medium ID $^{\mathbf{a}}$ & Glucose $(\mathrm{g} / \mathbf{1 0 0} \mathbf{~ m l})$ & GlcNAc $(\mathrm{g} / \mathbf{1 0 0 ~} \mathbf{~ m l})$ \\
\hline SH medium & 2000 & 2.0 & 0.0 \\
Modified SH medium & 1604 & 1.6 & 0.4 \\
Modified SH medium & 1010 & 1.0 & 1.0 \\
Modified SH medium & 0416 & 0.4 & 1.6 \\
Modified SH medium & 0020 & 0.0 & 2.0 \\
\hline
\end{tabular}

a A medium ID was given for the different sugar compositions. The BC produced from the different media are named after the corresponding medium ID.

Source: Adapted from T. T. Nge et al., Bacterial cellulose-based biomimetic composites. In: Biopolymers (ISBN 978-953-307-109-1, InTech, 2010), 345-368, with permission.

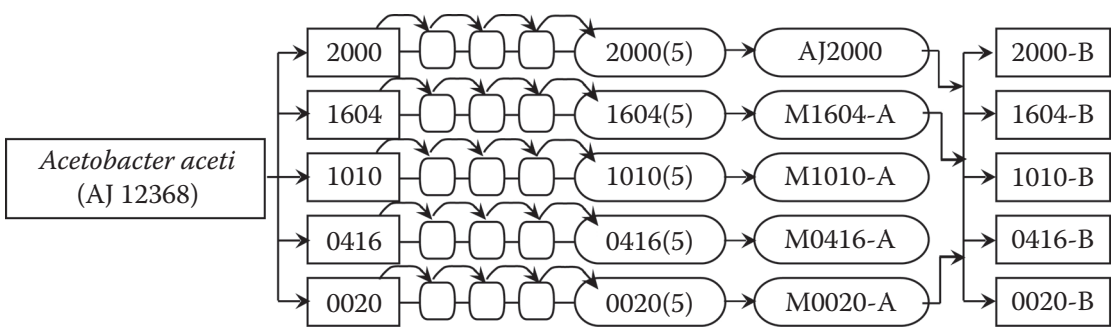

\section{FIGURE 11.1}

Experimental setup for $\mathrm{N}$-acetylglucosamine (GlcNAc) incorporation in BC. The first two digits represent the glucose concentration and the later two represent the GlcNAc concentration as described in Table 1. M: modified medium containing GlcNAc (starter culture obtained by 5 times repeated transferring of inoculum at 3 days of growth). A: GlcNAc-containing medium/ GlcNAc-containing BC (inoculation using the same sugar contents as for the starter culture). B: GlcNAc-containing BC with different sugar content (inoculation from AJ2000, M1604-A, and M0020-A as starter cultures). Source: From Nge et al., Biopolymers (ISBN:978-953-307-109-1, InTech., 2010), 345-368, with permission.

until day 14. In the presence of mixed sugars (glucose and GlcNAc), the lag phase was shorter than that of AJ2000 medium as judged by a higher mass production at days 3 and 4, followed by an increasing mass phase until day 7 and leveling off. However, the mass produced over 14 days in the mixed sugars media was lower than AJ2000 medium, with 4.74, 3.33, and $1.29 \mathrm{mg}$ cellulose/ml for M1604-1604A, M1604-1010B, and M1604-0416B, respectively. The cellulose production in the medium containing GlcNAc only (M1604-0020B) was low $(0.19 \mathrm{mg} / \mathrm{ml})$ throughout the incubation period, most likely reflecting low cell growth. At a fixed total carbon source of $2 \%$, the decrease in cellulose mass with increasing GlcNAc concentrations reflects the limitation of cellulose formation, although GlcNAc enhances the initial cellulose production rate. There was greater consumption of glucose rather than GlcNAc by the cells. The mass therefore decreased with decreasing glucose 


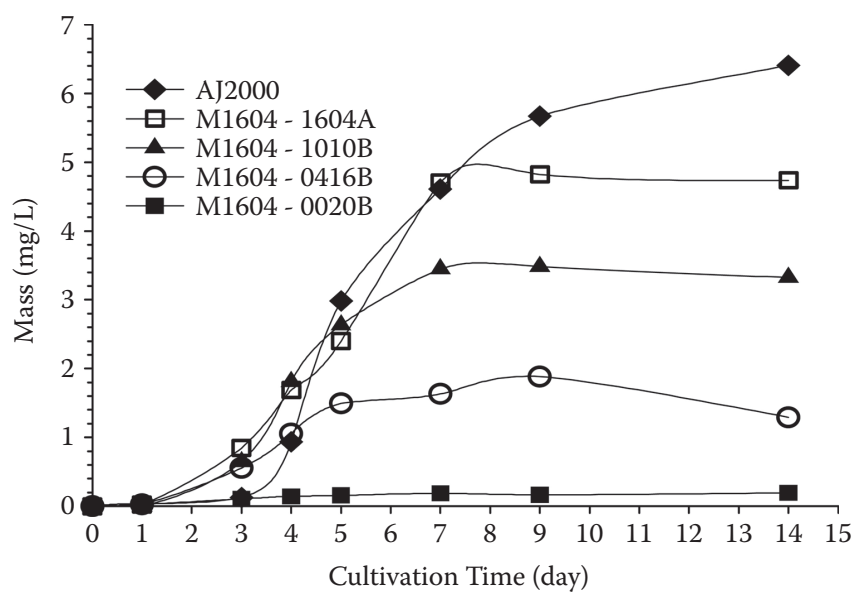

FIGURE 11.2a

Cellulose production in the presence of glucose, mixed sugars, or GlcNAc as a function of cultivation time. Source: From Nge et al., Biopolymers (ISBN:978-953-307-109-1, InTech., 2010), 345-368, with permission.

concentrations at a fixed total carbon source. Shirai et al. (1994) also reported the accumulation of GlcNAc during incubation in mixed sugar media. For their analysis, the authors determined the time course of sugar consumption by using Schales's modified procedure for total sugar and a glucose oxidase/peroxidase system for glucose.

Based on the results shown in Figure 11.2a, the cultivation time in further experiments was fixed to 7-9 days for the media containing GlcNAc of all starter cultures. Cellulose produced from AJ2000 and M1604A showed a similar mass profile, whereas a slightly lower mass profile was observed for cellulose produced from M0020A starter culture. Therefore cell adaptation was not critical in starter cultures containing small amounts of GlcNAc (M1604A). The amount of incorporated GlcNAc was determined by a labeling method based on the use of radioactive GlcNAc (1.85 MBq N-acetyl-D-[1-14 C] glucosamine; Moravek Biochemicals, Inc., Brea, CA, USA) and liquid scintillation counting (LSC; Packard Tri-Carb, Perkin Elmer, Inc.) (Nge et al. 2010a). Small-scale labeling was carried out in $1 \mathrm{ml}$ culture media containing different amounts of nonradioactive GlcNAc and inoculated using starter cultures AJ2000, M1604, M1010, M0416, and M0020. The molar concentration of added radioactive GlcNAc was negligible compared to the concentration of nonradioactive sugar in the culture medium. The static cultures were incubated for 7 days under the same conditions as for bulk production. The small pellicle formed was taken out at days 2, 3, 5, and 7 followed by purification as described for bulk production. After addition of the scintillation cocktail (Ultima Gold, Perkin Elmer) in the vials containing the purified BC pellicles, the radioactivity corresponding to the incorporated GlcNAc was 


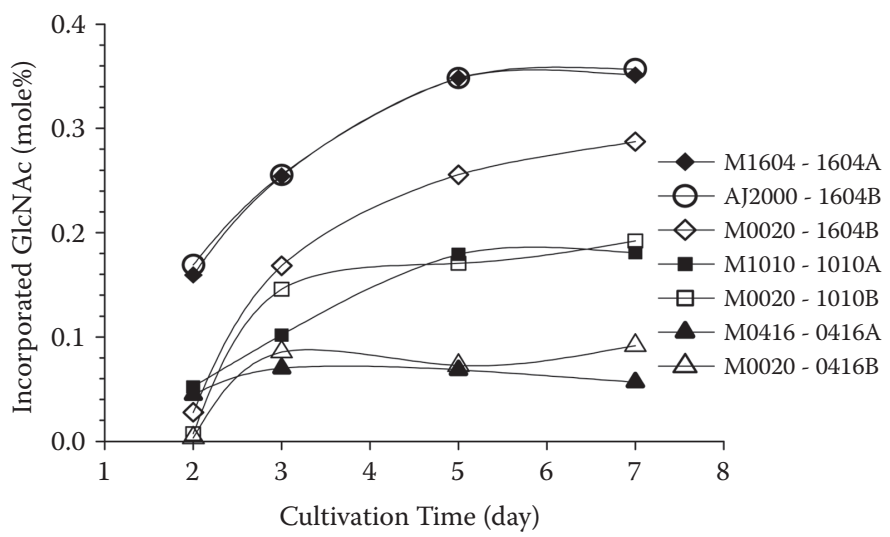

FIGURE $2 b$

Amount of incorporated GlcNAc in bacterial cellulose as a function of cultivation time. Source: From Nge et al., Biopolymers (ISBN:978-953-307-109-1, InTech., 2010), 345-368, with permission.

measured by liquid scintillation. The total amount of incorporated GlcNAc was then calculated from the levels of radioactivity measured.

The mole percentage of incorporated GlcNAc as a function of cultivation time from different starter cultures is shown in Figure 11.2b. A detectable level of GlcNAc incorporation was observed at day 2. The measured radioactivity increased until day 3 in M0416-0416A, M0020-M0416B, and M0020-M1010B; and day 5 in M1010-M1010A, M1604-M1604A, M0020-M1604B, and AJ2000-M1604B. They were fairly stable after 5 days, which indicated that the incorporation of GlcNAc occurred essentially in early incubation times, within 5 days. This finding was in agreement with the higher cellulose mass observed at an early incubation time in the media containing mixed sugars compared to the medium containing glucose only, as shown in Figure 11.2a. The level of GlcNAc incorporated after 7 days was in the range of $0.03-0.36 \mathrm{~mole} \%$, depending on the amount of nonradioactive GlcNAc present in the culture medium and the type of starter culture used.

The incorporation of GlcNAc decreased with increasing GlcNAc concentrations (decreasing glucose concentrations) in the culture medium. The presence of high GlcNAc concentrations with a concomitant decrease in glucose content may limit the activity of cellulose biosynthesis. Therefore the time course of cellulose production in the medium containing a high concentration of nonradioactive GlcNAc (M0020-0416B) showed a lower profile than that of M0020-1604B, although the same starter culture (M0020) was used for inoculation. In addition, the effect of the type of starter culture on GlcNAc incorporation was observed in the glucose GlcNAc 1010 and 1604 media. The starter cultures containing the same sugar content (e.g., inoculation of M1604 starter culture into 1604 medium) led to higher amounts of GlcNAc incorporation than inoculums from the medium containing GlcNAc only (i.e., inoculation 
of M0020 starter culture into 1604 medium). This effect was more significant for the 1604 medium than for the 1010 medium, where a higher incorporation rate was observed through the cultivation time. Moreover, the M1604-1604A cultures showed a similar trend as sample AJ2000-1604B (direct inoculation from a starter culture containing glucose only). This observation confirmed that no adaptation period in the presence of GlcNAc was necessary to obtain an increased GlcNAc incorporation into cellulose.

The 1604 medium is best suited for high GlcNAc incorporation and cellulose production under the conditions studied, although the maximum incorporation obtained ( $0.36 \mathrm{~mole} \%)$ is lower compared with other earlier studies (Lee et al. 2001; Shirai et al. 1994), which were based on the use of different Acetobacter strains and analysis methods. As only a small amount of GlcNAc can be incorporated during $\mathrm{BC}$ biosynthesis, we switched our strategy to the use of chitosan (a deacetylated form of chitin) instead of GlcNAc and fabricated BC/chitosan porous scaffolds by freeze-drying TEMPO-oxidized BC microfibril suspension in a chitosan solution (Nge et al. 2010b). By using this approach, one can control the ratio of $\mathrm{BC}$ and chitosan to obtain desired properties.

\section{Morphology of BC Templates}

The lateral dimensions of nascent BC microfibrils after ribbon assembly and before the formation of interwoven network mats (pellicle) from 2-day incubated culture medium were observed by transmission electron microscopy (TEM) (JEOL JEM 2000-EXII) (Nge et al. 2010a). The lateral dimension of the ribbons from standard culture media (AJ2000) was from 60 to $105 \mathrm{~nm}$, whereas a small increase in the lateral dimension of fibril aggregates (approximately 80-125 $\mathrm{nm}$ ) was observed in the GlcNAc-containing media, M1010-1010A. On the other hand, the network assembly had already formed in M1604-1604A and a wider range of fibril aggregates comprising 18-23 nm individual finer fibrils as well as $46-60 \mathrm{~nm}, 70-90 \mathrm{~nm}$, and approximately $110 \mathrm{~nm}$ bundles were observed.

The ultrafine network structures of BC pellicles observed by field emission scanning electron microscopy (FE-SEM) (JEOL JSM-6700F) are shown in Figure 11.3. Because oxygen is required for the aerobic growth of Acetobacter cells and for cellulose production, the formation of cellulose occurs only at an aerobic zone of the culture medium-air interface. Generally a dense surface layer is built at this interface, whereas the lower layer that remains in contact with the liquid medium is gelatinous and consists of a loose network. The middle compartment is a stack of several layers and network density is intermediate to that of the upper and lower surface layers. The cross-sectional surfaces of the freeze-dried BC pellicles exhibit a hierarchical structure of the physical three-dimensional (3D) microfibril network.

In particular, the dense surface layer was observed in native BC (AJ2000) and M1604, whereas only a thin surface layer was formed in M1010 and M0416. The network architecture of the frontier area between the surface 

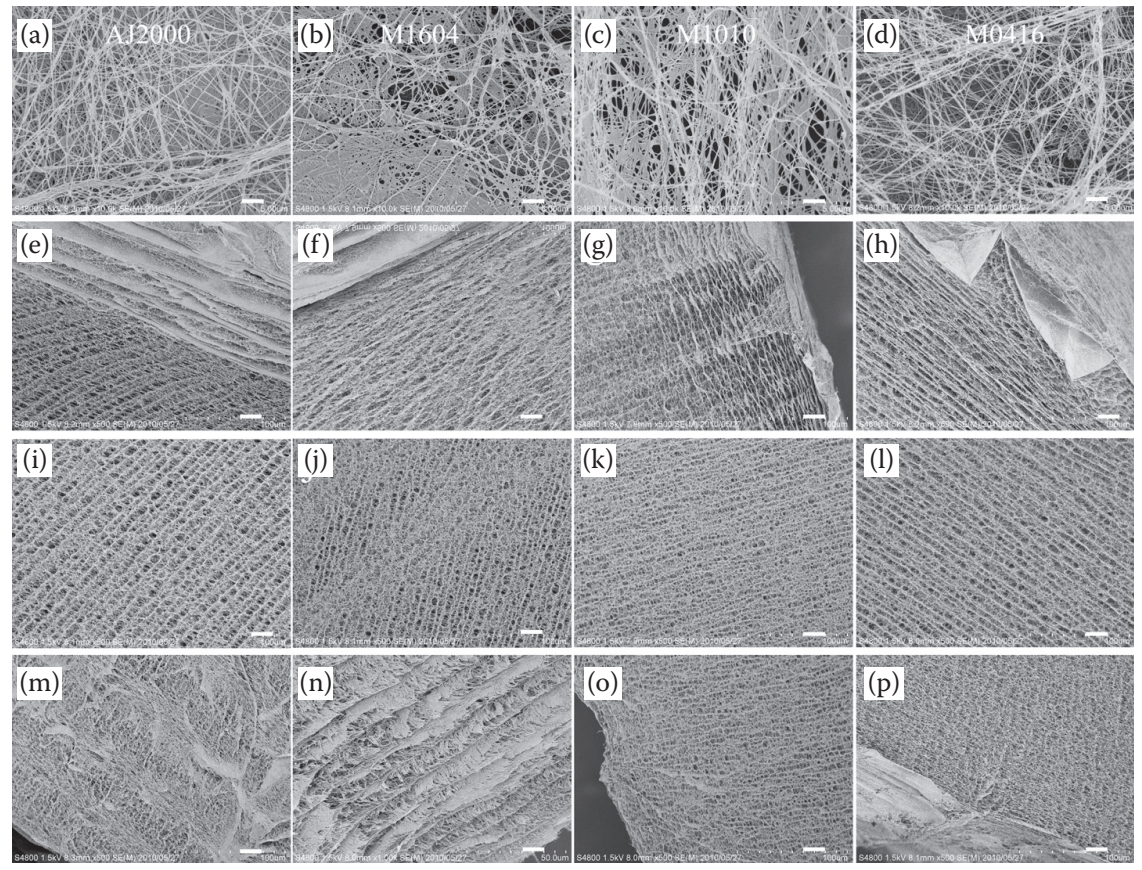

\section{FIGURE 11.3}

FE-SEM images of the upper surface layer (a-d) and cross-sectional surfaces (e-p) of freeze-dried BC (AJ2000) and BC-GlcNAc pellicles; the second row (e-h) represents the upper part (pellicle-air interface during cultivation), the third row (i-l) the middle part, and the fourth row (m-p) the lower part (pellicle-culture medium interface) of the pellicles; scale bars, $1 \mu \mathrm{m}$ for a-d and $20 \mu \mathrm{m}$ for e-p. Source: Adapted from Nge et al., Biopolymers (ISBN:978-953-307-109-1, InTech., 2010), 345-368, with permission.

layer and middle compartment also varied between samples obtained from media containing different GlcNAc concentrations. An array of specific network patterns was observed in M1010 and M0416 compared with M1604. The texture of the middle compartment reflects the highly swollen nature of the BC pellicle network in its cultivation state as the never-dried BC pellicle is known to contain approximately $99 \%$ water. This high water-holding ability, or high hydrophilicity, is explained by the presence of pores forming ultrafine 3D structures with abundant hydroxyl groups in the cellulose molecular structure. The hollow space or pore size as well as the stacking lamellar patterns were also different from each other in the middle compartment. Judging from the FE-SEM images, the decreasing order of pore size is AJ2000 $\geq$ M0416 > M1604 > M1010, with sizes of 4-8 $\mu \mathrm{m}, 4-7 \mu \mathrm{m}, 3-7 \mu \mathrm{m}$, and $2-5 \mu \mathrm{m}$, respectively. The thickness of the lower layer also varied from sample to sample, with a few layers of fibril mat in AJ2000 and M1604 and a thin layer in M1010 and M0416. Variations in the morphological formation 
of the dense surface layer, middle compartment, and lower layer as well as variations of their thickness reflect the activity of viable cells, which was influenced by the absence or presence of different GlcNAc concentrations in the medium.

\section{Biomimetic Mineralization of Apatite on BC Templates}

\section{Soaking BC Templates in an SBF (1.5 SBF)}

The biomimetic mineralization of a Ca-P coating was performed in a simple supersaturated ion solution, in which ion concentrations were 1.5 times those of the SBF developed by Kokubo et al. (1990). The 1.5 SBF was prepared by dissolving sequentially reagent-grade sodium chloride $(\mathrm{NaCl})$, sodium hydrogen carbonate $\left(\mathrm{NaHCO}_{3}\right)$, potassium chloride $(\mathrm{KCl})$, dipotassium hydrogen phosphate $\left(\mathrm{K}_{2} \mathrm{HPO}_{4} \cdot 3 \mathrm{H}_{2} \mathrm{O}\right)$, magnesium chloride hexahydrate $\left(\mathrm{MgCl}_{2} \cdot 6 \mathrm{H}_{2} \mathrm{O}\right)$, calcium chloride $\left(\mathrm{CaCl}_{2}\right)$, and sodium sulfate $\left(\mathrm{Na}_{2} \mathrm{SO}_{4}\right)$ (Wako Pure Chemical, Osaka, Japan) in distilled water. The $\mathrm{pH}$ was adjusted to $\mathrm{pH}$ $7.4 \pm 0.01$ with tris-hydroxymethyl aminomethane $\left(\mathrm{NH}_{2} \mathrm{C}\left(\mathrm{CH}_{2} \mathrm{OH}\right)_{3}\right)$ and $1 \mathrm{M}$ $\mathrm{HCl}$ while maintaining the solution temperature at $36.5^{\circ} \mathrm{C}$. The stock solution was filtered through a $0.2 \mu \mathrm{m}$ cellulose acetate filter.

Freeze-dried BC templates, namely native BC, BC-TEMPO, and BC-TEMPO-Ca with a size of $10 \mathrm{~mm} \times 10 \mathrm{~mm} \times 5 \mathrm{~mm}$ and known weight were soaked in $10 \mathrm{ml}$ of $1.5 \mathrm{SBF}$ at $37^{\circ} \mathrm{C}$ for 3 weeks. The SBF solution was renewed every 7 days. Samples were taken out at 2, 7, 14, and 21 days, washed thoroughly with distilled water, and freeze-dried. The freeze-dried samples were weighed to determine the extent of mineral deposition. Four to five pieces were used for each BC sample for each designated soaking time (Nge and Sugiyama 2007).

\section{Soaking BC Templates in Calcium and Phosphate Solutions}

The calcium solution (0.05 $\mathrm{M} \mathrm{CaCl}_{2}$ in $50 \mathrm{mM}$ Tris- $\mathrm{HCl}$ buffer at $\left.\mathrm{pH} 7.4\right)$ and the phosphate solution $\left(0.03 \mathrm{M} \mathrm{NaH}_{2} \mathrm{PO}_{4}\right)$ were prepared to obtain $\mathrm{Ca}: \mathrm{P}$ ratio of 1.67 similar to the $\mathrm{Ca}$ :P ratio of synthetic hydroxyapatite $\left(\mathrm{Ca}_{10}\left(\mathrm{PO}_{4}\right)_{6}(\mathrm{OH})_{2}\right)$. Freeze-dried BC samples of native BC, BC-TEMPO, BC-TEMPO-Ca, and $\mathrm{BC}-\mathrm{GlcNAc}$ were alternatively soaked in the calcium and phosphate solutions at $37^{\circ} \mathrm{C}$ for $30 \mathrm{~min}$ each in a reciprocal shaking incubator (50 strokes $/ \mathrm{min}$ ). The samples were rinsed thoroughly with distilled water between each soaking solution. A total of five soaking cycles were performed. Samples were withdrawn from each alternate soaking cycle, gently and thoroughly washed with distilled water, and freeze-dried to determine the weight of mineralized Ca-P that deposited on different BC templates (Nge et al. 2010a) 


\section{Morphology and Structural Analyses of the Mineralized Ca-P Phase}

The morphology of deposited minerals on BC templates was examined by FE-SEM (S 4100B, Hitachi Co., Japan, and JEOL, JSM-6700F, Japan) operating at an accelerating voltage of $1.5 \mathrm{kV}$. The $\mathrm{BC}$ specimens were sputter-coated with platinum-paladium (Pt-Pd) and platinum for the Hitachi and JEOL microscopes, respectively. Formation of the mineralized Ca-P phase was determined by Fourier transform infrared spectroscopy (FTIR) (Spectrum 2000, Perkin Elmer) equipped with an attenuated total reflection (ATR) accessory using a single reflection diamond crystal ATR top plate. The Ca:P ratio was measured by inductively coupled plasma atomic emission spectroscopy (ICP-AES) (SPS7800, Seiko Instrument, Japan). A known amount of apatite-deposited $\mathrm{BC}$ templates were soaked in $2 \%$ nitric acid $\left(\mathrm{HNO}_{3}\right)$ for 1 day to dissolve the Ca-P mineral, followed by filtration through a $0.2 \mu \mathrm{m}$ cellulose acetate filter and dilution to a concentration of about 5 ppm (detectable range by ICP-AES). The determination of the Ca:P ratios of all samples was based on calibration using synthetic hydroxyapatite (Wako Pure Chemical Ltd., Japan) as a standard (Nge and Sugiyama 2007).

\section{BC Templates Soaked in 1.5 SBF}

Native BC, BC-TEMPO, and BC-TEMPO-Ca were soaked in 1.5 SBF to study the effect of surface functional groups on apatite formation on respective BC templates (Nge and Sugiyama 2007). The SEM images of the apatite-deposited surfaces of native BC and BC-TEMPO-Ca after 21 days of soaking in 1.5 SBF are shown in Figure 11.4. The island-like hemispherical globules of apatite crystals were formed on the BC microfibril surfaces, where the globule sizes were clearly different between native BC and BC-TEMPO-Ca, with approximately $3 \mu \mathrm{m}$ and less than $1 \mu \mathrm{m}$, respectively. From TEM observation, each apatite globule was composed of a cluster of numerous thin-film flakes, with a needlelike appearance, with the relative individual crystal size
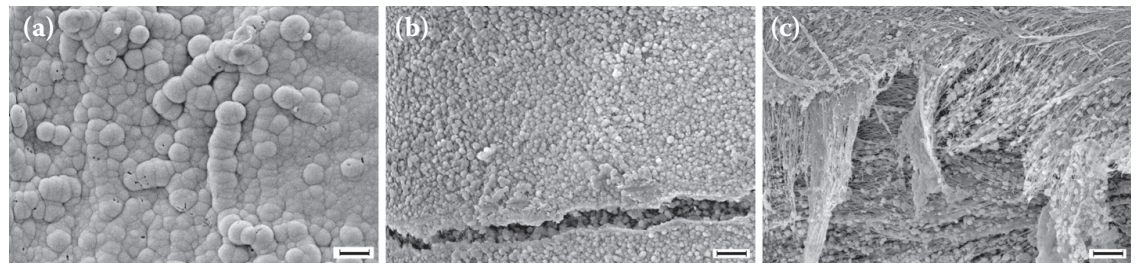

\section{FIGURE 11.4}

SEM images of apatite-deposited BC surfaces: (a) Native-BC, (b and c) BC-TEMPO-Ca after 21 days of soaking in 1.5 SBF. Source: From Nge et al., Biopolymers (ISBN:978-953-307-109-1, InTech., 2010), 345-368, with permission. 
ranging from 60 to $140 \mathrm{~nm}$ long (Nge and Sugiyama 2007). The mineralized apatite globules firmly adhered on the microfibril surfaces and formed a uniform Ca-P layer, followed by further crystal growth upon the existing layer. The variation in size of the apatite globules under the same soaking condition revealed the influence of the microfibril surface structure at the initial nucleation process of apatite formation (Sato et al. 2001; Uchida et al. 2003). The globule size formed on the microfibril surfaces of the BC-TEMPO template was about $1.5 \mu \mathrm{m}$. The presence of carboxylate groups on the $\mathrm{BC}$ microfibril surface (BC-TEMPO) and further ion exchange treatment with calcium (BC-TEMPO-Ca) significantly affect the rate of apatite nucleation by a rapid consumption of calcium and phosphate ions from the surrounding supersaturated fluid. A large number of nuclei thus grew competitively on BC-TEMPO-Ca, leading to a smaller average size than for the BC-TEMPO and native $\mathrm{BC}$ templates. In other words, an increasing amount of mineral phase or more crystals may occur principally by multiplication in BC-TEMPO-Ca, whereas progressive mineralization with an increase in size of the crystals or crystal growth may occur in native $\mathrm{BC}$, like in bone matrix mineralization and calcification of enamel (Rey et al. 1991a).

The profiles of relative weight gain of mineralized Ca-P on BC templates as a function of soaking time also reflect the growth behavior of apatite crystal with respective BC templates. The BC-TEMPO-Ca samples, which exhibited the smallest apatite globules, showed the highest deposited weight, whereas the gradual increase of mineral deposition that occurred in native $\mathrm{BC}$ was characterized by the lowest deposited weight throughout the soaking time, and the profile of BC-TEMPO samples displayed between BC-TEMPO-Ca and native BC. Because calcium ion is one of the main mineral components of the apatite crystal, a preexisting ionic complex like $\mathrm{BC}-\mathrm{COOCa}^{+}$in BC-TEMPO-Ca makes it easier and faster for subsequent $\mathrm{Ca}-\mathrm{P}$ phase formation than with other $\mathrm{BC}$ templates. The Ca:P molar ratio analyzed by ICP-AES was $1.52 \pm 0.09,1.50 \pm 0.08$, and $1.45 \pm 0.04$ for native BC, BC-TEMPO, and BC-TEMPO-Ca, respectively, after 21 days soaking in $1.5 \mathrm{SBF}$, while it was only $1.37 \pm 0.08,1.39 \pm 0.02$, and $1.31 \pm 0.05$ for a soaking time of 2 days. The Ca:P ratio of about 1.3 at day 2 indicated that the Ca-P phase nucleated on the surface of $\mathrm{BC}$ microfibrils was octacalcium phosphate (OCP; $\left.\mathrm{Ca}_{8} \mathrm{H}_{2}\left(\mathrm{PO}_{4}\right)_{6} \cdot 5 \mathrm{H}_{2} \mathrm{O}\right)$ or OCP-like Ca-P phase. Although the Ca:P ratio increased with the soaking time, the values for all the $\mathrm{BC}$ templates were lower than the theoretical value of 1.67 for synthetic hydroxyapatite $\left(\mathrm{Ca}_{10}\left(\mathrm{PO}_{4}\right)_{6}(\mathrm{OH})_{2}\right)$. The mineralized Ca-P phase thus formed on BC templates was calcium-deficient hydroxyapatite. The lower Ca:P ratio was due to the substitution of the trivalent $\mathrm{PO}_{4}{ }^{3-}$ ions by divalent ions such as acid phosphate groups $\left(\mathrm{HPO}_{4}{ }^{2-}\right)$ and carbonate groups $\left(\mathrm{CO}_{3}{ }^{2-}\right)$. As a result, vacancies at $\mathrm{Ca}^{2+}$ cationic sites in the apatite structure occurred during the formation of the Ca-P phase (Elliott et al. 1985; Rey et al. 1989).

A number of hypotheses have been proposed stating that the nature of the initial deposits, a solid phase of Ca-P in bone tissue and enamel, are 
TABLE 11.2

Assignment of ATR-FTIR Spectra for BC Templates Soaking in 1.5 SBF Solution for 21 Days

\begin{tabular}{lccccc}
\hline & \multicolumn{5}{c}{ Wavenumber $\left(\mathbf{c m}^{-1}\right)$} \\
\cline { 2 - 5 } Assignment & 0 Days & 2 Days & 7 Days & 14 Days & 21 Days \\
\hline $\mathrm{OH}, \mathrm{v}(\mathrm{BC})$ & $\sim 3448$ & $\sim 3448$ & $\sim 3448$ & $\sim 3448^{\mathrm{a}}$ & $\sim 3448^{\mathrm{a}}$ \\
$\mathrm{HPO}_{4}{ }^{2-}(\mathrm{OCP})$ & & $1295-1297$ & $1295-1297$ & & \\
$\mathrm{PO}_{4}{ }^{3-}, v_{3(\text { asym })}$ & & $1020-1024$ & $1020-1024$ & $1020-1024^{\mathrm{b}}$ & $1020-1024^{\mathrm{b}}$ \\
$\mathrm{PO}_{4}{ }^{3-}, v_{1(\text { sym) }}$ & & & $960-962^{\mathrm{b}}$ & $960-962^{\mathrm{b}}$ \\
$\mathrm{HPO}_{4}{ }^{2-}(\mathrm{OCP})$ & & & & \\
$\mathrm{CO}_{3}{ }^{2-}$ & & & $810-911$ & & \\
$\mathrm{PO}_{4}{ }^{3-}, v_{4 a}$ & & $600-602$ & $600-602^{\mathrm{b}}$ & $600-602^{\mathrm{b}}$ \\
$\mathrm{PO}_{4}{ }^{3-}, v_{4 \mathrm{c}}$ & & $558-560$ & $558-560^{\mathrm{b}}$ & $558-560^{\mathrm{b}}$ \\
\hline
\end{tabular}

a The $\mathrm{OH}$ absorption band of $\mathrm{BC}$ became very weak with increasing soaking time.

b The $\mathrm{PO}_{4}^{3-}$ absorption band of apatite became strong with increasing soaking time.

Source: Data from T. T. Nge and J. Sugiyama, "Surface functional groups dependent apatite formation on bacterial cellulose microfibrils network in a simulated body fluid," J. Biomed. Mater. Res. 81A (2007): 124-134.

precursor or metastable phases of $\mathrm{Ca}-\mathrm{P}$ such as amorphous calcium phosphate (ACP) (Termine and Posner 1966), brushite (dicalcium phosphate dihydrate [DCPD]) (Francis and Webb 1971), and octacalcium phosphate (OCP) (Houllé et al. 1998) because they are kinetically favorable to deposit under the same conditions with respect to apatite. One or a combination of these precursor phases eventually transform into a mature Ca-P phase of biological hydroxyapatite, which is regarded as the most thermodynamically stable Ca-P phase in the physiological environment.

The detailed information relative to the Ca-P phases obtained with soaking times of 2, 7, 14, and 21 days was revealed by ATR-FTIR spectral features (Nge and Sugiyama 2007) and the characteristics spectral data are described in Table 11.2. The characteristic phosphate absorption bands of the Ca-P phase (Barrère et al. 2003; Klee and Engel 1970; Koutsopoulos 2002) at 1020-1024/cm $\left(\mathrm{PO}_{4}{ }^{3-}, \mathrm{v}_{3}\right), 600-602 / \mathrm{cm}\left(\mathrm{PO}_{4}{ }^{3-}, \mathrm{v}_{4 \mathrm{a}}\right)$, and 558-560/cm $\left(\mathrm{PO}_{4}{ }^{3-}, \mathrm{v}_{4 \mathrm{c}}\right)$ were eventually detected with increasing soaking time. The phosphate absorption band at $1020-1024 / \mathrm{cm}$ is attributed to the presence of crystalline imperfection in the apatite structure, that is, nonstoichiometric apatite containing $\mathrm{HPO}_{4}{ }^{2-}$ and/or $\mathrm{CO}_{3}{ }^{2-}$ groups (Rey et al. 1991b), whereas the characteristic phosphate absorption band of stoichiometric hydroxyapatite usually displays at approximately $1030 / \mathrm{cm}$. Moreover, the characteristic $\mathrm{HPO}_{4}{ }^{2-}$ bands of OCP at $1295-1297 / \mathrm{cm}$ and $910-911 / \mathrm{cm}$ corresponding to $\mathrm{OH}$ in-plane bending and P-OH stretching mode of $\mathrm{HPO}_{4}$ (6) (Barrère et al. 2003; Fowler et al. 1993) were detected at 2-day and 7-day spectra and became weak and/or disappeared at the 14-day and 21-day spectra. It was reported (Fowler et al. 1993) that the $\mathrm{HPO}_{4}(6)$ in OCP preferentially decreased during the progressive hydrolysis 
of OCP to HA. In addition, the appearance of the $\mathrm{CO}_{3}{ }^{2-}$ band at $873-875 / \mathrm{cm}$ starting from the 7-day spectra indicated the formation of carbonated apatitic phase (type $\mathrm{B}$ carbonate), where $\mathrm{CO}_{3}{ }^{2-}$ groups were replaced at the site of $\mathrm{PO}_{4}{ }^{3-}$ groups in the apatitic lattice (Elliott et al. 1985; Rey et al. 1989). The spectral feature of 21 days was typical of a calcium-deficient carbonated apatite, which has been widely reported as a bonelike apatite because of its similar spectral features with those of bone tissues (Elliott et al. 1985; Rey et al. 1989, 1991a, 1991b). From ATR-FTIR spectral data and the value of the $\mathrm{Ca}: \mathrm{P}$ ratio, the crystalline phase nucleated on the $\mathrm{BC}$ microfibril surface was calcium-deficient carbonated apatite through the initial formation of $\mathrm{OCP}$ or OCP-like Ca-P phase.

\section{BC Templates Alternatively Soaked in Calcium and Phosphate Solutions}

Templates of native BC, BC-TEMPO, BC-TEMPO-Ca, and BC-GlcNAc were used for five alternate soaking cycles (Nge et al. 2010a). The alternate soaking method showed a similar result as that of soaking in $1.5 \mathrm{SBF}$, where the newly formed Ca-P globules varied in size between different $\mathrm{BC}$ templates (Figure 11.5). The globule size formed on native BC and BC-TEMPO were 4-6 $\mu \mathrm{m}$ and 1.5-2 $\mu \mathrm{m}$, respectively, which were larger than those formed in $1.5 \mathrm{SBF}$, with approximately $3 \mu \mathrm{m}$ and $1.5 \mu \mathrm{m}$ for native BC and BC-TEMPO, respectively. The globules on BC-TEMPO-Ca, however, have a similar size $(<1 \mu \mathrm{m})$ with both soaking methods. The distinctively larger size formed on the microfibril surface of native $\mathrm{BC}$ may be due to a relatively higher ion concentration as well as to a more rapid soaking cycle ( $30 \mathrm{~min}$ interval for a total of about $5 \mathrm{~h}$, as opposed to 21 days for the $1.5 \mathrm{SBF}$ soaking method), which accelerates the increase in size of the crystals and the progressive mineralization nature of native BC.

The nature of the multiplication mineralization that occurred in the BC-TEMPO and BC-TEMPO-Ca templates, however, seems independent of the soaking methods. A more defined morphology of apatite globule was observed on the lower surface of the BC templates, as the lower surface layer of the $\mathrm{BC}$ pellicle consists in general of a looser network than the upper surface layer. For example, the apatite globule with a honeycomb-like 3D structure composed of thin platelike crystals was observed in BC-TEMPO, and a cluster of thin platelike crystals spreading upward in all directions was observed in BC-TEMPO-Ca, as shown in Figures $11.5 \mathrm{~g}$ and $11.5 \mathrm{~h}$. It should be noted that the surface functional groups or surface structure plays an important role in the initial step of apatite nucleation and governs the following mineralization process by either multiplication or progressive mineralization.

On the other hand, the apatite-deposited BC-GlcNAc surface in Figures $11.5 \mathrm{~d}-\mathrm{f}$ showed a different morphology, where the Ca-P mineralization may take place along the surface with the initial formation of a monolayer followed by the formation of apatite globules $(1-1.5 \mu \mathrm{m})$ on the existing monolayer. The proportion of incorporated GlcNAc and the type of starter culture used for the inoculation also affect the morphology of apatite 

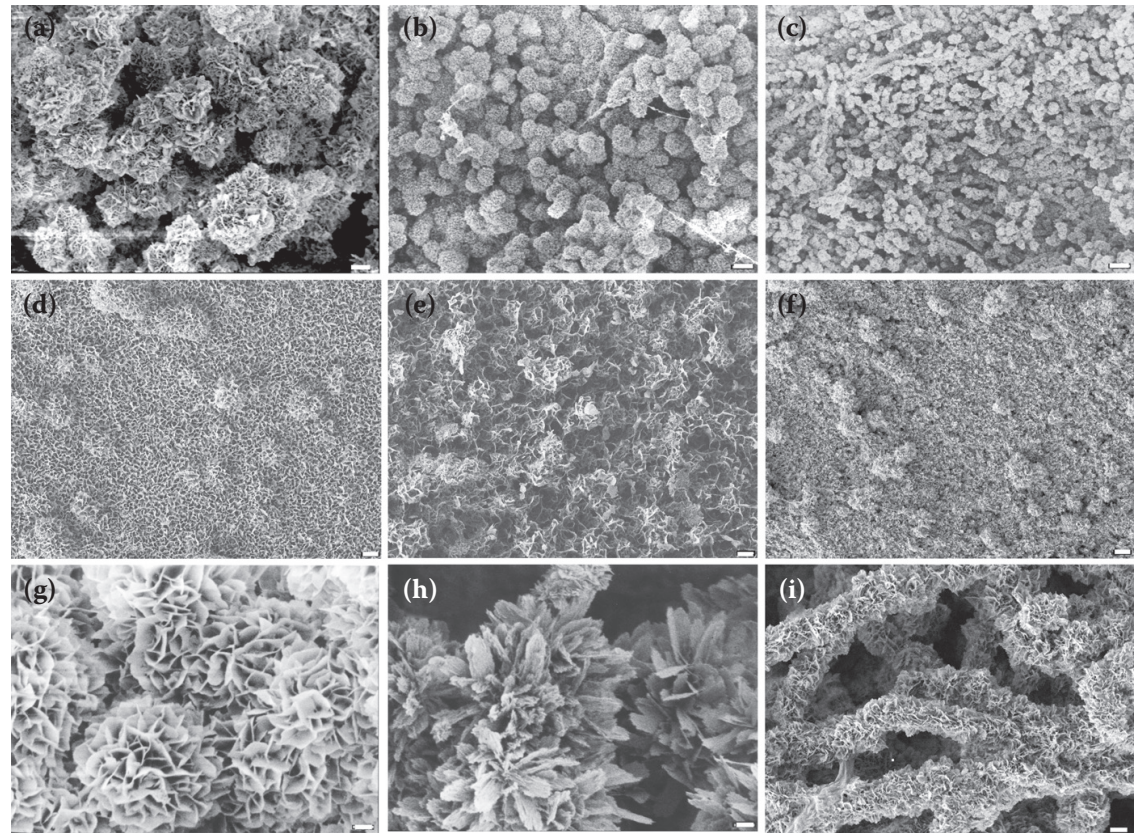

\section{FIGURE 11.5}

FE-SEM images of apatite-deposited BC surfaces after 5 cycles of alternate soaking in calcium and phosphate solutions. (a) Native-BC, (b) BC-TEMPO, (c) BC-TEMPO-Ca, (d) BC-GlcNAc (M1604-1604A), (e) BC-GlcNAc (M1010-1010A), (f) BC-GlcNAc (M0416-0416A) and magnified view of apatite globules formed on the lower surfaces of the (g) BC-TEMPO, (h) BC-TEMPO-Ca, and (i) BC-GlcNAc (M0020-1010B) template. Scale bars, $2 \mu \mathrm{m}$ for a-f and $500 \mathrm{~nm}$ for g-i. Source: Adapted from Nge et al., Biopolymers (ISBN:978-953-307-109-1, InTech., 2010), 345-368, with permission.

formed on the BC-GlcNAc templates. Figure 11.5i shows the typical morphology of apatite formed on the lower surface layer in all BC-GlcNAc templates, where the mineral nucleation took place around the surface of each microfibril/bundle. The apatite-coated microfibril diameter (width) was found to be approximately $1 \mu \mathrm{m}$. Based on the observed lateral dimensions of fibril aggregates or ribbons of about $100-110 \mathrm{~nm}$ and a $1 \mu \mathrm{m}$ width after five alternate soaking cycles (approximately $5 \mathrm{~h}$ ) of apatite coating, the mineral deposition rate can be estimated to be $3 \mathrm{~nm} / \mathrm{min}$. These interesting observations of apatite morphology with respective $\mathrm{BC}$ templates indicate that the surface properties of $\mathrm{BC}$ can be tailored by modifying the $\mathrm{BC}$ during biosynthesis.

The Ca:P molar ratios of BC templates determined by ICP-AES are shown in Table 11.3. The OCP or OCP-like Ca-P phase was likely to form after three cycles. All Ca:P ratios after five cycles were lower than the theoretical value of hydroxyapatite (1.67), similar to the values of BC templates soaked in 1.5 SBF, and the $\mathrm{Ca}-\mathrm{P}$ phase formed on all $\mathrm{BC}$ templates was a calcium-deficient hydroxyapatite. The BC-GlcNAc samples that were produced from the M0416 medium (glucose:GlcNAc $=0.4: 1.6 \mathrm{~g} / 100 \mathrm{ml}$ ) inoculated with any 


\section{TABLE 11.3}

Ca:P Molar Ratios of Different BC Templates after Five Cycles of Alternate Soaking in Calcium and Phosphate Solutions, as Measured by ICP-AES

\begin{tabular}{lccc}
\hline & Cycle 1 & Cycle 3 & Cycle 5 \\
\hline Native BC & $1.25 \pm 0.002$ & $1.29 \pm 0.003$ & $1.41 \pm 0.03$ \\
BC-TEMPO & $1.23 \pm 0.001$ & $1.31 \pm 0.002$ & $1.46 \pm 0.01$ \\
BC-TEMPO-Ca & $1.23 \pm 0.001$ & $1.32 \pm 0.003$ & $1.39 \pm 0.01$ \\
BC-GlcNAc & & & \\
M1604-1604A & NA & NA & $1.39 \pm 0.01$ \\
M1604-1010B & NA & NA & $1.46 \pm 0.03$ \\
M1604-0416B & NA & NA & $1.46 \pm 0.01$ \\
& & & \\
M0020-1604B & NA & NA & $1.42 \pm 0.02$ \\
M0020-1010B & NA & NA & $1.41 \pm 0.04$ \\
M0020-0416B & NA & NA & $1.45 \pm 0.07$ \\
M1010-1010A & & & \\
M0416-0416A & NA & NA & $1.38 \pm 0.04$ \\
\hline
\end{tabular}

Source: Adapted from T. T. Nge et al., Bacterial cellulose-based biomimetic composites. In: Biopolymers (ISBN 978-953-307109-1, InTech, 2010), 345-368, with permission.

type of starter culture (M1604, M0020, M0416) showed a Ca:P ratio (1.45-1.51) higher than that of other BC-GlcNAc templates. The highest $\mathrm{Ca}$ :P ratio of this sample correlated with its highest deposit weight, as shown in Figure 11.6.

Figure 11.6 shows the weight gain of Ca-P that mineralized on various BC templates as a function of the number of soaking cycles. The largest deposit amounts were observed in the BC-TEMPO, BC-TEMPO-Ca, and native BC templates throughout the soaking cycles. Compared to the BC-GlcNAc templates, the surface-modified BC-TEMPO and BC-TEMPO-Ca templates were characterized by the largest deposit amounts. The presence of surface carboxylate groups significantly affects not only the crystal nucleation process, but also the amount of mineral deposited.

It was found that the presence of GlcNAc on the surface of BC microfibrils limits the rate of Ca-P nucleation, where the weight gain decreased with increasing incorporated GlcNAc. Generally the ultrafine network with highly hydrophilic nature of BC favors the infiltration of calcium and phosphorus ions during the first soaking cycle and facilitates Ca-P nucleation at the microfibril surface. The presence of incorporated GlcNAc somehow hinders mineral nucleation, although the detailed mechanism remains to be elucidated. As a consequence, the overall rate of mineral nucleation on the surface of BC microfibrils was slow and the mineral deposition observed at the first 


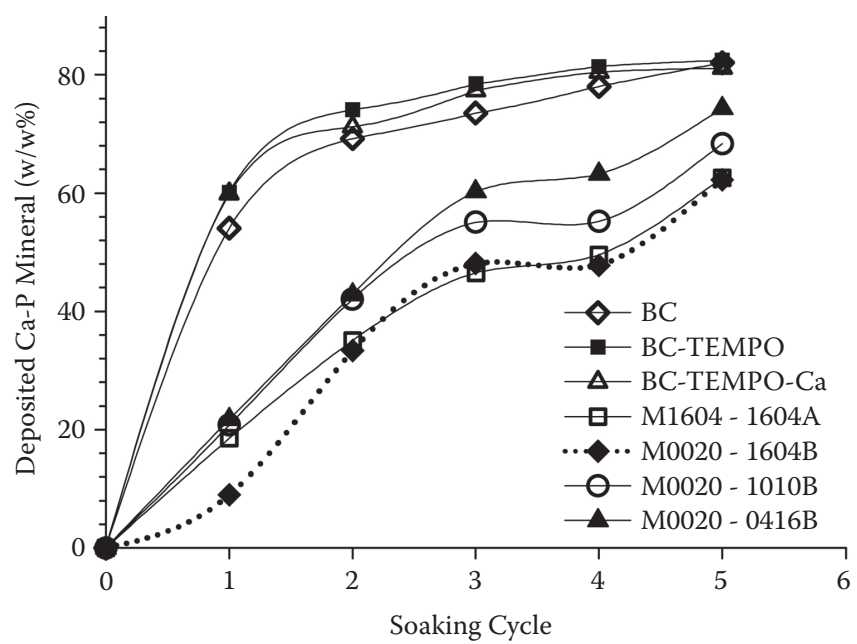

FIGURE 11.6

Relative weight of Ca-P mineral deposit on different BC templates as a function of the number of soaking cycles. Source: From Nge et al., Biopolymers (ISBN:978-953-307-109-1, InTech., 2010), $345-368$, with permission.

soaking cycle was very low. After a gradual increase of the mineral deposition during the first three cycles, the deposition became fairly stable between the third and fourth cycles in all BC-GlcNAc templates. The entire BC surface was covered with a Ca-P mineral at this stage, which was in agreement with the observation of the formation of Ca-P layers in the FE-SEM images. The increase of Ca-P deposition between the fourth and fifth cycles reflects the further growth of apatite globules visible in the FE-SEM images (Figure 11.5).

The surface of the $\mathrm{BC}$ microfibril network may be considered a matrix of chemical groups. Therefore every monomer unit at the surface is a potential nucleation site for interaction with calcium and/or phosphate ions. The accessibility of these units at the surface to the ions in $1.5 \mathrm{SBF}$ or calcium and phosphate solutions depends on the orientation of the polymer chains making up the matrix as well as the pore size and the pore distribution within the network. Subtle morphological changes at the surfaces of various $\mathrm{BC}$ templates during apatite formation as well as varying weight deposits and Ca:P ratios are observed accordingly.

\section{Conclusion}

Various biodegradable and biocompatible polymeric materials have recently been investigated to fabricate inorganic-organic hybrid composites by 
mimicking the mineralization system of natural bone, with some successful outcomes. However, the search for an ideal biomaterial with properties and functionalities similar to natural bone is a continuing process because no single material can satisfy all the requirements for creating optimal scaffolding properties, such as strength, toughness, osteoconductivity, osteoinductivity, controlled degradation, inflammatory response, and deformability. In this study, the ultrafine 3D BC network structure with its native unique properties is exploited to fabricate hybrid materials analogous to natural bone. Our study showed that the formation of apatite is dependent on the presence and type of surface functional groups in the BC microfibrillar network, that is, the hydroxyl $(\mathrm{OH})$ functional groups induced the progressive mineralization process and carboxylate $\left(\mathrm{COO}^{-}\right)$functional groups induced the multiplication mineralization process, whereas the presence of incorporated $\mathrm{N}$-acetylglucosamine (GlcNAc) hindered the rate of the nucleation process.

Modification of BC by the incorporation of lysozyme-susceptible sugar units (GlcNAc) was performed during microbial synthesis, considering the analogue of biomimetic extracellular matrix as well as enhancing the degradation property of $\mathrm{BC}$. It is expected that the modified $\mathrm{BC}$ will become more relevant for end use, for instance, in the biomedical area. However, even a small amount (0.03-0.36 mole\%) of GlcNAc-incorporated BC showed a rather slow mineralization process compared with the mineralization process of $\mathrm{BC}$ having surface functional groups like $\mathrm{OH}$ and $\mathrm{COO}^{-}$. Further investigations are needed to tailor the surface properties of $\mathrm{BC}$ to meet the main criteria of mineralized collagen composites such as bone and teeth, and also should be complemented by in vitro and in vivo degradation studies.

\section{Acknowledgments}

This research was supported by the Japan Society for the Promotion of Science (JSPS) (Grant-in-aid for Scientific Research 16004160) and the RISH-Mission project (2006-2007 fiscal years), the Center for Exploratory Research on Humanosphere, and the Research Institute for Sustainable Humanosphere at Kyoto University. Shigeru Yamanaka from Shinshu University is thanked for the generous gift of the Acetobacter strain used in this study. T. T. Nge thanks Akira Isogai and Tsuguyki Saito (University of Tokyo) for their guidance in conducting conductimetric titration; Vincent Bulone (KTH, Sweden) and Takefumi Hattori (RISH, Kyoto University) for their guidance in conducting the radiolabeling experiments; Yuji Imamura, Tsuyoshi Yoshimura, and Won-Joung Hwang (RISH, Kyoto University) for their help in using the ICP-AES facility; and Teruo Kawada (Graduate School of Agriculture, Kyoto University) and Hiroyuki Yano (RISH, Kyoto University) for the use of FE-SEM imaging. 


\section{References}

Barrère, F., C. M. van der Valk, R. A. J. Dalmeijer, C. A. van Blitterswijk, K. de Groot., and P. Layrolle. 2003. In vitro and in vivo degradation of biomimetic octacalcium phosphate and carbonated apatite coating on titanium implants. J. Biomed. Mater. Res. 64A:378-387.

Bigi, A., E. Boanini, S. Panzavolta, N. Roveri, and K. Rubini. 2002. Bonelike apatite growth on hydroxyapatite-gelatin sponges from simulated body fluid. J. Biomed. Mater. Res. 59:709-714.

Czaja, W., A. Krystynowicz, S. Bielecki, and R. M. Brown, Jr. 2006. Microbial cellulosethe natural power to heal wounds. Biomaterials 27:145-151.

Di Martino, A., M. Sittinger, and M. V. Risbud. 2005. Chitosan: a versatile polymer for orthopaedic tissue engineering (review). Biomaterials 26:5983-5990.

Elliott, J. C., D. W. Holcomb, and R. A. Young. 1985. Infrared determination of the degree of substitution of hydroxyl by carbonate ions in human dental enamel. Calcif. Tissue Int. 37:372-375.

Fowler, B. O., M. Marković, and W. E. Brown. 1993. Octacalcium phosphate. Part 3: infrared and Raman vibrational spectra. Chem. Mater. 5:1417-1423.

Francis, M. D., and N. C. Webb. 1971. Hydroxyapatite formation from a hydrated calcium monohydrogen phosphate precursor. Calcif. Tissue Res. 6:335-342.

Gilmcher, M. J. 1998. The nature of the mineral phase in bone: biological and clinical applications. In: Metabolic Bone Disease and Related Disorders, ed. L. V. Avioli and S. M. Krane, 23-50. San Diego: Academic Press.

Granja, P. L., M. A. Barbosa, L. Pouységu, B. De Jéso, F. Rouais, and C. Baquey. 2001a. Cellulose phosphates as biomaterials. Mineralization of chemically modified regenerated cellulose hydrogels. J. Mater. Sci. 36:2163-2172.

Granja, P. L., C. C. Ribeiro, B. De Jéso, C. Baquey, and M. A. Barbosa. 2001b. Mineralization of regenerated cellulose hydrogels. J. Mater. Sci. Mater. Med. 12:785-791.

Helenius, G., H. Bäckdahl, A. Bodin, U. Nannmark, P. Gatenholm, and B. Risberg. 2006. In vivo biocompatibility of bacterial cellulose. J. Biomed. Mater. Res. 76A:431-438.

Hestrin, S., and M. Schramm. 1954. Synthesis of cellulose by Acetobacter xylinum. Part 2: preparation of freeze-dried cells capable of polymerizing glucose to cellulose. Biochem. J. 58:345-352.

Houllé, P. B., P. Steuer, J. C. Voegel, and F. J. G. Cuisinier. 1998. First experimental evidence for human dentine crystal formation involving conversion of octacalcium phosphate to hydroxyapatite. Acta Crystallogr. D Biol. Crystallogr. 54:1377-1381.

Jonas, R., and L. F. Farah. 1998. Production and application of microbial cellulose. Polym. Degrad. Stabil. 59:101-106.

Klee, W. E., and G. Engel. 1970. Infrared spectra of the phosphate ions in various apatites. J. Inorg. Nucl. Chem. 32:1837-1843.

Klemm, D., D. Schumann, U. Udhardt, and S. Marsch. 2001. Bacterial synthesized cellulose-artificial blood vessels for microsurgery. Prog. Polym. Sci. 26:1561-1603.

Kokubo, T. 1991. Bioactive glass ceramics, properties and applications. Biomaterials 12:155-163. 
Kokubo, T. 1993. Bioactivity of glasses and glass-ceramics. In: Bone bonding biomaterials, ed. P. Ducheyne, T. Kokubo, and C. A. van Blitterswijk, 31-46. Leiderdorp, Netherlands: Reed Healthcare Communications.

Kokubo, T., H. Kushitani, S. Sakka, T. Kitsugi, and T. Yamamuro. 1990. Solutions able to reproduce in vivo surface-structure changes in bioactive glass-ceramic A-W. J. Biomed. Mater. Res. 24:721-734.

Koutsopoulos, S. 2002. Synthesis and characterization of hydroxyapatite crystals: a review study on the analytical methods. J. Biomed. Mater. Res. 62:600-612.

Lee, J. W., F. Deng, W. G. Yeomans, A. L. Allen, R. A. Gross, and D. L. Kaplan. 2001. Direct incorporation of glucosamine and $\mathrm{N}$-acetylglucosamine into exopolymers by Gluconacetobacter xylinus (Acetobactor xylinum) ATCC 10245: production of chitosan-cellulose and chitin-cellulose exopolymers. Appl. Environ. Microbiol. 67:3970-3975.

Maeda,H., T. Kasuga, and M. Nogami. 2002. Biomimetic apatite formation on poly(lactic acid) composites containing calcium carbonates. J. Mater. Res. 17:727-730.

Montanari, S., M. Roumani, L. Heux, and M. R. Vignon. 2005. Topochemistry of carboxylated cellulose nanocrystals resulting from TEMPO-mediated oxidation. Macromolecules 38:1665-1671.

Nge, T. T., and J. Sugiyama. 2007. Surface functional groups dependent apatite formation on bacterial cellulose microfibrils network in a simulated body fluid. J. Biomed. Mater. Res. 81A:124-134.

Nge, T. T., J. Sugiyama, and B. Vincent. 2010a. Bacterial cellulose-based biomimetic composites. In: Biopolymers, ed. M. Elnashar, 345-368. ISBN 978-953-307-109-1. InTech. Available at http: / / www.intechopen.com/articles/show/title/bacterialcellulose-based-biomimetic-composites

Nge, T. T., M. Nogi, H. Yano, and J. Sugiyama. 2010b. Microstructure and mechanical properties of bacterial cellulose/chitosan porous scaffold. Cellulose 17:349-363.

Rey, C., B. Collins, T. Goehl, I. R. Dickson, and M. J. Glimcher. 1989. The carbonate environment in bone mineral: a resolution-enhanced Fourier transform infrared spectroscopy study. Calcif. Tissue Int. 45:157-164.

Rey, C., V. Renugopalakrishnan, M. Shimizu, B. Collins, and M. J. Glimcher. 1991a. A resolution-enhanced Fourier transform infrared spectroscopic study of the environment of the $\mathrm{CO}_{3}^{2-}$ ion in the mineral phase of enamel during its formation and maturation. Calcif. Tissue Int. 49:259-268.

Rey, C., M. Shimizu, B. Collins, and M. J. Glimcher. 1991b. Resolution-enhanced Fourier transform infrared spectroscopy study of the environment of phosphate ion in the early deposits of a solid phase of calcium phosphate in bone and enamel and their evolution with age. Part 2: investigations in the $v_{3} \mathrm{PO}_{4}$ domain. Calcif. Tissue Int. 49:383-388.

Roberts, G. A. F. 1992. Chitin Chemistry. London: Macmillan.

Saito, T., and A. Isogai. 2004. TEMPO-mediated oxidation of native cellulose. The effect of oxidation conditions on chemical and crystal structures of the water-insoluble fractions. Biomacromolecules 5:1983-1989.

Saito, T., and A. Isogai. 2005. Ion-exchange behavior of carboxylate groups in fibrous cellulose oxidized by the TEMPO-mediated system. Carbohyd. Polym. 61:183-190.

Sato, K., T. Kogure, Y. Kumagai, and J. Tanaka. 2001. Crystal orientation of hydroxyapatite induced by ordered carboxyl groups. J. Colloid Interface Sci. 240:133-138. 
Shirai, A., M. Takahashi, H. Kaneko, S. I. Nishimura, M. Ogawa, N. Nishi, and S. Tokura. 1994. Biosynthesis of a novel polysaccharide by Acetobacter xylinum. Int. J. Biol. Macromol. 16:297-300.

Suh, J. F. K., and H. W. T. Matthew. 2000. Application of chitosan-based polysaccharide biomaterials in cartilage tissue engineering (review). Biomaterials 21:2589-2598.

Takeuchi, A., C. Ohtsuki, T. Miyazaki, H. Tanaka, M. Yamazaki, and M. Tanihara. 2003. Deposition of bone-like apatite on silk fiber in a solution that mimics extracellular fluid. J. Biomed. Mater. Res. 65A:283-289.

Termine, J. D., and A. S. Posner. 1966. Infrared analysis of rat bone: age dependency of amorphous and crystalline mineral fractions. Science 153:1523-1525.

Uchida, M., H. M. Kim, T. Kokubo, S. Fujibayashi, and T. Nakamura. 2003. Structural dependence of apatite formation on titania gels in a simulated body fluid. J. Biomed. Mater. Res. 64A:164-170.

Yamanaka, S., K. Watanabe, N. Kitamura, M. Iguchi, S. Mitsuhashi, Y. Nishi, and M. Uryu. 1989. The structure and mechanical properties of sheet prepared from bacterial cellulose. J. Mater. Sci. 24:3141-3145. 


\title{
12
}

\section{Bacterial Nanocellulose as a Structured Platform for Conductive Biopolymers}

\author{
Fernando Dourado, ${ }^{1}$ Daliana Muller, ${ }^{2}$ Catarina Nunes, ${ }^{1}$ \\ Carlos Rambo, ${ }^{3}$ and Guilherme Mariz de Oliveira Barra ${ }^{2}$ \\ ${ }^{1}$ Centre for Biological Engineering, Institute for Biotechnology and \\ Bioengineering, University of Minho, Braga, Portugal \\ ${ }^{2}$ Department of Mechanical Engineering, Federal University of \\ Santa Catarina, Campus Universitário, Santa Catarina, Brazil \\ ${ }^{3}$ Department of Electrical Engineering, Federal University \\ of Santa Catarina, Santa Catarina, Brazil
}

\section{CONTENTS}

Introduction

Conductive Polymer Fibrous Templates ………………………………….... 243

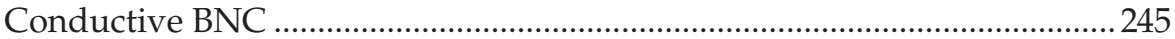

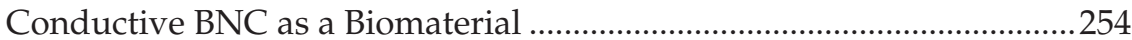

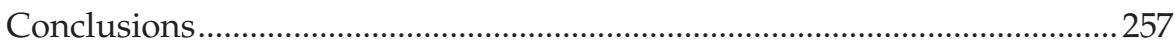

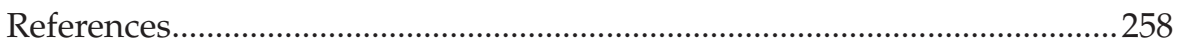

Since the discovery that polyacetylene could be doped to the metallic state more than 3 decades ago, an ever-growing body of a multidisciplinary approach to material design, synthesis, and system integration has been evidenced. The present chapter will primarily review the emerging field of intrinsically conducting polymer and conductive polymer blends, with polyaniline and polypyrrole as the major representatives of conducting polymers. This survey will also address some of the potential areas for applications of such conductive polymer blends. Also, current results concerning the chemical polymerization of conducting polymers on bacterial nanocellulose (BNC) will be presented, including brief remarks on the rationale for the use of conductive BNC blends. This will be followed by a discussion on their properties and potential applications. 


\section{Introduction}

Intrinsically conducting polymers (ICPs), also referred to as synthetic metals or organic semiconductors, are materials that display the electrical and optical properties of metals or semiconductors while retaining some of the mechanical properties and processing advantages of polymers. Indeed, ICPs allow a wide range of freedom in the formulation of materials with varying degrees of conductivity, from very low to very high, ranges unmatched with the use of metals. This special class of polymers has been studied extensively due to their scientific importance in many research areas and emerging technological applications, with some conducting polymers already being produced on a commercial scale by several industries (Bernhard 1997; Song and Palmore 2006). Their distinct intrinsic properties and characteristics have shown strong potential for use in a wide range of applications that can be grouped into two main areas. The first concerns the use of their electrically conductive properties to produce electromagnetic shielding (Joo and Epstein 1994), printed circuit boards (Meyer et al. 1994), artificial nerves (Baughman 1996), and antistatic clothing (Dall'Acqua et al. 2004). In a second area, their electroactive properties are called into play for applications such as ion exchange membranes (Nagarale et al. 2006), energy storage devices (Song and Palmore 2006; Wang et al. 2010), molecular electronics (Akkerman et al. 2006), electrical displays (Mortimer et al. 2006), electrochemical actuators (Lewis et al. 1999), drug release systems (Svirskis et al. 2010), sensory/biosensory devices (Becerik and Kadirgan 2001; Ramanavičius et al. 2006), tissue engineering scaffolds (Kim et al. 2004) and neural probes (Guimard et al. 2007; Ravichandran et al. 2010). A graphic representation (Figure 12.1) of a query using the term "conducting polymers" under "topic" on the Thomson Reuters (formerly ISI) Web of Knowledge database, as well as on EspaceNet, shows the increasing number of published manuscripts and patents in the last decade. Further breakdown of the search term according to specific categories (Figure 12.2) shows that during this decade the major area of (potential) applications of ICPs concerns the development of sensors. This is followed by the development of membrane units almost in parallel with corrosion prevention systems. A third place would be occupied by energy storage devices (batteries).

The unique electronic structural feature of ICPs is characterized by the occurrence of a conjugated $\pi$-electron system along the main chain (Figure 12.3a). The designation of "conjugated" polymer is related to the presence of alternating single and double bonds in the polymer chain. Doping is the unique, central, and unifying concept that distinguishes conducting polymers from all other types of polymers: the bonding $\pi$ system of the polymer backbone can be oxidized ( $\mathrm{p}$-doping) or reduced (n-doping) chemically or electrochemically to the metallic state more easily than with conventional polymers. During the doping process (Figure 12.3b), counterions called dopants are inserted into the polymer backbone and the conjugated polymer (nondoped 


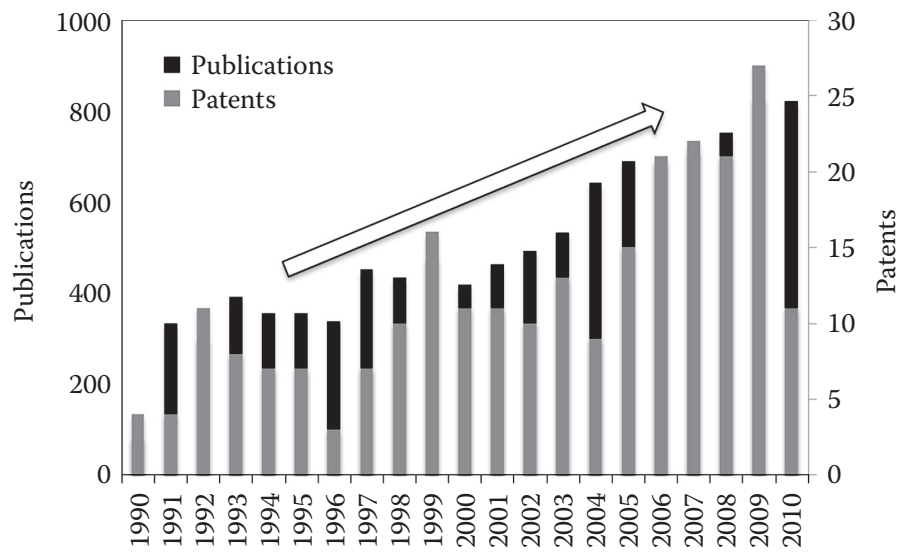

FIGURE 12.1

Number of journal articles and patents published annually relating to "conductive polymers." For "publications," data was gathered from ISI Web of Knowledge (http://wokinfo.com/); for "patents" data was gathered from EspaceNet (http://worldwide.espacenet.com/).

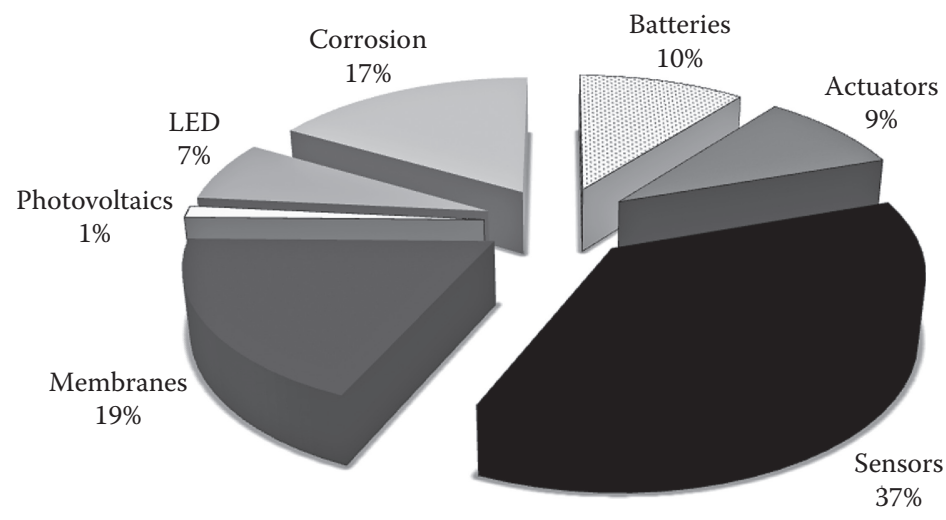

\section{FIGURE 12.2}

Proportion of scientific journals (from 1990 to 2010) grouped under specific categories. Data was gathered from ISI Web of Knowledge (http://wokinfo.com/), combining each of the above categories with "conductive polymers," both under the "topic" search query.

state) is converted into a doped state. Transitory doping techniques such as photodoping, charge-injection doping, or nonredox doping, which introduce no dopant ions, are also known. The doping process induces substantial changes in the polymers' structure and physicochemical properties, such as mechanical, electronic, optic, and magnetic properties of the polymer. Upon doping a conjugated polymer, its electrical conductivity can be increased by several orders of magnitude, from an originally insulating or semiconducting material $\left(10^{-15}-10^{-5} \mathrm{~S} / \mathrm{cm}\right)$ to a doped form of the polymer, in many cases with near metallic conductivity $\left(1-10^{4} \mathrm{~S} / \mathrm{cm}\right)$. Also, their electrical conductivity 
poly(p-phenylene) (PPP)

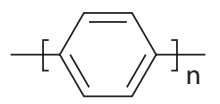

polypyrrole (PPy)

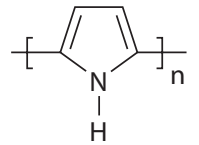

polyacetylene (PA)

$-(\mathrm{CH}=\mathrm{CH}) \overline{\mathrm{n}}$

polythiophene (PPt)

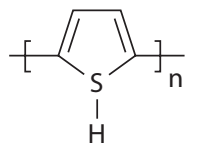

(a)

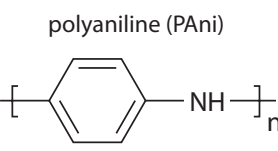

poly(3,4-ethylene dioxythiophene) (PEDOT)

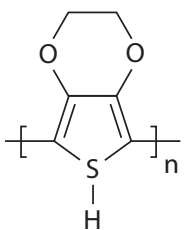

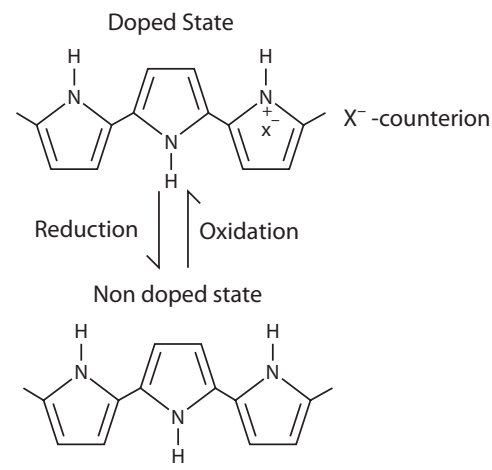

(b)

\section{FIGURE 12.3}

Chemical repeating units of nondoped forms for several intrinsically conducting polymers (a) and idealized representation of a reversible redox reaction system corresponding to the doping-dedoping process of polypyrrole (b); the oxidation (p-doping) of the polypyrrole backbone induces the insertions of anionic dopants as counterions).

can be reversibly controlled by adjusting the doping level, thus allowing fine-tuning of the best properties for each type of polymer.

The highest known electrical conductivity value $\left(10^{5} \mathrm{~S} / \mathrm{cm}\right)$ is that of iodine-doped polyacetylene (Tsukamoto 1990). However, and despite the excellent mechanical properties, its high instability in air precludes its potential use in many technological applications. In fact, the major and widely known limitation of doped ICPs concerns their poor processability; that is, these materials decompose during conventional polymer processing techniques and exhibit very low solubility even in highly polar solvents. Also, ICPs have relatively poor mechanical properties and low thermal stability, which limits their commercial applications. To overcome these problems, several alternative processing techniques have been proposed, among which is the dispersion of ICPs in insulating polymer matrices. This 
extrinsic approach has been receiving increasing attention, as it allows combining the processability and mechanical properties of the polymer matrices with the electronic, magnetic, and optic properties of ICPs (Barra et al. 2001). Also, since the mid-1980s, several air-stable ICPs, such as polypyrrole (PPy), poly(3,4-ethylene dioxythiophene) (PEDOT), polyaniline (PAni), and poly(thiophene) $(\mathrm{Pt})$ have been explored in many areas due to their environmental stability, ease of synthesis, and physicochemical properties that can be controlled by changing the doping degree.

A large number of excellent reviews on ICPs and their blends with insulating polymers have been published (Bhadra et al. 2009; De Paoli 1997). The following review is confined to conductive polymer blends for medical and biological applications. Research on ICPs for use in biomedical applications has been greatly expanding, given the discovery that these materials could be (bio)compatible with many biological molecules such as those used in biosensors. ICPs have also been shown to modulate cellular activities via electrical stimulation. This effect includes cell adhesion, migration, DNA synthesis, and protein secretion. Specifically, many of these studies involved nerve, bone, muscle, and cardiac cells, which respond to electrical impulses. Also, along with biocompatibility, ICPs have been shown to be capable of entrapping and controllably releasing biological molecules to transfer charge from biochemical reactions (Guimard et al. 2007; Ravichandran et al. 2010).

Polypyrrole and polyaniline will be discussed here because they are probably the most extensively studied conducting polymers. Current results concerning the preparation, characterization, and properties of conductive porous nanofibrous blends comprised of bacterial nanocellulose (BNC) containing polypyrrole or polyaniline are also presented and compared with other conductive porous nanofibrous blends used for biological and medical applications.

\section{Conductive Polymer Fibrous Templates}

Conductive polymer scaffolds having fibers in the nanoscale range should mimic, up to a determined level, the structural and biological functions of native cells' extracellular matrix. These materials show great potential for tissue engineering and therapeutic applications given their biocompatibility, efficient electrical charge transfer from bioreactions, potential for carrying current in biological environments, and the ability to undergo expansion and contraction during electrochemical redox reactions. The in vitro and in vivo cell and tissue compatibility of PPy and PAni scaffolds has been demonstrated. Earlier studies also showed that PAni supports the adhesion and proliferation of $\mathrm{H} 9 \mathrm{c} 2$ cardiac myoblasts, COS fibroblast cells, and PC12 cells, while PPy is cytocompatible with mouse fibroblasts and neuroblastoma cells (Ghasemi-Mobarakeh et al. 2009; Gizdavic-Nikolaidis et al. 2010; Li et al. 2006). 
Insulating fibrous polymer membranes can be obtained through many techniques, such as phase separation (Fu et al. 2005), drawing (Williamson and Coombes 2004), template synthesis (Lu et al. 2005), self-assembly (Kweon et al. 2003), and electrospinning (MacDiarmid 2001a, 2001b; MacDiarmid et al. 2001; Picciani et al. 2009; Wei et al. 2005). Among these, electrospinning has been the most easily adopted technique to produce conductive nanofibrous membranes due to its versatility, relatively low cost, and simplicity of production. Conductive nanofibrous films obtained through electrospinning can be divided basically into two categories: (1) those obtained by a direct method, in which conducting and insulating polymers are previously blended in a solution and then electrospun to create a conductive nanofibrous membrane-the main disadvantage of this approach lies in the difficulty in producing a stable and homogeneous conductive polymer solution-and (2) those obtained by a two-step technique in which previously electrospun membranes are coated with polyaniline or polypyrrole by in situ polymerization. Conductive nanofibrous membranes of polyaniline or polypyrrole and insulating polymers have been fabricated through electrospinning polymer mixture solutions by tailoring the conducting and insulating polymer concentrations, in combination with the use of several solvents, including water. Poly(ethylene oxide) (PEO), polyacrylonitrile (PAN), polystyrene (PS), poly(L-lactic acid) (PLA), poly(methyl methacrylate) (PMMA), polycarbonate (PC), polystyrene/polybutadiene (PS/PB) (75:25), and poly(ع-caprolactone)/gelatin (PCL/PG) (70:30) are among the most representative examples of insulating polymers used in the preparation of conductive nanofibers (MacDiarmid 2001a, 2001b; MacDiarmid et al. 2001; Picciani et al. 2009; Wei et al. 2005).

Several reports have demonstrated the potential of polyaniline and polypyrrole for the production of conductive porous nanofibrous blends as scaffolds to improve nerve cell attachment and proliferation (Ghasemi-Mobarakeh et al. 2009, 2011; Gizdavic-Nikolaidis et al. 2010; Lee et al. 2009; Xie et al. 2009). Recently Ghasemi-Mobarakeh et al. (2009) reported the preparation of conductive scaffolds based on PCL/PG and doped PAni through an electrospinning process. The prepared scaffolds were used to electrically stimulate nerve cells, resulting in a substantial increase in cell proliferation and neurite outgrowth as compared to a control using nonstimulated nanofibrous templates. Li et al. (2006) prepared (in vivo) biocompatible fibrous scaffolds comprised of PAni/cross-linked gelatin endowed with an electrical conductivity of $0.01 \mathrm{~S} / \mathrm{cm}$; this nanostructured composite was also shown to support in vitro the growth and proliferation of $\mathrm{H} 9 \mathrm{c} 2$ cardiac myoblasts. Conductive membranes of poly(aniline-co-3-aminobenzoic acid) copolymer and PLA fabricated through electrospinning using a range of relative proportions of both components have also been demonstrated to have good biocompatibility. These blends did not allow enhanced in vitro cell growth, but displayed antimicrobial capacity against Staphylococcus aureus (Gizdavic-Nikolaidis et al. 2010). 
The second categorical method, the two-step technique, involves the in situ (aniline or pyrrole) oxidative polymerization on an insulating polymer nanofiber surface previously obtained by electrospinning. Lui et al. (2008) demonstrated that nanofibrous polypyrrole/poly(styrene- $\beta$-isobutylene- $\beta$ styrene) (PPy/SIS) scaffold is an excellent template for PC12 cell attachment and growth. In that study, SIS was first blended with iron III p-toluene sulfonate in tetrahidrofuran and subsequently electrospun onto gold-coated Mylar in a sealed box filled with pyrrole vapors that were in situ (surface) polymerized on the SIS fibrous membrane. Polypyrrole-coated electrospun poly(lactic-coglycolic acid) (PLGA) obtained through in situ pyrrole oxidative polymerization on electrospun PLGA nanofibers displayed an electrical resistivity value of about $2.4 \times 10^{4} \Omega$ /square. PPy-coated PLGA scaffolds demonstrated comparable growth and differentiation of PC12 cells and hippocampal neurons to PLGA scaffolds without PPy. The electrical stimulation of PC12 cells on PPy-PLGA scaffold improved neurite outgrowth when compared with nonstimulated cells (Lee et al. 2009). Zhang et al. (2007) produced poly(D,L-lactide-co- $\varepsilon$-caprolactone) (PDLLA/CL) coated with polypyrrole through in situ pyrrole oxidative polymerization on PDLLA/CL nanofibrous blends. Their studies showed that electrical stimulation applied through the conductive polymer scaffolds enhanced neurite outgrowth in a currentdependent fashion. It was also demonstrated that PPy-coated PDLLA/CL scaffolds can support the proliferation and differentiation of PC12 cells into neuronal phenotypes and sciatic nerve regeneration in rats (Zhang et al. 2007). Core-sheath nanofibers with polypyrrole as the sheath and PCL or poly(L-lactide) (PLLA) as the core were prepared through in situ polymerization of pyrrole on electrospun PCL or PLLA nanofibers. In this study, Xie et al. (2009) observed that electrical stimulation increases the rate of neurite extension in either random or aligned conductive nanofibers. Other works have also applied electrical stimulation to different cells types through conductive nanofibrous scaffolds based on polyaniline or polypyrrole blends prepared by the direct or two-step electrospinning process. Similarly these studies revealed significant enhanced cell attachment and proliferation on the conductive polymer nanofibrous templates (Ghasemi-Mobarakeh et al. 2011; Jeong et al. 2008).

\section{Conductive BNC}

A literature overview shows that conductive paper materials, where cellulose fibers are encapsulated with the conducting polymer, have been proposed as promising materials for several high-tech applications (electromagnetic interference shielding material, sensing elements, heating elements, and packaging of electronic components and equipment) (Kelly et al. 2007) given 
the relevance of the cellulose's abundance, its well-established industrial use, environmental friendliness, mechanical flexibility, high surface area, and low cost, matched by the interesting electrical, optical, magnetic, chemical conductivity, and efficient electroactive behavior of the ICPs. The cellulose-based conducting polymer composites are thus typically flexible conductive paper materials, which can be molded into different shapes. Further, given recent advances in the production of nanofibers from cellulosic substrates (mostly from vegetable origin) the properties of the ICPs could be enhanced, not only by mechanically reinforcing their brittle polymeric structure, but also by increasing the surface area for coating (Chen et al. 2010; Johnston et al. 2006; Kelly et al. 2007; Richardson et al. 2006).

In recent work, PPy has been coated onto high surface area Cladophora algae cellulose (Mihranyan et al. 2008). This type of cellulose displays distinct properties in comparison to plant cellulose (Mihranyan and Stromme 2004), properties perhaps only rivaled with BNC. In previous chapters, the unique properties of this biopolymer have already been addressed. In this view, conductive blends composed of BNC fibers coated with either polypyrrole or polyaniline emerge as new promising conducting polymer templates.

The first and recent (2007-2010) reports concerning the production of conductive BNC dealt with the use of carbon nanotubes (Chen et al. 2010; Yan et al. 2008; Yoon et al. 2006) and also silver nanoparticles (Barud et al. 2008; Maneerung et al. 2008; Maria et al. 2009) for additional antimicrobial properties. This year (2011), four independent reports focusing on the preparation of electrically conducting blends of BNC as the polymer matrix, and ICPs as the conductive filler were published (Hu et al. 2011; Lee et al. 2012; Marins et al. 2011; Muller et al. 2011a). Muller et al. (2011b) synthesized polypyrrole-coated $\mathrm{BNC}$ through in situ oxidative polymerization of pyrrole in aqueous solution by using iron III hydrochloride $\left(\mathrm{FeCl}_{3} \cdot 6 \mathrm{H}_{2} \mathrm{O}\right)$ as an oxidizing agent. Figure 12.4 summarizes the effects of the reaction time on the $\mathrm{PPy} \cdot \mathrm{FeCl}_{3}$ content and electrical conductivities of $\mathrm{BNC} / \mathrm{PPy} \cdot \mathrm{FeCl}_{3}$ blends.

The amount of pyrrole deposited on the BNC surface and the electrical conductivity of $\mathrm{BNC} / \mathrm{PPy} \cdot \mathrm{FeCl}_{3}$ increases with the reaction time. The electrical conductivity reaches a maximum and constant value of $3.1 \mathrm{~S} / \mathrm{cm}$ at 90 min reaction time, while weight increase tends to plateau after $150 \mathrm{~min}$. Comparative study of Fourier transform infrared attenuated total reflectance mode (FTIR-ATR) spectra of polypyrrole powder, $\mathrm{BNC}$ and $\mathrm{BNC} / \mathrm{PPy} \cdot \mathrm{FeCl}_{3}$ blends provides direct support that the delocalized $\pi$-electrons of the polypyrrole backbone are affected by the presence of $\mathrm{BNC}$, indicating the formation of chemical bonds probably originated from chemical interactions of $\mathrm{PPy} \cdot \mathrm{FeCl}_{3}$ and $\mathrm{BNC}$ chains. The field emission gun scanning electron micrographs (FEG-SEMs) (Figure 12.5a) showed that BNC is formed by a three-dimensional (3D) network structure with fiber diameters of 30-100 nm. The $\mathrm{BNC} / \mathrm{PPy} \cdot \mathrm{FeCl}_{3}$ blend micrograph (Figure $12.5 \mathrm{~b}$ ) showed that $\mathrm{PPy} \cdot \mathrm{FeCl}_{3}$ particle agglomerates with a mean size diameter of $35 \mathrm{~mm}$ are entangled within BNC fibers, forming a uniform and continuous coating nanolayer, 


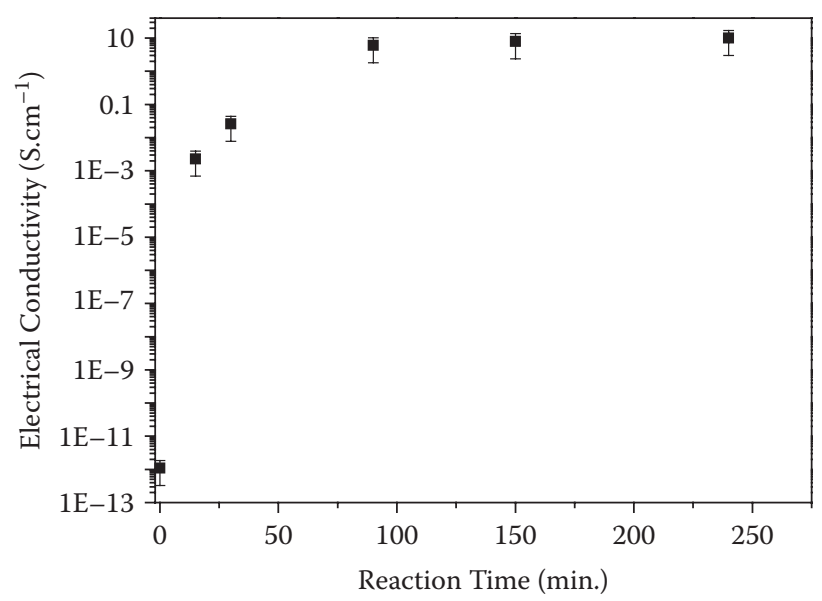

(a)

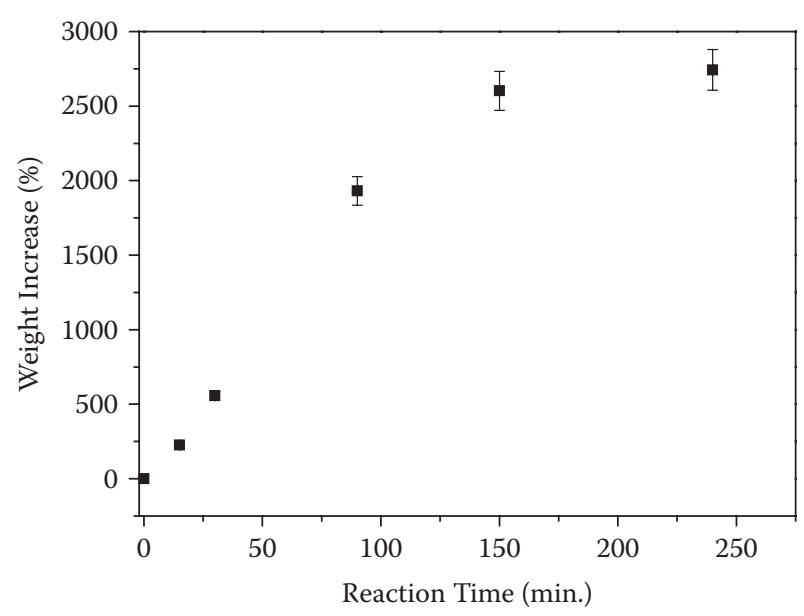

(b)

\section{FIGURE 12.4}

The effect of (a) reaction time on electrical conductivity and (b) weight increase for 0.03 mol.L-1 pyrrole (Py) concentration by using $\mathrm{FeCl} 3 / \mathrm{Py}$ molar ratio of 1.3. (adapted from Muller et al. 2011a).

which is responsible for the high electrical conductivity values. The mean diameter of $\mathrm{BNC} / \mathrm{PPy} \cdot \mathrm{FeCl}_{3}$ fibers is fourfold higher than that of the uncoated BNC. Muller et al. (2011b) also demonstrated that the morphology and electrical conductivity of BNC/PPy blends are strongly influenced by the oxidizing agent (iron III hydrochloride $\left[\mathrm{FeCl}_{3} \cdot 6 \mathrm{H}_{2} \mathrm{O}\right]$ or ammonium persulfate [APS]). As also observed in Figure 12.5, the morphology of the dried blends varies according to the oxidizing agent. BNC/PPy.APS micrographs revealed that deposited PPy.APS particles onto BNC have a spherical shape with a mean size of $0.16 \pm 0.03 \mu \mathrm{m}$, differing from $\mathrm{PPy}^{\mathrm{FeCl}} \mathrm{Fe}_{3}$ deposited particles. 

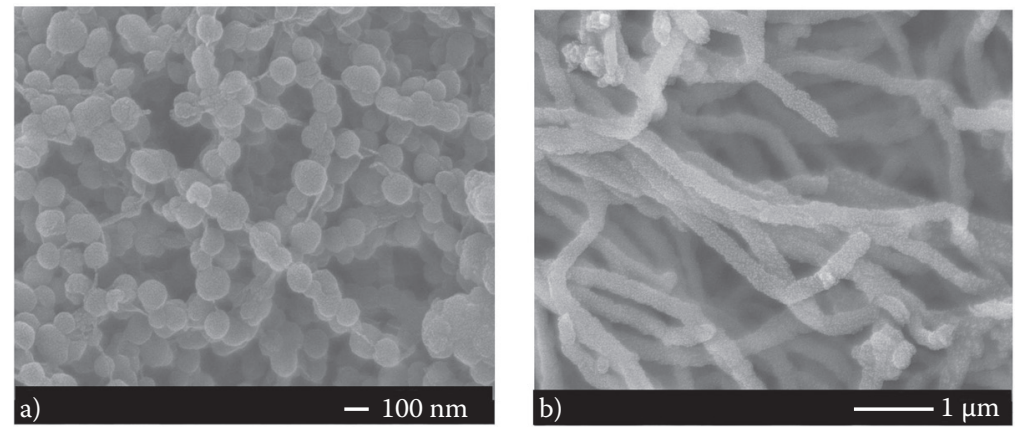

FIGURE 12.5

FEG-SEM micrographs of BNC (a) and BNC blends obtained (b) by using FeC13.

In addition, for a fixed PPy concentration, the electrical conductivity value for the BNC/PPy.APS blend was $1.7 \times 10^{-5} \mathrm{~S} / \mathrm{cm}, 10^{4}$-fold lower than those for the $\mathrm{BNC} / \mathrm{PPy} \cdot \mathrm{FeCl}_{3}$ blend $(0.2 \mathrm{~S} / \mathrm{cm})$. This behavior is probably attributed to the higher doping degree of $\mathrm{PPy} \cdot \mathrm{FeCl}_{3}$ particles and/or the interaction level between $\mathrm{BNC}$ and $\mathrm{PPy} \cdot \mathrm{FeCl}_{3}$ (Muller et al. 2011b).

Conductive BNC/polyaniline blends have also been synthesized through in situ oxidative polymerization of aniline onto BNC by using APS as an oxidizing agent and different protonic acids (Hu et al. 2011; Lee et al. 2012; Marins et al. 2011). Hu et al. (2011) studied the influence of the reaction time and different types of protonic acids on the electrical conductivity of BNC/PAni blends. As expected, the amount of polyaniline deposited on the BNC nanofibers and electrical conductivity values of BNC/PAni blends increased with the reaction time, reaching a maximum value of $5 \times 10^{-2} \mathrm{~S} / \mathrm{cm}$ at $90 \mathrm{~min}$, thus demonstrating that the nanofibrils worked as a good template for the polymerization. The electrical conductivities were also shown to be affected by the protonic acid. BNC/hydrochloric acid-doped polyaniline blends (BNC/PAni. $\mathrm{HCl}$ ) displayed significantly higher conductivity than blends composed of BNC/PAni-dodecylbenzene sulfonic acid (DBSA), $\mathrm{BNC} / \mathrm{PAni} \cdot \mathrm{p}$-toluene sulfonic acid (PTSA), BNC/PAni-sulfuric acid $\left(\mathrm{H}_{2} \mathrm{SO}_{4}\right)$, BNC/PAni-phosphoric acid $\left(\mathrm{H}_{3} \mathrm{PO}_{4}\right)$, and BNCPAni-sulfamic acid $\left(\mathrm{NH}_{2} \mathrm{SO}_{3} \mathrm{H}\right)$. According to $\mathrm{Hu}$ et al. (2011), this behavior is attributed to the fact that PAni- $\mathrm{HCl}$ probably has a higher doping degree than the others.

Conductive BNC/PAni.DBSA blends prepared through in situ polymerization of aniline by using DBSA and ammonium persulfate were also reported by Marins et al. (2011). The morphology, crystalinity degree, and electrical conductivity are affected by the DBSA concentration. The optimum DBSA/aniline molar ratio to produce BNC/PAni with electrical conductivity quite similar to that found by Hu et al. (2011) was 1.5.

Lee et al. (2012) obtained BNC/PAni blends through direct in situ polymerization of aniline on $\mathrm{BNC}$ fibers by using $\mathrm{HCl}$ solution containing ammonium persulfate and PTSA. Electrical conductivity values of BNC/PAni 


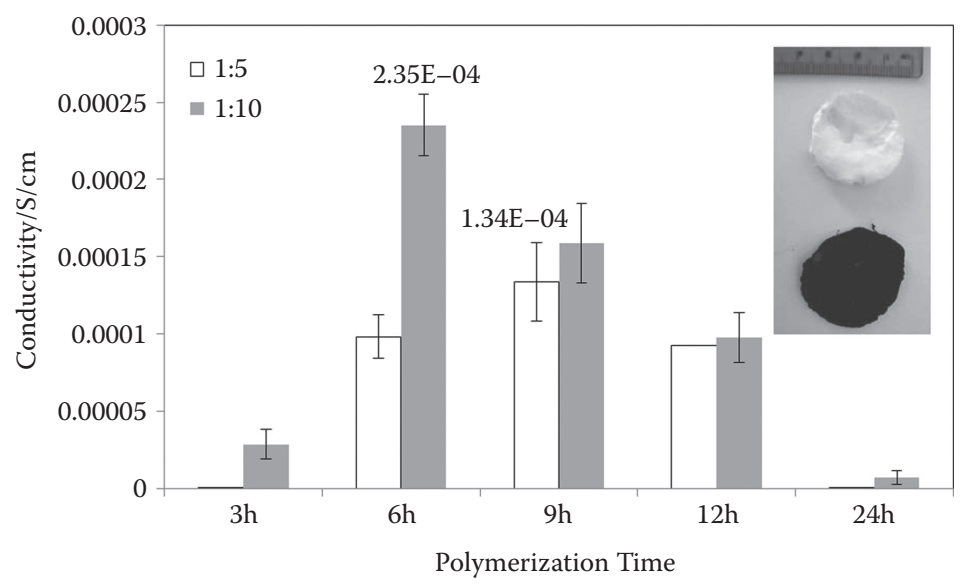

FIGURE 12.6

(See color insert.) Effects of the reaction time on the electrical conductivity of BNC/PAni.HCl composites.

doped with and without PTSA were $1.3 \mathrm{~S} / \mathrm{cm}$ and $0.8 \mathrm{~S} / \mathrm{cm}$, respectively. Cyclic voltammetry used to measure the electrochemical redox properties of the BNC/PAni sample revealed that it was thermodynamically stable.

Figure 12.6 shows results obtained by our research group on the effect of the polymerization time on the electrical conductivity of BNC/Ani. $\mathrm{HCl}$ composites. BNC (from Gluconacetobacter xylinus ATCC 53582) was produced as described elsewhere (Lopes et al. 2011). One hundred milligrams of hydrated BNC were immersed in $50 \mathrm{ml}$ previously distilled aniline dissolved in $0.2 \mathrm{M} \mathrm{HCl}$ at room temperature. Under constant stirring, a solution of APS in $0.2 \mathrm{M} \mathrm{HCl}$ was slowly added, thus totaling $100 \mathrm{ml}$ of volume reaction. At specific intervals, the reaction was stopped by removing demodified BNC from the reaction media. This was followed by extensive washing with acidified $(0.2 \mathrm{M} \mathrm{HCl})$ distilled water and freeze-drying. The following molar proportions of BNC:Ani were assayed: 1:1, 1:5, and 1:10. Figure 12.6 also shows a control disk of BNC (white) and a modified disk, obtained following polymerization with Ani (dark green). Hydrated disks had an average diameter of $2.5 \mathrm{~cm}$ and a thickness of about $5 \mathrm{~mm}$, on average. The electrical conductivity of the composites was measured at room temperature by the standard four-probe method. These results (Figure 12.6) showed an increasing conductivity with increasing amounts of the conducting monomer. The conductivity of the composites obtained using the proportions of 1:1 were too low (data not shown). In the remaining cases, conductivity reached a peak, with a tendency to decrease with the polymerization time. According to Yin et al. (1997), with the increase in the reaction time, PAni units in the emeraldine form may be further oxidized to the pernigraniline state, which is less conductive, thus decreasing the conductivity. Qaiser et al. (2009) also reported that when APS was used, from an initially dark green color, the 

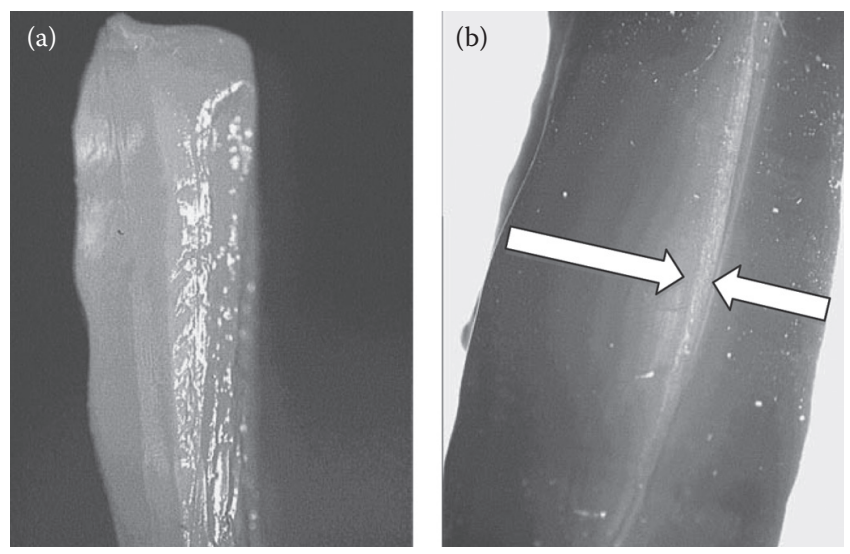

\section{FIGURE 12.7}

Cross section photographs of hydrated $\mathrm{BNC}$ and $\mathrm{BNC} / \mathrm{PAni}$.HCl composite. In situ polymerization of Ani onto BNC (a) induces an inward concentration gradient of PAni (b) on the BNC. The white arrows mark the direction of the decreasing concentration gradient of polymerized PAni.

solution from the reaction mixture later turned purple, and on prolonged polymerization it turned blackish-brown due to PAni degradation, thus showing overoxidation.

In our work, BNC disks of $2.5 \mathrm{~cm}$ diameter and $5 \mathrm{~mm}$ thickness were used for the in situ polymerization of aniline. As observed in Figure 12.7, given the thickness of the BNC, PAni was not uniformly distributed across the substrate, the outer regions of the disks having a higher concentration of PAni. It is known that when polymerization occurs in the presence of a high surface area substrate (as is the case of $\mathrm{BNC}$ ), polymerization occurs on the substrate's surface, given the limited solubility of aniline in water (Qaiser et al. 2009). It is also know that aniline forms aggregates (with varying morphologies according to the experimental conditions used [Stejskal et al. 2008]) that precipitate from solution and/or aggregate onto the surface of a fibrillar template. It is possible that, with the polymerization of Ani onto the surface of BNC fibers, the accumulation of aggregates on the outer surface of the cellulose disks (fibrils) exerted a plug effect characterized by a reduction in the porosity of the 3D matrix, thus restraining further diffusion of Ani oligomers into the interior of the nanofibrillar matrix. The lower electrical conductivity values obtained here as compared to other works may thus be ascribed to the greater thickness of our samples. Also, while freeze-drying allows for a better preservation of the native 3D matrix of BNC (Cao 2011; Clasen et al. 2006), it lowers the number of contact points between the polymerized fibers, which in turn may account for the lower conductivity values obtained here. A similar effect was independently observed by Marins et al. (2011). Contrary to our work, Hu et al. (2011) used thin films (0.03 mm thickness) and, and this is noteworthy, they used oven-dried BNC for the polymerization. This 

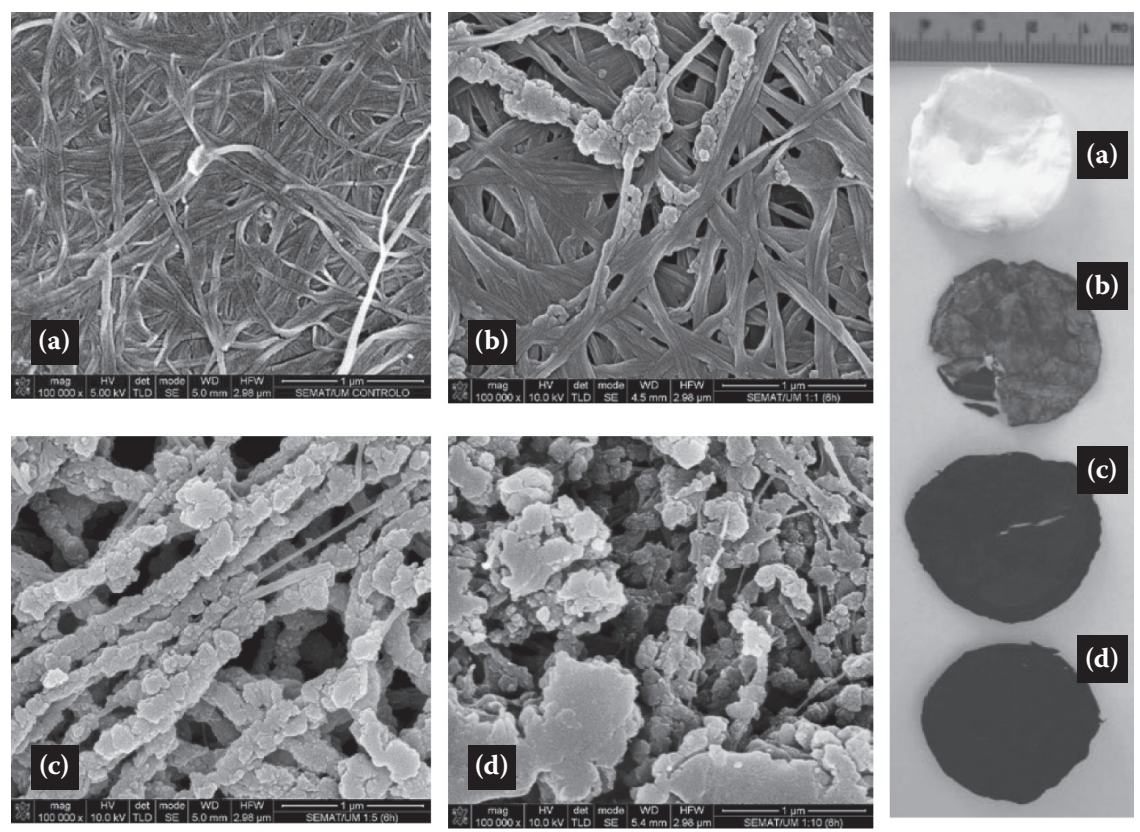

\section{FIGURE 12.8}

(See color insert.) Scanning Electron Microscopy (SEM) with EDS X-ray analysis of BNC and $\mathrm{BNC} / \mathrm{PAni} \mathrm{HCl}$ composites at different BNC:Ani molar proportions. (a) - BNC (Control); (b) - 1:1; (c) - 1:5; (d) - 1:10. All micrographs correspond to a polymerization time of $6 \mathrm{hr}$. Samples were coated with Au.

allowed for greater fiber compaction after drying and hence a more uniform distribution of the conductive aggregates.

The morphologies of $\mathrm{BC}$ and PANI/BC composites were analyzed using scanning electron microscopy (SEM). Figure 12.8 shows SEM-energy dispersive spectroscopy (EDS) images of BNC and BNC/PAni. $\mathrm{HCl}$ composites at different BNC:Ani molar proportions at $6 \mathrm{~h}$ polymerization time. Figure 12.8a shows the typical morphology of BNC from G. xylinus where a 3D matrix consisting of continuous agglomerates of nanofibrils can be observed. By increasing the monomer concentration, the amount of spherical aggregates of PAni significantly increases (Figures 12.8b and 12.8c), as opposed to a low concentration of Ani, where only disconnected PAni particles can be observed (Figure 12.8a). With a molar proportion of 1:10 (BNC:Ani) there are sections at the outer surface of the BNC disks containing almost continuous layer fragments of PAni, as a result of extensive particle deposition and fusion. It was interesting to observe that, with the polymerization, the deposition of PAni promoted separation of the bundles of fibers, as observed by the smaller diameter of uncoated sections of the fibers (Figures 12.8c and 12.8d). This suggests a strong interaction between the deposited PAni and the nanofibers, to the point of disrupting hydrogen 


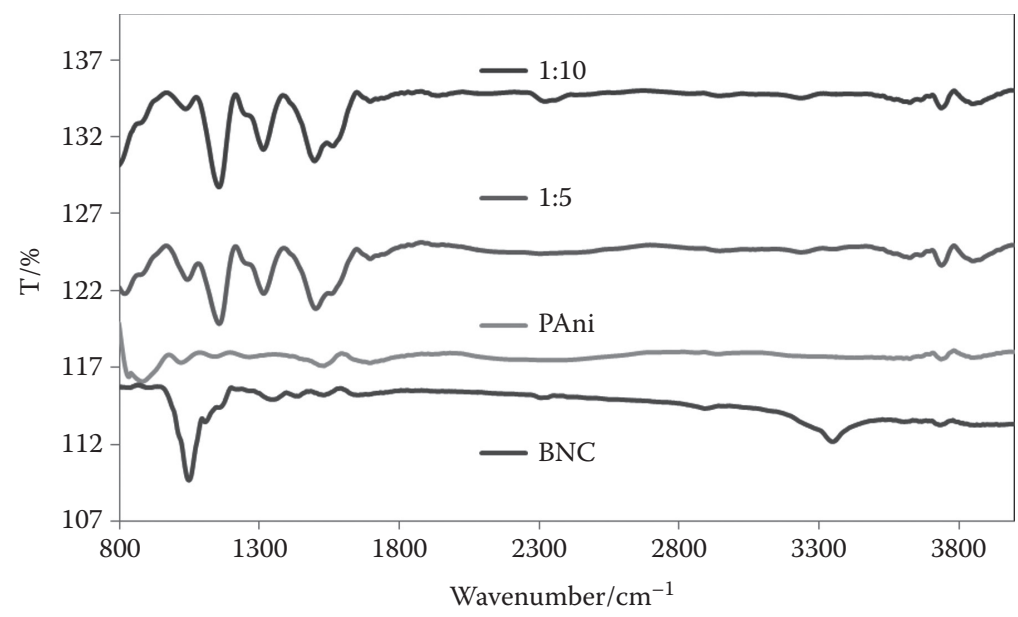

FIGURE 12.9

Fourier Transform Infra-Red Spectrometry-Attenuated Total Reflectance (FTIR-ATR) of BNC, PAni and composites of BNC/PAni.HCl, as prepared with different proportions of BNC:Ani.

bonds between the fiber agglomerates. This is in accordance with Hu et al. (2011), where BNC/PAni. $\mathrm{HCl}$ composites showed weaker mechanical properties, as displayed by the lower values of the tensile strength and Young's modulus of the blends.

The impregnation of the BNC fibers with PAni was confirmed by FTIR-ATR. Spectra were recorded with a Perkin-Elmer Spectrum One IR spectrometer within the range between 4000 and $400 / \mathrm{cm}$ at ambient temperature at a resolution of $8 / \mathrm{cm}$ and 32 times scanning. The samples (disks) were first pressed using a hydraulic system in order to increase the analyzed superficial area. Figure 12.9 compares FTIR-ATR spectra of BNC, PAni, and composites of $\mathrm{BNC} / \mathrm{PAni} \cdot \mathrm{HCl}$ as prepared with different proportions of BNC:Ani. The stretching vibration corresponding to $\mathrm{OH}$ groups of $\mathrm{BNC}$ can be observed at $3300 / \mathrm{cm}$ (due to the stretching vibration of $\mathrm{OH}$ groups). Stretching vibration of the $\mathrm{C}-\mathrm{H}$ bond in the BNC pyranoid ring is slightly noticed at $2840 / \mathrm{cm}$. The $\mathrm{H}-\mathrm{O}-\mathrm{H}$ bending of absorbed water is located at $1614 / \mathrm{cm}$. The peak at $1494 / \mathrm{cm}$ is attributed to the $\mathrm{O}-\mathrm{H}$ bending, and at $1136 / \mathrm{cm}$ to the asymmetric bridge stretching of C-O bonds. The strong band at 1040/cm can be assigned to the $\mathrm{C}-\mathrm{O}-\mathrm{C}$ pyranose ring skeletal vibration (Grande et al. 2009; Hu et al. 2011). Figure 12.9 also displays a typical feature of pure PAni. The peaks in the range of $1200 / \mathrm{cm}$ can be attributed to stretching of the $\mathrm{C}-\mathrm{N}$ band and vibration of $\mathrm{C}-\mathrm{H}$ in the benzene ring of PAni. In comparison to BNC, the absorption peak at $3300 / \mathrm{cm}^{\prime}$ which is related to the OH groups, is shifted to $3200 / \mathrm{cm}$ following PAni deposition (at 1:5 and 1:10 BNC:Ani molar proportions), with the intensity of the band decreasing following PAni polymerization. This suggests some sort of hydrogen bond interaction between $\mathrm{OH}$ groups of $\mathrm{BNC}$ 
and amine groups of PAni. Hu et al. (2011) and Marins et al. (2011) also evidenced a shift on the BNC-OH absorption band following PAni deposition. The emergence of a new band at 2100/cm (Marins et al. 2011) on PAni-DBSA composites was proposed to be an indication of the formation of new chemical bonds, probably originated from grafting reactions of PAni-DBSA on the $\mathrm{BC}$ chains. Also, the X-ray diffraction pattern of BNC/PAni.DBSA blends showed the characteristic peaks related for both undiluted components, but one peak at $2 \theta=14.5^{\circ}$ was shifted for higher $2 \theta$ values, suggesting the presence of new crystals forms, probably due to the interactions of components (Marins et al. 2011). These observations suggest that BNC is activated by acids and the intermolecular hydrogen bands are broken during the in situ polymerization of Ani. As more OH groups become accessible, more PAni can be deposited in the nanofibrous membrane.

The thermal behavior of BNC, PAni and BNC/PAni.HCl was studied using thermogravimetric analysis (TGA) and differential scanning calorimetry (DSC) (Izasa, S.A., Portugal) (Figure 12.10). Acquisition of the results was done using TA-50WS software (version 1.14). For the assays, $5 \mathrm{mg}$ were weighted (the exact masses were recorded) into aluminum pans (Izasa, S.A., Portugal). Open pans were used for the assays. Samples were heated over the temperature range $25^{\circ} \mathrm{C}-500^{\circ} \mathrm{C}$ at a scanning rate of $5^{\circ} \mathrm{C} / \mathrm{min}$. All samples showed a small amount of adsorbed water $(5 \%-8 \% \mathrm{w} / \mathrm{w}$, on average) that was removed at up to $125^{\circ} \mathrm{C}$. This occurred mostly as a two-stage process, where a weakly adsorbed water fraction ("free water") was lost at up to $100^{\circ} \mathrm{C}$, while a more strongly adsorbed ("freezing bound water") was lost at up to $125^{\circ} \mathrm{C}$. This two-step stage was followed by a third step in weight loss. For BNC, this occurred at $200^{\circ} \mathrm{C}$, while for PAni and 1:10 BNC/PAni.HCl this was at $260^{\circ} \mathrm{C}$ and represents the oxidative degradation of the polymers (BNC and PAni). These results also show that the polymerization of Ani onto BNC improves its thermal properties, as observed by a shift to higher temperatures on the profile of weight loss. The water desorption registered in the TGA profile coincides with a small endothermic peak at $50^{\circ} \mathrm{C}$ in the DSC assays. Degradation of BNC begins at $200^{\circ} \mathrm{C}$, producing a single and broad exothermic peak at $273^{\circ} \mathrm{C}$. A similar profile can be observed for $1: 10 \mathrm{BNC} / \mathrm{PAni} \cdot \mathrm{HCl}$, only shifted to $300^{\circ} \mathrm{C}$, in agreement with the shift observed for the weight loss from TGA results. Finally, the chemical process associated with PAni's exothermic peak (at $285^{\circ} \mathrm{C}$ ) was proposed to be due to cross-linking reactions (Scherr et al. 1991). This reaction resulted from a coupling of two neighboring $-\mathrm{N}-\mathrm{Q}-\mathrm{N}-$ groups (where $\mathrm{Q}$ stands for the quinoid ring) to yield two $-\mathrm{NH}-\mathrm{B}-\mathrm{NH}$ - groups (where B stands for the benzenoid ring) through a link of the $\mathrm{N}$ with its neighboring quinoid ring. Interestingly, in a second run, the DSC thermogram of the emeraldine base of PAni powder indicated no significant endothermic or exothermic peaks, given the removal of moisture and destruction of PAni intermolecular cross-links. 

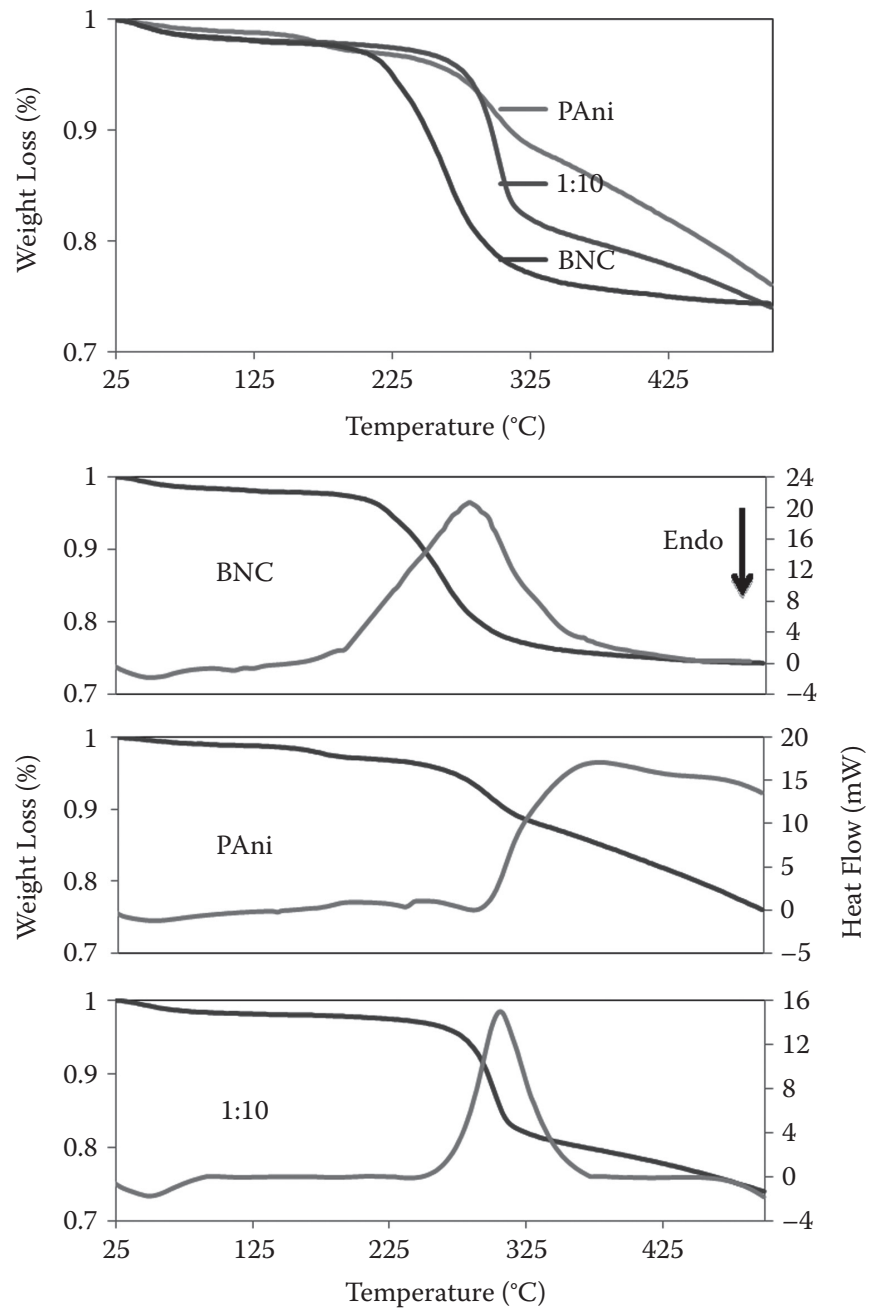

FIGURE 12.10

DSC and TGA profiles of BNC, PANI and BNC/PAni $\mathrm{HCl}$ samples.

\section{Conductive BNC as a Biomaterial}

Among all possible technological applications for BNC-based hydrogels, their application as a engineered tissue biomaterial or as a platform for drug administration and other therapeutic approaches has increased in the last few years. As discussed previously, BNC hydrogels may find useful industrial and biomedical applications. Three-dimensional synthesized biocompatible architectures are key components in the development of novel medical bioproducts, particularly in the field of regenerative medicine and tissue and organ engineering. 
Major challenges include the production of self-assembled semipermeable thick membranes; anisotropic internal nanofiber arrangement; adequate porosity and appropriate internal channel network; full compatibility with hydrophilic fluids; biointegration with polysaccharides, proteins, and other biologically active structural molecules and polymers; suitability to sustain cell attachment and growth; moldability; and scaling-up. Advances in innovative biomaterials design are moving toward feasible and low-cost processes of mimicking extracellular matrices (ECMs) (Bierwolf et al. 2009; Chung et al. 2008; Place et al. 2009). In the last few years a large variety of synthetic and natural approaches have being successfully applied to produce new biocompatible ECM-like materials (Gillette et al. 2008; Kopecek and Yang 2009; Mitragotri and Lahann 2009; Stalling et al. 2009; Zeugolis et al. 2008). Therefore the design of novel scaffolds (supports that allow surrounding cells to migrate into them, regenerating the damaged tissue) with adequate micromorphology are the main challenge in the actual tissue engineering concept, which includes specific properties for cell adhesion and new tissue formation.

Recent advances of biomaterials in tissue engineering include the development of functionalized ECM-like scaffolds coated with biomaterials, such as hydroxyapatite, polyaniline, or polypyrrole (Kamalesh et al. 2000; Li et al. 2006; Muller et al. 2010; Recouvreux et al. 2009) to name a few. Conductive PPy coatings on nanofibers acting as an active interface for tissue engineering may be used to regulate cell activity through electrical stimulation.

To demonstrate the potential of $\mathrm{BNC} / \mathrm{PPy} \cdot \mathrm{FeCl}_{3}$ membranes produced by in situ polymerization as scaffold for tissue engineering applications, cell seeding, and attachment they were used as templates for seeding L929 mouse connective tissue, which are fibroblast-like cells. Cell suspension was directly seeded $\left(8 \times 10^{5}\right.$ cells) onto the surfaces of the BNC/PPy membranes. After $72 \mathrm{~h}$ incubation, the membranes with attached cells were washed with phosphate buffered saline (PBS) and fixed with glutaraldehyde $2.5 \%$. The samples were subsequently dehydrated with ethanol and dried. The scaffolds were gold sputtered and examined by SEM to evaluate the cellular morphology and surface behavior of the L929 cells.

SEM micrographs of cell-seeded BNC/PPy (Figure 12.11) membranes revealed that the fibroblasts are homogeneously distributed over the surface (Figures 12.11a and 12.11b). Fibroblasts with connected nascent daughter cells are also observed (Figures 12.11c and 12.11d), indicating that cells on $\mathrm{BNC} / \mathrm{PPy}$ fibers are being actively divided. Image analyses showed that cells are being attached and grown throughout the BNC/PPy membranes, which suggests an inducible tissue reconstruction. The unique properties of BNC combined with the conductive properties of PPy may be interesting for tissue engineering applications that require electrical conductivity. BNC fibrous membranes coated with conductive polymers may potentially be implanted directly in neuronal tissue-deficient regions or used as cell-carrier scaffolds for therapeutic and regenerative tissue applications. The BNC/PPy composite can also be used as platforms for 3D cell cultures for in vitro human cell 

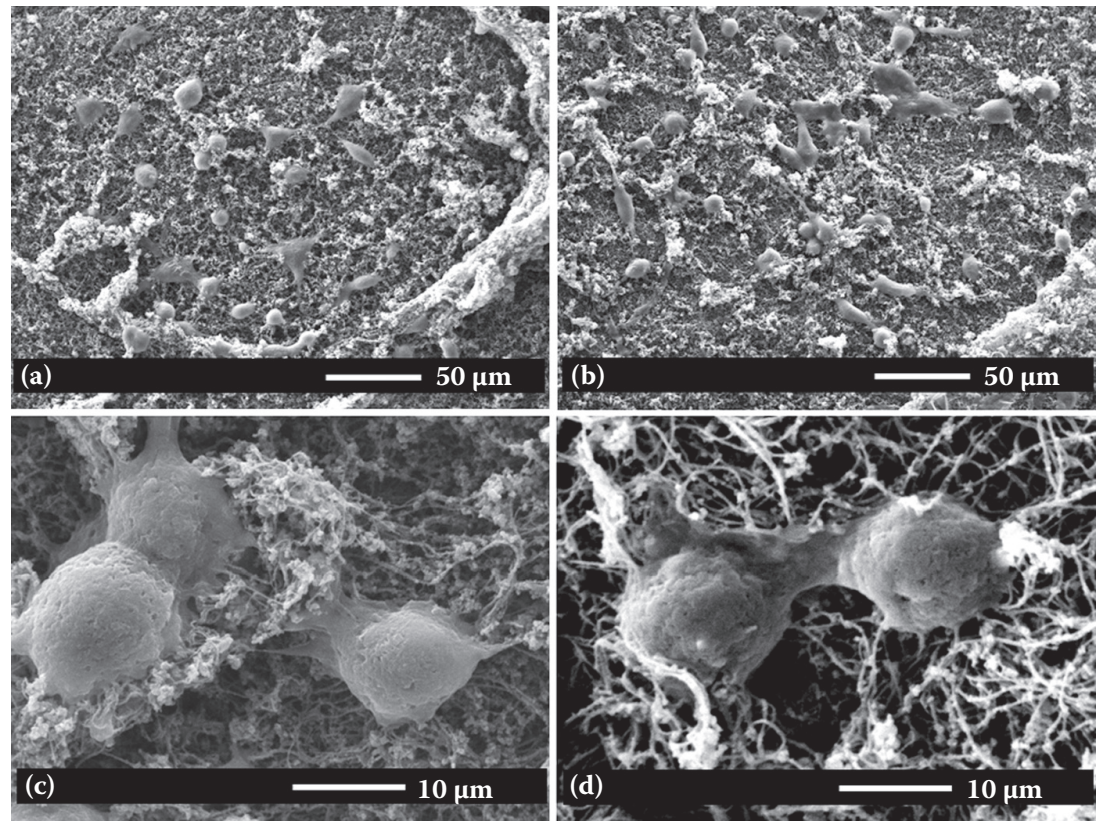

FIGURE 12.11

SEM micrographs of L929, fibroblast cells attached on the surface of BNC/PPy membranes.

therapy research, stem cell 3D differentiation, cancer and stem cell research, and different cell type cultures and cocultures.

Compared to PPy, few groups have considered PAni as potential conductive substrates for tissue engineering applications (Bidez et al. 2006; Kamalesh et al. 2000; Li et al. 2006). In the past 2 decades, the ability to tailor the oxidative state of ICPs has caused the study of PAni in a wide range of research fields such as for biosensors or as scaffolds in tissue engineering (Karyakin et al. 1996; Wei et al. 2004). Figure 12.12 shows the cell proliferation assays by MTS on BNC and BNC/PAni.HCl. 3T3 cells were cultured in Dulbecco's Modified Eagle's Medium (DMEM) supplemented with 10\% calf serum and $1 \%$ penicillin/streptomycin and maintained at $37^{\circ} \mathrm{C}$ in a humidified incubator containing $5 \% \mathrm{CO}_{2}$. They were fed every 2 days and subcultured once they reached $70 \%-90 \%$ confluence by treatment with a trypsin/ethylenediaminetetraacetic acid (EDTA) solution.

On the day of the experiment, cells were seeded $\left(2 \times 10^{5}\right.$ cells $\left./ \mathrm{ml}\right)$ on the top of polyaniline-treated or nontreated bacterial discs previously placed on the bottom of 96-well plates. The effects of the different treatments on cell viability were assessed after 24,48 , and $96 \mathrm{~h}$ by using the Cell Titer 96 Aqueous ONE Solution Reagent (MTS [3-(4,5-dimethylthiazol-2-yl)-5(3-carboxymethoxyphenyl)-2-(4-sulfo-phenyl)-2H-tetrazolium] colorimetric 


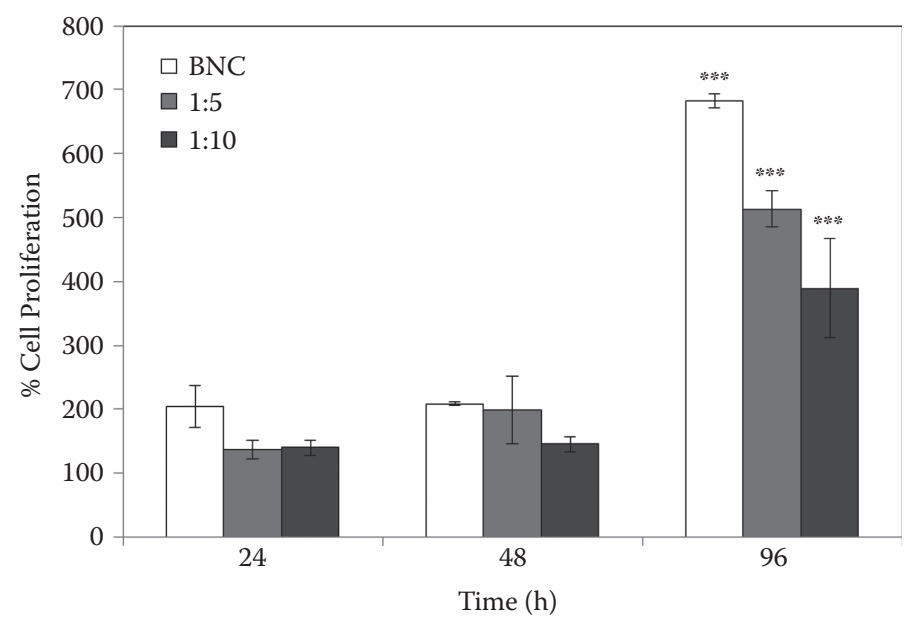

FIGURE 12.12

Cell proliferation assessed by MTS assay on BNC and BNC/PAni.HCl.

assay (Promega, Madison, WI), according to the instructions provided by the manufacturer. One hundred milliliters from each well were transferred to clean wells and the absorbance read at $490 \mathrm{~nm}$. Results were expressed relatively to $t=0 \mathrm{~h}$, which was considered as $100 \%$ of cell proliferation

As can be observed (Figure 12.12), coating BNC with PAni did not result in any toxic effect to the cells, as none of them resulted in a decrease of cell viability to values below the initial ones (considered as $100 \%$ ). Moreover, none of the PAni-treated BNC affected cell proliferation up to $48 \mathrm{~h}$. However, at $96 \mathrm{~h}$, a statistically significant decrease in cell proliferation was detected for the modified celluloses. These results suggest that $\mathrm{BNC} / \mathrm{PAni} \cdot \mathrm{HCl}$ might provide a novel conductive material well suited as biocompatible scaffolds for tissue engineering.

\section{Conclusions}

In today's world, plastic and electrical devices walk hand in hand. Since the discovery in the late 1970s of an electrically conductive, quasi one-dimensional organic polymer, polyacetylene, a wide variety of conducting polymers have been developed. The high hopes raised by these new classes of polymers can easily be evidenced by the ever-growing attraction of researchers from diverse areas of interest such as synthetic chemistry, electrochemistry, solid-state physics, materials science, polymer science, electronics, and electrical engineering. Given the availability of methods to 
circumvent the insolubility, infusibility, and poor mechanical properties of ICPs, new composites can be obtained by controlling the doping level of the conducting polymers any where between that of the fully doped (conducting) and that of the nondoped (insulating or semiconducting) polymer. In fact, their unique electrical properties have allowed consideration of a wide range of applications in the microelectronics industry, including battery technology, photovoltaic devices, light emitting diodes, and electrochromic displays, and more recently in the biological/biomedical field. Research on ICPs for biomedical applications expanded greatly with the discovery in the 1980s that these materials were compatible with many biological molecules, such as those used in biosensors. A range of biomedical applications for ICPs are currently being considered, including the development of artificial muscles, controlled drug release, neural recording, and the stimulation of nerve regeneration. Moreover, electrically active tissues including the brain, heart, and skeletal muscle provide opportunities to couple electronic devices and computers with human or animal tissues to create therapeutic body-machine interfaces. Within the biomedical domain, natural materials endowed with a nanofibrillar nature, flexibility, biocompatibility, mechanical strength, and degradability have demonstrated numerous advantages for biomedical (as well as nonbiomedical) applications. In this context, BNC has been demonstrated to be a biopolymer of choice. Only recently, however, have the potential applications of BNC as a structured platform for conductive biopolymers been tapped. New hybrid materials of BNC with conducting polymers have been successfully produced in which individual nanofibers have been coated with PPy and PAni spheres, fused together. The bond between the fibers and polymer coating is believed to be hydrogen bonding between the respective polymer $(\mathrm{NH}, \mathrm{N})$ and the surface $\mathrm{OH}$ groups from the cellulose. The electrical conductivity of the composites was shown to increase substantially from that of $\mathrm{BNC}$, the values varying according to the preparation method. This could be ascribed to the variable electrical contact between adjacent conductive nanofibers and the variable coverage extension of ICP coating. The biocompatibility of the conductive $\mathrm{BNC}$ has also been demonstrated.

While a few potential applications have already been put forward, considerable efforts are still needed, focused on polymer chemistry, fermentation technology, and engineering to ensure the development of new composite products.

\section{References}

Akkerman, H. B., P. W. M. Blom, D. M. de Leeuw, and B. de Boer. 2006. Towards molecular electronics with large-area molecular junctions. Nature 441(7089):69-72. 
Barra, G. M. O., M. E. Leyva, M. M. Gorelova, B. G. Soares, and M. Sens. 2001. X-ray photoelectron spectroscopy and electrical conductivity of polyaniline doped with dodecylbenzenesulfonic acid as a function of the synthetic method. J. Appl. Polym. Sci. 80(4):556-565.

Barra, G. M. O., R. R. Matins, K. A. Kafer, R. Paniago, C. T. Vasques, and A. T. N. Pires. 2008. Thermoplastic elastomer/polyaniline blends: evaluation of mechanical and electromechanical properties. Polym. Test. 27(7):886-892.

Barud, H., C. Barrios, T. Regiani, R. Marques, M. Verelst, J. Dexpertghys, Y. Messaddeq, and S. Ribeiro. 2008. Self-supported silver nanoparticles containing bacterial cellulose membranes. Mater. Sci. Eng. C 28(4):515-518.

Baughman, R. H. 1996. Conducting polymer artificial muscles. Synthetic Metals 78(3):339-353.

Becerik, I., and F. Kadirgan. 2001. Glucose sensitivity of platinum-based alloys incorporated in polypyrrole films at neutral media. Synthetic Metals 124(2-3):379-384.

Bernhard, W. 1997. Scientific and commercial breakthrough for organic metals. Synthetic Metals 85(1-3):1313-1318.

Bhadra, S., D. Khastgir, N. K. Singha, and J. H. Lee. 2009. Progress in preparation, processing and applications of polyaniline. Prog. Polym. Sci. 34(8):783-810.

Bidez, P. R., 3rd, S. Li, A. G. Macdiarmid, E. C. Venancio, Y. Wei, and P. I. Lelkes. 2006. Polyaniline, an electroactive polymer, supports adhesion and proliferation of cardiac myoblasts. J. Biomater. Sci. Polym. Ed. 17(1-2):199-212.

Bierwolf, J., J. P. Erbes, S. Deichmann, C. Stieglitz, E. Toronyi, M. Luetgehetmann, B. Nashan, K. Feng, P. X. Ma, and J. N. Pollok. 2009. Tissue engineering of primary rat hepatocytes using nano-fibrous 3D polymeric scaffolds for application in ADME studies in pharmaceutical research. Liver Transplant. 15(7):S177-S177.

Cao, Z. 2011. Effect of drying methods on structure and mechanical properties of bacterial cellulose films. Adv. Mater. Res. 239-242:2667-2670.

Chen, P., Y. S. Yun, H. Bak, S. Y. Cho, and H. J. Jin. 2010. Multiwalled carbon nanotubes-embedded electrospun bacterial cellulose nanofibers. Mol. Cryst. Liq. Cryst. 519:169-178.

Chung, S. E., W. Park, S. Shin, S. A. Lee, and S. Kwon. 2008. Guided and fluidic self-assembly of microstructures using railed microfluidic channels. Nat. Mater. 7(7):581-587.

Clasen, C., B. Sultanova, T. Wilhelms, P. Heisig, and W. M. Kulicke. 2006. Effects of different drying processes on the material properties of bacterial cellulose membranes. Macromol. Symp. 244(1):48-58.

Dall'Acqua, L., C. Tonin, R. Peila, F. Ferrero, and M. Catellani. 2004. Performances and properties of intrinsic conductive cellulose-polypyrrole textiles. Synthetic Metals 146(2):213-221.

De Paoli, M.-A. 1997. Conductive polymer blends and composites. In: Organic conductive molecules and polymers, ed. H. S. Nalwa. New York: John Wiley \& Sons.

Fu, X., H. Matsuyama, M. Teramoto, and H. Nagai. 2005. Preparation of hydrophilic poly(vinyl butyral) hollow fiber membrane via thermally induced phase separation. Sep. Purif. Technol. 45(3):200-207.

Ghasemi-Mobarakeh, L., M. P. Prabhakaran, M. Morshed, M. H. Nasr-Esfahani, H. Baharvand, S. Kiani, S. Al-Deyab, and S. Ramakrishna. 2011. Application of conductive polymers, scaffolds and electrical stimulation for nerve tissue engineering. J. Tissue Eng. Regener. Med. 5(4):E17-E35. 
Ghasemi-Mobarakeh, L., M. P. Prabhakaran, M. Morshed, M. H. Nasr-Esfahani, and S. Ramakrishna. 2009. Electrical stimulation of nerve cells using conductive nanofibrous scaffolds for nerve tissue engineering. Tissue Eng. A 15(11):3605-3619.

Gillette, B. M., J. A. Jensen, B. X. Tang, G. J. Yang, A. Bazargan-Lari, M. Zhong, and S. K. Sia. 2008. In situ collagen assembly for integrating microfabricated three-dimensional cell-seeded matrices. Nat. Mater. 7(8):636-640.

Gizdavic-Nikolaidis, M., S. Ray, J. R. Bennett, A. J. Easteal, and R. P. Cooney. 2010. Electrospun functionalized polyaniline copolymer-based nanofibers with potential application in tissue engineering. Macromol. Biosci. 10(12):1424-1431.

Grande, C. J., F. G. Torres, C. M. Gomez, and M. C. Bano. 2009. Nanocomposites of bacterial cellulose/hydroxyapatite for biomedical applications. Acta Biomater. 5(5):1605-1615.

Guimard, N. K., N. Gomez, and C. E. Schmidt. 2007. Conducting polymers in biomedical engineering. Prog. Polymer Sci. 32(8-9):876-921.

Hu, W. L., S. Y. Chen, Z. H. Yang, L. T. Liu, and H. P. Wang. 2011. Flexible electrically conductive nanocomposite membrane based on bacterial cellulose and polyaniline. J. Phys. Chem. B 115(26):8453-8457.

Jeong, S. I., I. D. Jun, M. J. Choi, Y. C. Nho, Y. M. Lee, and H. Shin. 2008. Development of electroactive and elastic nanofibers that contain polyaniline and poly(L-lactideco- $\varepsilon$-caprolactone) for the control of cell adhesion. Macromol. Biosci. 8(7):627-637.

Johnston, J. H., F. M. Kelly, J. Moraes, T. Borrmann, and D. Flynn. 2006. Conducting polymer composites with cellulose and protein fibres. Curr. Appl. Phys. 6(3):587-590.

Joo, J., and A. J. Epstein. 1994. Electromagnetic-radiation shielding by intrinsically conducting polymers. Appl. Phys. Lett. 65(18):2278-2280.

Kamalesh,S.,P.C. Tan, J.J. Wang, T. Lee, E. T.Kang, and C.H. Wang. 2000. Biocompatibility of electroactive polymers in tissues. J. Biomed. Mater. Res. 52(3):467-478.

Karyakin, A. A., O. A. Bobrova, L. V. Luckachova, and E. E. Karyakina. 1996. Potentiometric biosensors based on polyaniline semiconductor films. Sensor. Actuat. B Chem. 33(1-3):34-38.

Kelly, F. M., J. H. Johnston, T. Borrmann, and M. J. Richardson. 2007. Functionalised hybrid materials of conducting polymers with individual fibres of cellulose. Eur. J. Inorg. Chem. 2007(35):5571-5577.

Kim, D. H., M. Abidian, and D. C. Martin. 2004. Conducting polymers grown in hydrogel scaffolds coated on neural prosthetic devices. J. Biomed. Mater. Res. A 71(4):577-585.

Kopecek, J., and J. Y. Yang. 2009. Peptide-directed self-assembly of hydrogels. Acta Biomater. 5(3):805-816.

Kweon, H., M. K. Yoo, I. K. Park, T. H. Kim, H. C. Lee, H. S. Lee, J. S. Oh, T. Akaike, and C. S. Cho. 2003. A novel degradable polycaprolactone networks for tissue engineering. Biomaterials 24(5):801-808.

Lee, B.-H., H.-J. Kim, and H.-S. Yang. 2012. Polymerization of aniline on bacterial cellulose and characterization of bacterial cellulose/polyaniline nanocomposite films. Curr. Appl. Phys. 12(1):75-80.

Lee, J. Y., C. A. Bashur, A. S. Goldstein, and C. E. Schmidt. 2009. Polypyrrole-coated electrospun PLGA nanofibers for neural tissue applications. Biomaterials 30(26):4325-4335.

Lewis, T. W., L. A. P. Kane-Maguire, A. S. Hutchison, G. M. Spinks, and G. G. Wallace. 1999. Development of an all-polymer, axial force electrochemical actuator. Synthetic Metals 102(1-3):1317-1318. 
Li, M. Y., Y. Guo, Y. Wei, A. G. MacDiarmid, and P. I. Lelkes. 2006. Electrospinning polyaniline-contained gelatin nanofibers for tissue engineering applications. Biomaterials 27(13):2705-2715.

Liu, Y., X. Liu, J. Chen, K. J. Gilmore, and G. G. Wallace. 2008. 3D bio-nanofibrous PPy/SIBS mats as platforms for cell culturing. Chem. Commun. 2008(32):3729-3731.

Lopes, J. L., J. M. Machado, L. Castanheira, P. L. Granja, F. M. Gama, F. Dourado, and J. R. Gomes. 2011. Friction and wear behaviour of bacterial cellulose against articular cartilage. Wear 271(9-10):2328-2333.

Lu, H., L. Zhang, W. Xing, H. Wang, and N. Xu. 2005. Preparation of $\mathrm{TiO}_{2}$ hollow fibers using poly(vinylidene fluoride) hollow fiber microfiltration membrane as a template. Mater. Chem. Phys. 94(2-3):322-327.

MacDiarmid, A. G. 2001a. Nobel lecture: "Synthetic metals": a novel role for organic polymers. Rev. Mod. Phys. 73(3):701-712.

MacDiarmid, A. G. 2001b. "Synthetic metals": a novel role for organic polymers (Nobel lecture). Angew. Chem. Int. Ed. 40(14):2581-2590.

MacDiarmid, A. G., W. E. Jones, I. D. Norris, J. Gao, A. T. Johnson, N. J. Pinto, J. Hone, B. Han, F. K. Ko, H. Okuzaki, and M. Llaguno. 2001. Electrostatically-generated nanofibers of electronic polymers. Synthetic Metals 119(1-3):27-30.

Maneerung, T., S. Tokura, and R. Rujiravanit. 2008. Impregnation of silver nanoparticles into bacterial cellulose for antimicrobial wound dressing. Carbohyd. Polym. 72(1):43-51.

Maria, L. C. D., A. L. C. Santos, P. C. Oliveira, H. S. Barud, Y. Messaddeq, and S. J. L. Ribeiro. 2009. Synthesis and characterization of silver nanoparticles impregnated into bacterial cellulose. Mater. Lett. 63(9-10):797-799.

Marins, J. A., B. G. Soares, K. Dahmouche, S. J. L. Ribeiro, H. Barud, and D. Bonemer. 2011. Structure and properties of conducting bacterial cellulose-polyaniline nanocomposites. Cellulose 18(5):1285-1294.

Meyer, H., R. J. Nichols, D. Schröer, and L. Stamp. 1994. The use of conducting polymers and colloids in the through hole plating of printed circuit boards. Electrochim. Acta 39(8-9):1325-1338.

Mihranyan, A., L. Nyholm, A. E. G. Bennett, and M. Stromme. 2008. Novel high specific surface area conducting paper material composed of polypyrrole and Cladophora cellulose. J. Phys. Chem. B 112(39):12249-12255.

Mihranyan, A., and M. Stromme. 2004. Capillary condensation of moisture in fractal pores of native cellulose powders. Chem. Phys. Lett. 393(4-6):389-392.

Mitragotri, S., and J. Lahann. 2009. Physical approaches to biomaterial design. Nat. Mater. 8(1):15-23.

Mortimer, R. J., A. L. Dyer, and J. R. Reynolds. 2006. Electrochromic organic and polymeric materials for display applications. Displays 27(1):2-18.

Muller, D., D. O. S. Recouvreux, F. V. Berti, L. M. Porto, G. M. O. Barra, and C. R. Rambo. 2010. Fibroblasts adhesion on polypyrrole coated bacterial cellulose membranes. Paper presented at the 6th Latin American Congress of Artificial Organs and Biomaterials, COLAOB 2010, August 17-27, Gramado, Brazil.

Muller, D., C. R. Rambo, D. O. S. Recouvreux, L. M. Porto, and G. M. O. Barra. 2011a. Chemical in situ polymerization of polypyrrole on bacterial cellulose nanofibers. Synthetic Metals 161(1-2):106-111. 
Muller, D., J. P. Silva, F. O. Q. Dourado, C. R. Rambo, G. M. O. Barra, and M. Gama. 2011b. Desenvolvimento de Suportes Condutores de Celulose Bacteriana com Polipirrol para Aplicações em Células Neuronais. Paper presented at the 11th Congresso Brasileiro de Polímeros, CBPol, October 16-20, Campos do Jordão, Brazil.

Nagarale, R. K., G. S. Gohil, and Vinod K. Shahi. 2006. Recent developments on ion-exchange membranes and electro-membrane processes. Adv. Colloid Interf. Sci. 119(2-3):97-130.

Picciani, P. H. S., E. S. Medeiros, Z. L. Pan, W. J. Orts, L. H. C. Mattoso, and B. G. Soares. 2009. Development of conducting polyaniline/poly(lactic acid) nanofibers by electrospinning. J. Appl. Polym. Sci. 112(2):744-753.

Place, E. S., N. D. Evans, and M. M. Stevens. 2009. Complexity in biomaterials for tissue engineering. Nat. Mater. 8(6):457-470.

Qaiser, A. A., M. M. Hyland, and D. A. Patterson. 2009. Control of polyaniline deposition on microporous cellulose ester membranes by in situ chemical polymerization. J. Phys. Chem. B 113(45):14986-14993.

Ramanavičius, A., A. Ramanavičienè, and A. Malinauskas. 2006. Electrochemical sensors based on conducting polymer-polypyrrole. Electrochim. Acta 51(27):6025-6037.

Ravichandran, R., S. Sundarrajan, J. R. Venugopal, S. Mukherjee, and S. Ramakrishna. 2010. Applications of conducting polymers and their issues in biomedical engineering. J. R. Soc. Interface 7:S559-S579.

Recouvreux, D. O. S., F. V. Berti, C. R. Rambo, R. M. Ribeiro-do-Valle, R. V. Antônio, P. F. Dias, and L. M. Porto. 2009. Spatial distribution of embedded human vein endothelial cell growth in multilayered BC-HAp hydrogel. In XXII Congresso de Microscopia e Microanálises, SBMM. Belo Horizonte, MG.

Richardson, M. J., J. H. Johnston, and T. Borrmann. 2006. Electronic properties of intrinsically conducting polymer-cellulose based composites. Curr. Appl. Phys. 6(3):462-465.

Scherr, E. M., A. G. MacDiarmid, S. K. Manohar, J. G. Masters, Y. Sun, X. Tang, M. A. Druy, P. J. Glatkowski, V. B. Cajipe, J. E. Fischer, K. R. Cromack, M. E. Jozefowicz, J. M. Ginder, R. P. Mccall, and A. J. Epstein. 1991. Polyaniline: oriented films and fibers. Synthetic Metals 41(1-2):735-738.

Song, H. K., and G. T R Palmore. 2006. Redox-active polypyrrole: toward polymer-based batteries. Adv. Mater. 18(13):1764-1768.

Stalling, S. S., S. O. Akintoye, and S. B. Nicoll. 2009. Development of photocrosslinked methylcellulose hydrogels for soft tissue reconstruction. Acta Biomater. 5(6):1911-1918.

Stejskal, J., I. Sapurina, M. Trchova, and E. N. Konyushenko. 2008. Oxidation of aniline: polyaniline granules, nanotubes, and oligoaniline microspheres. Macromolecules 41(10):3530-3536.

Svirskis, D., J. Travas-Sejdic, A. Rodgers, and S. Garg. 2010. Electrochemically controlled drug delivery based on intrinsically conducting polymers. J. Control. Release 146(1):6-15.

Tsukamoto, J. 1990. Structure and electrical properties of polyacetylene yielding a conductivity of 105 S/cm. Jpn. J. Appl. Phys. 29:125-130.

Wang, C. Y., G. Tsekouras, P. Wagner, S. Gambhir, C. O. Too, D. Officer, and G. G. Wallace. 2010. Functionalised polyterthiophenes as anode materials in polymer/polymer batteries. Synthetic Metals 160(1-2):76-82.

Wei, M., J. Lee, B. W. Kang, and J. Mead. 2005. Preparation of core-sheath nanofibers from conducting polymer blends. Macromol. Rapid Commun. 26(14):1127-1132. 
Wei, Y., P. I. Lelkes, A. G. MacDiarmid, E. Guterman-Tretter, S. Cheng, K. Palouian, and P. Bidez. 2004. Electroactive polymers and nanostructured materials for neural tissue engineering. In: Contemporary Topics in Advanced Polymer Science and Technology, ed. Z. Qi-Feng and S. Z. D. Cheng. Beijing: Peking University Press.

Williamson, M. R., and A. G. Coombes. 2004. Gravity spinning of polycaprolactone fibres for applications in tissue engineering. Biomaterials 25(3):459-465.

Xie, J., M. R. MacEwan, S. M. Willerth, X. Li, D. W. Moran, S. E. Sakiyama-Elbert, and Younan Xia. 2009. Conductive core-sheath nanofibers and their potential application in neural tissue engineering. Adv. Funct. Mater. 19(14):2312-2318.

Yan, Z. Y., S. Y. Chen, H. P. Wang, B. Wang, C. S. Wang, and J. M. Jiang. 2008. Cellulose synthesized by Acetobacter xylinum in the presence of multi-walled carbon nanotubes. Carbohyd. Res. 343(1):73-80.

Yin, W. S., J. Li, Y. M. Li, Y. P. Wu, T. R. Gu, and C. Y. Liu. 1997. Conducting IPN based on polyaniline and crosslinked cellulose. Polym. Int. 42(3):276-280.

Yoon, S. H., H. J. Jin, M. C. Kook, and Y. R. Pyun. 2006. Electrically conductive bacterial cellulose by incorporation of carbon nanotubes. Biomacromolecules 7(4):1280-1284.

Zeugolis, D. I., S. T. Khew, E. S. Y. Yew, A. K. Ekaputra, Y. W. Tong, L. Y. L. Yung, D. W. Hutmacher, C. Sheppard, and M. Raghunath. 2008. Electro-spinning of pure collagen nano-fibres: just an expensive way to make gelatin? Biomaterials 29(15):2293-2305.

Zhang, Z., M. Rouabhia, Z. X. Wang, C. Roberge, G. X. Shi, P. Roche, J. M. Li, and L. H. Dao. 2007. Electrically conductive biodegradable polymer composite for nerve regeneration: electricity-stimulated neurite outgrowth and axon regeneration. Artif. Organs 31(1):13-22. 



\section{Index}

A

AAm monomer concentration, 79-80

Acetan, 25

Acetobacter, 74

Acetobacter aceti, 144, 220

Acetobacter xylinum, 32, 99, 113, 197, 218.

See also Gluconacetobacter xylinus

nata de coco production and, 144-148

wound dressings and, 168

Acetylation, 105

Acs $A B, 7-8$

AcsAII, 7

Adenosine-5' -triphosphate (ATP), 27-28

vitreoscilla and, 30

Adhesion, cell, 91-97

Agitated cultivation, 46-47, 48. See also

Static cultivation

airlift reactors and, 54-56

batch fermentation under stirring using plastic composite supports, 53-54

benefits and limits of, 48-50

reactor types, 50-51

by shaking, 51-52

by stirring, 52-53

Agrobacterium tumefaciens, 14

Airlift reactors, 54-56

Amino acids in CSL, 37-38

Aminoethyl-BC (AE-BNC), 94

Antimicrobial agents, 100-101

in BNC wound dressings, 164-166

Antioxidant activities, 166-167

Applications of BNC, 20-21, 44, 73-74, 92-97, 157-158, 170-172

Arg-Gly-Asp (RGD), 38-39, 92-97, 180

Artificial blood vessel production, 31-32, 92-97

cellulose production yield and cellulose content, 35-36

tube bioreactor, 32-35
ATP. See Adenosine-5'-triphosphate

(ATP)

Autologous grafts, 20-21

\section{B}

Bacillus subtilis, 166

Bacteria. See also Gluconacetobacter xylinus proteins involved in cellulose synthesis in, 9-10

that produce cellulose, 13-15

Bacterial cellulose (BC) templates

biomimetic mineralization of apatite on, 227

morphology and structural analyses of mineralized Ca-P phase, 228-234

morphology of, 225-227

preparation, 220-227

Bacterial nanocellulose (BNC), 2, 14-15.

See also Agitated cultivation; Hydrogels, BNC; Nata de coco; Nematic ordered cellulose (NOC); Static cultivation; Wound dressings

antimicrobial agents and, 100-101, 164-166

antioxidant and protease-binding activities, 166-167

applications of, 20-21, 44, 73-74, 92-97, 157-158, 170-172

bioactivity, 179-180

biocompatibility, 177-179

biodegradation, 85-87, 97-100, 180-181

carbon metabolism in G. xylinus and, 22-23

carbon substrate and, 28

cell adhesion, 91-97

cellulose production yield and content, 35-36

characteristics, 20-21, 33-35, 218-220

conductive, 245-257 
controlled biotechnological production of, 184-186

corn steep liquor and, 28-29, 37-38

cosmetic applications of, 170-172

degree of polymerization (DP) in, 24-25

density, 34-35

effect of growth medium components on production of, 26-29, 47, 220-225

enzyme immobilization, 101-103

ethanol and, 28

flat, $44-46$

genetic modifications of G. xylinus for enhanced production of, 29-31

hydrophobic derivatives, 105-106

immobilization of cells in, 103-104

as implant material for regeneration of chondral defects, $186-188$

influence of oxygen availability on tube formation in production of, 36-37

investigations on cell adherence of, 38-39

lactate and, 27-28

mechanical properties in biomaterials, 183

medical implants from, 186-193

meniscus microweaver, $210-212$

modifications for optoelectronic devices, 104-105

network formation factors, 24-26

$\mathrm{pH}$ and production of, 25

porosity, 181-183

precipitation of metal ions, 106-107

scale-dependent properties of, 202-207

semicontinuous generation of flat, 63-65

static cultivation, $45-47$

sterilization, 183-184

suitability as a biomaterial, 177-186

variety of generation processes, 44-48, 184-186

as vascular graft material, $189-193$

vascular system and, 31-38, 189-193

as viable candidate for tissue

engineering scaffolds, 199-202 water-soluble polysaccharides and, 25-26

Bacterial nanocellulose (BNC) gels application as replacement of biomaterials, $82-88$

bNC-gelatin DN, 74-78

bNC-PAAm DN, 78-82

effect of AAm monomer concentration in, 79-80

effect of cross-linking in, 77-78, 80-81

effect of DN in, 74-77, 78, 79

effect of water content in, 78, 81-82

mechanical properties, 74-82

wear property, 83-84

Bacterium xylinum, 13

Batch fermentation under stirring using PCSs, 53-54

Bcs operon, 7, 9-10, 11

Benzalkonium, 101

Bioactive wound dressings, 160

Bioactivity, 179-180

Biocompatibility, 177-179, 244

Biodegradation, 85-87, 97-100

and BNC suitability for biomaterials, 180-181

Biodirected epitaxial nanodeposition on molecular tracks of NOC template, 128-131

critical factors in, 131-134

Biomaterials, 20-21, 175-176

application of BNC-based DN gels as replacement of, $82-88$

chondral defects and, 186-188

conductive BNC as, 254-257

investigations on cell adherence of, 38-39

medical implants from BNC, 186-193

production, 31-38

suitability of BNC as, 177-186

vascular graft, 189-193

Biomimetic mineralization of apatite, 217-218

on BC templates, 227

introduction to, 218-220

morphology and structural analyses of mineralized Ca-P phase, 228-234

preparation of $\mathrm{BC}$ templates for, 220-227 
Biomimic mineralization, 138-140

Bioreactors. See also Agitated cultivation airlift, 54-56

influence of oxygen availability on, 36-37

L spherical-type bubble-column, 56

non-static-film, 61-63

scale up and, 48

tube, 32-35

types, $50-51$

Bioresorbable BNC wound dressings, 167

Blood vessels. See Artificial blood vessel production

BNC. See Bacterial nanocellulose (BNC)

BNC-gelatin DN gel, 74-78

BNC-PAAm DN gel, 78-82

Burns, 168-169

C

Calcium phosphates, 138-140, 219 soaking of BC templates in, 227 , 228-234

Carbon dioxide, 24

Carbon metabolism in G. xylinus, 22-23

Carbon substrate, 28

Carboxymethyl-BC (CM-BNC), 94-95

Cardiovascular disease, 20-21, 189-193. See also Artificial blood vessel production

Cartilage tissue, 82-83, 95-96

C-di-GMP. See Cyclic diguanylate (c-di-GMP)

Cel+. See Mutation, spontaneous

Cel-. See Mutation, spontaneous

Cell adhesion, 91-97

Cell-directed assembly of glucan chains, 4-5

Cellobiose, 116

Cellulose, bacterial. See Bacterial nanocellulose (BNC)

Cellulose biosynthesis. See also Bacterial nanocellulose (BNC)

in bacteria catalyzed by membrane-associated cellulose synthase, 5-6

carbon metabolism in G. xylinus and, $22-23$ cell-directed assembly of glucan chains and, 4-5

characteristics of cellulose molecule and, 116-117

composition and structure, $2-3$

genes for, 1-2

"guilt by association" model of G. xylinus complex for, 11,12

proteins involved in, 9-10

unanswered questions about, 12-13

Cellulose-chitin blends, 115, 124-126

Chitin, 115, 124-126

ordered templates, 134-138

Chondral defects, regeneration of, 186-188

CMCase, 9

Conductive BNC, 245-253

as a biomaterial, 254-257

potential applications, 257-258

Conductive polymer fibrous templates, 243-245

Conduits, PDMS, 207-210

Corn steep liquor (CSL), 28-29 properties and components of, 37-38

Cosmetic applications of bacterial cellulose, $170-172$

Cross-linking, 77-78, 80-81

Crystallization, 5

CSL. See Corn steep liquor (CSL)

Cultivation of BNC. See Agitated cultivation; Static cultivation

Culture media, 26-29, 47, 220-225

Cyclic diguanylate (c-di-GMP), 6, 7, 14

carbon metabolism and, 23

diguanylate cyclase gene for synthesis of, 9

phosphodiesterase-A gene for cleavage of, 9

Cyclic guanosine monophosphate (cGMP), 39

\section{D}

Dacron, 31

Degree of polymerization (DP), 24-25

Degree of substitution (DS), 105

Density, cellulose, 34-35

Dielectrophoresis, 205-206

Diethyl aminoethyl-BNC (DEAE-BNC), 94 
Doping, 239, 240-243, 258. See also Conductive $\mathrm{BNC}$

Double-network (DN) gel, BNC-gelatin, 74-75

biodegradation after implantation into subcutaneous tissue, $85-87$

biological responses after implantation into muscle and subcutaneous tissue, $87-88$

wear property of, 83-84

Double-network (DN) gel, BNC-PAAm, 78-82

E

E. coli. See Escherichia coli

Electrical conductivity. See Conductive $\mathrm{BNC}$

Electric charge, 94-95

Electrokinetics, 203-204

Electronic paper, 104

Electrophoresis, 204-205

Enzyme immobilization, 101-103

Epitaxial deposition, 131-133

EPTFE, 31

Escherichia coli, 3, 14, 100

BNC wound dressings and, 166

vitreoscilla and, 29-30

Ethanol, 28

Extracellular matrix (ECM), 33, 179-180, 220

F

Facial masks, 170-172 nata de coco, 152-153

Fed-batch cultivation, 58-60

Flat BNC, 44-46 semicontinuous generation of, 63-65

Fourier transform infrared spectroscopy (FTIR), 94, 121

Fructose, 23

level in cultivation medium, 35

Fruit juice, 145-148

G

G. xylinus. See Gluconacetobacter xylinus

Gate with turbine impeller, 51
Gels, BNC

application as replacement of biomaterials, $82-88$

biodegradation after implantation into subcutaneous tissue, 85-87

biological responses after implantation into muscle and subcutaneous tissue, $87-88$

BNC-gelatin DN gel, 74-78

BNC-PAAm DN, 78-82

effect of AAm monomer concentration in, 79-80

effect of cross-linking in, 77-78, 80-81

effect of DN in, 74-77, 78, 79

effect of water content in, 78, 81-82

mechanical properties, 74-82

Genes for cellulose biosynthesis, 1-2

bacteria with ability and, 13-15

classification based on function of codified proteins, 6-9

Genetic modifications of G. xylinus for enhanced production of bacterial cellulose, 29-31

Glucoamylase, 102-103

Gluconacetobacter hansenii, 63

Hestrin-Schramm medium and, 98

Gluconacetobacter xylinus, 1, 2, 113, 197-198, 218

agitated cultivation and, 49-56

bcs genes in, 9-10

biomaterial applications of cellulose produced by, 20-21

BNC wound dressings and, 160, 167

carbon metabolism in, 22-23

cell envelope, 5

compared to other bacteria with genes for cellulose synthesis, 13-15

discovery of, 44,144

effect of growth medium components on bacterial cellulose production in, 26-29

electrophoresis and, 204-205

factors involved during nanocellulose network formation by, 24-26

genes for cellulose biosynthesis, 7-9 genetic modifications of, 29-31 
guanosine triphosphate (GTP) and,

$$
\text { 5-6 }
$$

"guilt by association" model of cellulose-synthesizing complex in, 11,12

modification of cellulose synthesis in, 15

moving harvesting and, 60-61

nematic ordered cellulose and, 114-115, 129-140

production of artificial blood vessels by, 31-38

proteins involved in cellulose synthesis in, 9-10

spontaneous mutation in, 30-31

structure of, 3-4

transfection with pBla-VHb-122 plasmid, 29-30

as a unique cellulose-producing bacterium, 3-4

utilization of sugar in synthesis of cellulose, 4

Glucose

in carbon metabolism, 22-23

conversion into gluconate, 22

in nata de coco production, 145

Gly-Arg-Gly-Asp-Tyr (GRGDY), 92-93, 180

Gold nanoparticles-BC nanofibers, 103

Grafts, vascular, 189-193

Growth medium components and bacterial cellulose production, 26-29

GTP. See Guanosine triphosphate (GTP)

Guanosine triphosphate (GTP), 6

$\mathbf{H}$

Harvesting, moving, 60-61

Hernia, 169

Hestrin-Schramm medium, 98, 134, 220

Hierarchical order and molecular tracks, 131

Hydrogels, BNC, 74, 175-176. See also Bacterial nanocellulose (BNC) gels

conductive, 254-257

introduction to, 176

mechanical properties, 183 porosity, 181-183

as scaffold for tissue engineering, 200-202

Hydrophobic derivatives, 105-106

I

ICPs. See Intrinsically conducting polymers (ICPs)

Immobilization of cells, 103-104

enzyme, 101-103

Implants, medical, 186-193

Indonesia and nata de coco production, 151-152

Intrinsically conducting polymers (ICPs) conductive BNC, 245-257

introduction to, 240-243

J

JeNaCell®, 65

Joint function, 82-83, 95-96

K

Ketogluconic acids, 23

L

Lactate, $27-28$

Levan, 25

Liquid crystal displays (LCDs), 104

L spherical-type bubble-column bioreactor, 56

M

Maxblend impeller, 51

MBAA. See N,N'-methylenebis acrylamide (MBAA)

Mechanical properties of BNC gels, 74-82, 183

Medical implants from BNC, 186-193

Meniscus microweaver, 210-212

Metal ions, precipitation of, 106-107

Methionine, 28-29 
Microbial synthesis of bacterial cellulose from modified culture medium, 221-225

from standard culture medium, 220-221

Microvessels, BNC scaffolds with, 212, 213

Mineralization, biomimic, 138-140

Modified NOC, 126-128

Morphology

of bacterial cellulose templates, 225-227

and structural analyses of mineralized Ca-P phase, 228-234

Moving cultivation, $60-65$

Moving harvesting, 60-61

Muscle, biological responses of DN gels after implantation into, $87-88$

Mutation, spontaneous, 30-31 agitated cultivation and, 49-50

$\mathbf{N}$

N-acetylglucoasmine (GlcNAc), 221-225

$\mathrm{N}$-acetylglucosamine (GlcNAc), 98-99

Nanocellulose. See Bacterial nanocellulose (BNC); Nematic ordered cellulose (NOC)

Nanocomposite BNC dressings, 167-168

Nanodeposition process imaging, 136-138

Nanosilver-coated bacterial cellulose, $165,199-200$

Nata de coco, 143

facial mask, 152-153

food products, 144

markets, producers, and companies, 149-152

production, $144-148$

Native BC, 220-221

Nematic ordered cellulose (NOC), 113-114 biodirected epitaxial nanodeposition on molecular tracks of, 128-131 characteristics of, 116-124 chitin templates, 134-138 critical factors in biodirected epitaxial nanodeposition on molecular tracks, 131-134

future of, 140 introduction to, 114-116

modified, 126-128

nematic ordered chitin and chitin/cellulose blends, 124-126

structural stability in water, 122-123

structure, 118-124

surface properties and applications of, 128-131

templates and modified, 124-128

templates mediating order patterned deposition accompanied by synthesis of calcium phosphates as biomimic mineralization, $138-140$

transformation into polymorphs and, 123-124

transmission electron microscopy (TEM), 118-120, 124-126

wide-angle $X$-ray diffraction (WAXD), 121-122, 124

N,N-dimethylacetamide (DMAc)/LiCL solution, 117-118

$\mathrm{N}, \mathrm{N}^{\prime}$-methylenebis acrylamide (MBAA), 80-81

Non-static-film bioreactors, 61-63

Nostoc, 14

\section{O}

1-ethyl-3-(3-dimethylaminopropyl) carbodiimide hydrochloride (EDC), 75, 77, 78

Optoeletronic devices, 104-105

ORF1, 9

ORF2, 9

Organic light emitting diode (OLED) displays, 104

Oxygen

agitated cultivation and, 50

airlift reactors and, 54-56

availability in tube bioreactors, 36-37

static cultivation and, 58

\section{$\mathbf{P}$}

Passive wound dressings, 159-160

PBla-VHb-122 plasmid, 29-30 
PCSs. See Plastic composite supports (PCSs)

$\mathrm{PH}$ and cellulose production, 25

Philippines and nata de coco production, 150-151

Phosphate-buffered saline (PBS), 100

Phosphate-NOC, 126-128, 138-140

Phosphodiesterase A and B, 23

Piezoelectric crystals, 59

Pin-on flat test, 83-84

Planar BNC material, 63-65

Plasma, 96-97

Plastic composite supports (PCSs), 53-54

Polyacetylene, 239, 242

Poly(acrylamide) (PAAm). See

BNC-PAAm DN gel

Polyaniline, 239, 243, 244

conductive BNC and, 245-253

Polydimethylsiloxane (PDMS), 205-210

Polyester, 31

Polylactide (PLLA) nanocomposites, 106

Polymerization, 5

Polypyrrole, 239, 243, 244, 246

conductive BNC as biomaterial and, 255-257

Polysaccharides

in vivo degradable, 98-99

water-soluble, 25-26

Porosity, 181-183

Precipitation of metal ions, 106-107

Production of artificial blood vessels by G. xylinus in bioreactors, $31-38$

Protease-binding activities, 166-167

Proteins

bioactivity of BNC and, 180

corn steep liquor, 37-38

involved in cellulose synthesis, 9-10

RGD, 38-39, 92-97, 180

Prototype BNC meniscus microweaver, 210-212

Pseudomonas stutzeri, 103-104

\section{$\mathbf{R}$}

Reactors. See Bioreactors, tube

Regeneration of chondral defects, 186-188 RGD. See Arg-Gly-Asp (RGD)

Rotating rollers or discs, 61-62

\section{S}

Saccharomyces cerevisiae, 104

Salmonella, 3

Scaffolds, 198-199

$\mathrm{BNC}$ as viable candidate for tissue engineering, 199-202

conductive polymer, 243-245

with microvessels, 212, 213

Scale-dependent properties of BNC, 202-207

Second harmonic generation (SHG) microscopy, 34

Semicontinuous generation of flat BNC, 63-65

Shaking, agitated cultivation by, 51-52

Skin tears, 170

Soaking of BC templates, 227

Spontaneous mutation in G. xylinus, 30-31

agitated cultivation and, 49-50

Staphylococcus aureus, 100, 244

Static cultivation, 45-47, 57-58. See also Agitated cultivation

benefits and limitations of, 58

combination with moving cultivation, $60-65$

fed-batch cultivation, 58-60

Sterilization, 183-184

Stirring, agitated cultivation by, 52-53

batch fermentation under stirring using plastic composite supports, 53-54

Subcutaneous tissue

biocompatibility of BNC and, 178

biodegradation of $\mathrm{DN}$ gels after implantation into, 85-87

biological responses of DN gels after implantation into, $87-88$

Sucrofermentans, 54

Suitability of BNC as a biomaterial, 177-186

Sulfated-BNC, 96

Surface modification, bacterial cellulose. See also Bacterial nanocellulose (BNC)

antimicrobial agents incorporation, 100-101

biodegradation, 97-100

cell adhesion, 91-97 
enzyme immobilization, 101-103

hydrophobic derivatives, 105-106

immobilization of cells, 103-104

for optoelectronic devices, 104-105

precipitation of metal ions, 106-107

by TEMPO-mediated oxidation, 221

Synthase, 5-6

$\mathrm{T}$

T. viride. See Trichoderma viride

Tears, skin, 170

TEM. See Transmission electron microscopy (TEM)

Templates, nematic ordered, 124-128

TEMPO-mediated oxidation, 220, 221

Terminal complex (TC) subunits, 5, 11, 12

Tetratricopeptide repeat (TPR) motifs, 10,11

Tissue engineering, 197-198

BNC as viable candidate for, 199-202

BNC meniscus microweaver and, 210-212

BNC scaffolds with microvessels, 212,213

introduction to, 198-199

PDMS conduits and, 207-210

scale-dependent properties of BNC and, 202-207

TPR. See Tetratricopeptide repeat (TPR) motifs

Transfection of G. xylinus with pBla-VHb-122 plasmid, 29-30

Transmission electron microscopy (TEM), 118-120

nematic ordered chitin and chitin/cellulose blends, 124-126

Trichoderma viride, 99, 103

Trimethyl ammonium betahydroxy propyl-BC (TMAHP-BNC), 94

\section{U}

UDP-glucose. See Uridine diphosphate glucose (UDP-glucose)

as substrate, 6

Ultrahigh molecular weight polyethylene (UHMWPE), 83-84
Ultraviolet graft polymerization, 107

Uridine diphosphate glucose

(UDP-glucose), 4, 13

genes for, 7

production, 23

V

Vascular grafts, 189-193

Venous leg ulcers, 170

Vitreoscilla, 29-30

W

Water content

BNC-gelatin DN gel, 78

BNC-PAAm DN gel, 81-82

Water-soluble polysaccharides, 25-26

Water-swollen fixed gel of cellulose

(WSC), 118, 126-127

Wear property of $\mathrm{DN}$ gels in pin-on flat test, $83-84$

Wide-angle $X$-ray diffraction (WAXD), 121-122, 124

Wound dressings, 157-158

with antimicrobial properties, 164-166

with antioxidant and protease-binding activities, 166-167

applications of BNC, 168-170

biocompatibility of, 162

bioresorbable BNC, 167

burns and, 168-169

categorization of, 159-160

hernias and, 169

manufacturers of BNC, 161-162

nanocomposite BNC, 167-168

pain and, 163

performance requirements of, 158-160

properties of BNC, 160-168

skin tears and, 170

venous leg ulcers and, 170

X

Xyloglucan-Gly-Arg-Gly-Asp-Ser

(XG-GRGDS), 92 